\title{
Amine promoted asymmetric cascade synthesis of highly functionalized heterocycles
}

\author{
Cheng Zhong \\ West Virginia University
}

Follow this and additional works at: https://researchrepository.wvu.edu/etd

\section{Recommended Citation}

Zhong, Cheng, "Amine promoted asymmetric cascade synthesis of highly functionalized heterocycles" (2010). Graduate Theses, Dissertations, and Problem Reports. 3098.

https://researchrepository.wvu.edu/etd/3098

This Dissertation is protected by copyright and/or related rights. It has been brought to you by the The Research Repository @ WVU with permission from the rights-holder(s). You are free to use this Dissertation in any way that is permitted by the copyright and related rights legislation that applies to your use. For other uses you must obtain permission from the rights-holder(s) directly, unless additional rights are indicated by a Creative Commons license in the record and/ or on the work itself. This Dissertation has been accepted for inclusion in WVU Graduate Theses, Dissertations, and Problem Reports collection by an authorized administrator of The Research Repository @ WVU.

For more information, please contact researchrepository@mail.wvu.edu. 


\title{
Amine Promoted Asymmetric Cascade Synthesis of Highly Functionalized Heterocycles
}

\author{
钟诚 \\ ZHONG Cheng
}

Dissertation Submitted to the Eberly College of Arts and Sciences at West Virginia University in partial fulfillment of the requirements for the degree of Doctor of Philosophy in Organic Chemistry

\author{
Xiaodong M. Shi, Ph.D., Chair \\ Peter M. Gannett, Ph.D. \\ George A. O'Doherty, Ph.D. \\ Björn C. G. Söderberg, Ph.D. \\ Kung K. Wang, Ph.D. \\ C. Eugene Bennett Department of Chemistry \\ Morgantown, West Virginia \\ 2010
}

Keyword: Lewis Base Catalysis, Dual catalysis, Isoxazoline-N-oxide, Dihydrofuran, Piperidine, Guanosine, Self-Assembly 


\title{
Abstract \\ Amine Promoted Asymmetric Cascade Synthesis of Highly Functionalized Heterocycles
}

\section{ZHONG Cheng}

\begin{abstract}
Enantioselective Intermolecular Crossed-Conjugate Additions between Nitroalkenes and $\alpha, \beta$-Enals through a Dual

Enantioselective intermolecular crossed-conjugated additions between nitroalkenes and enals were developed through Lewis base and iminium cation dual activation strategy. The optimal condition was suitable for a large group of nitroalkenes and enals with excellent yields and e.e.. Further derivatization led two diastereomers into single enantiomers of substituted pyrrolidine with excellent stereochemistry retention.
\end{abstract}

One-pot cascade Michael-Michael-Aldol condensation for Diastereoselective Synthesis of Nitro-Substituted Cyclohexanes

A one-pot Michael-Michael-Aldol cascade reaction of proline Lewis base activated nitroalkenes and enones was developed for the preparation of nitro-vinylsubstituted cyclohexanes. Good yields and diastereoselectivity were observed. Further simple transformations easily lead to poly-N-heterocycles, which have great potential for the backbone synthesis of bioactive natural products.

Concise Asymmetric Synthesis of Fully Substituted Isoxazoline-N-Oxide through Lewis Base Catalyzed Nitroalkene Activation

A concise asymmetric synthesis of isoxazoline-N-oxides is reported through secondary amine Lewis base catalyzed nitroalkenes activation and sequential intermolecular condensation with aldehydes and ylides. Through the further application of camphor derived sulfur ylide, the enantiomeric enriched isoxazoline-N-oxides were 
prepared with excellent yields (up to 95\%) and stereoselectivity (single trans isomers and up to $96 \%$ e.e.). The limitation of previous study had been successfully overcome. Further transformations of isoxazoline-N-oxide led to the gram-scale synthesis of a Clausenamide analogue, which has great potential for brain damage repair (Alzheimer's disease). Notably, this strategy had provided the all-cis $\gamma$-lactam with four stereogenic centers in 4 steps, with excellent stereochemistry control. Asymmetric synthesis of other related analogues is underway.

\section{One-Step Synthesis of Substituted Di-Hydrofurans from Lewis Base Catalyzed Three-Component Condensation}

A one-pot synthesis of substituted di-hydrofuran was developed through Lewis base catalyzed three components cascade condensation between nitroalkene, aldehyde and 1,3-dicarbonyl compounds. The di-nucleophile moieties (1,3-dicarbonyl compounds) readily reacted with the nitro diene intermediate to provide the desired cyclization products with large substrate scope ( $>20$ examples) and excellent diastereoselectivity (only trans isomers) in good to excellent yields (up to 96\%). Further application of the dihydrofuran towards useful synthetic building blocks is being studied with preliminary results.

\section{One-Pot Asymmetric Synthesis of Substituted Piperidines by Exocyclic Chirality Induction}

A highly efficient one-pot synthesis of substituted piperidines was developed through nitroalkene, amine, and enone condensation. The transformation was suitable for a large group of substrates, giving excellent yields and diastereoselectivity. Reactions using chiral amines revealed a chirality induction through the unusual exo-cyclic stereochemistry control, producing enantiomeric pure piperidines in simple steps. A sixmember ring chair transition state was proposed to account for the stereoselectivity. Reactions involving chiral amine for the similar transition state are being investigated to extend the exo-cyclic chirality transfer strategy. 


\section{Anion Bridged Nanosheet from Self-Assembled G-Quadruplexes}

A novel, non-covalent polymeric nanosheet has been produced through functionalized Guanosine self-assembly via the cooperation of cations and corresponding anions. The nanosheet material was $3.0 \mathrm{~nm}$ in height $200 \mathrm{~nm}$ in average diameter. Its composition and structure have been characterized by solution NMR, solid-state NMR, powder XRD and AFM. Functionalized Guanosine had also been synthesized for further construction of nano scale supramolecular structures. 


\section{DEDICATED TO}

my dearest love 廖文晏 (LIAO Wenyan) 


\section{ACKNOWLEDGEMENTS}

I would like to express my sincere gratitude to my advisor, Dr. Xiaodong (Michael) Shi, with whom I have been learning, growing and fighting. He, with his support, patience, enthusiasm and encouragement throughout my graduate studies, has witnessed my growth as an organic chemist.

I am deeply thankful for the valuable assistance and suggestions provided by Dr. Peter Gannett, Dr. George O’Doherty, Dr. Björn Söderberg and Dr. Kung Wang.

I want to thank my father 钟胜富 (ZHONG Shengfu) and mother 孙丽娟 (SUN Lijuan) for their endless parental love and preliminary education throughout the early years of my life. Also, I would like to extend my appreciation to all my family members in China, for their encouragements and loves. Especially, I want to thank my dearest baby daughter Sophia (钟子杉 ZHONG Zishan), who has been guiding me how to be a good father and carry on all the responsibilities that I am supposed to.

The great helps from Dr. Shi's group have always been most valuable for my graduate studies. I would like to thank Dr. Weibing Lu, Dr. Xiaohua Sun, Dr. Haifeng Duan, Dr. Yunfeng Chen, Dr. Yuxiu Liu, Dr. Dawei Wang, Sujata Sengupta, Wuming Yan, Tao Liao, Lekh Nath S. Gautam, Qiaoyi Wang and Xiaohan Ye.

Also, I would like to thank the C. Eugene Bennett department of Chemistry, especially all my colleagues in organic division for their kind helps in the last five years. I would like express my special thanks to Dr. Jeffrey Petersen for his greatest help of the X-ray crystallography studies, and also Dr. Novruz Akhmedov for his NMR studies.

Last, but essentially, I want to express my deepest gratitude to my dearest love 廖文晏 (LIAO Wenyan), without whose love I could have never reach this far. It is love that brought us together, not looking at each other but looking at the same direction of our intersected life. The bond between us will never be broken, and the chemistry will always work, with the further fruition predictable. 


\section{TABLE OF CONTENT}

Title Page

Abstract

ii

Dedication

V

Acknowledgements vi vi

Table of Contents vii

List of Schemes xii

List of Tables xiv

List of ORTEP Drawings of the Crystal Structures xvi

\section{Part I}

Enantioselective Intermolecular Crossed-Conjugate Additions between Nitroalkenes and $\alpha, \beta$-Enals through a Dual Activation Strategy

1.1 Introduction 1

1.1.1 Organocatalyzed cascade reaction in organic synthesis 1

1.1.2 Amine Lewis base catalysis in organic synthesis 3

1.1.3 Previous study on L-proline Lewis base activation of nitro alkene 3

$\begin{array}{lll}1.2 & \text { Research Objective } & 7\end{array}$

1.2.1 Lewis-base catalyzed enantioselective cross conjugate addition 7

1.2.2 Synthesis of pyrrolidine derivatives and solution the d.r. problem 7

$\begin{array}{lll}1.3 & \text { Results and Discussion } & 7\end{array}$

1.3.1 Reaction optimization of the crossed conjugate addition 7

1.3.2 Reaction substrate scope 9

1.3.3 Diastereoselective disubstituted pyrrolidine synthesis 10

1.4. Conclusion 12 


\section{Part II}

\section{One-pot cascade Michael-Michael-Aldol condensation for Diastereoselective Synthesis of Nitro-Substituted Cyclohexanes}

2.1 Introduction

2.1.1 Lewis base catalyzed cascade reaction 13

$\begin{array}{lll}2.2 & \text { Research Objective } & 13\end{array}$

2.2.1 Lewis-base catalyzed cascade synthesis of cyclohexanes 13

2.2.2 Further mechanistic investigation and N-heterocycles synthesis 15

$\begin{array}{lll}2.3 & \text { Results and Discussion } & 15\end{array}$

2.3.1 Mechanistic study of the cross conjugate addition 15

2.3.2 Reaction condition optimization 16

2.3.3 Cyclohexane synthesis through cascade condensation 18

2.3.4 Nitrogen containing heterocycles synthesis 20

$\begin{array}{lll}2.4 & \text { Conclusions } & 20\end{array}$

\section{Part III}

\section{Concise Asymmetric Synthesis of Fully Substituted Isoxazoline-} N-Oxide through Lewis Base Catalyzed Nitroalkene Activation

$\begin{array}{lll}3.1 & \text { Introduction } & 21\end{array}$

3.1.1 Literature reported synthesis of isoxazoline-N-oxide 21

3.1.2 Application of isoxazoline-N-oxide 23

3.1.3 Previous study in our group 24

3.2 Research Objectives 26

3.2.1 Asymmetric three-component synthesis of isoxazoline-N-oxide 26

3.2.2 Further application towards the synthesis of natural products 26

3.3 Results and Discussion 26

3.3.1 Diversity of C-4 position in isoxazoline-N-oxides 26

$\begin{array}{ll}3.3 .1 .1 \text { Reaction optimization } & 27\end{array}$ 
3.3.2 Differentiation of C-3 and C-5 position in isoxazoline-N-oxides 30

3.3.2.1 Characterization of diene intermediate and cross cyclization 30

3.3.2.2 Chemical difference of vinyl groups on C-3 and C-5 31

3.3.3 Three-component cascade synthesis of isoxazoline-N-oxide 32

3.3.3.1 Probing for the appropriate third component 32

3.3.3.2 Substrate scope of three-component the designed cascade reaction $\quad 34$

3.3.4 Asymmetric cascade synthesis of isoxazoline-N-oxides 35

3.3.4.1 Attempts of chiral Lewis base catalysis 35

3.3.4.2 Attempts of chiral auxiliary and condition optimization 35

3.3.4.3 Substrate scope of asymmetric three-component cascade synthesis of $\begin{array}{ll}\text { isoxazoline-N-oxides } & 38\end{array}$

3.3.5 Further application of isoxazoline-N-oxide product in total synthesis of (-) Clausenamide derivatives $\quad 39$

3.3.5.1 Asymmetric synthesis of substituted $\gamma$-lactam 39

3.3.5.2 Asymmetric synthesis Clausenamide analogue 41

3.4 Conclusion 44

\section{Part IV}

\section{One-Step Synthesis of Substituted Di-Hydrofurans from Lewis Base Catalyzed Three-Component Condensation}

4.1 Introduction 45

4.1.1 Literature reported furan and di-hydrofuran synthesis 45

4.1.2 Proposal for DHF synthesis inspired by our previous studies 47

4.2 Research Objectives 48

$\begin{array}{lll}4.3 & \text { Results and Discussion } & 48\end{array}$

4.3.1 Reaction condition screening 48

$\begin{array}{lll}\text { 4.3.2 Substrate scope } & 50\end{array}$

4.3.3 Mechanistic study of the proposed mechanism 52

4.3.4 Attempts to enantioselective synthesis and further transformation 52 
4.3.4.1 Enantioselective synthesis study 52

4.3.4.2 Further transformations 53

4.4 Conclusion $\quad 54$

\section{Part V}

\section{One-Pot Asymmetric Synthesis of Substituted Piperidines by}

\section{Exocyclic Chirality Induction}

5.1 Introduction 56

5.1.1 Piperidines syntheses in literature and their bioactivity values 56

5.1.2 Rationale of piperidine synthesis based on our previous studies 57

5.2 Research Objectives 58

5.3 Results and Discussion $\quad 58$

5.3.1 Reaction condition optimization $\quad 59$

$\begin{array}{lll}\text { 5.3.2 Substrate scope } & 60\end{array}$

5.3.3 Rationale for the high diastereoselectivity 61

5.3.4 Enantiomeric piperidine synthesis 61

5.3.4.1 Chiral amine chiral induction and the NMR evidence 61

5.3.4.2 Enhanced exo-cyclic stereochemistry control by amino esters 63

5.3.5 Extension of the exo-cyclic chirality transfer concept 64

$\begin{array}{lll}5.4 \text { Conclusion } & 66\end{array}$

\section{Part VI}

\section{Anion Bridged Nanosheet from Self-Assembled G-Quadruplexes}

$\begin{array}{lll}6.1 & \text { Introduction } & 67\end{array}$

6.1.1 Self-assembly study involving Guanosine 67

6.2 Research Objectives 69

$\begin{array}{lll}6.3 & \text { Results and Discussion } & 69\end{array}$ 
6.3.1 Preparation of complex and the composition study

6.3.2 Atomic force microscope (AFM) study, solid phase NMR study and powder X-ray characterization

6.4. Conclusion

References

\section{Part VII}

\section{Experimental Section}

Section A: General Methods and Materials $\quad 81$

Section B: General Experimental Procedures 83

$\begin{array}{ll}\text { Section C: Compound characterization } & 113\end{array}$

\section{Part VIII}

\section{${ }^{1}$ H NMR, ${ }^{13}$ C NMR, HPLC Spectra}




\section{List of Schemes}

Scheme 1. Iminium and enamine cascade catalysis 1

Scheme 2. Combination of enamine catalysis with transition-metal catalysis 2

Scheme 3. Bifunctional or dual catalysis of organocatalyst 2

Scheme 4. Amine Lewis base catalysis by organocatalysts 3

Scheme 5. Evolution of the cross conjugate addition 4

Scheme 5. Mechanistic rationale for proline catalysis as Lewis base 6

Scheme 6. Proposed secondary amine activation of nitro alkene 7

Scheme 7. Enantioselective synthesis of disubstituted pyrrolidines 11

$\begin{array}{lll}\text { Scheme 8. Unfavored Henry-Michael addition } & 13\end{array}$

Scheme 9. Proposed Lewis base catalyzed cascade synthesis of cyclohexanes 14

Scheme 10. Decomposition of $\gamma$-keto allylic nitro compounds towards base 16

Scheme 11. Synthesis of complex N-heterocycles 20

Scheme 12. Synthesis of complex isoxazoline-N-oxide via [3+2] cyclization 21

Scheme 13. Synthesis of complex isoxazoline-N-oxide via O-alkylation 22

Scheme 14. Synthesis of complex isoxazoline-N-oxide through ylide auxiliary 23

Scheme 15. Derivatization of isoxazoline-N-oxide through reduction 23

Scheme 16. Derivatization of isoxazoline-N-oxide through [3+2] cyclization 24

Scheme 17. Isoxazoline-N-oxide synthesis in previous study and mechanism 25

Scheme 18. Proposed isoxazoline-N-oxide synthesis through proline promoted Henry-aldol Condensation 26

Scheme 19. Experimental confirmation of Lewis base catalysis mechanism 28

Scheme 20. Deuterium exchange experiment 30

Scheme 21. Cross condensation with nitro-diene 31

Scheme 22. Chemoselective differentiation of vinyl groups on $\mathbf{C - 3}$ and $\mathbf{C - 5} \quad 31$

Scheme 23. Proposed three-component reaction involving a Nu-LG moiety 32

Scheme 24. Perfect chemoselectivity among the three components 33

Scheme 25. X-ray structure of related camphor-derived ylide aux-e 37

Scheme 26. Diastereoselective ring opening to reach $\gamma$-lactam 40

Scheme 27. Retrosynthetic design of Clausenamide derivatives $20 \quad 40$

Scheme 28. First attempt based on the proposed synthetic pathway 41 
Scheme 29. Another two alternative routes 42

Scheme 30. $\mathrm{Zn} / \mathrm{H}^{+}$reduction of vinyl isoxazoline-N-oxide 19a 43

Scheme 31. Synthesis of 3-ent-6-ent-Clausenamide $33 \quad 43$

Scheme 32. First examples of furan derivatives synthesis known in literature 43

Scheme 33. Oxidative coupling and Pd (II) catalysis in di-hydrofuran syntheses

Scheme 34. Formal [4+1] annulation strateggies for the synthesis of dihydrofurans 46

Scheme 35. Nitro group as leaving group in previous studies 47

Scheme 36. Reported removal of (allylic) nitro group by nucleophilic $\begin{array}{ll}\text { substitution } & 47\end{array}$

Scheme 37. Proposed di-hydrofuran synthesis 48

Scheme 38. Possible [4+1] formal annulation pathway of the synthesis of DHF 52

Scheme 39. Attempts towards asymmetric catalysis 53

Scheme 40. Further Transformation and $\operatorname{Pd}(0)$ catalyzed dihydropyran synthesis from di-hydrofurans $\quad 54$

Scheme 41. Biologically active piperidine moieties containing natural molecules 56

Scheme 42. Literature reported piperidines syntheses 57

Scheme 43. Proposed piperidine synthesis 58

Scheme 44. Account for the high diastereoselectivity: the equilibrium of azaMichael addition of amine to nitroalkene and enone 61

Scheme 45. Proposed exo-cyclic chirality induction 63

Scheme 46. Enhanced chirality transfer by amino esters and enantiomeric pure piperidine synthesis $\quad 64$

Scheme 47. Six-member ring transition-state and Claisen rearrangement 65

Scheme 48. Proposed exo-cylcic chirality transfer in aza-Claisen re$\begin{array}{ll}\text { arrangement } & 65\end{array}$

Scheme 49. Guanosine self-assembly and its nano size 67

$\begin{array}{lll}\text { Scheme 50. Proposed nano sheet through Guanosine self-assembly } & 69\end{array}$

Scheme 51. Formation of nano size Guanosine complex 69 
Scheme 52. Solution NMR study of the composition of the complex

Scheme 53. AFM images of the prepared Guanosine nano-sheet

Scheme 54. $\quad{ }^{23} \mathrm{Na}$ solid phase NMR comparison 


\section{List of Tables}

Table 1. Brief reaction optimization of cross conjugate addition 5

Table 2. Optimization of reaction conditions 8

Table 3. Substrate scope of cross conjugate addition of nitroalkenes to enals 10

Table 4. Michael-aldol condensation of allylic nitro compounds 17

Table 5. Cascade Michael-Aldol condensation of 8 and enals 18

Table 6. Cascade Michael-Michael-Aldol condensation 19

Table. 7 One-pot synthesis of homo-isoxazoline-N-oxide by Lewis base activated nitroalkene-aldehyde condensation $\quad 27$

Table 8. Substrate scope of "homo" isoxazoline-N-oxide 29

Table 9. Substrates screening for the third component (Nu-LG) 33

Table 10. Representative isoxazoline-N-oxides syntheses via the designed three-component strategy 34

Table 11. Summarized chiral Lewis base catalysis for the asymmetric synthesis

Table 12. Condition optimization of different auxiliaries asymmetric synthesis

Table 13. Substrate scope of asymmetric three-component synthesis 38

Table 14. Reaction condition optimization 49

$\begin{array}{lll}\text { Table 15. } & \text { Substrate scope } & 51\end{array}$

Table 16. Reaction condition screening 59

$\begin{array}{lll}\text { Table 17. Representative substrates } & 60\end{array}$

Table 18. Piperidine synthesis with chiral amines and NMR study $\quad 62$ 


\section{List of Figures}

Figure 1. ORTEP Drawing of the X-ray Crystal Structure 4e-cis 225

Figure 2. ORTEP Drawing of the X-ray Crystal Structure 4e-trans 225

Figure 3. ORTEP Drawing of the X-ray Crystal Structure 6a-cis 226

Figure 4. ORTEP Drawing of the X-ray Crystal Structure 6a-trans 226

Figure 5. ORTEP Drawing of the X-ray Crystal Structure 6a-trans 227

Figure 6. ORTEP Drawing of the X-ray Crystal Structure 12g-major 227

Figure 7. ORTEP Drawing of the X-ray Crystal Structure 12a-minor 228

Figure 8. ORTEP Drawing of the X-ray Crystal Structure 11a-major 228

Figure 9. ORTEP Drawing of the X-ray Crystal Structure 12f-major 229

Figure 10. ORTEP Drawing of the X-ray Crystal Structure 3b 229

Figure 11. ORTEP Drawing of the X-ray Crystal Structure 15c 230

Figure 12. ORTEP Drawing of the X-ray Crystal Structure 11a-major 230

Figure 13. ORTEP Drawing of the X-ray Crystal Structure aux-g 231

Figure 14. ORTEP Drawing of the X-ray Crystal Structure 33

Figure 15. ORTEP Drawing of the X-ray Crystal Structure 39a-major 232

Figure 16. ORTEP Drawing of the X-ray Crystal Structure 39a-minor 232

Figure 17. ORTEP Drawing of the X-ray Crystal Structure 39e 233

Figure 18. ORTEP Drawing of the X-ray Crystal Structure 41d-major 233

Figure 19. ORTEP Drawing of the X-ray Crystal Structure 41d-minor 234

Figure 20. ORTEP Drawing of the X-ray Crystal Structure 41a-minor 234 


\section{Part I}

\section{Enantioselective Intermolecular Crossed-Conjugate Additions between Nitroalkenes and $\alpha, \beta$-Enals through a Dual Activation Strategy}

\subsection{Introduction}

\subsubsection{Organocatalyzed cascade reaction in organic synthesis}

In modern organic syntheses, efficient construction of complex frameworks with multiple stereogenic centers continues to be of great importance and interests in both academic and industrial laboratories. ${ }^{1}$ As one of the most popular techniques to date, asymmetric multi-component organocatalytic cascade reaction (or domino reaction, tandem reaction) has been widely used to construct the backbones of natural products. ${ }^{2}$ The high atom economy, great stereoselectivity and its biomimic pathway make it a prevalent strategy in organic syntheses yet with challenges. ${ }^{3}$ The iminium and enamine cascade reactions first reported by MacMillan and Jørgensen are perfect examples to functionalize enals with both nucleophiles and electrophiles in one pot (Scheme 1). ${ }^{4}$

\section{Scheme 1. Iminium and enamine cascade catalysis}
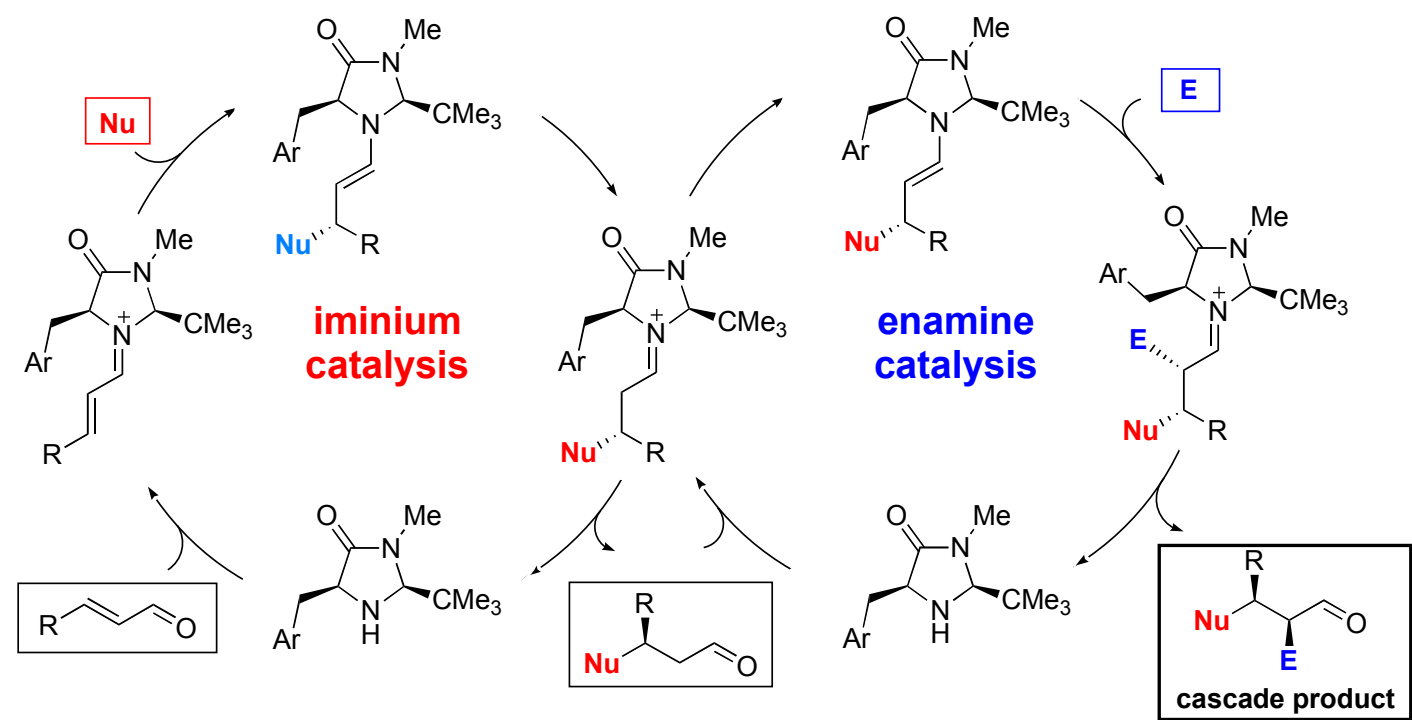
Moreover, the enamine chemistry has been successfully fused with different catalysis systems for the complex yet efficient cascade transformations. For example, the combination of enamine nucleophiles with transition-metal activated electrophiles had been well studied through the last decade (Scheme 2). ${ }^{5}$

\section{Scheme 2. Combination of enamine catalysis with transition-metal catalysis}

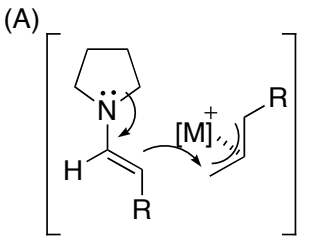

(B)
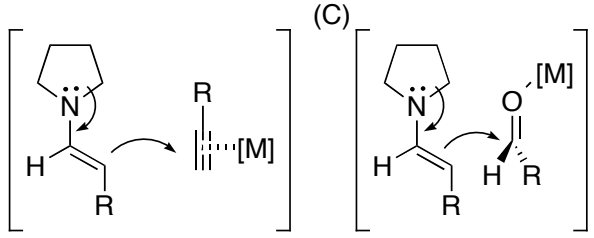

Another interesting and robust area is the bifunctional catalysis or dual catalysis of small organic molecules, which also falls into the cascade concept (Scheme 3). ${ }^{6}$ Extensive studies had been well documented. In this case, one organocatalyst molecule can activate both of the substrate (as electrophile and nucleophile) and promote the new bond formation, also referred to as dual catalysis. For example, when a secondary amine is tethered to a thiourea functionality through a chiral scaffold, an aldol-Michael reaction will occur with excellent chemical yield, diastereoselectivity and enantioselectivity. ${ }^{7}$ Proline is also widely considered as a bifunctional catalyst during the aldol reactions between aldehyde functional groups, inter- or intramolecularly. ${ }^{8}$

\section{Scheme 3. Bifunctional or dual catalysis of organocatalyst}
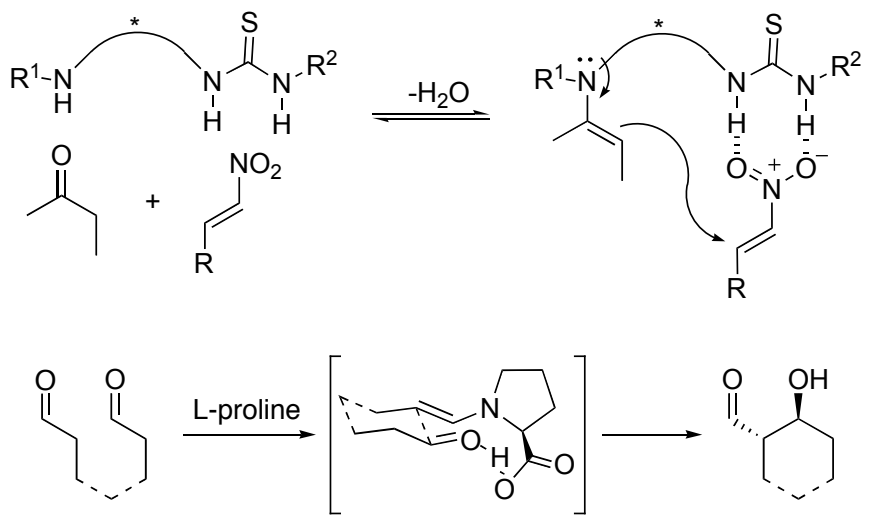


\subsubsection{Amine Lewis base catalysis in organic synthesis}

Predominantly, chiral secondary amines were used for asymmetric enamine and iminium chemistry. Although there have been a few reports about the aza-Michael addition of aliphatic amine (primary and secondary) to electron deficient alkenes, few exploration of their nucleophilicity as Lewis basic catalyst had been reported. In the nitrogen containing Lewis base catalysis category, only two types of reactions have been well developed (Scheme 4). One is that the tertiary amines $\left(\mathrm{Et}_{3} \mathrm{~N}\right.$ or DABCO) are typically used for the activation of enones/enals for Baylis-Hillman reaction; ${ }^{9}$ the other is that the aromatic amines like DMAP or pyridine are used in the esterification and Aldol condensation.

\section{Scheme 4. Amine Lewis base catalysis by organocatalysts}

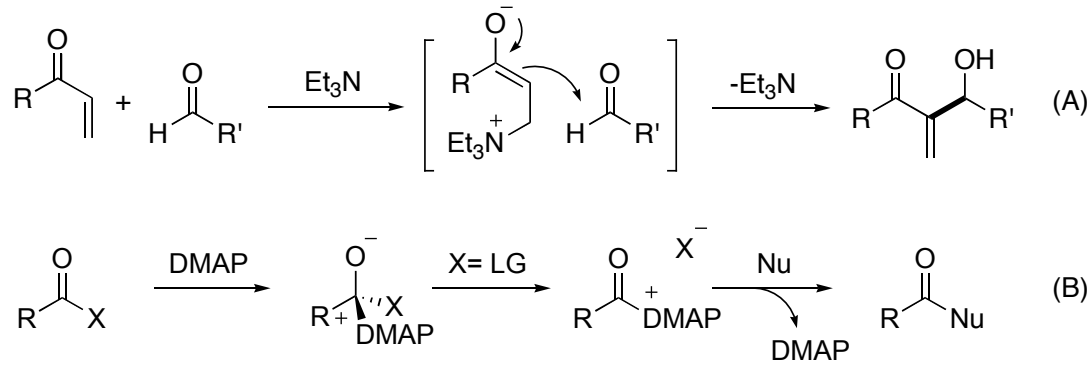

\subsubsection{Previous study on L-proline Lewis base activation of nitro alkene}

In early 2007, a new reaction mode of L-proline was discovered in our research group. The concept started from Morita-Baylis-Hillman reaction, ${ }^{10}$ in which Lewis base activated electron deficient alkene to form a carbanion in situ, serving as the nucleophile and react with the aldehyde, followed by the elimination of Lewis base to yield the allylic alcohol product (Scheme 5A). We then wondered what would happen if we have another electron deficient alkene to substitute the aldehyde as electrophile, hoping for a cascade cross-conjugate addition (Scheme 5B). A simple model study was then set up between $\beta$ nitro styrene and cyclohexanone, under the condition of proline $(0.2$ eq. $)$ and $\mathrm{NaN}_{3}(1.0$ eq.) in DMSO at room temperature. The reaction finished in 3 hours with only $32 \%$ isolated yield of the desired product (only $E$ isomer), which was quite difficult to isolate 
(Scheme 5C). The possible problem was that the cyclohexanone is a much weaker Michael receptor comparing to the $\beta$ nitro styrene, which ended up with selfpolymerization rather than the cross addition, giving this messy reaction with low yield. To circumvent this problem, a methyl group was introduced to the $\alpha$ position of the nitro alkene. Surprisingly, an allylic nitro functionality was obtained in high chemical yield, which indicated the proline Lewis base activation of the nitro alkene and the $\beta$ elimination of the Lewis base to relocate the carbon double bond (Scheme 5D). ${ }^{11}$

\section{Scheme 5. Evolution of the cross conjugate addition}
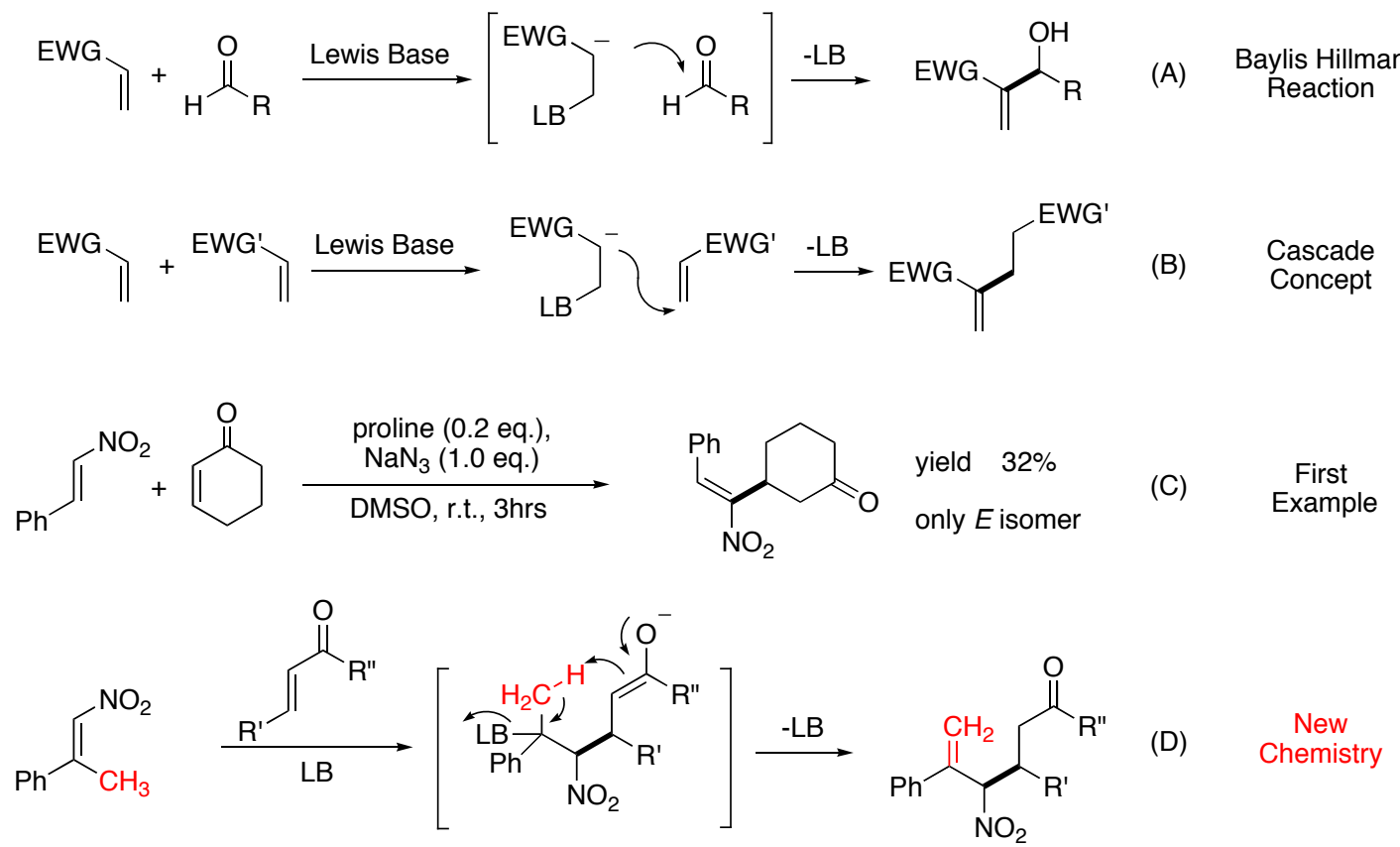

In the brief condition optimization (Table 1), the reaction went smooth with only catalytic amount of proline ( 0.2 eq.) (entry 1$)$. The rationale was that the reaction would have never happen with only the iminium activation of the enone but without the activation of nitro alkene. Thus, we proposed that proline would be the Lewis base to activate the nitro alkene. Even the combination of pyrrolidine and $\mathrm{AcOH}$ (both were 0.2 equivalent in order to mimic proline) gave similar performance (entry 4). However, $\mathrm{NaN}_{3}$ (1.0 eq.) by itself, which was supposed to serve as Lewis base, did not efficiently promote the reaction as expected (entry 2$)$. In the presence of both proline ( 0.2 eq.) and 
$\mathrm{NaN}_{3}$ (1.0 eq.), the reaction went smooth and gave $87 \%$ isolated yield within 4 hours, which suggested $\mathrm{NaN}_{3}$ might provide a buffer media for the optimal performance of proline as Lewis base to activate nitroalkene and also the iminium electrophile formation (entry 3). Classic Lewis bases, such as $\mathrm{Ph}_{3} \mathrm{P}$ and DMAP were tested, presenting moderate yields yet confirming the nitro alkene Lewis base activation mechanism (entries 6,7). Basic conditions were not suitable for the reaction (entries 8,9), even in the presence of proline (entry 5). Other solvents were also tested indicating DMSO as the best.

Table 1. Brief reaction optimization of cross conjugate addition

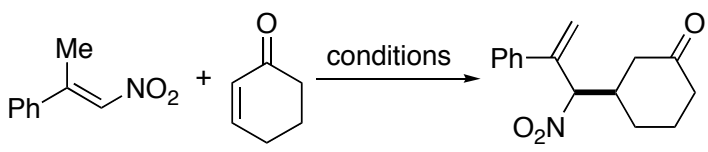

\begin{tabular}{|c|c|c|c|c|c|c|}
\hline & sol. & $\begin{array}{l}\text { Lewis Base } \\
(0.2 \text { eq. })\end{array}$ & $\begin{array}{l}\text { Additive } \\
\text { (1.0 eq.) }\end{array}$ & $\begin{array}{l}\text { time } \\
\text { (h) }\end{array}$ & $\begin{array}{c}\text { conv. }^{b} \\
(\%)\end{array}$ & $\begin{array}{c}\text { yield }^{c} \\
(\%)\end{array}$ \\
\hline 1 & DMSO & proline & -- & 4 & 93 & $80(78)$ \\
\hline 2 & DMSO & $\mathrm{NaN}_{3}(1.0)$ & -- & 10 & 61 & 8 \\
\hline 3 & DMSO & proline & $\mathrm{NaN}_{3}$ & 4 & 100 & $90(87)$ \\
\hline 4 & DMSO & pyrrolidine & $\mathrm{AcOH}(0.2)$ & 4 & 100 & $81(78)$ \\
\hline 5 & DMSO & proline & $\mathrm{Et}_{3} \mathrm{~N}$ & 4 & 89 & 41 \\
\hline 6 & DMSO & $\mathrm{Ph}_{3} \mathrm{P}$ & -- & 4 & 63 & 48 \\
\hline 7 & DMSO & DMAP & -- & 4 & 70 & 38 \\
\hline 8 & DMSO & -- & $t-\mathrm{BuONa}$ & 4 & 86 & $<5$ \\
\hline 9 & DMSO & -- & $\mathrm{Et}_{3} \mathrm{~N}$ & 4 & 74 & 25 \\
\hline 10 & $\mathrm{MeOH}$ & proline & $\mathrm{NaN}_{3}$ & 4 & 69 & 54 \\
\hline 11 & THF & proline & $\mathrm{NaN}_{3}$ & 4 & 51 & 39 \\
\hline 12 & $\mathrm{MeNO}_{2}$ & proline & $\mathrm{NaN}_{3}$ & 4 & 57 & 48 \\
\hline
\end{tabular}

During the investigation, we found that in the presence of proline, nitro alkene a and its isomer $\mathbf{b}$ were in equilibrium under the reaction condition. Another concern was then raised, was it really the Lewis base activation of the nitroalkene a, or just simple deprotonation of allylic nitro compound $\mathbf{b}$ to conduct the nucleophilic addition to the 
enone? For clarification, the comparison of molecule a exposed to proline and molecule $\mathbf{b}$ under basic condition was conducted, suggesting the validity of Lewis base activation pathway. On the other hand, the deprotonation pathway apparently disagreed with the pKa theory, since the allylic nitro carbanion was far more acidic than the carbonyl carbanion (pKa 11 v.s. pKa 20), which would drive the reaction go backwards to the starting materials, which was then later confirmed in later part of this document. Furthermore, computational studies also verified the viability of Lewis base activation nitro alkenes by proline (Scheme 5).

\section{Scheme 5. Mechanistic rationale for proline catalysis as Lewis base}

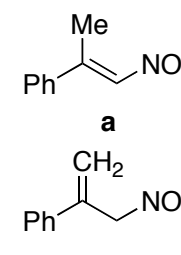

b

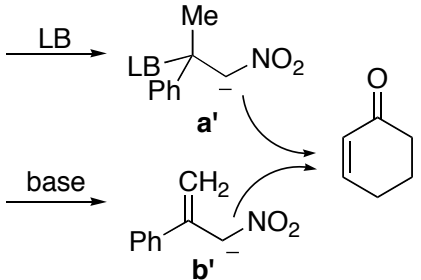

$\mathbf{b}^{\prime}$

\begin{tabular}{ccc}
\hline reactant & time $(\mathrm{h})$ & yield $(\%)$ \\
\hline $\mathbf{a}$ & 4 & 90 \\
$\mathbf{b}$ & 4 & 17 \\
$\mathbf{b}$ & 14 & 51 \\
\hline
\end{tabular}

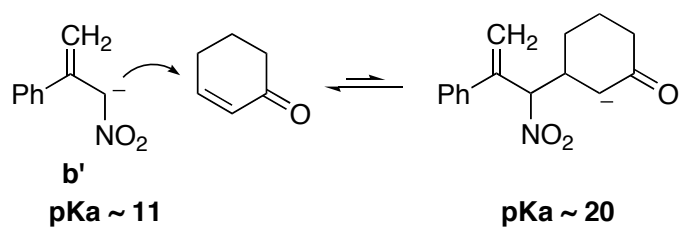

Through almost three years of extensive study, several reactions between proline (or secondary amine) activated $\beta$ alkyl nitro alkene and different electrophiles have been well developed. A more logical mechanism of secondary amine Lewis base activation of nitro alkenes was then proposed based on our studies (Scheme 6). Generally, after the nucleophilic addition of secondary amine to nitro alkene, a zwetterion species $\mathbf{A}$ is created, which is actually the resonance structure of the neutral nitronate $\mathbf{B}$. Due to the stabilization of the secondary amine, nitronate $\mathbf{B}$ serves as a more general and milder nucleophile. In the presence of electrophile, a new covalent bond is formed, which is then followed by the $\beta$ elimination to release the secondary amine and yield the allylic nitro product. 


\section{Scheme 6. Proposed secondary amine activation of nitro alkene}
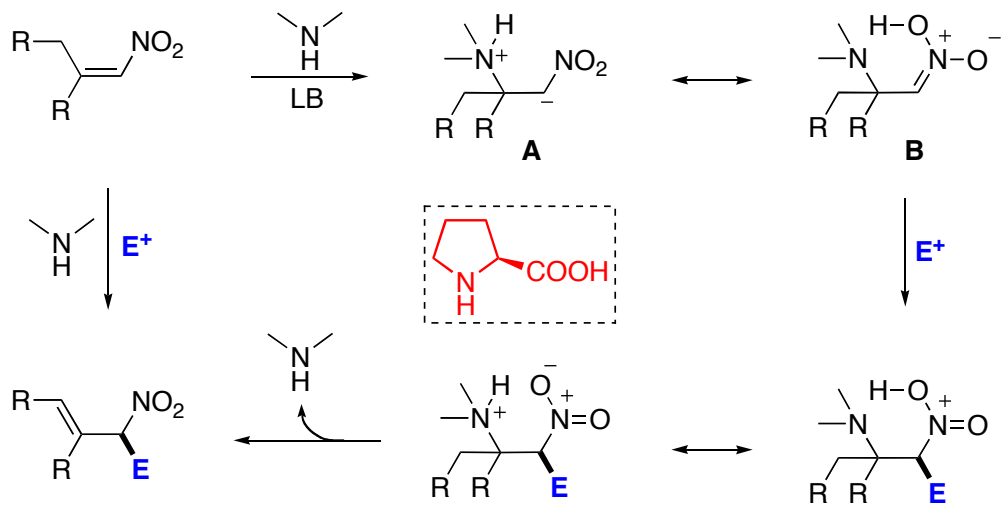

\subsection{Research Objective}

\subsubsection{Lewis-base catalyzed enantioselective cross conjugate addition}

Based on our previous study, chiral cyclic secondary amines were then tested for the asymmetric cross conjugate addition between $\beta$ alkyl nitro alkenes and enals. As proposed, the chiral secondary amine should activate both enals as iminium electrophiles and also the nitro alkene as stabilized nitronates (or nitro carbanions) to serve as mild nucleophiles. Good yields and enantioselectivity were expected.

\subsubsection{Synthesis of pyrrolidine derivatives and solution the $d . r$. problem}

The diastereoselective ratio might be low due to the low $\mathrm{pKa}$ of the allylic nitro functionality. To reach to a high diastereoselective radio, further transformations were desired to solve the d.r. problem.

\subsection{Results and Discussion}

\subsubsection{Reaction optimization of the cross conjugate addition}

The reaction condition screening was summarized in Table 2. Conducting the reaction in DMSO gave the desired product 3a in modest yield (polymerization of starting material) with poor stereoselectivity (entry 1). Switching the solvent to $\mathrm{MeOH}$ when only L-proline (0.2 eq.) was used as catalyst, $78 \%$ yield and $11 \%$ e.e. of 3a was 
observed (entry 2). This strongly supported the proposed dual activation mechanism, that is iminium cation carbonyl activation and nitroalkene Lewis base activation.

Table 2. Optimization of reaction conditions ${ }^{a}$

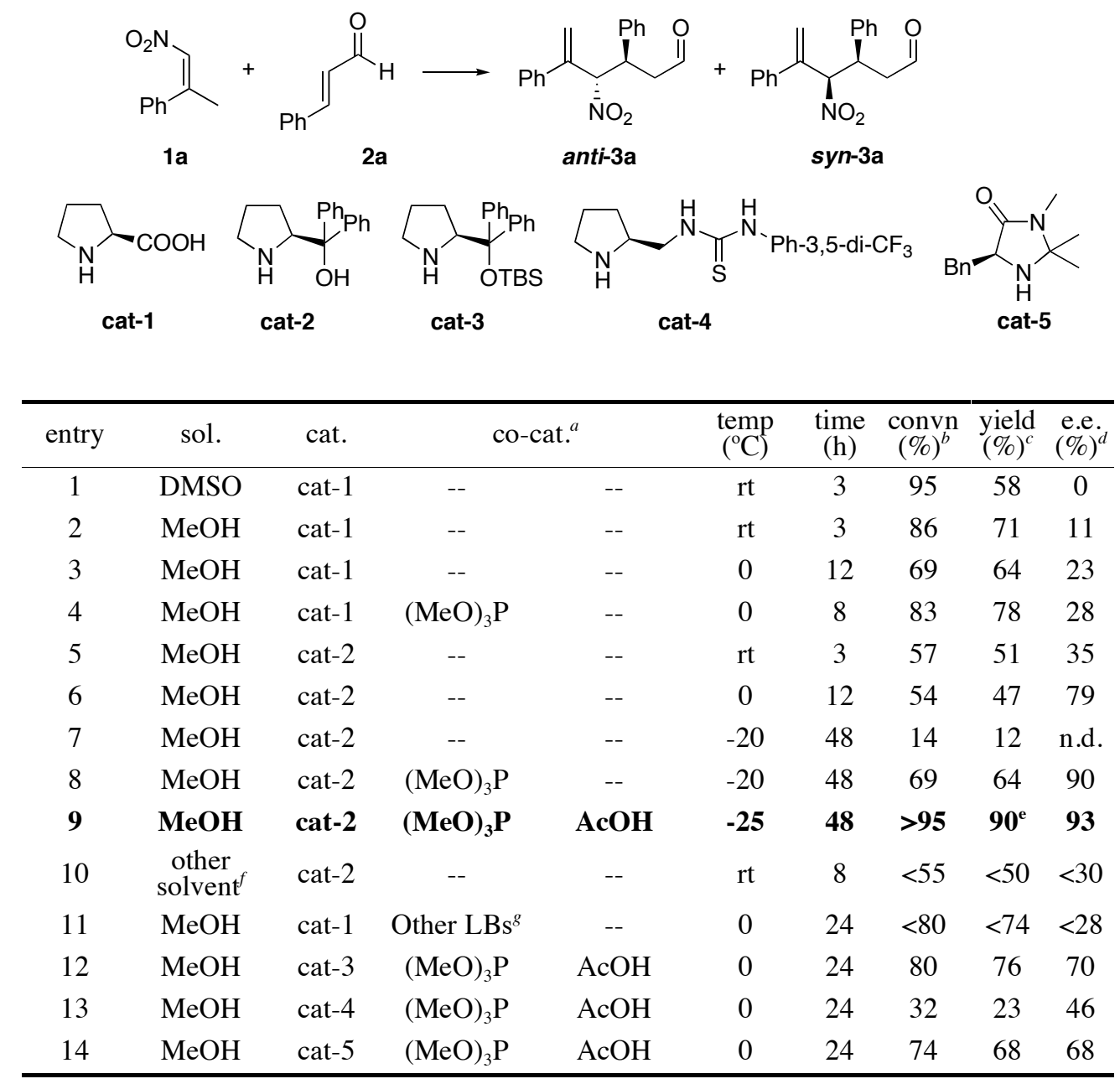

${ }^{a} \mathbf{1 a : 2 a}=1: 2$, concentration of $\mathbf{1}$ is $0.2 \mathrm{M}$, catalyst was $20 \%, \mathrm{LB}$ and $\mathrm{AcOH}$ co-catalysts were 1 equiv.; ${ }^{b}$ based on the consumption of 1 by NMR; ${ }^{c}$ NMR yield with $1,3,5-$ trimethoxybenzene as internal standard; ${ }^{d}$ the two diastereomers were separated and e.e. were determined by chiral HPLC (anti isomer only). The poor $d . r$. selectivity (less than 2:1) is all cases favoring the anti isomer; ${ }^{e}$ isolated yields of both isomers; ${ }^{f}$ includes DCM, EtOAc, Acetone, toluene, MeCN, THF. ${ }^{g}$ includes $\mathrm{Ph}_{3} \mathrm{P}$, DMAP, imidazole, NMI.

When lower temperature $\left(0{ }^{\circ} \mathrm{C}\right)$ was applied, better enantioselectivity was received though longer reaction time was required to reach a reasonable yield (entry 3 , $63 \%$ yield, $23 \%$ e.e. $)$. With the additional Lewis base $\left(\mathrm{MeO}_{3} \mathrm{P}, 1.0\right.$ eq.) added in the reaction system, better yield (78\%) was observed with slightly improved e.e. $(28 \%)$, 
indicating the release of proline for the proposed Lewis base nitroalkene activation, which then helped the better formation of chiral iminium electrophile (entry $4,78 \%, 28 \%$ e.e.). Application of cat-2 at lower temperature greatly improved the enantioselectivity up to $79 \%$ e.e. (entries 3 and 6). But further lowering reaction temperature caused significant decrease of reaction rate (entry $7,-20^{\circ} \mathrm{C}, 12 \%$ yield in 48 hours), which might be caused by the slow release of amine catalyst from nitroalkene addition (resulting in the lack of iminium activation) at low temperature. To help the release of the amine, various Lewis bases were tested as the co-catalysts, including $\mathrm{Ph}_{3} \mathrm{P}$, DMAP, imidazole, $\mathrm{P}(\mathrm{OMe})_{3}$ and NMI, $\mathrm{P}(\mathrm{MeO})_{3}$ was identified as the best at $-25^{\circ} \mathrm{C}$. As expected, both reaction rate and enantioselectivity increased (entry 8, 64\% yield, 90\% e.e.). Further addition of AcOH promoted the iminium cation formation, gave 3a in excellent yield and slightly improved enantioselectivity (entry 9, 90\% yield, 93\% e.e.). Different solvents were also tested, including DCM, EtOAc, Acetone, toluene, MeCN, THF, all of which did not provide comparable performance of the reaction than in $\mathrm{MeOH}$. Other typical chiral secondary catalysts were also tested under the same condition, but no better performance was observed.

\subsubsection{Reaction substrate scope}

With the optimized condition, this transformation is subjected to a wide range of substrates, with good yield and excellent enantioselectivity. Various $\beta$-substituted enals, including aryl, alkyl and heterocycles, were all suitable for this reaction. The aldehyde products 4 were all reduced into alcohol, which showed were more stable and easier for both isolation and the examination of enantioselectivity. Only two product aldehydes (4n and 4o) were not reduced due to their instability under reductive conditions.

Moreover, both alkyl/aryl and di-alkyl substituted nitroalkenes could be promoted in this transformation. Mono-alkyl substituted nitroalkene, although possible for the $\beta$ elimination, gave low yield due to significant polymerization. The substrate scope is summarized in Table 3. 
Table 3. Substrate scope of cross conjugate addition of nitro alkenes to enals ${ }^{a}$
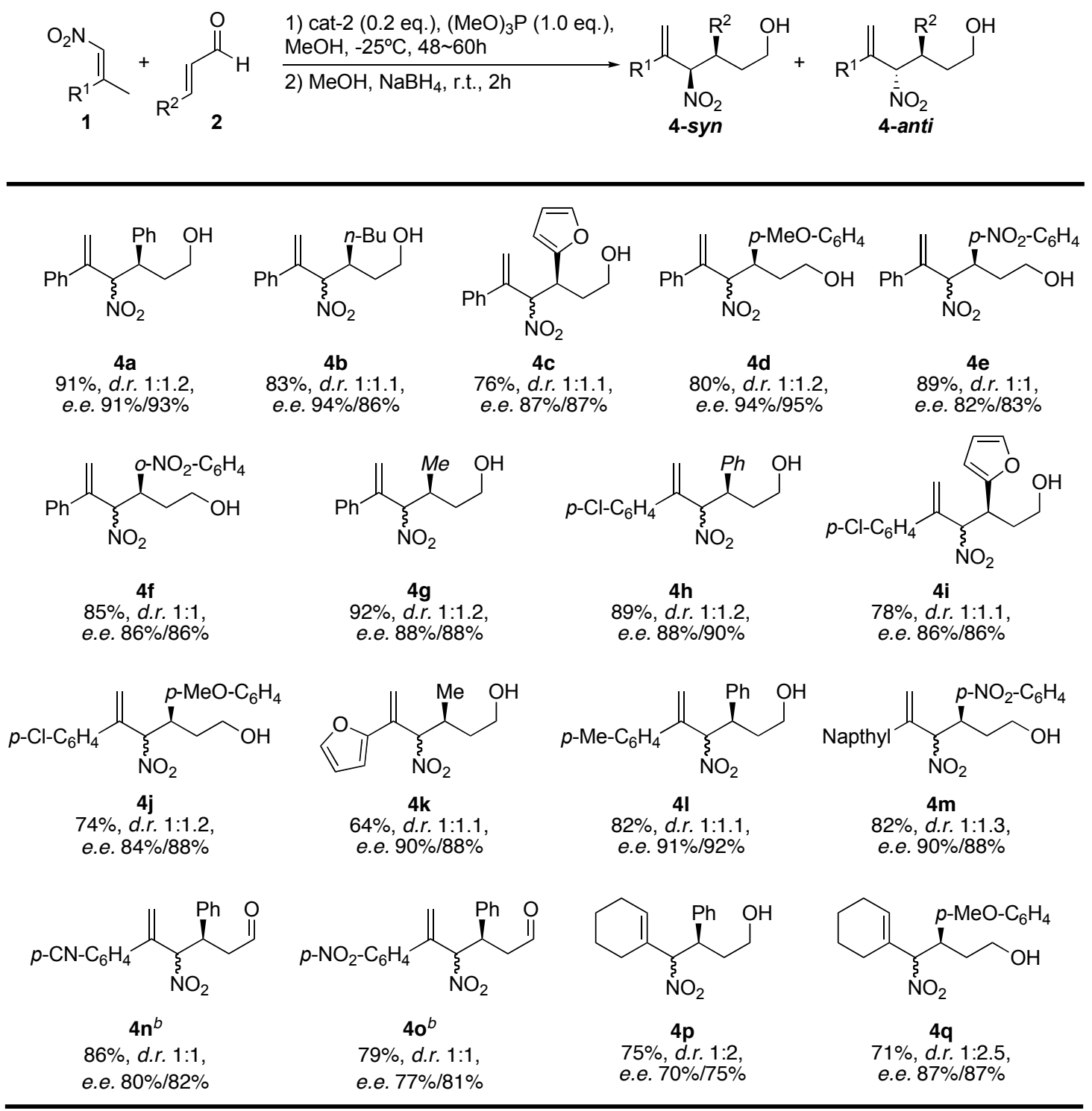

${ }^{a} \mathbf{1}: \mathbf{2}=1: 3$, concentration of $\mathbf{1}$ is $0.2 \mathrm{M}$; isolated yield of both isomers; $d . r$. values were determined by crude ${ }^{1} \mathrm{H}-\mathrm{NMR}$; e.e. were determined by chiral HPLC; ${ }^{b}$ yields of aldehydes, no further reduction.

\subsubsection{Diastereoselective disubstituted pyrrolidine synthesis}

Although the reported reaction had reached high yield and enantioselectivity, the diastereo ratio was always low due to the acidic proton (pKa 11) at the allylic nitro position. Further transformation was then required to convert both diastereomers into one to reach high atom efficiency. It had been known in our previous study that the nitro group $\left(\mathrm{NO}_{2}\right)$ could be reduced into amine $\left(\mathrm{NH}_{2}\right)$ via Zinc reduction under acidic 
condition. Since intramolecular imine formation was predictable, a reductive cyclization would yield a disubstituted pyrrolidine derivative, which might improve the $d . r$.

\section{Scheme 7. Enantioselective synthesis of disubstituted pyrrolidines}

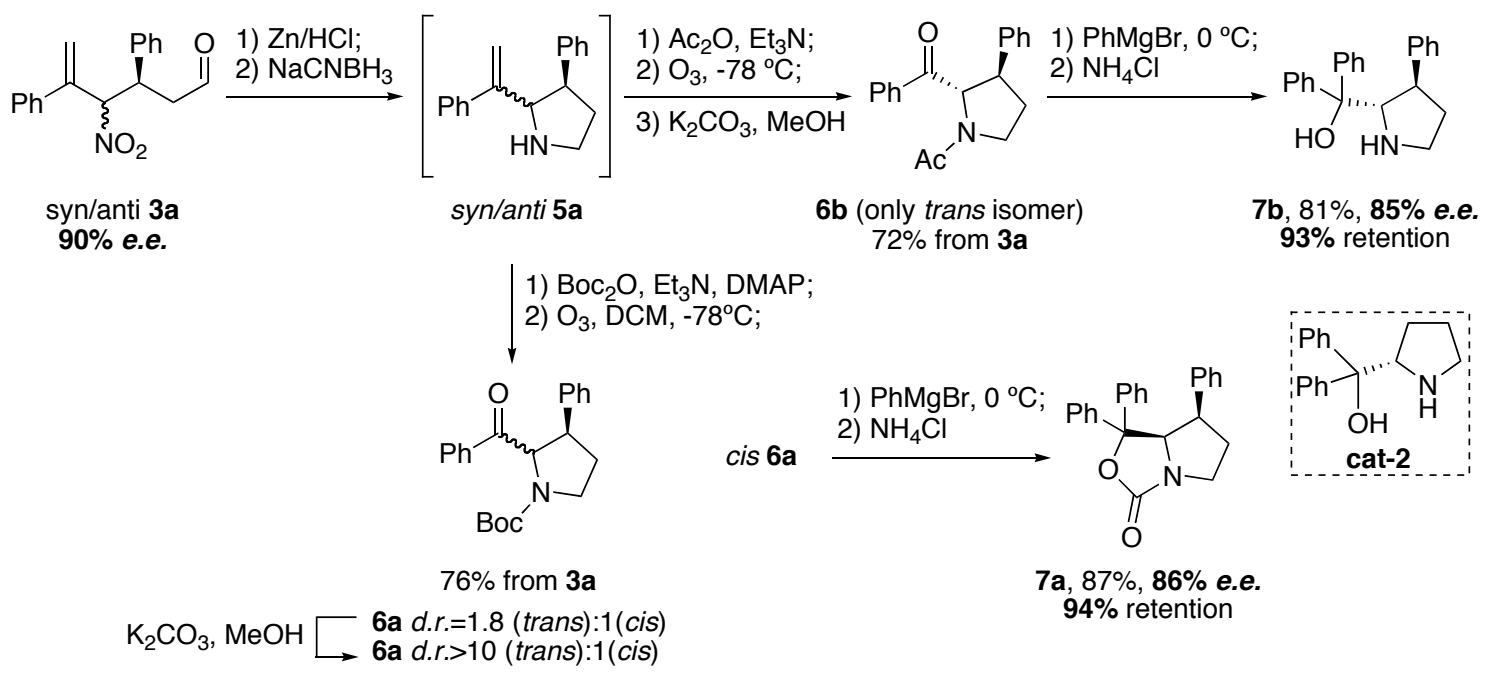

As shown in Scheme 7, concise syntheses of disubstituted pyrrolidine derivatives had been developed towards nitrogen containing heterocycles. Reductive cyclization of syn/anti 3a gave pyrrolidines 5a, which were then further converted into carbamate or amide protected $\mathbf{6 a}$ and $\mathbf{6 b}$ through amine protection and ozonolysis. Mixtures of diastereomers were obtained for both $\mathbf{6 a}$ and $\mathbf{6 b}$ with d.r. $<2: 1$. However, upon treatment of $\mathrm{K}_{2} \mathrm{CO}_{3}$ in $\mathrm{MeOH}$, the cis isomer was successfully converted into trans isomers, with d.r. $>10: 1$ in $6 \mathbf{a}$ and only trans isomer observed in $\mathbf{6 b}$. The trans $\mathbf{6 b}$ was then converted into $\mathbf{7 b}$ with excellent yield and stereochemistry retention. Interestingly, treating the cis carbamate 6a with $\mathrm{PhMgBr}$ gave the cis-cyclo-carbamate, which significantly extended the application of this transformation: with the different protecting groups, both cis and trans substituted pyrrolidine can be achieved with excellent stereochemistry retention. More interestingly, pyrrolidine $\mathbf{7 b}$ is fairly similar to the catalyst cat-2 with one more trans substitute group $(\mathrm{Ph})$ at the $\mathrm{C}-3$ position. Further application of $\mathbf{7 b}$ as organocatalyst is of great interest in our research group. 


\subsection{Conclusion}

A novel Lewis base catalysis mode of proline or secondary amines had been discovered and studied. The enantioselective crossed-conjugate addition of nitroalkene and enals was successfully developed. The wide substrate scope, excellent yields and enantioselectivity, and unique activation approach provided great potential of this new $\mathrm{C}$ C bond formation strategy. Simple derivatization had converted the diastereo mixtures into single enantiomers. Both cis and trans disubstituted pyrrolidine derivatives had been synthesized in great yields and stereochemistry retention.

The allylic nitro functionality produced through our proline Lewis base catalysis has great potentials for further transformation, targeting interesting and useful building blocks. The nitrogen containing heterocycles can be easily synthesized which may possess great attractive chemical and biological activities. Also, studies of $\mathbf{7 b}$ as organocatalyst are currently underway in our group.

This project was a collaborating work with Dr. Yunfeng Chen, Dr. Jeffrey Petersen and Dr. Novruz Akhmedov. Dr. Chen helped with the part of the derivatization of two diastereomers and the NMR spectra organization. Dr. Petersen helped with the Xray crystallography study to determine the relative stereochemistry and structures of target compounds. Dr. Akhmedov carried out the extensive NMR studies of the derivatization products $7 \mathbf{a}$ and $\mathbf{7 b}$. 


\section{Part II}

\section{One-pot cascade Michael-Michael-Aldol condensation for Diastereoselective Synthesis of Nitro-Substituted Cyclohexanes}

\subsection{Introduction}

\subsubsection{Lewis base catalyzed cascade reaction}

Compared to the Lewis acid, radical and transition metal promoted cascade reactions, the Lewis base mediated cascade transformations were much less documented in literature. However, with the unique reaction mechanism, successful Lewis base mediated cascade processes could provide feasible approaches to complex molecules construction that are difficult using other methods. ${ }^{12}$ Our interest in developing Lewis base mediated cascade reactions was initiated by the investigation of the Henry-Aldol condensation. With the presence of more acidic proton, the addition of nitro compounds to enone is thermodynamically unfavored (formation of stronger base), if no other reagents applied (Scheme 8). Thus, few successful Henry-Michael additions have been reported. However, novel 1, 4-nitro, ketone di-functionalities building blocks would be constructed that can be further converted to synthetic useful complex molecules. ${ }^{13}$

\section{Scheme 8. Unfavored Henry-Michael addition}

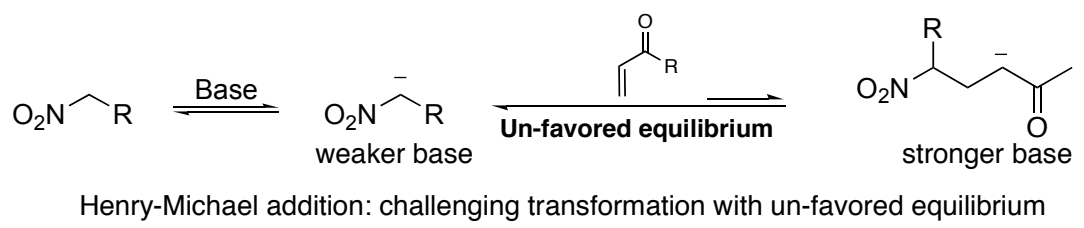

\subsection{Research Objective}

\subsubsection{Lewis-base catalyzed cascade synthesis of cyclohexanes}

Based on our previous study of the proline promoted cross conjugate addition of nitroalkene to enones and enals, we postulated a Michael-Michael-Aldol cascade 
condensation of nitroalkenes and enones for the synthesis of functionality enriched cyclohexanes.

Nitroalkene is one important synthetic building block for producing diverse functional groups through easy transformations. ${ }^{14}$ In literature, nitroalkenes were commonly used as electrophiles or electron-deficient dienophiles. ${ }^{15}$ The Lewis base activation of nitroalkene provided a new interesting strategy, whereby the resulting nitro carbanion A could serves as nucleophile (Scheme 9). The challenge is the homocondensation of nitroalkenes, leading to the polymerization. ${ }^{16}$ Our recently reported alternative $\beta$-elimination approach had successfully solved the problem, producing the allylic nitro compound $\mathbf{C}$. The key for the success of this new strategy is the introduction of one irreversible step in the overall equilibrium system.

\section{Scheme 9. Proposed Lewis base catalyzed cascade synthesis of cyclohexanes}

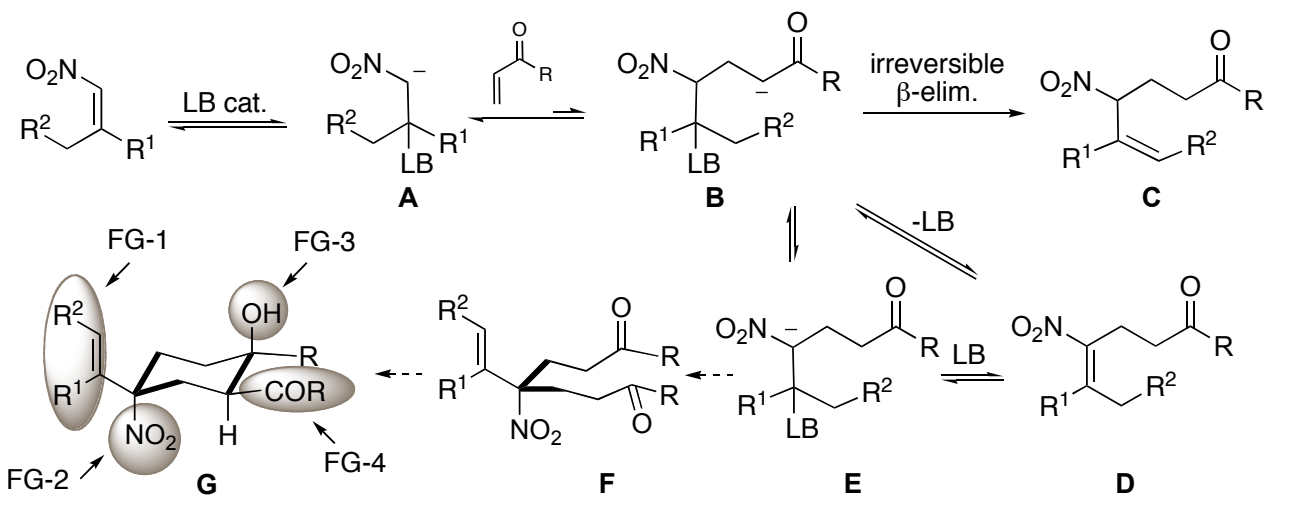

Encouraged by this result, we wondered whether this new cascade process could be further extended to a second Michael addition and sequential intramolecular aldol reaction, leading to nitro-substituted cyclohexane $\mathbf{G}$ in one-pot as shown in Scheme $\mathbf{9}$. We envisioned this proposed cascade condensation would result in nitro-substituted cyclohexanes that can be readily converted into functional group enriched N-heterocycles through simple steps. Moreover, the mechanism revealed would help us understanding the key factors that control this complex multi-component. 
As indicated in the reaction path, the key for success Michael-Michael-Aldol condensation that converts simple nitroalkene into cyclohexane $\mathbf{G}$ is to switch the irreversible $\beta$-elimination step (B to $\mathbf{C}$ ) into equilibrium and expect the intramolecular aldol reaction (formation of $\mathbf{G}$ ) as the new irreversible step under proper reaction conditions. Since the $\alpha$-proton of the allylic nitro compound $\mathbf{C}$ is acidic, deprotonation will be one simple approach in converting the B-C transformation as equilibrium. Experiments were then designed to test this hypothesis.

\subsubsection{Further mechanistic investigation and N-heterocycles synthesis}

We envisioned this proposed cascade condensation would result in nitrosubstituted cyclohexanes that can be readily converted into highly functionalized Nheterocycles through simple steps. Moreover, the mechanism revealed through this process would certainly help us in understanding the key factors that control this complex multi-component condensation and lead to the discovery of other new cascade reactions.

\subsection{Results and Discussion}

\subsubsection{Mechanistic study of the cross conjugate addition}

The reactions between allylic nitro compounds $\mathbf{8}$ and strong base $t \mathrm{BuOK}$ were then investigated. As shown in Scheme 10, treatment of nitroalkene with enone or $\alpha, \beta$ unsaturated ester gave the allylic nitroalkene $\mathbf{8}$ in excellent yields in the presence of Lproline as Lewis base. No cyclohexane products were observed due to the irreversible $\beta$ elimination. Reactions of $\mathbf{8}$ with $t \mathrm{BuOK}$ caused the decomposition of $\mathbf{8}$, returning both of the starting materials. However, no cyclization product was observed either and polymerization of nitroalkenes was detected when extending the reaction time. Since the base indeed led to the deprotonation of $\mathbf{8}$, converting the irreversible elimination to equilibrium, the fact that no cyclohexane was formed under this reaction may caused by either the equilibrium in the intramolecular aldol step (Scheme 9, $\mathbf{F}$ to $\mathbf{G}$ ) or the unfavored equilibrium between $\mathbf{A}$ and $\mathbf{B}$. 


\section{Scheme 10. Decomposition of $\gamma$-keto allylic nitro compounds towards base}
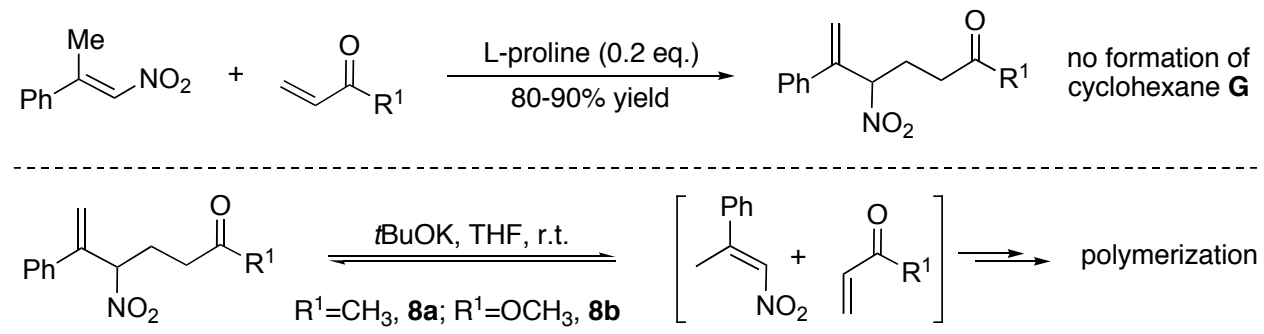

8

\subsubsection{Reaction condition optimization}

To evaluate these crucial factors that influence this transformation, reactions between 8 and carbonyl activated alkenes 9 were investigated. As indicated in Table 4, reactions between $\mathbf{8}$ and $\mathbf{9}$ would presumably form three different condensation products: the simple Michael addition product 10, the kinetic aldol condensation product 11 and the thermodynamic aldol condensation product 11' ${ }^{17}$ The Henry-Michael addition did not occur between 8 and 9 in the absence of base, even for acroline 9a with the presence of proline catalyst in DMSO (entries 1-2). Enal polymerization occurred when 9a was treated with base in DMSO (entry 3$)$. With the addition of the strong base $(t \mathrm{BuOK})$, the allylic nitro compound $\mathbf{8}$ tended to decompose (entry 5) through the reaction path shown in Scheme 10. Application of mild base with $\mathbf{8 b}$ and ester 9c, the Henry-Michael addition product 10a was observed with low yield (entry 6). However, the desired intramolecular aldol condensation did not occur, which was likely due to the poor reactivity of the ester group under the reaction condition. This result suggested that the Henry-aldol addition is possible if proper basic condition was applied (deprotonation of the more acidic nitro carbon hydrogen other than the carbonyl $\alpha$-proton).

Encouraged by this result, we then investigated the reaction of ketone 8a with different Michael receptors 9 in the presence of various mild bases. To our please, reactions between $8 \mathbf{a}$ and $\mathbf{9 a} / \mathbf{9 b}$ gave the desired Michael-Aldol product 11a/11b in the presence of $\mathrm{Et}_{3} \mathrm{~N}$ (entries 7 and 8), though with low reaction rates. The reaction rate for enal 9a were later improved with the addition of catalytic amount of proline (entry 9, carbonyl activation). Although $\mathrm{NaN}_{3}$ and $\mathrm{K}_{2} \mathrm{CO}_{3}$ could also promote this reaction, $\mathrm{Et}_{3} \mathrm{~N}$ gave the best results, where nitro cyclohexane 11a was produced in excellent yield. 
Table 4. Michael-aldol condensation of allylic nitro compounds ${ }^{a}$

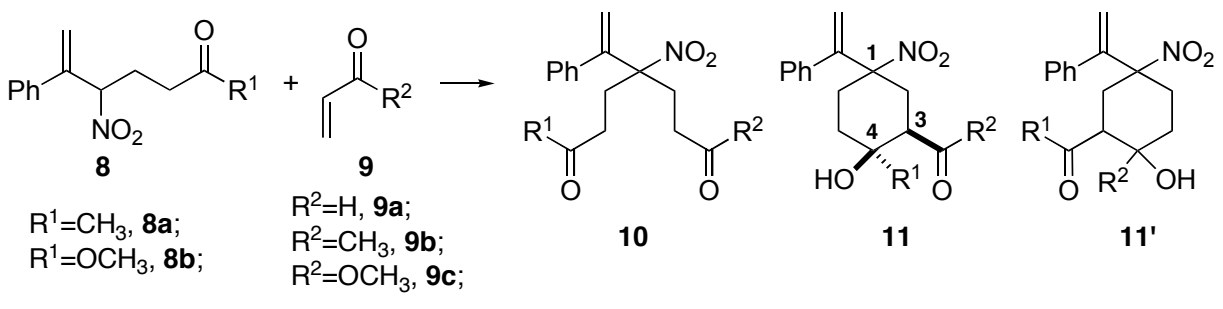

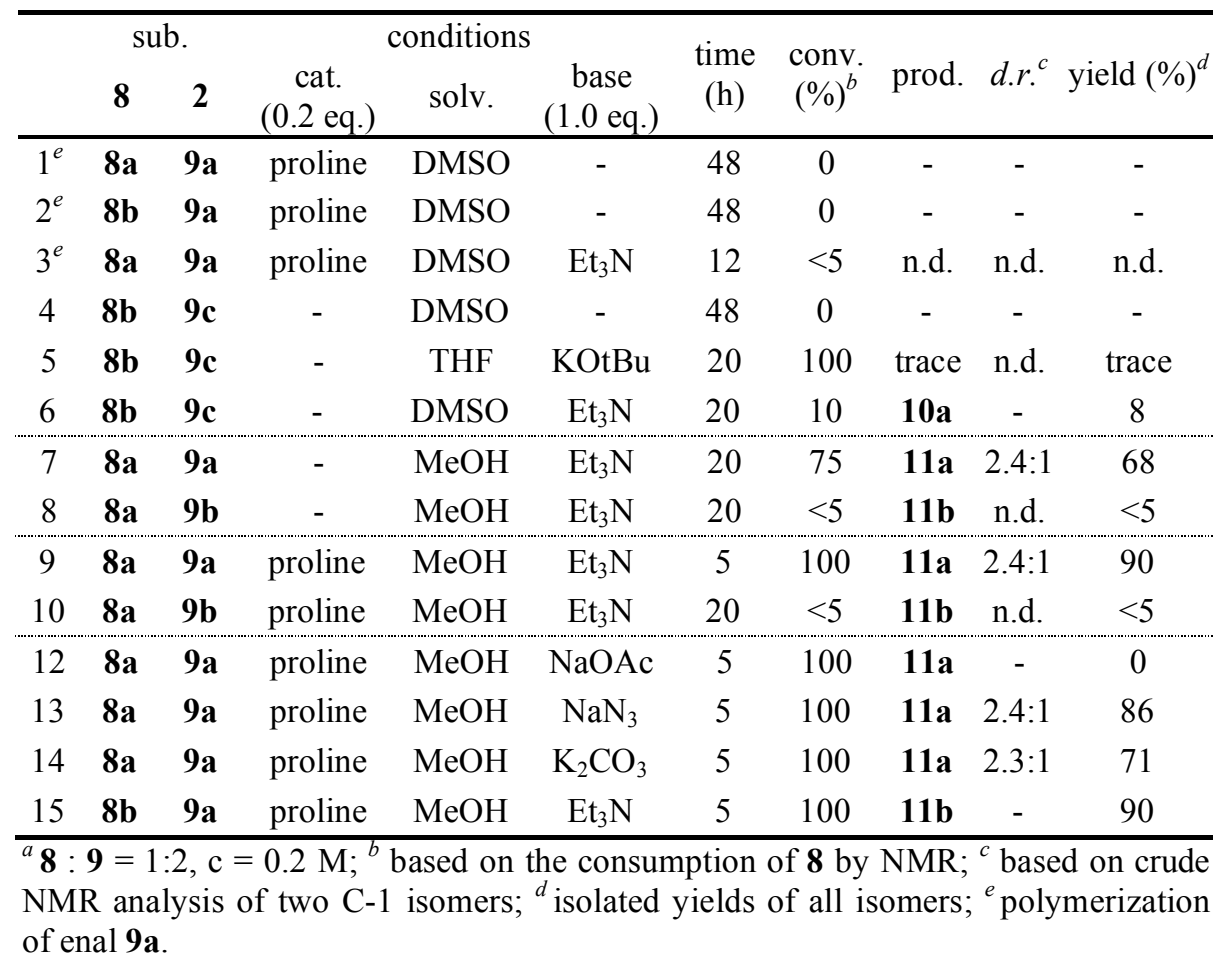

Notably, the thermodynamic product 11' (ketone $\alpha$-carbon addition to aldehyde) was not observed in all cases (even without proline activation, entry 7 ). ${ }^{18}$ This was also confirmed by the reaction between ester $\mathbf{8 b}$ and enal 9a, where Henry-Michael addition product 10b was received in excellent yield and no further cyclization product formed under the reaction condition (entry 15). Moreover, the Aldol product 11 was stable under even strong basic condition: treatment of 11 a with $t \mathrm{BuOK}$ at $60{ }^{\circ} \mathrm{C}$, no decomposition occurred for 24 hours. All these results strongly suggested that the intramolecular aldol condensation was a rapid irreversible step in this cascade process, which drove the equilibrium into the desired cyclohexane product. 


\subsubsection{Cyclohexane synthesis through cascade condensation}

Meanwhile, although three stereogenic centers were generated in product 11, only two C-1 diastereomers were obtained. This is likely caused by the insignificant size difference between the nitro and the vinyl groups on the $\mathrm{C}-1$ position in the six-member ring chair-like transition state. Various enals were applied to the reactions with allylic nitro compound $\mathbf{8}$ and the desired nitro-substituted cyclohexanes $\mathbf{1 1}$ were obtained with good yields and diastereoselectivity (Table 5).

Table 5. Cascade Michael-Aldol condensation of 8 and enals ${ }^{a}$
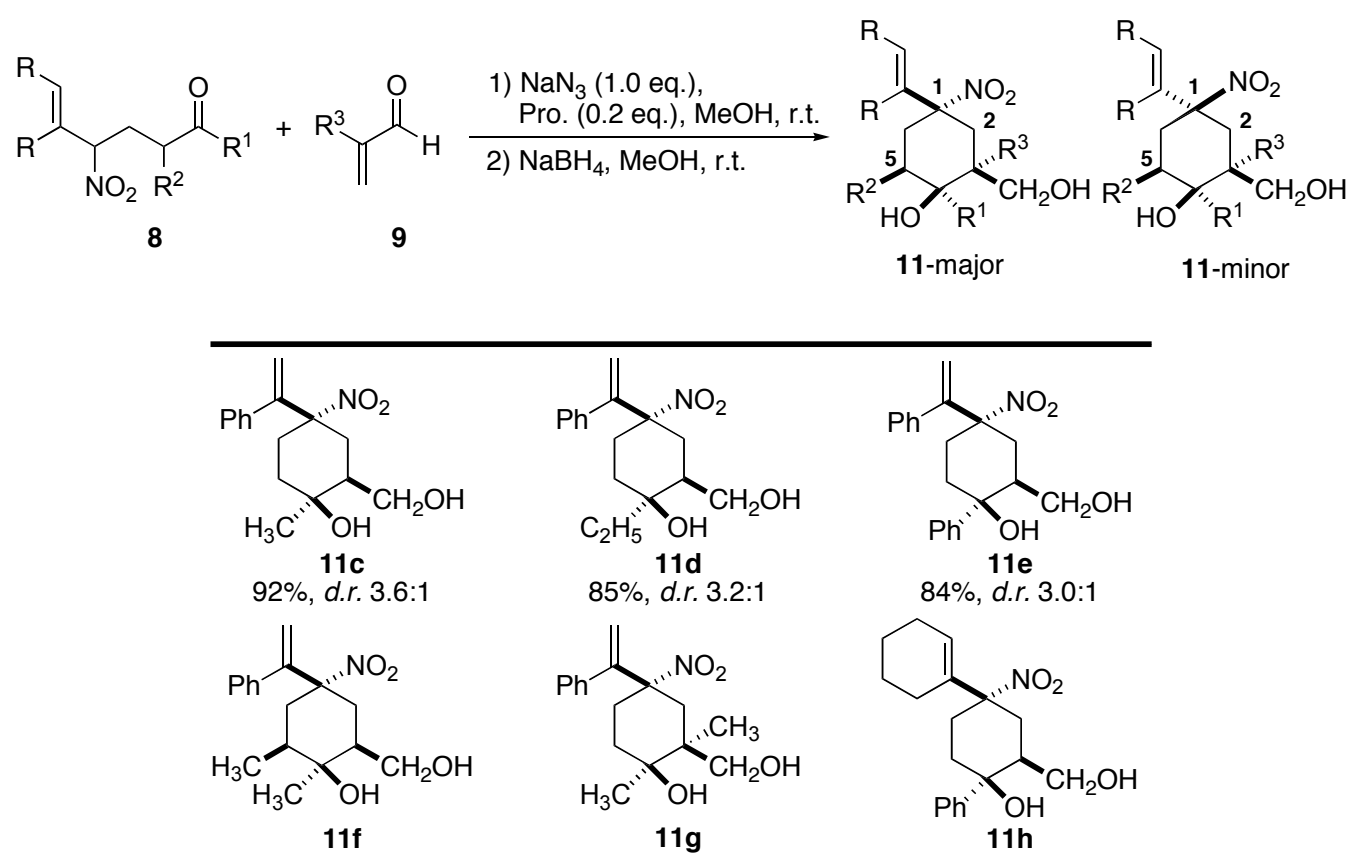

78\%, d.r. $4.0: 1$

$82 \%$, d.r. $2: 2: 1$

$83 \%$, d.r. $12: 1$

${ }^{a} \mathbf{1 : 2}=1: 2, \mathrm{c}=0.2 \mathrm{M} ;$ d.r. values are based on crude ${ }^{1} \mathrm{H}$ NMR analysis; isolated yields of all isomers.

With this optimal condition for the intramolecular aldol reaction, we then investigated the direct condensation between nitroalkenes and enones. As expected the one-pot Michael-Michael-aldol condensation was successfully achieved with excellent yield and good diastereoselectivity. The substrate scope is summarized in Table 6.

The proline catalyst could successfully promote the cascade Michael-MichaelAldol reaction with the addition of mild bases $\left(\mathrm{Et}_{3} \mathrm{~N}\right.$ or $\left.\mathrm{NaN}_{3}\right)$. Application of $\alpha$, $\beta$ - 
unsaturated ester gave good yield of Michael-Michael addition product 10a without further Dieckmann condensation. ${ }^{19}$ Although enantioselective intramolecular Aldol condensation of 1,7-di-aldehyde has been reported in literature using proline as catalyst, the condensation reported by us gave rather low enantioselectivity $(<20 \%$ e.e. in all cases). We postulated the reason could be the difficult formation of $\mathrm{H}$-bond between proline carboxylate group and carbonyl oxygen under basic condition.

Table 6. Cascade Michael-Michael-Aldol condensation ${ }^{a}$
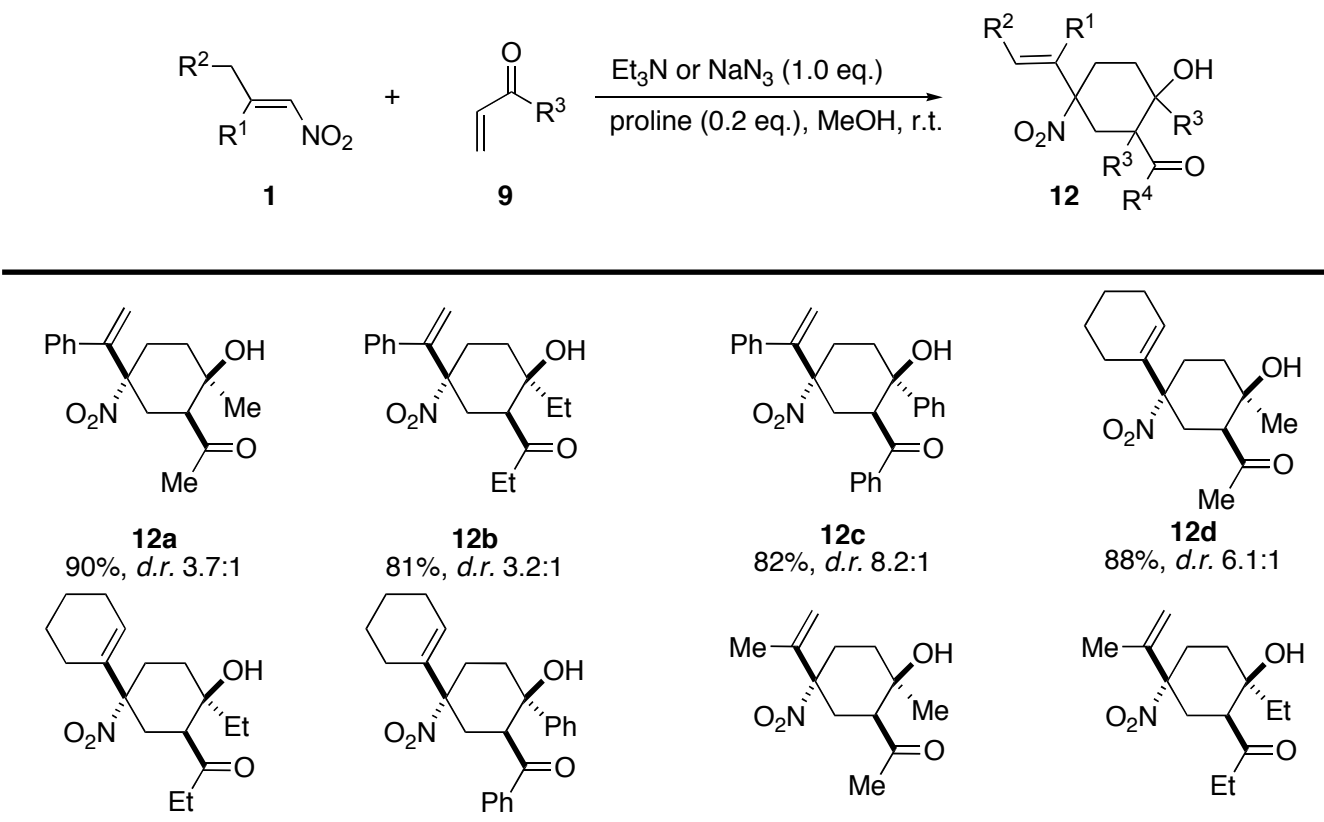

$88 \%$, d.r. $6.4: 1$
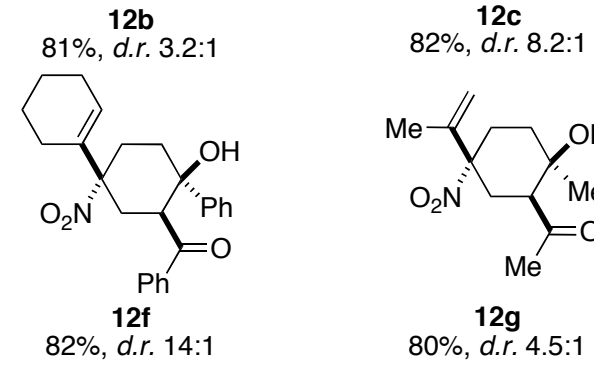

$88 \%$, d.r. $6.1: 1$

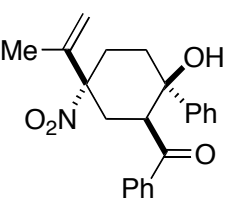

12i
$77 \%$, d.r. $12: 1$
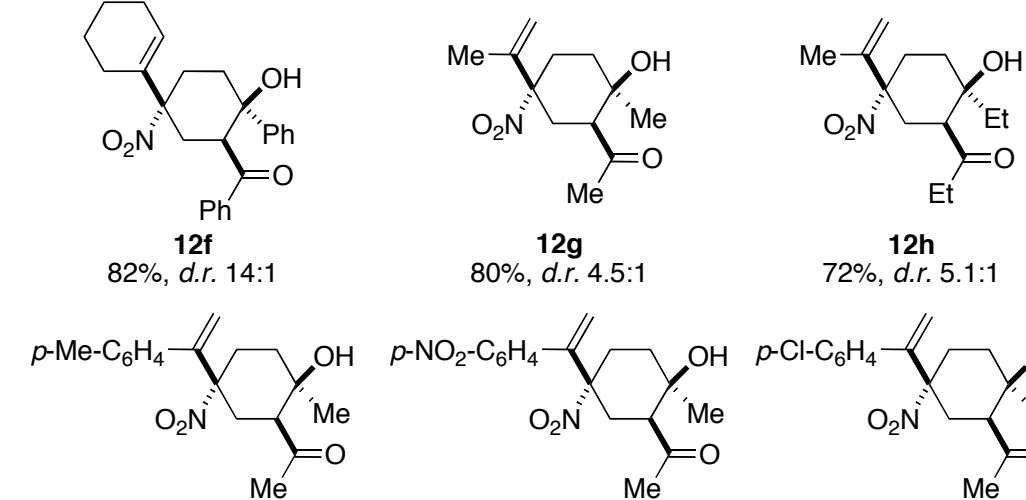

$80 \%$, d.r. $4.5: 1$

$72 \%$, d.r. $5.1: 1$

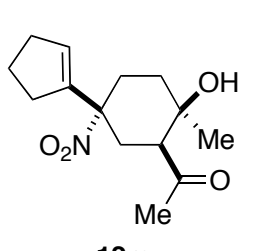

76\%, d.r. $5.0: 1$
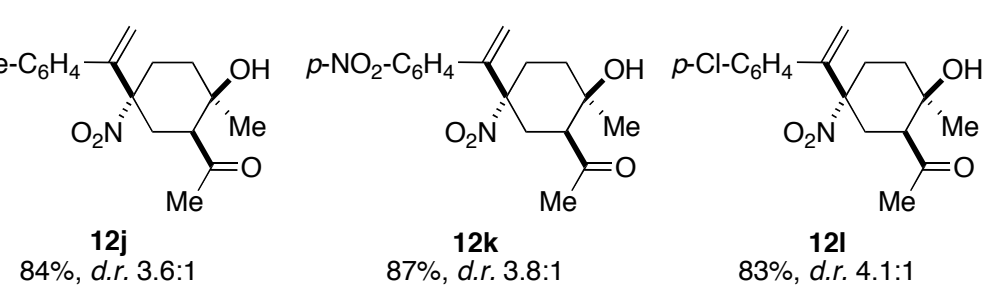

$87 \%$, d.r. $3.8: 1$

$83 \%$, d.r. $4.1: 1$
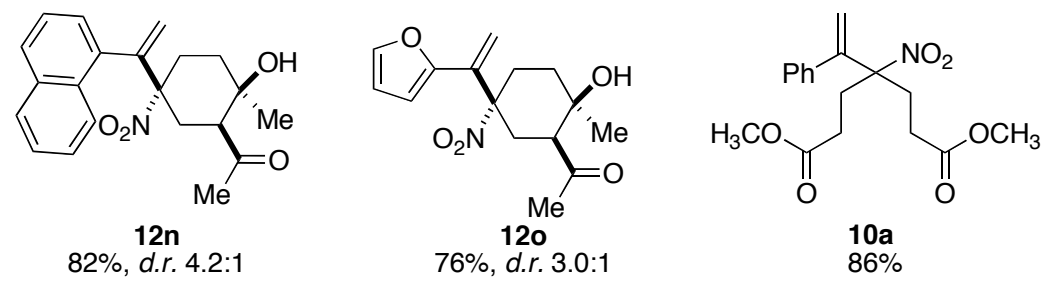

${ }^{a} \mathbf{1 : 2}=1: 4, \mathrm{c}=0.2 \mathrm{M}$, reaction generally finished in 10-20 hours; $d . r$. determined by crude ${ }^{1} \mathrm{H}$ NMR analysis; isolated yields of all isomers. 


\subsubsection{Nitrogen containing heterocycles synthesis}

Simple reduction of nitro group into amine, two effective derivatizations were then carried out and summarized in Scheme 11, which further empathize the strength of the reported method as highly efficient new approach in complex molecule construction.

\section{Scheme 11. Synthesis of complex N-heterocycles}

A) Transfermation 1,7-diester to N-hetero-bi-cycle

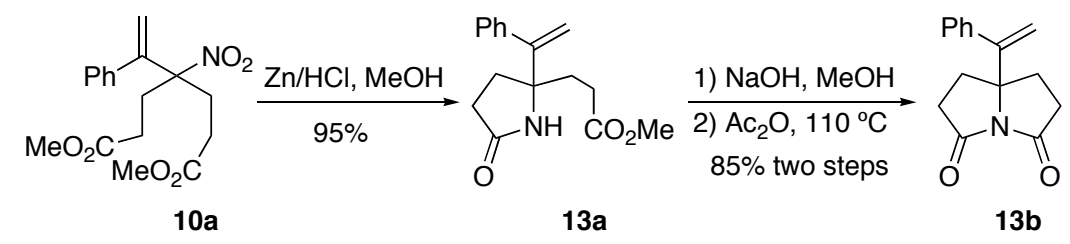

B) Efficient synthesis of poly-N-hetereocycle
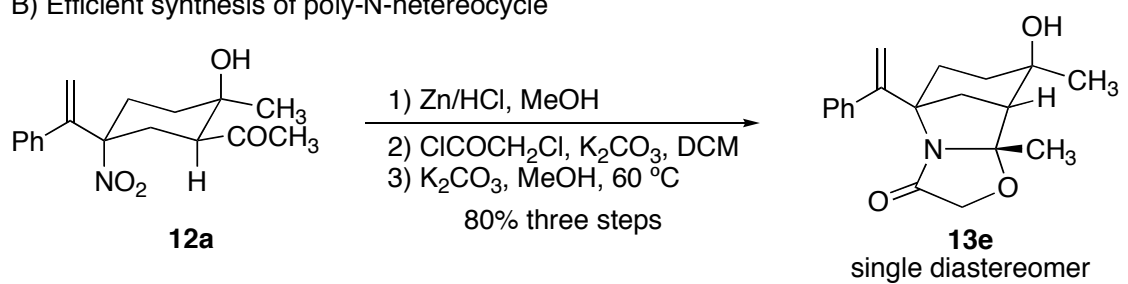

\subsection{Conclusions}

In conclusion, a one-pot condensation of nitroalkenes and carbonyl-activated alkenes was developed. The optimal condition was developed with the application of both Lewis base and mild bases. Through this process functional group enriched cyclohexanes were prepared with excellent yields and good diastereoselectivity. The asymmetric transformation and application of this approach in natural product total synthesis are currently under investigation.

This project is a collaboration work with Dr. Yunfeng Chen. I helped with the substrate scope (Table 6) and the $\mathrm{N}$ containing heterocycle synthesis. 


\section{Part III}

\section{Concise Asymmetric Synthesis of Fully Substituted Isoxazoline-N-Oxide through Lewis Base Catalyzed Nitroalkene Activation}

\subsection{Introduction}

\subsubsection{Literature reported synthesis of Isoxazoline-N-Oxide}

Isoxazoline $\mathrm{N}$-oxides and their derivatives are frequently used as intermediates in the synthesis of complex molecules in chemical ${ }^{20}$ and biological field ${ }^{21}$.

Scheme 12. Synthesis of complex isoxazoline-N-oxide via $[3+2]$ cyclization

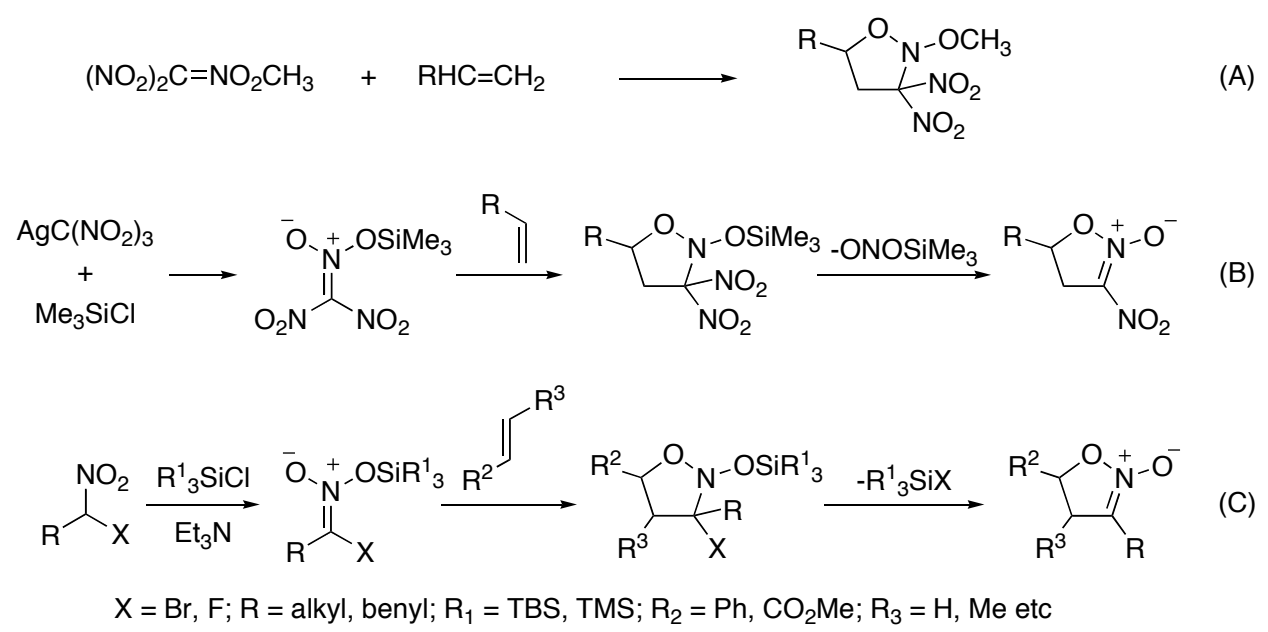

Back in the year 1964, nitronic esters were found to undergo 1,3-dipolar cycloaddition to alkenes to afford isoxazolidine. Representative works had been reported by Tartakovskii and co-workers ${ }^{22 \mathrm{a}}$ (Scheme 12A). Later on, the same group also developed another similar strategy for the synthesis of isoxazoline $\mathrm{N}$-oxides (Scheme 
12B). ${ }^{22 b}$ Trinitromethane silver salt reacted with trimethylsilylchloride to form silylnitronate intermediate, which then went through a [3+2] cycloaddition with alkene to yield the isoxazolidine product. However, in this case, one molecule of silylnitronate was eliminated to give the final product. A more recent synthesis was done using the similar strategy targeting the isoxazoline-N-oxide molecules (Scheme 12C). ${ }^{23 \mathrm{c}}$ Halides were used in this case to better the elimination process after the cycloaddition, which was nitronate previously. In the 1990's, Denmark extended this [3+2] strategy into remarkable complex heterocycles synthesis and natural product synthesis. ${ }^{23}$

Another important synthesis pathway of isoxazoline-N-oxide molecules is the Oalkylation of $\mathrm{NO}_{2}$ containing molecule, pioneer works had been reported (Scheme 13). ${ }^{24}$ The nucleophilicity of $\alpha$ carbon was well studied to react with different electrophiles, such as aldehydes, epoxides and haloenones, which then followed by designed Oalkylation to complete the cascade synthesis of isoxazoline-N-oxides.

\section{Scheme 13. Synthesis of complex isoxazoline-N-oxide via O-alkylation}

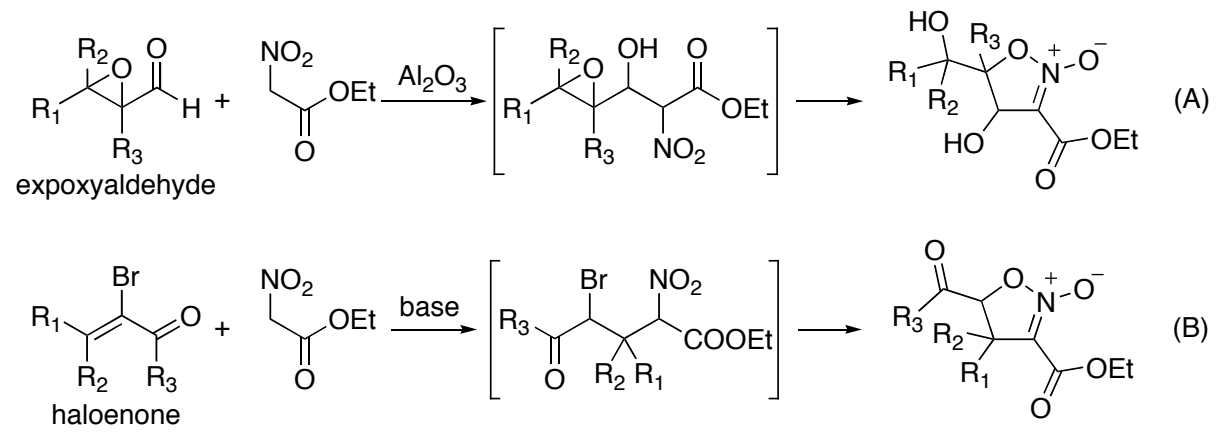

The combination of ylide auxiliaries with nitroalkenes had also been well studied as one possible route. In 1976, Holy reported that dimethylsulfoxanium methylide could react with nitroalkene to afford isozaxoline- $N$-oxides in the presence of copper(I $)^{25}$ (Scheme 14 A). More recently, Tang and coworkers had successfully developed a more practical and simple method, using sulfur ylide through a much milder condition (Scheme 14 B). ${ }^{26 a}$ Later on, the same group reported the asymmetric synthesis of isoxazoline-N-oxide with good yields and excellent enantioselectivity (Scheme 14 C). ${ }^{26 \mathrm{~b}}$ 


\section{Scheme 14. Synthesis of complex isoxazoline-N-oxide through ylide auxiliary}
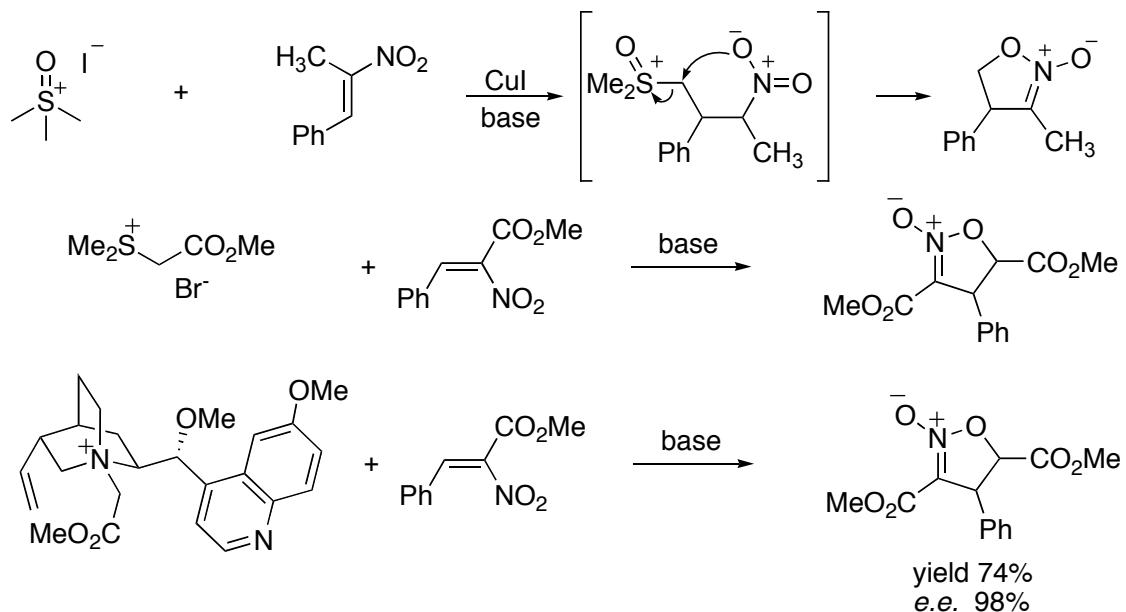

\subsubsection{Application of Isoxazoline-N-Oxide}

The stereoselective synthesis of isoxazoline $N$-oxide is an important task in the context of synthetic strategies toward the preparation of biological target molecules such as polyhydroxylated amino acids, aminopolyols and amino sugars ${ }^{27}$. Through chemoselective reductions, $\mathrm{N}-\mathrm{O}$ bond could be easily cleaved, followed by hydrolytic workup to give $\beta$-hydroxyketone, which provides an alternate route to reach aldol product. The dehydrogenation of intermediate iminoalcohol arising from N-O bond cleavage affords $\beta$-hydroxy nitriles. Complete reduction of the isoxazoline ring delivers $\gamma$-aminoalcohols. Base-mediated ring opening would provide $\alpha, \beta$-unsaturated oximes. Chemoselective reduction could also yield $\alpha$-hydroxyl, $\gamma$-lactam functionality in one step. All the hydroxyl compounds can be easily dehydrated or the other functional group can be transformed further to obtain synthetically useful molecules (Scheme 15).

\section{Scheme 15. Derivatization of isoxazoline-N-oxide through reduction}

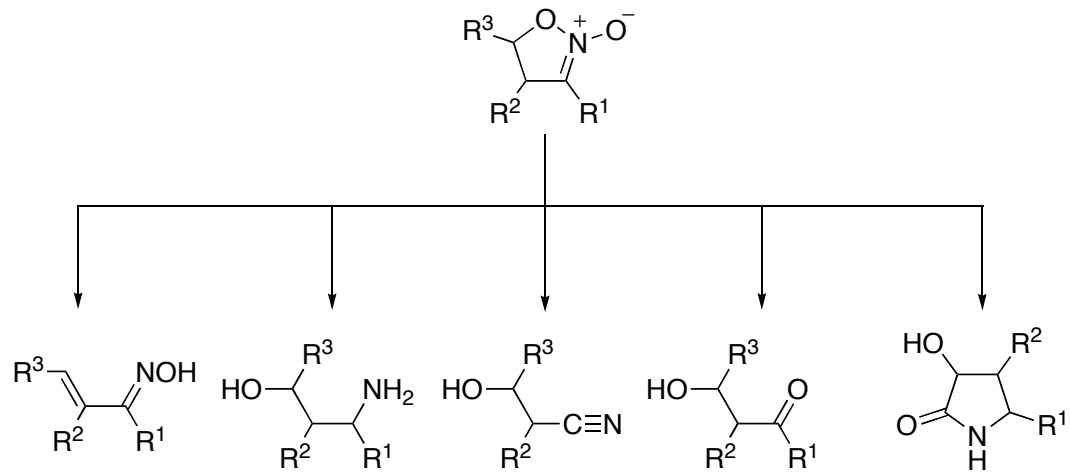


The dipolar character of these cyclic nitronates can be exploited for further 1,3dipolar cycloaddition with olefins to afford multi-cyclic structure moiety in total synthesis. The placement of the tether at the $\mathrm{C} 3$ position of the nitronate provides access to the spiro mode cycloadditions. This class has been evaluated for the preparation of five- and six-membered spiro ring systems ${ }^{28}$ (Scheme 16 A).

\section{Scheme 16. Derivatization of isoxazoline-N-oxide through $[3+2]$ cyclization}

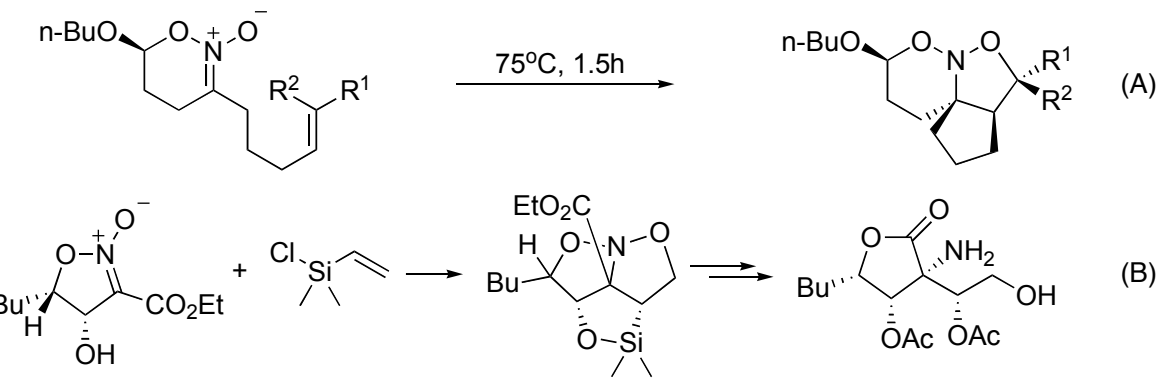

Rosini's group reported the first example of a silicon-tethered intermolecular 1,3dipolar cycloaddition ${ }^{29}$ (Scheme 16 B). The tricyclic compound was converted to the corresponding bicyclic diol by an oxidative removal of the temporary silicon linker. The unstable diol was protected immediately as the diacetate. Reductive ring opening of the nitroso acetal moiety was performed with hygrogen and a catalytic amount of Raney $\mathrm{Ni}$, to afford the polyhydroxylated aminolactone derivative.

\subsubsection{Previous study in our group}

In 2008, an alternate synthesis pathway was discovered in our group based on the Lewis base activation of nitroalkenes, using $\beta$-alkyl nitroalkene and vinyl ester as the simple starting materials. ${ }^{30} \mathrm{~A}[3+2]$ cyclization between proline activated nitroalkene and vinyl ester was proposed, yet to be confirmed, followed by a series of transformation to reach the diene intermediate $\mathbf{A}$. Sequential conjugate addition by the second nitro carbanion led to the di-nitro intermediate $\mathbf{B}$ and intramolecular cyclization gave the substituted isoxazoline-N-oxide as single trans isomer in moderate to good yields (Scheme 17). 


\section{Scheme 17. Isoxazoline-N-oxide synthesis in previous study and its mechanism}
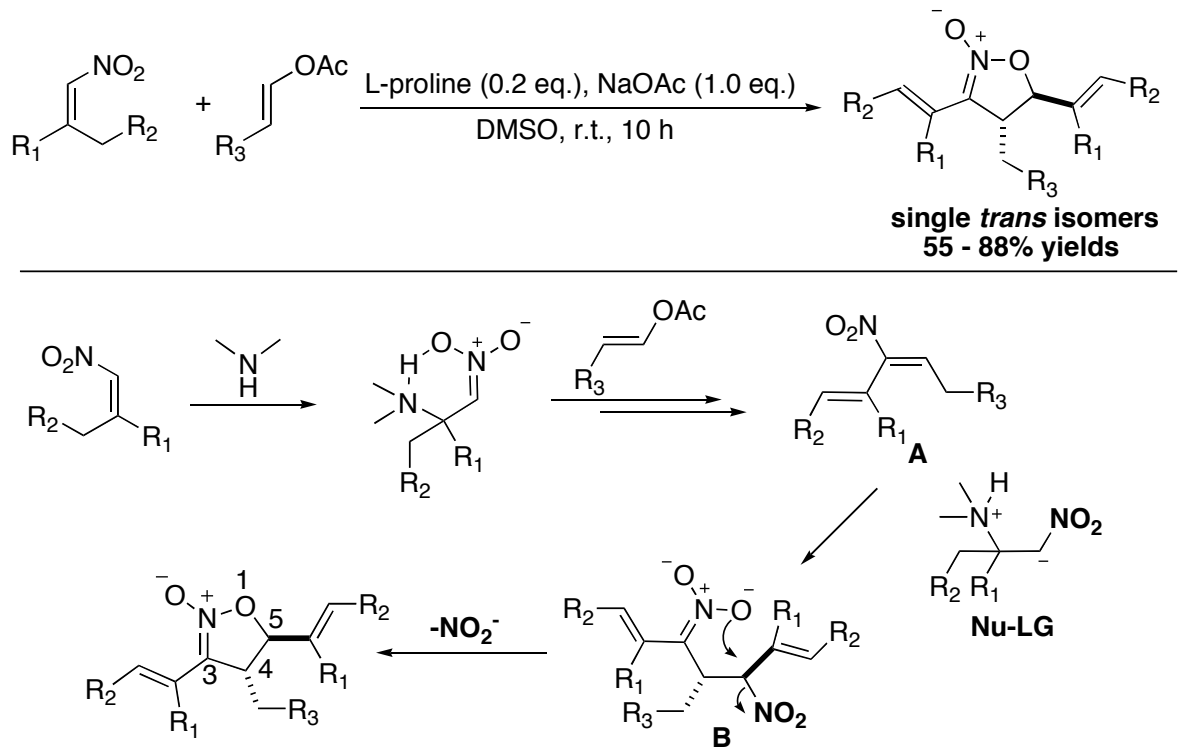

This cascade approach was attractive since it revealed a new strategy in producing active diene intermediate $\mathbf{A}$ in-situ, which allowed the preparation of isoxazoline N-oxide without going through the challenging nitronate intermediate synthesis in literature reported studies. Moreover, the reaction condition tended to be milder and two vinyl groups were set up at the $\mathrm{C} 3$ and $\mathrm{C} 5$ positions for further easy derivatizations.

However, despite the high efficiency, this cascade reaction did suffer from three obvious problems:

a) The C-4 substitution was limited to only aliphatic groups, since vinyl esters were used as starting materials.

b) Two identical vinyl groups on both C-3 and C-5 positions, which lowers the synthetic value of the molecules.

c) Poor enantioselectivity when chiral secondary amines were applied as catalysts. $(<15 \%$ e.e. was observed in all cases).

Owing to the unique mechanism and high efficiency, it is expected that successful strategies in overcoming the above-mentioned limitations will lead to the discovery of concise synthesis of the isoxazoline-N-oxide motifs. 


\subsection{Research Objectives}

\subsubsection{Asymmetric three-component synthesis of isoxazoline-N-oxide}

As described in the last section, three limitations need to be extended. First, more various functionalities are supposed to be suitable for $\mathrm{C} 4$ position, instead of only aliphatic groups. Second, in order to differentiate the functional groups at C3 and C5 positions, further modifications are needed. Or, a third component needs to be introduced into the reaction system. Third, asymmetric synthesis needs to be investigated to reach optical active molecules for further natural product derivatives syntheses.

\subsubsection{Further application towards the synthesis of natural products}

With the asymmetric three-component synthesis of the isoxazoline- $\mathrm{N}$-oxide motifs, a wide range of natural molecules or their backbones are expected to be readily realized, due to the well set-up of the multi-functionality at the very beginning.

\subsection{Results and Discussion}

\subsubsection{Diversity of $\mathrm{C}-4$ position in isoxazoline-N-oxides}

Since we had known that the key step for the isoxazoline-N-oxide synthesis was the formation of diene intermediate $\mathbf{A}$. Besides the [3+2] cycloaddition approach (Scheme 17), we postulated that the intermediate could be accessible through the Lewis based mediated Henry aldol condensation between nitroalkene 1 and aldehyde 14a. Thus, the desired homo-isoxazoline-N-oxide 15a would be prepared through a more general process with broader substrate scopes on the C-4 position.

Scheme 18. Proposed isoxazoline-N-oxide synthesis through proline promoted Henry-aldol Condensation

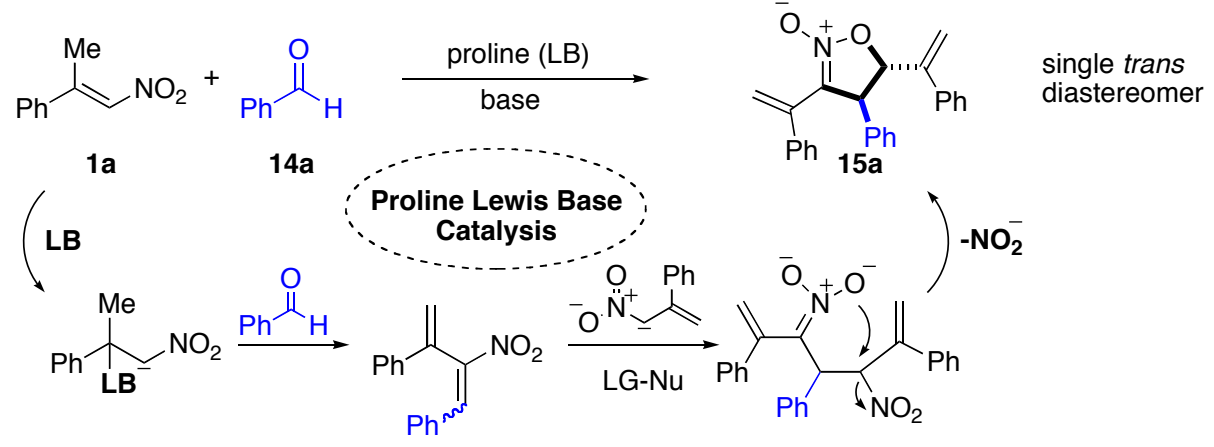




\subsubsection{Reaction optimization}

The reactions between 1a and benzyl aldehyde 14a were investigated. As expected, isoxazoline-N-oxide 15a was obtained as single trans diastereomer. The reaction condition optimization is summarized in Table 7.

Table. 7 One-pot synthesis of homo-isoxazoline-N-oxide by Lewis base activated nitroalkene-aldehyde condensation ${ }^{a}$

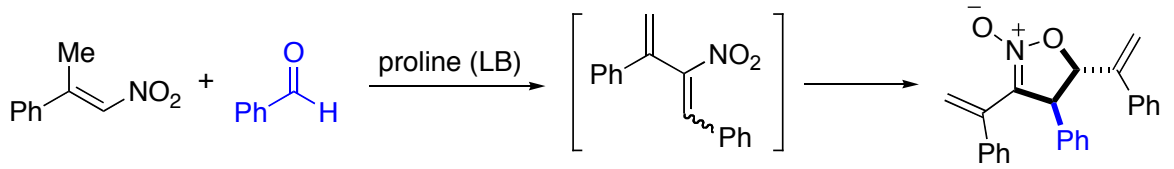

\begin{tabular}{|c|c|c|c|c|c|c|}
\hline 1a & \multicolumn{2}{|c|}{$14 a$} & \multicolumn{2}{|c|}{ Active diene intermediate } & \multicolumn{2}{|c|}{$\begin{array}{c}\text { single diasteromer } \\
15 a\end{array}$} \\
\hline entry & sol. & $\overline{L B}(0.2$ eq. $)$ & additive & $\mathrm{t}(\mathrm{h})$ & conv. $(\%)^{b}$ & $\overline{\text { yield }(\%)^{c}}$ \\
\hline 1 & DMSO & proline & - & 12 & 46 & 11 \\
\hline 2 & DMSO & -- & $\mathrm{NaOAc}(1.0 \mathrm{eq})$ & 12 & 79 & $<5$ \\
\hline 3 & DMSO & proline & $\mathrm{NaOAc}(1.0 \mathrm{eq})$ & 3 & $>95$ & $87^{d}$ \\
\hline 4 & DMSO & proline & $\mathrm{K}_{2} \mathrm{CO}_{3}(0.5 \mathrm{eq})$ & 3 & $>95$ & $92^{d}$ \\
\hline 5 & DMSO & proline & $\mathrm{NaO} t \mathrm{Bu}(1.0 \mathrm{eq})$ & 3 & 84 & $70^{d}$ \\
\hline 6 & DMSO & proline & $\mathrm{Et}_{3} \mathrm{~N}(1.0 \mathrm{eq})$ & 12 & 89 & $65^{d}$ \\
\hline 7 & DMSO & others $^{e}$ & $\mathrm{~K}_{2} \mathrm{CO}_{3}(0.5 \mathrm{eq})$ & 3 & $>95$ & $<50$ \\
\hline 8 & THF & proline & $\mathrm{K}_{2} \mathrm{CO}_{3}(0.5 \mathrm{eq})$ & 12 & 23 & 19 \\
\hline 9 & THF & proline & $\mathrm{BF}_{3}-\mathrm{THF}(1.0 \mathrm{eq})$ & 12 & 35 & trace \\
\hline 10 & others $^{f}$ & proline & $\mathrm{K}_{2} \mathrm{CO}_{3}(0.5 \mathrm{eq})$ & $3 \sim 12$ & $45 \sim 90$ & $13 \sim 49$ \\
\hline 11 & $\mathrm{MeOH}$ & proline & $\mathrm{K}_{2} \mathrm{CO}_{3}(0.5 \mathrm{eq})$ & 12 & $>95$ & 43 \\
\hline 12 & DMSO & pyrrolidine & $\mathrm{K}_{2} \mathrm{CO}_{3}(0.5 \mathrm{eq})$ & 3 & $>95$ & 88 \\
\hline
\end{tabular}

${ }^{a}$ general reaction condition: $1 \mathbf{a}(2.1$ eq.), 14a $(1.0$ eq., $0.1 \mathrm{M})$ and catalysts were mixed in solvents. The reactions were monitored by TLC; ${ }^{b}$ based on the consumption of 14a; ${ }^{c}$ NMR yield with 1,3,5-trimethoxybenzene as internal standard; ${ }^{d}$ isolated yield; ${ }^{e}$ other Lewis bases include DMAP, DABCO, NMI, $\mathrm{Ph}_{3} \mathrm{P} .{ }^{f}$ other solvents include EtOAc, DCM, Toluene, MeCN, $i \mathrm{PrOH}$.

Similar to the previous reported cases, the reaction did not precede well when only proline was present since base was required for neutralization of the $\mathrm{HNO}_{2}$ generated in the reaction (entry 1); nor only weak base $\mathrm{NaOAc}$ was applied without Lewis base activation of nitroalkene (entry 2). The major side reaction was the polymerization of 1a. However, the desired isoxazoline-N-oxide 14a was obtained in good yield when both proline ( 0.2 eq.) and $\mathrm{NaOAc}(1.0$ eq.) were used and the reaction finished within 3 hours (entry 3), much faster than the previous vinyl ester version of 
synthesis, which required at least 7 hours. Strong base, such as $t \mathrm{BuONa}$, caused significant polymerization of $1 \mathrm{a}$ (entries 5,6 ) and 0.5 equivalent of $\mathrm{K}_{2} \mathrm{CO}_{3}$ was identified as the optimal choice with minimum polymerization of nitroalkene and the maximum yield (entry 4). Other common nucleophilic base catalysts, such as DMAP, DABCO, NMI etc. have also been tested, giving only moderate yields (entry 7), yet confirming the Lewis base catalysis mechanism. These results highlighted the unique reaction nature of proline nucleophilic addition to nitroalkenes: the problematic nitroalkene polymerization associated with the Lewis base catalyzed mechanism was successfully avoided by forming a rather stable H-bond intermediate (Part I, 1.3, Scheme 6). Pyrrolidine (secondary amine) was also tested to be effective LB catalyst for this transformation (entry $12,88 \%$ yield) and proline was selected in this study because of its practical nature (low cost and easy to handle). Other solvents were also tested, including EtOAc, DCM, toluene, $\mathrm{MeCN}, i \mathrm{PrOH}$, giving lower yields compared to DMSO (entry 10).

\section{Scheme 19. Experimental confirmation of Lewis base catalysis mechanism}

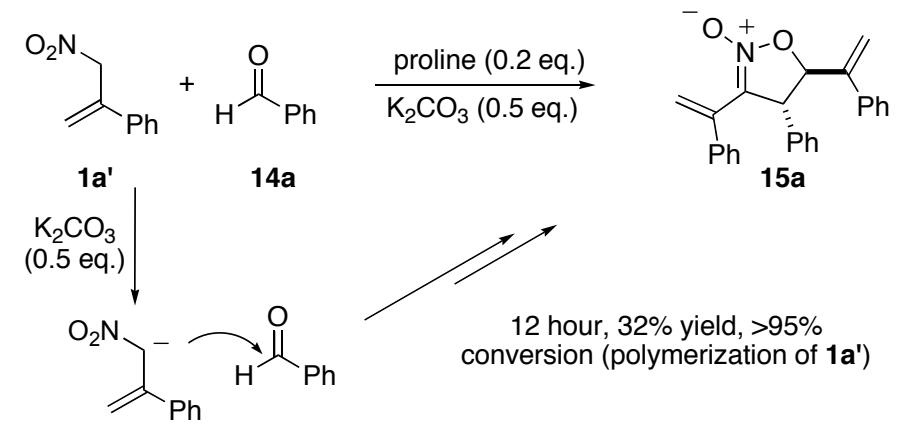

Furthermore, to confirm the Lewis base addition mechanism in this transformation, the allylic nitro compound (1/a') was prepared and. As expected, a much slower reaction was observed with low yield (32\% yield while 95\% starting material was consumed in 12 hours). This significant difference between $\mathbf{1 a}$ and $\mathbf{1 \mathbf { a } ^ { \prime }}$ as the starting materials provided strong evidence for the proposed Lewis base addition mechanism other than the alternative base deprotonation path. 


\subsubsection{Substrate scope}

With the optimal reaction conditions in hand, various isoxazoline-N-oxides were prepared with different substitution on C-4 position from corresponding aldehydes as shown in Table 8.

Table 8. Substrate scope of "homo" isoxazoline-N-oxide

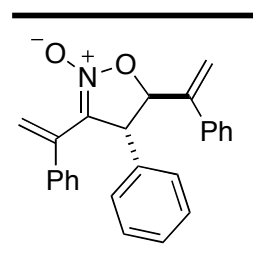

15a, $92 \%$<smiles>C=C(C1=[N+]([O-])O[C@H](C(=C)c2ccccc2)[C@H]1c1ccc(C)cc1)c1ccccc1</smiles>

15f, $93 \%$

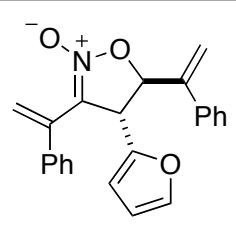

15b, $85 \%$

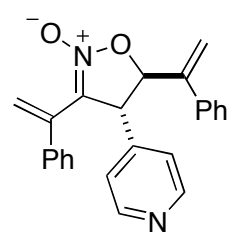

15c, $91 \%$

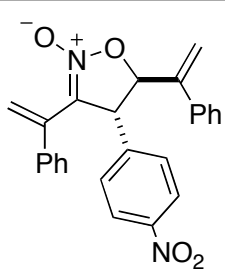

15d, $95 \%$

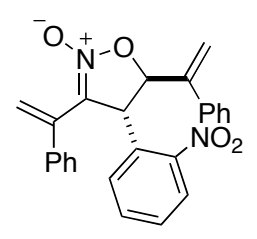

$15 e, 86 \%$

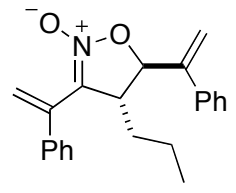

15g, $61 \%$

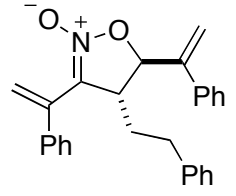

15h, $57 \%$

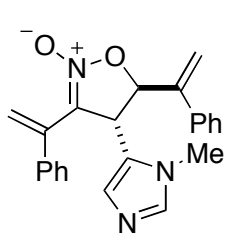

$15 i, 81 \%$

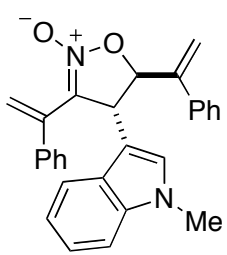

15j, 53\%

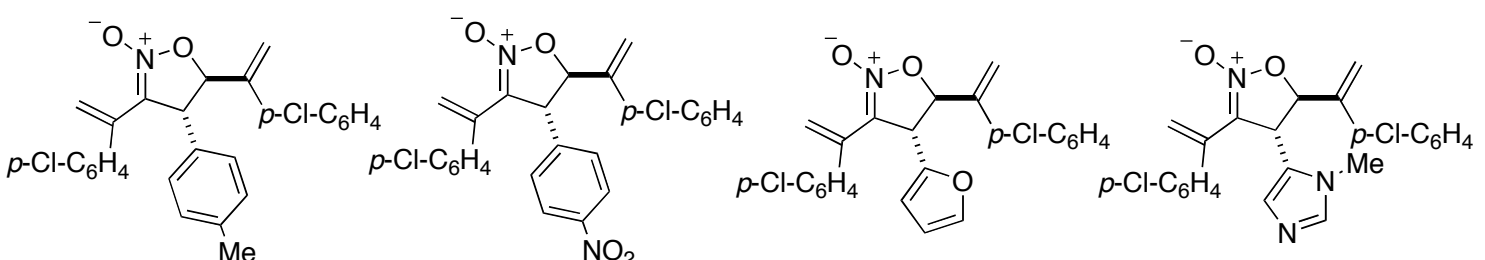

15k, 95\%

15I, 93\%

15m, 87\%

15n, $87 \%$

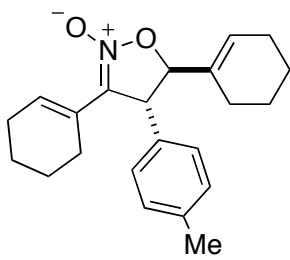

15o, $94 \%$

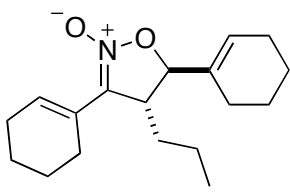

15s, $64 \%$

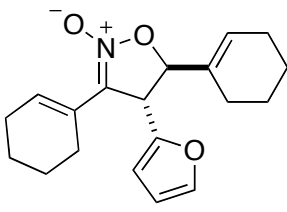

15p, $89 \%$

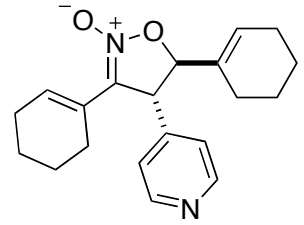

15q, 92\%

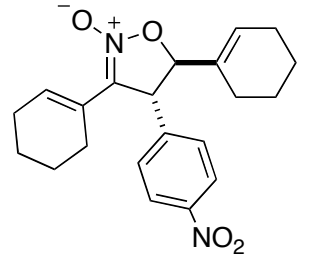

15r, 92\%

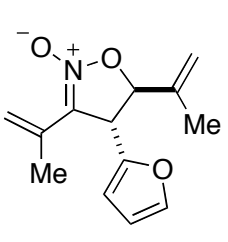

$15 w, 71 \%$

Among all these substrates, excellent diastereoselectivity were achieved and only trans isomers were obtained. This reaction was not only subjected to the simple aromatic 
aldehydes with excellent yields, but also the heterocyclic aromatic aldehydes with good yields. For aliphatic aldehydes, the reaction also worked though lower yields. This might be caused by the side reactions resulting from the formation of enamines in the presence of proline. In these cases, the previously reported [3+2] cyclization strategy could be an alternative route for the synthesis of desired products. Therefore, combining these two strategies, large variety of functional groups could be readily introduced into the C-4 position with high efficiency.

\subsubsection{Differentiation of $\mathrm{C}-3$ and $\mathrm{C}-5$ position in isoxazoline-N-oxides}

\subsubsection{Characterization of diene intermediate and cross cyclization}

As discussed earlier, the nitro-diene $\mathbf{A}$ was the key intermediate that accounted for the formation of the desired product. Interestingly, previous experiment of deuterium exchange suggested that the C-4 stereogenic center was not epimerizable (Scheme 20). Therefore, the absolute stereochemistry of the products would be determined by the second nucleophilic addition to the diene. Efforts were then made in obtaining the diene intermediate.

\section{Scheme 20. Deuterium exchange experiment}
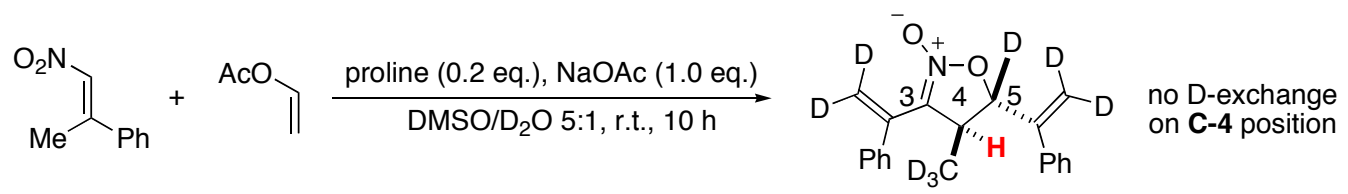

As show in Scheme 21, diene $\mathbf{A}^{\prime}$ was successfully isolated even though as a mixture of isomers $(Z / E=1: 1.5$, conformations were determined by 2D NMR, see experimental section). The formation of $Z / E$ isomer mixture raised concern for the potential asymmetric synthesis, since it was hard to achieve good enantioselectivity with two diastereomeric intermediates $(Z / E$ isomers) present in such a similar ratio. To investigate the diene's reactivity, a cross-condensation between diene $\mathbf{A}^{\prime}$ and nitroalkene 1c was then performed. Interestingly, mixtures of all the four possible isoxazoline-Noxides were obtained in the crude NMR (Scheme 21), which indicated the equilibrium between nitroalkene $\mathbf{A}^{\prime}$ and starting materials (nitroalkene $\mathbf{1 b}$ and aldehyde $\mathbf{1 4 b}$ ). 


\section{Scheme 21. Cross condensation with nitro-diene}

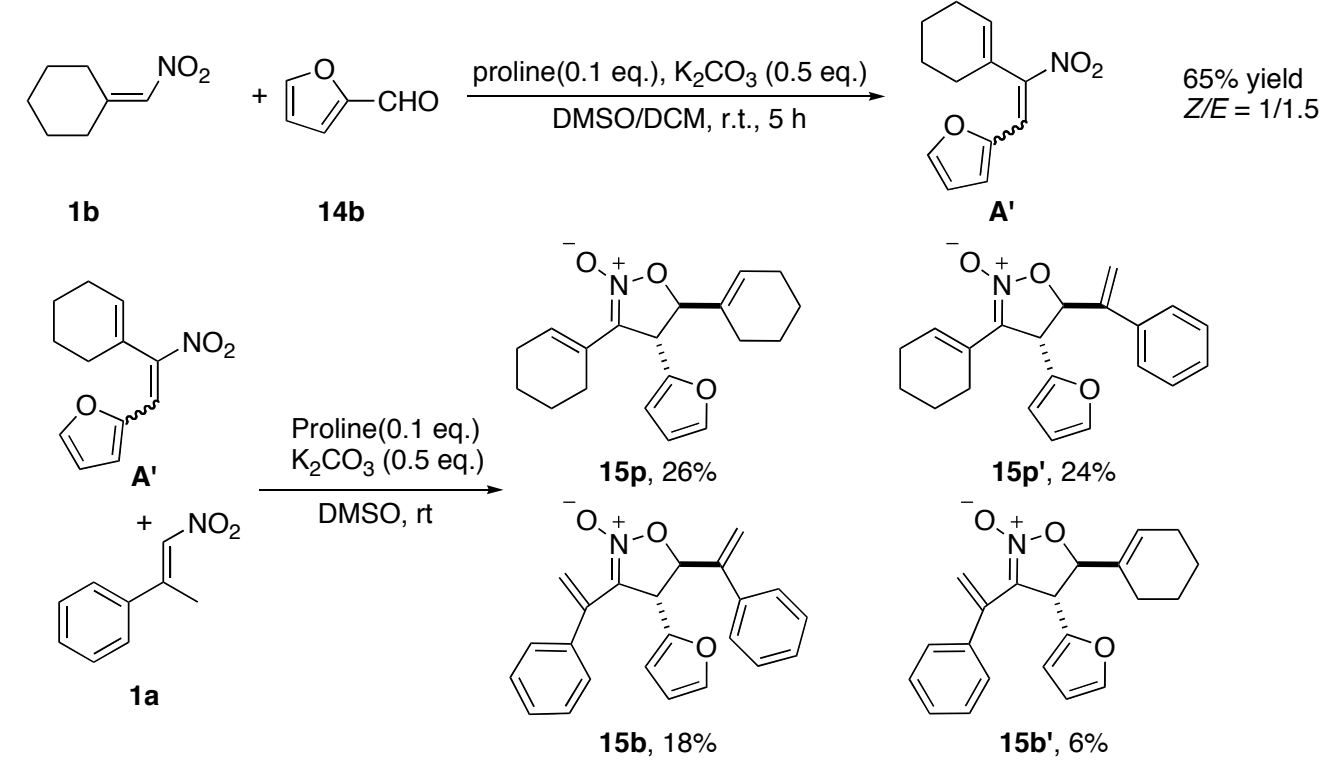

Another important information from these two experiments is that the enantioselective synthesis of the desired isoxazoline-N-oxides relied on the different reactivity of the two isomers, under the assumption that these two isomers could interconvert into each other (dynamic kinetic resolution). Then, it is feasible to control the C-4 stereogenic center with proper nucleophile with dynamic kinetic resolution (vide infra).

\subsubsection{Chemical difference of vinyl groups on $\mathrm{C}-3$ and $\mathrm{C}-5$}

\section{Scheme 22. Chemoselective differentiation of vinyl groups on C-3 and C-5}

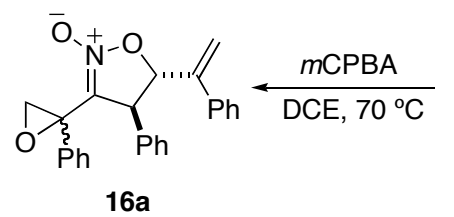

$65 \%$, d.r. $1: 1$

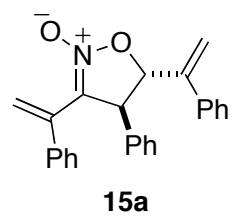

$15 a$

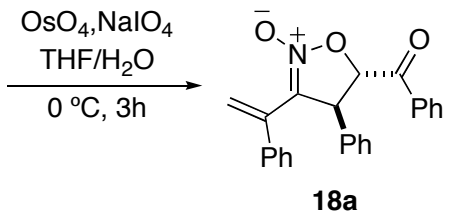

$84 \%$

Although there are two exactly same vinyl groups on the C-3 and C-5 positions of the "homo" isoxazoline-N-oxide molecules, they are different by electronic effects. One tends to accept electrons (C-3 position), while the other tends to give electrons (C-5 position). Thus, chemoselective oxidation would be possible to differentiate these two vinyl groups. When treated with $m$-CPBA, C-3 vinyl group of 15a was readily converted 
into epoxide 16a with 1:2 d.r. When $\mathrm{OsO}_{4} / \mathrm{NaIO}_{4}$ condition was applied, C-5 vinyl group was oxidized into ketone 18a in good yield. Other chemoselective operations could be applied for the differentiation of C-3 and C-5 vinyl groups, however, multi-steps are required which makes the described strategy less practical. Thus, the desire to develop a three-component cascade synthesis of isoxazoline-N-oxide drove us ahead.

\subsubsection{Three-component cascade synthesis of isoxazoline-N-oxide}

\subsubsection{Probing for the appropriate third component}

As demonstrated by our previous work, the diene $\mathbf{A}$ was the key intermediate for this synthesis. We postulated an alternative path that involved the third component with the featured functionality as Nu-LG moiety (nucleophile tethered leaving group) as shown in Scheme 23. Based on this hypothesis, compounds with Nu-LG functionality were applied to react with nitroalkene 1a and aldehyde 14a. The results are summarized in Table 9.

Scheme 23. Proposed three-component reaction involving a Nu-LG moiety

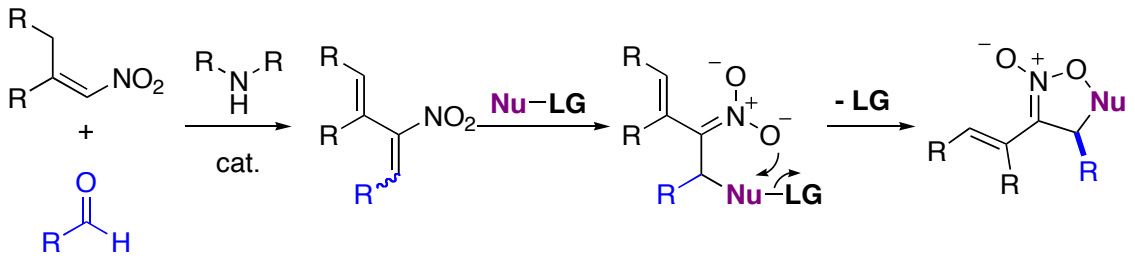

As shown in Table 9, application of $\mathbf{1 7} \mathbf{a}$ and $\mathbf{1 7} \mathbf{b}$, under the previous developed optimal condition, produced only the homo-isoxazoline-N-oxide 15a as the major product, which was probably due to the low reactivity of the sulfonate nucleophiles (entries 1 and 2). The phosphine ylide 17e and amine ylide 17d did generate the desired product 18, although in low yields. Also, the reaction suffered from significant competition of homo-condensation (entries 3 and 4). To our delight, application of sulphur ylides 17e and $\mathbf{1 7 f}$ gave compound $\mathbf{1 8}$ as the dominant product in excellent yields (entries 5 and 6) with only trace amount of 15a observed. 
Table 9. Substrates screening for the third component (Nu-LG)
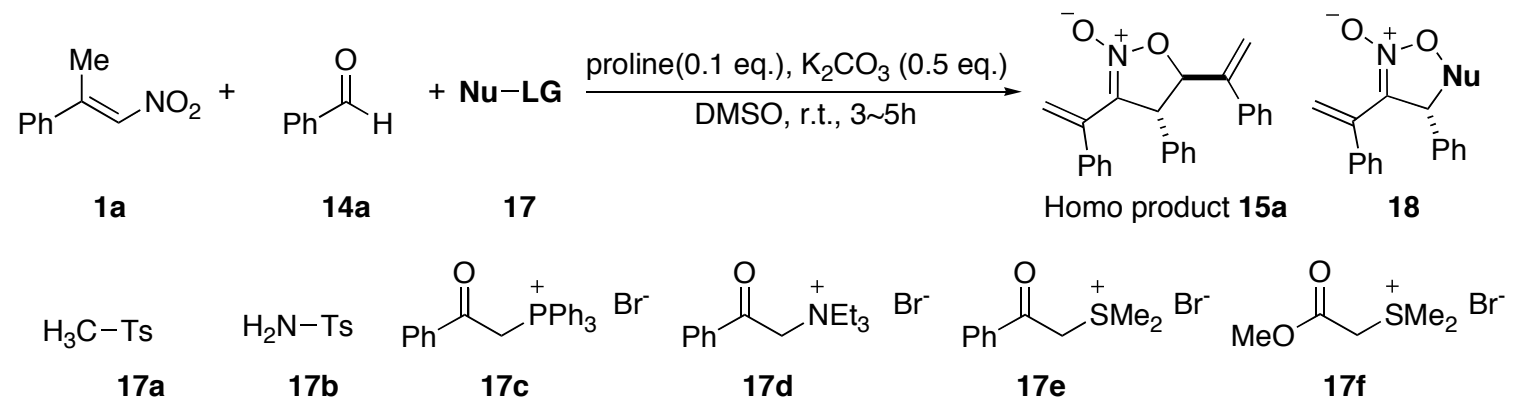

\begin{tabular}{cccc|cccc}
\hline \multirow{2}{*}{ entry } & \multirow{2}{*}{ Nu-LG } & \multicolumn{2}{|c|}{ product } & \multirow{2}{*}{ entry } & \multirow{2}{*}{ Nu-LG } & \multicolumn{2}{c}{ product } \\
& & $\mathbf{1 5 a}(\%)$ & $\mathbf{1 8}(\%)$ & & & $\mathbf{1 5 a}(\%)$ & $\mathbf{1 8}(\%)$ \\
\hline 1 & $\mathbf{1 7 a}$ & $76^{b}$ & $<5$ & 4 & $\mathbf{1 7 d}$ & $74^{b}$ & $18^{b}$ \\
2 & $\mathbf{1 7 b}$ & $85^{b}$ & $<5$ & 5 & $\mathbf{1 7}$ & $<5^{b}$ & $93^{c}$ \\
3 & $\mathbf{1 8 c}$ & $58^{b}$ & $35^{b}$ & 6 & $\mathbf{1 7 f}$ & $<5^{b}$ & $91^{c}$ \\
\hline
\end{tabular}

${ }^{a}$ reaction condition: 1a (1.0 eq.), 14a $(1.0$ eq., $0.15 \mathrm{M})$ and catalysts were mixed in solvents. The reactions were monitored by TLC; ${ }^{b}$ NMR yields with 1,3,5trimethoxybenzene as internal standard; ${ }^{c}$ isolated yield.

Notably, as demonstrated in Scheme 24, it is well known that reaction between aldehyde 14a and sulfur ylide 17e could produce the corresponding epoxide 15' in great yield under basic conditions. Without the involvement of the sulfur ylide, the dominant product would be 15a, as the "homo" isoxazoline-N-oxide product. However, under current reaction conditions, no significant amount of epoxide or the homo product was observed. This information further highlighted the superior chemoselectivity among the three designated components. The significance of the reported cascade process had also been emphasized: in the presence of alternative reaction paths (formation of homocondensation product 15a or epoxide $\mathbf{1 5}^{\prime}$ ), well-designed cascade process could direct the reaction pathway to products that would not be achieved from the step-wise approach.

\section{Scheme 24. Perfect chemoselectivity among the three components}

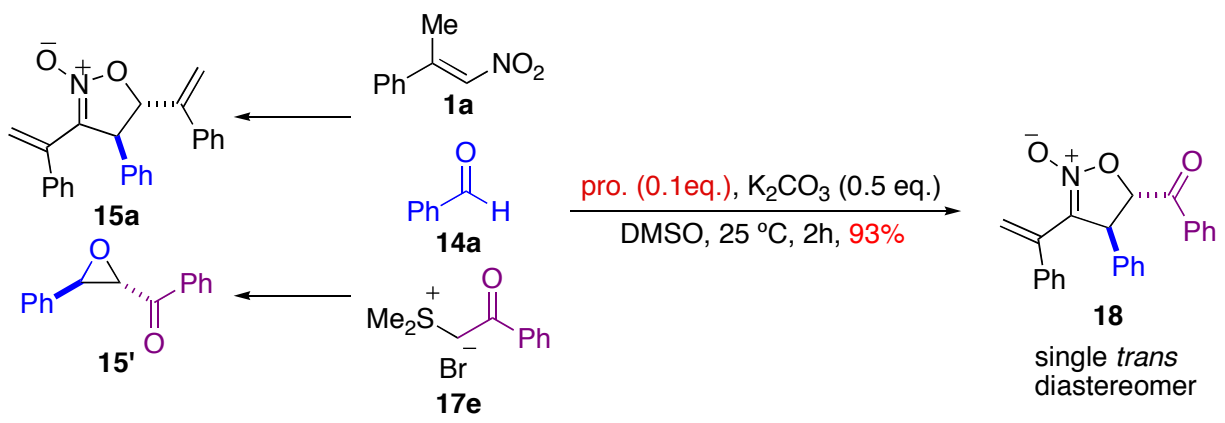




\subsubsection{Substrate scope of the designed cascade reaction}

Table 10. Representative isoxazoline-N-oxides syntheses via the designed threecomponent strategy ${ }^{a}$

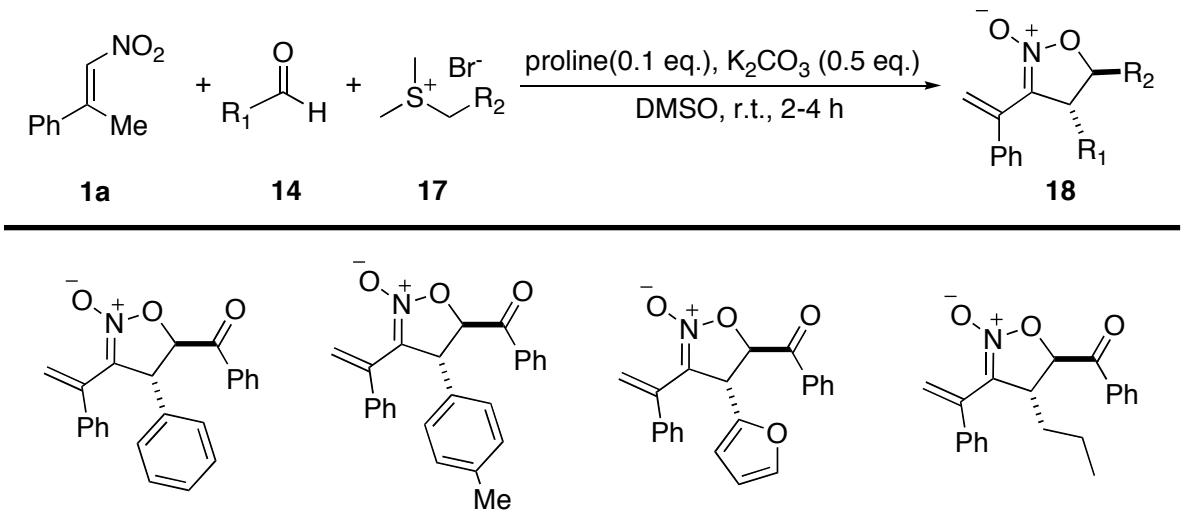

$18 a, 91 \%$

18b, $92 \%$

$18 c, 73 \%$

18d, $68 \%$<smiles>C=C(P)C1=C(C(C)C)OC(C(=O)c2ccccc2)C1C(=O)[O-]</smiles><smiles>C=C([PH2+])C1=[N+]([O-])OC(C(=O)c2ccccc2)C1c1ccccc1</smiles>

$18 f, 92 \%$

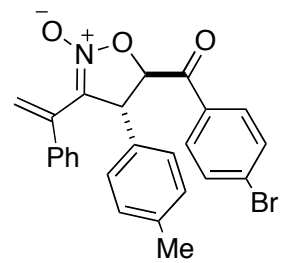

$18 \mathbf{i}, 91 \%$

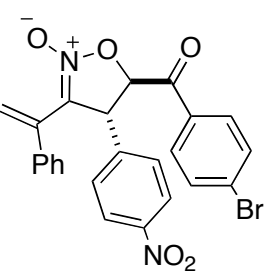

18j, $73 \%$

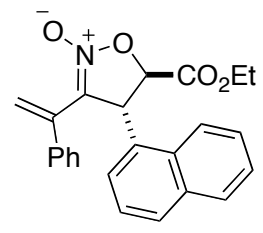

$18 g, 88 \%$

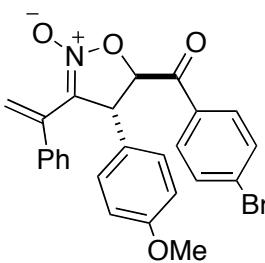

18k, $92 \%$

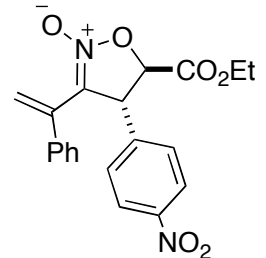

18h, $92 \%$

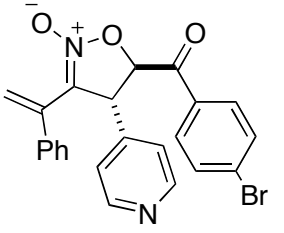

$181,92 \%$

${ }^{a}$ reaction condition: 1a (1.1 eq.), 14 (1.0 eq., $\left.0.15 \mathrm{M}\right)$ and ylide $\mathbf{1 7}$ (1.1 eq.) were mixed with proline (0.1 eq.) and $\mathrm{K}_{2} \mathrm{CO}_{3}(0.5$ eq.) in DMSO; isolated yields.

Up to this stage, the isoxazoline-N-oxide synthesis had been successfully developed into a real three-component cascade reaction. The summarized substrate scope investigation is shown in Table 10. Various aldehydes were tested, including simple aromatic aldehydes and heterocyclic aromatic aldehydes $(\mathbf{1 8 c}, \mathbf{1 8 1})$, good to excellent yields were received. The aliphatic aldehydes, linear and branched (18d, 18e), were also subjected to the designed cascade reaction system. Different sulfur ylides were also investigated, giving the desirable performance. 


\subsubsection{Asymmetric cascade synthesis of isoxazoline-N-oxides}

\subsubsection{Attempts of chiral Lewis base catalysis}

Table 11. Summarized chiral Lewis base catalysis for the asymmetric synthesis ${ }^{a}$

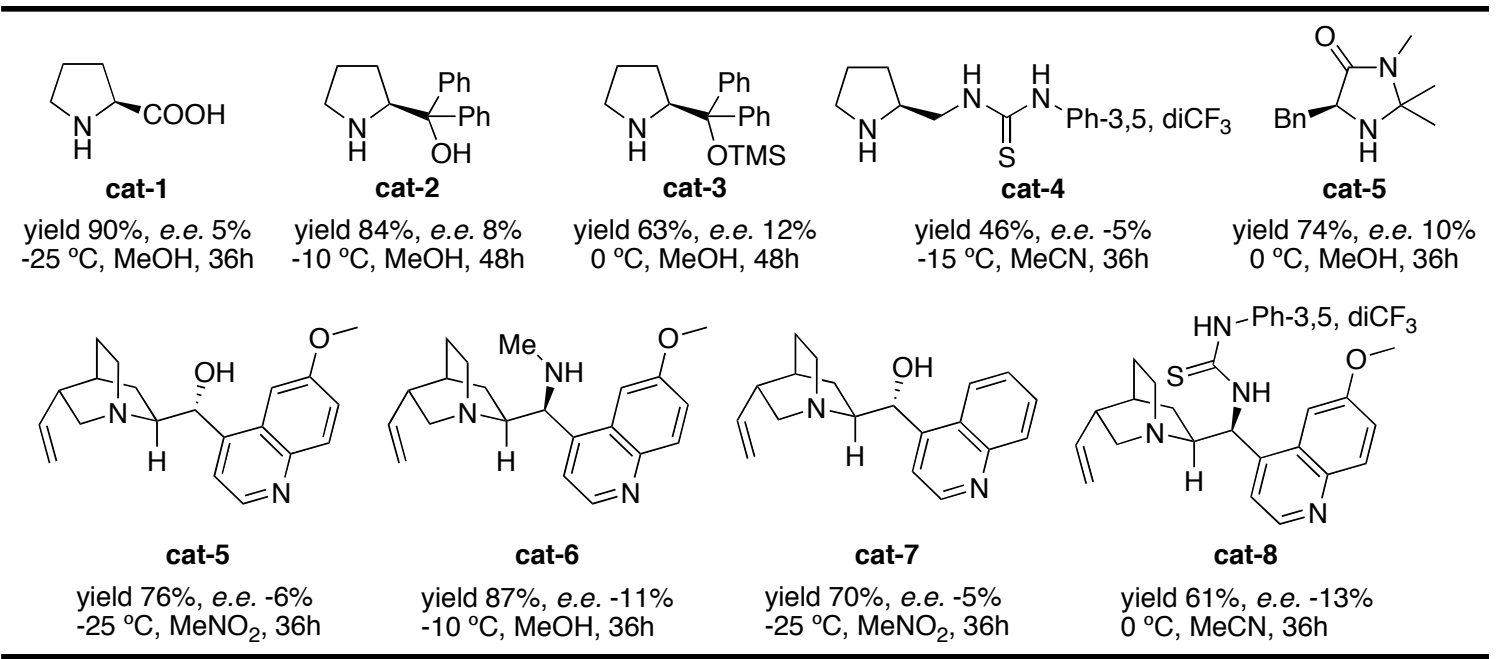

a reaction condition: 1a $(1.1$ eq. $), 14 a(1.0$ eq., $0.15 \mathrm{M})$ and ylide $\mathbf{1 7 b}(1.1$ eq.) were mixed with catalyst ( 0.2 eq.) and $\mathrm{K}_{2} \mathrm{CO}_{3}$ ( 0.5 eq.) in solvents; NMR yields of $\mathbf{1 8 h}$; data presented were the best combinations of yield and e.e. under the listed conditions.

Enantioselective synthesis of isoxazoline derivatives is considered as one challenging task and few asymmetric syntheses of this compound have been reported. ${ }^{31,32}$ Discussed in previous sections, although the Lewis base catalyzed cascade approach gave excellent trans diastereoselectivity. However, during the screening of chiral Lewis bases as catalysts, no encouraging enantioselectivity was observed (Table 11, $<15 \%$ e.e. in all cases), which might be due to the poor involvement of the catalysts in the stereochemistry-determining step (formation of the C-4 stereogenic center).

\subsubsection{Attempts of chiral auxiliary and condition optimization}

Based on previous discussion (2.3.2.1, Part II), one valid approach for asymmetric synthesis of fully substituted isoxazoline-N-oxides is to develop stereoselective nucleophilic addition of ylides to the diene intermediates (A in Table 7), through dynamic kinetic resolution. Thus, it is possible to deliver the chirality from the chiral auxiliary type of ylides. Several literature reported chiral ylides were then synthesized and applied (Table 12). 
Table 12. Condition optimization of different auxiliaries asymmetric synthesis ${ }^{a}$

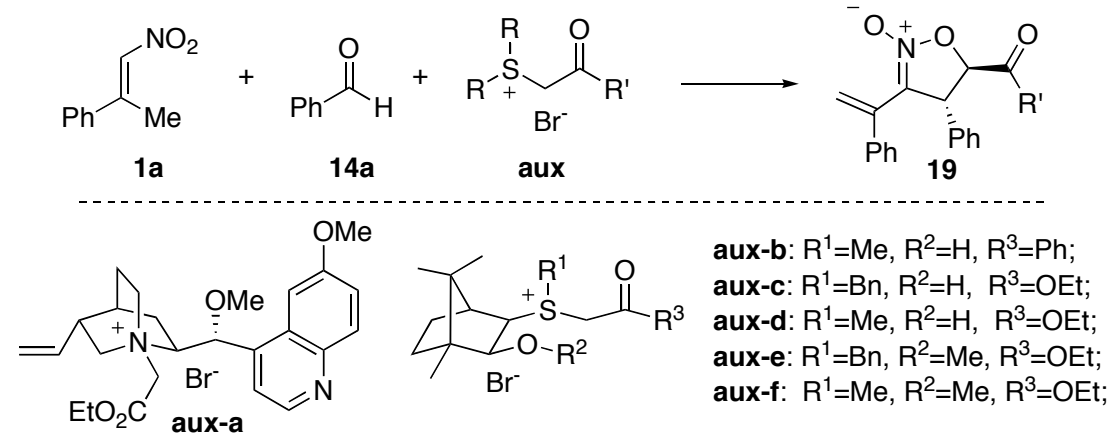

\begin{tabular}{|c|c|c|c|c|c|c|c|}
\hline entry & sol. & LB (0.2 eq.) & base (1.0 eq.) & aux & temp $\left({ }^{\circ} \mathrm{C}\right)$ & time $(\mathrm{h})$ & yield $\%^{b}(e . e . \%)$ \\
\hline 1 & DMSO & L-proline & $\mathrm{K}_{2} \mathrm{CO}_{3}$ & aux-a & r.t. & 12 & $<5$ (n.d.) $^{d}$ \\
\hline 2 & DMSO & L-proline & $\mathrm{K}_{2} \mathrm{CO}_{3}$ & aux-b & r.t. & 12 & $<5$ (n.d.) ${ }^{d}$ \\
\hline 3 & DMSO & L-proline & $\mathrm{K}_{2} \mathrm{CO}_{3}$ & aux-c & r.t. & 6 & 19 (n.d.) \\
\hline 4 & Acetone & L-proline & $\mathrm{K}_{2} \mathrm{CO}_{3}$ & aux-c & r.t. & 6 & 16 (n.d.) \\
\hline 5 & $\mathrm{THF}$ & L-proline & $\mathrm{K}_{2} \mathrm{CO}_{3}$ & aux-c & r.t. & 24 & 13 (n.d.) \\
\hline 6 & $\mathrm{MeOH}$ & L-proline & $\mathrm{K}_{2} \mathrm{CO}_{3}$ & aux-c & r.t. & 6 & $60^{e}(36)$ \\
\hline 7 & $\mathrm{MeOH}$ & L-proline & $\mathrm{K}_{2} \mathrm{CO}_{3}$ & aux-d & r.t. & 6 & $81^{e}(75)$ \\
\hline 8 & $\mathrm{MeOH}$ & L-proline & $\mathrm{K}_{2} \mathrm{CO}_{3}$ & aux-e & r.t. & 6 & $23(40)$ \\
\hline 9 & $\mathrm{MeOH}$ & L-proline & $\mathrm{K}_{2} \mathrm{CO}_{3}$ & aux-f & r.t. & 6 & $43(59)$ \\
\hline 10 & $\mathrm{MeOH}$ & L-proline & $\mathrm{Cs}_{2} \mathrm{CO}_{3}$ & aux-d & r.t. & 6 & $54(76)$ \\
\hline 11 & $\mathrm{MeOH}$ & L-proline & $\mathrm{Cs}_{2} \mathrm{CO}_{3}$ & aux-d & -25 & 48 & $87^{e}(82)$ \\
\hline 12 & $\mathrm{MeOH}$ & L-proline & $\mathrm{Cs}_{2} \mathrm{CO}_{3}$ & aux-d & -40 & 60 & $62^{e}(82)$ \\
\hline 13 & $\mathrm{MeOH}$ & D-proline & $\mathrm{Cs}_{2} \mathrm{CO}_{3}$ & aux-d & -25 & 48 & $90^{e}(86)$ \\
\hline 14 & МеОН & pyrrolidine & $\mathrm{Cs}_{2} \mathrm{CO}_{3}$ & aux-d & -25 & 48 & $86^{e}(91)$ \\
\hline 15 & $\mathrm{MeOH}$ & N-Me-Gly & $\mathrm{Cs}_{2} \mathrm{CO}_{3}$ & aux-d & -25 & 48 & $51^{e}(89)$ \\
\hline
\end{tabular}

${ }^{a}$ general reaction condition: 1a (1.2 eq.), 14a (1.0 eq., $\left.0.15 \mathrm{M}\right)$ and aux (1.1 eq.) . Fast ester exchange happened in $\mathrm{MeOH}$, only methyl ester was detected. ${ }^{b}$ NMR yields with 1,3,5trimethoxybenzene as internal standard; ${ }^{c}$ determined by chiral HPLC; ${ }^{d}$ major products were homo-isoxazoline 15a and the epoxide $\mathbf{1 5}^{\prime} ;{ }^{e}$ isolated yield.

As shown in Table 12, the quinine derived chiral tertiary amine ylide aux-a, well developed by Tang et. al., ${ }^{31 \mathrm{a}}$ did not promote the hetero coupling reaction (entry 1), giving the homo coupling product 15a ( $>85 \%$ yield). This was also suggested by our previous study (Table 9). Then we switch to camphor derived thio ether ylides, since sulfur ylides gave excellent yields for the three-component synthesis of isoxazoline-Noxides. However, similar result was observed with ketone ylide aux-b (entry 2), which was likely caused by the reduced nucleophilicity associated with more hindered chiral ylide (comparing with the simple ketone ylide 17e, which gave the desired hetero 
coupling product in excellent yields). The ester modified ylide aux-c did give the heterocoupling product 19 though with poor yield (entry 3). Solvent screening indicated that $\mathrm{MeOH}$ was the optimal solvent (entries 4-6). Further modification on the ylides revealed aux-d $\left(\mathrm{R}^{1}=\mathrm{Me}\right.$ and $\left.\mathrm{R}^{2}=\mathrm{H}\right)$ as the optimal auxiliary (entry 7 ) and $\mathrm{Cs}_{2} \mathrm{CO}_{3}$ as preferred choice of base (entry 10), producing the desired product 19 in excellent yield. In addition, to our great please, good enantioselectivity was also received ( $75 \%$ e.e.). Considering that diene intermediate would likely give low $Z / E$ selectivity, the success in obtaining good enantioselectivity supported the hypothesis that chiral ylide nucleophile reacted with the diene intermediate with good stereochemistry control and the asymmetric synthesis achieved through dynamic kinetic resolution (DKR).

Interestingly, the secondary amine Lewis base catalyst also indicated influence on the stereochemistry of the product. With either L-proline or D-proline, the absolute stereochemistry of the product 19 was identical (entries 11 and 13), which indicated that the ylide auxiliary dominated the stereochemistry control in the diene nucleophilic addition. However, to our surprise, slightly improved stereoselectivity was observed when D-proline was applied as catalyst (entry 13). Further investigation revealed that achiral secondary amine Lewis base, such as pyrrolidine, gave the best stereoselectivity. The desired product 19 was also received in excellent yield (entry 14). Application of Nmethyl-glycine gave similar good enantioselectivity, though with lower yield (entry 15). Notably, $>50 \%$ of the camphor auxiliary was recovered during the purification.

\section{Scheme 25. X-ray structure of related camphor-derived ylide aux-g}
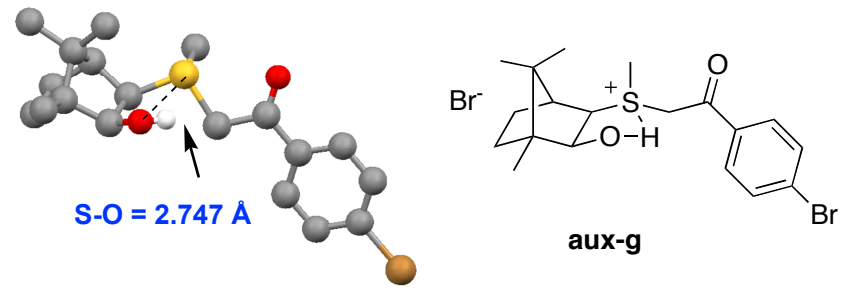

Moreover, one related camphor-derived auxiliary aux-g presented us an interesting finding (Scheme 25). An intramolecular hydrogen bonding between the alcohol and sulfonium was proposed in the X-ray crystallography analysis ( $\mathrm{S}-\mathrm{O}$ bond distance 2.747 å), which might account for the optimal yield and stereoselectivity. 


\subsubsection{Substrate scope of asymmetric three-component cascade synthesis of isoxazoline- $\mathrm{N}$-oxides}

Table 13. Substrate scope of asymmetric three-component synthesis

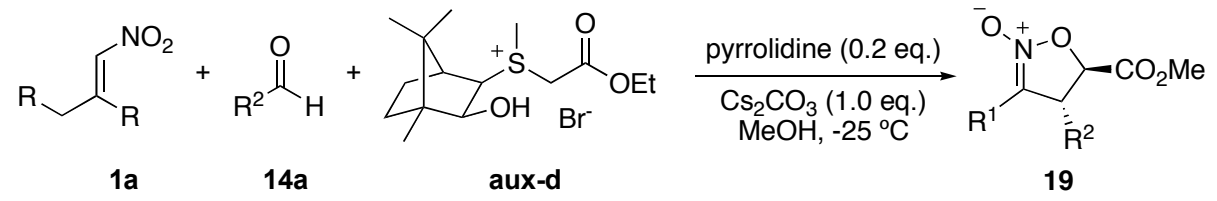

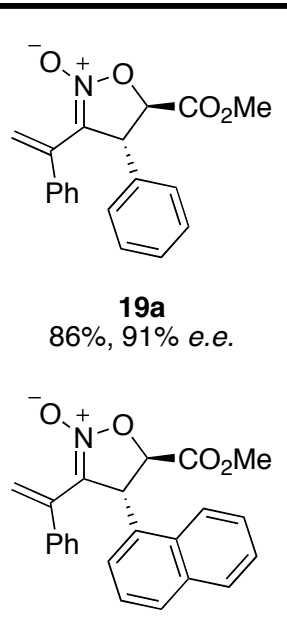

$19 f$

$86 \%, 96 \%$ e.e.<smiles>C=C(C1=[N+]([O-])O[C@@H](C(C)=O)[C@@H]1c1ccc(C)cc1C)c1ccccc1</smiles>

$79 \%, 92 \%$ e.e.

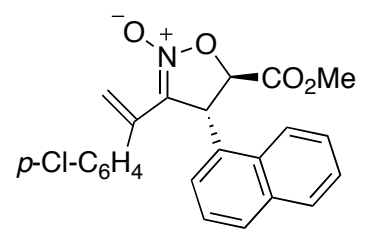$$
91 \%, 93 \% \text { e.e. }
$$

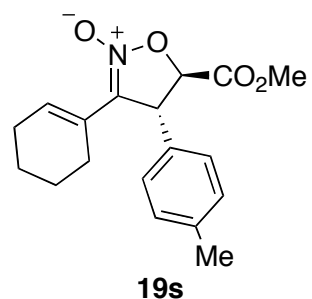

$80 \%, 90 \%$ e.e.

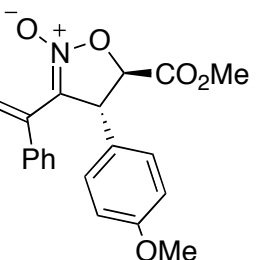

$19 b$ $82 \%, 94 \%$ e.e.

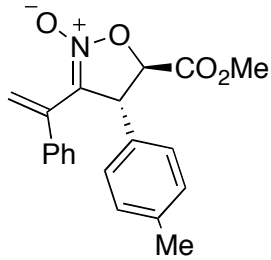

$19 \mathrm{c}$

$89 \%, 91 \%$ e.e.<smiles>C=C(C1=[N+]([O-])O[C@H](C(C)=O)[C@H]1c1ccccn1)c1ccccc1</smiles><smiles>C=C(C1=[N+]([O-])O[C@H](C(=O)OC)[C@H]1c1ccco1)c1ccccc1</smiles>

$19 d$

$90 \%, 80 \%$ e.e.

$19 \mathrm{e}$

$71 \%, 90 \%$ e.e.<smiles>C=C(C1=[N+]([O-])O[C@H](C(C)=O)[C@H]1c1ccc([N+](=O)[O-])cc1)c1ccccc1</smiles>

$19 \mathrm{~h}$ $63 \%, 88 \%$ e.e. $\quad 92 \%, 81 \%$ e.e. $\quad 82 \%, 70 \%$ e.e.

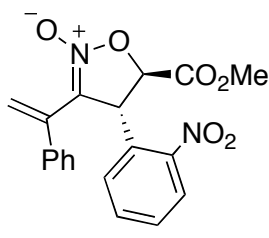

$19 \mathbf{i}$

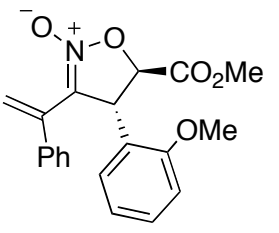

19j $84 \%, 88 \%$ e.e.

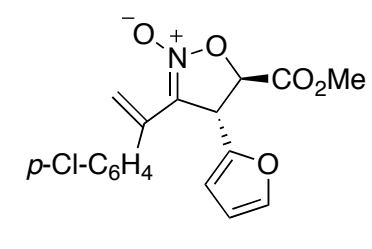

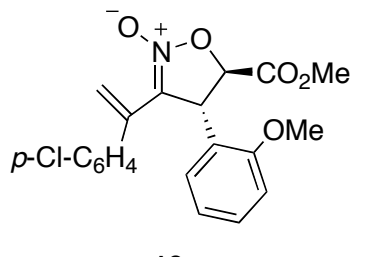

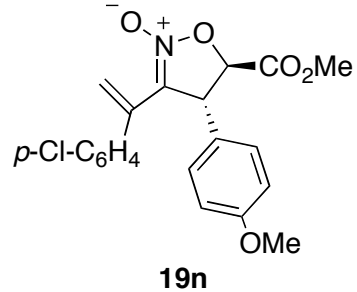

$81 \%, 76 \%$ e.e.

88\%, $81 \%$ e.e.

$81 \%$, $92 \%$ e.e.

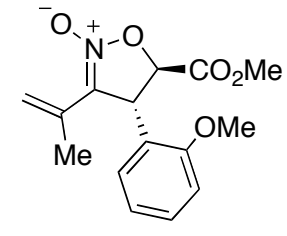

19p

$75 \%$, $81 \%$ e.e.

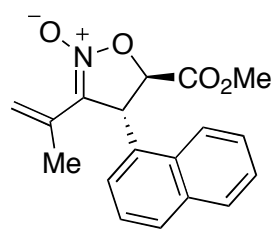

$19 q$ 70\%, 93\% e.e.

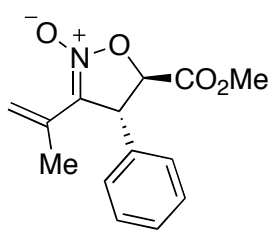

$19 r$ $80 \%, 85 \%$ e.e.

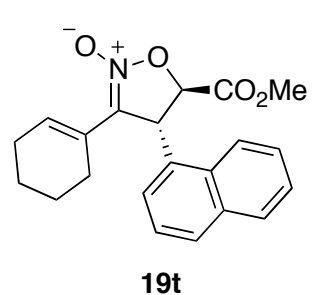

56\%, $91 \%$ e.e.

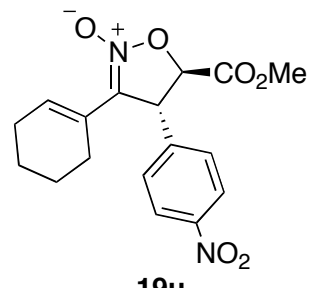

$19 u$

$93 \%, 65 \%$ e.e. 
As shown in Table 13, the reaction tolerates various nitroalkenes, including aryl, alkyl and cyclic structures. Moreover, various aldehydes, including aromatic, aliphatic and heteroaromatic substrates, were also suitable for this transformation. Compared to the nitroalkenes, the aldehyde substrates indicated stronger influence to the overall yield and stereoselectivity. With electron deficient aldehyde (i.e. 19h), excellent yield was obtained. However, the enantioselectivity was slightly decreased. This could be caused by the higher reactivity of the diene intermediate, which led to the lower efficiency in the DKR step. Sterically hindered aldehydes (i.e. 19f), on the other hand, promoted the stereoselectivity by providing better spatial control (larger difference of the reaction rates for the two $Z / E$ isomers). As a result, excellent enantioselectivity were obtained. With the same reason, good enantioselectivity was also received for aliphatic aldehyde (19g), though in moderate yield. Similar steric-electronic effect of the aldehyde was observed for all the other substrates tested.

\subsubsection{Further application of isoxazoline- $\mathrm{N}$-oxide product in total synthesis of (-) Clausenamide derivatives}

\subsubsection{Asymmetric synthesis of substituted $\gamma$-lactam}

This new enantioselective isoxazoline-N-oxides synthesis not only provided a general, highly efficient approach in preparing isoxazolines, but also gave opportunity to reach complex molecules in simple steps. One particularly interesting class of molecules is the $\gamma$-lactams due to their significance in biological and pharmatheutical research.

As shown in Scheme 25A, during our previous study, the reductive opening of isoxazoline ring could be highly diastereoselective, while treating homo isoxazoline derivative with $\mathrm{LiAlH}_{4}$ gave the ring opening product $\beta$-amino alcohol as a single

diastereomers. ${ }^{30}$ This stereoselective reduction was probably achieved through a diastereoselective reduction of imine intermediate with possible metal chelated sixmember-ring transition state. Encouraged by this result, we envisioned that compounds 19 could be applied as common precursors for the stereoselective construction of $\gamma$ lactams 20 through one simple reduction step (Scheme 26B). The key concern for this 
transformation is the diastereoselectivity, especially on the C-5 position, which will be determined by the imine reduction.

\section{Scheme 26. Diastereoselective ring opening to reach $\gamma$-lactam}
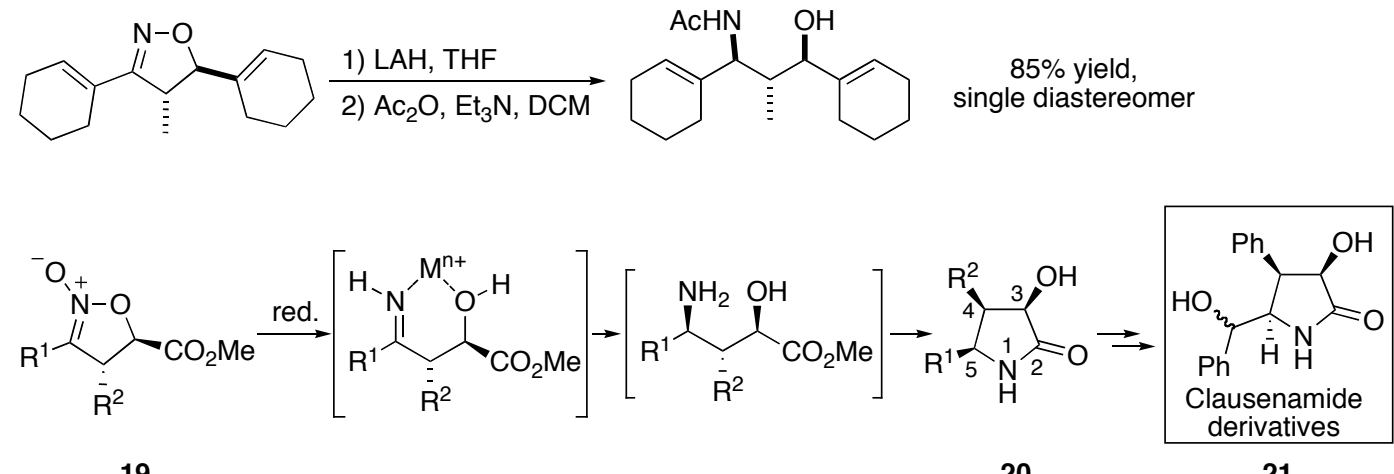

19

20

21

Furthermore, we expected simple transformations from $\mathbf{2 0}$ would lead to the Clausenamide derivatives $\mathbf{2 1},{ }^{33}$ which are a class of natural products with $\gamma$-lactam moieties and concise but complicated stereochemistry. As a model study, different reduction methods were applied to 19a targeting the $\gamma$-lactam 20 and the Clausenamide derivatives 21. The proposed synthetic pathway was described in Scheme 27.

\section{Scheme 27. Retrosynthetic design of Clausenamide derivatives 20}

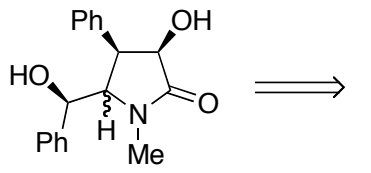

21

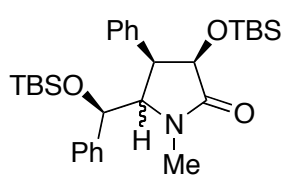

25'<smiles>O=C1N[C@H]([C@H](O)c2ccccc2)[C@@H]([PH2+])[C@H]1O</smiles>

25<smiles>C=CCCC=CCCC=CC</smiles>

A few simple steps were expected to reach the target molecule. The final product 21 could be obtained from the TBAF deprotection of di-TBS silyl ether 25', which was methylated after TBS protection from 25. The $\gamma$-lactam 25 could be chemoselectively reduced from the hydroxyl isoxazoline-N-oxide $\mathbf{2 3}$ to open the heterocycle and generate the amino alcohol 24 followed by the expected intramolecular amino-ester exchange. Ozonolysis or $\mathrm{OsO}_{4} / \mathrm{NaIO}_{4}$ oxidation could readily convert the terminal double bond into 
ketone, after which diastereoselective reduction would be applied to reach to $\mathbf{2 3}$ from isoxazoline-N-oxide 19a.

\subsubsection{Asymmetric synthesis Clausenamide analogue}

\section{Scheme 28. First attempt based on the proposed synthetic pathway}

(A)
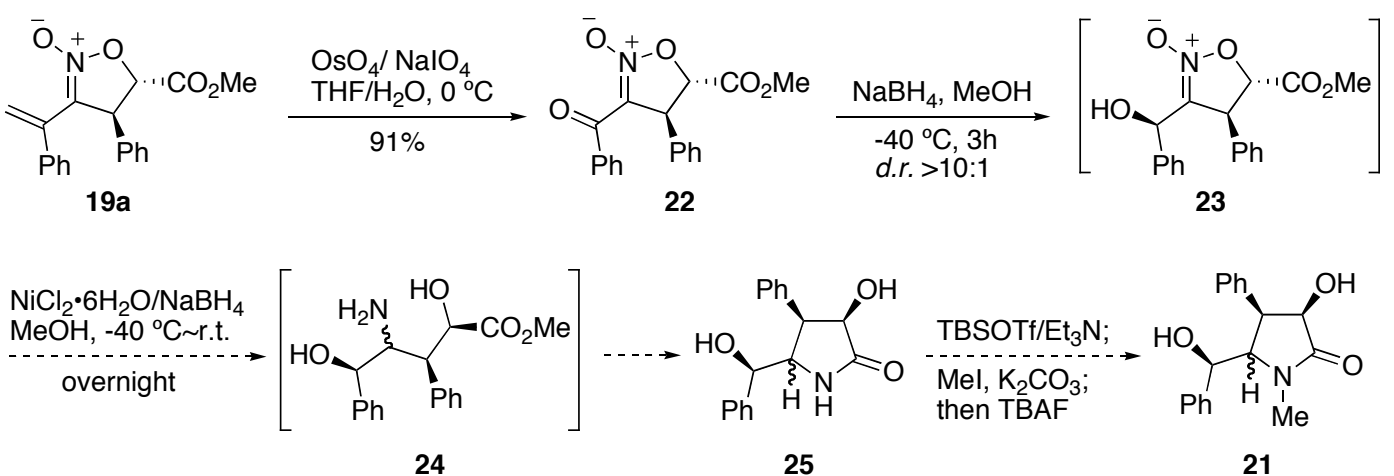

Efforts were then put in to the designed synthesis pathway. (Scheme 28A) As expected, 19a was oxidized to reach ketone 22 in 91\% yield. Simple $\mathrm{NaBH}_{4}$ reduction was applied under low temperature $\left(-40^{\circ} \mathrm{C}\right)$ to reach alcohol 23 . The reaction revealed a high diastereo ratio $(>10: 1)$, based on both TLC detection and in situ NMR study. However, this hydroxyl isoxazoline-N-oxide was quite unstable toward room temperature and acidic conditions. Thus, one more reductive operation was followed up in one pot. $\mathrm{NiCl}_{2} \cdot 6 \mathrm{H}_{2} \mathrm{O} / \mathrm{NaBH}_{4}$ was reported as one of the typical choices to chemoselectively reduce isoxazoline type of heterocycles. But the performance of this reduction failed to yield the desired di-ol $\gamma$-lactam, whose hydroxyl groups were supposed to be protected by TBSOTf. The problem could be, also confirmed in later studies, the sensitivity of the $\alpha$ oxy ester moiety to $\mathrm{NaBH}_{4}$. So, alternative routes were then planned and carried out (Scheme29).

Since the alcohol $\mathbf{2 3}$ in previous attempt was quite fragile, we thought it might be due to the high valence of the nitrogen in the isoxazoline-N-oxide heterocycle (Scheme 29B). Thus, 19a was reduced to isoxazoline using $(\mathrm{MeO})_{3} \mathrm{P}$ as solvent under $115^{\circ} \mathrm{C}$ reflux, after which $\mathrm{OsO}_{4} / \mathrm{NaIO}_{4}$ oxidation was then applied to give ketone 26 in $72 \%$ yield for 2 steps. $\mathrm{NaBH}_{4}$ reduction under low temperature, alcohol 27 was obtained in 
$86 \%$ yield with $d . r .8: 1$. Notably, the $\alpha$-oxy ester moiety was very sensitive to $\mathrm{NaBH}_{4}$ environment. Any reductant in excess tended to reduce the ester into alcohol (see experimental section). Three different reductive conditions were tested for the ringopening step to reach the amino alcohol 28, but no product was observed. $\mathrm{NiCl}_{2} \bullet 6 \mathrm{H}_{2} \mathrm{O} / \mathrm{NaBH}_{4}$ reduction presented the previous problem, while $\mathrm{Pd} / \mathrm{C} \mathrm{H}_{2}$ reduction stopped at the diol oxime. During the treatment of $\mathrm{Zn} / \mathrm{H}^{+}, 27$ did disappear on TLC, but no desired product was successfully isolated. The desired product $\mathbf{2 5}$ is a diol lactam that is extremely polar and the $\mathrm{Zn}$ dust was used in great excess (20 eq.), both of which increased the difficulty for isolation process.

\section{Scheme 29. Another two alternative routes}

(B)
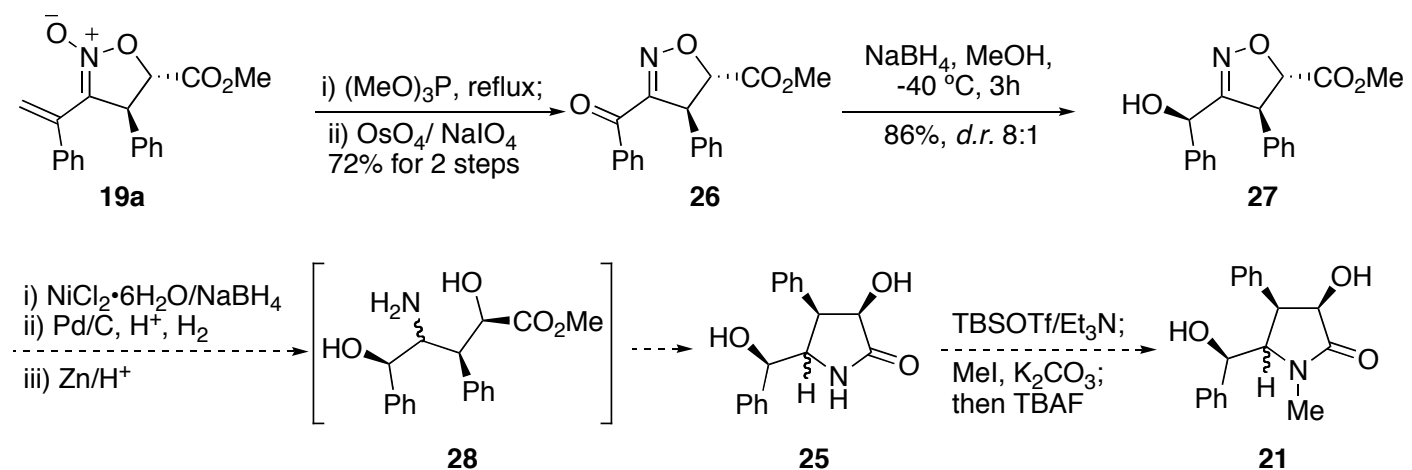

(C)

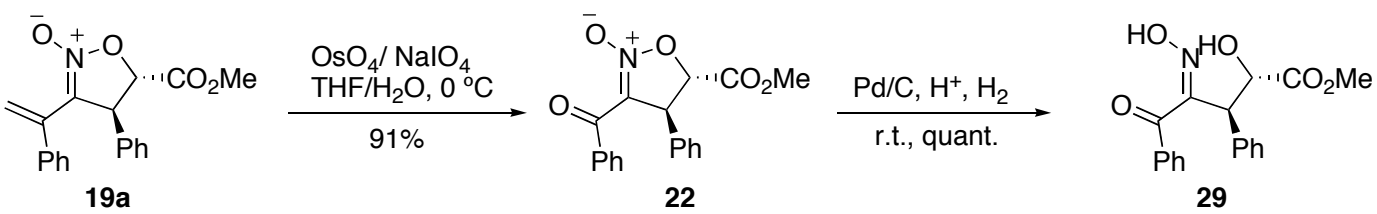

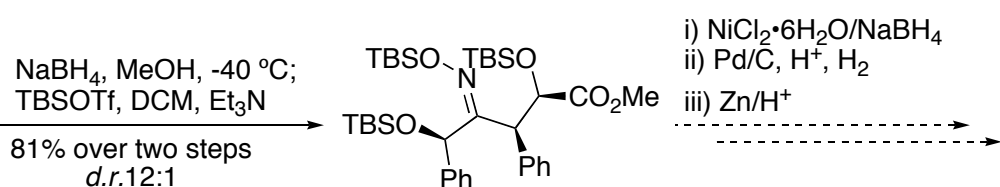

30

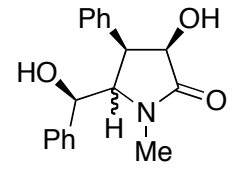

21

Another attempt started from $\mathrm{Pd} / \mathrm{C}, \mathrm{H}_{2}$ reduction of ketone isoxazoline-N-oxide 22. Hydroxyl oxime 29 was prepared in excellent yield from 19a. $\mathrm{NaBH}_{4}$ reduction followed by TBSOTf was then applied for the protection of alcohols, yielding the tri-TBS 
silyl ether in great yield. However, further reduction of the oxime silyl ether moiety failed to generate the amine group (Scheme 29C).

\section{Scheme 30. $\mathrm{Zn} / \mathrm{H}^{+}$reduction of vinyl isoxazoline-N-oxide 19a}
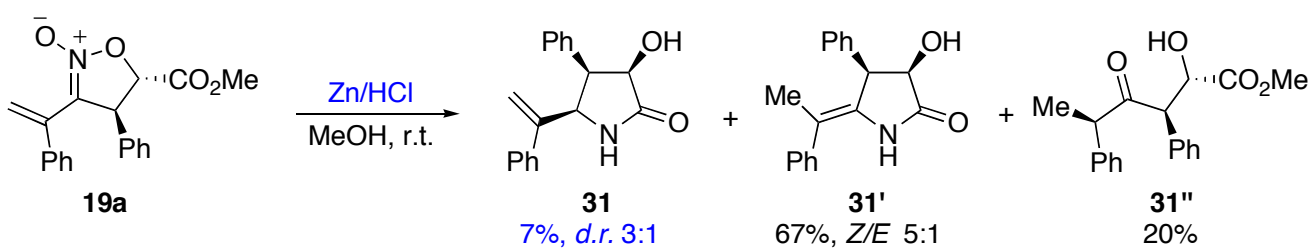

Although it requires only a few oxidation and reduction steps to reach the target molecule, the myth of this five-membered heterocycle certainly has a secret but specific map, which had to be probed by different combination of available operation techniques. Not long after, when attempt was put in for the reduction of $19 \mathrm{a}$ by $\mathrm{Zn} / \mathrm{H}^{+}$, two $\gamma$-lactams were tested after the full conversion of the starting material (Scheme 30). One is the vinyl $\gamma$-lactam 31' which was the major product (67\% yield, $Z / E 5: 1$ ), the other is the allylic $\gamma$ lactam 31. Although only 7\% yield (d.r. 3:1), this finding certainly lit up our hope towards the target molecule 21.

\section{Scheme 31. Synthesis of 3-ent-6-ent-Clausenamide 33}

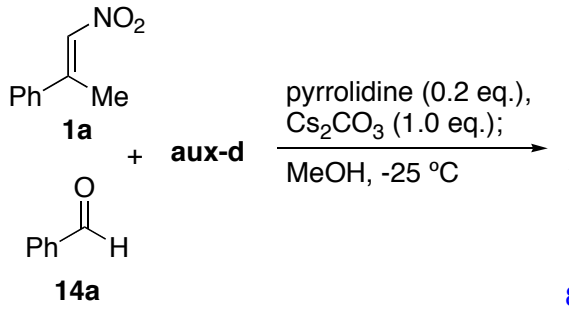

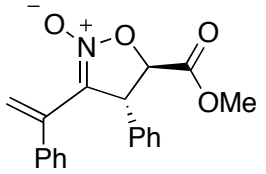

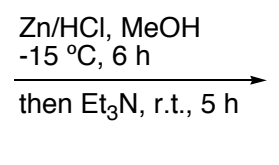

$19 a, 81 \%$ yield, $89 \%$ e.e., gram scale

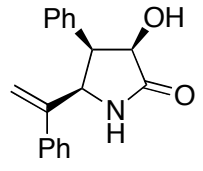

31

$57 \%$ isolated yield

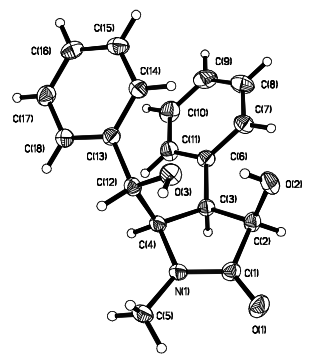

Crystal structure of $\mathbf{3 3}$

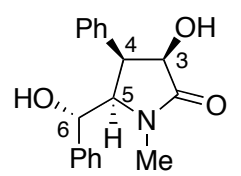

$33,85 \%$ e.e.

3-ent-6-ent-Clausenamide

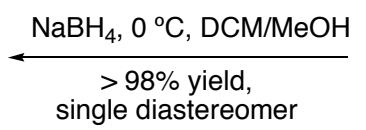

1. Mel, tBuONa, Acetone, $0^{\circ} \mathrm{C}$ 2. $\mathrm{OsO}_{4} / \mathrm{NaIO}_{4}, \mathrm{THF} / \mathrm{H}_{2} \mathrm{O}$.
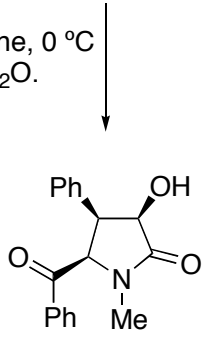

32

$79 \%$ isolated yield over two steps 
After careful condition optimization, the yield of the desired molecule $\mathbf{3 1}$ raised up to 57\% yield. As shown in Scheme 31, the Clausenamide derivatives 33 was prepared within 5 steps from nitroalkene 1a with $38 \%$ overall yield, in gram scale. The synthesis involved neither exotic reagents nor protecting groups, which gave the high atomic efficiency. Moreover, excellent stereochemistry retention was achieved in this transformation, where 33 was prepared in $85 \%$ e.e.. One recrystallization operation easily improved the purity to $>99 \%$ e.e. (determined by chiral HPLC, experimental section). The reduction of $\mathbf{3 2}$ was highly diastereoselective and the relative stereochemistry of $\mathbf{3 3}$ was undoubtedly confirmed by X-ray crystallography, as an all-cis- $\gamma$-lactam.

Notably, the Clausenamide derivative $\mathbf{3 3}$ is one of the most challenging isomers in the Clausenamide family due to its all-cis-substitution on the $\gamma$-lactam ring. With the reported synthetic route, preparation of different Clausenamide derivatives, including different substitute groups on C-4 and C-5 position and different stereoisomers at all four stereogenic centers, is accessible with simple modifications. Construction of compound library based on the Clausenamide core skeleton is currently undergoing in our group.

\subsection{Conclusion}

Although a facile proline Lewis base catalyzed isoxazoline-N-oxide synthesis was previously discovered in our group, three limitations needed to be extended in order to make the overall protocol more general and accessible towards the asymmetric synthesis of complex building blocks or even natural products.

The overall methodology was based on Lewis base catalyzed multi-component condensation of nitroalkene, aldehyde and sulfur ylide. This had allowed easy derivatization to achieve various substitutions at selected positions. Excellent yields and enantioselectivity were obtained when camphor derived sulfur ylide was applied. With the high efficiency and excellent chemo and enantioselectivity, this reported method had provided a new strategy for the preparation of isoxazoline derivatives, which could be applied either for the new drug candidate discovery or for the construction of complex building blocks. Successful transformation of isoxazoline-N-oxide 19a into the all-cis $\gamma$ lactam in a highly stereoselective fashion and gram-scale synthesis of Clausenamide 
analogue 9e further highlighted the advantage of the reported method as a powerful approach in complex molecules preparation with high atom efficiency and good stereoselectivity.

This project is collaborated with Lekh Nath S. Gautam, Dr. Jeffrey Petersen and Dr. Novruz Akhmedov. Lekh Nath helped with part of the substrates synthesis and supporting information preparation. Dr. Petersen helped with the X-ray crystallography study to determine the relative stereochemistry and structures of target compounds. Dr. Akhmedov carried out the extensive NMR studies of the derivatization products. 


\section{Part IV}

\section{One-Step Synthesis of Substituted Di-Hydrofurans from Lewis Base Catalyzed Three-Component Condensation}

\subsection{Introduction}

\subsubsection{Literature reported furan and di-hydrofuran synthesis}

Furans and their derivatives are an important class of compounds in chemical and biological research. ${ }^{34}$ The first documented approaches for the synthesis of substituted furan was the Feist-Bénary reaction, which was developed in the early $20^{\text {th }}$ century. 1,3di-carbonyl compounds were used to react with a-halo-ketones under basic conditions (Scheme 32). ${ }^{35}$ Some modified conditions have also been reported (often referred as "interrupted" Feist-Bénary reaction) to avoid the water elimination, giving the hydroxyl substituted hydrofurans. In 2005, Calter et. al. successfully developed a asymmetric pathway of the "interrupted" Feist-Bénary furan synthesis. ${ }^{36}$

\section{Scheme 32. First examples of furan derivatives synthesis known in literature}

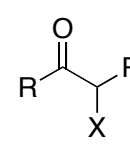

Feist-Bénary reaction

$+$

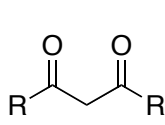

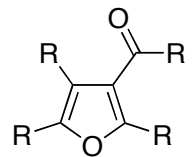

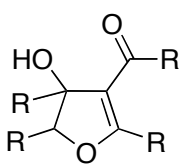

Meanwhile, oxidative coupling strategies had also been well studied. Two representative examples are Ce (IV) or Mn (III) oxidative coupling of 1,3 diketo functionalities and alkenes ${ }^{37}$ (Scheme 33A) and the palladium (II) catalysis via propargyl-allenyl isomerization and Alder-Ene transformation ${ }^{38}$ (Scheme 33B). 


\section{Scheme 33. Oxidative coupling and Pd (II) catalysis in di-hydrofuran syntheses}

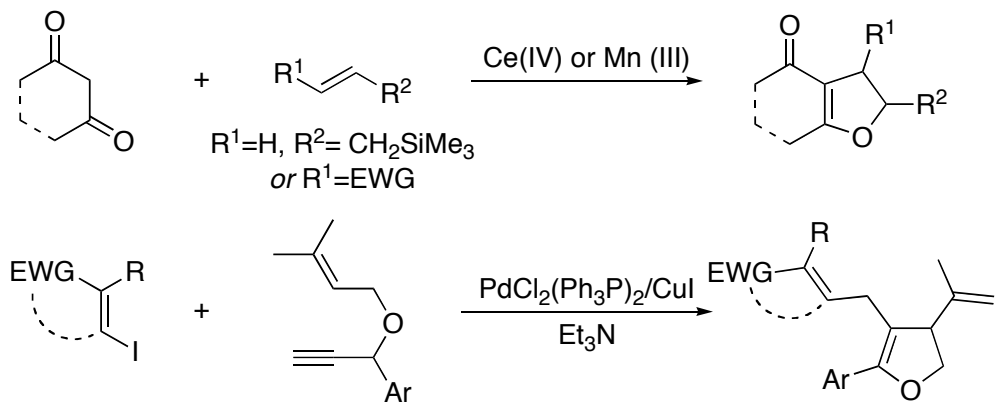

In 1967, during the investigation of cyclopropanation of $\alpha, \beta$-enones and sulfur ylides, Payne discovered a di-hydrofuran byproduct through a formal [4+1] annulation pathway. ${ }^{39}$ This finding had been well studied in recent years by Ma, ${ }^{40 \mathrm{a}}$ Liang $^{40 \mathrm{~b}}$ and Piras $^{40 c}$ (Scheme 34A). However, these strategies suffered either low diastereoselectivity problems or the competitive cyclopropanation side reaction. More recently, Tang and coworkers ${ }^{41}$ had reported an enantioselective synthesis of optical active di-hydrofurans via camphor derived ylide auxiliary. Excellent yields and enantioselectivity were received, although the substrate scope was limited (Scheme 34B). Moreover, these methods usually involved rather specific $\alpha$-ylidene- $\beta$-diketones and ylide auxiliaries, most of which were not readily available and lowered the atomic efficiency of the overall methods.

\section{Scheme 34. Formal $[4+1]$ annulation strategy for the synthesis of di-hydrofurans}

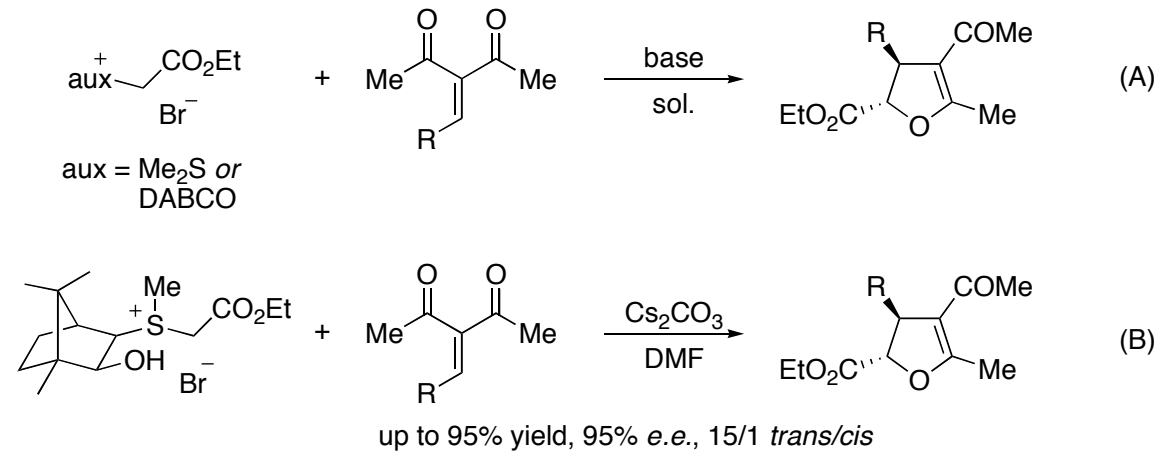

Thus, there is a strong desire for new method that overcomes all the previous problems and allows the easy preparation of dihydrofurans (DHF) with high atom efficiency and, more importantly, feasibility to assemble various substitution patterns. 


\subsubsection{Proposal for DHF synthesis inspired by our previous studies}

In our previous studies, several amine catalyzed new multi-component condensations had led to the formation of various heterocycles with good to excellent yields and stereoselectivity, represented by the isoxazoline-N-oxide synthesis (Scheme35A) and the 4,5-disubstituted 1,2,3-NH triazole synthesis (Scheme 35B).

\section{Scheme 35. Nitro group as leaving group in previous studies}

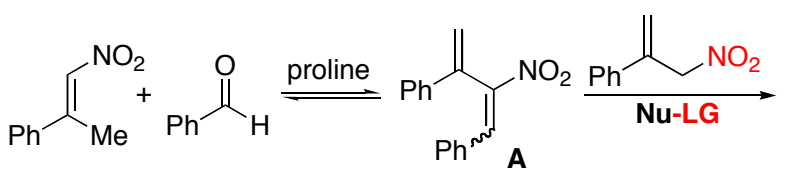<smiles>C=C(c1ccccc1)C1ON([O-])C(=C)C1c1ccccc1</smiles>

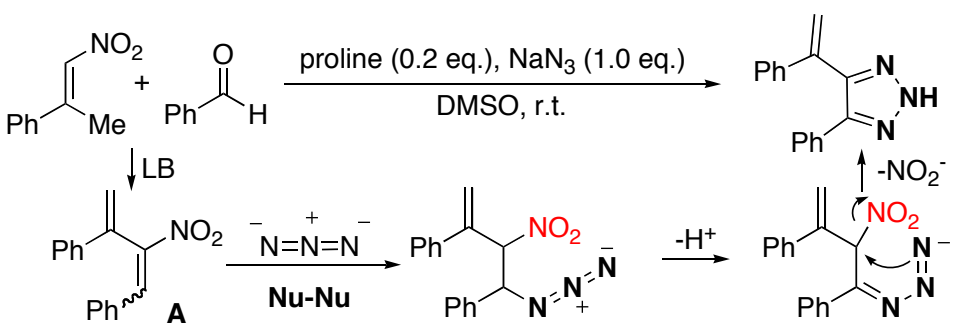

Both reactions required the formation of the nitro-diene intermediate $\mathbf{A}$ via the Henry-Aldol condensation of Lewis base (proline) activated nitroalkene and aldehyde. This highly reactive intermediate could then react with proper nucleophiles and precede the cascade processes. Mechanistically, one important message was that the allylic nitro groups were knocked away by $\mathrm{O}$ or $\mathrm{N}$ nucleophiles through intramolecular $\mathrm{SN}_{2}$ substitutions, under relatively mild conditions. As a leaving group, the elimination of $\mathrm{NO}_{2}{ }^{-}$usually requires harsher conditions (such as high temperature ${ }^{42}$, transition-metal assistance $^{43}$ or strong Lewis acids activation ${ }^{44}$ (Scheme 36).

Scheme 36. Reported removal of (allylic) nitro group by nucleophilic substitution<smiles>[R]C([N+]=O)C1=CCCCC1C</smiles>

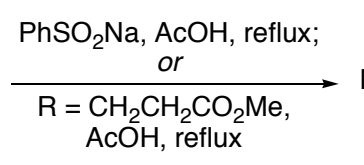<smiles>[R]C(C1=CCCCC1C)S(=O)(=O)Oc1ccccc1</smiles><smiles>CC1CCCC=C1C1CCC(=O)O1</smiles>

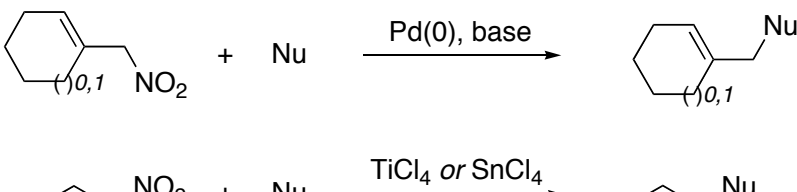

$$
\leadsto \mathrm{NO}_{2}+\mathrm{Nu} \stackrel{\mathrm{TiCl}_{4} \text { or } \mathrm{SnCl}_{4}}{\longrightarrow} \rightleftharpoons \mathrm{Nu}
$$


Thus, the effective substitution of allylic nitro group under mild conditions in our previous studies provided an appealing approach to further extend the cascade strategy for the stereoselective synthesis of complex building blocks.

Combining these mechanistic discoveries, we postulated that the treatment of reactants with "di-nucleophile" moieties to the nitro-diene intermediate A could lead to the facile synthesis of complex functionalized cyclic molecules in a "one-pot" fashion (Scheme 37A). When the 1,3-diketo functionality was applied, we envisioned that an $\mathrm{O}$ substitution of allylic nitro group would present the di-hydrofuran moieties under mild basic conditions. (Scheme 37B)

\section{Scheme 37. Proposed di-hydrofuran synthesis}
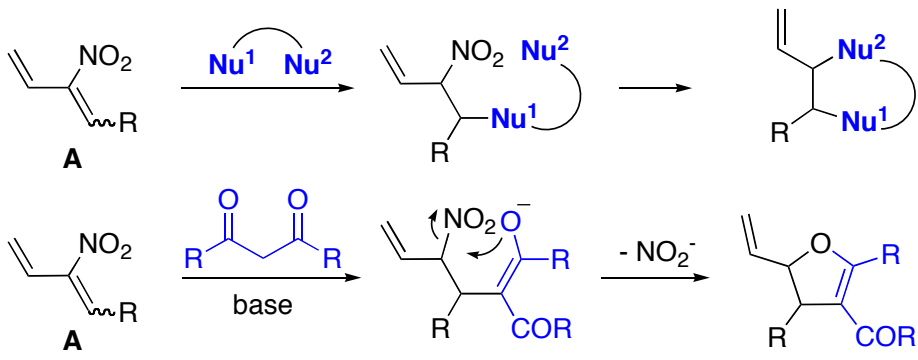

\subsection{Research Objectives}

As proposed in last section, the Lewis base catalyzed three-component cascade synthesis of di-hydrofuran will be examined. The validity of 1,3-diketo molecules as dinucleophiles will be tested. The proposed elimination of nitro group will be studied during the condition screening. Different nitroalkenes, aldehydes and di-nucleophiles will be applied as the substrate scope exploration. More importantly, the Lewis base catalysis mechanism should be tested to confirm the secondary amines are effective catalysts.

\subsection{Results and Discussion}

\subsubsection{Reaction condition screening}

To testify our hypothesis, reactions between nitroalkene 1a, aldehyde $\mathbf{2 a}$ and cyclohexane-1,3-dione 3a were investigated (Table 14). The competing side reactions for this three-component condensation included the formation of isoxazoline $1 \mathbf{5 f}$ and 
nitroalkene polymerization. To our please, the desired DHF 35a was formed when treating the three starting materials with proline, though with low yield ( $30 \%$, entry 1$)$. Significant nitroalkene polymerization was observed in this case. Since one equivalent of $\mathrm{HNO}_{2}$ would be generated in this process, various bases were added to balance the acidity of the overall reaction. With the application of 1.0 equivalent of $\mathrm{K}_{2} \mathrm{CO}_{3}$, the yield of DHF was significantly improved ( $71 \%$, entry 2$)$.

Table 14. Reaction condition optimization ${ }^{a}$

\begin{tabular}{|c|c|c|c|c|c|c|c|}
\hline & & & & $35 a$ & & $15 f$ & \\
\hline \multirow{2}{*}{ entry } & \multirow{2}{*}{ cat. (eq.) } & \multirow{2}{*}{ base (eq.) } & \multirow{2}{*}{ sol } & \multirow{2}{*}{ time (h) } & \multirow{2}{*}{$\operatorname{conv}^{b}(\%)$} & \multicolumn{2}{|c|}{ yield $(\%)^{c}$} \\
\hline & & & & & & $4 a$ & $5 \mathrm{a}$ \\
\hline 1 & proline ( 0.2 eq. $)$ & -- & DMSO & 2 & 77 & 30 & 11 \\
\hline 2 & proline ( 0.2 eq.) & $\mathrm{K}_{2} \mathrm{CO}_{3}(1.0)$ & DMSO & 2 & 100 & $71^{d}$ & 8 \\
\hline 3 & -- & $\mathrm{K}_{2} \mathrm{CO}_{3}(1.0)$ & DMSO & 2 & 90 & 22 & $<5$ \\
\hline 4 & proline ( 0.2 eq.) & $\mathrm{K}_{2} \mathrm{CO}_{3}(0.5)$ & DMSO & 2 & 100 & $80^{d}$ & $<5$ \\
\hline 5 & proline ( 0.1 eq.) & $\mathrm{K}_{2} \mathrm{CO}_{3}(0.5)$ & DMSO & 3 & 100 & $87^{d}$ & $<5$ \\
\hline 6 & proline ( 0.05 eq.) & $\mathrm{K}_{2} \mathrm{CO}_{3}(0.5)$ & DMSO & 4 & 100 & $87^{d}$ & $<5$ \\
\hline 7 & proline ( 0.02 eq.) & $\mathrm{K}_{2} \mathrm{CO}_{3}(0.5)$ & DMSO & 8 & 100 & $74^{d}$ & $<5$ \\
\hline 8 & proline ( 0.05 eq.) & $\mathrm{K}_{2} \mathrm{CO}_{3}(0.5)$ & DMSO & 4 & 100 & $91^{e}$ & $<5$ \\
\hline 9 & proline ( 0.05 eq.) & other bases ${ }^{f}$ & DMSO & 4 & 100 & $<85^{e}$ & $<5$ \\
\hline 10 & proline ( 0.05 eq.) & $\mathrm{K}_{2} \mathrm{CO}_{3}(0.5)$ & solvents $^{\mathrm{g}}$ & 4 & 100 & $<72^{e}$ & $<5$ \\
\hline 11 & $\mathrm{PPh}_{3}$ (0.2 eq.) & $\mathrm{K}_{2} \mathrm{CO}_{3}(0.5)$ & DMSO & 3 & 100 & 26 & 18 \\
\hline 12 & $\mathrm{Et}_{3} \mathrm{~N}(0.2 \mathrm{eq})$ & $\mathrm{K}_{2} \mathrm{CO}_{3}(0.5)$ & DMSO & 8 & 100 & 37 & $<5$ \\
\hline 13 & DMAP (0.2 eq.) & $\mathrm{K}_{2} \mathrm{CO}_{3}(0.5)$ & DMSO & 8 & 100 & 30 & 21 \\
\hline 14 & glycine (0.2 eq.) & $\mathrm{K}_{2} \mathrm{CO}_{3}(0.5)$ & DMSO & 4 & 100 & $69^{e}$ & $<5$ \\
\hline
\end{tabular}

${ }^{a}$ 1a:14f:34a $=1: 1: 1$, concentration of $14 f$ is $0.15 \mathrm{M} ;{ }^{b}$ based on the consumption of nitroalkene 1a. ${ }^{c}$ NMR yield with 1,3,5-trimethoxybenzene as internal standard. ${ }^{d}$ isolated yields. ${ }^{e}$ isolated yield based on aldehyde, with the loading of 1a:14f:34a = 1.2:1.0:1.1, concentration of $\mathbf{1 4 f}$ is $0.15 \mathrm{M} .{ }^{f}$ other bases include $\mathrm{Cs}_{2} \mathrm{CO}_{3}, \mathrm{NaOAc}$, $t \mathrm{BuONa}, \mathrm{Et}_{3} \mathrm{~N}$ and DIPEA. ${ }^{g}$ solvents include DCM, EtOAc, acetone, toluene and THF.

Notably, a much significantly lower yield was received when only $\mathrm{K}_{2} \mathrm{CO}_{3}$ was applied (22\%, entry 3$)$. These results were consistent with our previously reported proline-nitroalkene activation mechanism. Considering that strong basic conditions usually favored the undesired nitroalkene polymerization, the amount of $\mathrm{K}_{2} \mathrm{CO}_{3}$ was 
reduced to 0.5 equivalent. Higher yield of $\mathbf{3 5 a}$ was received as expected ( $80 \%$, entry 4$)$. Interestingly, decreasing the loading of proline resulted in better performance with only a slight increase in reaction time (entries 5-7). This could be explained by the relatively slower nitroalkene polymerization associated with the lower Lewis base loading. With this optimal condition, excellent yield of DHF 35a was received as the single trans isomer, when slightly excess amount (1.2 equiv.) of nitroalkene was applied ( $91 \%$, entry 8). Different bases (such as DIPEA, $\mathrm{Et}_{3} \mathrm{~N}$ and $\mathrm{Cs}_{2} \mathrm{CO}_{3}$ ) and various solvents have also been investigated and $\mathrm{K}_{2} \mathrm{CO}_{3}$ (0.5 eq.) in DMSO was confirmed as the optimal choice. Different Lewis base catalysts, such as $\mathrm{PPh}_{3}$ and DMAP, have also been applied to catalyze this reaction. However, much lower yields were obtained (entries 11, 12) along with the formation of significant amounts of $\mathbf{1 5 f}$.

In addition, no cis diastereo isomers or cyclopropanation products were detected in all cases. The competing side reaction, homo-isoxazoline-N-oxide formation, was also successfully diminished. These results highlighted the unique reaction nature of proline catalyzed nitroalkene activation in cascade syntheses.

\subsubsection{Substrate scope}

Various nitroalkenes, aldehydes and 1,3-diketones/ $\beta$-keto-esters were then applied to investigate the reaction substrate scope (Table 15). This new method worked great for a big variety of substrates, giving the desired trans DHF in good to excellent yields. Both aromatic nitroalkene and aliphatic nitroalkene were suitable for this transformation, giving good diversity on the $\mathrm{C}-5$ position. Meanwhile, the allylic ether functionality was introduced, providing a new "synthetic handle" to the product. A large group of different aldehydes, including aromatic (with either electron donating groups or electron withdrawing groups), aliphatic and heterocyclic structures, were all suitable for this reaction, which provided an efficient strategy to introduce different carbon-substitute groups on the $\mathrm{C}-4$ position. The efficient assembly of readily available diverse groups on the C-4 and C-5 position made the reported method highly efficient for the preparation of functional substituted DHF that would be challenging to reach via other methods. 
Table 15. Substrate scope ${ }^{a}$

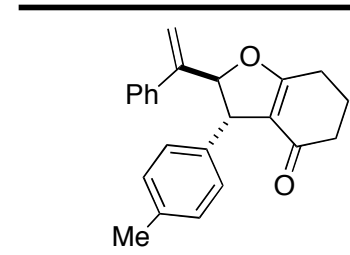

35a, $91 \%$

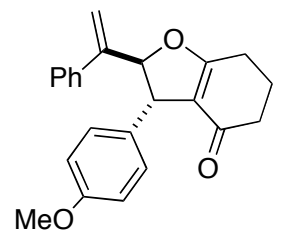

$35 \mathrm{~b}, 82 \%$

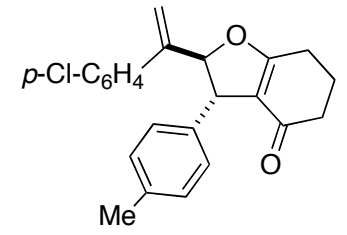

35c, $92 \%$<smiles>C=C(c1ccccc1)C1OC(C)=C(C(C)=O)[C@H]1c1ccc(C)cc1</smiles>

35 e, $86 \%$<smiles>C=C(c1ccccc1)C1OC(C)=C(C(C)=O)C1c1ccc([N+](=O)[O-])cc1</smiles>

35f, $94 \%$

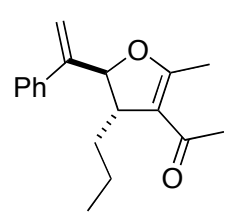

$35 \mathrm{~g}, 65 \%$

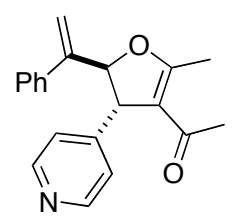

35h, $93 \%$<smiles>C=C(c1ccccc1)[C@H]1OC2=C(C(=O)CCC2)[C@H]1c1cccs1</smiles>

35d, $81 \%$<smiles>C=C(c1ccccc1)[C@H]1OC(C)=C(C(C)=O)[C@H]1c1ccco1</smiles><smiles>C=C(c1ccccc1)C1OC(C)=C(C(C)=O)[C@H]1c1ccc(C)cc1</smiles>

$35 \mathbf{j}, 89 \%$<smiles>C=C(c1ccco1)C1OC(C)=C(C(C)=O)[C@H]1c1ccc(OC)cc1</smiles>

$350,83 \%$<smiles>COC(=O)C1=C(C)OC(C2=CCCCC2)[C@H]1c1ccc(C)cc1</smiles>

35s, $85 \%$<smiles>C=C(c1ccccc1)C1OC(C)=C(C(=O)OC)[C@H]1c1ccc(F)cc1</smiles>

35k, $90 \%$<smiles>C=C(c1ccccc1)C1OC(C)=C(C(C)=O)[C@H]1CCc1ccccc1</smiles>

35I, $71 \%$<smiles>C=C(c1ccccc1)C1OC(C)=C(C(=O)OCC)[C@H]1c1ccc(C)cc1</smiles>

$35 \mathrm{~m}, 86 \%$

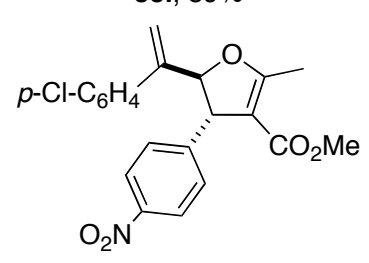

35n, $95 \%$

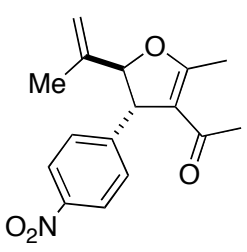

$35 q, 85 \%$

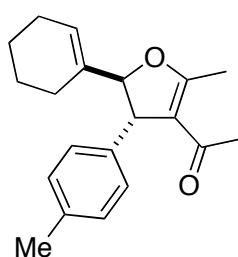

$35 t, 83 \%$

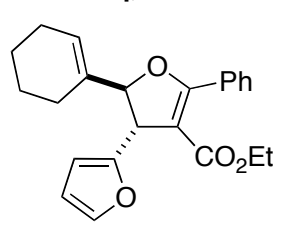

$35 u, 75 \%$

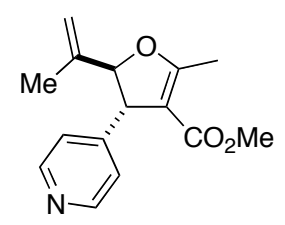

$35 r, 80 \%$

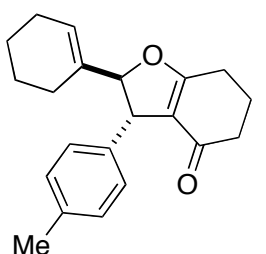

$35 v, 92 \%$

\footnotetext{
${ }^{a} \mathbf{1 a}: 14 a: 34 a=1.2: 1.0: 1.1$, concentration of $14 a$ is $0.15 \mathrm{M}$; yields are isolated yields; only trans isomers were observed.
}

The application of different 1,3-di-ketone and $\beta$-keto-esters further extended this method for easy introduction of various functional groups on C-2 and C-3 positions. In the case of $\beta$-keto-esters, good chemoselectivity was achieved, giving only the ketone cyclization products. This result gave advantage to selectively introduce different groups on either $\mathrm{C}-2$ or $\mathrm{C}-3$ positions through the reaction with corresponding di-nucleophiles (i.e. 4u). Notably, excellent diastereoselectivity was achieved in all cases, while only trans isomers were observed. Thus, with the great diversity, high efficiency and excellent 
diastereoselectivity, this reported method could be applied as a new general protocol for the synthesis of various functional di-hydrofuran building blocks.

\subsubsection{Mechanistic study of the proposed mechanism}

Although the proposed mechanism in Scheme 36 looked valid, another possibility was that the 1,3-diketones $\mathbf{3 4 b}$ would go through condensation with aldehyde $\mathbf{1 4 f}$ to give the di-carbonyl activated alkenes $\mathbf{B}$ as the intermediate. Nucleophilic addition of Lewis base activated nitroalkenes to $\mathbf{B}$ followed by the intramolecular $\mathrm{SN}_{2}$ substitution would then reach the product 35e (Scheme 38B). Simple experiment was then carried out to test the possible formal $[4+1]$ annulation pathway.

Scheme 38. Possible $[4+1]$ formal annulation pathway of the synthesis of DHF

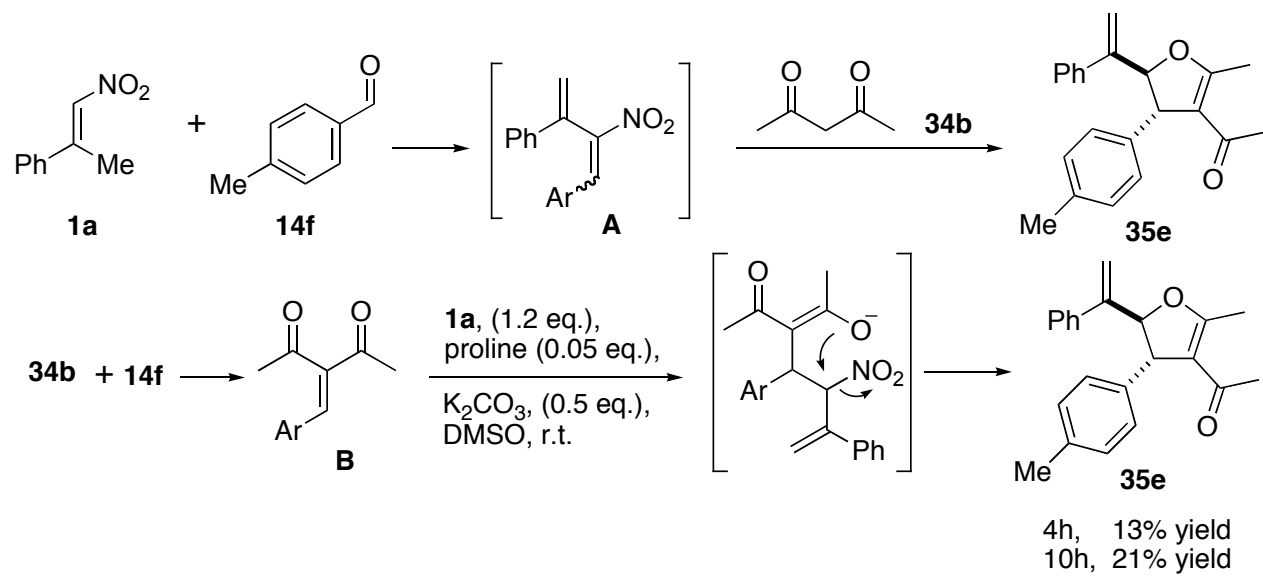

When the enone B (prepared from 34b and 14f) was treated with nitroalkene 1a under the optimal condition, only 13\% NMR yield was detected after 4 hours and 21\% yield after 10 hours, with large amount of $\mathbf{B}$ was left and $\mathbf{1 a}$ polymerized.

\subsubsection{Attempts to enantioselective synthesis and further transformation}

\subsubsection{Enantioselective synthesis study}

Efforts had been also put into the enantioselective synthesis of the highly functionalized di-hydrofurans. The catalysts tested are listed below in Scheme 38, however, no obvious enantiomeric excess was received. Similar to the previous asymmetric catalysis in the isoxazoline-N-oxide synthesis, the chiral secondary amines 
(cat-1, 2, 3, 5, 6) did catalyze the reaction and give moderate to good yields. The poor enantioselectivity ( $>10 \%$ e.e.) might be due to the poor involvement of the chiral amines as Lewis base with the diene intermediate $\mathbf{A}$.

\section{Scheme 39. Attempts towards asymmetric catalysis}

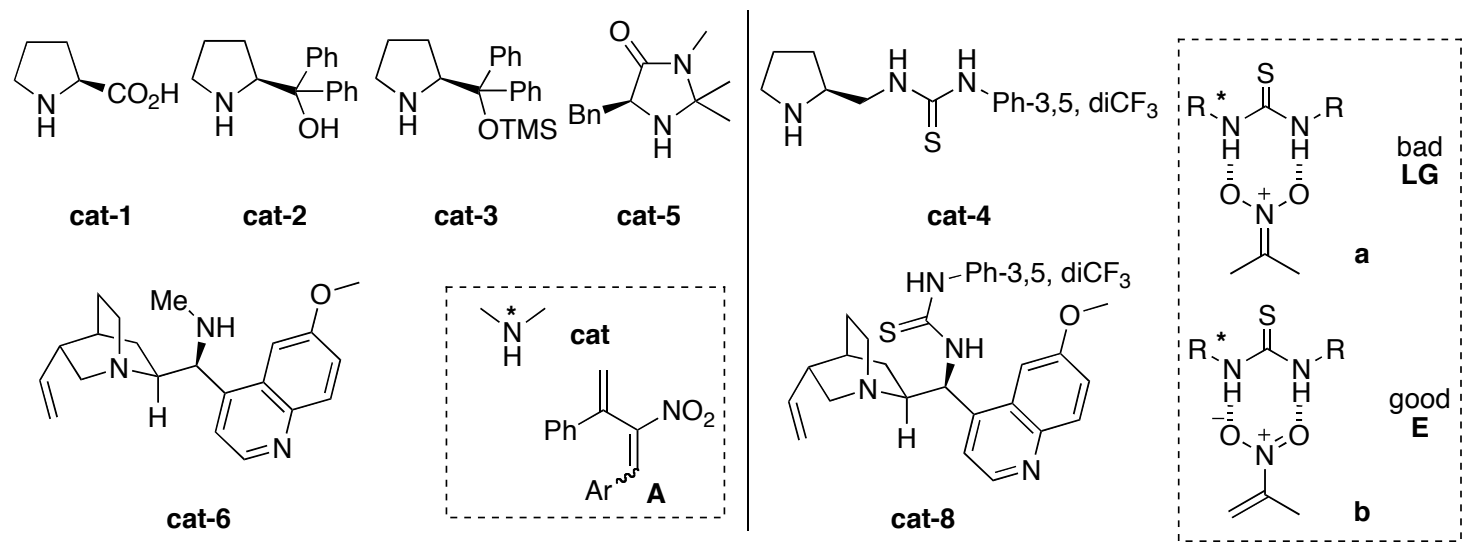

For the thio urea catalysts (cat-4 and cat-8), however, the reaction was dramatically shut down, only trace amount of product could be observed in the crude NMR. This could be reasoned that with the strong coordination of thiourea and nitro group, the $\mathrm{NO}_{2}$ containing moieties were either locked up (Scheme 39 a) and changed into a ineffective leaving group, or activated by the similar interaction to be a perfect electrophile (Scheme 39 b). Further attempts of other effective catalysts are underway.

\subsubsection{Further transformations}

With the highly functionalized di-hydrofurans, facile transformations could easily lead to natural product backbones and useful building blocks (Scheme 39). the allylic ether moieties could be readily applied to palladium chemistry to form the $\mathrm{Pd} \pi$-allyl complex a. the b-ether enone could be transformed into Danishefsky's diene b for inter(or intra-) molecular Diels-Alder reactions. Also, the integrated allylic vinyl ether moieties could be used to reach the Claisen rearrangement products c. On the other hand, functionalities on the aldehydes could also be utilized to reach the tricyclic (or tetracyclic) structures d (via intramolecular Heck coupling) or lectam moieties e (via amino ester exchange). Moreover, 2-keto-furan derivatives f could be easily prepared, 
which would be starting materials for the de novo synthesis of functionalized carbohydrates.

\section{Scheme 40. Possible Transformation and $\operatorname{Pd}(0)$ catalyzed dihydropyran synthesis}
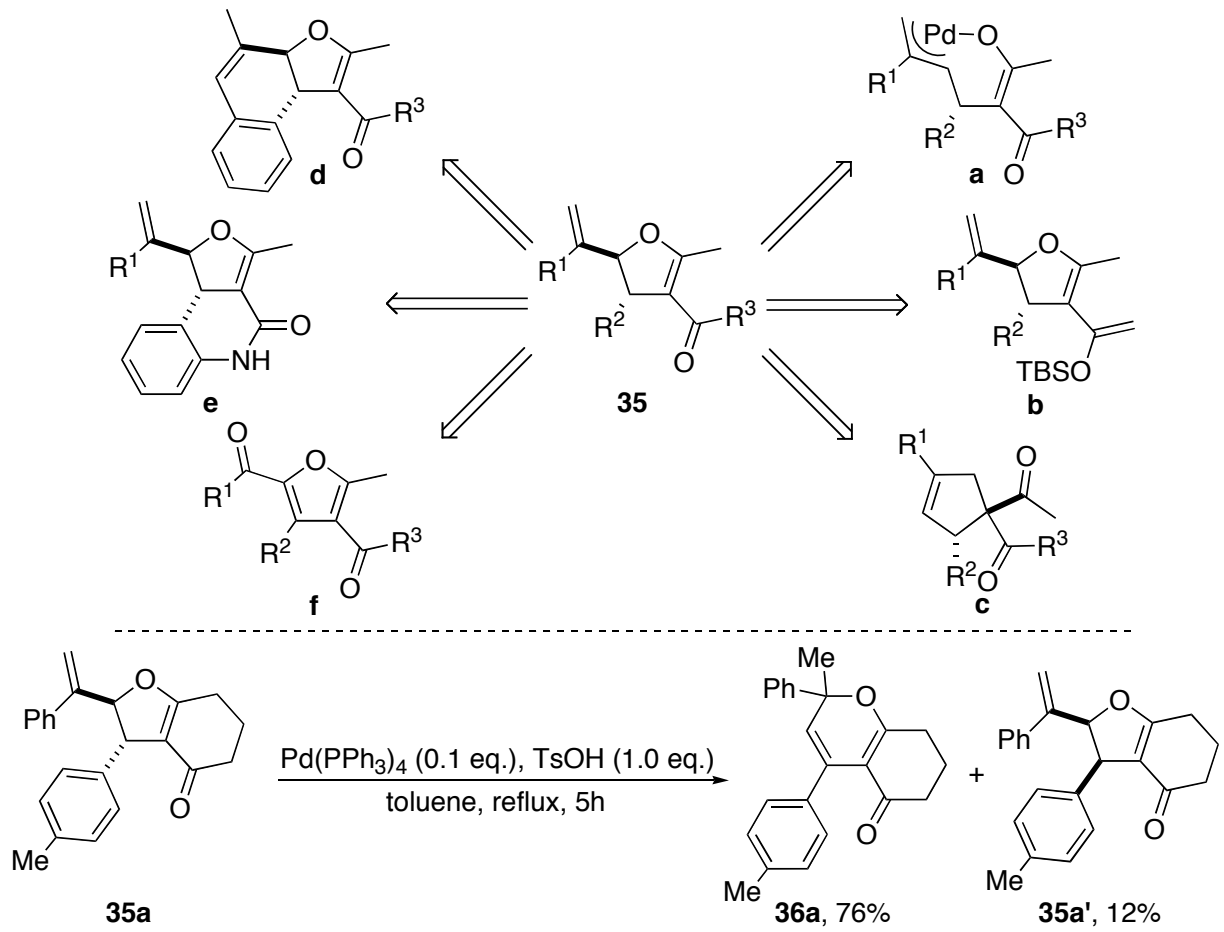

Up to date, the only one derivatization of the three-component di-hydrofurans is the palladium catalysis, which had presented unexpected results. When 35 was treated with $\mathrm{Pd}\left(\mathrm{PPh}_{3}\right)_{4}(0.1$ eq. $)$ under acidic condition (TsOH ,1.0 eq.) in toluene reflux for 5 hours, a dihydropyran 36a was obtained in good yield (76\%) with partial 35a transformed into its cis isomer 35a'. The reaction did not proceed without acid, nor only acid but no palladium catalyst. Detailed mechanistic study is underway to investigate this unexpected 1,4-hydride shift observation.

\subsection{Conclusion}

In conclusion, a highly efficient cascade synthesis of di-hydrofuran was developed through proline catalyzed one-pot three-component condensation of nitro alkene, aldehyde and 1,3-di-ketones/ $\beta$-keto-esters. This reaction used readily available starting materials under mild conditions and gave the desired products in excellent yields, 
excellent chemoselectivity and diastereoselectivity. Substituted groups on all the four positions of furan could be readily controlled with the applications of corresponding starting materials. With the great atomic efficiency and functional group tolerability, the reported methods would be of great interests for chemical and pharmaceutical researchers by providing a readily available compound library. In addition, the success of this method provided another strong support for the proposed secondary amine nucleophilic addition to nitroalkene. Further extension of this strategy with other plausible di-nucleophiles for new transformations is being examined. Enantioselective version of DHF synthesis and application in natural product synthesis are also under investigation in our group.

This project is a collaborated work with Tao Liao and Odbadrakh Tuguldur. They helped with some of the substrates preparation and the spectra organization. 


\section{Part V}

\section{One-Pot Asymmetric Synthesis of Substituted Piperidines by Exocyclic Chirality Induction}

\subsection{Introduction}

\subsubsection{Piperidines syntheses in literature and their bioactivity values}

Functionalized piperidines are one very important group of heterocycles in chemistry and biological research due to their unique chemical properties and important biological activity. ${ }^{45}$ Many biologically active natural compounds share the piperidines moieties in their skeletons, such like anopterine, scopolamine, himbacine and morphine ${ }^{45 \mathrm{~d}}$ (Scheme 41).

\section{Scheme 41. Biologically active piperidine moieties containing natural molecules}

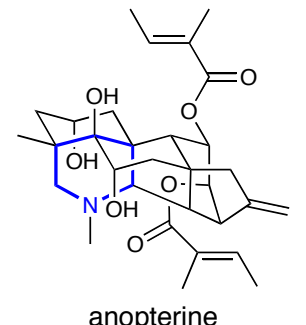

anopterine

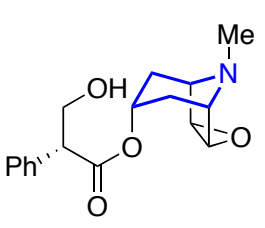

scopolamine

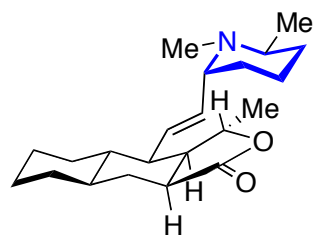

himbacine

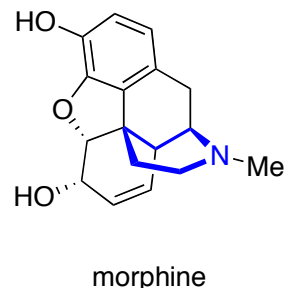

morphine

As a consequence, considerable interest is centered on the synthesis of this heterocycle with the focus on high efficiency and good stereoselectivity. ${ }^{46}$ This need is particularly emphasized by the fast growing call for enantiomeric pure, diverse piperidines in alkaloids ${ }^{47}$ and aza-sugar ${ }^{48}$ researches. Three examples are listed below, representing the recent efforts put in synthesizing piperidines, including the enantioselective total syntheses of natural product. (+)-nankakurines A and B had been successfully synthesized by Overman's group in $2008^{49 a}$ (Scheme 42A). The key step was the intramolecular azomethine imine [3+2] cycloaddition to reach the tetracyclic pyrazolidine, which then went thorough several steps to get the final product. Another elegant natural product synthesis via the piperidine intermediates was reported by Denmark's group in $2006^{49 b}$ (Scheme 42B). A tandem double intramolecular [4+2]/[3+2] cyclization of nitroalkene was developed with great diastereoselectivity. Catalyzed by 
$\mathrm{AlMe}_{3}$, at $-78^{\circ} \mathrm{C}$ in toluene, nitroalkene which contained a diene as the dienophile served well in the tandem cyclization to yield the nitroso acetal product in good yield. Subsequent functional group manipulations allowed for high yield synthesis of diphnilactone B. Also, an interesting [4+2] hetero-cycloaddition strategy was well developed by Nelson's group, targeting the diastereoselective synthesis of piperidines ${ }^{49 c}$ (Scheme 42C). Through the Lewis acid catalysis, the $\mathrm{N}$-alkenyl iminium was easily formed. In the presence of electron rich alkenes, a facile [4+2] cyclization was observed to yield the iminium product, which could be readily transformed into fully substituted piperidine in the presence of appropriate nucleophiles.

\section{Scheme 42. Literature reported piperidines syntheses}

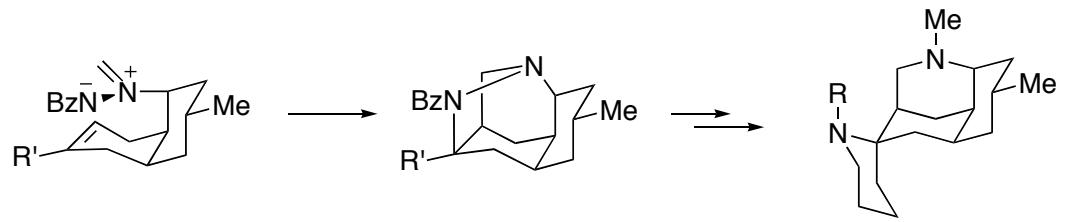

(+)-nankakurine $\mathrm{A}: \mathrm{R}=\mathrm{H}$
(+)-nankakurine $\mathrm{B}: \mathrm{R}=\mathrm{Me}$
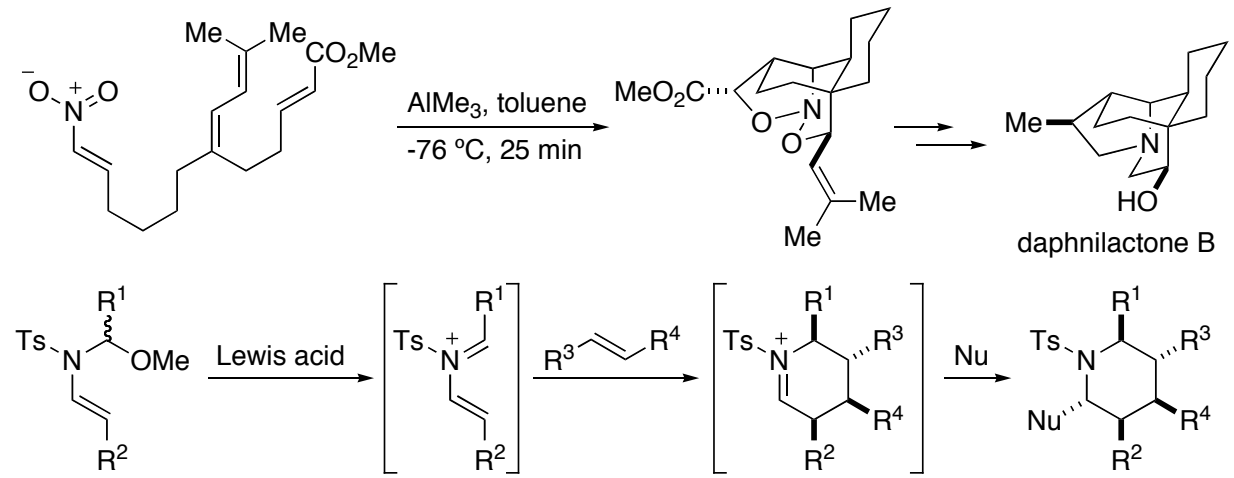

\subsubsection{Rationale of piperidine synthesis based on our previous studies}

Our group has recently focused on the secondary amine Lewis base activation of nitroalkenes. Related studies had been discussed in previous parts. Generally, secondary amine served as Lewis base to activate the $\beta$-alkyl nitroalkene and provided a stabilized $\alpha$-nitro carbanion (or nitronate) to react with electrophiles. After the $\beta$-elimination to release the Lewis base, a new covalent bond was formed with an allylic-nitro functionality (Scheme 43A) 


\section{Scheme 43. Proposed piperidine synthesis}

(A) $\mathrm{NH}$-amine conjugate addition promoted new cascade reactions

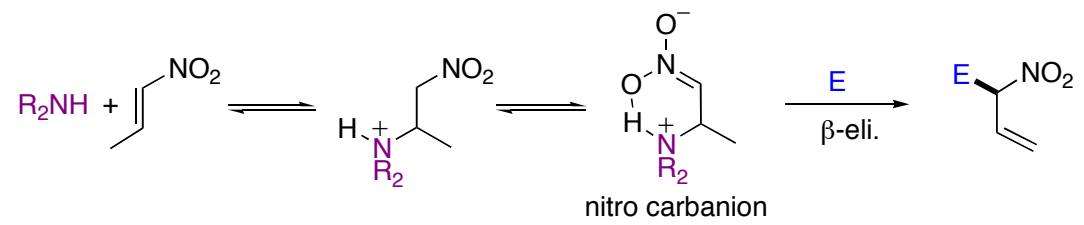

(B) Proposed one-pot cascade piperidine synthesis

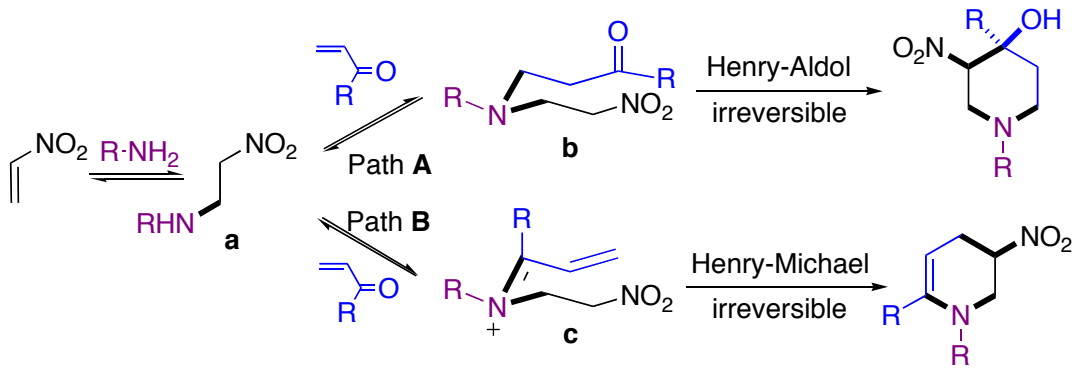

Revealed by our previous experimental and computational studies, the addition of amine to nitroalkene is a fast equilibrium. We then postulated that the amine-nitroalkene adduct a might be suitable for sequential Michael addition and ring closure, providing the substituted piperidines through a one-pot cascade process (Scheme 43B). From the azaMichael adduct a, tow ring-closing pathways were proposed, one was the Henry-Aldol reaction after the aza-Michael reaction between a and MVK (Path A); the other was the Henry-Michael reaction after the iminium formation from a and MVK (Path B).

\subsection{Research Objectives}

The three-component reaction between primary amine, nitroalkene and enones would be investigated. The two pathways proposed in Scheme 43B would be examined, or there might be other unpredicted reactions between these three components. The mechanism of the reaction should be explained with further understanding, which might be useful in our further research.

\subsection{Results and Discussion}

To test our hypothesis, reactions between nitroalkene 37a, benzyl amine 38a and enone 9b were set up. Surprisingly, the condensation between thess three components 
dominantly favored Path A, giving piperidine 39a in 65\% yield with d.r. 5:1 in $\mathrm{MeOH}$ after 14 hours, upon the first try. Notably, 30\% 37a was recovered.

\subsubsection{Reaction condition optimization ${ }^{a}$}

Table 16. Reaction condition screening

\begin{tabular}{|c|c|c|c|c|c|c|}
\hline & $37 a$ & $38 a$ & $9 b$ & & $39 a-m$ & \\
\hline entry & solvent & & base & time (h) & yield $(\%)^{b}$ & $d . r^{c}$ \\
\hline 1 & $\mathrm{MeOH}$ & & -- & 14 & 66 & $5: 1$ \\
\hline 2 & $\mathrm{MeOH}$ & & $\mathrm{K}_{2} \mathrm{CO}_{3}$ & 14 & 5 & -- \\
\hline 3 & $\mathrm{MeOH}$ & & DMAP & 14 & 65 & $5: 1$ \\
\hline 4 & $\mathrm{MeOH}$ & & $\mathrm{Et}_{3} \mathrm{~N}$ & 14 & 60 & $4: 1$ \\
\hline 5 & $\mathrm{MeOH}$ & & -- & 36 & 70 & $5: 1$ \\
\hline 6 & $\mathrm{MeCN}$ & & -- & 14 & 6 & -- \\
\hline 7 & DMSO & & -- & 14 & 19 & $4: 1$ \\
\hline 8 & $\mathrm{CH}_{2} \mathrm{Cl}_{2}$ & & -- & 14 & 40 & $10: 1$ \\
\hline 9 & THF & & -- & 14 & $82^{d}$ & $7: 1$ \\
\hline 10 & THF & & $\mathrm{Et}_{3} \mathrm{~N}$ & 14 & $80^{d}$ & $6: 1$ \\
\hline 11 & THF & & DMAP & 14 & $78^{d}$ & $7: 1$ \\
\hline 12 & THF & & -- & 36 & $97^{d}$ & $7: 1$ \\
\hline
\end{tabular}

${ }^{a}$ nitroalkene 37a:38a:9b $=1.0: 1.5: 2.0,\left(0.2 \mathrm{M}\right.$ for 37a). Bases were 0.2 eq.; ${ }^{b}$ yields of both isomers were determined by ${ }^{1} \mathrm{H}$ NMR with 1,3,5-trimethoxybenzene as internal standard; ${ }^{c}$ d.r. determined by ${ }^{1} \mathrm{H}$ NMR integration. ${ }^{d}$ isolated yields.

During the condition optimization (Table 16), we found that additional base $\left(\mathrm{K}_{2} \mathrm{CO}_{3}, 0.2\right.$ eq.) dramatically shut down the reaction (entry 2). However, when organic base like DMAP (0.2 eq.) or $\mathrm{Et}_{3} \mathrm{~N}$ (0.2 eq.) were applied, no great differences were observed in term of yield and $d . r$. . (entries 3,4) Extending the reaction time from 14 hours to 36 hours, slightly improved yield (70\%) was received. (entry 5) MeCN, DMSO and DCM were also tested as solvent, with much lower yields although the d.r. jumped up to 10:1 in DCM. (entries 6-8) When we switch to THF as solvent, $82 \%$ isolated yield and 7:1 d.r. were observed. (entry 9) Notably, the reaction performed in THF was much cleaner than other solvents, after which the product 39a-major could be even purified by simple crystallization. Additional DMAP and $\mathrm{Et}_{3} \mathrm{~N}$ did not improve the yields (entries 10, 
11). However, extending the reaction time to 36 hours in THF at room temperature, almost quantitative yield was received (97\%) with similar d.r. (7:1). (entry 12)

\subsubsection{Substrate scope}

\section{Table 17. Representative substrates ${ }^{a}$}

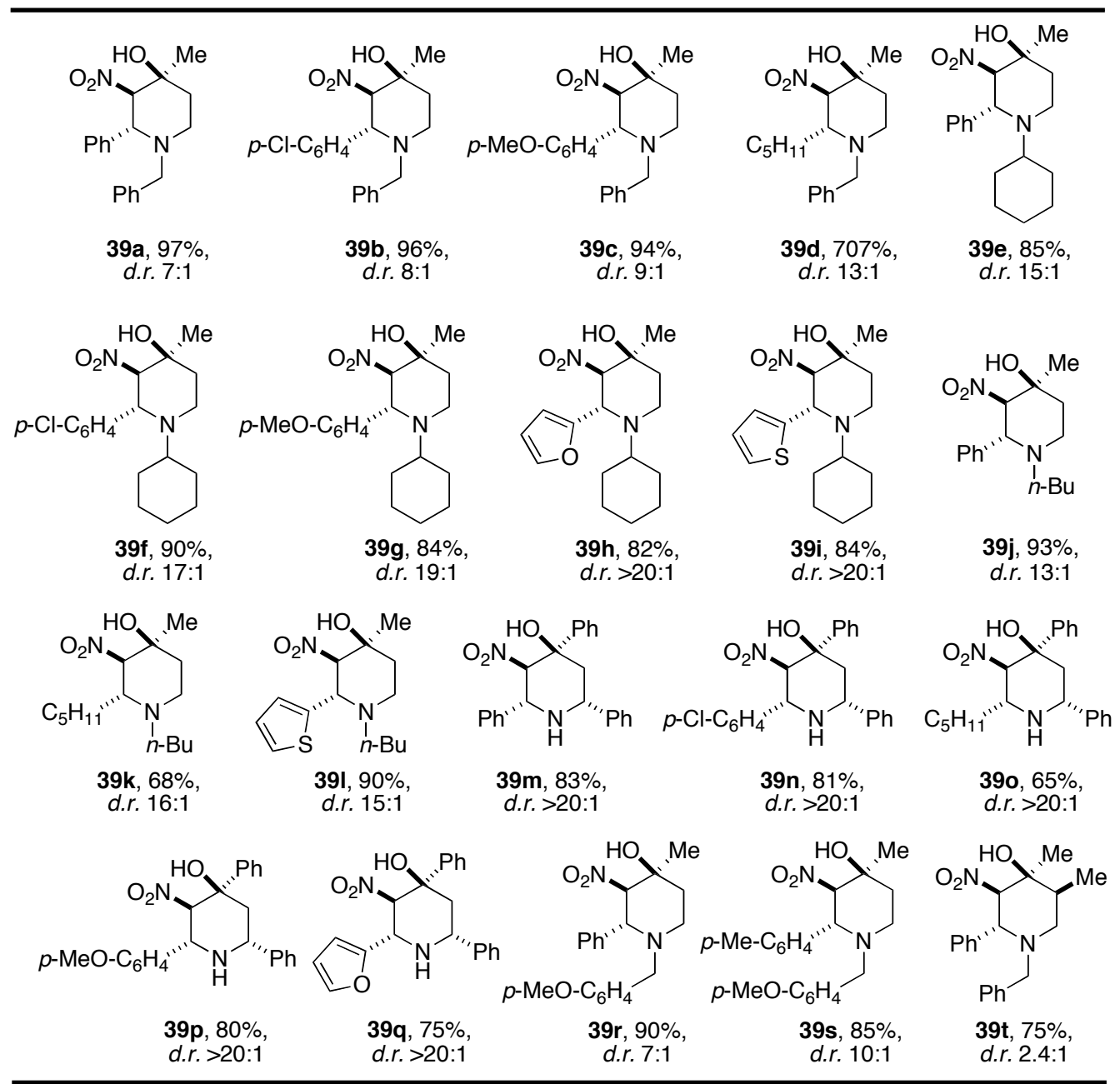

${ }^{a}$ General reaction conditions: compounds 37 (1.0 eq.), 38 (1.5 eq.), and $\mathbf{9}$ (2.0 eq.) were mixed in THF $(0.2 \mathrm{M}$ of 1). The reactions were monitored by TLC till $\mathbf{3 7}$ was totally consumed. ${ }^{b}$ Isolated yields of both isomers. ${ }^{c}$ d.r. determined by NMR of crude reaction mixtures. ${ }^{d}$ Structure and relative stereochemistry determined by X-ray crystallography.

As summarized in Table 17, a large group of substrates were suitable for this transformation, including alkyl and aryl nitroalkenes, primary amine even ammonia, and $\alpha / \beta$ substituted enones. Good to excellent diastereoselectivity was observed in all cases with only C-4 isomers obtained. The diastereo-selectivity can be improved by different amines, where cyclohexyl amine and $\mathrm{NH}_{3}$ gave excellent $d . r .(>20: 1)$. The wide scope of 
substrates, excellent yields and diastereo-selectivity made this method promising new protocol for the synthesis of substituted piperidines.

\subsubsection{Rationale for the high diastereoselectivity}

In all the substrates, although at least three stereogenic centers were set up at the same time, only C-4 isomers were detected, which was confirmed by the X-ray crystal structures (Scheme 43).

Scheme 44. Account for the high diastereoselectivity: the equilibrium of azaMichael addition of amine to nitroalkene and enone

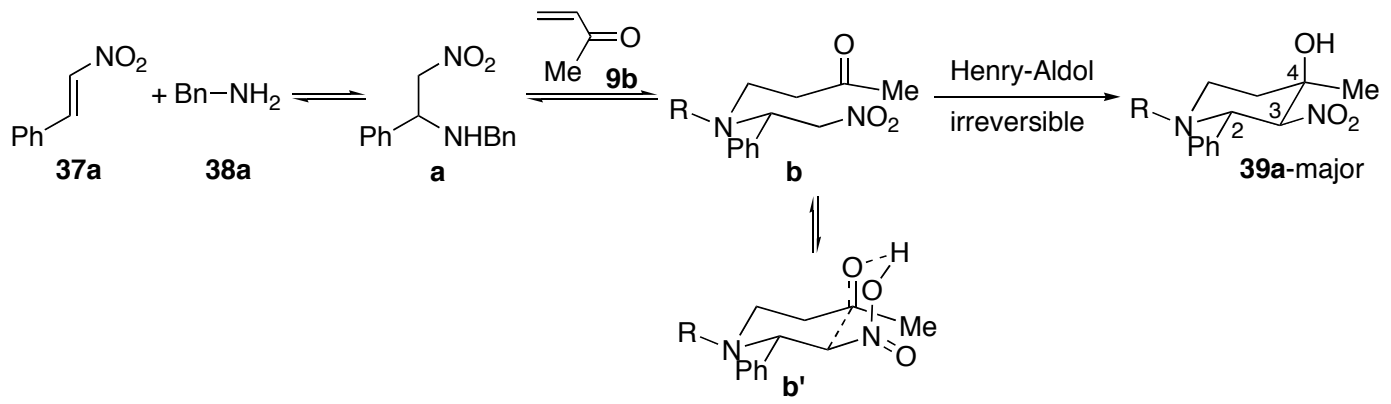

As we expected, cis isomer between C-4 hydroxyl and C-3 nitro was the major product due to the intramolecular H-bonding. All the stereochemistry was driven by the six-member ring transition state, in which the big substitute groups (phenyl and nitro) located at equatorial positions. This result was consistent with our proposal, the final Henry-Aldol reaction was the rate determining irreversible step, before which both the aza-Michael addition to nitroalkene and enone were in equilibrium. This was also confirmed by the NMR study of the first step, amine addition to nitroalkene. The adduct a could be isolated from the reaction, but not stable at all. Recharging it back into solvents, the nitroalkene $\mathbf{3 7} \mathbf{a}$ and amine $\mathbf{3 8 a}$ were released.

\subsubsection{Enantiomeric piperidine synthesis}

\subsubsection{Chiral amine chiral induction and the NMR evidence}

With this efficient new method available, the enantioselective transformation became highly desirable. Since the rate determining chair transition state led to the excellent diastereoselectivity, we therefore wondered whether chiral amines could be 
applied in this transformation to deliver the chirality into the piperidine ring via the spatial arrangement in the six-member ring transition state.

To investigate the chirality induction by chiral amines, arylethanamines 40a-c were applied (Table 18). As expected, piperidines 41a-c were obtained in good yields. With the formation of two possible C-4 isomers, introduction of new stereogenic center on C-7 position could result in the formation of four diastereomers. Excellent diastereoselectivity on C-4 position was obtained in 41a and 41b (comparing P1 to P3 and $\mathbf{P 2}$ to P4), giving d.r. $>10: 1$. For amine 40c, $\mathrm{MeOH}$ was required for optimal performance, due to the slow reaction rate in THF, which caused a slight decrease of $d . r$. on C-4 selectivity. However, to evaluate the chirality transfer by chiral amine, the relative stereochemistry of the exocyclic C-7 and the piperidine ring is the key (comparing the C7 isomers: P1 to $\mathbf{P 2}$ or $\mathbf{P 3}$ to $\mathbf{P 4}$ ). If the exocyclic N-C bond is free rotation of the, one may not expect any stereochemistry control. To our surprise, modest chirality induction was obtained in all three cases, giving C-7 isomers around 4:1 d.r.

Table 18. Piperidine synthesis with chiral amines and NMR study ${ }^{a}$

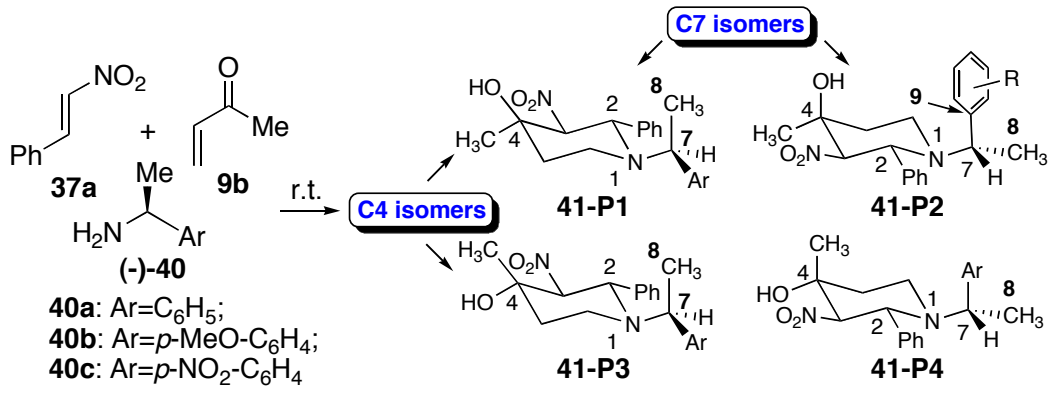

\begin{tabular}{|c|c|c|c|c|c|c|c|c|c|}
\hline \multirow[b]{2}{*}{ product } & \multirow{2}{*}{$\begin{array}{c}\text { d.r. (\%) } \\
\text { P1:P2:P3:P4 }\end{array}$} & \multicolumn{4}{|c|}{${ }^{13} \mathrm{C}$ chemical shift of $\mathrm{C}-8$} & \multicolumn{4}{|c|}{${ }^{13} \mathrm{C}$ chemical shift of C-9 } \\
\hline & & $\mathrm{P} 1$ & $\mathrm{P} 2$ & P3 & P4 & P1 & P2 & P3 & $\mathrm{P} 4$ \\
\hline $41 a$ & 82:18:0:0 & 8.6 & 18.8 & -- & -- & 143.3 & 137.9 & -- & -- \\
\hline $41 b$ & $78: 15: 7: 0$ & 8.7 & 19.1 & 8.3 & -- & 135.3 & 130.0 & 135.0 & -- \\
\hline $41 \mathrm{c}$ & $73: 16: 11: 0$ & 8.8 & 18.4 & 8.6 & -- & 151.1 & 145.8 & 150.8 & -- \\
\hline
\end{tabular}

${ }^{a}$ General reaction condition as described in Table 17. ${ }^{b}$ Combined isolated yields of all isomers. Structures of all reported isomers were determined by either X-ray crystallography or comprehensive 1D and 2D NMR experiments. ${ }^{c}$ d.r. determined by NMR of crude reaction mixtures.

Based on the X-ray crystallography and NMR spectroscopy, structures of all obtained isomers were identified. Interestingly, as shown in all crystal structures, the 
exocyclic C-7 adopted a perfect staggered conformation with the piperidine ring. Moreover, the chemical shifts of the C-8 and C-9 carbons were significantly shifted upfield when they were at the antiparallel positions relative to the nitrogen lone pair electrons (C-8 in P1/P3 and C-9 in P2, Table 18). Therefore, it strongly suggested that the N1-C7 $\sigma$-bond rotation was restricted. Considering all these results, a Henry-aldol cyclization chair transition state with the exo-cyclic chiral amine induction was proposed in Scheme 45.

\section{Scheme 45. Proposed exo-cyclic chirality induction}
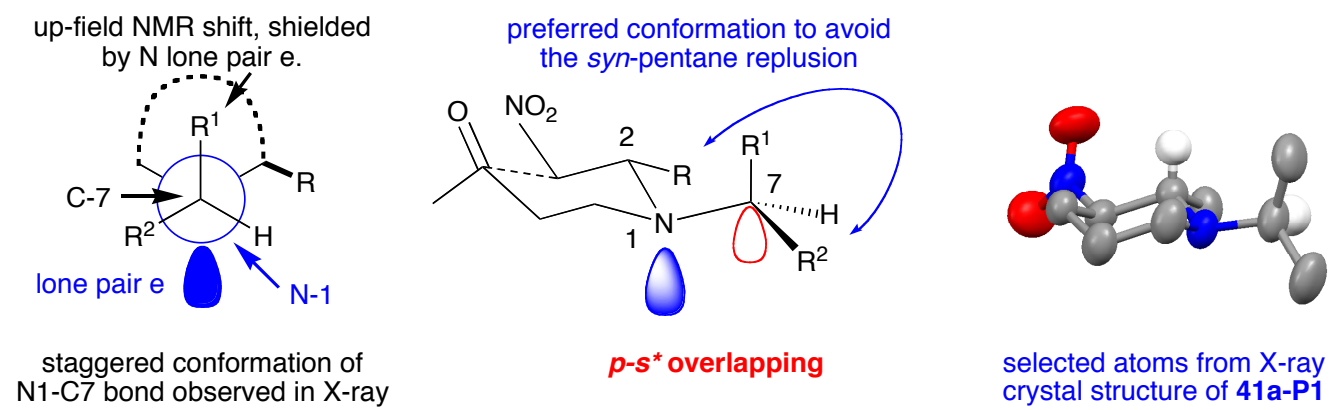

As part of the six-membered ring, the N-1 nitrogen lone pair electrons adopted the axial position. Thus, with the preferred staggered N1-C7 conformation, three syn-pentane interactions would be generated in the transition state: $[\mathrm{C}-2-\mathrm{R}$ to $\mathrm{C}-7-\mathrm{H}]$ and $[\mathrm{C}-2 / \mathrm{C}-6-\mathrm{H}$ to $\mathrm{C}-7-\mathrm{R}^{2}$ ]. This proposed transition-state model was consistent with the experimental observation in the synthesis of piperidine 41a-c, where in the major stereoisomers the less bulky methyl group (relative to the aryl groups) was placed on the $\mathrm{R}^{1}$ position to generate the energetically favored transition state by minimizing the $s y n$-pentane steric repulsion. However, besides the steric interaction, the stability of the chair transition state could also be influenced by the $\mathrm{p}-\sigma^{*}$ electronic interaction between nitrogen lone pair electrons and exocyclic axial $\mathrm{R}^{2}$ group. Compared to the methyl group, the more bulky phenyl group could form stronger $\mathrm{p}-\sigma^{*}$ interactions if it were placed on the $\mathrm{R}^{1}$ position.

\subsubsection{Enhanced exo-cyclic stereochemistry control by amino esters}

Therefore, we wondered whether this chirality induction could be further enhanced by application of the chiral amine with a less bulky group that would produce stronger $\mathrm{p}-\sigma^{*}$ interaction (Scheme 46). 


\section{Scheme 46. Enhanced chirality transfer by amino esters and enantiomeric pure piperidine synthesis}

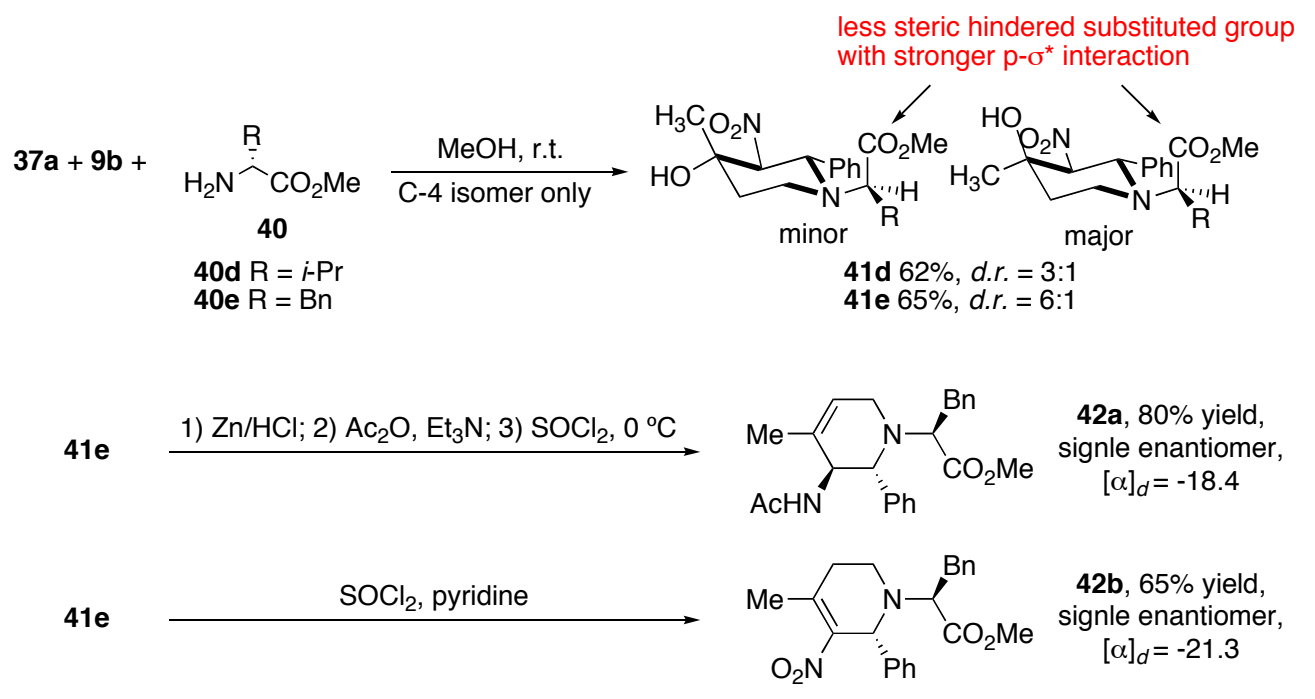

The readily available amino acid derivatives become ideal chiral amines for this application with the smaller $\mathrm{CO}_{2} \mathrm{Me}$ group that provides stronger $\mathrm{p}-\sigma^{*}$ interaction, giving energetically a more favored transition state than other different alternatives (Scheme 45A). As expected, reactions among 37a, 9b and amino esters 40d and 40e generated the desired enantiomeric pure piperidine 41d and 41e with only C-4 isomers observed: 100\% chirality induction was achieved through the chirality control by exocyclic asymmetry. Based on the reaction crude NMR, the C-4 isomers were the only piperidine products observed. Under the reaction conditions, $25 \%$ of unreacted 37 a was recovered in both cases, making the theoretical isolated yields of 41d and 41e to $83 \%$ and $87 \%$. No epimerization occurred under the reaction conditions and compounds 41d and 41e were indeed prepared with $100 \%$ enantiomeric purity. Through some simple transformations, the two C-4 isomers were converted into single enantiomers 42a and 42b (Scheme 45B and $\mathbf{C}$ ).

\subsubsection{Extension of the exo-cyclic chirality transfer concept}

In the history of organic synthesis, a lot of classic and elegant reactions are based on the six-member ring chair transition state (Zimmerman-Traxler transition state). It had explained the excellent diastereo involved in Aldo reactions and all the Claisen type of rearrangement reactions (Scheme 47). 


\section{Scheme 47. Six-member ring transition-state and Claisen rearrangement}

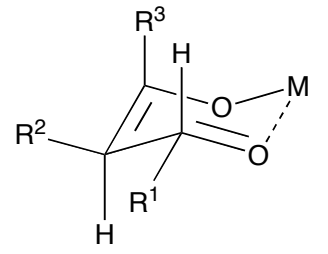

six-member ring transition state in Aldol reaction ( $E$ enolate favored conformation, anti product)

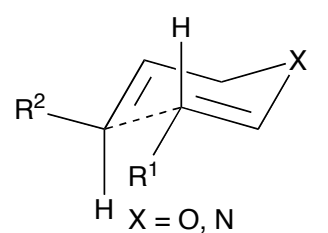

six-member ring transition state

in Claisen rearrangment

\section{Scheme 48. Proposed exo-cylcic chirality transfer in aza-Claisen rearrangement}

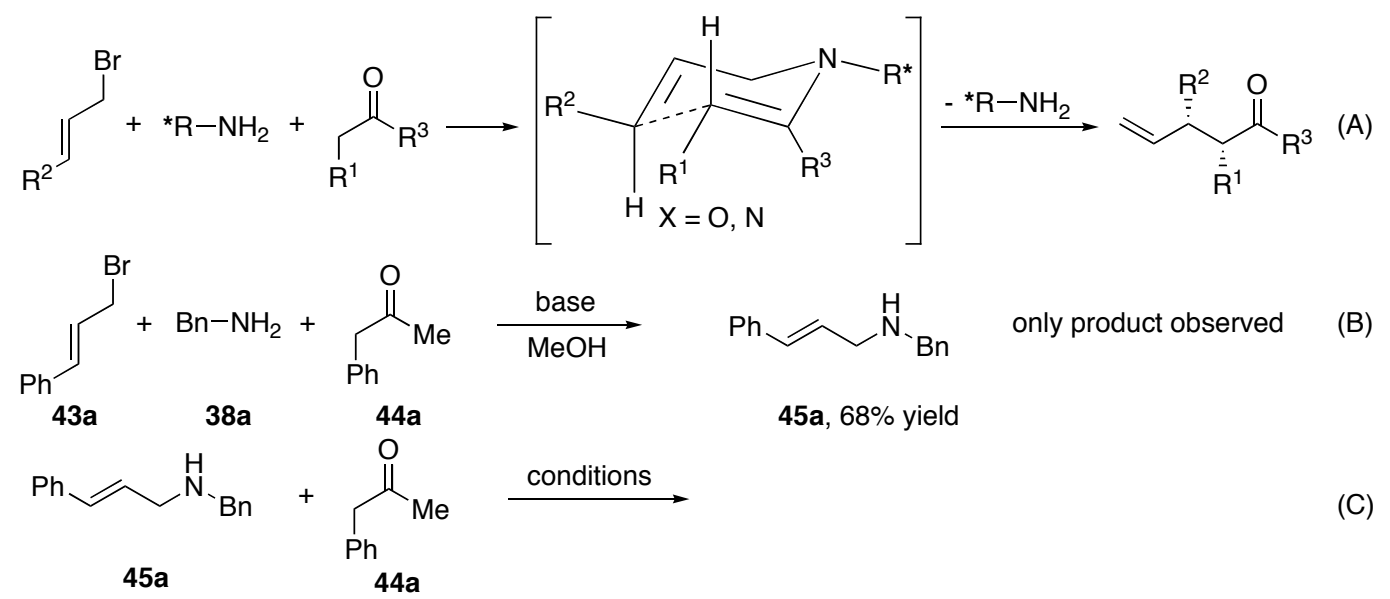

Inspired by our three-component syntheses of piperidines with chiral amines, which also shared the same transition state, the extension of the exo-cylcic chirality transfer certainly had caught our strong attention. Further exploration was then carried out. What we had hoped is to reach the allylic vinyl amine from the condensation of allyl bromide, chiral amine and ketone, which then might go through a six-member ring azaClaisen type of transition state to give the rearrangement product with the chirality transferred from the amine (Scheme 48A). Also, we wished the reaction required only catalytic amount of chiral amine. A racemic model reaction was then set up between cinnamyl bromide 43a, benzyl amine 38a and ketone 44a (Scheme 48B). However, only the allyl amine 45a was observed under basic condition. We reasoned it that the basic condition did not help the formation of enamine to reach the allylic vinyl amine intermediate. Therefore, 45a was treated with ketone 44a under various conditions, including acidic and high temperature to reach the proposed intermediate and overcome the kinetic barriers of the rearrangement process (Scheme 48C). No desired product was 
observed, with either large amount of 45a left intact or decomposition under high temperature.

However, it is our belief that the exo-cyclic chirality transfer will fruit in organic synthesis in the future, rather than just a concept.

\subsection{Conclusion}

In conclusion, applying the one-pot cascade strategy, substituted piperidines were prepared with excellent yields and great stereoselectivity. The transformation was suitable for a large group of substrates and selective functionalization on each position of the piperidine ring have been achieved. Moreover, based on an exocyclic stereochemistry control, $100 \%$ chirality induction was achieved with amino esters, giving enantiomeric pure substituted piperidines in less than three steps. This new stereochemistry control strategy will be further applied into similar systems for the synthesis of other chemical and biological important heterocycles.

This project is a collaboration work with Dr. Yunfeng Chen. I helped with the substrate scope (Table 17) and the exo-cyclic chirality transfer expriments. 


\section{Part VI}

\section{Anion Bridged Nanosheet from Self-Assembled G- Quadruplexes}

\subsection{Introduction}

\subsubsection{Self-assembly study involving Guanosine}

Bottom-up nanotechnology, or so-called "molecular manufacturing" or "molecular nanotechnology", has attracted explosive attention in recent years. The bottom-up approach has been demonstrated to create larger scale architectures through the precisely controlled assembly of specific atoms or molecules. Self-assembly has been recognized as one of the enabling strategies to prepare the functionalized molecular $\operatorname{architectures}^{50}$, which has been applied in many areas of nanotechnology, including nanoparticle synthesis ${ }^{51}$, surface modification ${ }^{52}$ and device development ${ }^{53}$. However, selfassembly involving organic molecules did not receive enough attention in nanotechnology researches.

\section{Scheme 49. Guanosine self-assembly and its nano size}
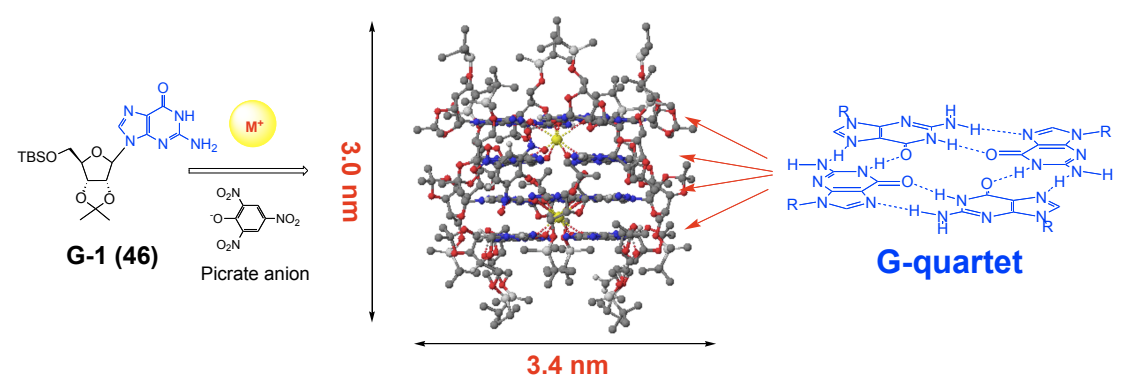

Crystal structures of G-hexadecamer

$(\mathrm{G}-1)_{16}-\mathrm{M}_{4}-\mathrm{Pic}_{4}$
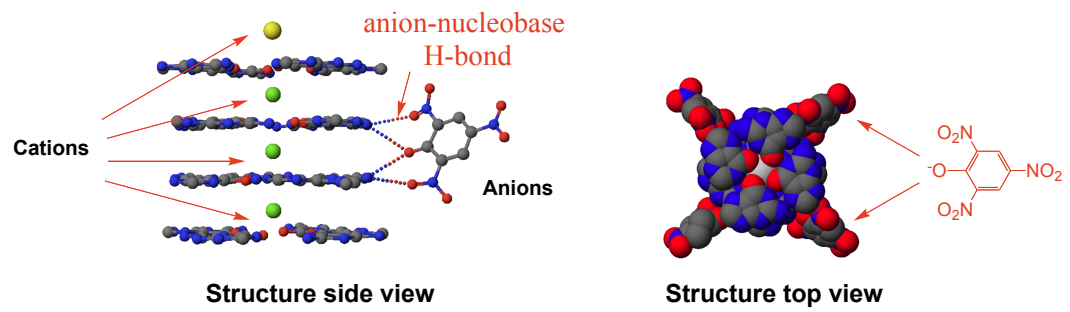
Guanosine, well known as self-assembly precursor, has been found for a long time in nature as the guanosine quartet (G-quartet), a motif formed through selfcomplementary hydrogen bonding of the purine base ${ }^{54}$ (Scheme 49). In the presence of proper cations $\left(\mathrm{Ba}^{2+}, \mathrm{K}^{+}, \mathrm{Na}^{+}\right.$etc.) and counter anions (like picrate), four of the tetramer Guanosine complexes, which are flat structures, would be easily piled up as a bigger hexadecamer called G-quadruplex. This type of complex usually contains at least two sandwich subunits with the metal cations in the middle of the layers, binding with the four carbonyl groups in each layer; while the counter anions were bonding with the $\mathrm{N}-\mathrm{H}$ between the two middle layers. The size of the cylinder shape of complex is $3.0 \mathrm{~nm}$ in height and $3.4 \mathrm{~nm}$ long as the diameter.

Substantial work had been done in recent years; influence of different cations and different anions had been studied in detail. Amazingly, the lipophilic Guanosine G-1 (46) form self-assembled hexadecamer in solid state, solution and even gas phase, ${ }^{55}$ through alkali and alkali earth metal cation templation, whose structure, composition and properties could be easily characterized by NMR or even X-ray crystallography. It was important to notice that picrate anions bound at the side of the quadruplex through $\mathrm{H}$ bonds. Extensive studies have been made for this unique anion binding properties and various anions, including 2,6-DNP, $\mathrm{SCN}^{-}$, p-OMe-2,6-DNP etc, were found promoting the formation of same hexadecamer structures with different kinetic and thermodynamic properties.

Intrigued by this unique anion binding property, we wondered whether the anionbinding site could serve as a new synthetic handle to extend the supramolecular architecture of the G-quadruplex. In the crystal structure of the G-hexadecamer, picrate anions coordinate with the exocyclic amino group of the central two G- quartets through the anion's phenolate oxygen and the two nitro groups at the ortho position. The para position, which is solvent-exposed from the G-quadruplex, provides an ideal synthetic handle for the extension of the supermolecule without disturbing the G-quartet's key noncovalent interactions. Thus, we proposed that with the 2,6,2',6'- tetranitrobiphenolate (TNBP) dianion, which might serve as the bridging anion, could be used to tether 
individual G-hexadecamers in a two dimensional pattern. In this case, a nano size sheet structure would be possibly prepared wit a height of $3.0 \mathrm{~nm}$ (Scheme 50).

Scheme 50. Proposed nano sheet through Guanosine self-assembly

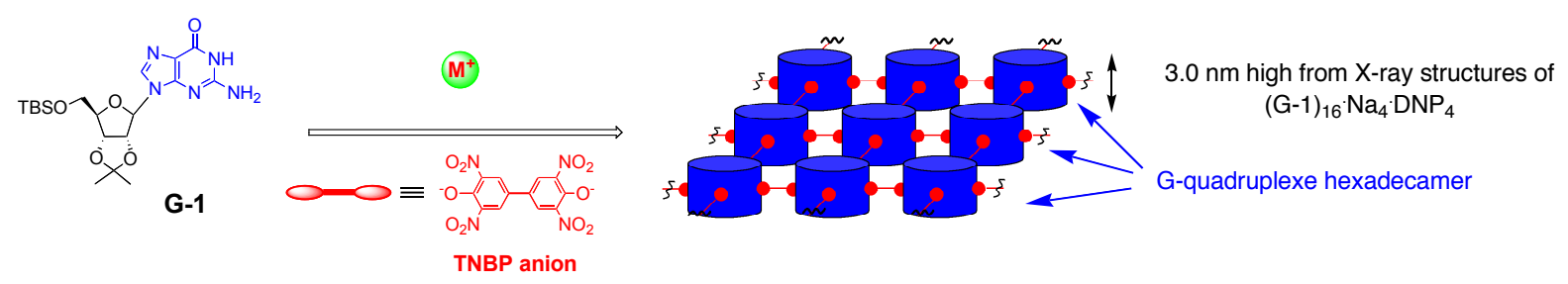

\subsection{Research Objectives}

The proposed Guanosine self-assembly nano sheet structure would be prepared for characterization. The composition and the structure of the complex would be studied. Different cations would be applied and tested. Further application of this non-covalent nano size supramolecular complex would be performed.

\subsection{Results and Discussion}

\subsubsection{Preparation of complex and the composition study}

Both G-1 (46) and $\mathrm{Na}_{2}$ TNBP (47) were prepared using literature procedures. ${ }^{56}$ The preparation of the polymeric G-quadruplexes was performed in two methods: 1) direct treatment of G-1 (46) with $\mathrm{Na}_{2} \mathrm{TNBP}$ (47), and 2) anion exchange of preformed Ghexadecamer $(\mathbf{G}-1)_{16} \cdot \mathrm{Na}_{4} \bullet \mathrm{SCN}_{4}$ with $\left(\mathrm{Bu}_{4} \mathrm{~N}\right)_{2} \cdot \mathrm{TNBP}(\mathbf{4 8})$. In the first approach, the G-1 was dissolved in $\mathrm{CH}_{2} \mathrm{Cl}_{2}$, and the $\mathrm{Na}_{2} \mathrm{TNBP}$ was dissolved in water. Notably, the $\mathrm{Na}_{2}$ TNBP salt is not soluble in $\mathrm{CH}_{2} \mathrm{Cl}_{2}$ while G-1 has little solubility in water. Mixing two clear solutions yielded a red solid at the interface of the two solutions (Scheme 51).

\section{Scheme 51. Formation of nano size Guanosine complex}

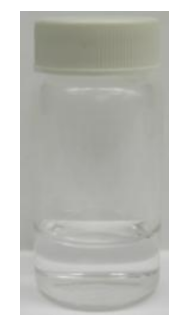

G-1 (46) in DCM

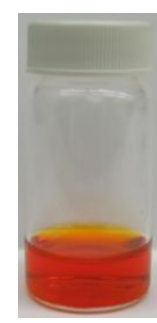

$\mathrm{Na}_{2}$ TNBP in $\mathrm{H}_{2} \mathrm{O}$

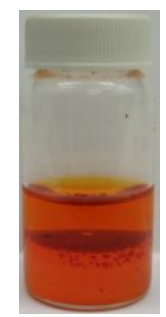

Red precipitate at the interface 
The same polymeric complexes were also obtained through the anion exchange between preformed G-hexadecamer, $(\mathbf{G}-1)_{16} \cdot \mathrm{Na}_{4} \cdot \mathrm{SCN}_{4}$, and TNBP anions. It has been demonstrated previously that sodium thiocyanate promotes the formation of Ghexadecamer by directing self-assembly of G-1. Because it is a relatively poor H-bond receptor, the $\mathrm{SCN}^{-}$anion binding with the G-quartets in $(\mathbf{G}-1)_{16} \cdot \mathrm{Na}_{4} \cdot \mathrm{SCN}_{4}$ is highly dynamic. Compared to $\mathrm{SCN}^{-}$, the 2,6-DNP anion forms stronger $\mathrm{H}$-bonds with the Gquartets, resulting in the formation of kinetically and thermodynamically more stable guanosine complexes. ${ }^{11 \mathrm{a}}$ The $\left(\mathrm{Bu}_{4} \mathrm{~N}\right)_{2} \bullet$ TNBP salt was prepared to perform this anion exchange study due to its solubility in $\mathrm{CH}_{2} \mathrm{Cl}_{2}$ and the weak binding between the $\mathrm{Bu}_{4} \mathrm{~N}^{+}$ cation and the G-quartet. Upon mixing of $(\mathbf{G}-\mathbf{1})_{16} \bullet \mathrm{Na}_{4} \bullet \mathrm{SCN}_{4}$ with $\left(\mathrm{Bu}_{4} \mathrm{~N}\right)_{2} \bullet \mathrm{TNBP}$ solutions in $\mathrm{CH}_{2} \mathrm{Cl}_{2}$, a red precipitate was again observed within 5 minutes.

\section{Scheme 52. Solution NMR study of the composition of the complex}

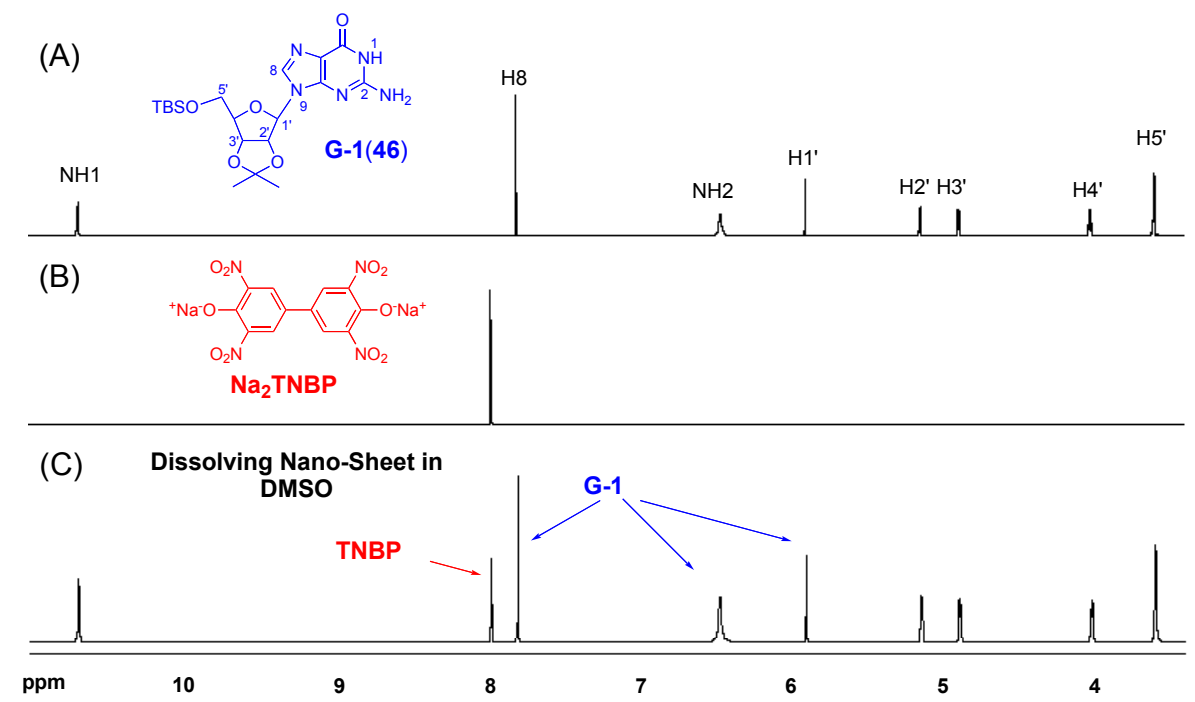

The precipitate was easily filtered and obtained as red floppy solid. DMSO could break the non-covalent H-bonds, which held up the molecules as one piece. As revealed by the ${ }^{1} \mathrm{H}$ NMR in DMSO in Scheme 52, the signals for the H8 proton of G-1 (46) and for the $\mathrm{Na}_{2} \mathrm{TNBP}(\mathbf{4 7})$ protons gave a 2:1 ratio, indicating a 16:4 ratio between $\mathbf{G - 1}$ (46) and TNBP anions. This result was consistent with the proposed nano-sheet structure. The ${ }^{13} \mathrm{C}$ NMR comparison of these three compounds was also performed Both ${ }^{1} \mathrm{H}$ NMR and 
${ }^{13} \mathrm{C}$ NMR confirm that the nano-sheets are formed from G-1 (46) and $\mathrm{Na}_{2} \mathrm{TNBP}$ (47) through non-covalent interactions.

\subsubsection{Atomic force microscope (AFM) study, solid phase NMR study and powder X-ray characterization}

Furthermore, the size and shape of the G-quadruplex polymer formed upon crosslinking by TNBP were also measured by atomic force microscope (AFM). The resulting nanoparticles were about $90-200 \mathrm{~nm}$ in lateral size with the height of $3.0 \pm 0.05 \mathrm{~nm}$, which is consistent with the size of guanosine hexadecamers. This result confirmed that the TNBP anion bridges link the non-covalent G-quadruplexes together and produce even bigger, highly ordered supramolecular architectures (Scheme 53).

\section{Scheme 52. AFM images of the prepared Guanosine nano-sheet}

(A)

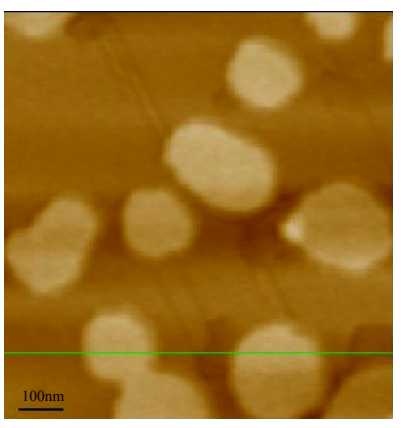

(B)

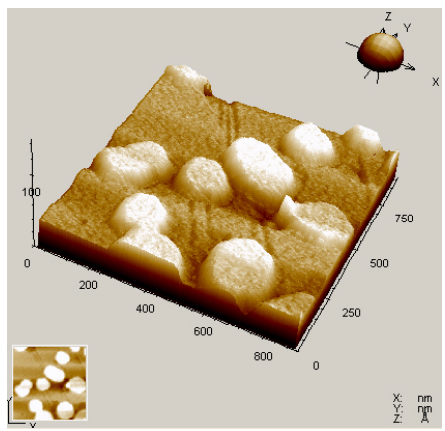

(C)

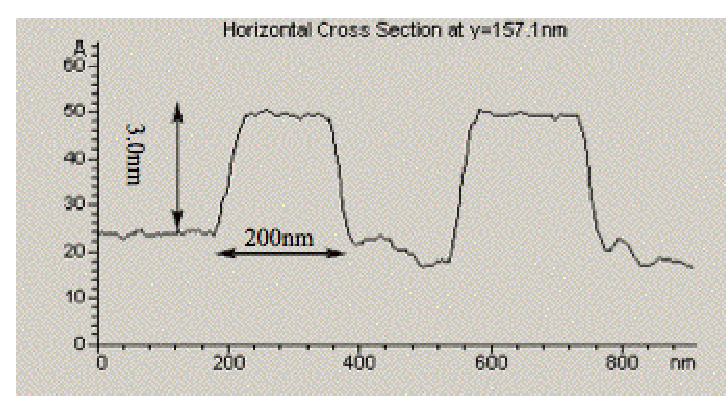

(A) 2D topography AFM image of $(\mathrm{G}-1)_{16} \cdot \mathrm{Na}^{+} \cdot{ }_{4} \cdot \mathrm{TNBP}^{2-}{ }_{2}$ complex; (B) 3D topography AFM image of the same sampling area as B; (C) cross sectional profile of $(\mathrm{G}-1)_{16} \mathrm{Na}^{+}{ }_{4} \mathrm{TNBP}^{2-}{ }_{2}$ complex over the line indicated in B.

Since we used $\mathrm{Na}_{2}$ TNBP (47) to prepare the nano-sheet, it is important to characterize the $\mathrm{Na}^{+}$cation within the non-covalent nano-sheet structure. Recently, solid 
state NMR techniques have been successfully developed for the determination of cation coordination within the G-quartet. Wu's group has studied the solid-state ${ }^{23} \mathrm{Na},{ }^{39} \mathrm{~K}$, and ${ }^{87} \mathrm{Rb}$ NMR of guanosine complexes and clearly demonstrated the cation-G-quartet binding in the solid state. ${ }^{57}$ Several ${ }^{23} \mathrm{Na}$ solid-state NMR experiments were then performed on the polymeric nanosheet (Scheme 53).

\section{Scheme 54. $\quad{ }^{23} \mathrm{Na}$ solid phase NMR comparison}

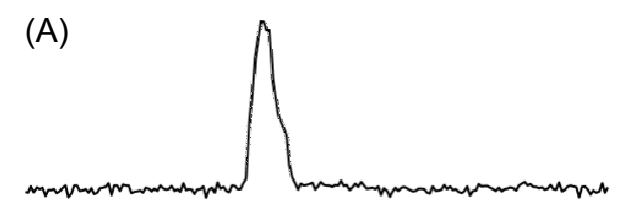

(B)

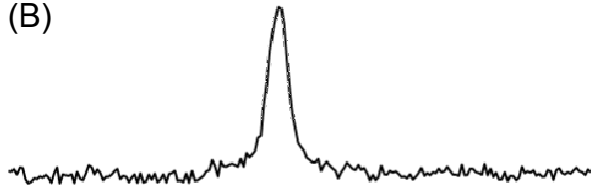

(C)

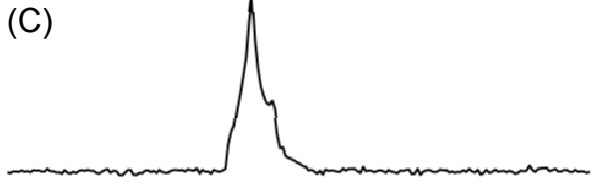

(D)

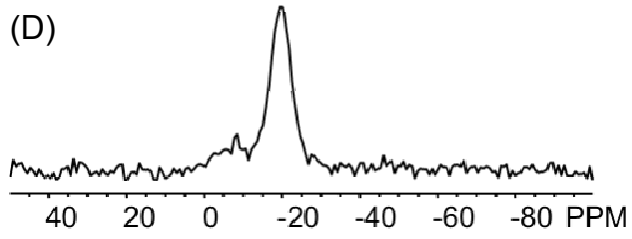

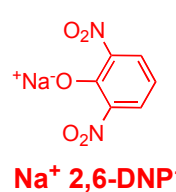

$(\mathrm{G}-1){ }_{16} \cdot \mathrm{Na}^{+} \cdot(2,6-\mathrm{DNP})_{4}$

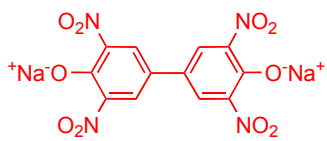

$\mathrm{Na}^{+}{ }_{2}$ TNBP $^{2-}$

Self-Assembled "Nano-Sheet"

The $\mathrm{Na}_{2}(2,6-\mathrm{DNP})$ salt was examined to simulate the ion's chemical environment within the nano-sheet complexes. The G- hexadecamer formed from G-1 and $\mathrm{Na}(2,6-$ DNP) has been previously characterized by X-ray and solution NMR studies. Solid state ${ }^{23} \mathrm{Na}$ NMR spectra yielded different chemical shifts for "free" $\mathrm{Na}^{+}$and G-quartet bound $\mathrm{Na}^{+}$(Scheme 54A and B). Through the direct comparison of these standard samples, more than $90 \%$ of the $\mathrm{Na}^{+}$cations are bound to the G-quartets in the nano-sheet complexes (Scheme 54D).

Moreover, the powder X-ray diffraction of the nano-sheet materials has been performed. The powder X-ray pattern reveals two low angle peaks observed at d-spacing $47.5 \AA$ and $23.75 \AA$, which indicating the presence of large unit cell. As 
reported previously, the typical guanosine hexadecamer crystal has the unit cell around $47 \AA$ (for example, $(\mathbf{G}-1)_{16} \cdot \mathrm{Ba}_{2} \cdot(\mathrm{P}-\mathrm{OME}-2,6-\mathrm{DNP})_{4}$ has a unit cell as $\mathrm{a}=46.662 \AA$, $\mathrm{b}=24.259 \AA, \mathrm{c}=45.111 \AA$ ). Therefore, the large unit cell revealed by the powder X-ray is consistent with Guanosine hexadecamer and support the formation proposed nano-sheet structure.

\subsection{Conclusion}

In conclusion, the covalent linked dianion, $\mathrm{TNBP}^{2-}$, promotes the formation of a non-covalent polymer by cross-linking lipophilic G-quadruplexes. The AFM images, the solution NMR data, the solid state ${ }^{23} \mathrm{Na}$ NMR analysis and powder X-ray measurement, all strongly support the formation of novel non-covalent network as proposed. This novel supramolecular architecture exhibits a highly ordered structure. Notably, this novel nano-scale non-covalent complex is produced simply through the self-assembly of small molecules (G-1 and $\left.\mathrm{Na}_{2} \mathrm{TNBP}\right)$ in one step. Since the complex construction is based on non-covalent interactions, the molecular architecture is dynamic and potentially reversible, and may provide unique properties as novel nanomaterial. Meanwhile, the hydrophobic stacking of G-quartet and cation transport properties of G-quadruplexes makes this complex potentially interesting in the studies of electron and ion transfer and nano-device development. Screening studies of various anions and spacer linkers and deposition of the nano-sheet on metal surfaces for device development are currently under investigation in our group.

This project was collaborated with Dr. Jin Wang, Dr. Nianqiang Wu, Dr. Gang Wu and Dr. Peter Y. Zavalij. Dr. Wang and Dr. Nianquang Wu helped with the AFM image characterization. Dr. Gang Wu helped with solid-state NMR experiments of ${ }^{23} \mathrm{Na}$ of the complex. Dr. Zavalij helped with the powder X-ray analysis of the complex. 


\section{Reference:}

1 Nicolaou, K. C.; Montagnon, T.; Snyder, S. A. Chem. Commun. 2003, 551.

2 For reviews, see: a) Dondoni, A.; Massi, A. Angew. Chem. Int. Ed. 2008, 47, 4638-4660. b) de Figueiredo R. M.; Christmann, M. Eur. J. Org. Chem. 2007, 2575-2600. For recent examples, see: c) Simmons, B.; Walji, A. M.; MacMillan, D. W. C. Angew. Chem. Int. Ed. 2009, 48, 4349-4353. d) Jakubec, P.; Cockfield, D. M.; Dixon, D. J. J. Am. Chem. Soc. 2009, 131, 16632-16633.

3 a) Enantioselective Organocatalysis: Reactions and Experimental Procedures (Ed.: Dalko, P. I.), Wiley-VCH, Weinheim, 2007. b) Seayad, J.; List, B. Org. Biomol. Chem. 2005, 3, 719-724. c) MacMillan, D. W. C. Nature, 2008, 455, 304-308.

4 a) Huang, Y.; Walji, A. M.; Larsen, C. H.; MacMillan, D. W. C. J. Am. Chem. Soc. 2005, 127, 15051-15053. b) Marigo, M.; Schulte, T.; Franzén, J.; Jørgensen, K. A. J. Am. Chem. Soc. 2005, 127, 15710-15711.

5 For reviews, see: a) Zhong, C.; Shi, X. Eur. J. Org. Chem. 2010, accepted. b) Shao, Z.; Zhang, H. Chem. Soc. Rev. 2009, 38, 2745-2755.

6 For review, see: a) Akiyama, T.; Itoh, J.; Fuchibea, K. Adv. Synth. Catal. 2006, 348, 999-1010. b) Zhang, Z.; Schreiner, P. R. Chem. Soc. Rev. 2009, 38, 11871198.

7 For recent examples: a) Yoon, T. P.; Jacobsen, E. N. Angew. Chem. Int. Ed. 2005, 44, 466-468. b) Fuerst, D. E.; Jacobsen, E. N. J. Am. Chem. Soc. 2005, 127, 8964-8965. c) Xu, H.; Zuend, S. J.; Woll, M. G.; Tao, Y.; Jacobsen, E. N. Science, 2010, 327, 986-990.

8 a) Pidathala, C.; Hoang, L.; Vignola, N.; List, B. Angew. Chem. Int. Ed. 2003, 42, 2785-2788. b) Bahmanyar, S.; Houk, K. N.; Martin, H. J.; List, B. J. Am. Chem. Soc. 2003, 125, 2475-2479.

9 For recent studies on Baylis-Hillman reaction, see: a) Aggarwal, V. K.; Emme, I.; Fulford, S. Y. J. Org. Chem. 2003, 68, 692-700. b) Price, K. E.; Broadwater, S. J.; Jung, H. M.; McQuade, D. T. Org. Lett. 2005, 7, 147-150. c) Robiette, R.; Aggarwal, V. K; Harvey, J. N. J. Am. Chem. Soc. 2007, 129 ,15513-15525. 
10 a) Baylis, A. B.; Hillman, M. E. D. German Patent 2155113, 1972. B) Morita, K.; Suzuki, Z.; Hirose, H. Bull. Chem. Soc. Jpn. 1968, 41, 2815-2815.

11 Sun, X.; Sengupta, S.; Petersen, J. L.; Wang, H.; Lewis, J. P.; Shi, X. Org. Lett. 2007, 9, 4495-4498.

12 For examples: a) Xie, H.; Zu, L.; Li, H.; Wang, J.; Wang, W. J. Am. Chem. Soc. 2007, 129, 10886-10894. b) Tran, Y. S.; Kwon, O. J. Am. Chem. Soc. 2007, 129, 12632-12633. c) Tozawa, T.; Fujisawa, H.; Mukaiyama, T. Chem. Lett. 2004, 33, 1454-1455. d) Chapuis, C.; Büchi, G.; Wüest, H. Helv. Chim. Acta, 2005, 88, 3069-3088.

13 For review, see: Ballini, R.; Bosica, G.; Fiorini, D.; Palmieri, A.; Petrini, M. Chem. Rev. 2005, 105, 933-971.

14 Ono, N. The Nitro Group in Organic Synthesis, Wiley-VCH, Weinheim, Germany, 2005.

15 For reviews, see: a) Berner, O. M. ; Tedeschi, L.; Enders, D. Eur. J. Org. Chem. 2002, 1877-1894. b) Denmark, S. E.; Thorarensen, A. Chem. Rev. 1996, 96, 137165.

16 Dadwal, M.; Mohan, R.; Panda, D.; Mobinc, S. M. Namboothiri, I. N. N. Chem. Commun. 2006, 338-340.

17 For examples: a) Halland, N.; Aburel, P. S.; Jørgensen, K. A. Angew. Chem., Int. Ed. 2004, 43, 1272-1277. b) Campanà, A. G.; Fuentes, N.; Gómez-Bengoa, E.; Mateo, C.; Enrique Oltra, J.; Echavarren, A. M.; Cuerva, J. M. J. Org. Chem. 2007, 72, 8127-8130. c) Correc, O.; Guillou, K.; Hamelin, J.; Paquin, L.; TexierBoullet, F.; Toupet, L. Tetrahedron Lett. 2004, 45, 391-395.

18 Hayashi, Y.; Sekizawa, J.; Yamaguchi, H. Gotoh, H. J. Org. Chem. 2007, 72, 6493-6499.

19 For examples: a) Reddy, C.; Reddy, V.; Verkade, J. G. J. Org. Chem. 2007, 72, 3093-3096. b) Hirano, M.; Hirai, M.; Ito, Y.; Tsurumaki, T.; Baba, A.; Fukuoka, A.; Komiya, S. J. Organomet. Chem. 1998, 569, 3-14.

20 a) Righi, P.; Marotta, E.; Landuzzi, A.; Rosini, G. J. Am. Chem. Soc. 1996, 118, 9446-9447. b) Trost, B. M.; Chupak, L. S.; Lubbers, T. J. Am. Chem. Soc. 1998, 
120, 1732-1740. c) Fuller, A. A.; Chen, B.; Minter, A. R.; Mapp, A. K. J. Am. Chem. Soc. 2005, 127, 5376-5385.

21 a) Barbachyn, M. R.; Cleek, G. J.; Dolak, L. A.; Garmon, S. A.; Morris, J.; Seest, E. P.; Thomas, R. C.; Toops, D. S.; Watt, W.; Wishka, D. G.; Ford, C. W.; Zurenko, G. E.; Hamel, J. C.; Schaadt, R. D.; Stapert, D.; Yagi, B. H.; Adams, W. J.; Friis, J. M.; Slatter, J. G.; Sams, J. P.; Oien, N. L.; Zaya, M. J.; Wienkers, L. C.; Wynalda, M. A. J. Med. Chem. 2003, 46, 284-302. b) Simoni, D.; Grisolia, G.; Giannini, G.; Roberti, M.; Rondanin, R.; Piccagli, L.; Baruchello, R.; Rossi, M.; Romagnoli, R.; Invidiata, F. P.; Grimaudo, S.; Jung, M. K.; Hamel, E.; Gebbia, N.; Crosta, L.; Abbadessa, V.; Cristina, A. D.; Dusonchet, L.; Meli, M.; Tolomeo, M. J. Med. Chem., 2005, 48, 723-736.

22 a) Tartakovskii, V. A.; Onishchenko, A. A.; and Novikov, S. S. Zh. Org. Khim. 1967, 3, 588. b) Chlenov, I. E.; Kashutina, M. V.; Ioffe, S. L.; Novikov, S. S.; Tartakovsky, V. A. Bull. Acad. Sci. USSR, Div. Chem. Sci. (Engl. Transl.), 1969, 18, 1948. c) Kunetsky, R. A.; Dilman, A. D.; Ioffe, S. L.; Struchkova, M. I.; Strelenko, Y. A.; Tartakovsky, V. A. Org. Lett. 2003, 5, 25, 4907-4909.

23 Denmark, S. E.; Middleton, D. S. J. Org. Chem. 1998, 63, 1604-1618.

24 a) Scardovi, N.; Casalini, A.; Francesca, P.; Righi, P. Org. Lett. 2002, 4, 9653968. b) Galli, C.; Marotta, E.; Righi, P.; Rosini, G. J. Org. Chem. 1995, 60, 6624-6626.

25 Clagett, M.; Gooch, A.; Gzaham, P.; Holy, N.; Mains, B.; Strunk, J. J. Org. Chem. 1976, 41, 25, 4033-4034.

26 a) Zhu, C.; Sun, X.; Deng, X.; Zheng, J.; Tang, Y. Tetrahedron. 2008, 64, 55835589. b) Zhu, C.; Deng, X.; Sun, X.; Zheng, J.; Tang, Y. Chem. Commun. 2008, $738-740$.

27 a) Marotta, E.; Baravelli, M.; Maini, L.; Righi, P.; Rosini, G. J. Org. Chem. 1998, 63, 8235-8246. b) Jiang, H.; Elsner, P.; Jensen, K. L.; Falcicchio, A.; Marcos, V.; Jørgensen, K. A. Angew. Chem. Int. Ed. 2009, 48, 6844-6848.

28 Denmark, S. E.; and Middleton, D. S. J. Org. Chem., 1998, 63, 1604.

29 Righi, P.; Marotta, E.; Landuzzi, A.; and Rosini, G. J. Am. Chem. Soc., 1996, 118 , 
9446-9447.

30 Duan, H.; Sun, X.; Liao, W.; Petersen, J. L.; Shi, X. Org. Lett. 2008, 10, 41134116.

31 a) Zhu, C.; Deng, X.; Sun, X.; Zheng, J.; Tang, Y. Chem. Commun. 2008, 738740. b) Zhu, C.; Sun, X.; Deng, X.; Zheng, J.; Tang, Y. Tetrahedron. 2008, 64, $5583-5589$.

32 Jiang, H.; Elsner, P.; Jensen, K. L.; Falcicchio, A.; Marcos, V.; Jørgensen, K. A. Angew. Chem. Int. Ed. 2009, 48, 6844-6848.

33 For the bioactivity of Clausenamide: a) Wang, X. Y.; Zhang, J. T. J. Asian. Nat. Prod. Res. 2005, 5, 1-4. b) Wang, X. Y.; Zhang, J. T. Acta Pharmacologica Sinica 2001, 22, 1099-1102. c) Xu, L.; Liu, S. L.; Zhang, J. T. Chirality 2005, 17, 239-244. For the synthesis of Clausenamide: a) Yang, L.; Deng, G.; Wang, C. X.; Huang, Z. T.; Zhu, J. P.; Wang, M. X. Org. lett. 2007, 9, 1387-1390. b) Yakura, T.; Matsumura, Y.; Ikeda, M. Synlett. 1991, 343-344.

34 For reviews, see: a) Figadère, B. Acc. Chem. Res. 1995, 28, 359-365. b) Roy, A.; Saraf, S. Biol. Pharm. Bull. 2006, 29, 191-201.

35 a) Feist, F. Chem. Ber. 1902, 35, 1537-1544. b) Bénary, E. Chem. Ber. 1911, 44, $489-492$.

36 For recent examples, see: a) Calter, M. A.; Zhu, C. Org. Lett. 2002, 4, 205-208. b) Calter, M. A.; Phillips, R. M.; Flaschenriem, C. J. Am. Chem. Soc. 2005, 127, $14566-14567$.

37 a) Zhang, Y.; Raines, A. J.; Flowers, R. A., II Org. Lett. 2003, 5, 2363-2365. b) Wang, G.-W.; Dong, Y.-W.; Wu, P.; Yuan, T.-T.; Shen, Y.-B. J. Org. Chem. 2008, 73, 7088-7095.

38 a) Shen, R.; Huang, X. Org. Lett. 2008, 10, 3283-3286. b) Shen, R.; Zhu, S.; Huang, X. J. Org. Chem. 2009, 74, 4118-4123.

39 Payne, G. B. J. Org. Chem. 1967, 35, 3351-3355.

40 a) Jiang, Y.; Ma, D. Tetrahedron: Asymmetry 2002, 13, 1033-1038. b) Yang, Z.; Fan, M.; Mu, R.; Liu, W.; Liang, Y. Tetradedron 2005, 61, 9140-9146. c) 
Bernard, A. M.; Frongia, A.; Piras, P. P.; Secci, F.; Spiga, M. Org. Lett. 2005, 7, 4565-4568.

41 Zheng, J.-C.; Zhu, Y.-C.; Sun, X.-L.; Tang, Y.; Dai, L.-X. J. Org. Chem. 2008, 73, 6909-6912.

42 Barlaam, B.; Boivin, J.; Zard, S. Z. Tetrahedron Lett. 1990, 31, 7429- 7432.

43 For examples, see: a) Tamura, R.; Hegedus, L. S. J. Am. Chem. Soc. 1982, 104, 3727-3729. b) Ono, N.; Hamamoto, I.; Kaji, A. J. Chem. Soc., Chem. Commun. 1982, 821-822. c) Tamura, R.; Kai, Y.; Kakihana, M.; Hayashi, K.; Tsuji, M.; Nakamura, T.; Oda, D. J. Org. Chem. 1986, 51, 4375-4385.

44 For selected examples, see: (a) Miyake, H.; Yamamura, K. Tetrahedron Lett. 1986, 27, 3025-3028. (b) Ono, N.; Kamimura, A.; Sasatani, H.; Kaji, A. J. Org. Chem. 1987, 52, 4133-4135.

45 For reviews, see: a) Kallstrom, S.; Leino, R. Bioorg. Med. Chem. 2008, 16, 601635. b) Yamashita, T.; Yasuda, K.; Kizu, H.; Kameda, Y.; Watson, A. A.; Nash, R. J.; Fleet, G. W. J.; Asano, N. J. Nat. Prod. 2002, 65, 1875-1881. For recent example, see: c) Mochizuki, A.; Nakamoto, Y.; Naito, H.; Uoto, K.; Ohta, T. Bioorg. Med. Chem. Lett. 2008, 18, 782-787. d) Buffat, M. G. P. Tetrahedron, 2004, 60, 1701-1729.

46 For recent examples, see: a) Sarkar, N.; Banerjee, A.; Nelson, S. G. J. Am. Chem. Soc. 2008, 130, 9222-9223. b) Denmark, S. E.; Baiazitov, R. Y. J. Org. Chem. 2006, 71, 593-605. c) Kobayashi, T.; Nakashima, M.; Hakogi, T.; Tanaka, K.; Katsumur, S. Org. Lett. 2006, 8, 3809-3812.

47 For review, see: a) Felpin, F. X.; Lebreton, J. Eur. J. Org. Chem. 2003, 19, 36933712.

48 For selected reviews, see: a) Cipolla, L.; La Ferla, B.; Nicotra, F. Curr. Top. Med. Chem. 2003, 3, 485-511. b) Okitsu, O.; Suzuki, R.; Kobayashi, S. J. Org. Chem. 2001, 66, 809-863. c) Pearson, M. S. M.; Mathe-Allainmat, M.; Fargeas, V.; Lebreton, J. Eur. J. Org. Chem. 2005, 11, 2159-2191.

49 a) Nilsson, B. L.; Overman, L. E.; de Alaniz, J. R.; Rohde, J. M. J. Am. Chem. Soc. 2008, 130, 11297-11299. b) Denmark, S. E.; Baiazitov, R. Y. J. Org. Chem. 2006, 71, 593-605. c) Sarkar, N.; Banerjee, A.; Nelson, S. G. J. Am. Chem. Soc. 
2008, 130, 9222-9223.

50 a) Whitesides, G. M.; Simanek, E. E.; Mathias, J. P.; Seto, C. T.; Chin, D. N.; Mammen, M.; Gordon, D. M. Acc. Chem. Res. 1995, 28, 37-44. b) Stang, P. J.; Olenyuk, B. Acc. Chem. Res. 1997, 30, 502-518. c) Prins, L. J.; Reinhoudt, D. N.; Timmerman, P. Angew. Chem. Int. Ed. 2001, 40, 2383-2426. d) Reinhoudt, D. N.; Crego-Calama, M. Science, 2002, 295, 2403-2407. e) Stoddart, J. F.; Tseng, H. Proc. Natl. Acad. Sci. U. S. A. 2002, 99, 4797-4800.

51 a) Rodriguez-Hernandez, J.; Checot, F.; Gnanou, Y.; Lecommandoux, S. Prog. Polym. Sci. 2005, 30, 691-724. b) Shimizu, T. Polym. J. 2003, 35, 1-22.

52 a) Hamilton, W. A. Curr. Opin. Colloid Interface Sci. 2005, 9, 390-395. b) Smith, R. K.; Lewis, P. A.; Weiss, P. S. Prog. Surf. Sci. 2004, 75, 1-68. c) Maenosono, S.; Okubo, T.; Yamaguchi, Y. J. Nanopart. Res. 2003, 5, 5-15.

53 Greig, L. M.; Philp, D. Chem. Soc. Rev. 2001, 30, 287-302.

54 a) Davis, J. T. Angew. Chem. Int. Ed. 2004, 43, 668-698. b) Sessler, J. L.; Sathiosatham, M. ; Doerr, K.; Lynch, V.; Abboud, K. A. Angew. Chem. Int. Ed. 2000, 39, 1300-1303.

55 a) Shi, X.; Mullaugh, K. M.; Fettinger, J. C.; Jiang, Y.; Hofstadler, S. A.; Davis, J. T. J. Am. Chem. Soc. 2003, 125, 10830-10841. b) Shi, X.; Fettinger, J. C.; Davis, J. T. Angew. Chem. Int. Ed. 2001, 40, 2827-2831. c) Kotch, F. W.; Fettinger, J. C.; Davis, J. T. Org. Lett. 2000, 2, 3277-3280.

56 Nishimura, K.; Kondo, T.; Drozdova, O. O.; Yamochi, H.; Saito, G. J. Mater. Chem. 2000, 10, 911-919.

57 Solid-state NMR in the determination of metal-G-quartet binding: a) Liu, X. Y.; Kwan, I. C. M.; Wang, S. N.; Wu, G. Org. Lett. 2006, 8, 3685-3688. b) Wong, A.; Ida, R.; Wu, G. Biochem. Biophys. Res. Commun. 2005, 337, 363-366. c) Ida, R.; Wu, G. Chem. Commun. 2005, 4294-4296. d) Wong, A.; Wu, G. J. Am. Chem. Soc. 2003, 125, 13895-13905. 


\section{Part VII \\ Experimental Section}

\section{Section A: General Methods and Materials}

Unless otherwise noted, all commercial reagents and solvents were obtained from the commercial provider and used without further purification. Air and/or moisture-sensitive reactions were carried out under an atmosphere of nitrogen using oven/flame-dried glassware and standard syringe/septa techniques. ${ }^{1} \mathrm{H}-\mathrm{NMR}$ and ${ }^{13} \mathrm{C}-\mathrm{NMR}$ spectra were recorded on Joel 270 and Varian $600 \mathrm{MHz}$ spectrometers. Chemical shifts were reported relative to internal tetramethylsilane $(\delta 0.00 \mathrm{ppm})$ or $\mathrm{CDCl}_{3}(\delta 7.26 \mathrm{ppm})$ for ${ }^{1} \mathrm{H}$ and $\mathrm{CDCl}_{3}(\delta 77.0 \mathrm{ppm})$ or DMSO $(\delta 39.5 \mathrm{ppm})$ for ${ }^{13} \mathrm{C}$. Flash column chromatography was performed on 230-430 mesh silica gel. Analytical thin layer chromatography was performed with precoated glass baked plates $(250 \mu)$ and visualized by fluorescence and by charring after treatment with potassium permanganate or ninhydrin stain. Rf values were obtained by elution in the stated solvent ratios. Optical rotations were measured with a Jasco DIP- 370 digital polarimeter in the solvent specified. High Pressure Liquid chromatography (HPLC) was performed on a SHIMADZU SPD-M20A (LC-20AB) chromatographs using a chiral column $(50 \mathrm{~cm})$ and guard column $(5 \mathrm{~cm})$ as noted for each compound. Mass spectra were obtained from the Micro-Mass/Analytical Facility operated by the Department of Chemistry, West Virginia University. Atomic force microscope (AFM) measurement was carried out on Molecular Imaging (PICOPLUS) in Department of Mechanical \& Aerospace Engineering. Solid-state NMR spectra were recorded at $11.75 \mathrm{~T}$ on a Bruker Avance-500 spectrometer. XRD measurement was performed on Bruker D8 Advance diffractometer using CuKa radiation (scan rate 0.1 $\mathrm{deg} / \mathrm{min}$, scan step $0.02 \mathrm{deg})$.

The nitroalkene starting materials 1a-1e were prepared according to reported procedures: Ohta, H.; Kobayashi, N.; Ozaki, K. J. Org. Chem. 1989, 54, 1802-1804. 
Matin, N. J. A.; Ozores, L.; List, B. J. Am. Chem. Soc. 2007, 129, 8976-8977. Nitroalkene 1f-1h were prepared according to reported procedure: Jang, Y. J.; Lin, W. W.; Shih, Y. K.; Liu, J. T.; Hwang, M. H.; Yao, C. F. Tetrahedron, 2003, 59, 4979-4992. Allylic nitro compounds 1a-1e were synthesized according to the literature: X. Sun, S. Sengupta, J. L. Petersen, H. Wang, J. P. Lewis, X. Shi, Org. Lett. 2007, 9, 4495-4498.

The catalysts (cat-1 to cat-8) were either purchased directly from Aldrich or prepared based on the reported literature.

The corresponding quinine tertiary amine ylide (aux-a) and camphor derived sulfur ylides (aux-b-f) were prepared according to related literature.

Zheng, J.; Zhu, C.; Sun, L.; Tang, Y.; Dai, L. J. Org. Chem. 2008, 73, 6909-6912.

Zhu, C.; Deng, X.; Sun, X.; Zheng, J.; Tang, Y. Chem. Commun. 2008, 738-740.

Nitroalkenes 37 was synthesis according to the literature as below:

Bowman, R. K.; Johnson, J. S. J. Org. Chem. 2004, 69, 8537-8540.

Trost, B. M.; Mueller, C. J. Am. Chem. Soc. 2008, 130, 2438-2439. 


\section{Section B: General Experimental Procedures}

\section{General procedure of crossed conjugate addition of nitroalkene to enal:}

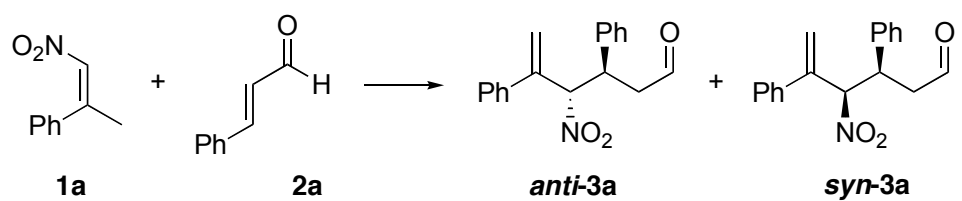

\section{1) Racemic sample preparation:}

The nitroalkene 1a (1.0 mmol, 1.0 eq.) was added to a solution of $\alpha, \beta$-unsaturated aldehyde $2 \mathrm{a}$ (1.5 mmol, 1.5 eq.), L-Proline (0.2mmol, $20 \mathrm{~mol} \%$ ), AcOH (0.2 mmol, 0.2 eq) and $\mathrm{NaN}_{3}$ (1.0 eq.) in $\mathrm{MeOH}$, with a concentration of $0.1 \mathrm{M}$ for 1a. The resulting reaction mixture was stirred at room temperature for $5 \sim 8$ hours followed by the dilution with dichloromethane. The organic layer was washed with $\mathrm{NaHCO}_{3}$ sat. solution and brine and then dried over anhydrous sodium sulfate. The solvent was removed under reduced pressure with flash silica gel chromatography to give the pure product. The two diastereomers were difficult to separate. $\mathrm{NaBH}_{4}$ (1.0 eq.) was then applied to reduce aldehydes quantitatively into alcohols, which were then separated and examined for e.e.

\section{2) Optical sample preparation:}

The $\alpha, \beta$-unsaturated aldehyde $2 \mathrm{a}(3.0 \mathrm{mmol}, 3.0$ eq.) was added to (S)-(-)-2(Diphenylhydroxymethyl)pyrrolidine (0.2 mmol, $20 \mathrm{~mol} \%$ ), AcOH (1.0 mmol, 1.0 eq.) and $(\mathrm{MeO})_{3} \mathrm{P}(1.0 \mathrm{mmol}, 1.0$ eq. $)$ in $5 \mathrm{~mL} \mathrm{MeOH}$. Stir at room temperature for 30 minutes, cool down to $-25{ }^{\circ} \mathrm{C}$, stir for another 15 minutes, nitroalkene 1a $(1.0 \mathrm{mmol}, 1.0$ eq., $0.2 \mathrm{M}$ ) was added in and keep stirring for 48 60 hours. The resulting reaction mixture was diluted with dichloromethane. The organic layer was washed with sat. $\mathrm{NaHCO}_{3}$ solution and brine and then dried over anhydrous sodium sulfate. The solvent was removed under reduced pressure with flash silica gel chromatography to give the pure product. $\mathrm{NaBH}_{4}$ (1.0 eq.) was then applied to reduce aldehydes quantitatively into alcohols, which were then separated and examined for e.e. 


\section{Synthesis of compound $6 \mathrm{~b}$}

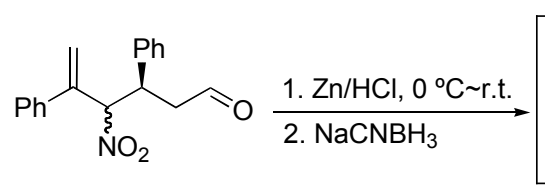

$3 a$

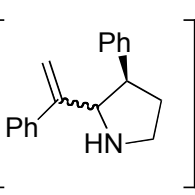

syn/anti 5a

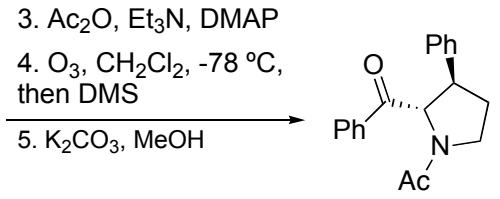

6b

To a solution of aldehydes (anti-3a \& syn-3a) $(500 \mathrm{mg}, 1.69 \mathrm{mmol})$ in 2-propanol (50 $\mathrm{ml}), 1 \mathrm{~N} \mathrm{HCl}(25 \mathrm{ml}, 25 \mathrm{mmol})$ and activated $\mathrm{Zn}$ powder $(2.17 \mathrm{~g}, 33.9 \mathrm{mmol})$ were added at $0{ }^{\circ} \mathrm{C}$. And the mixture was stirring for another 3hours at room temperature. To the mixture, $\mathrm{NaCl}$ sat. solution $(50 \mathrm{ml})$ was added, followed by the extraction by $\mathrm{CH}_{2} \mathrm{Cl}_{2}$ ( $35 \mathrm{ml}$ x 3). The combined organic layer was washed with $\mathrm{NaHCO}_{3}$ (aq.) and brine, then dried over with $\mathrm{Na}_{2} \mathrm{SO}_{4}$. After filtration and evaporation, the residue was dissolved in $\mathrm{CH}_{2} \mathrm{Cl}_{2}(25 \mathrm{ml})$. To the solution, $\mathrm{Et}_{3} \mathrm{~N}(341 \mathrm{mg}, 3.38 \mathrm{mmol})$ and $\mathrm{Ac}_{2} \mathrm{O}(260 \mathrm{mg}, 2.54$ mmol) and DMAP $(10.3 \mathrm{mg}, 0.08 \mathrm{mmol})$ were added at $0^{\circ} \mathrm{C}$. The mixture was stirred at room temperature and monitored by TLC till the reaction was done in 3 hours. After removal of the solvent, the residue was diluted in $50 \mathrm{ml}$ ether and washed with $50 \mathrm{ml}$ water. The aqueous phase was then extracted with $50 \mathrm{ml}$ ether again. The combined organic solution was then washed with Brine and dried over $\mathrm{Na}_{2} \mathrm{SO}_{4}$. After filtration and evaporation, the residue was dissolved in $\mathrm{CH}_{2} \mathrm{Cl}_{2}(25 \mathrm{ml})$ in a $50 \mathrm{ml}$ two-neck flask equipped with a gas dispersing tube. A stream of ozone was bubbled through the solution at $-78{ }^{\circ} \mathrm{C}$. Ozone treatment was terminated when the starting material was gone. To the solution added $\mathrm{Me}_{2} \mathrm{~S}$ after which the solvent was removed under reduced pressure. $\mathrm{K}_{2} \mathrm{CO}_{3} \mathrm{MeOH}$ solution was then applied and followed by flash silica gel chromatography to gave $356 \mathrm{mg}$ amide $\mathbf{6 b}$. The yield over these four steps is $72 \%$. Only one trans was obtained since.

\section{$\underline{\text { Synthesis of compound } 7 \mathrm{~b}}$}

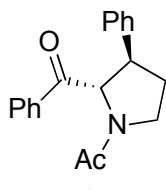

$6 \mathbf{b}$
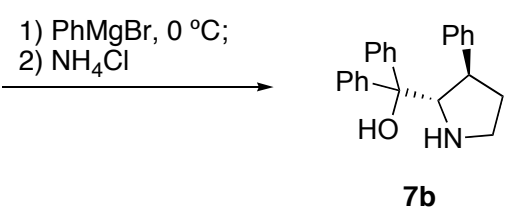
To a solution of amide $6 \mathbf{b}(150 \mathrm{mg}, 0.51 \mathrm{mmol})$ in dry THF under $\mathrm{N}_{2}$ atmosphere at $0{ }^{\circ} \mathrm{C}$, was added $\mathrm{PhMgBr}(0.18 \mathrm{~mL}, 2.8 \mathrm{M}$ in THF) dropwise. The mixture was stirred at room temperature and monitored by TLC till starting materials all gone. $\mathrm{NH}_{4} \mathrm{Cl}$ (aq.) $(5 \mathrm{~mL})$ was added to quench the reaction, the mixture was extracted with ether $(25 \mathrm{ml} \times 3)$, washed with brine and dried over with $\mathrm{Na}_{2} \mathrm{SO}_{4}$. After removal of the solvent, the residue was purified by flash silica gel chromatography to give $136 \mathrm{mg}$ as an oil, yield $81 \%$.

\section{Synthesis of compound 6a}

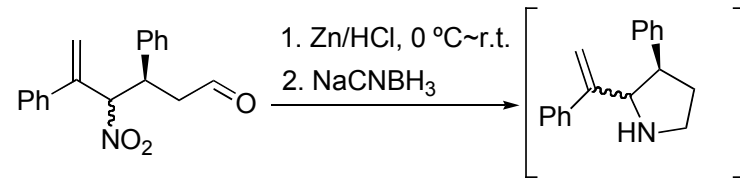

3a syn/anti 5a

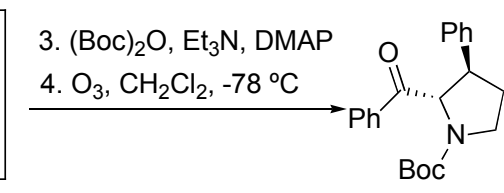

$6 a$

To a solution of aldehydes (anti-3a \& syn-3a) $(500 \mathrm{mg}, 1.69 \mathrm{mmol})$ in 2-propanol (50 $\mathrm{ml}), 1 \mathrm{~N} \mathrm{HCl}(25 \mathrm{ml}, 25 \mathrm{mmol})$ and activated $\mathrm{Zn}$ powder $(2.17 \mathrm{~g}, 33.9 \mathrm{mmol})$ were added at $0^{\circ} \mathrm{C}$. And the mixture was stirring for another $3 \mathrm{hr}$ at room temperature. To the mixture, $\mathrm{NaCl}$ sat. solution $(50 \mathrm{ml})$ was added, followed by the extraction by $\mathrm{CH}_{2} \mathrm{Cl}_{2}(35 \mathrm{ml} \times 3)$. The combined organic layer was washed with $\mathrm{NaHCO}_{3}(\mathrm{aq})$ and brine, then dried over with $\mathrm{Na}_{2} \mathrm{SO}_{4}$. After filtration and evaporation, the residue was dissolved in $\mathrm{CH}_{2} \mathrm{Cl}_{2}(25$ $\mathrm{ml})$. To the solution, $\mathrm{Et}_{3} \mathrm{~N}(341 \mathrm{mg}, 3.38 \mathrm{mmol})$ and $(\mathrm{Boc})_{2} \mathrm{O}(553 \mathrm{mg}, 2.54 \mathrm{mmol})$ and DMAP $(10.3 \mathrm{mg}, 0.08 \mathrm{mmol})$ were added at $0^{\circ} \mathrm{C}$. The mixture was stirred at room temperature and monitored by TLC till the reaction was done in 3 hours. After removal of the solvent, the residue was diluted in $50 \mathrm{ml}$ ether and washed with $50 \mathrm{ml}$ water. The aqueous phase was then extracted with $50 \mathrm{ml}$ ether again. The combined organic solution was then washed with Brine and dried over $\mathrm{Na}_{2} \mathrm{SO}_{4}$. After filtration and evaporation, the residue was dissolved in $\mathrm{CH}_{2} \mathrm{Cl}_{2}(25 \mathrm{ml})$ in a $50 \mathrm{ml}$ two-neck flask equipped with a gas dispersing tube. A stream of ozone was bubbled through the solution at $-78^{\circ} \mathrm{C}$. Ozone treatment was terminated when the starting material was gone. To the solution added $\mathrm{Me}_{2} \mathrm{~S}$ after which the solvent was removed under reduced pressure and followed by flash silica gel chromatography to gave carbamide $\mathbf{6 a} 462 \mathrm{mg}$ as white solid. A diastereo ratio 
of 1.8:1 (trans: cis) was detected. Treatment of $\mathrm{K}_{2} \mathrm{CO}_{3}$ (1.0 eq.) in $\mathrm{MeOH}(0.2 \mathrm{M})$ for 5 hours at room temperature improved d.r. to 10:1 (trans: cis), without loss of yield.

\section{Synthesis of compound $7 \mathbf{a}$}

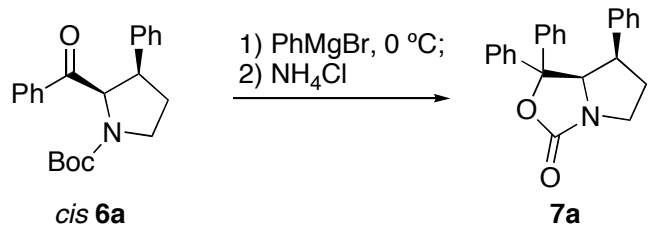

To a solution of carbamide cis-6a (100 mg, $0.28 \mathrm{mmol}$ )in dry THF under $\mathrm{N}_{2}$ atmosphere at $0^{\circ} \mathrm{C}$, was added $\mathrm{PhMgBr}(0.1 \mathrm{ml}, 2.8 \mathrm{M}$ in THF) dropwise. The mixture was stirred at room temperature and monitored by $\mathrm{TLC}$. $\mathrm{NH}_{4} \mathrm{Cl}$ (aq.) $(5 \mathrm{~mL})$ was added to quench the reaction, the mixture was extracted with ether $(25 \times 2 \mathrm{ml})$, washed with brine and dried over with $\mathrm{Na}_{2} \mathrm{SO}_{4}$. After removal of the solvent, the residue was purified by flash silica gel chromatography (EtOAc/Hexane, $\mathrm{V} / \mathrm{V}=1: 5)$ gave $87 \mathrm{mg}$ as a white solid, yield $87 \%$.

\section{Procedure for synthesis of cyclohexane derivatives 11}

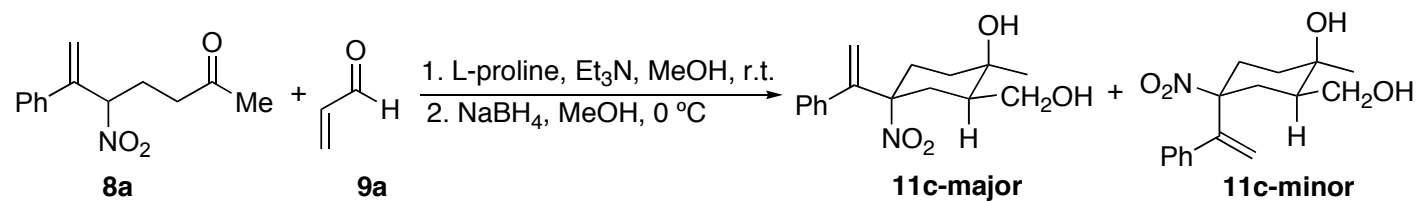

The allylic nitro compound $8 \mathbf{a}(233 \mathrm{mg}, 1 \mathrm{mmol})$ and L-proline $(23 \mathrm{mg}, 0.2 \mathrm{mmol})$ was dissolved in $\mathrm{MeOH}(5 \mathrm{~mL}, 0.2 \mathrm{M})$. To this solution was added the $\mathrm{Et}_{3} \mathrm{~N}(101 \mathrm{mg}, 1$ $\mathrm{mmol})$ and acrolein 9a $(117 \mathrm{mg}, 90 \%, 1.5 \mathrm{mmol})$ at room temperature. The resulting mixture was then stirred and checked by TLC. After the reaction had completed, the mixture was diluted with $\mathrm{MeOH}(10 \mathrm{~mL})$, the solution was passed through a short silica gel column and then treated with $\mathrm{NaBH}_{4}(152 \mathrm{mg}, 4 \mathrm{mmol})$ at $0{ }^{\circ} \mathrm{C} .15$ minuntes later, the solution was quenched with water, neutralized by dilute $\mathrm{HCl}(1 \mathrm{M})$ solution and extracted with EtOAc $(40 \mathrm{~mL}$ x 3). The combined organic layer was washed with brine and dried over with anhydrous $\mathrm{Na}_{2} \mathrm{SO}_{4}$. The solvent was removed under reduced pressure to give a 
residue, the residue was the purified by flash silica gel chromatography to obtain two diasteroisomers 11c as colorless oil, yield: 92\%.

\section{Procedure for synthesis of cyclohexane derivatives 12}

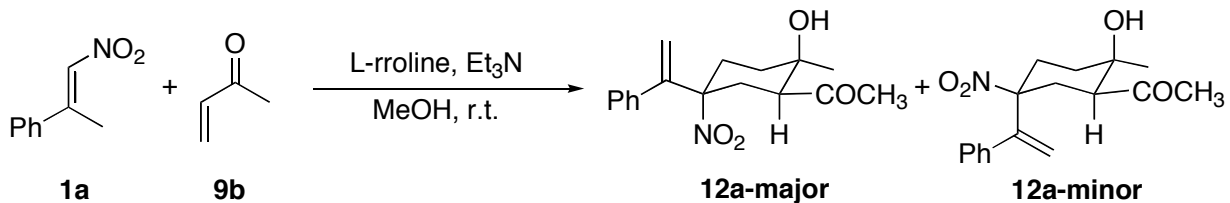

The nitroalkene 1a (163 mg, $1 \mathrm{mmol}, 1.0$ eq.) and L-proline (23 mg, $0.2 \mathrm{mmol}, 0.2$ eq.) was dissolved in $\mathrm{MeOH}(5 \mathrm{~mL}, 0.2 \mathrm{M})$. To this solution was added $\mathrm{Et}_{3} \mathrm{~N}(101 \mathrm{mg}, 1$ mmol) and MVK 9b (280 mg, 4 mmol, 4.0 eq.) at room temperature. The resulting mixture was then stirred at room temperature and checked by TLC. When the reaction had completed, the solvent was removed under reduced pressure to give a residue, the residue was then purified by flash silica gel chromatography to obtain two diasteroisomers (3.7:1) 12a as white solids, yield: 90\%.

\section{Procedure for synthesis of $10 a$}

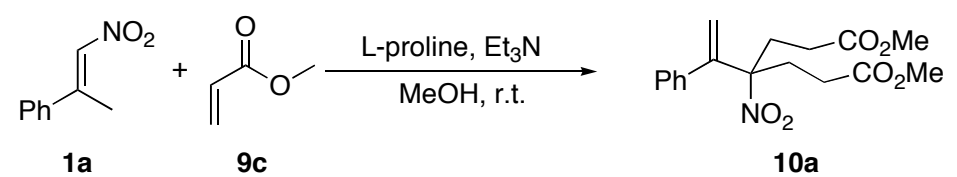

The nitroalkene 1a (163 mg, $1 \mathrm{mmol})$ and L-proline $(23 \mathrm{mg}, 0.2 \mathrm{mmol})$ was dissolved in $\mathrm{MeOH}$ (5 mL, $0.2 \mathrm{M})$. To this solution was added the $\mathrm{Et}_{3} \mathrm{~N}$ (101 mg, $1 \mathrm{mmol}$ ) and methyl acrylate 9c (344 mg, $4 \mathrm{mmol})$ at room temperature. The resulting mixture was then stirred and checked by TLC. After the reaction had completed, the solvent was removed under reduced pressure to give a residue, the residue was the purified by flash silica gel chromatography to obtain $\mathbf{1 0 a}(298 \mathrm{mg}, 0.89 \mathrm{mmol})$ as white solid, yield: $89 \%$.

\section{Procedure for synthesis of 13a}

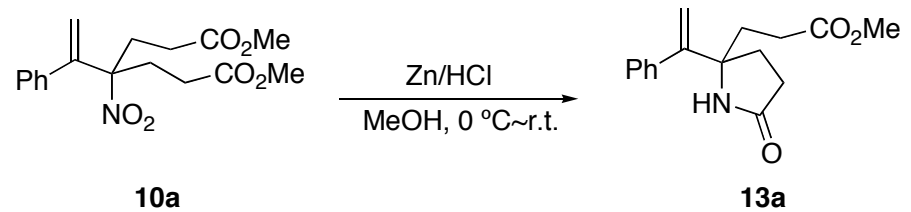


To a solution of 10a (200 mg, $0.6 \mathrm{mmol})$ in $\mathrm{MeOH}(40 \mathrm{~mL}), 1 \mathrm{~N} \mathrm{HCl}(6 \mathrm{~mL}, 6 \mathrm{mmol})$ and activated $\mathrm{Zn}$ powder $(585 \mathrm{mg}, 9 \mathrm{mmol})$ were added at $0{ }^{\circ} \mathrm{C}$. And the mixture was stirred at room temperature for 8 hours. To the mixture, was added $\mathrm{NaHCO}_{3}$ (aq.) until $\mathrm{pH}>10$, followed by the extraction with $\mathrm{CH}_{2} \mathrm{Cl}_{2}(30 \mathrm{~mL} \times 5)$. The combined organic layer was washed with brine and then dried over with anhydrous $\mathrm{Na}_{2} \mathrm{SO}_{4}$. After filtration and evaporation, the residue was purified by flash silica gel chromatography with EtOAc/Hexane (1:2, v/v) to get 13a $(140 \mathrm{mg}, 0.51 \mathrm{mmol})$ as a white solid, yield: $85 \%$.

\section{Procedure for synthesis of $13 \mathrm{~b}$}

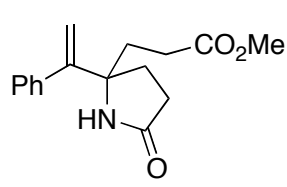

$13 a$

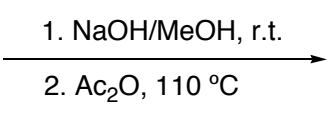

2. $\mathrm{Ac}_{2} \mathrm{O}, 110^{\circ} \mathrm{C}$

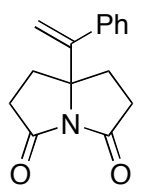

13b

To a solution of 13a $(100 \mathrm{mg}, 0.37 \mathrm{mmol})$ in $\mathrm{MeOH}(5 \mathrm{~mL})$ was added the $\mathrm{NaOH}(22$ $\mathrm{mg}, 0.56 \mathrm{mmol}$ ) at room temperature. The mixture was stirred for an hour. To this mixture was added $2 \mathrm{~N} \mathrm{HCl}(0.5 \mathrm{~mL})$ and then extract with EtOAc $(20 \mathrm{~mL} \times 3)$, the combined organic layer was washed with brine, dried over with anhydrous $\mathrm{Na}_{2} \mathrm{SO}_{4}$. The solvent was removed under reduced pressure to get a colorless solid. To this solid was added $\mathrm{Ac}_{2} \mathrm{O}(5 \mathrm{~mL})$, the solution was stirred for $3 \mathrm{hr}$ at $110{ }^{\circ} \mathrm{C}$. After returned to room temperature, the solution was poured into water $(20 \mathrm{~mL})$ and stirred for half an hour. Then neutralized by $\mathrm{NaHCO}_{3}$ (aq.), followed by extraction with EtOAc $(20 \mathrm{~mL} \times 3)$, the combined organic layer was washed with brine, dried with anhydrous $\mathrm{Na}_{2} \mathrm{SO}_{4}$. The solvent was removed under reduced pressure to get a residue. The residue can purified by recrystallization (EtOAc/Hexane, 1:5, v/v) or flash silica gel chromatography with EtOAc/Hexane $(1: 3, \mathrm{v} / \mathrm{v})$ to give $\mathbf{1 3 b}(70 \mathrm{mg}, 0.29 \mathrm{mmol})$ as colorless crystal, yield: $79 \%$.

\section{Procedure for synthesis of $13 \mathrm{c}$}

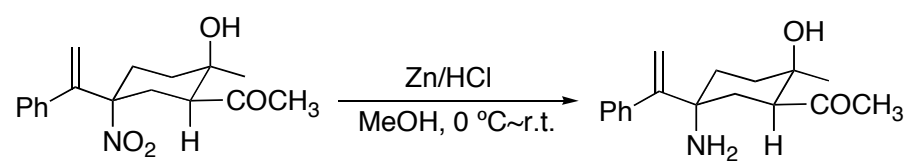


To a solution of 12a-major (303 mg, $1 \mathrm{mmol})$ in $\mathrm{MeOH}(25 \mathrm{~mL}), 1 \mathrm{~N} \mathrm{HCl}(10 \mathrm{~mL}, 10$ $\mathrm{mmol}$ ) and $\mathrm{Zn}$ powder $(975 \mathrm{mg}, 15 \mathrm{mmol})$ were added at $0{ }^{\circ} \mathrm{C}$. And the mixture was stirred at room temperature for $4 \mathrm{hr}$. To the mixture was added $\mathrm{NaHCO}_{3}(\mathrm{aq})$ until $\mathrm{pH}>$ 10 , followed by the extraction with $\mathrm{CH}_{2} \mathrm{Cl}_{2}(20 \mathrm{~mL} \times 5)$. The combined organic layer was washed with brine and dried over with anhydrous $\mathrm{Na}_{2} \mathrm{SO}_{4}$. After filtration and evaporation, the residue was purified by flash silica gel chromatography with EtOAc/hexane $(1: 1, \mathrm{v} / \mathrm{v})$ to give $\mathbf{1 3 c}(240 \mathrm{mg}, 0.88 \mathrm{mmol})$ as colorless oil, yield: $88 \%$.

\section{Procedure for synthesis of $13 d$}

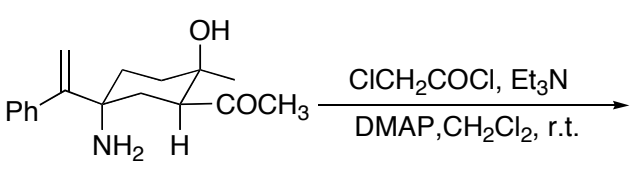

13c

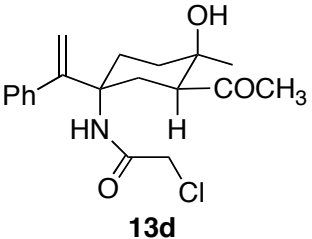

$13 d$

To a solution of 13c (273 mg, $1 \mathrm{mmol})$ DMAP (6 mg, $0.05 \mathrm{mmol})$ and $\mathrm{Et}_{3} \mathrm{~N}(150 \mathrm{mg}, 1.5$ mmol) in $\mathrm{CH}_{2} \mathrm{Cl}_{2}(10 \mathrm{~mL})$, 2-chloroacetyl chloride $(136 \mathrm{mg}, 1.2 \mathrm{mmol})$ was added by dropwise. The mixture solution was stirred at room temperature and monitored by TLC. The solution was diluted with EtOAc $(20 \mathrm{~mL})$, and poured into $\mathrm{NaHCO}_{3}(\mathrm{aq})$, then extracted with EtOAc $(20 \mathrm{~mL} \times 3)$. The combined organic layer was washed with brine, dried over with anhydrous $\mathrm{Na}_{2} \mathrm{SO}_{4}$. After filtration and evaporation, the residue was purified by flash silica gel chromatography with EtOAc/Hexane $(1: 5, \mathrm{v} / \mathrm{v})$ to give 13d (318 $\mathrm{mg}, 0.91 \mathrm{mmol}$ ) as white solid, yield: 91\%.

\section{Procedure for synthesis of $13 \mathrm{e}$}

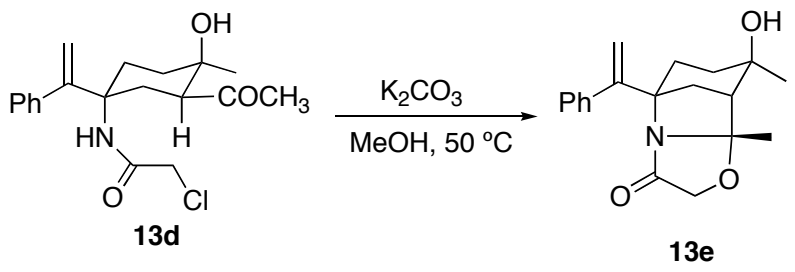

To a solution of 13d (50 mg, $0.14 \mathrm{mmol})$ in $\mathrm{MeOH}(2 \mathrm{~mL})$ was added the $\mathrm{K}_{2} \mathrm{CO}_{3}(29 \mathrm{mg}$, $0.21 \mathrm{mmol}$ ), the mixture solution was stirred at $50{ }^{\circ} \mathrm{C}$ and checked by TLC. The solvent 
was removed under reduced pressure and the residue was purified by flash silica gel chromatography with EtOAc/Hexane $(1: 2, \mathrm{v} / \mathrm{v})$ to obtain $13 \mathbf{e}(36 \mathrm{mg}, 0.12 \mathrm{mmol})$ as colorless oil, yield: $83 \%$.

\section{General Procedure for preparation of homo-isoxazoline-N-oxide (15):}

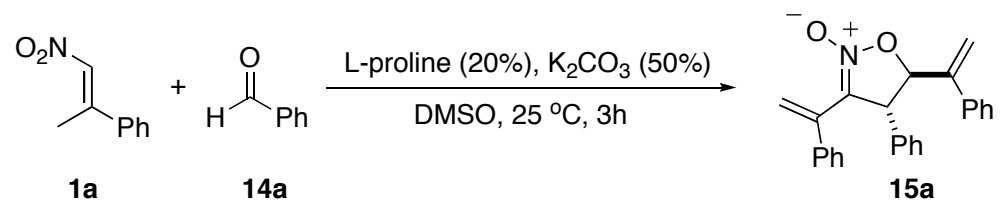

The nitroalkene 1a (1.1 mmol, 1.1 eq.) was added to a DMSO solution of aldehyde 14a (0.5 mmol, 0.5 eq.), L-proline ( $25 \mathrm{mg}, 0.22 \mathrm{mmol}, 0.2$ eq.), and $\mathrm{K}_{2} \mathrm{CO}_{3}$ ( $35 \mathrm{mg}, 0.25$ mmol, 0.5 eq.), with a concentration of $0.1 \mathrm{M}$ for aldehyde. The resulting reaction mixture was stirred at room temperature for $2 \sim 5$ hours monitored by TLC. Upon the aldehyde was all consumed, the mixture was diluted with EtOAc $(100 \mathrm{~mL})$. The organic phase was washed by $\mathrm{HCl}$ solution $(1.0 \mathrm{M})$, saturated $\mathrm{NaHCO}_{3}$ (aq.) and brine and then dried over anhydrous $\mathrm{Na}_{2} \mathrm{SO}_{4}$. The solvent was removed under reduced pressure to give a residue. Flash silica gel chromatography was then applied to give the product.

\section{Procedure for the synthesis of diene intermediate $A^{\prime}$ :}

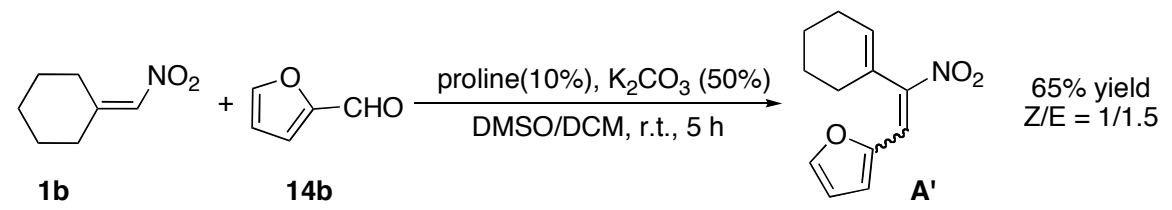

The nitroalkene $1 \mathbf{b}(1.0 \mathrm{mmol}, 0.1 \mathrm{M}, 1.0$ eq.) DMSO solution was added to a DCM solution of aldehyde 14b (1.5 mmol, 0.15 M. 1.5 eq.), L-Proline (11 mg, $0.1 \mathrm{mmol}, 0.1$ eq.), and $\mathrm{K}_{2} \mathrm{CO}_{3}$ ( $35 \mathrm{mg}, 0.25 \mathrm{mmol}, 0.5$ eq.). Upon the DCM solution was mixed well, the nitroalkene was added dropwise through $5 \mathrm{~min}$. The resulting reaction mixture was stirred at room temperature for 2 hours monitored by TLC. Upon the nitroalkene was all consumed, the mixture was diluted with EtOAc $(100 \mathrm{~mL})$. The organic phase was washed by brine and then dried over anhydrous $\mathrm{Na}_{2} \mathrm{SO}_{4}$. The solvent was removed under reduced pressure to give a residue. Flash silica gel chromatography was then applied to give the 
$\mathrm{Z} / \mathrm{E}$ isomers $\mathbf{A}^{\prime}$ as a mixture, $\mathrm{Z} / \mathrm{E}=1 / 1.5,143 \mathrm{mg}, 65 \%$ yield based on the nitroalkene. Detailed NMR study of $\mathbf{A}^{\prime}$ is shown as below.

${ }^{1} \mathrm{H}$ NMR

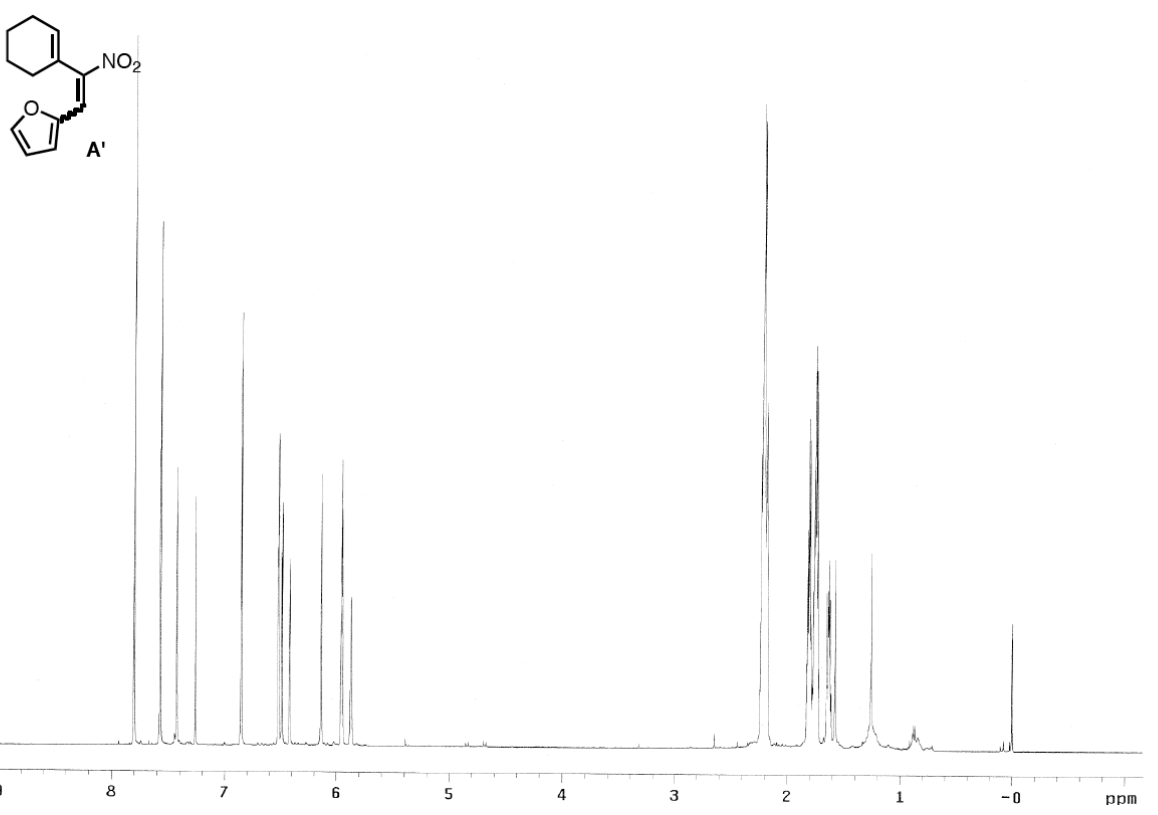

${ }^{13} \mathrm{C}$ NMR<smiles>O=[N+]([O-])C(=Cc1ccco1)C1=CCCCC1</smiles>

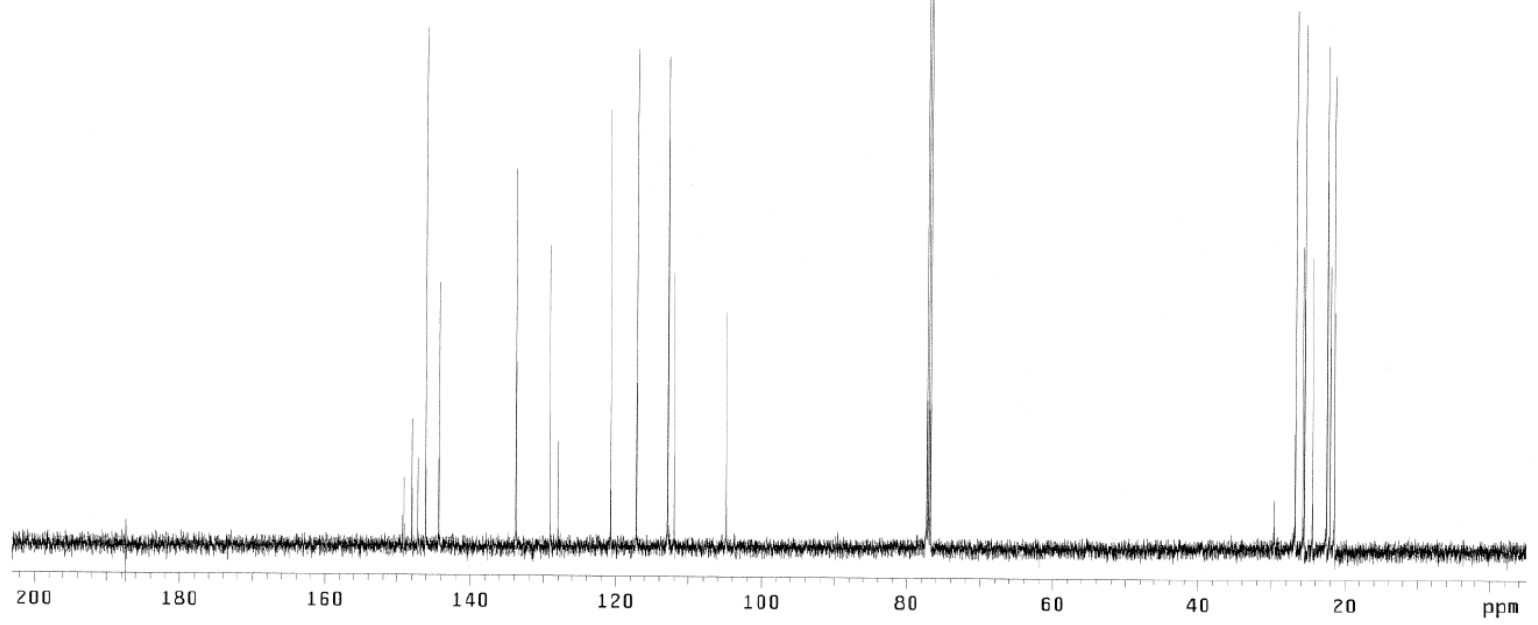

91 

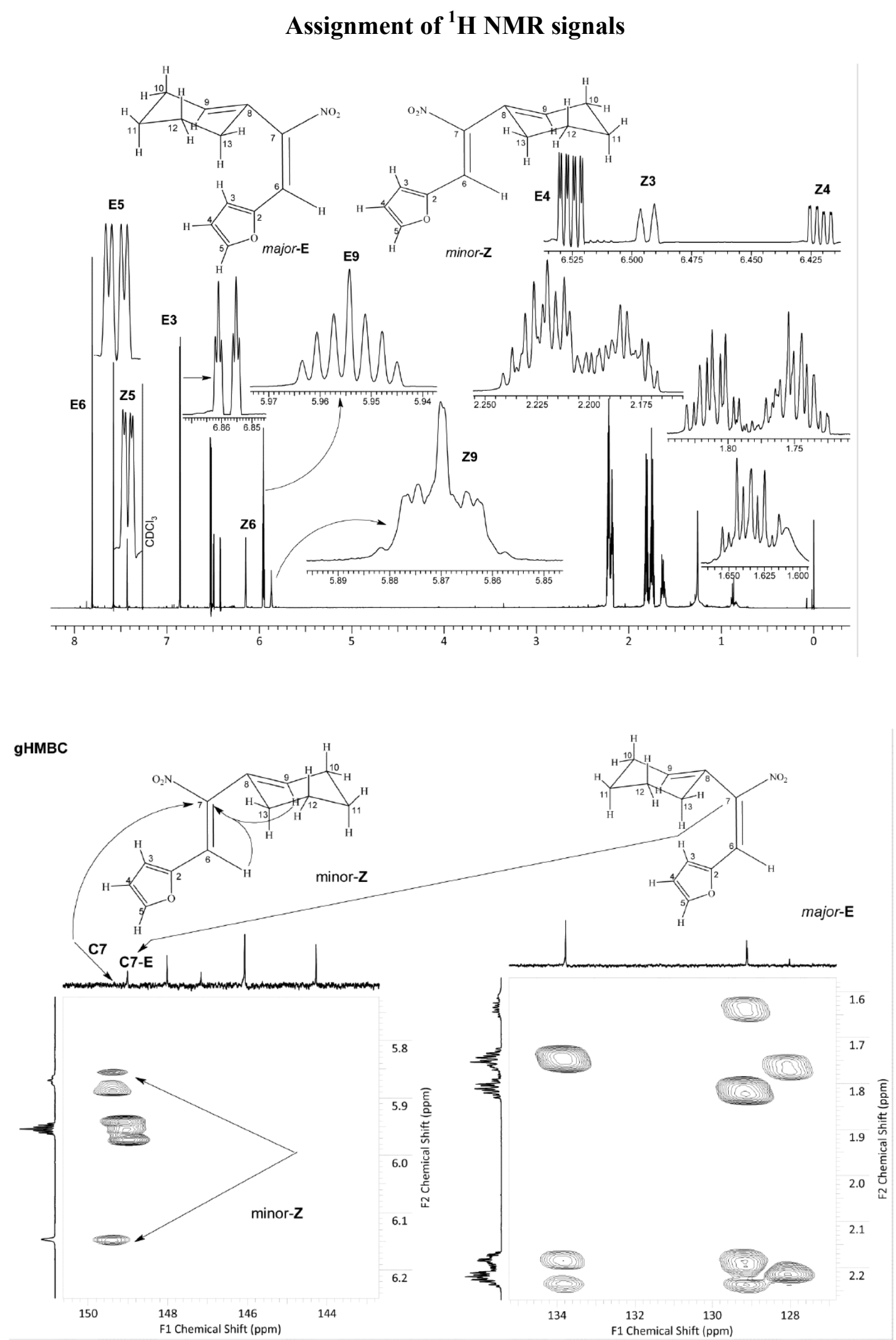


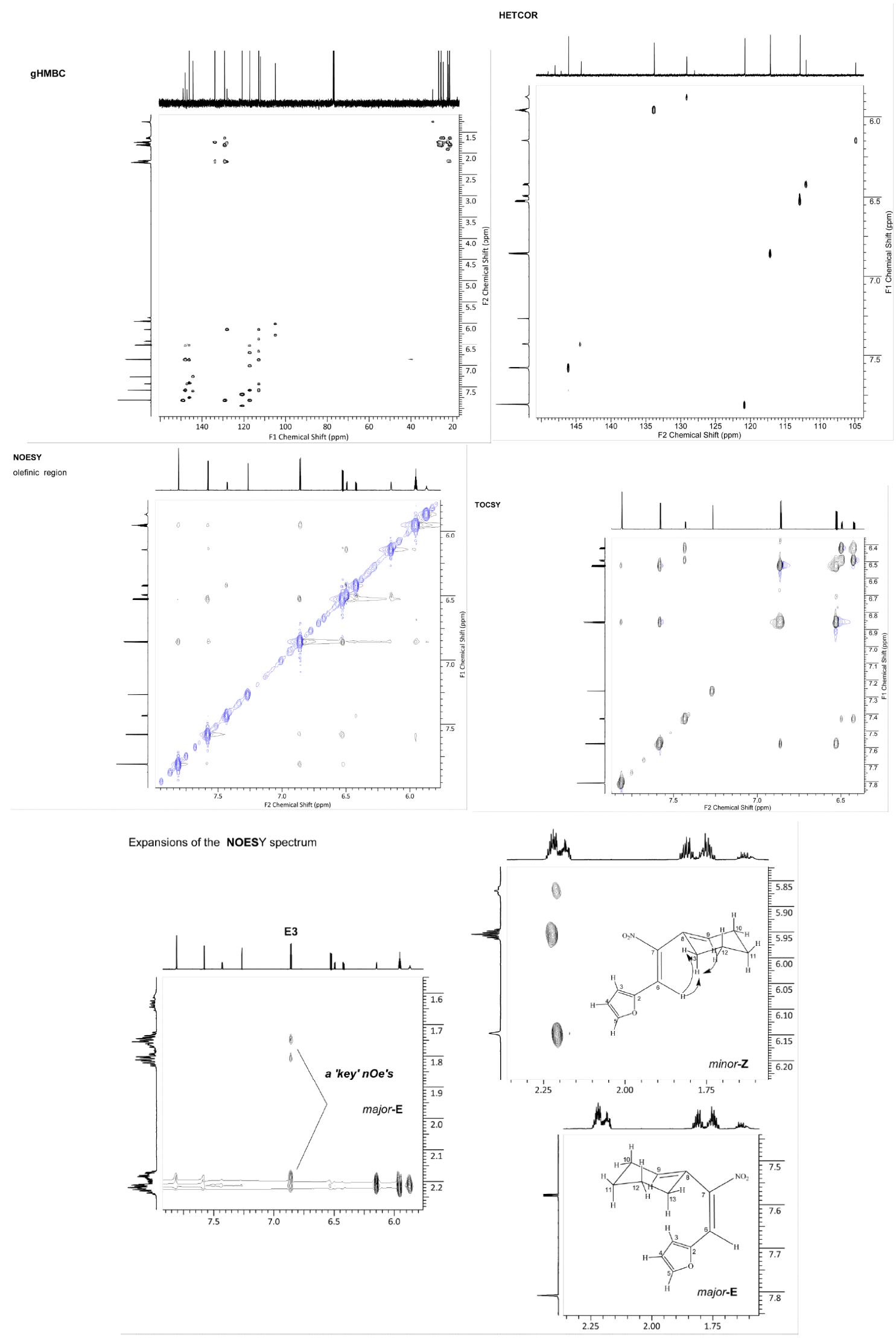




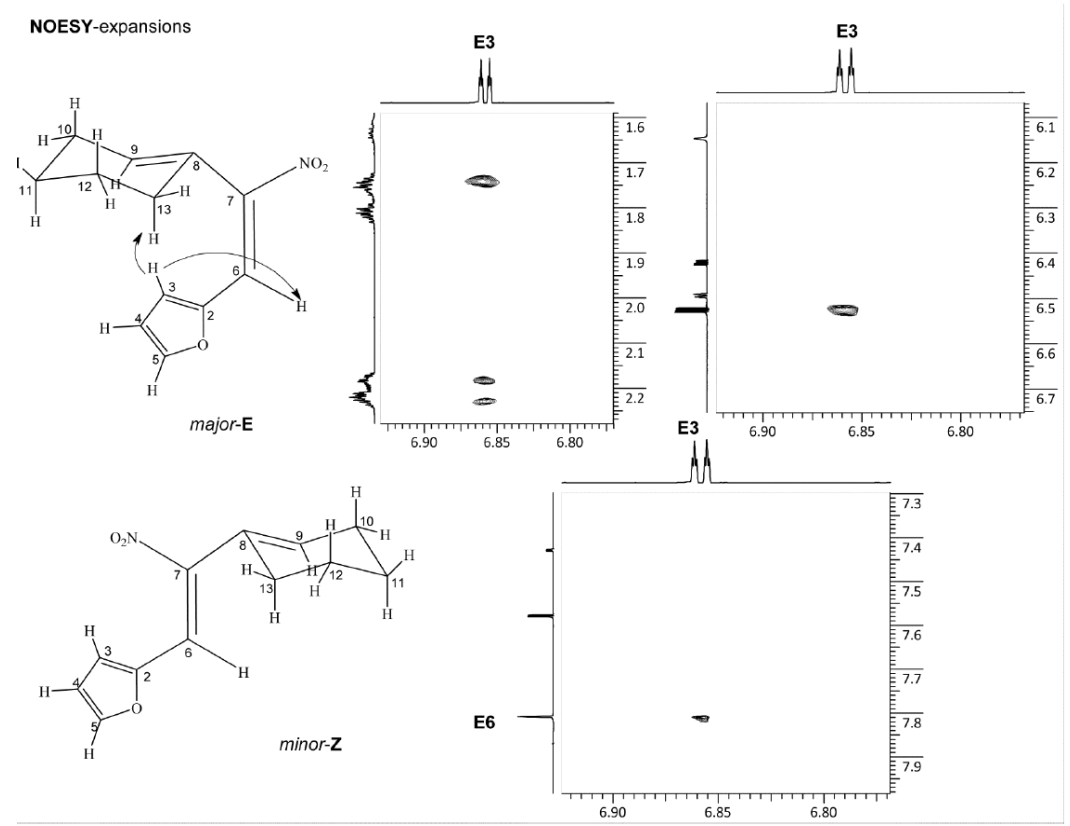

\section{Procedure for the cross condensation through diene intermediate $A^{\prime}$ and 1a:}

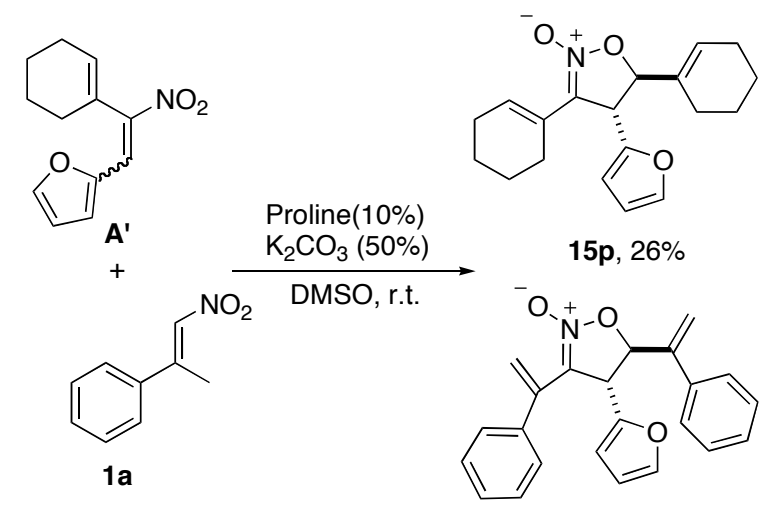

15b, $18 \%$

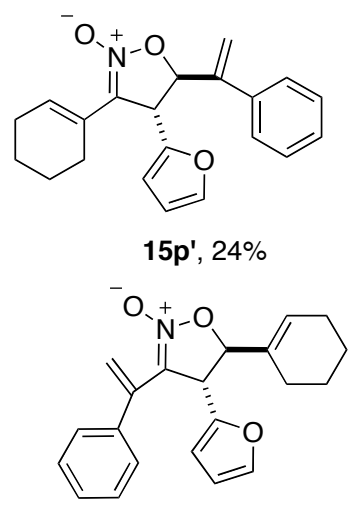

15b', $6 \%$

The diene $\mathbf{A}^{\prime}$ (0.6 mmol, 1.0 equiv.) was then mixed with nitroalkene 1a (0.65 mmol, 1.1 equiv.) in DMSO, with $\mathbf{A}^{\prime}$ as $0.1 \mathrm{M}$. Proline (14 $\mathrm{mg}, 0.12 \mathrm{mmol}, 0.2$ equiv.) and $\mathrm{K}_{2} \mathrm{CO}_{3}$ (41 mg, $0.30 \mathrm{mmol}, 0.5$ equiv.) were added in the solution. Keep stirring and monitored by TLC. Two hrs later, when all the nitroalkene was consumed, the mixture was diluted with EtOAc $(100 \mathrm{~mL})$. The organic phase was washed by saturated $\mathrm{NaHCO}_{3}$ (aq.) and brine and then dried over anhydrous $\mathrm{Na}_{2} \mathrm{SO}_{4}$. The solvent was removed under reduced pressure to give a residue. Flash silica gel chromatography was then applied to give the crude product 3b (18\%), 3b' (6\%), 3s (26\%), 3s' (24\%). These four compounds could not be separated by chromatography. 


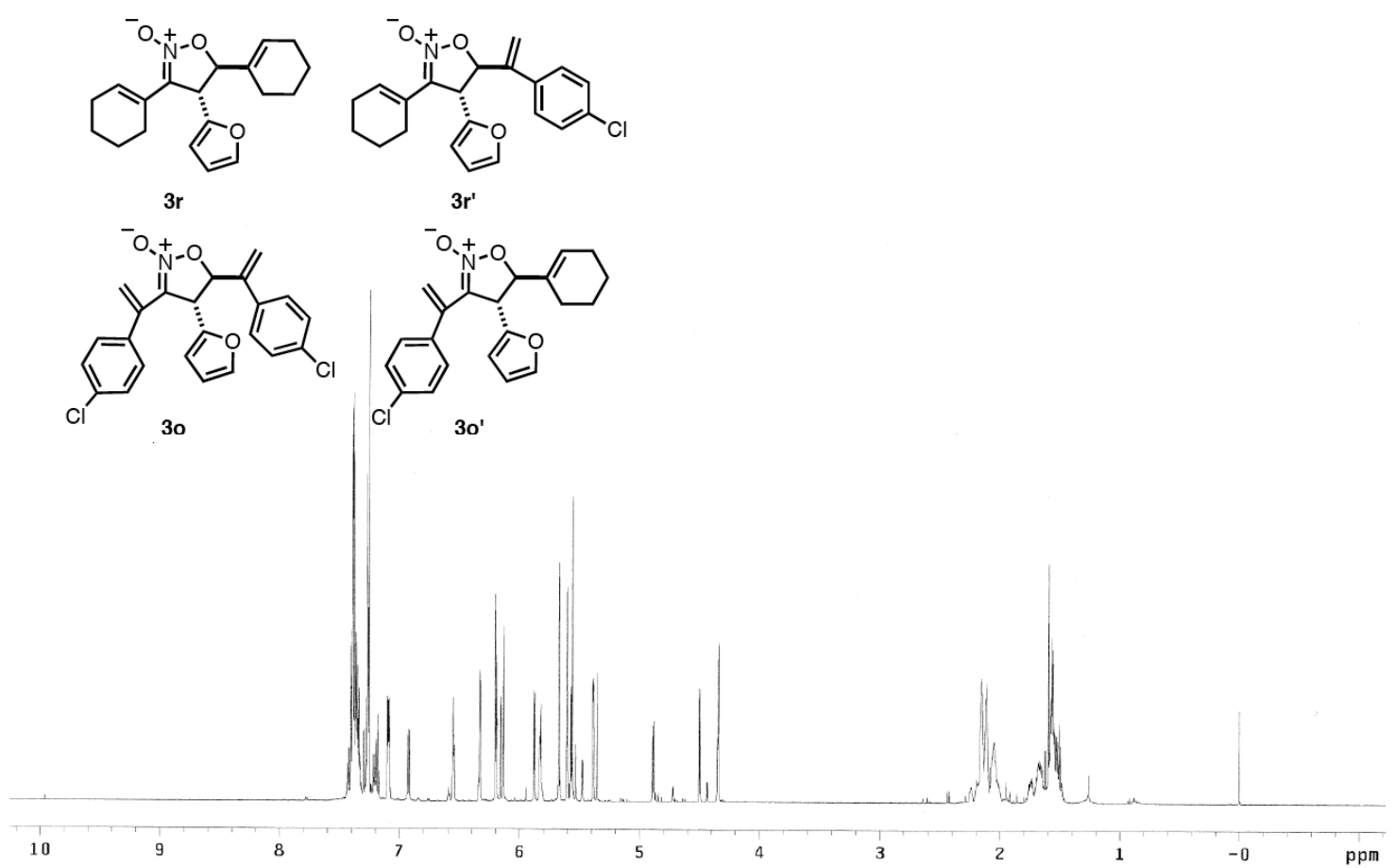

General Procedure for preparation of three-component isoxazoline-N-oxide (18):

\section{1) Racemic sample preparation:}
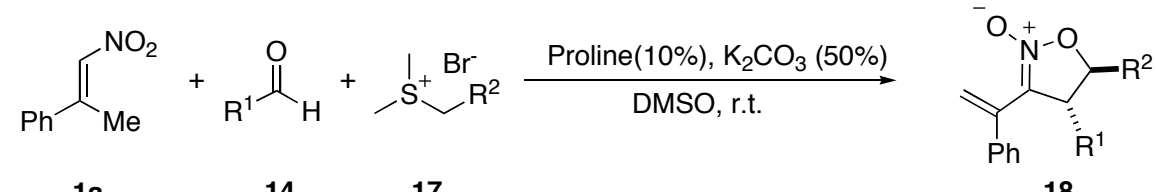

The nitroalkene 1a $(0.6 \mathrm{mmol}, 1.2$ eq.) was added to a solution of the corresponding sulfur ylide (0.55 mmol, 1.1 eq.) DMSO (0.2 $\mathrm{M}$ for nitroalkene) solution, till the ylide is all dissolved. Proline (12 mg, $0.1 \mathrm{mmol}, 0.2$ eq.) and $\mathrm{K}_{2} \mathrm{CO}_{3}(35 \mathrm{mg}, 0.25 \mathrm{mmol}, 0.5$ eq.) were then added in and stir for 5 minutes. The aldehyde $(0.5 \mathrm{mmol}, 1.0$ eq.) DMSO solution $(0.2 \mathrm{M})$ was added drop wise through 5 minutes. The resulting reaction mixture was stirred at room temperature for 1 5 hrs monitored by TLC. Upon the aldehyde was all consumed, the mixture was diluted with EtOAc $(100 \mathrm{~mL})$. The organic phase was washed by $\mathrm{HCl}$ solution $(1.0 \mathrm{M})$, saturated $\mathrm{NaHCO}_{3}$ (aq.) and brine and then dried over anhydrous $\mathrm{Na}_{2} \mathrm{SO}_{4}$. The solvent was removed under reduced pressure to give a residue. Flash silica gel chromatography was then applied to give the product. 


\section{2) Optical sample preparation:}

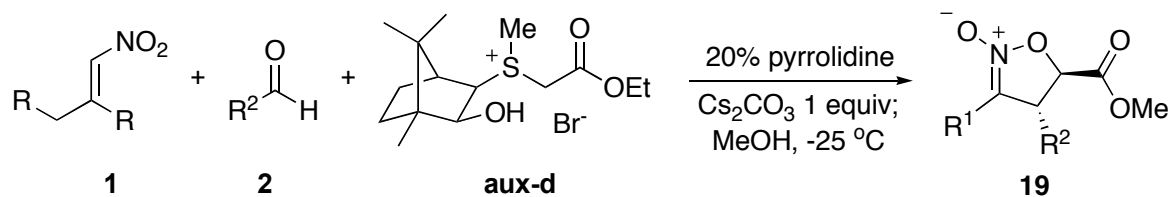

The nitroalkene $1(0.7 \mathrm{mmol}, 1.4 \mathrm{eq}$ ) $)$ was added to a solution of the sulfur ylide (0.6 mmol, 1.2 eq.) $\mathrm{MeOH}$ solution ( $0.4 \mathrm{M}$ for nitroalkene), till the ylide is all dissolved. The mixture was cooled down to $-25{ }^{\circ} \mathrm{C}$. pyrrolidine $\left(7 \mathrm{mg}, 0.1 \mathrm{mmol}, 0.2\right.$ eq.) and $\mathrm{K}_{2} \mathrm{CO}_{3}(35$ $\mathrm{mg}, 0.25 \mathrm{mmol}, 0.5$ eq.) were then added in and stir for 30 minutes. The aldehyde ( 0.5 mmol, 1.0 eq.) $\mathrm{MeOH}$ solution $(0.4 \mathrm{M})$ was added drop wise through 10 minutes. The resulting reaction mixture was stirred at $-25{ }^{\circ} \mathrm{C}$ for $48 \sim 60$ hours, monitored by TLC. The mixture was then diluted with EtOAc $(20 \mathrm{~mL})$ and the water phase was extracted with EtOAc $(20 \mathrm{~mL} \times 3)$. The organic phase was washed by $\mathrm{HCl}$ solution $(1.0 \mathrm{M})$, saturated $\mathrm{NaHCO}_{3}$ (aq.) and brine and then dried over anhydrous $\mathrm{Na}_{2} \mathrm{SO}_{4}$. The solvent was removed under reduced pressure to give a residue. Flash silica gel chromatography was then applied to give the product, which were then examined for e.e values.

\section{Procedure for preparation of ketone 22}

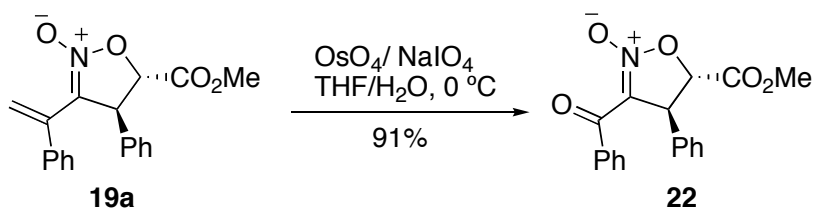

The isozaxoline-N-oxide 19a ( $323 \mathrm{mg}, 1.0 \mathrm{mmol}, 1.0$ equiv.) was dissolved in $\mathrm{THF} / \mathrm{H}_{2} \mathrm{O}$ (1:1) $(10 \mathrm{ml})$ at $0{ }^{\circ} \mathrm{C}$ and keep stirring for 10 minutes. $\mathrm{OsO}_{4}(13 \mathrm{mg}, 0.05 \mathrm{mmol}, 0.05$ equiv.) was added in the solution followed by the addition of $\mathrm{NaIO}_{4}(1065 \mathrm{mg}, 5.0 \mathrm{mmol}$, 5.0 equiv.). The reaction was stirred for 5 hours until the starting material 19a was all gone. $\mathrm{Na}_{2} \mathrm{~S}_{2} \mathrm{O}_{8}$ saturated solution $(20 \mathrm{ml})$ was then added in to quench the reaction. EtOAc was used for extraction $(20 \mathrm{ml} \times 3)$. The combined organic solution was then dried over anhydrous $\mathrm{Na}_{2} \mathrm{SO}_{4}$ and evaporated by rotavapor after a simple filtration of celite column. The residue was then purified by column. $294 \mathrm{mg}$ of ketone 22 was obtained as solid. 


\section{Procedure for preparation of ketone 26}

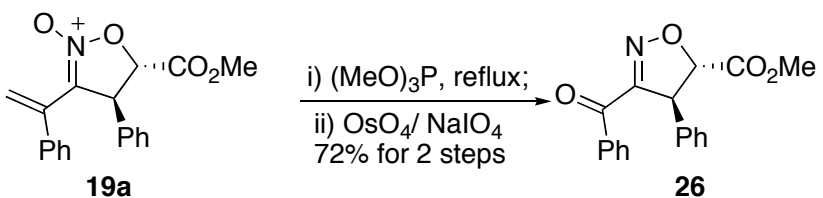

The isozaxoline-N-oxide 19a (323 mg, $1.0 \mathrm{mmol}, 1.0$ equiv.) was dissolved in $(\mathrm{MeO})_{3} \mathrm{P}$ $(20 \mathrm{ml})$ and degassed by argon, the reaction was heated up to $120^{\circ} \mathrm{C}$ for 8 hours until the starting material was all gone. The reaction was then cooled down to $0^{\circ} \mathrm{C}$ and $\mathrm{HCl}(5 \mathrm{~N})$ was added in to quench the reaction. EtOAc $(20 \mathrm{ml})$ was added in for extraction. $\mathrm{NaHCO}_{3}$ (aq.) $(30 \mathrm{ml})$ was then applied another $20 \mathrm{ml}$ of EtOAc was added. The organic layer was then dried over $\mathrm{Na}_{2} \mathrm{SO}_{4}$ and condensed down on rotapavor for next step. The crude product was dissolved in $\mathrm{THF} / \mathrm{H}_{2} \mathrm{O}(1: 1)(10 \mathrm{ml})$ at $0{ }^{\circ} \mathrm{C}$ and keep stirring for 10 minutes. $\mathrm{OsO}_{4}(13 \mathrm{mg}, 0.05 \mathrm{mmol}, 0.05$ equiv.) was added in the solution followed by the addition of $\mathrm{NaIO}_{4}(1065 \mathrm{mg}, 5.0 \mathrm{mmol}, 5.0$ equiv.). The reaction was stirred for 5 hours until the starting material was all gone. $\mathrm{Na}_{2} \mathrm{~S}_{2} \mathrm{O}_{8}$ saturated solution $(20 \mathrm{ml})$ was then added in to quench the reaction. EtOAc was used for extraction $(20 \mathrm{ml} \times 3)$. The combined organic solution was then dried over anhydrous $\mathrm{Na}_{2} \mathrm{SO}_{4}$ and evaporated by rotavapor after a simple filtration of celite column. The residue was then purified by column. $226 \mathrm{mg}$ of ketone $\mathbf{2 6}$ was obtained as oil.

\section{Procedure for preparation of alcohol 27}

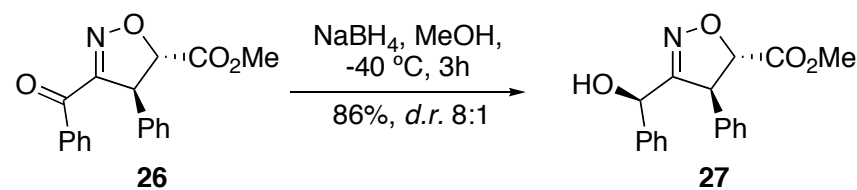

The ketone 26 (226 mg, $0.72 \mathrm{mmol}, 1.0$ equiv.) was dissolved into $\mathrm{MeOH}$ (10 ml) at -40 ${ }^{\circ} \mathrm{C}$ and $\mathrm{NaBH}_{4}$ (28 mg, $0.72 \mathrm{mmol}, 1.0$ equiv.) was applied. 3 hours later, once all the ketone was consumed, the reaction was quenched by $\mathrm{HCl}(1 \mathrm{~N})$ at low temperature. $\mathrm{NaHCO}_{3}$ (aq.) was added in to remove the $\mathrm{HCl}$ in excess. EtOAc $(20 \mathrm{ml}$ x 2) was used for extraction and the organic layer was dried over $\mathrm{Na}_{2} \mathrm{SO}_{4}$ and evaporated to have the residue. Crude NMR gave an 8:1 diastereo ratio. Column was then applied to purify the product alcohol 27 (183 mg) as solid. 


\section{Procedure for preparation of $\gamma$-oxime alcohol 29}

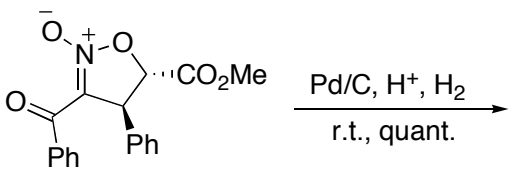

22

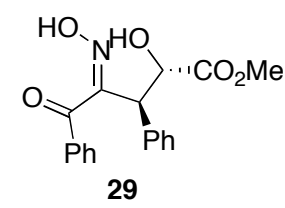

29

The ketone 26 (226 mg, $0.72 \mathrm{mmol}$ ) was dissolved into $\mathrm{MeOH}(10 \mathrm{ml})$ at room temperature. $\mathrm{Pd} / \mathrm{C}(10 \%)$ was added in, followed by the $\mathrm{H}_{2}$ gas applied to the reaction. The reaction went smooth and yielded the product 29 in quantitative yield $(226 \mathrm{mg})$. The reaction was quenched and filtered through celite. No further purification required.

\section{Procedure for preparation of tri-TBS-silyl ether 30}

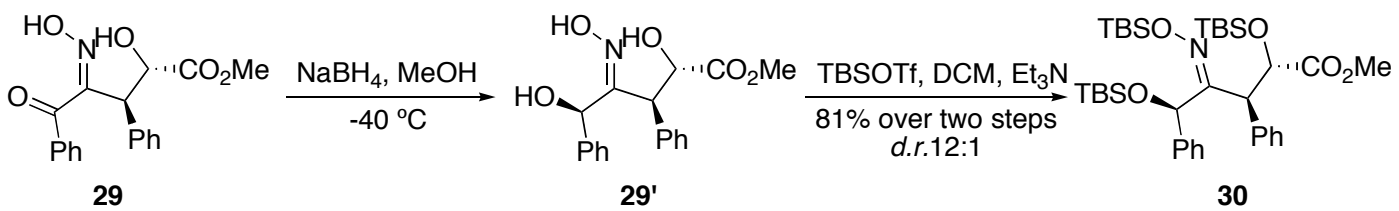

Ketone 29 (226 mg, $0.72 \mathrm{mmol}, 1.0$ equiv.) was treated with $\mathrm{NaBH}_{4}$ (28 mg, $0.72 \mathrm{mmol}$, 1.0 equiv.) in $\mathrm{MeOH}$ under $-40^{\circ} \mathrm{C} .3$ hours later, once all the ketone was consumed, the reaction was quenched by $\mathrm{HCl}(1 \mathrm{~N})$ at low temperature. $\mathrm{NaHCO}_{3}$ (aq.) was added in to remove the $\mathrm{HCl}$ in excess. EtOAc $(20 \mathrm{ml} \times 2)$ was used for extraction and the organic layer was dried over $\mathrm{Na}_{2} \mathrm{SO}_{4}$ and evaporated to have the residue. Crude NMR gave a 12:1 diastereo ratio. Without further purification, TBSOTf (792 mg, $3.0 \mathrm{mmol}, 4$ equiv.) was added in the DCM $(20 \mathrm{ml})$ solution of crude product at room temperature. 4 hours later, when the oxime diol 29' was all consumed, $30 \mathrm{ml}$ DCM was added and wash the reaction with $\mathrm{HCl}(1 \mathrm{~N}), \mathrm{NaHCO}_{3}$ (aq.) and brine. The organic layer was dried over $\mathrm{Na}_{2} \mathrm{SO}_{4}$ and evaporated to have the residue. Purification by column give the product $\mathbf{3 0} 550 \mathrm{mg}$ as colorless oil.

\section{Procedure for preparation of $\gamma$-lactam 31}
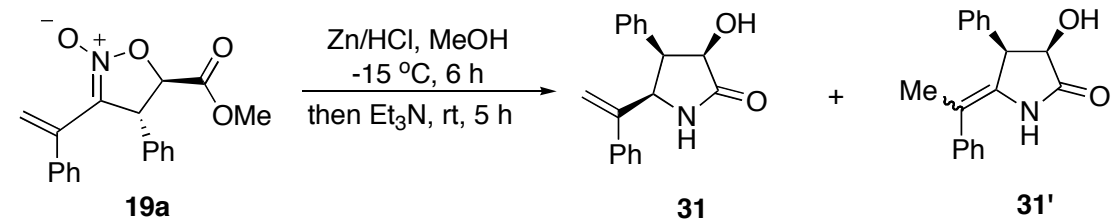
The isozaxoline-N-oxide 19a (323 mg, $1.0 \mathrm{mmol}, 1.0$ equiv.) was dissolved in $\mathrm{MeOH}$ (20 $\mathrm{ml})$ at $-15{ }^{\circ} \mathrm{C}$ and keep stirring for 10 minutes. Zn dust (975 mg, $15 \mathrm{mmol}, 15$ equiv., activated) was added in the solution slowly. Five molar $\mathrm{HCl}$ and $\mathrm{MeOH}(5 \mathrm{ml}, 25 \mathrm{mmol}$, 25 equiv.) solution was added in drop wise through 10 minutes. Keep cold and stir for 6 hours. $\mathrm{Et}_{3} \mathrm{~N}$ ( $2500 \mathrm{mg}, 25 \mathrm{mmol}, 25$ equiv.) was then added in to form white precipitate. Warm up to room temperature and keep stirring for another 5 hours. The reaction mixture was put under the vacuum to remove most of the solvent. The mixture was then diluted with EtOAc $(50 \mathrm{~mL})$ and the water phase was extracted with EtOAc $(20 \mathrm{~mL} \times 3)$. The organic phase was washed by $\mathrm{HCl}$ solution $(1.0 \mathrm{M})$, saturated $\mathrm{NaHCO}_{3}$ (aq.) and brine and then dried over anhydrous $\mathrm{Na}_{2} \mathrm{SO}_{4}$. The solvent was removed under reduced pressure to give a residue. Flash silica gel chromatography was then applied to give the product $\gamma$ lactam 31 as white solid $160 \mathrm{mg}$, in 57\% yield. The byproduct 31' was isolated in $37 \%$ yield. A 11:1 diastereo ratio was observed.

\section{Procedure for preparation of $\gamma$-lactam ketone 32}
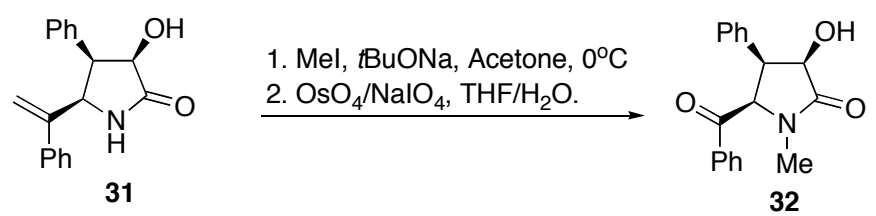

The $\gamma$-lactam 31 (160 mg, $0.57 \mathrm{mmol}, 1.0$ equiv.) was then dissolved into $10 \mathrm{ml}$ acetone at $0^{\circ}$ C. $t \mathrm{BuONa}(55 \mathrm{mg}, 0.57 \mathrm{mmol}, 1.0$ equiv.) was added in the solution and stir for 20 minutes. MeI ( $81 \mathrm{mg}, 0.58 \mathrm{mmol}, 1.0$ equiv.) acetone $(5 \mathrm{ml})$ solution was added in the reaction drop wise, through 5 minutes. The reaction was monitored by TLC and quenched by adding $\mathrm{H}_{2} \mathrm{O}$ after 5 hours. Carefully vacuumed out most of the acetone and dilute the mixture with EtOAc $20 \mathrm{ml}$ for extraction. The water phase was extracted with EtOAc $(20 \mathrm{~mL} \times 3)$. The organic phase was washed by $\mathrm{HCl}$ solution $(1.0 \mathrm{M})$, saturated $\mathrm{NaHCO}_{3}$ (aq.) and brine and then dried over anhydrous $\mathrm{Na}_{2} \mathrm{SO}_{4}$. After vacuum, the crude product was then dissolved in THF and $\mathrm{H}_{2} \mathrm{O}$ (1:1) solution (10 $\mathrm{ml}$ in total). At room temperature, $\mathrm{NaIO}_{4}(487 \mathrm{mg}, 2.28 \mathrm{mmol}, 4.0$ equiv.) was added in the solution and keep stirring till all dissolved. $\mathrm{OsO}_{4}(8 \mathrm{mg}, 0.029 \mathrm{mmol}, 0.05$ equiv.) was added in the reaction mixture. Monitored by TLC, the reaction was quenched by $\mathrm{Na}_{2} \mathrm{~S}_{2} \mathrm{O}_{3}$ saturated solution 
after 4 hours. $50 \mathrm{ml}$ of EtOAc and $20 \mathrm{ml}$ of brine were then added in the reaction mixture for extraction. The water phase was extracted with EtOAc $(20 \mathrm{~mL} \times 3)$. The organic phase was washed brine and then dried over anhydrous $\mathrm{Na}_{2} \mathrm{SO}_{4}$. The solvent was removed under reduced pressure to give a residue. Flash silica gel chromatography was then applied to give $79 \%$ (132 $\mathrm{mg}$ ) of the product $\mathbf{3 2}$ as white solid.

\section{Procedure for preparation of target molecule 33}

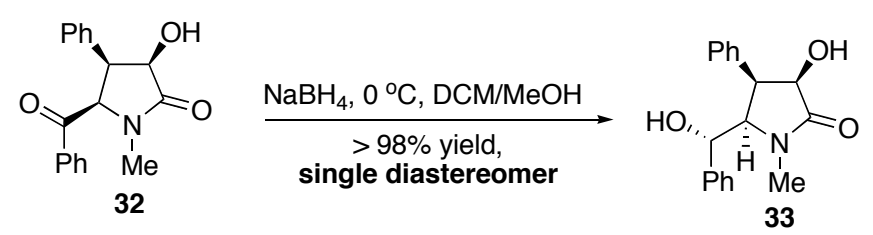

The ketone 32 (132 mg, $0.45 \mathrm{mmol}, 1.0$ equiv.) was dissolved in $5 \mathrm{ml}$ of MeOH/DCM (1:1) solution and cooled down to $0^{\circ} \mathrm{C} . \mathrm{NaBH}_{4}(20 \mathrm{mg}, 0.52 \mathrm{mmol}, 1.1$ equiv.) was added in the solution portion wise through 5 minutes. 2 hours later, TLC indicated the ketone 9d all consumed, warm up the reaction and dilute in EtOAc $(50 \mathrm{ml}), 50 \mathrm{ml}$ of brine was added in for extraction. The water phase was extracted with EtOAc $(20 \mathrm{~mL} \times 2)$. The organic phase was washed by $\mathrm{HCl}$ solution $(1.0 \mathrm{M})$, saturated $\mathrm{NaHCO}_{3}$ (aq.) and brine and then dried over anhydrous $\mathrm{Na}_{2} \mathrm{SO}_{4}$. The solvent was removed under reduced pressure to give a residue. Flash silica gel chromatography was then applied to give $\mathbf{3 3}$ (132 mg, $>98 \%$ yield) as white crystals. 
${ }^{1} \mathrm{H}$ NMR of 33

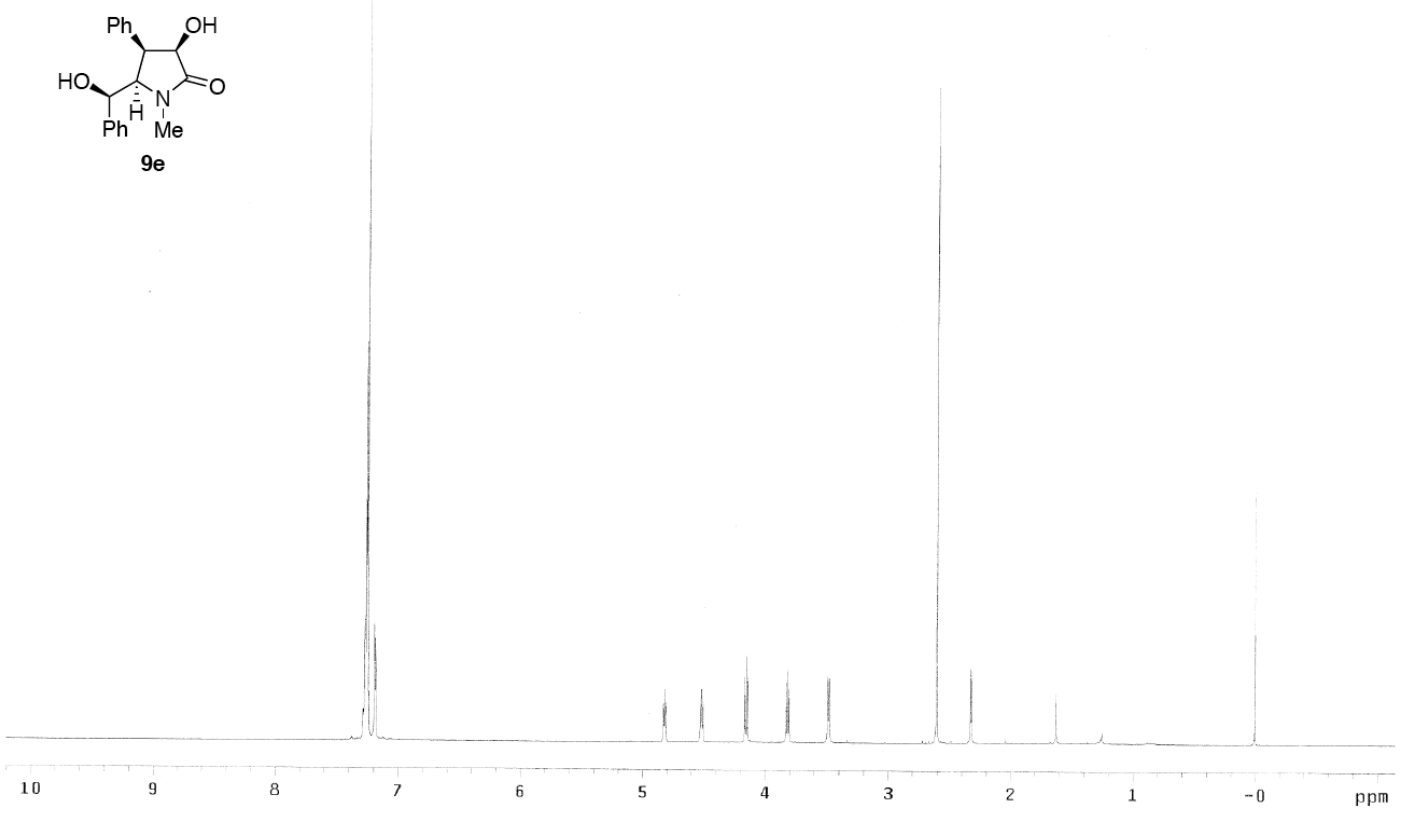

${ }^{13} \mathrm{C}$ NMR of 33

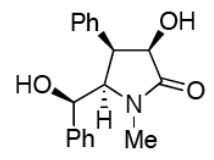

9 e

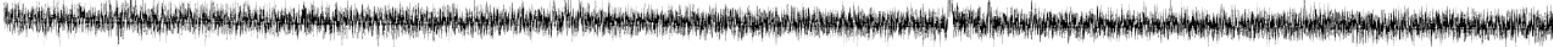

200

180

160

140

120

100

80

60

40

20

ppm 
NOE
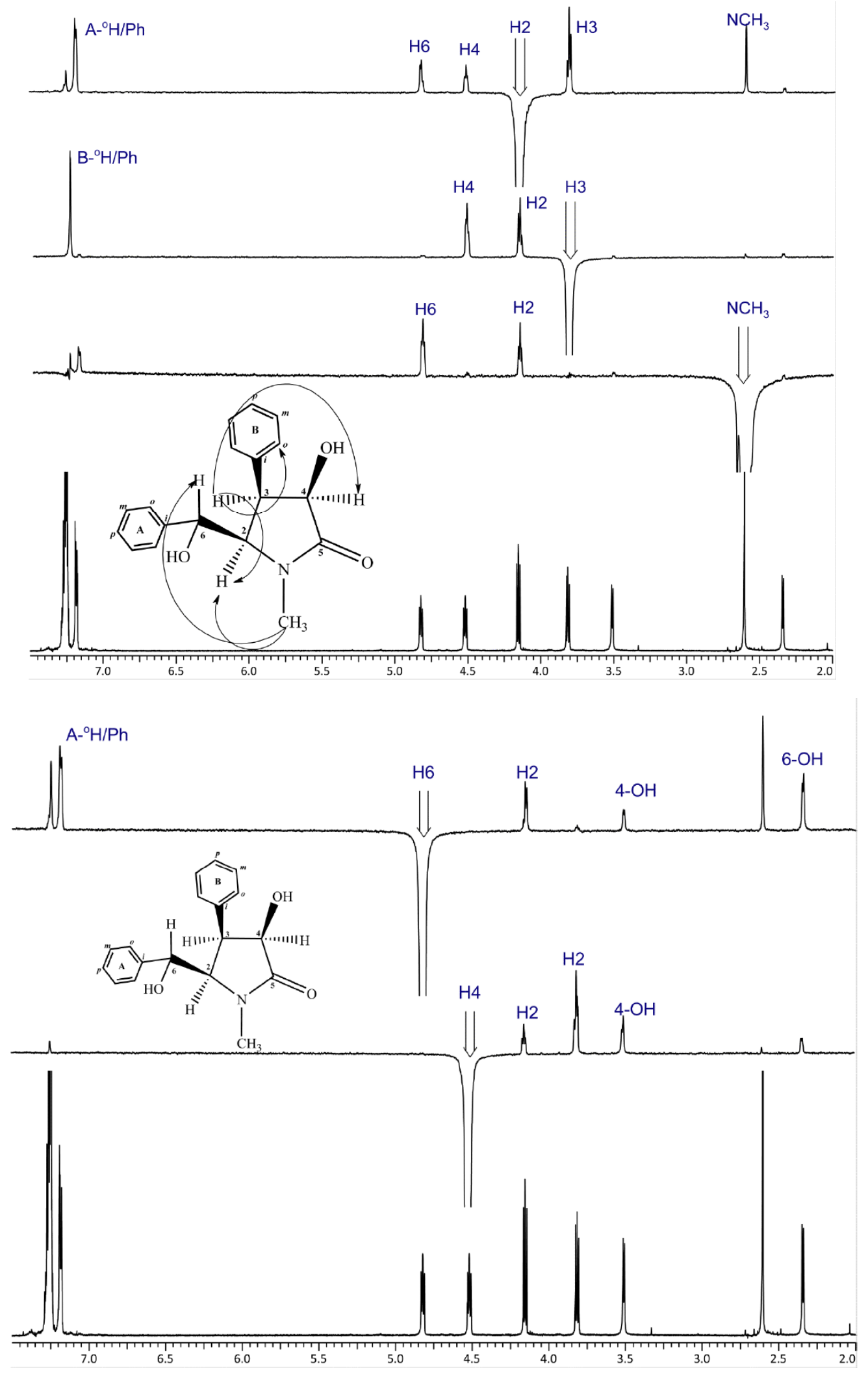

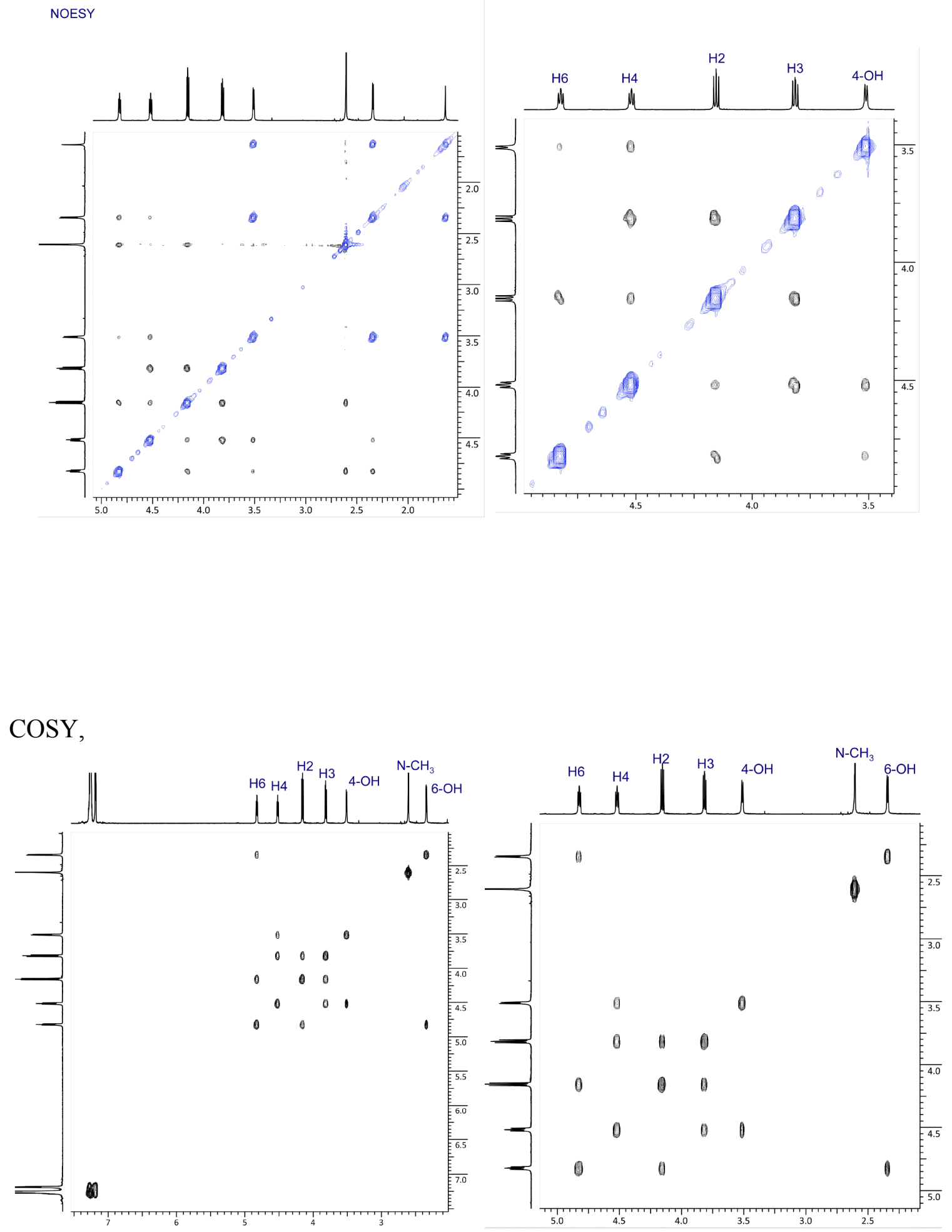


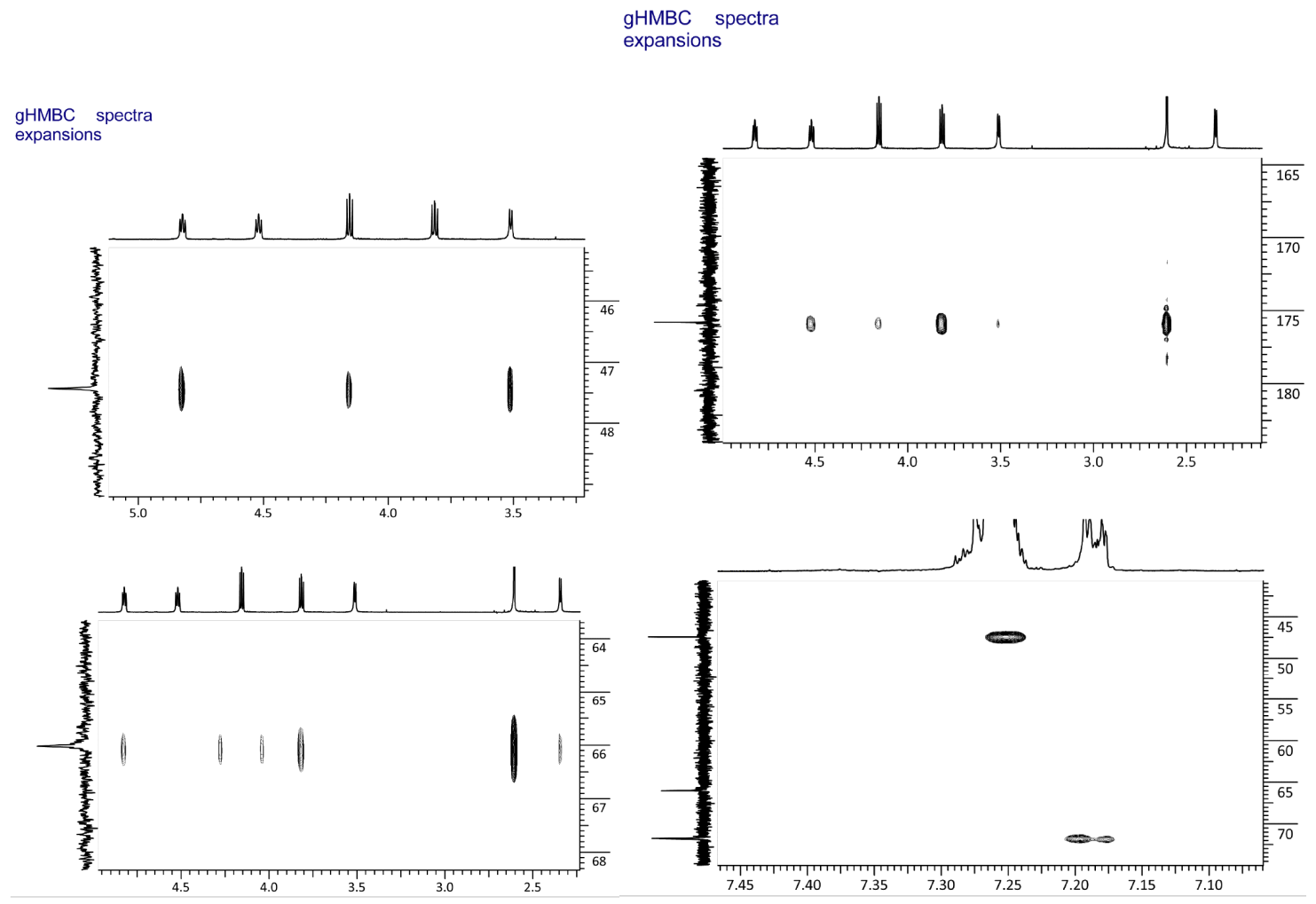

gHMBC

spectrum-

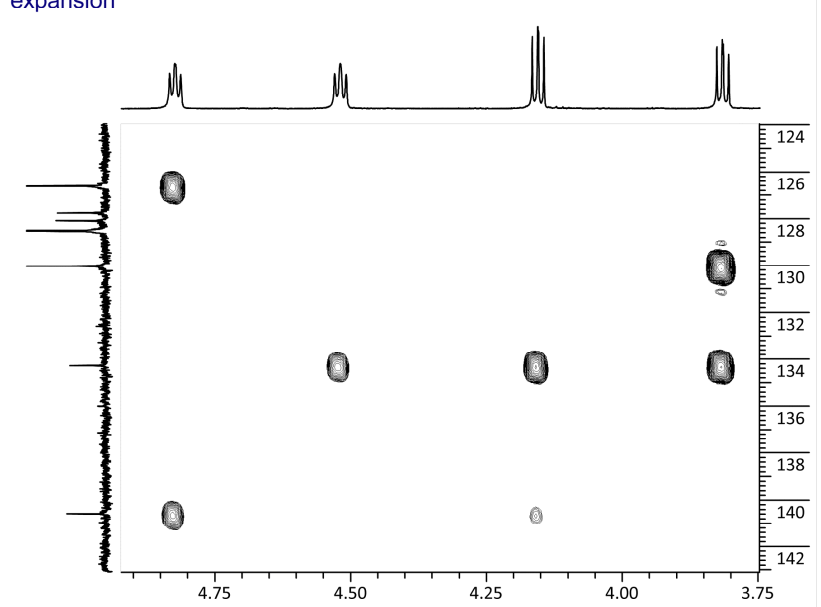




\section{General Procedure for preparation of dihydrofuran (35a):}

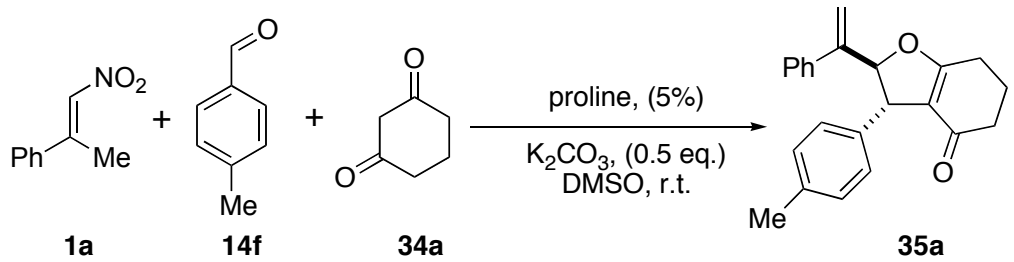

The nitroalkene 1a (202 mg, $1.2 \mathrm{mmol}, 1.2$ equiv.) was added to a DMSO solution of aldehyde $14 \mathbf{f}$ (1.0 mmol, 1.0 equiv.), cyclohexane-1, 3-dione 34a (1.1 mmol, 1.1 equiv.) and L-proline ( $6 \mathrm{mg}, 0.05 \mathrm{mmol}, 0.05$ equiv.), with a concentration of $0.15 \mathrm{M}$ for aldehyde. The reaction mixture was stirred for 5 10 minutes to have the clear solution; $\mathrm{K}_{2} \mathrm{CO}_{3}$ (69 $\mathrm{mg}, 0.5 \mathrm{mmol}, 0.5$ equiv.) was then added. The resulting reaction mixture was stirred at room temperature for 4 hours monitored by TLC. Upon the aldehyde was all consumed, the mixture was diluted with EtOAc $(100 \mathrm{~mL})$. The organic phase was washed by $\mathrm{HCl}$ solution $(1.0 \mathrm{M})$, saturated $\mathrm{NaHCO}_{3}$ (aq.) and brine and then dried over anhydrous $\mathrm{Na}_{2} \mathrm{SO}_{4}$. The solvent was removed under reduced pressure to give a residue. Flash silica gel chromatography was then applied to give the product $\mathbf{3 5 a}$.

\section{General Procedure for Palladium catalyzed rearrangement (36a):}
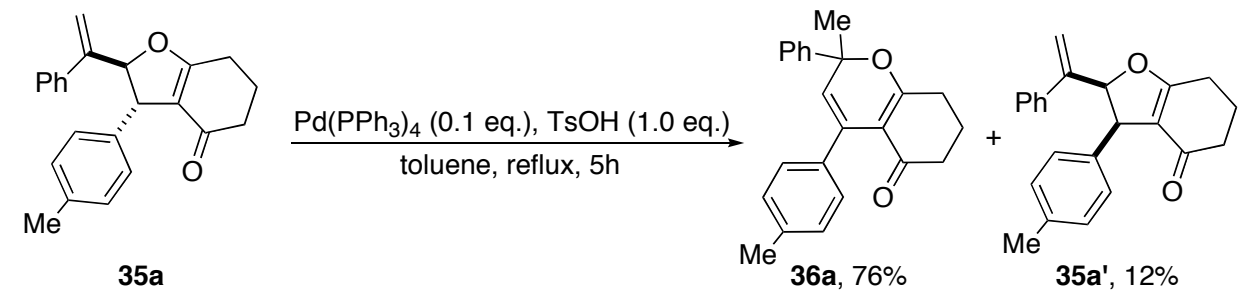

$330 \mathrm{mg}$ of 35a (1.0 mmol, 1.0 equiv.) was dissolved in distilled toluene $20 \mathrm{ml}$, followed by the addition of $\mathrm{Pd}\left(\mathrm{PPh}_{3}\right)_{4}(115 \mathrm{mg}, 0.1$ equiv. $)$ and TsOH (172 mg, $1.0 \mathrm{mmol}, 1.0$ equiv.). The reaction was then degassed by argon for 10 minutes and heated in oil bath to reflux. After 5 hours, the reaction was cooled down to room temperature and $\mathrm{NaHCO}_{3}$ (aq.) was applied to remove the acid. Routine extraction was applied after which the organic phase was dried over anhydrous $\mathrm{Na}_{2} \mathrm{SO}_{4}$. The solvent was removed under reduced pressure to give a residue. Flash silica gel chromatography was then applied to give the product 36a $(250 \mathrm{mg}, 0.76 \mathrm{mmol})$ in $76 \%$ yield and 35a' $(40 \mathrm{mg}, 0.12 \mathrm{mmol})$ in $12 \%$ yield. 


\section{Representative Procedure for Synthesis of Piperidine (39a)}

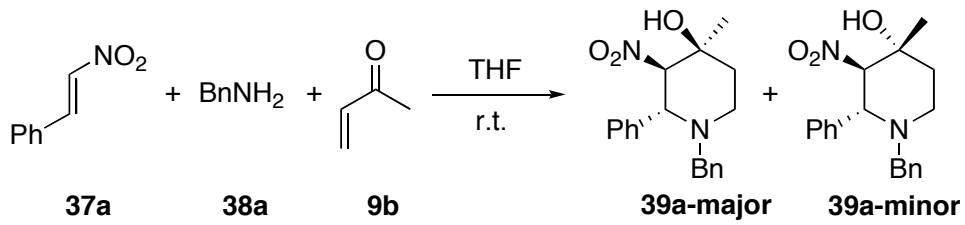

To a solution of nitroalkene $\mathbf{3 7 a}$ (149 mg, $1 \mathrm{mmol}, 1$ eq.) in dry THF (5 mL), was added successively phenylmethanamine 38a (158 mg, $1.5 \mathrm{mmol}, 1.5$ eq.) and MVK 9b (140 $\mathrm{mg}, 2.0 \mathrm{mmol}, 2.0$ eq.) under $\mathrm{N}_{2}$ atmosphere. The mixture was stirred at room temperature and monitored by TLC. After removing the solvent, the residue was purified by flash silica gel chromatography (Hexane-EtOAc v/v 10:1) gave two diastereomers piperidines 39a-major (275 mg, $0.85 \mathrm{mmol}$, yiled: 85\%) and 39a-minor (38 $\mathrm{mg}, 0.12$ mmol, yiled: $12 \%$ ) as white solids.

\section{$\underline{\text { Representative Procedure for Synthesis of Piperidine (39m) }}$}

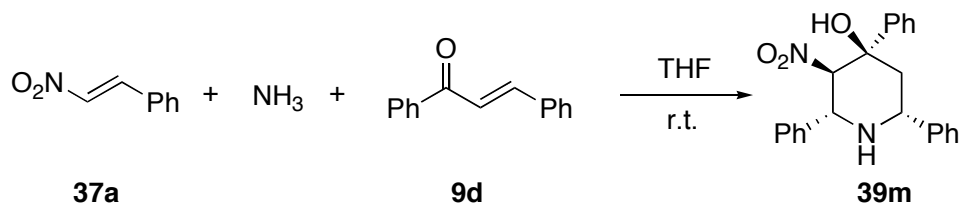

Nitroalkene 37a (149 mg, $1 \mathrm{mmol}, 1$ eq) was dissolved in dry THF (10 mL) in a $25 \mathrm{~mL}$ two-neck flask equipped with a gas dispersing tube. A stream of $\mathrm{NH}_{3}$ gas was bubbled into the solution at room temperature. After the color of solution turned colorless, the $\mathrm{NH}_{3}$ gas was stopped and then chalcone 9d (312 mg, $\left.1.5 \mathrm{mmol}, 1.5 \mathrm{eq}\right)$ was added. The mixture was stirred at room temperature and monitored by TLC, $\mathrm{NH}_{3}$ gas was refilled 12 hr later. After removing the solvent, the residue was purified by flash silica gel chromatography (Hexane-EtOAc v/v 10:1) gave piperidines 39m (310 mg, $0.83 \mathrm{mmol}$, yield: $83 \%$ ) as white solid.

\section{Representative Procedure for Synthesis of Piperidine (41d)}

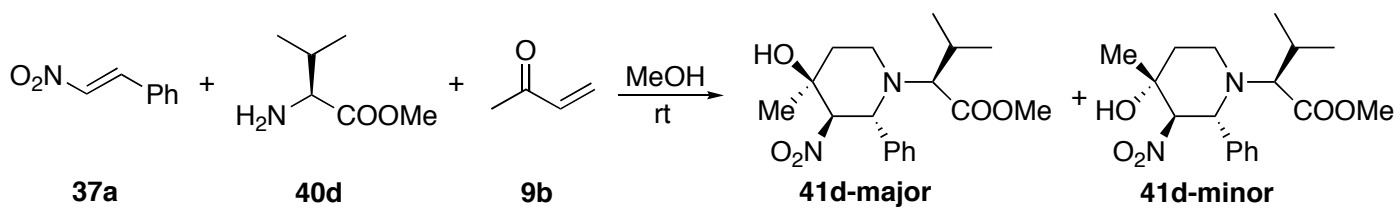


To a solution of nitroalkene 37a (149 $\mathrm{mg}, 1 \mathrm{mmol}, 1 \mathrm{eq})$ in $\mathrm{MeOH}(10 \mathrm{~mL})$, was added successively L-Valine methyl ester 40d (196 mg, $1.5 \mathrm{mmol}, 1.5 \mathrm{eq}$ ) and MVK $9 b$ (140 $\mathrm{mg}, 2.0 \mathrm{mmol}, 2.0 \mathrm{eq}$ ) under $\mathrm{N}_{2}$ atmosphere. The mixture was stirred at room temperature for 36h. After removing the solvent, the residue was purified by flash silica gel chromatography (Hexane-EtOAc v/v 5:1) gave two diastereomers piperidines 41d-major (168 mg, $0.48 \mathrm{mmol}$, yield: 48\%) and 41d-minor (49 mg, $0.14 \mathrm{mmol}$, yield: $14 \%$ ) as white solids.

\section{Synthesis of Compound 42a}

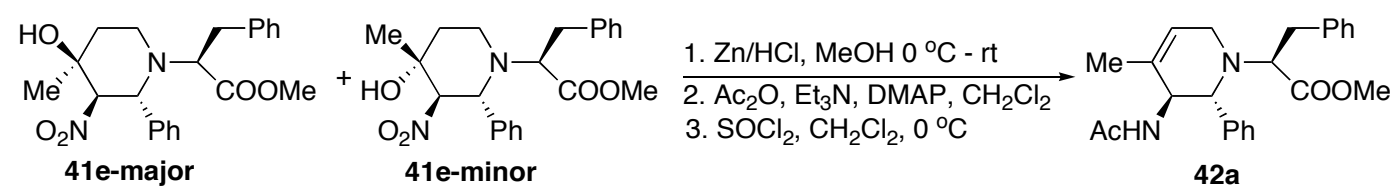

To a solution of $41 \mathrm{e}$ (major \& minor) $(200 \mathrm{mg}, 0.5 \mathrm{mmol}, 1 \mathrm{eq})$ in $\mathrm{MeOH}(25 \mathrm{~mL}), 1 \mathrm{~N}$ $\mathrm{HCl}(5 \mathrm{~mL}, 5 \mathrm{mmol}, 10 \mathrm{eq})$ and $\mathrm{Zn}$ powder $(488 \mathrm{mg}, 7.5 \mathrm{mmol}, 15 \mathrm{eq})$ were added at $0^{\circ} \mathrm{C}$ . The mixture was then stirred at room temperature and monitored by TLC. To the mixture, $\mathrm{NaHCO}_{3}$ (aq) was added until $\mathrm{pH}>10$, followed by the extraction with $\mathrm{CH}_{2} \mathrm{Cl}_{2}$ (30 mL x 5). The combined organic layer was washed with brine and then dried over anhydrous $\mathrm{Na}_{2} \mathrm{SO}_{4}$. After filtration and evaporation, the residue was dissolved in $\mathrm{CH}_{2} \mathrm{Cl}_{2}$ $(25 \mathrm{~mL})$. To the solution, Et3N (101 mg, $1 \mathrm{mmol}, 2 \mathrm{eq})$ and $\mathrm{Ac}_{2} \mathrm{O}(78 \mathrm{mg}, 0.75 \mathrm{mmol}$, $1.5 \mathrm{eq}$ ) and DMAP ( $3 \mathrm{mg}, 0.025 \mathrm{mmol}, 0.05 \mathrm{eq})$ were added at $0^{\circ} \mathrm{C}$. The mixture was stirred at room temperature and monitored by TLC. After removal of the solvent, the residue was diluted with EtOAc $(20 \mathrm{~mL})$ and washed with water. The aqueous phase was then extracted with EtOAc $(20 \mathrm{~mL} \times 3)$. The combined organic solution was then washed with brine and dried over anhydrous $\mathrm{Na} 2 \mathrm{SO} 4$. After filtration and evaporation, the residue was dissolved in $\mathrm{CH}_{2} \mathrm{Cl}_{2}(10 \mathrm{~mL})$ at $0{ }^{\circ} \mathrm{C}$. To this solution was added thionyl chloride (298 mg, $2.5 \mathrm{mmol}, 5$ equiv) by dropwise. The mixture was stirred at $0^{\circ} \mathrm{C}$ and monitored by TLC. To this solution was added $\mathrm{NaHCO}_{3}$ (aq.) to quench the reaction and then extract with EtOAc $(30 \mathrm{~mL} \times 3)$. The combined organic solution was then washed with brine and dried over anhydrous $\mathrm{Na} 2 \mathrm{SO} 4$. After filtration and evaporation, the residue was purified by flash silica gel chromatography (Hexane-EtOAc v/v 3:1) gave 
42a (156 mg, $0.40 \mathrm{mmol}$, yield: $80 \%$ ) as white solid.

\section{Synthesis of compound $7 \mathrm{~b}$}

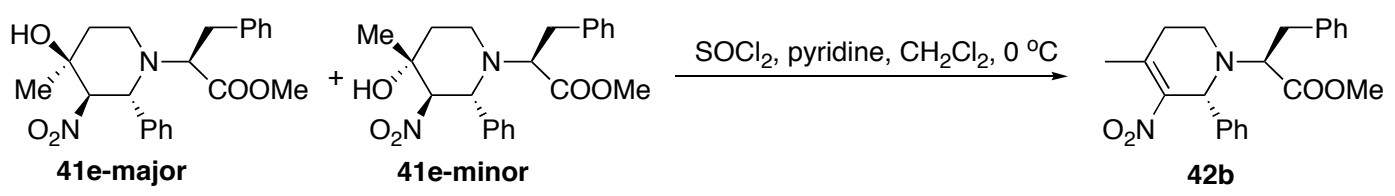

To a solution of 41 (100 mg, $0.25 \mathrm{mmol}, 1 \mathrm{eq})$ in dry pyridine $(1 \mathrm{~mL})$ and dry $\mathrm{CH}_{2} \mathrm{Cl}_{2}(3$ $\mathrm{mL}$ ) at $0{ }^{\circ} \mathrm{C}$, thionyl chloride $(119 \mathrm{mg}, 1 \mathrm{mmol}, 5 \mathrm{eq})$ was added dropwise. The mixture was stirred at $0{ }^{\circ} \mathrm{C}$ and monitored by TLC. To this solution was added $\mathrm{NaHCO} 3$ (aq) to quench the reaction and then extract with EtOAc $(30 \mathrm{~mL} \times 3)$. The combined organic solution was then washed with brine and dried over anhydrous $\mathrm{Na} 2 \mathrm{SO} 4$. After filtration and evaporation, the residue was purified by flash silica gel chromatography (HexaneEtOAc v/v 10:1) gave 42b (62 mg, $0.16 \mathrm{mmol}$, yield: $65 \%$ ) as a colorless oil.

\section{Synthesis of compound 41'}

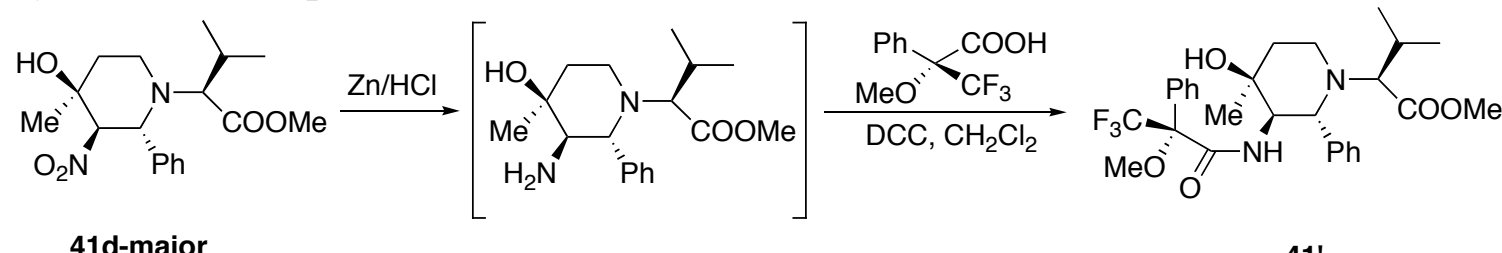

41d-major

$41^{\prime}$

To a solution of 41d-major (70 mg, $0.2 \mathrm{mmol}$, 1eq) in $\mathrm{MeOH}(25 \mathrm{~mL}), 1 \mathrm{~N} \mathrm{HCl}(2 \mathrm{~mL}, 2$ mmol, $10 \mathrm{eq}$ ) and $\mathrm{Zn}$ powder $(195 \mathrm{mg}, 3 \mathrm{mmol}, 15 \mathrm{eq})$ were added at $0^{\circ} \mathrm{C}$. And the mixture was stirred at room temperature and monitored by TLC. To the mixture, was added $\mathrm{NaHCO}_{3}$ (aq.) until $\mathrm{pH}>10$, followed by the extraction with $\mathrm{CH}_{2} \mathrm{Cl}_{2}(20 \mathrm{~mL}$ x 5). The combined organic layer was washed with brine and then dried over with anhydrous $\mathrm{Na}_{2} \mathrm{SO}_{4}$. After filtration and evaporation, the residue was dissolved in dry $\mathrm{CH}_{2} \mathrm{Cl}_{2}(10$ $\mathrm{mL}$ ). To this solution was added Acid (56 mg, $0.24 \mathrm{mmol}, 1.2 \mathrm{eq}$ ) and DCC (50 mg, 0.24 mmol, 1.2 equiv.) at $0^{\circ} \mathrm{C}$. The mixture was stirred at room temperature for $5 \mathrm{hr}$. After removal the solvent, the residue was diluted with EtOAc $(30 \mathrm{~mL})$, the mixture was got through a short column to remove most of insoluble solid and get a clear solution. After removal of the solvent, the residue was purified by flash silica gel chromatography (Hexane-EtOAc v/v 2:1) gave 41' (87 mg, 0.16 mmol, yiled: $81 \%$ ) as a colorless oil. 


\section{General procedures for nano-sheet preparation:}

Guanosine G-1 (46) and Na2TNBP were prepared following published methods.

Approach I: The guanosine monomer G-1 (200 mg, 428 umol) was dissolved in $\mathrm{CH}_{2} \mathrm{Cl}_{2}$ $(5 \mathrm{~mL}$ ) forming a colorless, clear solution. The bridging salt Na2TNBP (51 mg, $125 \mathrm{umol}$ ) was dissolved in distilled water $(5 \mathrm{~mL})$ as a transparent, dark red solution. The two resulting solutions were mixed together at room temperature and a red precipitate was observed at the interface of two the solutions after 10 minutes. The heterogeneous reaction mixture was left overnight and the solid was filtered followed by the washing with $\mathrm{CH}_{2} \mathrm{Cl}_{2}(20 \mathrm{ml} \times 2)$ and distilled water (20 $\left.\mathrm{ml} \times 2\right)$ respectively. The remaining red solid (233 mg, 93\%) was dried under vacuum and used for the AFM measurement and NMR studies.

Approach II: The guanosine monomer G-1 (200 mg, 428 umol) was dissolved in $\mathrm{CH}_{2} \mathrm{Cl}_{2}$ $(5 \mathrm{~mL})$ forming a clear solution, followed by the addition of NaSCN aqueous solution $(5 \mathrm{ml}, 25.2 \mathrm{mmol} / \mathrm{L})$. The mixture was stirred for $5 \mathrm{hrs}$. The organic layer was separated and washed by distilled water $(10 \mathrm{ml} \times 2)$. The organic solution was treated by flowing through a pipette column filled with $\mathrm{Na}_{2} \mathrm{SO}_{4}$ to remove the trace amount of water. The solution of $(\mathbf{G}-1){ }_{1} \cdot{ }^{*} \mathrm{Na}_{4} \bullet \mathrm{SCN}_{4}$ complex was then prepared. Dissolving the $(\mathrm{Bu} 4 \mathrm{~N}){ }_{2} \bullet \mathrm{TNBP}$ (106 mg, $125 \mathrm{umol}$ ) in $\mathrm{CH}_{2} \mathrm{Cl}_{2}(5 \mathrm{~mL})$ giving the dark-red TBA-TNBP solution. The two solutions of $(\mathbf{G}-\mathbf{1})_{1} \bullet^{\bullet} \mathrm{Na}_{4} \bullet \mathrm{SCN}_{4}$ and $(\mathrm{Bu} 4 \mathrm{~N})_{2} \bullet \mathrm{TNBP}$ were mixed together. Within 1 min, the clear solution turned turbid. The red precipitate suspension was formed in 5 hours. After vacuum filtration, the red precipitate was washed by $\mathrm{CH}_{2} \mathrm{Cl}_{2}(20 \mathrm{ml} \times 4)$ to remove the remaining free $(\mathrm{Bu} 4 \mathrm{~N}) 2)_{2} \cdot \mathrm{SCN}$ and $(\mathbf{G}-1){ }_{1} \bullet \mathrm{Na}_{4} \bullet \mathrm{SCN}_{4}$. The red solid was dried under vacuum (198 $\mathrm{mg}, 88 \%$ ) and used for the AFM measurement and NMR studies.

\section{$\underline{\text { AFM measurement }}$}

The nano-sheets were observed with an atomic force microscope (AFM) (Molecular Imaging, PICOPUS). The suspensions of nano-sheet particles were prepared in various 
solvents, including $\mathrm{CH}_{2} \mathrm{Cl}_{2}, \mathrm{CH}_{3} \mathrm{CN}, \mathrm{MeOH}$ and deionized $\mathrm{H}_{2} \mathrm{O}$. The AFM images shown below were prepared in the deionized water, which produced best quality of pictures of nano-sheet without severe aggregation. The nano-sheet was first suspended in deionized water and stirred for 3 hrs. The suspension was then filtered through a tissuepacked pipette. This process removed the large aggregated nano-particles and the nonaggregated/less-aggregated nano-particle can go through to form a nano-particle "solution" (suspension). The resulting clear "solution" was transferred to the surface of mica substrate (SPI, V4 grade). The mica with sample was then dried in the desiccator prior to AFM measurement. AFM measurement was performed on the nano-sheet sample under tapping mode with the Si tip (Applied Nanostructure, Model ACT) with a resonance frequency of $\sim 300 \mathrm{kHz}$ and spring constant of $40 \mathrm{~N} / \mathrm{m}$. During scanning, the tip was kept at a distance from the sample at which the oscillation amplitude was dampened by the intermolecular repulsive force with sample to $90 \%$ of its free amplitude. The scanning speed was $2 \mathrm{um} / \mathrm{s}$.

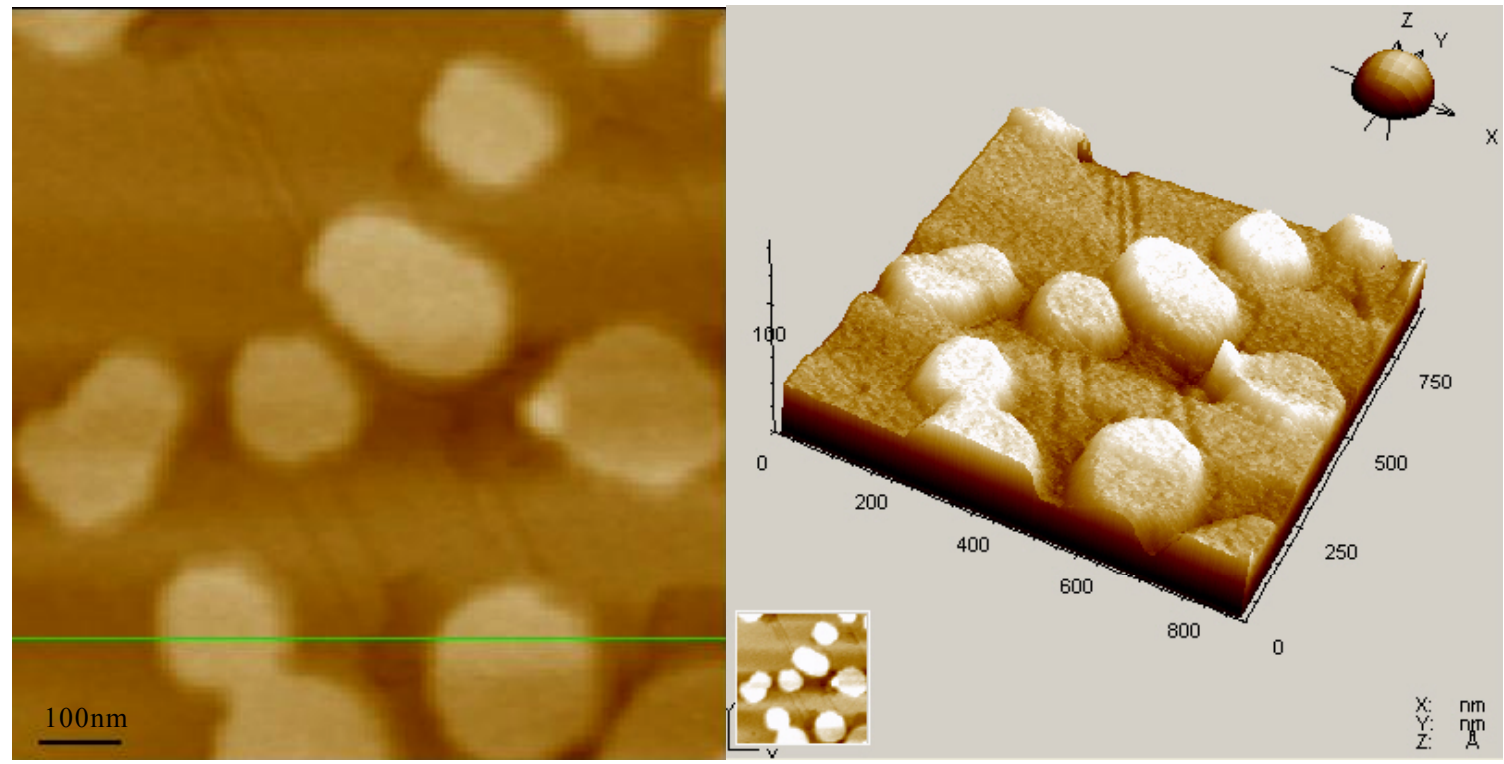

AFM 2D image

AFM 3D image 


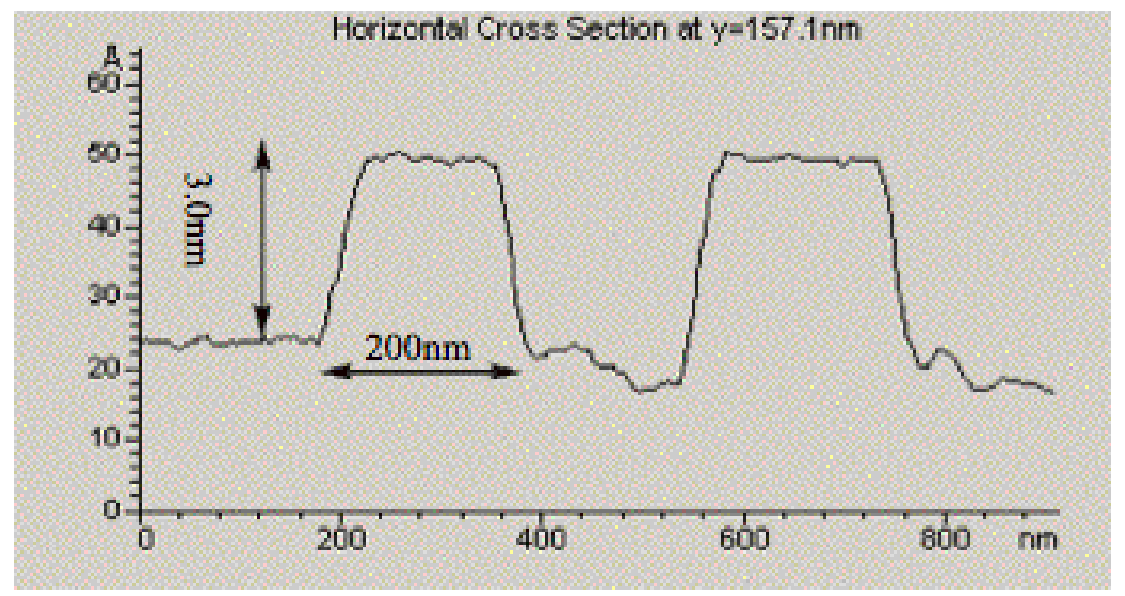

Cross section profile over the line indicated (green line)

\section{Powder X-ray determination}

The powder X-ray diffraction was measured using Bruker D8 Discover equipped with HiStar 2D detector and Göbel mirror. Two 2D frames were measured using $\mathrm{CuKa}$ radiation for 10 min each and integrated resulting XRD pattern shown below. The powder $\mathrm{X}$-ray pattern reveals two low angles peaks observed at d-spacing $47.5 \AA$ and $23.75 \AA$, which suggest 1 st and 2 nd orders (e.g. 100 and 200). This result indicates the presenting of large unit cell. The other smaller peaks cannot be interpreted from this pattern due to broadening and overlapping of the peaks. In the supporting information of reference $\mathbf{3}$, the unit cells of guanosine hexadecamer have been determined, which is $47 \AA$. For example, the unit cell for $(\mathbf{G}-1) 16 \cdot \mathrm{Ba} \cdot(\mathrm{P}-\mathrm{OME}-2,6-\mathrm{DNP}) 4$ hexadecamer is:

$\mathrm{a}=46.662 \AA \alpha=90$ o

$\mathrm{b}=24.259 \AA \beta=93.139$ 。

$\mathrm{c}=45.111 \AA \gamma=90$ 。

In the crystal structure, the dihedral angle between a G-quartet plane and the edge of unit cell is about 45o. The diameter of the G-quartet formed by G1 is around $33 \AA$. Therefore, the correlation is $33 \times 1.414=47 \AA$, determining the size of the unit cell. 


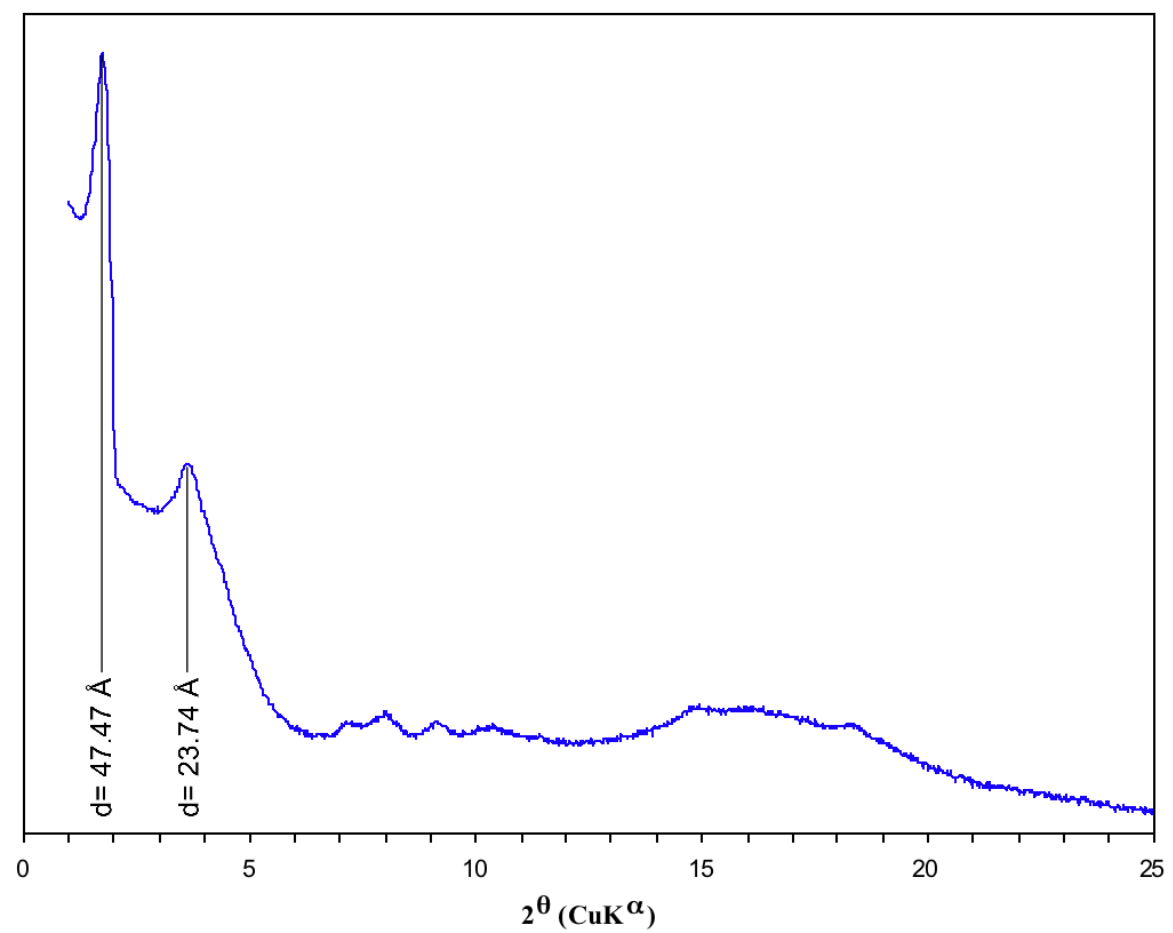




\section{Section C: Compound characterization}

\section{Part I}

4a was purified by flash chromatography (Hexane-EtOAc, v/v 7/1) as white oil with overall isolated yield: $91 \%$ for both isomers.<smiles>C=C(c1ccccc1)[C@H](C(CCO)c1ccccc1)[N+](=O)[O-]</smiles>

4a-syn

(3S,4R)-4-nitro-3,5-diphenylhex-5-en-1-ol (4a-syn) ${ }^{1} \mathrm{H}$ NMR (270 $\left.\mathrm{MHz}, \mathrm{CDCl}_{3}\right) \delta$ (ppm) 1.29 (br s, 1H), 1.90-2.00 (m, 2H), 3.31-3.40 (m, 1H), 3.48-3.56 (m, 1H), 3.70$3.80(\mathrm{~m}, 1 \mathrm{H}), 5.42(\mathrm{~s}, 1 \mathrm{H}), 5.67(\mathrm{~d}, J=11.4 \mathrm{~Hz}, 1 \mathrm{H}), 5.75(\mathrm{~s}, 1 \mathrm{H}), 6.97-7.02(\mathrm{~m}, 4 \mathrm{H})$, 7.14-7.22 (m, 6H); ${ }^{13} \mathrm{C}$ NMR (67.5 MHz, $\left.\mathrm{CDCl}_{3}\right) \delta(\mathrm{ppm}) 36.1,46.5,60.0,94.5,119.7$, 126.7.9, 127.6, 128.1, 128.4, 128.8, 129.0, 137.4, 140.3, 143.0. HRMS Calculated for $\left[\mathrm{C}_{18} \mathrm{H}_{19} \mathrm{NO}_{3} \mathrm{Na}\right]^{+}:$320.1263, Found: 320.1257. $[\alpha]_{\mathrm{D}}{ }^{26}=-67.8^{\circ}\left(\mathrm{c}=1.0 \mathrm{CHCl}_{3}\right)$. Enantiomeric excess was determined by HPLC with a Chiralcel OD-H column; $\lambda=254$ $\mathrm{nm}$; eluent: Hexane/Isopropanol $=90 / 10$; Flow rate: $0.5 \mathrm{~mL} / \mathrm{min}$; $\mathrm{t}_{\text {minor }}=9.184 \mathrm{~min}, \mathrm{t}_{\text {major }}$ $=31.704 \mathrm{~min} ; \mathrm{ee} \%=91 \%$.<smiles>C=C(c1ccccc1)C(CCO)C(CCO)c1ccccc1</smiles>

\section{4a-anti}

(3S,4S)-4-nitro-3,5-diphenylhex-5-en-1-ol (4a-anti) ${ }^{1} \mathrm{H}$ NMR $\left(270 \mathrm{MHz}, \mathrm{CDCl}_{3}\right) \delta$ (ppm) 1.22 (br s, 1H), 1.53-1.66 (m, 1H), 1.94-2.07 (m, 1H), 3.22-3.31 (m, 1H), 3.403.47 (m, 1H), 3.76 (dt, J =11.7, 3.0 Hz, 1H), 5.69 (d, J=11.6 Hz, 1H), 5.74 (s, 1H), 5.97 $(\mathrm{s}, 1 \mathrm{H}), 7.25-7.53(\mathrm{~m}, 10 \mathrm{H}) ;{ }^{13} \mathrm{C} \mathrm{NMR}\left(67.5 \mathrm{MHz}, \mathrm{CDCl}_{3}\right) \delta(\mathrm{ppm}) 34.3,46.8,59.8,94.7$, $119.5,125.9,126.8,128.0,128.2$, 128.7, 129.0, 138.6, 140.1, 142.7. HRMS Calculated for $\left[\mathrm{C}_{18} \mathrm{H}_{19} \mathrm{NO}_{3} \mathrm{Na}\right]^{+}$: 320.1263, Found: 320.1257. $[\alpha]_{\mathrm{D}}{ }^{26}=76.0^{\circ}\left(\mathrm{c}=1.0 \mathrm{CHCl}_{3}\right)$. Enantiomeric excess was determined by HPLC with a Chiralcel OD-H column; $\lambda=254$ $\mathrm{nm}$; eluent: Hexane/Isopropanol $=50 / 50$; Flow rate: $1.0 \mathrm{~mL} / \mathrm{min}$; $\mathrm{t}_{\text {minor }}=4.584 \mathrm{~min}, \mathrm{t}_{\text {major }}$ 
$=12.044 \mathrm{~min} ; \mathrm{ee} \%=92 \%$.

4b was purified by flash chromatography (Hexane-EtOAc, v/v 10/1) as white oil with overall isolated yield $83 \%$ for both isomers.<smiles>C=C(c1ccccc1)C(CCCCC)C(CCO)CCO</smiles>

4b-syn

(S)-3-((R)-1-nitro-2-phenylallyl)octan-1-ol (4b-syn): ${ }^{1} \mathrm{H}$ NMR $\left(270 \mathrm{MHz}, \mathrm{CDCl}_{3}\right) \delta$ (ppm) $0.78(\mathrm{t}, J=6.9 \mathrm{~Hz}, 3 \mathrm{H}), 1.04-1.80(\mathrm{~m}, 11 \mathrm{H}), 2.40-2.51(\mathrm{~m}, 1 \mathrm{H}), 3.62-3.73(\mathrm{~m}$, 2H), $5.49(\mathrm{~d}, J=9.9 \mathrm{~Hz}, 1 \mathrm{H}), 5.63(\mathrm{~s}, 1 \mathrm{H}), 5.71(\mathrm{~s}, 1 \mathrm{H}), 7.35-7.44(\mathrm{~m}, 5 \mathrm{H}) ;{ }^{13} \mathrm{C}$ NMR $\left(67.5 \mathrm{MHz}, \mathrm{CDCl}_{3}\right) \delta(\mathrm{ppm}) 14.0,22.9,28.1,28.7,32.7,38.7,60.3,93.6,118.6,126.7$, 128.6, 128.9, 140.0, 143.1. HRMS Calculated for $\left[\mathrm{C}_{17} \mathrm{H}_{2} 5 \mathrm{NO}_{3} \mathrm{Na}\right]^{+}: 314.1732$, Found: 314.1725. $[\alpha]_{\mathrm{D}}{ }^{26}=-69.5^{\circ}\left(\mathrm{c}=1.0 \mathrm{CHCl}_{3}\right)$. Enantiomeric excess was determined by HPLC with a Chiralcel OD-H column; $\lambda=254 \mathrm{~nm}$; eluent: Hexane/Isopropanol $=95 / 5$; Flow rate: $1.0 \mathrm{~mL} / \mathrm{min} ; \mathrm{t}_{\mathrm{minor}}=16.780 \mathrm{~min}, \mathrm{t}_{\text {major }}=18.372 \mathrm{~min} ; \mathrm{ee} \%=94 \%$.<smiles>C=C(c1ccccc1)C(CCCCC)C(CCO)C(=O)c1ccccc1</smiles>

4b-anti

(S)-3-((S)-1-nitro-2-phenylallyl)octan-1-ol (4b-anti): ${ }^{1} \mathrm{H}$ NMR $\left(270 \mathrm{MHz}, \mathrm{CDCl}_{3}\right) \delta$ (ppm) $0.88(\mathrm{t}, J=6.7 \mathrm{~Hz}, 3 \mathrm{H}), 1.10(\mathrm{br} \mathrm{s}, 1 \mathrm{H}), 1.22-1.72(\mathrm{~m}, 10 \mathrm{H}), 2.45-2.57(\mathrm{~m}, 1 \mathrm{H})$, 3.56-3.67 (m, 2H), $5.49(\mathrm{~d}, J=10.4 \mathrm{~Hz}, 1 \mathrm{H}), 5.66(\mathrm{~s}, 1 \mathrm{H}), 5.75(\mathrm{~s}, 1 \mathrm{H}), 7.33-7.50(\mathrm{~m}$, $5 \mathrm{H}) ;{ }^{13} \mathrm{C} \mathrm{NMR}\left(67.5 \mathrm{MHz}, \mathrm{CDCl}_{3}\right) \delta(\mathrm{ppm}) 14.1,23.0,28.0,31.4,38.8,60.2,93.4,119.0$, 126.7, 128.6, 129.0, 140.1, 143.0. HRMS Calculated for $\left[\mathrm{C}_{17} \mathrm{H}_{25} \mathrm{NO}_{3} \mathrm{Na}\right]^{+}$: 314.1732, Found: $314.1725 .[\alpha]_{\mathrm{D}}{ }^{26}=62.4^{\circ}\left(\mathrm{c}=1.0 \mathrm{CHCl}_{3}\right)$. Enantiomeric excess was determined by HPLC with a Chiralcel OD-H column; $\lambda=254 \mathrm{~nm}$; eluent: Hexane/Isopropanol $=$ $85 / 15$; Flow rate: $1.0 \mathrm{~mL} / \mathrm{min}$; $\mathrm{t}_{\text {minor }}=4.728 \mathrm{~min}$, $\mathrm{t}_{\text {major }}=13.240 \mathrm{~min} ; \mathrm{ee} \%=87 \%$.

4c was purified by flash chromatography (Hexane-EtOAc, v/v 5/1) as light yellow oil 
with overall isolated yield $76 \%$ for both isomers.<smiles>C=C(c1ccccc1)[C@H](C(CCO)c1ccco1)[N+](=O)[O-]</smiles>

4c-syn

(3S,4R)-3-(furan-2-yl)-4-nitro-5-phenylhex-5-en-1-ol (4c-syn): ${ }^{1} \mathrm{H}$ NMR $(270 \mathrm{MHz}$, $\left.\mathrm{CDCl}_{3}\right) \delta(\mathrm{ppm}) 1.30(\mathrm{br} \mathrm{s}, 1 \mathrm{H}), 1.83-2.07(\mathrm{~m}, 2 \mathrm{H}), 3.38-3.47(\mathrm{~m}, 1 \mathrm{H}), 3.57-3.65(\mathrm{~m}$, $1 \mathrm{H}), 3.91(\mathrm{dt}, J=10.8,3.7 \mathrm{~Hz}, 1 \mathrm{H}), 5.49(\mathrm{~s}, 1 \mathrm{H}), 5.70(\mathrm{~s}, 1 \mathrm{H}), 5.74$ (s, 1H), 6.07 (d, $J=$ $3.0 \mathrm{~Hz}, 1 \mathrm{H}), 6.18-6.21(\mathrm{~m}, 1 \mathrm{H}), 7.13-7.30(\mathrm{~m}, 6 \mathrm{H}) ;{ }^{13} \mathrm{C} \mathrm{NMR}\left(67.5 \mathrm{MHz}, \mathrm{CDCl}_{3}\right) \delta$ (ppm) 34.0, 40.1, 60.0, 92.3, 109.3, 110.4, 119.2, 126.7, 128.2, 128.5, 139.4, 142.2, 142.8, 150.6. HRMS Calculated for $\left[\mathrm{C}_{16} \mathrm{H}_{17} \mathrm{NO}_{4} \mathrm{Na}\right]^{+}: 310.1055$, Found: $310.1049 .[\alpha]_{\mathrm{D}}{ }^{26}$ $=-68.6^{\circ}\left(\mathrm{c}=1.0 \mathrm{CHCl}_{3}\right)$. Enantiomeric excess was determined by HPLC with a Chiralcel AS-H column; $\lambda=254 \mathrm{~nm}$; eluent: Hexane/Isopropanol = 80/20; Flow rate: $1.0 \mathrm{~mL} / \mathrm{min}$; $\mathrm{t}_{\text {minor }}=11.164 \mathrm{~min}, \mathrm{t}_{\text {major }}=7.232 \mathrm{~min} ; \mathrm{ee} \%=87 \%$.<smiles>C=C(c1ccccc1)C(CCO)[C@H](CCO)c1ccco1</smiles>

\section{4c-anti}

(3S,4S)-3-(furan-2-yl)-4-nitro-5-phenylhex-5-en-1-ol (4c-anti): ${ }^{1} \mathrm{H}$ NMR $(270 \mathrm{MHz}$, $\mathrm{CDCl} 3) \delta(\mathrm{ppm}) 1.20($ br s, $1 \mathrm{H}), 1.68-1.81(\mathrm{~m}, 1 \mathrm{H}), 1.87-1.99(\mathrm{~m}, 1 \mathrm{H}), 3.30-3.41(\mathrm{~m}$, $1 \mathrm{H}), 3.50-3.58(\mathrm{~m}, 1 \mathrm{H}), 3.96(\mathrm{dt}, J=11.4,3.5 \mathrm{~Hz}, 1 \mathrm{H}), 5.72-5.76(\mathrm{~m}, 2 \mathrm{H}), 5.89(\mathrm{~s}, 1 \mathrm{H})$, $6.24(\mathrm{~d}, J=3.0 \mathrm{~Hz}, 1 \mathrm{H}), 6.29-6.30(\mathrm{~m}, 1 \mathrm{H}), 7.35-7.50(\mathrm{~m}, 6 \mathrm{H}) ;{ }^{13} \mathrm{C}$ NMR $(67.5 \mathrm{MHz}$, CDCl3) $\delta(\mathrm{ppm}) 32.4,40.3,60.0,92.4,109.9,110.6,119.7,126.8,128.8,129.0,139.7$, 142.3, 142.6, 151.7. HRMS Calculated for $\left[\mathrm{C}_{16} \mathrm{H}_{17} \mathrm{NO}_{4} \mathrm{Na}\right]^{+}: 310.1055$, Found: 310.1049 . $[\alpha]_{\mathrm{D}}^{26}=63.7^{\circ}\left(\mathrm{c}=1.0 \mathrm{CHCl}_{3}\right)$. Enantiomeric excess was determined by HPLC with a Chiralcel OD-H column; $\lambda=254 \mathrm{~nm}$; eluent: Hexane/Isopropanol $=80 / 20$; Flow rate: 1.0 $\mathrm{mL} / \mathrm{min} ; \mathrm{t}_{\text {minor }}=5.676 \mathrm{~min}, \mathrm{t}_{\text {major }}=16.436 \mathrm{~min} ; \mathrm{ee} \%=87 \%$. 
4d was purified by flash chromatography (Hexane-EtOAc, v/v 5/1) as light yellow oil with overall isolated yield $80 \%$ for both isomers.<smiles>C=C(c1ccccc1)[C@H](C(CCO)c1ccc(OC)cc1)[N+](=O)[O-]</smiles>

4d-syn

(3S,4R)-3-(4-methoxyphenyl)-4-nitro-5-phenylhex-5-en-1-ol (4d-syn): ${ }^{1} \mathrm{H}$ NMR (270 $\left.\mathrm{MHz} \mathrm{CDCl}_{3}\right) \delta$ (ppm) 1.20 (br s, 1H), 1.82-2.04 (m, 2H), 3.32-3.41 (m, 1H), 3.47-3.57 (m, 1H), 3.64-3.74 (m, 4H), $5.42(\mathrm{~s}, 1 \mathrm{H}), 5.62(\mathrm{~d}, J=11.1 \mathrm{~Hz}, 1 \mathrm{H}), 5.73(\mathrm{~s}, 1 \mathrm{H}), 6.67-$ $6.73(\mathrm{~m}, 2 \mathrm{H}), 6.89-6.94(\mathrm{~m}, 2 \mathrm{H}), 6.99-7.04(\mathrm{~m}, 2 \mathrm{H}), 7.18-7.23(\mathrm{~m}, 3 \mathrm{H}) ;{ }^{13} \mathrm{C} \mathrm{NMR}(67.5$ $\mathrm{MHz}, \mathrm{CDCl3}) \delta(\mathrm{ppm}) 34.4,46.3,55.4,60.0,95.0,114.4,119.5,126.8,128.7,129.0$, 129.2, 130.4, 140.2, 142.8, 159.2. HRMS Calculated for $\left[\mathrm{C}_{19} \mathrm{H}_{21} \mathrm{NO}_{4} \mathrm{Na}\right]^{+}: 350.1368$, Found: $350.1362 .[\alpha]_{\mathrm{D}}{ }^{26}=-91.2^{\circ}\left(\mathrm{c}=1.1 \mathrm{CHCl}_{3}\right)$. Enantiomeric excess was determined by HPLC with a Chiralcel OD-H column; $\lambda=254 \mathrm{~nm}$; eluent: Hexane/Isopropanol = $80 / 20$; Flow rate: $1.0 \mathrm{~mL} / \mathrm{min} ; \mathrm{t}_{\text {minor }}=8.516 \mathrm{~min}, \mathrm{t}_{\text {major }}=22.676 \mathrm{~min} ; \mathrm{ee} \%=94 \%$.<smiles>C=C(c1ccccc1)[C@H](C(CCO)c1ccc(OC)cc1)[N+](=O)[O-]</smiles>

4d-anti

(3S,4S)-3-(4-methoxyphenyl)-4-nitro-5-phenylhex-5-en-1-ol (4d-anti): ${ }^{1} \mathrm{H}$ NMR (270 $\left.\mathrm{MHz}, \mathrm{CDCl}_{3}\right) \delta(\mathrm{ppm}) 1.21$ (br s, 1H), 1.53-1.64 (m, 1H), 1.94-2.05 (m, 1H), 3.25-3.34 (m, 1H), 3.42-3.51 (m, 1H), 3.66-3.80 (m, 4H), 5.63 (d, $J=11.6 \mathrm{~Hz}, 1 \mathrm{H}), 5.74(\mathrm{~s}, 1 \mathrm{H})$, $5.96(\mathrm{~s}, 1 \mathrm{H}), 6.82-6.88(\mathrm{~m}, 2 \mathrm{H}), 7.16-7.22(\mathrm{~m}, 2 \mathrm{H}), 7.40-7.52(\mathrm{~m}, 5 \mathrm{H}) ;{ }^{13} \mathrm{C} \mathrm{NMR}(67.5$ $\mathrm{MHz}, \mathrm{CDCl3}) \delta(\mathrm{ppm}) 34.2,46.0,55.2,59.8,94.8,114.2,119.3,126.6,128.5,128.8$, 129.0, 130.2, 140.0, 142.6, 159.0. HRMS Calculated for $\left[\mathrm{C}_{19} \mathrm{H}_{21} \mathrm{NO}_{4} \mathrm{Na}\right]^{+}: 350.1368$, 
Found: $350.1362 .[\alpha]_{\mathrm{D}}^{26}=124.1^{\circ}\left(\mathrm{c}=0.75 \mathrm{CHCl}_{3}\right)$. Enantiomeric excess was determined by HPLC with a Chiralcel AS-H column; $\lambda=254 \mathrm{~nm}$; eluent: Hexane/Isopropanol $=$ $60 / 40$; Flow rate: $1.0 \mathrm{~mL} / \mathrm{min} ; \mathrm{t}_{\text {minor }}=15.528 \mathrm{~min}, \mathrm{t}_{\text {major }}=11.180 \mathrm{~min} ; \mathrm{ee} \%=95 \%$.

4e was purified by flash chromatography (Hexane-EtOAc, v/v 5/1) as light yellow solid with overall isolated yield: $89 \%$ for both isomers.<smiles>C=C(c1ccccc1)C(C(CCO)c1ccc([N+](=O)[O-])cc1)[N+](=O)[O-]</smiles>

4e-syn

(3S,4R)-4-nitro-3-(4-nitrophenyl)-5-phenylhex-5-en-1-ol (4e-syn): ${ }^{1} \mathrm{H} \quad \mathrm{NMR} \quad(270$ $\left.\mathrm{MHz}, \mathrm{CDCl}_{3}\right) \delta(\mathrm{ppm}) 1.24$ (br s, $\left.1 \mathrm{H}\right), 1.84-2.10(\mathrm{~m}, 2 \mathrm{H}), 3.23-3.34(\mathrm{~m}, 1 \mathrm{H}), 3.50-3.59$ (m, 1H), 3.96 (dt, $J=10.8,3.5 \mathrm{~Hz}, 1 \mathrm{H}), 5.44(\mathrm{~s}, 1 \mathrm{H}), 5.68-5.73$ (m, 2H), 6.96-6.99 (m, $2 \mathrm{H})$, 7.14-7.24 (m, 5H), 7.97-8.01 (m, 2H) ; ${ }^{13} \mathrm{C}$ NMR (67.5 MHz, $\left.\mathrm{CDCl}_{3}\right) \delta$ (ppm) 34.1, 46.4, 59.2, 94.0, 120.0, 124.2, 126.8, 129.0, 129.2, 129.3, 139.7, 142.4, 146.6, 147.6. HRMS Calculated for $\left[\mathrm{C}_{18} \mathrm{H}_{18} \mathrm{~N}_{2} \mathrm{O}_{5} \mathrm{Na}\right]^{+}: 365.1113$, Found: 365.1107 . $[\alpha]_{\mathrm{D}}{ }^{26}=-81.1^{\circ}(\mathrm{c}=$ $1.5 \mathrm{CHCl}_{3}$ ). Enantiomeric excess was determined by HPLC with a Chiralcel OD-H column; $\lambda=254 \mathrm{~nm}$; eluent: Hexane/Isopropanol $=60 / 40$; Flow rate: $1.0 \mathrm{~mL} / \mathrm{min} ; \mathrm{t}_{\text {minor }}=$ $5.548 \mathrm{~min}, \mathrm{t}_{\text {major }}=13.250 \mathrm{~min} ; \mathrm{ee} \%=82 \%$.<smiles>C=C(c1ccccc1)C(C(CCO)c1ccc([N+](=O)[O-])cc1)[N+](=O)[O-]</smiles>

4e-anti

(3S,4S)-4-nitro-3-(4-nitrophenyl)-5-phenylhex-5-en-1-ol (4e-anti): ${ }^{1} \mathrm{H} \quad \mathrm{NMR} \quad(270$ $\left.\mathrm{MHz}, \mathrm{CDCl}_{3}\right) \delta(\mathrm{ppm}) 1.25(\mathrm{br} \mathrm{s}, 1 \mathrm{H}), 1.58-1.69(\mathrm{~m}, 1 \mathrm{H}), 2.01-2.13(\mathrm{~m}, 1 \mathrm{H}), 3.16-3.27$ (m, 1H), 3.45-3.54 (m, 1H), 3.98 (dt, $J=11.6,3.2 \mathrm{~Hz}, 1 \mathrm{H}), 5.71(\mathrm{~d}, J=11.6 \mathrm{~Hz}, 1 \mathrm{H})$, 
$5.78(\mathrm{~s}, 1 \mathrm{H}), 5.96(\mathrm{~s}, 1 \mathrm{H}), 7.41-7.53(\mathrm{~m}, 7 \mathrm{H}), 8.19-8.22(\mathrm{~m}, 2 \mathrm{H}) ;{ }^{13} \mathrm{C} \mathrm{NMR}(67.5 \mathrm{MHz}$, $\mathrm{CDCl} 3) \delta(\mathrm{ppm}) 35.9,46.3,59.3,93.5,119.9,123.8,126.5,128.5,128.7,129.9,139.6$, 142.7, 145.3, 147.2. HRMS Calculated for $\left[\mathrm{C}_{18} \mathrm{H}_{18} \mathrm{~N}_{2} \mathrm{O}_{5} \mathrm{Na}\right]^{+}$: 365.1113, Found: 365.1107. $[\alpha]_{\mathrm{D}}{ }^{26}=-81.1^{\circ}\left(\mathrm{c}=1.5 \mathrm{CHCl}_{3}\right)$. Enantiomeric excess was determined by HPLC with a Chiralcel OD-H column; $\lambda=254 \mathrm{~nm}$; eluent: Hexane/Isopropanol $=85 / 15$; Flow rate: $1.0 \mathrm{~mL} / \mathrm{min} ; \mathrm{t}_{\mathrm{minor}}=15.916 \mathrm{~min}, \mathrm{t}_{\mathrm{major}}=14.444 \mathrm{~min} ; \mathrm{ee} \%=83 \%$.

4f was purified by flash chromatography (Hexane-EtOAc, v/v 5/1) as yellowish oil with overall isolated yield: $85 \%$ for both isomers.<smiles>C=C(c1ccccc1)C(CCO)C(CCO)c1ccccc1[N+](=O)[O-]</smiles>

4f-syn

(3S,4R)-4-nitro-3-(2-nitrophenyl)-5-phenylhex-5-en-1-ol (4f-syn): ${ }^{1} \mathrm{H} \quad \mathrm{NMR} \quad(270$ $\left.\mathrm{MHz}, \mathrm{CDCl}_{3}\right) \delta(\mathrm{ppm}) 1.25$ (br s, $\left.1 \mathrm{H}\right), 1.66-1.78(\mathrm{~m}, 1 \mathrm{H}), 2.02-2.18(\mathrm{~m}, 1 \mathrm{H}), 3.44-3.55$ (m, 2H), 4.51-4.58 (m, 1H), 5.70 (d, $J=11.6 \mathrm{~Hz}, 1 \mathrm{H}), 5.75$ (s, 1H), 5.88 (s, 1H), 7.38$7.64(\mathrm{~m}, 8 \mathrm{H}), 7.79-7.83(\mathrm{~m}, 1 \mathrm{H}) ;{ }^{13} \mathrm{C} \mathrm{NMR}\left(67.5 \mathrm{MHz}, \mathrm{CDCl}_{3}\right) \delta(\mathrm{ppm}) 35.9,46.3,59.3$, 93.5, 119.9, 125.3, 126.8, 127.9, 128.9, 129.2, 133.4, 139.7, 142.5, 151.8. HRMS Calculated for $\left[\mathrm{C}_{18} \mathrm{H}_{18} \mathrm{~N}_{2} \mathrm{O}_{5} \mathrm{Na}\right]^{+}$: 365.1113, Found: 365.1108. $[\alpha]_{\mathrm{D}}{ }^{26}=-90.4^{\circ}(\mathrm{c}=1.3$ $\mathrm{CHCl}_{3}$ ). Enantiomeric excess was determined by HPLC with a Chiralcel AS-H column; $\lambda=254 \mathrm{~nm}$; eluent: Hexane/Isopropanol $=60 / 40$; Flow rate: $1.0 \mathrm{~mL} / \mathrm{min} ; \mathrm{t}_{\mathrm{minor}}=11.448$ $\min , \mathrm{t}_{\text {major }}=6.708 \mathrm{~min} ; \mathrm{ee} \%=86 \%$.<smiles>C=C(c1ccccc1)C(CCO)C(CCO)c1ccccc1[N+](=O)[O-]</smiles>

4f-anti

(3S,4S)-4-nitro-3-(2-nitrophenyl)-5-phenylhex-5-en-1-ol (4f-anti): ${ }^{1} \mathrm{H} \quad \mathrm{NMR} \quad(270$ 
$\left.\mathrm{MHz}, \mathrm{CDCl}_{3}\right) \delta(\mathrm{ppm}) 0.96(\mathrm{~d}, J=6.7 \mathrm{~Hz}, 3 \mathrm{H}), 1.25(\mathrm{br} \mathrm{s}, 1 \mathrm{H}), 1.42-1.54(\mathrm{~m}, 1 \mathrm{H}), 1.64-$ $1.78(\mathrm{~m}, 1 \mathrm{H}), 2.55-2.72(\mathrm{~m}, 1 \mathrm{H}), 3.63-3.81(\mathrm{~m}, 2 \mathrm{H}), 5.27(\mathrm{~d}, J=10.4 \mathrm{~Hz}, 1 \mathrm{H}), 5.64(\mathrm{~s}$, $1 \mathrm{H}), 5.73(\mathrm{~s}, 1 \mathrm{H}), 7.32-7.44(\mathrm{~m}, 5 \mathrm{H}) ;{ }^{13} \mathrm{C} \mathrm{NMR}\left(67.5 \mathrm{MHz}, \mathrm{CDCl}_{3}\right) \delta(\mathrm{ppm}) 34.8,40.6$, 59.6, 94.4, 119.8, 125.5, 126.1, 127.4, 128.3, 129.7, 133.5, 139.9, 142.1, 151.3. HRMS Calculated for $\left[\mathrm{C}_{18} \mathrm{H}_{18} \mathrm{~N}_{2} \mathrm{O}_{5} \mathrm{Na}\right]^{+}$: 365.1113, Found: $365.1108 .[\alpha]_{\mathrm{D}}{ }^{26}=-90.4^{\circ}(\mathrm{c}=1.3$ $\mathrm{CHCl}_{3}$ ). Enantiomeric excess was determined by HPLC with a Chiralcel AS-H column; $\lambda=254 \mathrm{~nm}$; eluent: Hexane/Isopropanol $=60 / 40$; Flow rate: $1.0 \mathrm{~mL} / \mathrm{min} ; \mathrm{t}_{\text {minor }}=11.448$ $\min , t_{\text {major }}=6.708 \mathrm{~min} ; \mathrm{ee} \%=86 \%$.

4g was purified by flash chromatography (Hexane-EtOAc, v/v 7/1) as white oil with overall isolated yield: $93 \%$ for both isomers.<smiles>C=C(c1ccccc1)C(C)C(CCO)[N+](=O)[O-]</smiles>

$4 \mathrm{~g}-\mathrm{syn}$

(3S,4R)-3-methyl-4-nitro-5-phenylhex-5-en-1-ol (4g-syn): ${ }^{1} \mathrm{H}$ NMR $\left(270 \mathrm{MHz}, \mathrm{CDCl}_{3}\right)$ $\delta(\mathrm{ppm}) 0.96(\mathrm{~d}, J=6.7 \mathrm{~Hz}, 3 \mathrm{H}), 1.25(\mathrm{br} \mathrm{s}, 1 \mathrm{H}), 1.42-1.54(\mathrm{~m}, 1 \mathrm{H}), 1.64-1.78(\mathrm{~m}, 1 \mathrm{H})$, 2.55-2.72 (m, 1H), 3.63-3.81 (m, 2H), 5.27 (d, $J=10.4 \mathrm{~Hz}, 1 \mathrm{H}), 5.64(\mathrm{~s}, 1 \mathrm{H}), 5.73$ (s, $1 \mathrm{H})$, 7.32-7.44 (m, 5H) ; ${ }^{13} \mathrm{C}$ NMR $\left(67.5 \mathrm{MHz}, \mathrm{CDCl}_{3}\right) \delta(\mathrm{ppm})$ 15.5, 34.4, 36.1, 60.1, 95.4, 118.9, 126.8, 128.5, 128.9, 140.1, 143.1. HRMS Calculated for $\left[\mathrm{C}_{13} \mathrm{H}_{17} \mathrm{NO}_{3} \mathrm{Na}\right]^{+}$: 258.1106, Found: $258.1106 .[\alpha]_{\mathrm{D}}{ }^{26}=-81.4^{\circ}\left(\mathrm{c}=1.2 \mathrm{CHCl}_{3}\right)$. Enantiomeric excess was determined by HPLC with a Chiralcel OD-H column; $\lambda=254 \mathrm{~nm}$; eluent: Hexane $/$ Isopropanol $=80 / 20$; Flow rate: $1.0 \mathrm{~mL} / \mathrm{min} ; \mathrm{t}_{\text {minor }}=8.232 \mathrm{~min}, \mathrm{t}_{\text {major }}=7.052$ $\min ; \mathrm{ee} \%=88 \%$.<smiles>C=C(c1ccccc1)C(C)C(C)CCO</smiles>

4g-anti

(3S,4S)-3-methyl-4-nitro-5-phenylhex-5-en-1-ol (4g-anti): ${ }^{1} \mathrm{H}$ NMR $\left(270 \mathrm{MHz}, \mathrm{CDCl}_{3}\right)$ $\delta(\mathrm{ppm}) 1.07(\mathrm{~d}, J=5.8 \mathrm{~Hz}, 3 \mathrm{H}), 1.22-1.35(\mathrm{~m}, 2 \mathrm{H}), 1.69-1.81(\mathrm{~m}, 1 \mathrm{H}), 2.54-2.70(\mathrm{~m}$, 
1H), 3.59-3.75 (m, 2H), 5.29 (d, $J=9.9 \mathrm{~Hz}, 1 \mathrm{H}), 5.67$ (s, 1H), $5.76(\mathrm{~s}, 1 \mathrm{H}), 7.33-7.45(\mathrm{~m}$, $5 \mathrm{H}) ;{ }^{13} \mathrm{C}$ NMR $\left(67.5 \mathrm{MHz}, \mathrm{CDCl}_{3}\right) \delta$ (ppm) $16.4,34.2,34.8,60.1,95.5,119.1,126.7$, 128.6, 128.9, 140.0, 142.7. HRMS Calculated for $\left[\mathrm{C}_{18} \mathrm{H}_{18} \mathrm{~N}_{2} \mathrm{O}_{5} \mathrm{Na}\right]^{+}: 365.1113$, Found: 365.1106. $[\alpha]_{\mathrm{D}}{ }^{26}=72.6^{\circ}\left(\mathrm{c}=1.0 \mathrm{CHCl}_{3}\right)$. Enantiomeric excess was determined by HPLC with a Chiralcel OD-H column; $\lambda=254 \mathrm{~nm}$; eluent: Hexane/Isopropanol $=60 / 40$; Flow rate: $1.0 \mathrm{~mL} / \mathrm{min} ; \mathrm{t}_{\mathrm{minor}}=4.928 \mathrm{~min}, \mathrm{t}_{\text {major }}=10.824 \mathrm{~min} ; \mathrm{ee} \%=88 \%$.

4h was purified by flash chromatography (Hexane-EtOAc, v/v 10/1) as white oil with overall isolated yield: $89 \%$ for both isomers.<smiles>C=C(c1ccc(Cl)cc1)C(c1ccccc1)C(CCO)[N+](=O)[O-]</smiles>

4h-syn

(3S,4R)-5-(4-chlorophenyl)-4-nitro-3-phenylhex-5-en-1-ol (4h-syn): ${ }^{1} \mathrm{H}$ NMR (600 $\left.\mathrm{MHz}, \mathrm{CDCl}_{3}\right) \delta(\mathrm{ppm}) 1.26(\mathrm{br} \mathrm{s}, 1 \mathrm{H}), 1.89-1.99(\mathrm{~m}, 2 \mathrm{H}), 3.34-3.38(\mathrm{~m}, 1 \mathrm{H}), 3.50-3.54$ (m, 1H), 3.73 (dt, $J=11.4,3.6 \mathrm{~Hz}, 1 \mathrm{H}), 5.39$ (s, 1H), 5.59 (d, $J=11.4 \mathrm{~Hz}, 1 \mathrm{H}), 5.76$ (s, $1 \mathrm{H})$, 6.88-6.90 (m, 2H), 6.97-6.99 (m, 2H), 7.14-7.17 (m, 5H); ${ }^{13} \mathrm{C}$ NMR (150 MHz, $\left.\mathrm{CDCl}_{3}\right) \delta$ (ppm) 35.9, 46.4, 59.7, 94.2, 120.1, 127.5, 127.9, 128.4, 128.6, 128.7, 134.0, 137.1, 138.5, 142.1. HRMS Calculated for $\left[\mathrm{C}_{18} \mathrm{H}_{18} \mathrm{ClNO}_{3} \mathrm{Na}\right]^{+}:$354.0872, Found: 354.0867. $[\alpha]_{\mathrm{D}}{ }^{26}=-74.3^{\circ}\left(\mathrm{c}=0.9 \mathrm{CHCl}_{3}\right)$. Enantiomeric excess was determined by HPLC with a Chiralcel OD-H column; $\lambda=254 \mathrm{~nm}$; eluent: Hexane/Isopropanol $=95 / 5$; Flow rate: $1.0 \mathrm{~mL} / \mathrm{min} ; \mathrm{t}_{\text {minor }}=23.852 \mathrm{~min}, \mathrm{t}_{\text {major }}=25.560 \mathrm{~min} ; \mathrm{ee} \%=89 \%$.<smiles>C=C(c1ccc(Cl)cc1)C(CCO)[C@H](CCO)c1ccccc1</smiles>

$4 \mathrm{~h}-$ anti

(3S,4S)-5-(4-chlorophenyl)-4-nitro-3-phenylhex-5-en-1-ol (4h-anti): ${ }^{1} \mathrm{H}$ NMR (600 $\left.\mathrm{MHz}, \mathrm{CDCl}_{3}\right) \delta(\mathrm{ppm}) 1.18$ (br s, $\left.1 \mathrm{H}\right), 1.58-1.64(\mathrm{~m}, 1 \mathrm{H}), 1.95-2.00(\mathrm{~m}, 1 \mathrm{H}), 3.26-3.30$ (m, 1H), 3.43-3.47 (m, 1H), 3.75 (dt, $J=11.4,3.0 \mathrm{~Hz}, 1 \mathrm{H}), 5.63$ (d, $J=12.0 \mathrm{~Hz}, 1 \mathrm{H})$, 
$5.74(\mathrm{~s}, 1 \mathrm{H}), 5.98(\mathrm{~s}, 1 \mathrm{H}), 7.25-7.27(\mathrm{~m}, 3 \mathrm{H}), 7.31-7.33(\mathrm{~m}, 2 \mathrm{H}), 7.37-7.40(\mathrm{~m}, 2 \mathrm{H})$, 7.43$7.45(\mathrm{~m}, 2 \mathrm{H}) ;{ }^{13} \mathrm{C} \mathrm{NMR}\left(150 \mathrm{MHz}, \mathrm{CDCl}_{3}\right) \delta(\mathrm{ppm}) 36.2,46.6,59.9,94.4,120.3,127.7$, $128.1,128.7,128.9,129.0,134.2,137.3,138.8,142.3$. HRMS Calculated for $\left[\mathrm{C}_{18} \mathrm{H}_{18} \mathrm{ClNO}_{3} \mathrm{Na}\right]^{+}:$354.0872, Found: 354.0867. $[\alpha]_{\mathrm{D}}{ }^{26}=84.1^{\circ}\left(\mathrm{c}=1.0 \mathrm{CHCl}_{3}\right)$. Enantiomeric excess was determined by HPLC with a Chiralcel OD-H column; $\lambda=254$ $\mathrm{nm}$; eluent: Hexane/Isopropanol $=95 / 5$; Flow rate: $1.0 \mathrm{~mL} / \mathrm{min}$; $\mathrm{t}_{\text {minor }}=7.240 \mathrm{~min}$, $\mathrm{t}_{\text {major }}=$ $10.480 \mathrm{~min} ; \mathrm{ee} \%=90 \%$.

$4 \mathbf{i}$ was purified by flash chromatography (Hexane-EtOAc, v/v 10/1) as light yellowish oil with overall isolated yield: $78 \%$ for both isomers.<smiles>C=C(c1ccc(Cl)cc1)C(C(CCO)c1ccco1)[N+](=O)[O-]</smiles>

$4 \mathbf{i}-s y n$

(3S,4R)-5-(4-chlorophenyl)-3-(furan-2-yl)-4-nitrohex-5-en-1-ol (4i-syn): ${ }^{1} \mathrm{H} \quad \mathrm{NMR}$ $\left(600 \mathrm{MHz}, \mathrm{CDCl}_{3}\right) \delta(\mathrm{ppm}) 1.32(\mathrm{br} \mathrm{s}, 1 \mathrm{H}), 1.86-1.91(\mathrm{~m}, 1 \mathrm{H}), 1.95-2.01(\mathrm{~m}, 1 \mathrm{H}), 3.39-$ $3.45(\mathrm{~m}, 1 \mathrm{H}), 3.60-3.62(\mathrm{~m}, 1 \mathrm{H}), 3.90(\mathrm{dt}, J=10.8,3.6 \mathrm{~Hz}, 1 \mathrm{H}), 5.46$ (s, $1 \mathrm{H}), 5.65(\mathrm{~d}, J=$ $10.8 \mathrm{~Hz}, 1 \mathrm{H}), 5.71(\mathrm{~s}, 1 \mathrm{H}), 6.05(\mathrm{~d}, J=3.0 \mathrm{~Hz}, 1 \mathrm{H}), 6.20-6.21(\mathrm{~m}, 1 \mathrm{H}), 7.06-7.08(\mathrm{~m}$, $2 \mathrm{H}), 7.15-7.16(\mathrm{~m}, 1 \mathrm{H}), 7.23-7.25(\mathrm{~m}, 2 \mathrm{H}) ;{ }^{13} \mathrm{C} \mathrm{NMR}\left(150 \mathrm{MHz}, \mathrm{CDCl}_{3}\right) \delta(\mathrm{ppm}) 33.9$, 40.1, 59.8, 92.1, 109.3, 110.3, 119.6, 128.0, 128.5, 134.2, 137.8, 141.9, 142.1, 150.4. HRMS Calculated for $\left[\mathrm{C}_{16} \mathrm{H}_{16} \mathrm{ClNO}_{4} \mathrm{Na}\right]^{+}: 344.0665$, Found: $344.0660 .[\alpha]_{\mathrm{D}}{ }^{26}=-78.2^{\circ}(\mathrm{c}$ $=1.0 \mathrm{CHCl}_{3}$ ). Enantiomeric excess was determined by HPLC with a Chiralcel AS-H column; $\lambda=254 \mathrm{~nm}$; eluent: Hexane/Isopropanol $=85 / 15$; Flow rate: $1.0 \mathrm{~mL} / \mathrm{min} ; \mathrm{t}_{\operatorname{minor}}=$ $11.084 \mathrm{~min}, \mathrm{t}_{\text {major }}=7.168 \mathrm{~min} ; \mathrm{ee} \%=87 \%$.<smiles>C=C(c1ccc(Cl)cc1)C(C(CCO)c1ccco1)[N+](=O)[O-]</smiles>

$4 \mathrm{i}-$ anti 
(3S,4R)-5-(4-chlorophenyl)-3-(furan-2-yl)-4-nitrohex-5-en-1-ol (4i-anti): ${ }^{1} \mathrm{H} \quad \mathrm{NMR}$ $\left(600 \mathrm{MHz}, \mathrm{CDCl}_{3}\right) \delta(\mathrm{ppm}) 1.23$ (br s, $\left.1 \mathrm{H}\right), 1.72-1.78(\mathrm{~m}, 1 \mathrm{H}), 1.87-1.92(\mathrm{~m}, 1 \mathrm{H}), 3.34-$ $3.39(\mathrm{~m}, 1 \mathrm{H}), 3.52-3.56(\mathrm{~m}, 1 \mathrm{H}), 3.94(\mathrm{dt}, J=11.4,3.6 \mathrm{~Hz}, 1 \mathrm{H}), 5.68$ (d, $J=11.4 \mathrm{~Hz}$, $1 \mathrm{H}), 5.73(\mathrm{~s}, 1 \mathrm{H}), 5.89(\mathrm{~s}, 1 \mathrm{H}), 6.23(\mathrm{~d}, J=3.0 \mathrm{~Hz}, 1 \mathrm{H}), 6.29-6.30(\mathrm{~m}, 1 \mathrm{H}), 7.35-7.38(\mathrm{~m}$, 3H), 7.40-7.42 (m, 2H); ${ }^{13} \mathrm{C}$ NMR (150 MHz, $\left.\mathrm{CDCl}_{3}\right) \delta(\mathrm{ppm})$ 32.2, 39.9, 59.7, 92.2, 108.9, 110.4, 120.3, 128.1, 129.0, 134.7, 137.8, 141.1, 142.4, 151.3. HRMS Calculated for $\left[\mathrm{C}_{16} \mathrm{H}_{16} \mathrm{ClNO}_{4} \mathrm{Na}\right]^{+}$: 344.0665 , Found: 344.0660. $[\alpha]_{\mathrm{D}}{ }^{26}=74.1^{\circ}\left(\mathrm{c}=0.9 \mathrm{CHCl}_{3}\right)$. Enantiomeric excess was determined by HPLC with a Chiralcel AS-H column; $\lambda=254$ $\mathrm{nm}$; eluent: Hexane/Isopropanol $=85 / 15$; Flow rate: $1.0 \mathrm{~mL} / \mathrm{min}$; $\mathrm{t}_{\text {minor }}=5.832 \mathrm{~min}, \mathrm{t}_{\text {major }}$ $=17.144 \mathrm{~min} ; \mathrm{ee} \%=86 \%$.

$\mathbf{4 j}$ was purified by flash chromatography (Hexane-EtOAc, v/v 5/1) as light yellowish oil with overall isolated yield: $74 \%$ for both isomers.

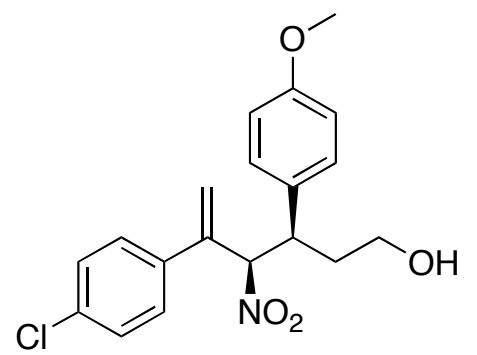

$4 \mathbf{j}-\mathbf{s y n}$

(3S,4R)-5-(4-chlorophenyl)-3-(4-methoxyphenyl)-4-nitrohex-5-en-1-ol (4j-syn): ${ }^{1} \mathrm{H}$ NMR (600 MHz, $\left.\mathrm{CDCl}_{3}\right) \delta(\mathrm{ppm}) 1.24$ (br s, 1H), 1.84-1.90 (m, 1H), 1.91-1.97 (m, 1H), 3.34-3.38 (m, 1H), 3.50-3.54 (m, 1H), 3.67 (dt, $J=11.1,3.0 \mathrm{~Hz}, 1 \mathrm{H}), 3.74(\mathrm{~s}, 3 \mathrm{H}), 5.40$ (s, 1H), $5.55(\mathrm{~d}, J=11.4 \mathrm{~Hz}, 1 \mathrm{H}), 5.74(\mathrm{~s}, 1 \mathrm{H}), 6.70-6.71(\mathrm{~m}, 2 \mathrm{H}), 6.89-6.93(\mathrm{~m}, 4 \mathrm{H})$, 7.16-7.17 (m, 2H); ${ }^{13} \mathrm{C}$ NMR (150 MHz, $\left.\mathrm{CDCl}_{3}\right) \delta(\mathrm{ppm})$ 35.9, 45.6, 55.2, 59.8, 94.4, $114.1,119.9,128.0,128.4,128.9,129.7,134.0,138.5,142.1$, 158.8. HRMS Calculated for $\left[\mathrm{C}_{19} \mathrm{H}_{20} \mathrm{ClNO}_{4} \mathrm{Na}\right]^{+}: 384.0979$, Found: $384.0974 .[\alpha]_{\mathrm{D}}{ }^{26}=-138.3^{\circ}\left(\mathrm{c}=0.62 \mathrm{CHCl}_{3}\right)$. Enantiomeric excess was determined by HPLC with a Chiralcel AS-H column; $\lambda=254$ $\mathrm{nm}$; eluent: Hexane/Isopropanol $=80 / 20$; Flow rate: $1.0 \mathrm{~mL} / \mathrm{min} ; \mathrm{t}_{\operatorname{minor}}=17.876 \mathrm{~min}$, $\mathrm{t}_{\text {major }}=8.460 \mathrm{~min} ; \mathrm{ee} \%=89 \%$. 
<smiles>C=C(c1ccc(Cl)cc1)[C@H](C(=C)[N+](=O)[O-])[C@@H](CCO)c1ccc(OC)cc1</smiles>

$4 \mathbf{j}-$ anti

(3S,4S)-5-(4-chlorophenyl)-3-(4-methoxyphenyl)-4-nitrohex-5-en-1-ol (4j-anti): ${ }^{1} \mathrm{H}$ NMR (600 MHz, $\left.\mathrm{CDCl}_{3}\right) \delta(\mathrm{ppm}) 1.12$ (br s, 1H), 1.55-1.60 (m, 1H), 1.93-1.98 (m, 1H), 3.29-3.33 (m, 1H), 3.44-3.47 (m, 1H), 3.70 (dt, $J=11.4,3.0 \mathrm{~Hz}, 1 \mathrm{H}), 3.78(\mathrm{~s}, 3 \mathrm{H}), 5.56$ $(\mathrm{d}, J=11.4 \mathrm{~Hz}, 1 \mathrm{H}), 5.73(\mathrm{~s}, 1 \mathrm{H}), 5.97(\mathrm{~s}, 1 \mathrm{H}), 6.84-6.86(\mathrm{~m}, 2 \mathrm{H}), 7.16-7.19(\mathrm{~m}, 2 \mathrm{H})$, 7.37-7.40 (m, 2H), 7.42-7.44 (m, 2H); $\left.{ }^{13} \mathrm{C} \mathrm{NMR} \mathrm{(150} \mathrm{MHz,} \mathrm{CDCl}_{3}\right) \delta(\mathrm{ppm}) 34.2,45.8$, $55.2,59.8,94.8,114.4,120.1,128.1,129.0,129.1,130.0,134.6,138.4,141.7,159.1$. HRMS Calculated for $\left[\mathrm{C}_{19} \mathrm{H}_{20} \mathrm{ClNO}_{4} \mathrm{Na}\right]^{+}: 384.0979$, Found: 384.0974 . $[\alpha]_{\mathrm{D}}{ }^{26}=68.3^{\circ}(\mathrm{c}$ $=1.1 \mathrm{CHCl}_{3}$ ). Enantiomeric excess was determined by HPLC with a Chiralcel OD-H column; $\lambda=254 \mathrm{~nm}$; eluent: Hexane/Isopropanol $=80 / 20$; Flow rate: $1.0 \mathrm{~mL} / \mathrm{min}$; $\mathrm{t}_{\mathrm{minor}}=$ $7.640 \mathrm{~min}, \mathrm{t}_{\text {major }}=8.936 \mathrm{~min} ; \mathrm{ee} \%=85 \%$.

4k was purified by flash chromatography (Hexane-EtOAc, v/v 7/1) as yellow oil with overall isolated yield: $64 \%$ for both isomers.

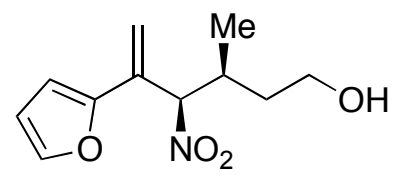

\section{$4 \mathrm{k}-\mathrm{syn}$}

(3S,4R)-5-(furan-2-yl)-3-methyl-4-nitrohex-5-en-1-ol (4k-syn): ${ }^{1} \mathrm{H}$ NMR $(600 \mathrm{MHz}$, $\left.\mathrm{CDCl}_{3}\right) \delta(\mathrm{ppm}) 1.11(\mathrm{~d}, J=6.6 \mathrm{~Hz}, 3 \mathrm{H}), 1.20(\mathrm{t}, J=4.8 \mathrm{~Hz}, 1 \mathrm{H}), 1.32-1.38(\mathrm{~m}, 1 \mathrm{H})$, 1.70-1.75 (m, 1H), 2.69-2.74 (m, 1H), 3.65-3.70 (m, 1H), 3.73-3.77 (m, 1H), $5.18(\mathrm{~d}, J=$ $10.8 \mathrm{~Hz}, 1 \mathrm{H}), 5.64(\mathrm{~s}, 1 \mathrm{H}), 5.96(\mathrm{~s}, 1 \mathrm{H}), 6.41(\mathrm{dd}, J=3.6,1.8 \mathrm{~Hz}, 1 \mathrm{H}), 6.53(\mathrm{~d}, J=3.6$ $\mathrm{Hz}, 1 \mathrm{H}), 7.40(\mathrm{~d}, J=1.8 \mathrm{~Hz}, 1 \mathrm{H}) ;{ }^{13} \mathrm{C} \mathrm{NMR}\left(150 \mathrm{MHz}, \mathrm{CDCl}_{3}\right) \delta(\mathrm{ppm}) 16.4,33.0,34.7$, 59.9, 93.2, 107.8, 111.6, 115.2, 131.3, 143.1, 151.7. HRMS Calculated for $\left[\mathrm{C}_{11} \mathrm{H}_{15} \mathrm{NO}_{4} \mathrm{Na}\right]^{+}:$248.0899, Found: 248.0894. $[\alpha]_{\mathrm{D}}{ }^{26}=-138.3^{\circ}\left(\mathrm{c}=0.56 \mathrm{CHCl}_{3}\right)$. 
Enantiomeric excess was determined by HPLC with a Chiralcel AS-H column; $\lambda=254$ $\mathrm{nm}$; eluent: Hexane/Isopropanol $=85 / 15$; Flow rate: $1.0 \mathrm{~mL} / \mathrm{min}$; $\mathrm{t}_{\text {minor }}=8.208 \mathrm{~min}$, $\mathrm{t}_{\text {major }}$ $=7.052 \mathrm{~min} ; \mathrm{ee} \%=89 \%$.<smiles>C=C(c1ccco1)[C@H](CCO)[N+](=O)[O-]</smiles>

$4 \mathrm{k}-$ anti

(3S,4S)-5-(furan-2-yl)-3-methyl-4-nitrohex-5-en-1-ol (4k-anti): ${ }^{1} \mathrm{H}$ NMR $(600 \mathrm{MHz}$, $\left.\mathrm{CDCl}_{3}\right) \delta(\mathrm{ppm}) 1.13(\mathrm{~d}, J=6.6 \mathrm{~Hz}, 3 \mathrm{H}), 1.24$ (t, $\left.J=4.8 \mathrm{~Hz}, 1 \mathrm{H}\right), 1.30-1.40(\mathrm{~m}, 1 \mathrm{H})$, 1.71-1.75 (m, $1 \mathrm{H}), 2.73-2.79(\mathrm{~m}, 1 \mathrm{H}), 3.60-3.67(\mathrm{~m}, 1 \mathrm{H}), 3.71-3.76(\mathrm{~m}, 1 \mathrm{H}), 5.19(\mathrm{~d}, J=$ $10.8 \mathrm{~Hz}, 1 \mathrm{H}), 5.70(\mathrm{~s}, 1 \mathrm{H}), 5.95(\mathrm{~s}, 1 \mathrm{H}), 6.43$ (dd, $J=3.6,1.8 \mathrm{~Hz}, 1 \mathrm{H}), 6.56(\mathrm{~d}, J=3.6$ $\mathrm{Hz}, 1 \mathrm{H}), 7.41(\mathrm{~d}, \mathrm{~J}=1.8 \mathrm{~Hz}, 1 \mathrm{H}) ;{ }^{13} \mathrm{C}$ NMR $\left(150 \mathrm{MHz}, \mathrm{CDCl}_{3}\right) \delta(\mathrm{ppm}) 16.3,33.4,34.2$, 59.9, 93.7, 106.4, 112.1, 115.9, 132.3, 144.5, 152.1. HRMS Calculated for $\left[\mathrm{C}_{11} \mathrm{H}_{15} \mathrm{NO}_{4} \mathrm{Na}\right]^{+}:$248.0899, Found: 248.0894. $[\alpha]_{\mathrm{D}}{ }^{26}=70.3^{\circ}\left(\mathrm{c}=0.95 \mathrm{CHCl}_{3}\right)$. Enantiomeric excess was determined by HPLC with a Chiralcel OD-H column; $\lambda=254$ $\mathrm{nm}$; eluent: Hexane/Isopropanol $=65 / 35$; Flow rate: $1.0 \mathrm{~mL} / \mathrm{min} ; \mathrm{t}_{\mathrm{minor}}=4.908 \mathrm{~min}, \mathrm{t}_{\text {major }}$ $=10.864 \mathrm{~min} ; \mathrm{ee} \%=88 \%$.

4l was purified by flash chromatography (Hexane-EtOAc, v/v 10/1) as white oil with overall isolated yield: $82 \%$ for both isomers.<smiles>C=C(c1ccc(C)cc1)C(c1ccccc1)C(CCO)[N+](=O)[O-]</smiles>

41-syn

(3S,4R)-4-nitro-3-phenyl-5-p-tolylhex-5-en-1-ol (4l-syn): ${ }^{1} \mathrm{H}$ NMR (600 MHz, $\left.\mathrm{CDCl}_{3}\right)$ $\delta(\mathrm{ppm}) 1.18$ (br s, $1 \mathrm{H}), 1.91-2.00(\mathrm{~m}, 2 \mathrm{H}), 2.29(\mathrm{~s}, 3 \mathrm{H}), 3.35-3.39(\mathrm{~m}, 1 \mathrm{H}), 3.50-3.54(\mathrm{~m}$, 1H), $3.74(\mathrm{dt}, J=11.1,4.2 \mathrm{~Hz}, 1 \mathrm{H}), 5.39$ (s, 1H), 5.66 (d, $J=11.4 \mathrm{~Hz}, 1 \mathrm{H}), 5.69(\mathrm{~s}, 1 \mathrm{H})$, 6.89-6.90 (m, 2H), 6.99-7.02 (m, 4H), 7.15-7.19 (m, 3H); ${ }^{13} \mathrm{C} \mathrm{NMR}\left(150 \mathrm{MHz}, \mathrm{CDCl}_{3}\right) \delta$ (ppm) 21.1, 35.9, 46.2, 59.9, 94.4, 119.0, 126.4, 127.4, 128.6, 128.7, 129.0, 137.2, 137.5, 
137.8, 142.6. HRMS Calculated for $\left[\mathrm{C}_{19} \mathrm{H}_{21} \mathrm{NO}_{3} \mathrm{Na}\right]^{+}: 334.1419$, Found: $334.1415 .[\alpha]_{\mathrm{D}}{ }^{26}$ $=-86.5^{\circ}\left(\mathrm{c}=1.0 \mathrm{CHCl}_{3}\right)$. Enantiomeric excess was determined by HPLC with a Chiralcel OD-H column; $\lambda=254 \mathrm{~nm}$; eluent: Hexane/Isopropanol $=85 / 15$; Flow rate: $1.0 \mathrm{~mL} / \mathrm{min}$; $t_{\text {minor }}=10.352$ min, $t_{\text {major }}=12.964 \mathrm{~min} ; \mathrm{ee} \%=90 \%$.<smiles>C=C(c1ccc(C)cc1)C(CCO)C(CCO)c1ccccc1</smiles>

4l-anti

(3S,4S)-4-nitro-3-phenyl-5-p-tolylhex-5-en-1-ol (4l-anti): ${ }^{1} \mathrm{H}$ NMR (600 MHz, $\left.\mathrm{CDCl}_{3}\right)$ $\delta(\mathrm{ppm}) 1.58-1.63(\mathrm{~m}, 1 \mathrm{H}), 1.98-2.18(\mathrm{~m}, 1 \mathrm{H}), 2.38(\mathrm{~s}, 3 \mathrm{H}), 3.26-3.30(\mathrm{~m}, 1 \mathrm{H}), 3.42-3.46$ (m, 1H), 3.75 (dt, $J=12.0,3.0 \mathrm{~Hz}, 1 \mathrm{H}), 5.68$ (d, $J=11.4 \mathrm{~Hz}, 1 \mathrm{H}), 5.71$ (s, 1H), 5.91 (s, 1H), 7.21-7.22 (m, 2H), 7.25-7.28 (m, 3H), 7.31-7.33 (m, 2H), 7.40-7.41 (m, $2 \mathrm{H}) ;{ }^{13} \mathrm{C}$ NMR (150 MHz, $\left.\mathrm{CDCl}_{3}\right) \delta(\mathrm{ppm}) 21.1,34.3,46.7,59.8,94.6,118.5,126.6,127.8,128.0$, $128.9,129.5,137.1,138.5,138.7,142.6$. HRMS Calculated for $\left[\mathrm{C}_{19} \mathrm{H}_{21} \mathrm{NO}_{3} \mathrm{Na}\right]^{+}$: 334.1419, Found: $334.1415 .[\alpha]_{\mathrm{D}}{ }^{26}=84.3^{\circ}\left(\mathrm{c}=1.0 \mathrm{CHCl}_{3}\right)$. Enantiomeric excess was determined by HPLC with a Chiralcel OD-H column; $\lambda=254 \mathrm{~nm}$; eluent: Hexane/Isopropanol $=85 / 15$; Flow rate: $1.0 \mathrm{~mL} / \mathrm{min} ; \mathrm{t}_{\mathrm{minor}}=8.524 \mathrm{~min}, \mathrm{t}_{\text {major }}=14.888$ $\min ; \mathrm{ee} \%=92 \%$.

4m was purified by flash chromatography (Hexane-EtOAc, v/v 5/1) as yellow oil with overall isolated yield: $82 \%$ for both isomers.

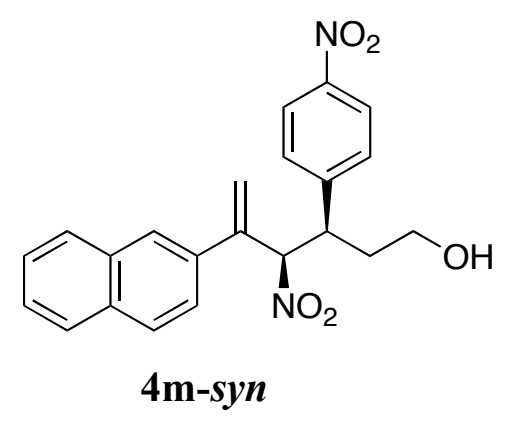

(3S,4R)-5-(naphthalen-2-yl)-4-nitro-3-(4-nitrophenyl)hex-5-en-1-ol $\quad$ (4m-syn): $\quad{ }^{1} \mathrm{H}$ NMR (600 MHz, $\left.\mathrm{CDCl}_{3}\right) \delta(\mathrm{ppm}) 1.28(\mathrm{t}, J=4.2 \mathrm{~Hz}, 1 \mathrm{H}), 1.92-1.98(\mathrm{~m}, 1 \mathrm{H}), 2.05-2.11$ (m, 1H), 3.29-3.33 (m, 1H), 3.54-3.58 (m, 1H), 4.03 (dt, $J=10.8,3.0 \mathrm{~Hz}, 1 \mathrm{H}), 5.57$ (s, 
1H), 5.83-5.85 (m, 2H), 6.96-6.99 (m, 2H), 7.08-7.10 (m, 1H), 7.16-7.18 (m, 2H), 7.46$7.49(\mathrm{~m}, 3 \mathrm{H}), 7.67-7.73(\mathrm{~m}, 2 \mathrm{H}), 7.76-7.78(\mathrm{~m}, 1 \mathrm{H}), 7.95-7.97(\mathrm{~m}, 2 \mathrm{H}) ;{ }^{13} \mathrm{C}$ NMR $(150$ $\left.\mathrm{MHz}, \mathrm{CDCl}_{3}\right) \delta$ (ppm) 35.8, 46.3, 59.2, 93.4, 120.1, 123.6, 124.1, 125.6, 126.6, 126.7, 127.6, 128.1, 128.4, 129.8, 132.8, 132.9, 136.8, 142.6, 145.2, 147.1. HRMS Calculated for $\left[\mathrm{C}_{22} \mathrm{H}_{20} \mathrm{~N}_{2} \mathrm{O}_{5} \mathrm{Na}\right]^{+}: 415.1270$, Found: $415.1265 .[\alpha]_{\mathrm{D}}^{26}=-147.5^{\circ}\left(\mathrm{c}=0.45 \mathrm{CHCl}_{3}\right)$. Enantiomeric excess was determined by HPLC with a Chiralcel OD-H column; $\lambda=254$ $\mathrm{nm}$; eluent: Hexane $/$ Isopropanol $=90 / 10$; Flow rate: $1.5 \mathrm{~mL} / \mathrm{min}$; $\mathrm{t}_{\text {minor }}=28.316 \mathrm{~min}$, $\mathrm{t}_{\text {major }}=20.900 \mathrm{~min} ; \mathrm{ee} \%=77 \%$.<smiles>C=C(c1ccc2ccccc2c1)[C@H](C(CCO)c1ccc([N+](=O)[O-])cc1)[N+](=O)[O-]</smiles>

$4 \mathrm{~m}-$ anti

(3S,4S)-5-(naphthalen-2-yl)-4-nitro-3-(4-nitrophenyl)hex-5-en-1-ol $\quad(4 \mathrm{~m}-a n t i): \quad{ }^{1} \mathrm{H}$ NMR (600 MHz, $\left.\mathrm{CDCl}_{3}\right) \delta(\mathrm{ppm}) 1.16(\mathrm{t}, J=4.2 \mathrm{~Hz}, 1 \mathrm{H}), 1.62-1.67(\mathrm{~m}, 1 \mathrm{H}), 2.10-2.15$ (m, 1H), 3.20-3.25 (m, 1H), 3.48-3.51 (m, 1H), 4.03 (dt, $J=11.4,3.0 \mathrm{~Hz}, 1 \mathrm{H}), 5.86(\mathrm{~d}, J$ $=11.4 \mathrm{~Hz}, 1 \mathrm{H}), 5.91(\mathrm{~s}, 1 \mathrm{H}), 6.05(\mathrm{~s}, 1 \mathrm{H}), 7.50-7.57(\mathrm{~m}, 4 \mathrm{H}), 7.61-7.63(\mathrm{~m}, 1 \mathrm{H}), 7.86-$ $7.91(\mathrm{~m}, 3 \mathrm{H}), 7.96-7.97(\mathrm{~m}, 1 \mathrm{H}), 8.20-8.22(\mathrm{~m}, 2 \mathrm{H}) ;{ }^{13} \mathrm{C} \mathrm{NMR}\left(150 \mathrm{MHz}, \mathrm{CDCl}_{3}\right) \delta$ (ppm) 34.0, 46.4, 59.1, 93.9, 120.0, 124.1, 124.4, 125.8, 126.8, 126.9, 127.7, 128.3, $128.9,129.1,133.1,133.2,136.8,142.3,146.4,147.5$. HRMS Calculated for $\left[\mathrm{C}_{22} \mathrm{H}_{20} \mathrm{~N}_{2} \mathrm{O}_{5} \mathrm{Na}\right]^{+}:$415.1270, Found: 415.1265. $[\alpha]_{\mathrm{D}}^{26}=139.2^{\circ}\left(\mathrm{c}=0.50 \mathrm{CHCl}_{3}\right)$. Enantiomeric excess was determined by HPLC with a Chiralcel OD-H column; $\lambda=254$ $\mathrm{nm}$; eluent: Hexane/Isopropanol $=75 / 25$; Flow rate: $1.0 \mathrm{~mL} / \mathrm{min}$; $\mathrm{t}_{\text {minor }}=11.436 \mathrm{~min}$, $\mathrm{t}_{\text {major }}=8.944 \mathrm{~min} ; \mathrm{ee} \%=74 \%$.

4n was purified by flash chromatography (Hexane-EtOAc, v/v 5/1) as white oil with overall isolated yield: $86 \%$ for both isomers. 
<smiles>C=C(c1ccc(C#N)cc1)[C@H](c1ccccc1)[C@H](CC=O)[N+](=O)[O-]</smiles>

4n-syn

(3S,4R)-4-nitro-5-(4-cyano)-3-phenylhex-5-enal (4n-syn): ${ }^{1} \mathrm{H} \mathrm{NMR}\left(600 \mathrm{MHz}, \mathrm{CDCl}_{3}\right)$ $\delta$ (ppm) 1.58 (br s, 1H), 2.81-2.84 (m, 1H), 2.99-3.04 (m, 1H), 4.11-4.15 (dt, $J=10.2,3.0$ $\mathrm{Hz}, 1 \mathrm{H}), 5.53$ (s, 1H), 5.64 (d, $J=11.4 \mathrm{~Hz}, 1 \mathrm{H}), 5.90$ (s, 1H), 7.00-7.01 (m, 2H), 7.05$7.06(\mathrm{~m}, 2 \mathrm{H}), 7.16-7.17(\mathrm{~m}, 3 \mathrm{H}), 7.48-7.49(\mathrm{~m}, 2 \mathrm{H}), 9.58-9.59(\mathrm{~m}, 1 \mathrm{H}) ;{ }^{13} \mathrm{C}$ NMR $(150$ $\left.\mathrm{MHz}, \mathrm{CDCl}_{3}\right) \delta(\mathrm{ppm}) 43.9,46.8,92.6,112.0,118.3,121.8,127.2,128.0,128.7,128.9$, 132.2, 136.4, 141.6, 144.1, 198.1. HRMS Calculated for $\left[\mathrm{C}_{19} \mathrm{H}_{16} \mathrm{~N}_{2} \mathrm{O}_{3} \mathrm{Na}\right]^{+}: 320.1161$, Found: $320.1157 .[\alpha]_{\mathrm{D}}{ }^{26}=-76.4^{\circ}\left(\mathrm{c}=0.60 \mathrm{CHCl}_{3}\right)$. Enantiomeric excess was determined by HPLC with a Chiralcel OD-H column; $\lambda=254 \mathrm{~nm}$; eluent: Hexane/Isopropanol = 91/10; Flow rate: $1.0 \mathrm{~mL} / \mathrm{min} ; \mathrm{t}_{\text {minor }}=10.192 \mathrm{~min}, \mathrm{t}_{\mathrm{major}}=8.804 \mathrm{~min} ; \mathrm{ee} \%=82 \%$.<smiles>C=C(c1ccc(C#N)cc1)[C@H](c1ccccc1)[C@H](CC=O)[N+](=O)[O-]</smiles>

4n-anti

(3S,4S)-4-nitro-5-(4-cyano)-3-phenylhex-5-enal (4n-anti): ${ }^{1} \mathrm{H}$ NMR $\left(600 \mathrm{MHz}, \mathrm{CDCl}_{3}\right)$ $\delta(\mathrm{ppm}) 1.58$ (br s, 1H), 2.68-2.80 (m, 2H), 4.18-4.22 (m, 1H), $5.73(\mathrm{~d}, J=11.4 \mathrm{~Hz}, 1 \mathrm{H})$, $5.86(\mathrm{~s}, 1 \mathrm{H}), 6.11(\mathrm{~s}, 1 \mathrm{H}), 7.27-7.34(\mathrm{~m}, 5 \mathrm{H}), 7.59-7.60(\mathrm{~m}, 2 \mathrm{H}), 7.72-7.74(\mathrm{~m}, 2 \mathrm{H}), 9.51$ $9.52(\mathrm{~m}, 1 \mathrm{H}) ;{ }^{13} \mathrm{C} \mathrm{NMR}\left(150 \mathrm{MHz}, \mathrm{CDCl}_{3}\right) \delta(\mathrm{ppm})$ 43.7, 45.7, 92.8, 112.8, 118.2, 122.7, 127.4, 127.9, 128.4, 129.2, 132.9, 137.6, 140.8, 143.7, 198.1. HRMS Calculated for $\left[\mathrm{C}_{19} \mathrm{H}_{16} \mathrm{~N}_{2} \mathrm{O}_{3} \mathrm{Na}\right]^{+}:$320.1161, Found: 320.1157. $[\alpha]_{\mathrm{D}}{ }^{26}=84.6^{\circ}\left(\mathrm{c}=0.55 \mathrm{CHCl}_{3}\right)$. Enantiomeric excess was determined by HPLC with a Chiralcel OD-H column; $\lambda=254$ $\mathrm{nm}$; eluent: Hexane/Isopropanol $=90 / 10$; Flow rate: $1.0 \mathrm{~mL} / \mathrm{min} ; \mathrm{t}_{\text {minor }}=14.124 \mathrm{~min}$, $\mathrm{t}_{\text {major }}=11.992 \mathrm{~min} ; \mathrm{ee} \%=80 \%$.

40 was purified by flash chromatography (Hexane-EtOAc, v/v 7/1) as yellowish oil with overall isolated yield: $79 \%$ for both isomers. 
<smiles>C=C(c1ccc([N+](=O)[O-])cc1)[C@@H](c1ccccc1)[C@@H](CC=O)CCCC</smiles>

(3S,4R)-4-nitro-5-(4-nitrophenyl)-3-phenylhex-5-enal (4o-syn): ${ }^{1} \mathrm{H}$ NMR $(600 \mathrm{MHz}$, $\left.\mathrm{CDCl}_{3}\right) \delta(\mathrm{ppm})$ 2.82-2.85 (m, 1H), 3.00-3.05 (m, 1H), 4.12-4.16 (m, 1H), $5.58(\mathrm{~s}, 1 \mathrm{H})$, $5.66(\mathrm{~d}, J=11.4 \mathrm{~Hz}, 1 \mathrm{H}), 5.95(\mathrm{~s}, 1 \mathrm{H}), 7.01-7.03(\mathrm{~m}, 2 \mathrm{H}), 7.11-7.12(\mathrm{~m}, 2 \mathrm{H}), 7.16-7.17$ $(\mathrm{m}, 3 \mathrm{H}), 8.04-8.06(\mathrm{~m}, 2 \mathrm{H}), 9.59(\mathrm{t}, J=1.2 \mathrm{~Hz}, 1 \mathrm{H})[\alpha]_{\mathrm{D}}{ }^{26}=-76.4^{\circ}\left(\mathrm{c}=0.60 \mathrm{CHCl}_{3}\right)$. Enantiomeric excess was determined by HPLC with a Chiralcel OD-H column; $\lambda=254$ nm; eluent: Hexane/Isopropanol = 95/5; Flow rate: $1.5 \mathrm{~mL} / \mathrm{min}$; tminor $=18.736 \mathrm{~min}$, $\mathrm{t}_{\text {major }}=17.144 \mathrm{~min} ; \mathrm{ee} \%=77 \%$.<smiles>C=C(c1ccc([N+](=O)[O-])cc1)[C@H]([C@@H](CC=O)c1ccccc1)[N+](=O)[O-]</smiles>

4o-anti

(3S,4S)-4-nitro-5-(4-nitrophenyl)-3-phenylhex-5-enal (4o-anti): ${ }^{1} \mathrm{H}$ NMR $(600 \mathrm{MHz}$, $\left.\mathrm{CDCl}_{3}\right) \delta(\mathrm{ppm}) 2.70-2.74(\mathrm{~m}, 1 \mathrm{H}), 2.78-2.83(\mathrm{~m}, 1 \mathrm{H}), 4.20-4.24(\mathrm{~m}, 1 \mathrm{H}), 5.77(\mathrm{~d}, J=$ $11.4 \mathrm{~Hz}, 1 \mathrm{H}), 5.91(\mathrm{~s}, 1 \mathrm{H}), 6.14(\mathrm{~s}, 1 \mathrm{H}), 7.25-7.28(\mathrm{~m}, 2 \mathrm{H}), 7.30-7.34$ (m, 3H), 7.64-7.66 $(\mathrm{m}, 2 \mathrm{H}), 8.26-8.28(\mathrm{~m}, 2 \mathrm{H}), 9.51(\mathrm{t}, J=1.2 \mathrm{~Hz}, 1 \mathrm{H}) ;{ }^{13} \mathrm{C} \mathrm{NMR}\left(150 \mathrm{MHz}, \mathrm{CDCl}_{3}\right) \delta$ (ppm) 43.6, 45.6, 92.8, 123.2, 124.2, 127.6, 127.9, 128.3, 129.1, 137.5, 140.5, 145.4, 147.9, 198.1. Enantiomeric excess was determined by HPLC with a Chiralcel OD-H column; $\lambda=254 \mathrm{~nm}$; eluent: Hexane/Isopropanol = 85/15; Flow rate: $1.0 \mathrm{~mL} / \mathrm{min}$; $\mathrm{t}_{\text {minor }}=$ $5.168 \mathrm{~min}, \mathrm{t}_{\text {major }}=5.860 \mathrm{~min} ; \mathrm{ee} \%=81 \%$.

4p was purified by flash chromatography (Hexane-EtOAc, v/v 15/1) as white oil with overall isolated yield: $75 \%$ for both isomers.

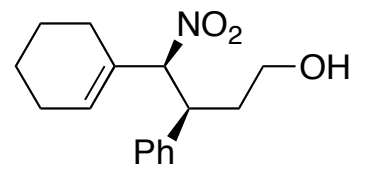

4p-syn 
(3S,4S)-4-cyclohexenyl-4-nitro-3-phenylbutan-1-ol (4p-syn): ${ }^{1} \mathrm{H}$ NMR $(600 \mathrm{MHz}$, $\left.\mathrm{CDCl}_{3}\right) \delta(\mathrm{ppm}) 1.21-1.45(\mathrm{~m}, 5 \mathrm{H}), 1.73-1.78(\mathrm{~m}, 1 \mathrm{H}), 1.85-1.95(\mathrm{~m}, 5 \mathrm{H}), 3.34-3.38(\mathrm{~m}$, $1 \mathrm{H}), 3.49-3.53(\mathrm{~m}, 1 \mathrm{H}), 3.66-3.71(\mathrm{~m}, 1 \mathrm{H}), 5.10(\mathrm{~d}, J=11.4 \mathrm{~Hz}, 1 \mathrm{H}), 6.75-6.77(\mathrm{~m}, 1 \mathrm{H})$, 7.13-7.14 (m, 2H), 7.22-7.31 (m, 3H); ${ }^{13} \mathrm{C}$ NMR (150 MHz, $\left.\mathrm{CDCl}_{3}\right) \delta$ (ppm) 21.5, 22.1, $24.3,25.2,36.1,43.4,60.0,98.7,127.3,128.4,128.6,130.9,132.9,138.1$. HRMS Calculated for $\left[\mathrm{C}_{16} \mathrm{H}_{21} \mathrm{NO}_{3} \mathrm{Na}\right]^{+}: 298.1419$, Found: 298.1415. $[\alpha]_{\mathrm{D}}{ }^{26}=-63.7^{\circ}(\mathrm{c}=0.75$ $\mathrm{CHCl}_{3}$ ). Enantiomeric excess was determined by HPLC with a Chiralcel OD-H column; $\lambda=254 \mathrm{~nm}$; eluent: Hexane/Isopropanol $=90 / 10$; Flow rate: $1.0 \mathrm{~mL} / \mathrm{min} ; \mathrm{t}_{\text {minor }}=7.536$ $\min , \mathrm{t}_{\text {major }}=12.184 \mathrm{~min} ; \mathrm{ee} \%=70 \%$.

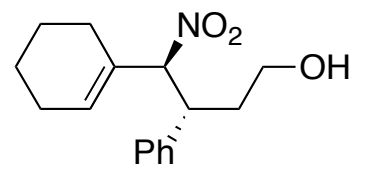

4p-anti

(3S,4R)-4-cyclohexenyl-4-nitro-3-phenylbutan-1-ol (4p-anti): ${ }^{1} \mathrm{H}$ NMR (600 MHz, $\left.\mathrm{CDCl}_{3}\right) \delta(\mathrm{ppm}) 1.23(\mathrm{br} \mathrm{s}, 1 \mathrm{H}), 1.58-1.74(\mathrm{~m}, 5 \mathrm{H}), 1.82-1.88(\mathrm{~m}, 1 \mathrm{H}), 2.12-2.25(\mathrm{~m}$, 4H), 3.32-3.36 (m, 1H), 3.47-3.51 (m, 1H), 3.63 (dt, $J=11.7,3 \mathrm{~Hz}, 1 \mathrm{H}), 5.12$ (d, $J=11.4$ $\mathrm{Hz}, 1 \mathrm{H}), 6.12-6.13(\mathrm{~m}, 1 \mathrm{H}), 7.22-7.31(\mathrm{~m}, 4 \mathrm{H}) ;{ }^{13} \mathrm{C} \mathrm{NMR}\left(150 \mathrm{MHz}, \mathrm{CDCl}_{3}\right) \delta(\mathrm{ppm})$ $21.8,22.3,23.5,25.6,34.4,42.4,59.9,99.5,127.6,128.0,128.8,131.0,133.7,138.8$. HRMS Calculated for $\left[\mathrm{C}_{16} \mathrm{H}_{21} \mathrm{NO}_{3} \mathrm{Na}\right]^{+}: 298.1419$, Found: $298.1415 .[\alpha]_{\mathrm{D}}{ }^{26}=85.4^{\circ}(\mathrm{c}=$ $0.70 \mathrm{CHCl}_{3}$ ). Enantiomeric excess was determined by HPLC with a Chiralcel OD-H column; $\lambda=254 \mathrm{~nm}$; eluent: Hexane/Isopropanol = 85/15; Flow rate: $1.0 \mathrm{~mL} / \mathrm{min}$; $\mathrm{t}_{\text {minor }}=$ $6.628 \mathrm{~min}, \mathrm{t}_{\text {major }}=10.056 \mathrm{~min} ; \mathrm{ee} \%=75 \%$.

4q was purified by flash chromatography (Hexane-EtOAc, v/v 10/1) as white oil with overall isolated yield: $79 \%$ for both isomers.

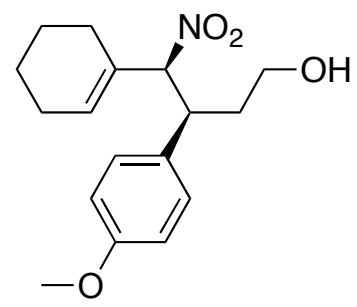

4q-syn 
(3S,4S)-4-cyclohexenyl-3-(4-methoxyphenyl)-4-nitrobutan-1-ol (4q-syn): ${ }^{1} \mathrm{H}$ NMR $\left(600 \mathrm{MHz}, \mathrm{CDCl}_{3}\right) \delta(\mathrm{ppm}) 1.14$ (br s, $\left.1 \mathrm{H}\right), 1.25-1.46(\mathrm{~m}, 4 \mathrm{H}), 1.76-1.93(\mathrm{~m}, 6 \mathrm{H}), 3.35-$ 3.39 (m, 1H), 3.49-3.53 (m, 1H), 3.63 (dt, $J=11.4,4.2 \mathrm{~Hz}, 1 \mathrm{H}), 3.79$ (s, 3H), 5.04 (d, $J=$ $12 \mathrm{~Hz}, 1 \mathrm{H}), 5.75-5.77(\mathrm{~m}, 1 \mathrm{H}), 6.82-6.84(\mathrm{~m}, 2 \mathrm{H}), 7.04-7.06(\mathrm{~m}, 2 \mathrm{H}) ;{ }^{13} \mathrm{C}$ NMR (150 $\left.\mathrm{MHz}_{\mathrm{CDCl}}\right) \delta(\mathrm{ppm})$ 21.5, 22.1, 24.3, 25.3, 36.2, 42.7, 55.2, 60.1, 98.9, 114.1, 129.4, 129.9, 131.1, 132.8, 158.7. HRMS Calculated for $\left[\mathrm{C}_{17} \mathrm{H}_{23} \mathrm{NO}_{4} \mathrm{Na}\right]^{+}$: 238.1525, Found: 238.1520. $[\alpha]_{\mathrm{D}}{ }^{26}=-72.0^{\circ}\left(\mathrm{c}=0.75 \mathrm{CHCl}_{3}\right)$. Enantiomeric excess was determined by HPLC with a Chiralcel AS-H column; $\lambda=254$ nm; eluent: Hexane/Isopropanol = 80/20; Flow rate: $1.0 \mathrm{~mL} / \mathrm{min} ; \mathrm{t}_{\text {minor }}=11.700 \mathrm{~min}, \mathrm{t}_{\text {major }}=8.452 \mathrm{~min} ; \mathrm{ee} \%=87 \%$.

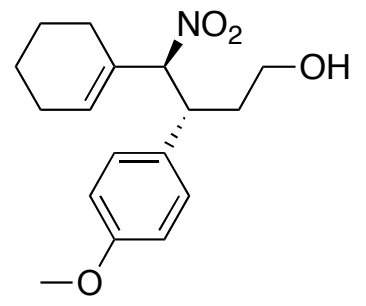

4q-anti

(3S,4R)-4-cyclohexenyl-3-(4-methoxyphenyl)-4-nitrobutan-1-ol (4q-anti): ${ }^{1} \mathrm{H}$ NMR $\left(600 \mathrm{MHz}, \mathrm{CDCl}_{3}\right) \delta(\mathrm{ppm}) 1.23($ br s, $1 \mathrm{H}), 1.56-1.72(\mathrm{~m}, 5 \mathrm{H}), 1.80-1.85(\mathrm{~m}, 1 \mathrm{H}), 2.12-$ 2.25 (m, 4H), 3.33-3.37 (m, 1H), 3.48-3.52 (m, 1H), 3.58 (dt, $J=12,3.0 \mathrm{~Hz}, 1 \mathrm{H}), 3.77$ (s, $3 \mathrm{H}), 5.06(\mathrm{~d}, J=12 \mathrm{~Hz}, 1 \mathrm{H}), 6.10-6.11(\mathrm{~m}, 1 \mathrm{H}), 6.82-6.84(\mathrm{~m}, 2 \mathrm{H}), 7.13-7.16(\mathrm{~m}, 2 \mathrm{H})$; ${ }^{13} \mathrm{C}$ NMR $\left(150 \mathrm{MHz}, \mathrm{CDCl}_{3}\right) \delta$ (ppm) 21.8, 22.3, 23.5, 25.6, 34.4, 41.7, 55.2, 60.0, 99.8, 114.3, 129.0, 130.6, 131.1, 133.6, 158.9. HRMS Calculated for $\left[\mathrm{C}_{17} \mathrm{H}_{23} \mathrm{NO}_{4} \mathrm{Na}\right]^{+}$: 238.1525, Found: $238.1520 .[\alpha]_{\mathrm{D}}{ }^{26}=61.4^{\circ}\left(\mathrm{c}=0.68 \mathrm{CHCl}_{3}\right)$. Enantiomeric excess was determined by HPLC with a Chiralcel AS-H column; $\lambda=254 \mathrm{~nm}$; eluent: Hexane/Isopropanol $=80 / 20$; Flow rate: $1.0 \mathrm{~mL} / \mathrm{min}$; $\mathrm{t}_{\text {minor }}=14.828 \mathrm{~min}, \mathrm{t}_{\text {major }}=10.184$ $\min ; \mathrm{ee} \%=87 \%$.

$4 \mathbf{z}$ was purified by flash chromatography (Hexane-EtOAc, v/v 10/1) as white oil with overall isolated yield: $90 \%$ for both isomers. 
<smiles>C=C(c1ccccc1)[C@H](CC(C)CO)[N+](=O)[O-]</smiles>

4z-syn

(2S,4R)-2-methyl-4-nitro-5-phenylhex-5-en-1-ol (4z-syn): ${ }^{1} \mathrm{H}$ NMR (270 MHz, $\left.\mathrm{CDCl}_{3}\right)$ $\delta(\mathrm{ppm}) 0.92(\mathrm{~d}, J=6.7 \mathrm{~Hz}, 3 \mathrm{H}), 1.25(\mathrm{br} \mathrm{s}, 1 \mathrm{H}), 1.67-1.77(\mathrm{~m}, 1 \mathrm{H}), 2.05-2.25(\mathrm{~m}, 2 \mathrm{H})$, 3.44-3.50 (m, 1H), 3.58-3.63 (m, 1H), $5.58(\mathrm{~s}, 1 \mathrm{H}), 5.59(\mathrm{~s}, 1 \mathrm{H}), 5.69-5.75(\mathrm{~m}, 1 \mathrm{H}), 7.33-$ $7.40(\mathrm{~m}, 5 \mathrm{H}) ;{ }^{13} \mathrm{C} \mathrm{NMR}\left(67.5 \mathrm{MHz}, \mathrm{CDCl}_{3}\right) \delta(\mathrm{ppm})$ 16.8, 32.7, 37.1, 67.4, 88.5, 117.9, 126.6, 128.4, 128.7, 138.6, 143.7. HRMS Calculated for $\left[\mathrm{C}_{13} \mathrm{H}_{17} \mathrm{NO}_{3} \mathrm{Na}\right]^{+}:$258.1106, Found: $258.1104 .[\alpha]_{\mathrm{D}}{ }^{26}=-19.4^{\circ}\left(\mathrm{c}=1.5 \mathrm{CHCl}_{3}\right)$. Enantiomeric excess was determined by HPLC with a Chiralcel OD-H column; $\lambda=254 \mathrm{~nm}$; eluent: Hexane/Isopropanol = 90/10; Flow rate: $1.0 \mathrm{~mL} / \mathrm{min} ; \mathrm{t}_{\text {minor }}=7.432 \mathrm{~min}, \mathrm{t}_{\text {major }}=13.276 \mathrm{~min} ; \mathrm{ee} \%=15 \%$.<smiles>C=C(c1ccccc1)[C@H](CC(C)CO)[N+](=O)[O-]</smiles>

4z-anti

(2S,4S)-2-methyl-4-nitro-5-phenylhex-5-en-1-ol (4z-anti): ${ }^{1} \mathrm{H}$ NMR (270 MHz, $\left.\mathrm{CDCl}_{3}\right)$ $\delta(\mathrm{ppm}) 0.99(\mathrm{~d}, J=6.7 \mathrm{~Hz}, 3 \mathrm{H}), 1.25$ (br s, 1H), 1.63-1.79 (m, 1H), 2.46-2.56 (m, 2H), 3.43-3.55 (m, 2H), $5.58(\mathrm{~s}, 2 \mathrm{H}), 5.62-5.67(\mathrm{~m}, 1 \mathrm{H}), 7.34-7.40(\mathrm{~m}, 5 \mathrm{H}) ;{ }^{13} \mathrm{C} \mathrm{NMR}(67.5$ $\left.\mathrm{MHz} \mathrm{CDCl}_{3}\right) \delta(\mathrm{ppm}) 16.0,32.9,36.8,67.7,88.1,117.9,126.6,128.5,128.7,138.7$, 143.8. HRMS Calculated for $[\mathrm{C} 13 \mathrm{H} 17 \mathrm{NO} 3 \mathrm{Na}]^{+}: 258.1106$, Found: $258.1104 .[\alpha]_{\mathrm{D}}{ }^{26}=$ $18.0^{\circ}\left(\mathrm{c}=1.5 \mathrm{CHCl}_{3}\right)$. Enantiomeric excess was determined by HPLC with a Chiralcel OD-H column; $\lambda=254 \mathrm{~nm}$; eluent: Hexane/Isopropanol =90/10; Flow rate: $1.0 \mathrm{~mL} / \mathrm{min}$; $\mathrm{t}_{\text {minor }}=7.276 \mathrm{~min}, \mathrm{t}_{\text {major }}=9.880 \mathrm{~min} ; \mathrm{ee} \%=19 \%$.<smiles>O=C(c1ccccc1)[C@@H]1[C@H](c2ccccc2)CCN1C(=O)O</smiles>

N-Boc protected phenyl $((2 R, 3 S)-3$-phenylpyrrolidin-2-yl)methanone, 6a was purified by flash chromatography (Hexane-EtOAc, v/v 10/1) as white solid with overall isolated 
yield: $76 \%$. 2 sets of signals ratio (1:1.6), HRMS Calculated for $\left[\mathrm{C}_{22} \mathrm{H}_{25} \mathrm{NO}_{3} \mathrm{Na}\right]^{+}$: 374.1732, Found: 374.1728.

Set 1: ${ }^{1} \mathrm{H}$ NMR $\left(600 \mathrm{MHz}, \mathrm{CDCl}_{3}\right) \delta(\mathrm{ppm}) 1.47(\mathrm{~s}, 9 \mathrm{H}), 2.10-2.15(\mathrm{~m}, 1 \mathrm{H}), 2.63-2.75$ (m, 1H), 3.52-3.58 (m, 1H), 3.73-3.82 (m, 1H), 3.93 (dt, $J=10.2,1.0 \mathrm{~Hz}, 1 \mathrm{H}), 5.64$ (d, $J$ $=8.4 \mathrm{~Hz}, 1 \mathrm{H}), 6.94-7.51(\mathrm{~m}, 10 \mathrm{H}) ;{ }^{13} \mathrm{C} \mathrm{NMR}\left(150 \mathrm{MHz}, \mathrm{CDCl}_{3}\right) \delta(\mathrm{ppm}) 28.2,28.5$, 46.0, 49.0, 64.0, 80.0, 127.2, 127.8, 128.0, 128.1, 128.3, 132.2, 136.1, 137.4, 153.6, 200.6

Set 2: ${ }^{1} \mathrm{H}$ NMR (600 MHz, $\left.\mathrm{CDCl}_{3}\right) \delta(\mathrm{ppm}) 1.24(\mathrm{~s}, 9 \mathrm{H}), 2.10-2.15(\mathrm{~m}, 1 \mathrm{H}), 2.63-2.75$ (m, 1H), 3.52-3.58 (m, 1H), 3.73-3.82 (m, 1H), 4.03 (dt, $J=10.2,1.2 \mathrm{~Hz}, 1 \mathrm{H}), 5.55(\mathrm{~d}, J$ $=8.4 \mathrm{~Hz}, 1 \mathrm{H}), 6.94-7.51(\mathrm{~m}, 10 \mathrm{H}) ;{ }^{13} \mathrm{C} \mathrm{NMR}\left(150 \mathrm{MHz}, \mathrm{CDCl}_{3}\right) \delta(\mathrm{ppm}) 27.6,28.4$, 46.2, 48.0, 64.0, 79.8, 127.1, 127.7, 127.8, 128.1, 128.3, 132.1, 136.2, 137.6, 154.4, 201.1 .<smiles>O=C1OC(c2ccccc2)(c2ccccc2)C2CCN3CCC123</smiles>

$7 \mathbf{a}$

(7R,7aS)-tetrahydro-1,1,7-triphenylpyrrolo[1,2-c]oxazol-3(1H)-one, 7a was purified by flash chromatography (Hexane-EtOAc, v/v 8/1) as white oil with overall isolated yield: $87 \%$. Only trans isomer.

${ }^{1} \mathrm{H}$ NMR $\left(600 \mathrm{MHz}, \mathrm{CDCl}_{3}\right) \delta(\mathrm{ppm})$ 2.14-2.18 (m, 1H), 2.46-2.53 (m, 1H), $3.29(\mathrm{t}, J=$ $6.6 \mathrm{~Hz}, 1 \mathrm{H}), 3.46-3.50(\mathrm{~m}, 1 \mathrm{H}), 4.06-4.11(\mathrm{~m}, 1 \mathrm{H}), 4.88-4.90(\mathrm{~m}, 1 \mathrm{H}), 6.43(\mathrm{~d}, J=7.8$ $\mathrm{Hz}, 2 \mathrm{H}), 6.86-7.05(\mathrm{~m}, 8 \mathrm{H}), 7.22-7.31(\mathrm{~m}, 3 \mathrm{H}), 7.50-7.52(\mathrm{~m}, 2 \mathrm{H}) ;{ }^{13} \mathrm{C} \mathrm{NMR}(150 \mathrm{MHz}$, $\left.\mathrm{CDCl}_{3}\right) \delta(\mathrm{ppm}) 34.5,44.8,46.2,72.4,84.8,124.5,125.2,125.7,126.7,127.6,127.9$, 128.1，128.5, 139.5, 140.1, 144.9, 159.8. HRMS Calculated for $\left[\mathrm{C}_{24} \mathrm{H}_{21} \mathrm{NO}_{2} \mathrm{Na}\right]^{+}$: 378.1470, Found: 378.1466. Enantiomeric excess was determined by HPLC with a Chiralcel OD-H column; $\lambda=254 \mathrm{~nm}$; eluent: Hexane/Isopropanol =90/10; Flow rate: 1.0 $\mathrm{mL} / \mathrm{min} ; \mathrm{t}_{\text {minor }}=10.124 \mathrm{~min}, \mathrm{t}_{\text {major }}=6.196 \mathrm{~min} ; \mathrm{ee} \%=84 \%$. 
<smiles>O=C(c1ccccc1)[C@@H]1[C@H](c2ccccc2)CCN1C(=O)c1ccccc1</smiles>

6b

N-acetate phenyl((2R,3S)-3-phenylpyrrolidin-2-yl)methanone, 6b was purified by flash chromatography (Hexane-EtOAc, v/v 5/1) as white oil with overall isolated yield: $72 \%$. Only trans isomer. 2 sets of signals ratio (1:4), HRMS Calculated for $\left[\mathrm{C}_{19} \mathrm{H}_{19} \mathrm{NO}_{2} \mathrm{Na}\right]^{+}:$316.1313, Found: 316.1309.

Set 1: ${ }^{1} \mathrm{H}$ NMR $\left(600 \mathrm{MHz}, \mathrm{CDCl}_{3}\right) \delta(\mathrm{ppm}) 2.18(\mathrm{~s}, 3 \mathrm{H}), 2.11-2.16(\mathrm{~m}, 1 \mathrm{H}), 2.46-2.51$ $(\mathrm{m}, 1 \mathrm{H}), 3.43(\mathrm{dt}, J=6.0,6.0 \mathrm{~Hz}, 1 \mathrm{H}), 3.75-3.79(\mathrm{~m}, 1 \mathrm{H}), 3.85-3.89(\mathrm{~m}, 1 \mathrm{H}), 5.61(\mathrm{~d}, J=$ $4.8 \mathrm{~Hz}, 1 \mathrm{H}), 7.20-7.79(\mathrm{~m}, 10 \mathrm{H}) ;{ }^{13} \mathrm{C} \mathrm{NMR}\left(150 \mathrm{MHz}, \mathrm{CDCl}_{3}\right) \delta$ (ppm) 22.2, 33.4, 47.3, $47.8,67.0,126.7,126.9,127.4,128.4,128.5,129.0,129.2,133.2,135.3,141.4,169.0$, 197.6.

Set 2: ${ }^{1} \mathrm{H}$ NMR $\left(600 \mathrm{MHz}, \mathrm{CDCl}_{3}\right) \delta(\mathrm{ppm}) 1.91(\mathrm{~s}, 3 \mathrm{H}), 2.11-2.16(\mathrm{~m}, 1 \mathrm{H}), 2.28-2.36$ $(\mathrm{m}, 1 \mathrm{H}), 3.52(\mathrm{dt}, J=4.8,4.8 \mathrm{~Hz}, 1 \mathrm{H}), 3.85-3.89(\mathrm{~m}, 1 \mathrm{H}), 3.90-3.94(\mathrm{~m}, 1 \mathrm{H}), 5.37$ (d, $J=$ 3.6 Hz, 1H), 7.26-7.83 (m, 10H); ${ }^{13} \mathrm{C}$ NMR (150 MHz, CDCl3) $\delta$ (ppm) 22.5, 36.4, 48.3, $47.1,66.0,126.7,126.8,127.3,128.2,128.4,129.1,129.3,133.2,135.4,141.8,169.0$, 197.9.

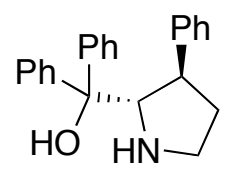

$7 \mathbf{b}$

diphenyl((2S,3R)-3-phenylpyrrolidin-2-yl)methanol, $7 \mathbf{b}$ was purified by flash chromatography (Hexane-EtOAc, v/v 10/1) as white oil with overall isolated yield: 81\%.

${ }^{1} \mathrm{H}$ NMR (600 MHz, $\left.\mathrm{CDCl}_{3}\right) \delta(\mathrm{ppm}) 1.22$ (br s, $\left.1 \mathrm{H}\right), 1.80-1.85(\mathrm{~m}, 1 \mathrm{H}), 2.22-2.29(\mathrm{~m}$, 1H), 3.16-3.20 (m, 2H), 3.30-3.34 (m, 1H), 4.12-4.13 (m, 1H), 6.68-6.70 (m, 2H), 7.00$7.07(\mathrm{~m}, 5 \mathrm{H}), 7.15-7.17(\mathrm{~m}, 1 \mathrm{H}), 7.25-7.29(\mathrm{~m}, 5 \mathrm{H}), 7.57-7.58(\mathrm{~m}, 2 \mathrm{H}) ;{ }^{13} \mathrm{C}$ NMR $(150$ 
$\mathrm{MHz}, \mathrm{CDCl} 3) \delta(\mathrm{ppm}) 36.4,44.8,47.0,72.4,77.8,125.4,125.8,125.9,126.2,126.5$, 127.4, 127.6, 128.0, 128.3, 143.9, 146.8, 148.1. HRMS Calculated for $\left[\mathrm{C}_{23} \mathrm{H}_{23} \mathrm{NONa}\right]^{+}$: 352.1677, Found: 352.1673 . 


\section{Pat II}<smiles>C=C(c1ccccc1)C(CCC(C)=O)[N+](=O)[O-]</smiles>

5-nitro-6-phenylhept-6-en-2-one (8a) was purified by flash silica gel chromatography (Hexane-EtOAc, v/v, 5/1) as colorless oil, yield: 87\%. ${ }^{1} \mathrm{H}$ NMR $\left(270 \mathrm{MHz}, \mathrm{CDCl}_{3}\right): \delta$ $7.33-7.38(\mathrm{~m}, 5 \mathrm{H}), 5.59(\mathrm{~s}, 1 \mathrm{H}), 5.48-5.57(\mathrm{~m}, 2 \mathrm{H}), 2.21-2.58(\mathrm{~m}, 4 \mathrm{H}), 2.11(\mathrm{~s}, 3 \mathrm{H}) .{ }^{13} \mathrm{C}$ NMR (67.5 MHz, $\left.\mathrm{CDCl}_{3}\right): \delta 206.6,143.4,138.4,128.8,128.7,126.7,118.1,88.9,39.2$, 30.1, 26.7. HRMS Calculated for $\mathrm{C}_{13} \mathrm{H}_{15} \mathrm{NO}_{3} \mathrm{Na}[\mathrm{M}+\mathrm{Na}]^{+}$: 256.09441, Found: 256.09446.<smiles>C=C(c1ccccc1)C(CCC(=O)OC)[N+](=O)[O-]</smiles>

$8 b$

Methyl 4-nitro-5-phenylhex-5-enoate (8b) was purified by flash silica gel chromatography (Hexane-EtOAc, v/v, 5/1) as colorless oil, yield: 82\%. ${ }^{1} \mathrm{H}$ NMR (270 $\left.\mathrm{MHz}_{\mathrm{CDCl}}\right)$ : 7.30-7.35(m, 5H), 5.50-5.67(m, 3H), 3.65(s, 3H), 2.01-2.62(m, 4H). ${ }^{13} \mathrm{C}$ NMR (67.5 MHz, $\left.\mathrm{CDCl}_{3}\right): \delta 172.4,143.3,138.3,128.8,128.7,126.7,118.3,88.9,52.0$, 30.1, 27.9. HRMS Calculated for $\mathrm{C}_{13} \mathrm{H}_{15} \mathrm{NO}_{4} \mathrm{Na}[\mathrm{M}+\mathrm{Na}]^{+}$: 272.08933, Found: 272.08918 .<smiles>C=C(c1ccccc1)C(CCC(=O)CC)[N+](=O)[O-]</smiles>

6-nitro-7-phenyloct-7-en-3-one (8c) was purified by flash silica gel chromatography (Hexane-EtOAc, v/v, 5/1) as colorless oil, yield: 86\% (combined all isomers). ${ }^{1} \mathrm{H}$ NMR $\left(600 \mathrm{MHz} \mathrm{CDCl}_{3}\right): \delta 7.27-7.39(\mathrm{~m}, 5 \mathrm{H}), 5.59(\mathrm{~s}, 1 \mathrm{H}), 5.51-5.59(\mathrm{~m}, 2 \mathrm{H}), 2.41-2.51(\mathrm{~m}$, $3 \mathrm{H}), 2.27-2.40(\mathrm{~m}, 3 \mathrm{H}), 1.02(\mathrm{t}, J=7.4 \mathrm{~Hz}, 3 \mathrm{H}) .{ }^{13} \mathrm{C} \mathrm{NMR}\left(150 \mathrm{MHz}, \mathrm{CDCl}_{3}\right): \delta 209.2$, 
143.3, 138.3, 128.7, 128.5, 126.8, 117.8, 88.8, 37.7, 36.0, 26.6, 7.6. HRMS Calculated for $\mathrm{C}_{14} \mathrm{H}_{18} \mathrm{NO}_{3}[\mathrm{M}+\mathrm{H}]^{+}: 248.12812$, Found: 248.12815.<smiles>C=C(c1ccccc1)C(CCC(=O)c1ccccc1)[N+](=O)[O-]</smiles>

$8 d$

4-nitro-1,5-diphenylhex-5-en-1-one and isomer (8d) was purified by flash silica gel chromatography (Hexane-EtOAc, v/v, 5/1) as colorless oil, yield: 83\% (combined all isomers). ${ }^{1} \mathrm{H}$ NMR $\left(600 \mathrm{MHz}, \mathrm{CDCl}_{3}\right): \delta 7.97-7.99(\mathrm{~m}, 0.26 \mathrm{H}), 7.90-7.92(\mathrm{~m}, 2 \mathrm{H}), 7.55-$ 7.61(m, 1.13H), 7.28-7.50(m, 7.65H), 7.15-7.17(m, 0.26H), 5.67(q, $J=4.8 \mathrm{~Hz}, 1 \mathrm{H})$, 5.63(s, 1H), 5.61(s, $1 \mathrm{H}), 3.28-3.31(\mathrm{~m}, 0.26 \mathrm{H}), 3.03-3.15(\mathrm{~m}, 2.26 \mathrm{H}), 2.62-2.70(\mathrm{~m}, 1 \mathrm{H})$, 2.48-2.54(m, 1H), 2.20(s, 0.39H). ${ }^{13} \mathrm{C}$ NMR (150 MHz, $\left.\mathrm{CDCl}_{3}\right): \delta 197.9,143.5,138.3$, 136.3, 133.4, 128.72, 128.66, 128.5, 127.9, 126.6, 118.1, 89.2, (35.9), 34.4, (31.6), 27.1, (24.6). HRMS Calculated for $\mathrm{C}_{18} \mathrm{H}_{18} \mathrm{NO}_{3}[\mathrm{M}+\mathrm{H}]^{+}: 294.12812$, Found: 294.12813.

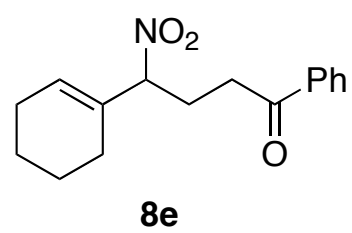

4-cyclohexenyl-4-nitro-1-phenylbutan-1-one (8e) was purified by flash silica gel chromatography (Hexane-EtOAc, v/v, 10/1) as white solid, yield: $85 \% .{ }^{1} \mathrm{H}$ NMR (270 $\left.\mathrm{MHz}, \mathrm{CDCl}_{3}\right): \delta 7.90-8.01(\mathrm{~m}, 2 \mathrm{H}), 7.51-7.63(\mathrm{~m}, 1 \mathrm{H}), 7.41-7.50(\mathrm{~m}, 2 \mathrm{H}), 5.96(\mathrm{~s}, 1 \mathrm{H})$, $5.00(\mathrm{dd}, J=9.0 \mathrm{~Hz}, J=6.0 \mathrm{~Hz}, 1 \mathrm{H}), 2.90-3.11(\mathrm{~m}, 2 \mathrm{H}), 2.50-2.62(\mathrm{~m}, 1 \mathrm{H}), 2.26-2.42(\mathrm{~m}$, $1 \mathrm{H}), 1.93-2.18(\mathrm{~m}, 4 \mathrm{H}), 1.46-1.76(\mathrm{~m}, 4 \mathrm{H}) .{ }^{13} \mathrm{C} \mathrm{NMR}\left(67.5 \mathrm{MHz}, \mathrm{CDCl}_{3}\right): \delta 198.0,136.4$, 131.9, 131.1, 128.7, 127.9, 92.8, 34.4, 25.3, 25.0, 24.1, 22.2, 21.7. HRMS Calculated for $\mathrm{C}_{16} \mathrm{H}_{19} \mathrm{NO}_{3} \mathrm{Na}[\mathrm{M}+\mathrm{Na}]^{+}: 296.12626$, Found: 296.12555. 


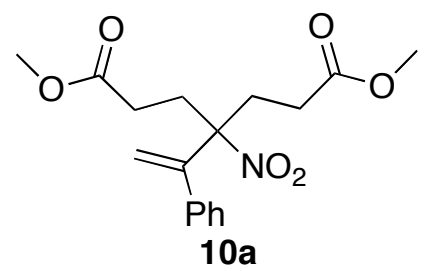

Dimethyl 4-nitro-4-(1-phenylvinyl)heptanedioate (10a) was purified by flash silica gel chromatography (Hexane-EtOAc, v/v, 5/1) as white solid, m. p: 85.5-86.6 ${ }^{\circ} \mathrm{C}$, yield: $89 \%$. ${ }^{1} \mathrm{H}$ NMR $\left(600 \mathrm{MHz}, \mathrm{CDCl}_{3}\right): \delta 7.26-7.30(\mathrm{~m}, 3 \mathrm{H}), 7.05-7.07(\mathrm{~m}, 2 \mathrm{H}), 5.59(\mathrm{~s}, 1 \mathrm{H}), 5.45(\mathrm{~s}$, $1 \mathrm{H}), 3.65(\mathrm{~s}, 6 \mathrm{H}), 2.51-2.57(\mathrm{~m}, 2 \mathrm{H}), 2.31-2.40(\mathrm{~m}, 4 \mathrm{H}), 2.18-2.26(\mathrm{~m}, 2 \mathrm{H}) .{ }^{13} \mathrm{C}$ NMR $(150$ $\left.\mathrm{MHz}, \mathrm{CDCl}_{3}\right): \delta 172.1,146.1,138.2,128.3,128.2,128.1,120.8,95.3,51.8,28.8,28.6$. HRMS Calculated for $\mathrm{C}_{17} \mathrm{H}_{21} \mathrm{NO}_{6} \mathrm{Na}[\mathrm{M}+\mathrm{Na}]^{+}: 358.12411$, Found: 358.12422 .

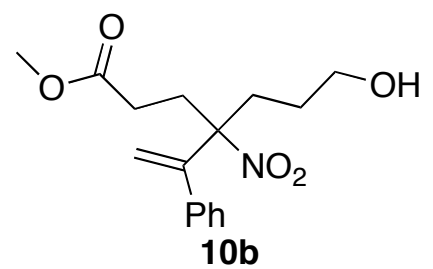

Methyl 7-hydroxy-4-nitro-4-(1-phenylvinyl)heptanoate (10b) was purified by flash silica gel chromatography (Hexane-EtOAc, v/v, 2/1) as colorless oil, yield: 90\%. ${ }^{1} \mathrm{H}$ NMR (600 MHz, $\left.\mathrm{CDCl}_{3}\right)$ : 7.28-7.50(m, 3H), 7.07-7.09(m, 2H), 5.63(s, 1H), 5.45(s, 1H), 3.68(s, 3H), 3.60-3.69(m, 2H), 2.57-2.63(m, 1H), 2.21-2.47(m, 4H), 2.04-2.09(m, 1H), 1.51-1.64(m, 2H), 1.35-1.43(m, 1H). ${ }^{13} \mathrm{C}$ NMR (150 MHz, $\left.\mathrm{CDCl}_{3}\right): \delta 172.6,146.7,138.7$, 128.4, 128.22, 128.15, 120.7, 95.9, 62.0, 52.0, 30.2, 29.0, 28.4, 27.0. HRMS Calculated for $\mathrm{C}_{16} \mathrm{H}_{21} \mathrm{NO}_{5} \mathrm{Na}[\mathrm{M}+\mathrm{Na}]^{+}$: 330.13119, Found: 330.13128 .

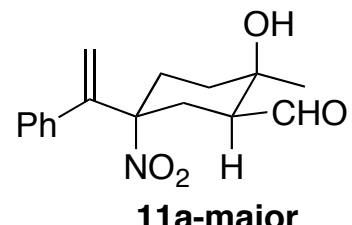


(1S,2R,5R)-2-hydroxy-2-methyl-5-nitro-5-(1-phenylvinyl)cyclohexanecarbaldehyde (11a-major) was purified by flash silica gel chromatography (Hexane-EtOAc, v/v, 5/1) then was recrystallized from $\mathrm{CH}_{2} \mathrm{Cl}_{2} / \mathrm{Hexane}$ (1:5) to get a colorless crystal, m.p: 103.1104.3 ${ }^{\circ} \mathrm{C} .{ }^{1} \mathrm{H}$ NMR $\left(600 \mathrm{MHz}, \mathrm{CDCl}_{3}\right): \delta$ 9.80(s, $\left.1 \mathrm{H}\right), 7.29-7.35(\mathrm{~m}, 3 \mathrm{H})$, 7.07-7.10(m, $2 \mathrm{H}), 5.66(\mathrm{~s}, 1 \mathrm{H}), 5.30(\mathrm{~s}, 1 \mathrm{H}), 2.75(\mathrm{dt}, J=14.4 \mathrm{~Hz}, J=3.6 \mathrm{~Hz}, 1 \mathrm{H}), 2.52-2.60(\mathrm{~m}, 2 \mathrm{H})$, $2.30(\mathrm{~s}, 1 \mathrm{H}), 2.21(\mathrm{td}, J=14.4 \mathrm{~Hz}, J=4.2 \mathrm{~Hz}, 1 \mathrm{H}), 2.06(\mathrm{dd}, J=14.4 \mathrm{~Hz}, J=13.2 \mathrm{~Hz}$, $1 \mathrm{H}), 1.69(\mathrm{dt}, J=15.0 \mathrm{~Hz}, J=3.6 \mathrm{~Hz}, 1 \mathrm{H}), 1.37-1.44(\mathrm{~m}, 1 \mathrm{H}), 1.36(\mathrm{~s}, 3 \mathrm{H}) .{ }^{13} \mathrm{C} \mathrm{NMR}(150$ $\left.\mathrm{MHz}, \mathrm{CDCl}_{3}\right): \delta 203.8,148.7,138.4,128.6,128.2,128.1,118.8,92.8,68.6,53.3,35.9$, 29.6, 29.0, 28.5. HRMS Calculated for $\mathrm{C}_{16} \mathrm{H}_{20} \mathrm{NO}_{4}[\mathrm{M}+\mathrm{H}]^{+}$: 290.13869, Found: 290.13879 .

11a-major and 11a-minor are unstable. At the same time, 11a-minor is hard to be separated to obtain pure product, so in situ reduction the mixture of 11a by treatment with $\mathrm{NaBH}_{4}$ give stable 11c.

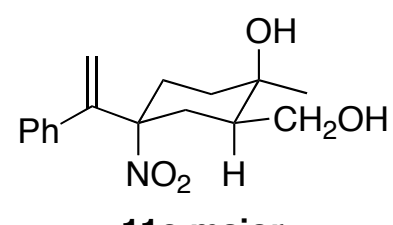

11c-major

(1R,2R,4R)-2-(hydroxymethyl)-1-methyl-4-nitro-4-(1-phenylvinyl)cyclohexanol

(11c-major) was purified by flash silica gel chromatography (Hexane-EtOAc, v/v, 3/11/1) as colorless oil, yield: $92 \%$ (combined two isomers). ${ }^{1} \mathrm{H}$ NMR (600 MHz, $\left.\mathrm{CDCl}_{3}\right): \delta$ 7.29-7.32(m, $3 \mathrm{H}), 7.08-7.11(\mathrm{~m}, 2 \mathrm{H}), 5.66(\mathrm{~s}, 1 \mathrm{H}), 5.26(\mathrm{~s}, 1 \mathrm{H}), 4.21(\mathrm{dd}, J=12.0 \mathrm{~Hz}, J=$ $3.0 \mathrm{~Hz}, 1 \mathrm{H}), 3.61(\mathrm{dd}, J=10.8 \mathrm{~Hz}, J=1.8 \mathrm{~Hz}, 1 \mathrm{H}), 2.39-2.58(\mathrm{~m}, 5 \mathrm{H}), 2.20(\mathrm{dt}, J=13.8$ $\mathrm{Hz}, J=3.6 \mathrm{~Hz}, 1 \mathrm{H}), 1.61-1.65(\mathrm{~m}, 1 \mathrm{H}), 1.40-1.47(\mathrm{~m}, 2 \mathrm{H}), 1.31(\mathrm{~s}, 3 \mathrm{H}) .{ }^{13} \mathrm{C}$ NMR $(150$ $\left.\mathrm{MHz}, \mathrm{CDCl}_{3}\right): \delta 149.4,138.6,128.7,128.0,127.8,118.3,94.6,70.9,64.1,41.5,36.6$, 32.9, 29.3, 28.3. HRMS Calculated for $\mathrm{C}_{16} \mathrm{H}_{22} \mathrm{NO}_{4}[\mathrm{M}+\mathrm{H}]^{+}:$292.15434, Found: 292.15444. 


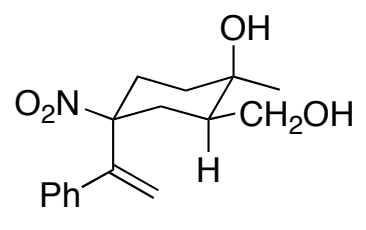

11c-minor

(1R,2R,4S)-2-(hydroxymethyl)-1-methyl-4-nitro-4-(1-phenylvinyl)cyclohexanol (11cminor) was purified by flash silica gel chromatography (Hexane-EtOAc, v/v, 3/1-1/1) as colorless oil, ${ }^{1} \mathrm{H}$ NMR $\left(600 \mathrm{MHz}, \mathrm{CDCl}_{3}\right): \delta$ 7.28-7.32(m, 3H), 7.08-7.11(m, 2H), 5.84(s, $1 \mathrm{H}), 5.63(\mathrm{~s}, 1 \mathrm{H}), 4.11(\mathrm{dd}, J=11.4 \mathrm{~Hz}, J=3.6 \mathrm{~Hz}, 1 \mathrm{H}), 3.48(\mathrm{dd}, J=10.8 \mathrm{~Hz}, J=2.4 \mathrm{~Hz}$, $1 \mathrm{H}), 2.69(\mathrm{t}, J=13.2 \mathrm{~Hz}, 1 \mathrm{H}), 2.38-2.50(\mathrm{~m}, 2 \mathrm{H}), 2.33(\mathrm{dt}, J=7.8 \mathrm{~Hz}, J=3.0 \mathrm{~Hz}, 1 \mathrm{H})$, $2.01(\mathrm{br}, 2 \mathrm{H}), 1.61(\mathrm{dt}, J=6.6 \mathrm{~Hz}, J=3.6 \mathrm{~Hz}, 1 \mathrm{H}), 1.40-1.50(\mathrm{~m}, 2 \mathrm{H}), 1.32(\mathrm{~s}, 3 \mathrm{H}) .{ }^{13} \mathrm{C}$ NMR (150 MHz, $\left.\mathrm{CDCl}_{3}\right): \delta 144.8,139.2,128.3,128.1,128.0,123.5,92.4,70.9,64.1$, 42.5, 37.1, 32.4, 29.0, 28.3. HRMS Calculated for $\mathrm{C}_{16} \mathrm{H}_{21} \mathrm{NO}_{4} \mathrm{Na}[\mathrm{M}+\mathrm{Na}]^{+}:$314.13628, Found: 314.13635.

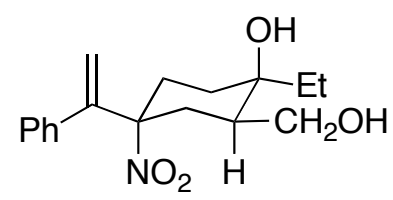

11d-major

(1R,2R,4R)-1-ethyl-2-(hydroxymethyl)-4-nitro-4-(1-phenylvinyl)cyclohexanol (11dmajor) was purified by flash silica gel chromatography (Hexane-EtOAc, v/v, 3/1-1/1) as colorless oil, yield: $85 \%$ (combined two isomers). ${ }^{1} \mathrm{H}$ NMR $\left(600 \mathrm{MHz}, \mathrm{CDCl}_{3}\right): \delta 7.28$ 7.33(m, 3H), 7.09-7.12(m, 2H), 5.66(s, 1H), 5.26(s, 1H), 4.19(dd, $J=10.8 \mathrm{~Hz} ; J=2.4$ $\mathrm{Hz}, 1 \mathrm{H}), 3.59(\mathrm{dd}, J=10.8 \mathrm{~Hz}, J=2.4 \mathrm{~Hz}, 1 \mathrm{H}), 2.47-2.60(\mathrm{~m}, 1 \mathrm{H}), 2.43-2.46(\mathrm{~m}, 2 \mathrm{H})$, $2.20(\mathrm{dt}, J=13.8 \mathrm{~Hz}, J=4.2 \mathrm{~Hz}, 1 \mathrm{H}), 2.12$ (br, 2H), 1.68-1.75(m, 1H), 1.57-1.64(m, 2H), $1.50-1.55(\mathrm{~m}, 1 \mathrm{H}), 1.40(\mathrm{dt}, J=13.8 \mathrm{~Hz}, J=4.2 \mathrm{~Hz}, 1 \mathrm{H}), 0.86(\mathrm{t}, J=7.8 \mathrm{~Hz}, 3 \mathrm{H}) .{ }^{13} \mathrm{C}$ NMR (150 MHz, $\left.\mathrm{CDCl}_{3}\right): \delta 149.5,138.9,128.7,128.0,127.8,118.3,94.5,73.1,63.9$, 39.3, 33.2, 33.0, 32.1, 29.1, 7.9. HRMS Calculated for $\mathrm{C}_{17} \mathrm{H}_{23} \mathrm{NO}_{4} \mathrm{Na}[\mathrm{M}+\mathrm{Na}]^{+}$: 328.15193, Found: 328.15215. 


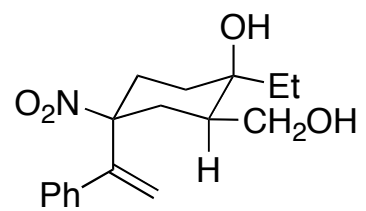

11d-minor

(1R,2R,4S)-2-(hydroxymethyl)-4-nitro-1-phenyl-4-(1-phenylvinyl)cyclohexanol (11dminor) was purified by flash silica gel chromatography (Hexane-EtOAc, v/v, 3/1-1/1) as colorless oil, hard to get purified product, NMR shows some major isomer included. ${ }^{1} \mathrm{H}$ NMR $\left(600 \mathrm{MHz}, \mathrm{CDCl}_{3}\right): \delta$ 7.30-7.32(m, 3H), 7.09-7.12(m, 2H), 5.85(s, 1H), 5.63(s, $1 \mathrm{H}), 4.07(\mathrm{dd}, J=10.8 \mathrm{~Hz}, J=3.0 \mathrm{~Hz}, 1 \mathrm{H}), 3.43(\mathrm{dd}, J=11.4 \mathrm{~Hz}, J=3.0 \mathrm{~Hz}, 1 \mathrm{H}), 2.74(\mathrm{t}$, $J=13.2 \mathrm{~Hz}, 1 \mathrm{H}), 2.32-2.50(\mathrm{~m}, 3 \mathrm{H}), 1.37-1.75(\mathrm{~m}, 7 \mathrm{H}), 0.88(\mathrm{t}, J=7.2 \mathrm{~Hz}, 3 \mathrm{H}) .{ }^{13} \mathrm{C} \mathrm{NMR}$ $\left(150 \mathrm{MHz}, \mathrm{CDCl}_{3}\right): \delta$ 145.0, $(139.3,128.7,128.3,128.1,128.03,127.99,127.8,123.4$, 118.3), 92.2, 73.1, (63.93), 63.89, 40.1, (39.4), (33.2), (33.0), 32.7, 32.4, (32.2), (29.2), 28.9, 8.0. HRMS Calculated for $\mathrm{C}_{21} \mathrm{H}_{23} \mathrm{NO}_{4} \mathrm{Na}[\mathrm{M}+\mathrm{Na}]^{+}$: 376.15193, Found: 376.15185.<smiles>C=C(c1ccccc1)C1([N+](=O)[O-])CCC(O)(c2ccccc2)C(CO)C1</smiles>

11e-major

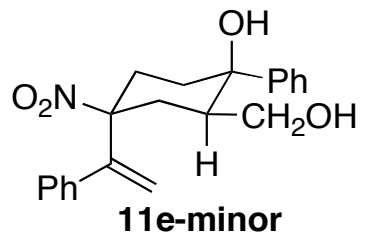

11e-minor

2-(hydroxymethyl)-4-nitro-1-phenyl-4-(1-phenylvinyl)cyclohexanol) (11e) was purified by flash silica gel chromatography (Hexane-EtOAc, v/v, 3/1-1/1) as colorless oil, yield: $84 \%$ (combined two isomers). Two disastereomers are hard to separated, NMR give d.r. $=3: 1$ isomers. ${ }^{1} \mathrm{H}$ NMR $\left(600 \mathrm{MHz}, \mathrm{CDCl}_{3}\right): \delta 7.12-7.38(\mathrm{~m}, 13 \mathrm{H}), 5.95(\mathrm{~s}$, $0.33 \mathrm{H}), 5.74(\mathrm{~s}, 0.33 \mathrm{H}), 5.70(\mathrm{~s}, 1 \mathrm{H}), 5.30(\mathrm{~s}, 1 \mathrm{H}), 3.57(\mathrm{dd}, J=10.2 \mathrm{~Hz}, J=3.0 \mathrm{~Hz}, 1 \mathrm{H})$, $3.45-3.48(\mathrm{~m}, 1.33 \mathrm{H}), 3.26(\mathrm{dd}, J=13.2 \mathrm{~Hz}, J=3.0 \mathrm{~Hz}, 0.33 \mathrm{H}), 2.87(\mathrm{t}, J=13.2 \mathrm{~Hz}$, $0.33 \mathrm{H}), 2.34-2.65(\mathrm{~m}, 5.32 \mathrm{H}), 1.66-2.00(\mathrm{~m}, 3.99 \mathrm{H}) .{ }^{13} \mathrm{C} \mathrm{NMR}\left(150 \mathrm{MHz}, \mathrm{CDCl}_{3}\right): \delta$ $149.5,146.6,146.4,145.1,139.3,138.9,128.7,128.44,128.42,128.1,127.9,127.0$, 126.9, 124.6, 124.5, 123.7, 118.4, 94.5, (92.2), (75.7), 75.6, 64.4, (42.6), 41.7, (37.5), 36.9, 32.8, (32.3), 29.6, (29.4). HRMS Calculated for $\mathrm{C}_{21} \mathrm{H}_{23} \mathrm{NO}_{4} \mathrm{Na}[\mathrm{M}+\mathrm{Na}]^{+}$: 376.15193, Found: 376.15212. 


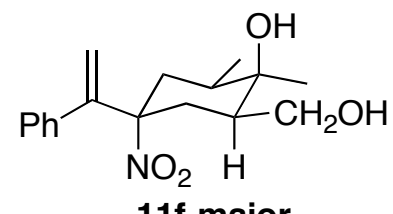

11f-major

(1R,2R,4R,6S)-2-(hydroxymethyl)-1,6-dimethyl-4-nitro-4-(1-phenylvinyl)cyclo-

hexanol (11f-major) was purified by flash silica gel chromatography (Hexane-EtOAc, $\mathrm{v} / \mathrm{v}, 5 / 1-2 / 1)$ as colorless oil, yield: 78\% (combined two isomers). ${ }^{1} \mathrm{H}$ NMR (600 MHz, $\left.\mathrm{CDCl}_{3}\right): \delta 7.28-7.32(\mathrm{~m}, 3 \mathrm{H}), 7.07-7.10(\mathrm{~m}, 2 \mathrm{H}), 5.65(\mathrm{~s}, 1 \mathrm{H}), 5.24(\mathrm{~s}, 1 \mathrm{H}), 4.24(\mathrm{dd}, J=$ $11.4 \mathrm{~Hz}, J=3.0 \mathrm{~Hz}, 1 \mathrm{H}), 3.61(J=11.4 \mathrm{~Hz}, J=3.0 \mathrm{~Hz}, 1 \mathrm{H}), 2.59(\mathrm{~s}, 2 \mathrm{H}), 2.50(\mathrm{dt}, J=$ $14.4 \mathrm{~Hz}, J=3.0 \mathrm{~Hz}, 1 \mathrm{H}), 2.40-2.46(\mathrm{~m}, 2 \mathrm{H}), 1.96(\mathrm{dd}, J=12.0 \mathrm{~Hz}, J=14.4 \mathrm{~Hz}, 1 \mathrm{H}), 1.41-$ 1.51(m, 2H), 1.29(s, 3H), 0.95(d, $J=6.6 \mathrm{~Hz}, 3 \mathrm{H}) .{ }^{13} \mathrm{C} \mathrm{NMR}\left(150 \mathrm{MHz}, \mathrm{CDCl}_{3}\right): \delta 149.4$, $138.8,128.7,128.0,127.8,118.3,94.4,72.8,64.3,42.4,37.6,37.4,33.0,24.4,14.3$. HRMS Calculated for $\mathrm{C}_{17} \mathrm{H}_{23} \mathrm{NO}_{4} \mathrm{Na}[\mathrm{M}+\mathrm{Na}]^{+}$: 328.15193 , Found: 328.15201 .

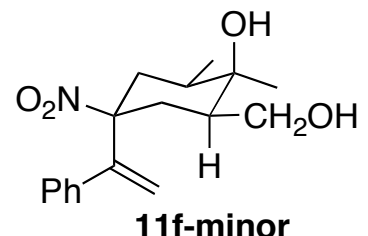

(1R,2R,4S,6S)-2-(hydroxymethyl)-1,6-dimethyl-4-nitro-4-(1-phenylvinyl)cyclohexanol (11f-minor) was purified by flash silica gel chromatography (Hexane-EtOAc, v/v, 5/1-2/1) as colorless oil. ${ }^{1} \mathrm{H}$ NMR $\left(600 \mathrm{MHz}, \mathrm{CDCl}_{3}\right): \delta$ 7.27-7.32(m, 3H), 7.067.10(m, 2H), 5.83(s, 1H), 5.61(s, 1H), 4.15(dd, $J=10.8 \mathrm{~Hz}, J=3.0 \mathrm{~Hz}, 1 \mathrm{H}), 3.48(\mathrm{dd}, J=$ $10.8 \mathrm{~Hz}, J=3.0 \mathrm{~Hz}, 1 \mathrm{H}), 2.68(\mathrm{t}, J=13.2 \mathrm{~Hz}, 1 \mathrm{H}), 2.26-2.35(\mathrm{~m}, 2 \mathrm{H}), 2.35(\mathrm{t}, J=13.2 \mathrm{~Hz}$, $1 \mathrm{H}), 2.06(\mathrm{~s}, 3 \mathrm{H}), 0.92(\mathrm{~d}, J=5.4 \mathrm{~Hz}, 3 \mathrm{H}) .{ }^{13} \mathrm{C} \mathrm{NMR}\left(150 \mathrm{MHz}, \mathrm{CDCl}_{3}\right): \delta 145.2,139.2$, (128.7), 128.3, 128.1, 128.02, 127.96, 127.8, 123.2, (118.3), (94.4), 92.0, 72.8, 64.4, (64.3), 43.4, (42.4), 38.4, (37.6), (37.4), 37.1, (33.0), 32.4, 24.6, (22.4), 14.3. HRMS Calculated for $\mathrm{C}_{17} \mathrm{H}_{24} \mathrm{NO}_{4}[\mathrm{M}+\mathrm{H}]^{+}:$306.16999, Found: 306.17019 . 


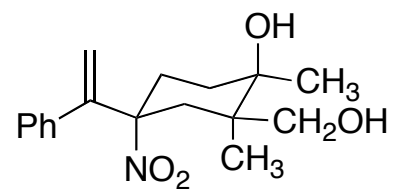

11g-isomer one

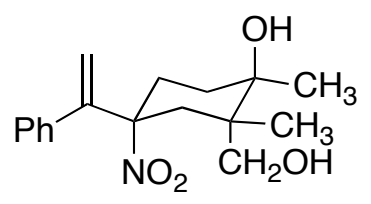

11g-isomer two

2-(hydroxymethyl)-1,2-dimethyl-4-nitro-4-(1-phenylvinyl)cyclohexanol (11g) was purified by flash silica gel chromatography (Hexane-EtOAc, v/v, 5/1-1/1) as colorless oil. NMR shows 1:1 ratio isomers (NMR show 2:2:1 ratio for three isomers), yield: 66\% (combined two isomers). ${ }^{1} \mathrm{H}$ NMR $\left(600 \mathrm{MHz}, \mathrm{CDCl}_{3}\right): \delta 7.28-7.32(\mathrm{~m}, 5.7 \mathrm{H}), 7.06-$ $7.10(\mathrm{~m}, 3.8 \mathrm{H}), 5.70(\mathrm{~s}, 1 \mathrm{H}), 5.68(\mathrm{~s}, 0.9 \mathrm{H}), 5.26(\mathrm{~s}, 0.9 \mathrm{H}), 5.23(\mathrm{~s}, 1 \mathrm{H}), 3.91(\mathrm{~d}, J=11.4 \mathrm{~Hz}$, $1 \mathrm{H}), 3.25(\mathrm{~d}, J=11.4 \mathrm{~Hz}, 1 \mathrm{H}), 3.22(\mathrm{~s}, 1.8 \mathrm{H}), 2.89(\mathrm{~d}, J=13.2 \mathrm{~Hz}, 1 \mathrm{H}), 2.69(\mathrm{dd}, J=15.6$ $\mathrm{Hz}, J=2.4 \mathrm{~Hz}, 1 \mathrm{H}), 1.82-2.62(\mathrm{~m}, 10.3 \mathrm{H}), 1.60-1.93(\mathrm{~m}, 1.9 \mathrm{H}), 1.57(\mathrm{dt}, J=14.4 \mathrm{~Hz}, J=$ $2.4 \mathrm{~Hz}, 1 \mathrm{H}), 1.23(\mathrm{~s}, 3 \mathrm{H}), 1.18(\mathrm{~s}, 2.7 \mathrm{H}), 1.12(\mathrm{~s}, 3 \mathrm{H}), 0.64(\mathrm{~s}, 2.7 \mathrm{H}) .{ }^{13} \mathrm{C}$ NMR $(150 \mathrm{MHz}$, $\left.\mathrm{CDCl}_{3}\right): \delta$ 150.3, (149.6), 138.9, (138.7), 128.8, 128.7, 128.2, 128.00, 127.96, 127.9, 127.8, (118.8), 118.3, (92.9), 92.1, 75.1, 71.0, 69.8, 65.4, 43.1, 40.2, 37.0, 36.0, 33.9, 32.9, (29.0), 28.8, 25.0, (24.8), 19.7, (19.3). HRMS Calculated for $\mathrm{C}_{17} \mathrm{H}_{23} \mathrm{NO}_{4} \mathrm{Na}$ $[\mathrm{M}+\mathrm{Na}]^{+}:$328.15193, Found: 328.15190.

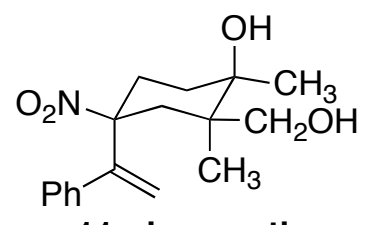

$11 \mathrm{~g}$-isomer three

\section{(1R,2R,4R)-2-(hydroxymethyl)-1,2-dimethyl-4-nitro-4-(1-phenylvinyl)cyclohexa-nol} (11g-isomer three) was purified by flash silica gel chromatography (Hexane-EtOAc, v/v, 5/1-1/1) as colorless oil, yield: 16\%. ${ }^{1} \mathrm{H}$ NMR (600 $\left.\mathrm{MHz}, \mathrm{CDCl}_{3}\right): \delta 7.28-7.33(\mathrm{~m}, 3 \mathrm{H})$, 7.04-7.07(m, 2H), 5.76(s, 1H), 5.22(s, 1H), 3.65(d, $J=10.2 \mathrm{~Hz}, 1 \mathrm{H}), 3.12(\mathrm{~d}, J=10.2 \mathrm{~Hz}$, $1 \mathrm{H}), 2.73(\mathrm{dq}, J=15.0 \mathrm{~Hz}, J=3.0 \mathrm{~Hz}, 1 \mathrm{H}), 2.54(\mathrm{dd}, J=3.0 \mathrm{~Hz}, J=15.0 \mathrm{~Hz}, 1 \mathrm{H})$, 2.40 (br, $2 \mathrm{H}), 2.02(\mathrm{td}, J=13.2 \mathrm{~Hz}, J=3.6 \mathrm{~Hz}, 1 \mathrm{H}), 1.64-1.71(\mathrm{~m}, 2 \mathrm{H}), 1.45(\mathrm{dt}, J=13.8$ $\mathrm{Hz}, J=3.6 \mathrm{~Hz}, 1 \mathrm{H}), 1.28(\mathrm{~s}, 3 \mathrm{H}), 1.03(\mathrm{~s}, 3 \mathrm{H}) .{ }^{13} \mathrm{C} \mathrm{NMR}\left(150 \mathrm{MHz}, \mathrm{CDCl}_{3}\right): \delta 145.0$, $138.7,128.6,128.1,128.0,118.4,90.5,73.9,70.9,41.0,93.3,33.4,31.2,23.1,16.8$. HRMS Calculated for $\mathrm{C}_{17} \mathrm{H}_{23} \mathrm{NO}_{4} \mathrm{Na}[\mathrm{M}+\mathrm{Na}]^{+}: 328.15193$, Found: 328.15215 . 


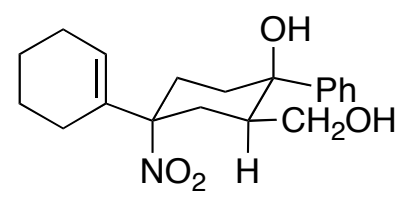

11h-major

(1R,2R,4R)-4-cyclohexenyl-2-(hydroxymethyl)-4-nitro-1-phenylcyclohexanol (11hmajor) was purified by flash silica gel chromatography (Hexane-EtOAc, v/v, 3/1) as colorless oil, yield: 83\%. ${ }^{1} \mathrm{H}$ NMR (600 MHz, $\left.\mathrm{CDCl}_{3}\right): \delta$ 7.37-7.41(m, 2H), 7.30-7.36(m, 2H), 7.23-7.26(m, 1H), 6.02-6.04(m, $1 \mathrm{H}), 3.62(\mathrm{dd}, J=10.8 \mathrm{~Hz}, J=3.0 \mathrm{~Hz}, 1 \mathrm{H}), 3.52(\mathrm{dd}$, $J=10.8 \mathrm{~Hz}, J=3.0 \mathrm{~Hz}, 1 \mathrm{H}), 2.64-2.68(\mathrm{~m}, 1 \mathrm{H}), 2.53(\mathrm{t}, J=14.4 \mathrm{~Hz}, 1 \mathrm{H}), 2.31(\mathrm{td}, J=$ $13.2 \mathrm{~Hz}, J=4.2 \mathrm{~Hz}, 1 \mathrm{H}), 2.10-2.14(\mathrm{~m}, 2 \mathrm{H}), 2.03-2.09(\mathrm{~m}, 2 \mathrm{H}), 1.98(\mathrm{dq}, J=12.6 \mathrm{~Hz}, J=$ $3.0 \mathrm{~Hz}, 1 \mathrm{H}), 1.82(\mathrm{td}, J=12.6 \mathrm{~Hz}, J=4.2 \mathrm{~Hz}, 1 \mathrm{H}), 1.74(\mathrm{dq}, J=14.4 \mathrm{~Hz}, J=2.4 \mathrm{~Hz}, 1 \mathrm{H})$, 1.62-1.67(m, 2H), 1.54-1.60(m, 2H). ${ }^{13} \mathrm{C} \mathrm{NMR}\left(150 \mathrm{MHz}, \mathrm{CDCl}_{3}\right): \delta 146.7,136.5,128.4$, $126.9,125.9,124.6,95.6,75.9,64.7,41.8,37.1,31.8,28.4,25.4,23.8,22.7,21.7$. HRMS Calculated for $\mathrm{C}_{19} \mathrm{H}_{25} \mathrm{NO}_{4} \mathrm{Na}[\mathrm{M}+\mathrm{Na}]^{+}$: 354.16758 , Found: 354.16756.

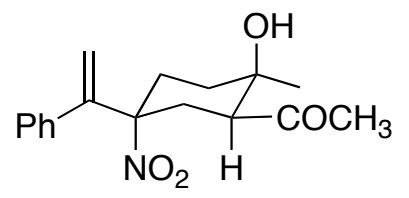

12a-major

\section{1-((1S,2R,5R)-2-hydroxy-2-methyl-5-nitro-5-(1-phenylvinyl)cyclohexyl)ethanone}

(12a-major) was purified by flash silica gel chromatography (Hexane-EtOAc, v/v, 5/1) as white solid, m.p: 98.2-98.9 ${ }^{\circ} \mathrm{C}$, yield: $66 \%$. ${ }^{1} \mathrm{H}$ NMR $\left(270 \mathrm{MHz}, \mathrm{CDCl}_{3}\right): \delta$ 7.227.36(m, 3H), 6.98-7.09(m, 2H), 5.60(s, 1H), 5.24(s, 1H), 3.40-3.90 (br, 1H), 2.47-2.67(m, $3 \mathrm{H}), 2.17-2.32(\mathrm{~m}, 4 \mathrm{H}), 2.08(\mathrm{t}, J=13.6 \mathrm{~Hz}, 1 \mathrm{H}), 1.69(\mathrm{dq}, J=14.6 \mathrm{~Hz}, J=1.5 \mathrm{~Hz}, 1 \mathrm{H})$, 1.19-1.33(m, 1H), 1.14(s, 3H). ${ }^{13} \mathrm{C}$ NMR (67.5 MHz, $\left.\mathrm{CDCl}_{3}\right): \delta 214.5,148.5,138.3$, 128.6, 128.1, 128.0, 118.7, 93.1, 68.3, 52.3, 34.8, 32.1, 31.5, 29.0, 28.2. HRMS Calculated for $\mathrm{C}_{17} \mathrm{H}_{21} \mathrm{NO}_{4} \mathrm{Na}[\mathrm{M}+\mathrm{Na}]^{+}$: 326.13683, Found: 326.13606 . 


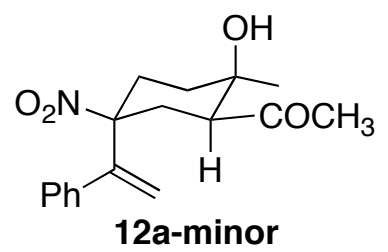

1-((1S,2R,5S)-2-hydroxy-2-methyl-5-nitro-5-(1-phenylvinyl)cyclohexyl)ethanone

(12a-minor) was purified by flash silica gel chromatography (Hexane-EtOAc, v/v, 5/1) as white solid, m.p: 75.6-77.1 ${ }^{\circ} \mathrm{C}$, yield: $24 \% .{ }^{1} \mathrm{H}$ NMR $\left(270 \mathrm{MHz}, \mathrm{CDCl}_{3}\right): \delta$ 7.327.43(m, 3H), 7.16-7.25(m, 2H), 5.92(s, 1H), 5.73(s, 1H), 3.88(d, $J=2.5 \mathrm{~Hz}, 1 \mathrm{H}), 2.24-$ 2.62(m, 5H), 1.69-1.81(m, 4H), 1.37-1.53(m, 1H), 1.17(s, 1H). ${ }^{13} \mathrm{C}$ NMR $(67.5 \mathrm{MHz}$, $\left.\mathrm{CDCl}_{3}\right): \delta 213.0,144.0,138.9,128.5,128.3,128.1,124.0,90.7,68.5,52.7,35.8,31.0$, 30.0, 29.6, 28.0. HRMS Calculated for $\mathrm{C}_{17} \mathrm{H}_{21} \mathrm{NO}_{4} \mathrm{Na}[\mathrm{M}+\mathrm{Na}]^{+}$: 326.13683, Found: 326.13608 .

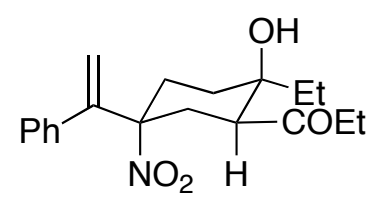

12b-major

\section{1-((1S,2R,5R)-2-ethyl-2-hydroxy-5-nitro-5-(1-phenylvinyl)cyclohexyl)propan-1-one}

(12b-major) was purified by flash silica gel chromatography (Hexane-EtOAc, v/v, 5/1) as white solid, m.p: 78.7-80.3 ${ }^{\circ} \mathrm{C}$, yield: $61 \%$. ${ }^{1} \mathrm{H}$ NMR $\left(270 \mathrm{MHz}, \mathrm{CDCl}_{3}\right): \delta$ 7.187.42(m, 3H), 6.96-7.14(m, 2H), 5.61(s, 1H), 5.25(s, 1H), 3.79(d, J=2.5 Hz, 1H), 2.06$2.76(\mathrm{~m}, 8 \mathrm{H}), 1.75(\mathrm{dt}, J=14.6 \mathrm{~Hz}, J=3.7 \mathrm{~Hz}, 1 \mathrm{H}), 1.10-1.54(\mathrm{~m}, 3 \mathrm{H}), 1.05(\mathrm{t}, J=7.2 \mathrm{~Hz}$, $3 \mathrm{H}), 0.74(\mathrm{t}, J=7.4 \mathrm{~Hz}, 3 \mathrm{H}) .{ }^{13} \mathrm{C} \mathrm{NMR}\left(67.5 \mathrm{MHz}, \mathrm{CDCl}_{3}\right): \delta 217.5,148.7,138.3,128.6$, $128.1,127.9,118.6,93.2,70.9,50.7,37.8,33.6,32.5,30.6,28.8,7.7,7.2$. HRMS Calculated for $\mathrm{C}_{19} \mathrm{H}_{25} \mathrm{NO}_{4} \mathrm{Na}[\mathrm{M}+\mathrm{Na}]^{+}:$354.16813, Found: 354.16738 . 


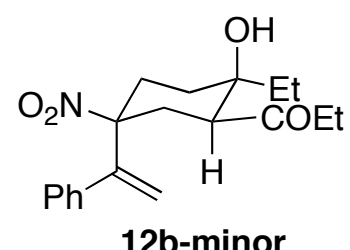

1-((1S,2R,5S)-2-ethyl-2-hydroxy-5-nitro-5-(1-phenylvinyl)cyclohexyl)propan-1-one

(12b-minor) was purified by flash silica gel chromatography (Hexane-EtOAc, v/v, 5/1) as colorless oil, yield: $20 \%$. ${ }^{1} \mathrm{H}$ NMR $\left(270 \mathrm{MHz}, \mathrm{CDCl}_{3}\right): \delta 7.30-7.44(\mathrm{~m}, 3 \mathrm{H}), 7.15-$ 7.25(m, 2H), 5.92(s, 1H), 5.71(s, 1H), 3.89(d, $J=2.7 \mathrm{~Hz}, 1 \mathrm{H}), 2.28-2.65(\mathrm{~m}, 5 \mathrm{H}), 1.88-$ 2.07(m, 2H), 1.75(dt, $J=14.1 \mathrm{~Hz}, J=3.7 \mathrm{~Hz}, 1 \mathrm{H}), 1.23-1.55(\mathrm{~m}, 3 \mathrm{H}), 0.78-0.92(\mathrm{~m}, 6 \mathrm{H})$. ${ }^{13} \mathrm{C}$ NMR (67.5 MHz, $\left.\mathrm{CDCl}_{3}\right): \delta 216.1,144.2,139.0,128.5,128.24,128.15,124.0,90.7$, 71.1, 50.3, 36.4, 33.4, 31.7, 31.4, 29.5, 29.4, 7.9, 7.1. HRMS Calculated for $\mathrm{C}_{19} \mathrm{H}_{25} \mathrm{NO}_{4} \mathrm{Na}[\mathrm{M}+\mathrm{Na}]^{+}:$354.16813, Found: 354.16739 .

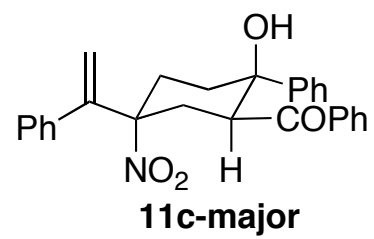

1-((1S,2R,5R)-2-hydroxy-5-nitro-2-phenyl-5-(1-phenylvinyl)cyclohexyl)(phenyl)

methanone (11c-major) was purified by flash silica gel chromatography (HexaneEtOAc, v/v, 10/1) as white solid, m.p: 163.2-164.8 ${ }^{\circ} \mathrm{C}$, yield: $72 \% .{ }^{1} \mathrm{H}$ NMR $(600 \mathrm{MHz}$, $\left.\mathrm{CDCl}_{3}\right): \delta 7.91-7.93(\mathrm{~m}, 2 \mathrm{H}), 7.55-7.59(\mathrm{~m}, 1 \mathrm{H}), 7.43-7.46(\mathrm{~m}, 2 \mathrm{H}), 7.30-7.36(\mathrm{~m}, 5 \mathrm{H})$, 7.17-7.21(m, 2H), 7.09-7.13(m, 3H), 5.66(s, 1H), 5.30(s, 1H), 5.12(d, J=3.0 Hz, 1H), $4.21(\mathrm{dd}, J=12.6 \mathrm{~Hz}, J=3.0 \mathrm{~Hz}, 1 \mathrm{H}), 2.93(\mathrm{dt}, J=14.4 \mathrm{~Hz}, J=3.0 \mathrm{~Hz}, 1 \mathrm{H}), 2.71(\mathrm{dq}, J=$ $14.4 \mathrm{~Hz}, J=3.0 \mathrm{~Hz}, 1 \mathrm{H}), 2.47-2.58(\mathrm{~m}, 2 \mathrm{H}), 1.90(\mathrm{dq}, J=14.4 \mathrm{~Hz}, J=3.0 \mathrm{~Hz}, 1 \mathrm{H}), 1.67-$ 1.75(m, 1H). ${ }^{13} \mathrm{C}$ NMR $\left(150 \mathrm{MHz}, \mathrm{CDCl}_{3}\right): \delta 204.6,148.8,146.3,138.4,135.5,134.2$, 129.0, 128.7, 128.4, 128.3, 128.2, 128.1, 127.0, 124.4, 118.8, 93.8, 73.2, 46.5, 36.9, 33.8, 29.9. HRMS Calculated for $\mathrm{C}_{27} \mathrm{H}_{25} \mathrm{NO}_{4} \mathrm{Na}[\mathrm{M}+\mathrm{Na}]^{+}: 450.16813$, Found: 450.16712. 


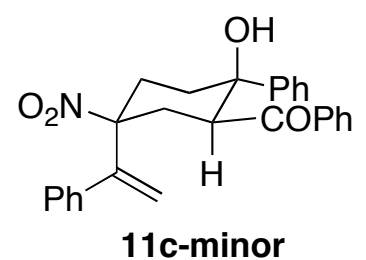

\section{1-((1S,2R,5S)-2-hydroxy-5-nitro-2-phenyl-5-(1-phenylvinyl)cyclohexyl)(phenyl)}

methanone (11c-minor) was purified by flash silica gel chromatography (HexaneEtOAc, v/v, 10/1-3/1) as colorless oil, yield: $10 \% .{ }^{1} \mathrm{H}$ NMR (600 MHz, $\left.\mathrm{CDCl}_{3}\right): \delta$ 7.417.48(m, 6H), 7.33-7.35(m, 2H), 7.09-7.23(m, 7H), 6.08(s, 1H), 5.88(s, 1H), 5.40(s, 1H), $3.85(\mathrm{dd}, J=12.0 \mathrm{~Hz}, J=4.2 \mathrm{~Hz}, 1 \mathrm{H}), 2.73-2.84(\mathrm{~m}, 4 \mathrm{H}), 1.88-1.92(\mathrm{~m}, 2 \mathrm{H}) .{ }^{13} \mathrm{C} \mathrm{NMR}$ $\left(150 \mathrm{MHz}, \mathrm{CDCl}_{3}\right): \delta 204.4,146.2,144.6,139.4,135.4,133.8,129.0,128.7,128.6$, $128.41,128.35,127.9,127.0,124.9,124.4,90.7,73.4,46.4,37.8,32.4,30.6$. HRMS Calculated for $\mathrm{C}_{27} \mathrm{H}_{25} \mathrm{NO}_{4} \mathrm{Na}[\mathrm{M}+\mathrm{Na}]^{+}: 450.16813$, Found: 450.16723 .

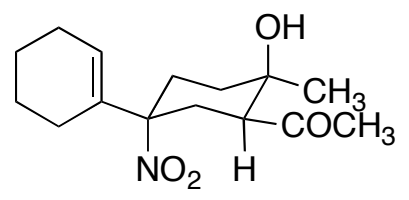

12d-major

1-((1S*,2R*,5R*)-5-cyclohexenyl-2-hydroxy-2-methyl-5-nitrocyclohexyl)ethanone

(12d-major) was purified by flash silica gel chromatography (Hexane-EtOAc, v/v, 5/1) as white solid, m.p: $129.5-130.8{ }^{\circ} \mathrm{C}$, yield: $74 \%$. ${ }^{1} \mathrm{H}$ NMR $\left(270 \mathrm{MHz}, \mathrm{CDCl}_{3}\right): \delta 5.84(\mathrm{~m}$, $1 \mathrm{H}), 3.81(\mathrm{~d}, J=2.4 \mathrm{~Hz}, 1 \mathrm{H}), 2.50-2.64(\mathrm{~m}, 2 \mathrm{H}), 2.43(\mathrm{dq}, J=14.4 \mathrm{~Hz}, J=3.0 \mathrm{~Hz}, 1 \mathrm{H})$, $2.20(\mathrm{~s}, 3 \mathrm{H}), 1.83-2.18(\mathrm{~m}, 6 \mathrm{H}), 1.37-1.35(\mathrm{~m}, 5 \mathrm{H}), 1.03-1.26(\mathrm{~m}, 1 \mathrm{H}), 1.09(\mathrm{~s}, 3 \mathrm{H}) .{ }^{13} \mathrm{C}$ NMR (67.5 MHz, $\left.\mathrm{CDCl}_{3}\right): \delta 214.4,135.5,125.9,94.0,68.3,52.6,31.2,31.1,27.6,25.1$, 23.5, 22.4, 21.3. HRMS Calculated for $\mathrm{C}_{15} \mathrm{H}_{23} \mathrm{NO}_{4} \mathrm{Na}[\mathrm{M}+\mathrm{Na}]^{+}$: 304.15248, Found: 304.15180. 


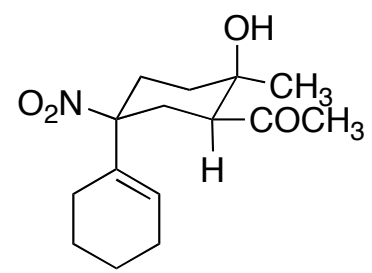

12d-minor

1-((1S,2R,5S)-5-cyclohexenyl-2-hydroxy-2-methyl-5-nitrocyclohexyl)ethanone (12dminor) was purified by flash silica gel chromatography (Hexane-EtOAc, v/v, 5/1) as white solid, m.p: $54.2-55.1{ }^{\circ} \mathrm{C}$, yield: $14 \% .{ }^{1} \mathrm{H}$ NMR $\left(270 \mathrm{MHz}, \mathrm{CDCl}_{3}\right): \delta 6.17(\mathrm{~m}, 1 \mathrm{H})$, $3.85(\mathrm{~d}, J=2.4 \mathrm{~Hz}, 1 \mathrm{H}), 2.15-2.60(\mathrm{~m}, 7 \mathrm{H}), 2.25(\mathrm{~s}, 3 \mathrm{H}), 1.94-2.05(\mathrm{~m}, 1 \mathrm{H}), 1.52-1.77(\mathrm{~m}$, $5 \mathrm{H}), 1.22-1.38(\mathrm{~m}, 1 \mathrm{H}), 1.17(\mathrm{~s}, 3 \mathrm{H}) .{ }^{13} \mathrm{C} \mathrm{NMR}\left(67.5 \mathrm{MHz}, \mathrm{CDCl}_{3}\right): \delta 213.2,131.3,130.6$, 92.2, 68.6, 53.5, 36.0, 30.9, 29.8, 27.4, 25.8, 24.1, 222.6, 21.4. HRMS Calculated for $\mathrm{C}_{15} \mathrm{H}_{23} \mathrm{NO}_{4} \mathrm{Na}[\mathrm{M}+\mathrm{Na}]^{+}:$304.15248, Found: 304.15177.

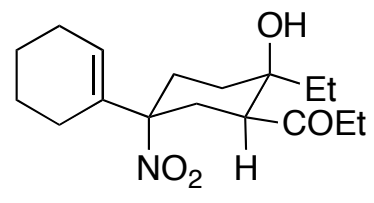

12e-major

\section{1-((1S,2R,5R)-5-cyclohexenyl-2-ethyl-2-hydroxy-5-nitrocyclohexyl)propan-1-one}

(12e-major) was purified by flash silica gel chromatography (Hexane-EtOAc, v/v, 5/1) as white solid, m.p: 91.8-92.9 ${ }^{\circ} \mathrm{C}$, yield: 71\%. ${ }^{1} \mathrm{H}$ NMR $\left(270 \mathrm{MHz}, \mathrm{CDCl}_{3}\right): \delta 5.80(\mathrm{~m}$, 1H), 3.70(d, $J=2.5 \mathrm{~Hz}, 1 \mathrm{H}), 2.29-2.67(\mathrm{~m}, 5 \mathrm{H}), 1.80-2.13(\mathrm{~m}, 6 \mathrm{H}), 1.61(\mathrm{dt}, J=13.6 \mathrm{~Hz}, J$ $=3.7 \mathrm{~Hz}, 1 \mathrm{H}), 1.14-1.54(\mathrm{~m}, 6 \mathrm{H}), 1.07(\mathrm{dt}, J=13.6 \mathrm{~Hz}, J=3.5 \mathrm{~Hz}, 1 \mathrm{H}), 0.95(\mathrm{t}, J=7.2$ $\mathrm{Hz}, 3 \mathrm{H}), 0.72(\mathrm{t}, J=8.6 \mathrm{~Hz}, 3 \mathrm{H}) .{ }^{13} \mathrm{C} \mathrm{NMR}\left(67.5 \mathrm{MHz}, \mathrm{CDCl}_{3}\right): \delta 217.2,135.5,125.7$, $93.9,70.8,50.5,37.4,33.4,31.4,30.5,27.4,25.1,23.4,22.3,21.3,7.5,6.9$. HRMS Calculated for $\mathrm{C}_{17} \mathrm{H}_{27} \mathrm{NO}_{4} \mathrm{Na}[\mathrm{M}+\mathrm{Na}]^{+}$: 332.18378, Found: 332.18303 . 


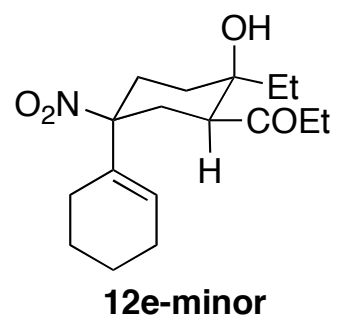

1-((1S,2R,5S)-5-cyclohexenyl-2-ethyl-2-hydroxy-5-nitrocyclohexyl)propan-1-one

(12e-minor) was purified by flash silica gel chromatography (Hexane-EtOAc, v/v, 6/1) as colorless oil, yield: $13 \% .{ }^{1} \mathrm{H}$ NMR $\left(270 \mathrm{MHz}, \mathrm{CDCl}_{3}\right): \delta 6.14(\mathrm{t}, J=1.0 \mathrm{~Hz}, 1 \mathrm{H})$, $3.82(\mathrm{~d}, J=2.5 \mathrm{~Hz}, 1 \mathrm{H}), 2.10-2.69(\mathrm{~m}, 9 \mathrm{H}), 1.88-2.02(\mathrm{~m}, 2 \mathrm{H}), 1.08-1.78(\mathrm{~m}, 8 \mathrm{H}), 1.02(\mathrm{t}, J$ $=7.2 \mathrm{~Hz}, 3 \mathrm{H}), 0.81(\mathrm{t}, J=7.4 \mathrm{~Hz}, 3 \mathrm{H}) .{ }^{13} \mathrm{C} \mathrm{NMR}\left(67.5 \mathrm{MHz}, \mathrm{CDCl}_{3}\right): \delta 216.3,131.2$, 130.6, 92.1, 71.0, 51.5, 37.2, 33.3, 31.7, 30.2, 27.1, 25.8, 24.0, 22.5, 21.4, 7.6, 7.1. HRMS Calculated for $\mathrm{C}_{17} \mathrm{H}_{27} \mathrm{NO}_{4} \mathrm{Na}[\mathrm{M}+\mathrm{Na}]^{+}: 332.18378$, Found: 332.18305 .

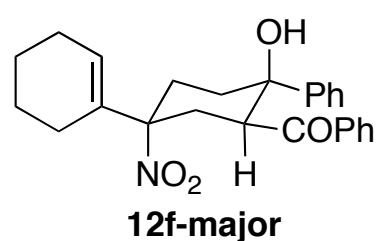

1-((1S,2R,5R)-5-cyclohexenyl-2-hydroxy-5-nitro-2-phenylcyclohexyl)(phenyl)methanone (12f-major) was purified by flash silica gel chromatography (Hexane-EtOAc, v/v, 6/1) as white solid, m.p: $133.1-134.6{ }^{\circ} \mathrm{C}$, yield: 82\%. ${ }^{1} \mathrm{H}$ NMR $\left(600 \mathrm{MHz}, \mathrm{CDCl}_{3}\right): \delta$ 7.96-7.98(m, 2H), 7.56-7.60(m, 1H), 7.45-7.48(m, 2H), 7.35-7.38(m, 2H), 7.19-7.22(m, 2H), 7.09-7.13(m, 1H), 5.98(m, 1H), 5.17(d, $J=1.8 \mathrm{~Hz}, 1 \mathrm{H}), 4.20(\mathrm{dd}, J=12.6 \mathrm{~Hz}, J=$ $3.0 \mathrm{~Hz}, 1 \mathrm{H}), 2.96(\mathrm{dt}, J=14.4 \mathrm{~Hz}, J=3.0 \mathrm{~Hz}, 1 \mathrm{H}), 2.70(\mathrm{dq}, J=14.4 \mathrm{~Hz}, J=3.0 \mathrm{~Hz}, 1 \mathrm{H})$, $2.48(\mathrm{td}, J=13.2 \mathrm{~Hz}, J=4.2 \mathrm{~Hz}, 1 \mathrm{H}), 2.39(\mathrm{dd}, J=13.2 \mathrm{~Hz}, J=12.6 \mathrm{~Hz}, 1 \mathrm{H}), 1.96-$ $2.12(\mathrm{~m}, 4 \mathrm{H}), 1.89(\mathrm{dq}, J=13.0 \mathrm{~Hz}, J=3.0 \mathrm{~Hz}, 1 \mathrm{H}), 1.51-1.71(\mathrm{~m}, 5 \mathrm{H}) .{ }^{13} \mathrm{C} \mathrm{NMR}(150$ $\left.\mathrm{MHz}, \mathrm{CDCl}_{3}\right): \delta 204.8,146.5,135.7,135.5,134.2,129.0,128.5,128.3,126.9,126.5$, 124.4, 94.7, 73.5, 46.6, 37.0, 32.9, 28.5, 25.4, 23.8, 22.6, 21.5. HRMS Calculated for $\mathrm{C}_{25} \mathrm{H}_{27} \mathrm{NO}_{4} \mathrm{Na}[\mathrm{M}+\mathrm{Na}]^{+}:$428.18378, Found: 428.18291. 


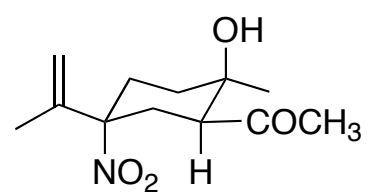

12g-major

1-((1S,2R,5R)-2-hydroxy-2-methyl-5-nitro-5-(prop-1-en-2-yl)cyclohexyl)ethan-one

(12g-major) was purified by flash silica gel chromatography (Hexane-EtOAc, v/v, 10/1) as white solid, m.p: $62.6-62.9{ }^{\circ} \mathrm{C}$, yield: $65 \% .{ }^{1} \mathrm{H}$ NMR $\left(270 \mathrm{MHz}, \mathrm{CDCl}_{3}\right): \delta 5.11(\mathrm{~s}, 1 \mathrm{H})$, $5.06(\mathrm{q}, J=1.2 \mathrm{~Hz}, 1 \mathrm{H}), 3.85(\mathrm{~d}, J=2.7 \mathrm{~Hz}, 1 \mathrm{H}), 2.56-2.69(\mathrm{~m}, 2 \mathrm{H}), 2.51(\mathrm{dq}, J=14.6 \mathrm{~Hz}$, $J=3.0 \mathrm{~Hz}, 1 \mathrm{H}), 2.26(\mathrm{~s}, 3 \mathrm{H}), 1.99-2.24(\mathrm{~m}, 2 \mathrm{H}), 1.64-1.78(\mathrm{~m}, 4 \mathrm{H}), 1.17-32(\mathrm{~m}, 1 \mathrm{H})$, 1.15(s, 3H). ${ }^{13} \mathrm{C}$ NMR (67.5 MHz, $\left.\mathrm{CDCl}_{3}\right): \delta 214.2,142.5,114.7,93.7,68.2,52.5,34.8$, 31.2, 31.1, 28.1, 28.0, 27.9. HRMS Calculated for $\mathrm{C}_{12} \mathrm{H}_{19} \mathrm{NO}_{4} \mathrm{Na}[\mathrm{M}+\mathrm{Na}]^{+}:$264.12118, Found: 264.12043.<smiles>C=C(C)C1([N+](=O)[O-])CCC(C(C)=O)C(C)(O)C1</smiles>

12g-minor

\section{1-((1S,2R,5S)-2-hydroxy-2-methyl-5-nitro-5-(prop-1-en-2-yl)cyclohexyl)ethanone}

(12g-minor) was purified by flash silica gel chromatography (Hexane-EtOAc, v/v, 10/1) as colorless oil, yield: $15 \% .{ }^{1} \mathrm{H}$ NMR $\left(270 \mathrm{MHz} \mathrm{CDCl}_{3}\right): \delta 5.18(\mathrm{~s}, 1 \mathrm{H}), 5.12(\mathrm{q}, J=1.5$ $\mathrm{Hz}, 1 \mathrm{H}), 2.69-2.86(\mathrm{~m}, 3 \mathrm{H}), 2.46(\mathrm{~s}, 1 \mathrm{H}), 2.32(\mathrm{~s}, 3 \mathrm{H}), 1.53-2.00(\mathrm{~m}, 8 \mathrm{H}), 1.18(\mathrm{~s}, 3 \mathrm{H}) .{ }^{13} \mathrm{C}$ $\operatorname{NMR}\left(67.5 \mathrm{MHz}, \mathrm{CDCl}_{3}\right): \delta 210.6,142.5,115.2,93.5,71.1,55.3,38.4,32.4,32.2,30.4$, 21.5, 21.4, 18.7. HRMS Calculated for $\mathrm{C}_{12} \mathrm{H}_{19} \mathrm{NO}_{4} \mathrm{Na}[\mathrm{M}+\mathrm{Na}]^{+}$: 264.12118, Found: 264.12051.

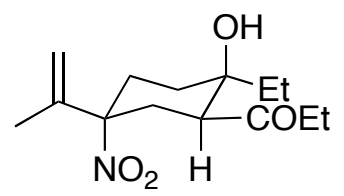

12h-major 
1-((1S,2R,5R)-2-ethyl-2-hydroxy-5-nitro-5-(prop-1-en-2-yl)cyclohexyl)propan-1-one (12h-major) was purified by flash silica gel chromatography (Hexane-EtOAc, v/v, 10/1) as white solid, m.p: $112.3-113.9{ }^{\circ} \mathrm{C}$, yield: $60 \% .{ }^{1} \mathrm{H}$ NMR $\left(600 \mathrm{MHz}, \mathrm{CDCl}_{3}\right): \delta 5.16(\mathrm{~s}$, $1 \mathrm{H}), 5.10(\mathrm{q}, J=1.2 \mathrm{~Hz}, 1 \mathrm{H}), 2.70-2.82(\mathrm{~m}, 4 \mathrm{H}), 2.44-2.53(\mathrm{~m}, 1 \mathrm{H}), 2.17(\mathrm{br}, 1 \mathrm{H}), 2.02(\mathrm{dd}$, $J=13.2 \mathrm{~Hz}, J=15.0 \mathrm{~Hz}, 1 \mathrm{H}), 1.96(\mathrm{td}, J=3.6 \mathrm{~Hz}, J=13.8 \mathrm{~Hz}, 1 \mathrm{H}), 1.77(\mathrm{~d}, J=0.6 \mathrm{~Hz}$, $3 \mathrm{H}), 1.66(\mathrm{dt}, J=4.2 \mathrm{~Hz}, J=15.0 \mathrm{~Hz}, 1 \mathrm{H}), 1.55-1.63(\mathrm{~m}, 1 \mathrm{H}), 1.28-1.40(\mathrm{~m}, 2 \mathrm{H}), 1.03(\mathrm{t}, J$ $=7.2 \mathrm{~Hz}, 3 \mathrm{H}), 0.87(\mathrm{t}, J=7.2 \mathrm{~Hz}, 3 \mathrm{H}) .{ }^{13} \mathrm{C} \mathrm{NMR}\left(150 \mathrm{MHz}, \mathrm{CDCl}_{3}\right): \delta 212.9,142.6$, 115.2, 93.6, 72.8, 55.5, 37.8, 32.5, 32.3, 29.9, 24.4, 18.7, 7.4, 6.8. HRMS Calculated for $\mathrm{C}_{14} \mathrm{H}_{24} \mathrm{NO}_{4}[\mathrm{M}+\mathrm{H}]^{+}: 270.16999$, Found: 270.17004 .

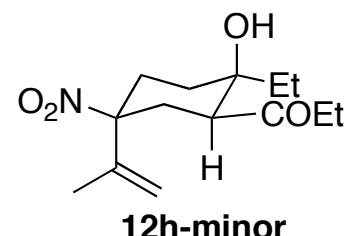

1-((1S,2R,5S)-2-ethyl-2-hydroxy-5-nitro-5-(prop-1-en-2-yl)cyclohexyl)propan-1-one (12h-minor) was purified by flash silica gel chromatography (Hexane-EtOAc, v/v, 10/1) as white solid, m.p: $77.6-78.9{ }^{\circ} \mathrm{C}$, yield: $12 \% .{ }^{1} \mathrm{H}$ NMR $\left(600 \mathrm{MHz}, \mathrm{CDCl}_{3}\right): \delta 5.34(\mathrm{~s}, 1 \mathrm{H})$, 5.32(s, 1H), 2.68-2.74(m, 2H), 2.35-2.62(m, 4H), 2.05-2.23(m, 3H), 1.81(d, J=0.6 Hz, $3 \mathrm{H}), 1.64-1.71(\mathrm{~m}, 1 \mathrm{H}), 1.42-1.52(\mathrm{~m}, 2 \mathrm{H}), 1.04(\mathrm{t}, J=7.2 \mathrm{~Hz}, 3 \mathrm{H}), 0.87(\mathrm{t}, J=7.2 \mathrm{~Hz}, 3 \mathrm{H})$. ${ }^{13} \mathrm{C}$ NMR $\left(150 \mathrm{MHz}, \mathrm{CDCl}_{3}\right): \delta 212.1,140.1,118.5,91.8,72.8,54.4,36.4,31.8,31.0$, 28.8, 27.1, 18.9, 7.5, 6.7. HRMS Calculated for $\mathrm{C}_{14} \mathrm{H}_{23} \mathrm{NO}_{4} \mathrm{Na}[\mathrm{M}+\mathrm{Na}]^{+}$: 292.15193, Found: 292.15205.

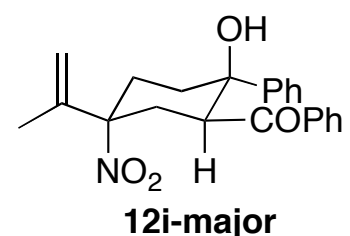

1-((1S,2R,5R)-2-hydroxy-5-nitro-2-phenyl-5-(prop-1-en-2-yl)cyclohexyl)phenylmethanone (12i-major) was purified by flash silica gel chromatography (Hexane- 
EtOAc, v/v, 10/1) as white solid, m.p: $141.8-142.7{ }^{\circ} \mathrm{C}$, yield: $77 \% .{ }^{1} \mathrm{H}$ NMR $(600 \mathrm{MHz}$, $\left.\mathrm{CDCl}_{3}\right): \delta 7.95-7.97(\mathrm{~m}, 2 \mathrm{H}), 7.58-7.61(\mathrm{~m}, 1 \mathrm{H}), 7.45-7.49(\mathrm{~m}, 2 \mathrm{H}), 7.35-7.38(\mathrm{~m}, 2 \mathrm{H})$, 7.19-7.23(m, 2H), 7.10-7.14(m, 1H), $5.21(\mathrm{~s}, 1 \mathrm{H}), 5.17(\mathrm{~d}, J=3.0 \mathrm{~Hz}, 1 \mathrm{H}), 5.13(\mathrm{q}, J=1.2$ $\mathrm{Hz}, 1 \mathrm{H}), 2.95(\mathrm{dt}, J=14.4 \mathrm{~Hz}, J=2.4 \mathrm{~Hz}, 1 \mathrm{H}), 2.72(\mathrm{dq}, J=14.4 \mathrm{~Hz}, J=3.0 \mathrm{~Hz}, 1 \mathrm{H})$, $2.54(\mathrm{td}, J=14.4 \mathrm{~Hz}, J=4.2 \mathrm{~Hz}, 1 \mathrm{H}), 2.45(\mathrm{dd}, J=14.4 \mathrm{~Hz}, J=13.2 \mathrm{~Hz}, 1 \mathrm{H}), 1.92(\mathrm{dq}, J$ $=14.4 \mathrm{~Hz}, J=3.0 \mathrm{~Hz}, 1 \mathrm{H}), 1.83(\mathrm{~s}, 3 \mathrm{H}), 1.67-1.74(\mathrm{~m}, 1 \mathrm{H}) .{ }^{13} \mathrm{C} \mathrm{NMR}\left(150 \mathrm{MHz}, \mathrm{CDCl}_{3}\right)$ : $\delta$ 204.7, 146.4, 142.6, 135.4, 134.2, 129.0, 128.5, 128.4, 127.0, 124.4, 115.2, 94.4, 73.4, $46.4,37.0,32.9,28.8,18.7$. HRMS Calculated for $\mathrm{C}_{22} \mathrm{H}_{23} \mathrm{NO}_{4} \mathrm{Na}[\mathrm{M}+\mathrm{Na}]^{+}: 388.15193$, Found: 388.15213 .

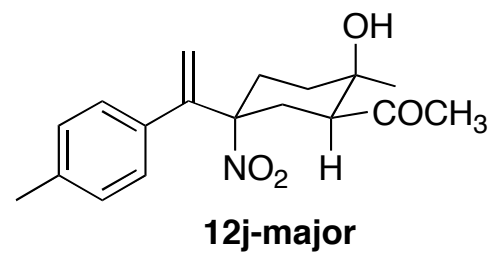

1-((1S*,2R*,5R*)-2-hydroxy-2-methyl-5-nitro-5-(1-p-tolylvinyl)cyclohexyl)ethanone (12j-major) was purified by flash silica gel chromatography (Hexane-EtOAc, v/v, 5/1) as colorless oil, yield: $63 \%$. ${ }^{1} \mathrm{H}$ NMR $\left(270 \mathrm{MHz}, \mathrm{CDCl}_{3}\right): \delta 7.11(\mathrm{~d}, J=8.0 \mathrm{~Hz}, 2 \mathrm{H}), 6.95(\mathrm{~d}$, $J=8.0 \mathrm{~Hz}, 2 \mathrm{H}), 5.59(\mathrm{~s}, 1 \mathrm{H}), 5.24(\mathrm{~s}, 1 \mathrm{H}), 3.86(\mathrm{~d}, J=2.7 \mathrm{~Hz}, 1 \mathrm{H}), 2.49-2.69(\mathrm{~m}, 3 \mathrm{H})$, 2.04-2.36(m, 8H), 1.66-1.76(m, $1 \mathrm{H}), 1.20-1.35(\mathrm{~m}, 1 \mathrm{H}), 1.16(\mathrm{~s}, 3 \mathrm{H}) .{ }^{13} \mathrm{C}$ NMR $(67.5$ $\left.\mathrm{MHz}, \mathrm{CDCl}_{3}\right): \delta 214.5,148.5,137.7,135.4,128.7,128.4,118.4,93.3,68.3,52.6,34.9$, 32.1, 31.5, 29.0, 28.2, 21.1. HRMS Calculated for $\mathrm{C}_{18} \mathrm{H}_{23} \mathrm{NO}_{4} \mathrm{Na}[\mathrm{M}+\mathrm{Na}]^{+}: 340.15248$, Found: 340.15170 .

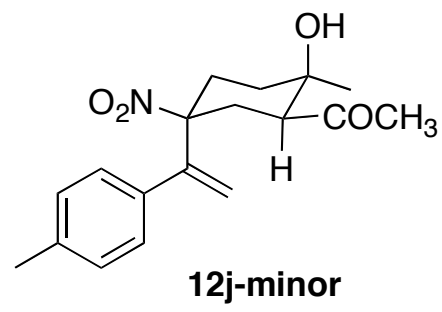

1-((1S,2R,5S)-2-hydroxy-2-methyl-5-nitro-5-(1-p-tolylvinyl)cyclohexyl)ethanone (12j-minor) was purified by flash silica gel chromatography (Hexane-EtOAc, v/v, 5/1) as 
colorless oil, yield: $21 \% .{ }^{1} \mathrm{H}$ NMR $\left(270 \mathrm{MHz}, \mathrm{CDCl}_{3}\right): \delta 7.17(\mathrm{~d}, J=8.2 \mathrm{~Hz}, 2 \mathrm{H}), 7.07(\mathrm{~d}$, $J=8.2 \mathrm{~Hz}, 2 \mathrm{H}), 5.88(\mathrm{~s}, 1 \mathrm{H}), 5.69(\mathrm{~s}, 1 \mathrm{H}), 3.88(\mathrm{~d}, J=2.5 \mathrm{~Hz}, 1 \mathrm{H}), 2.33-2.60(\mathrm{~m}, 8 \mathrm{H})$, $1.81(\mathrm{~s}, 3 \mathrm{H}), 1.74(\mathrm{dt}, J=14.4 \mathrm{~Hz}, J=3.8 \mathrm{~Hz}, 1 \mathrm{H}), 1.36-1.52(\mathrm{~m}, 1 \mathrm{H}), 1.17(\mathrm{~s}, 3 \mathrm{H}) .{ }^{13} \mathrm{C}$ NMR (67.5 MHz, $\left.\mathrm{CDCl}_{3}\right): \delta 213.1,143.9,138.2,136.0,129.1,128.0,123.6,90.9,68.5$, $52.8,35.9,31.0,30.2,29.5,28.1,21.1$. HRMS Calculated for $\mathrm{C}_{18} \mathrm{H}_{23} \mathrm{NO}_{4} \mathrm{Na}[\mathrm{M}+\mathrm{Na}]^{+}$: 340.15248, Found: 40.15169 .<smiles>C=C(c1ccc([N+](=O)[O-])cc1)C(C)(CC(O)C(=O)O)C(C)O</smiles>

\section{1-((1S,2R,5R)-2-hydroxy-2-methyl-5-nitro-5-(1-(4-nitrophenyl)vinyl)cyclohexyl)}

ethanone (12k-major) was purified by flash silica gel chromatography (Hexane-EtOAc, v/v, 8/1) as white solid, m.p: 106.9-108.1 ${ }^{\circ} \mathrm{C}$, yield: $62 \% .{ }^{1} \mathrm{H}$ NMR (600 MHz, $\mathrm{CDCl}_{3}$ ): $\delta$ 8.16(d, $J=9.0 \mathrm{~Hz}, 2 \mathrm{H}), 7.24(\mathrm{~d}, J=9.0 \mathrm{~Hz}, 2 \mathrm{H}), 5.74(\mathrm{~s}, 1 \mathrm{H}), 5.33(\mathrm{~s}, 1 \mathrm{H}), 3.69(\mathrm{br}, 1 \mathrm{H})$, $2.64(\mathrm{dd}, J=12.6 \mathrm{~Hz}, J=3.0 \mathrm{~Hz}, 1 \mathrm{H}), 2.59(\mathrm{dt}, J=14.4 \mathrm{~Hz}, J=3.0 \mathrm{~Hz}, 1 \mathrm{H}), 2.52(\mathrm{dq}, J=$ $14.4 \mathrm{~Hz}, J=3.0 \mathrm{~Hz}, 1 \mathrm{H}), 2.22-2.28(\mathrm{~m}, 4 \mathrm{H}), 2.11(\mathrm{t}, J=13.2 \mathrm{~Hz}, 1 \mathrm{H}), 1.71(\mathrm{dt}, J=14.4$ $\mathrm{Hz}, J=3.0 \mathrm{~Hz}, 1 \mathrm{H}), 1.28(\mathrm{td}, J=14.4 \mathrm{~Hz}, J=3.6 \mathrm{~Hz}, 1 \mathrm{H}), 1.16(\mathrm{~s}, 3 \mathrm{H}) .{ }^{13} \mathrm{C}$ NMR $(150$ $\left.\mathrm{MHz}, \mathrm{CDCl}_{3}\right): \delta 214.0,147.7,146.9,145.0,129.7,123.4,120.4,92.6,68.2,52.5,34.8$, 32.1, 31.6, 29.1, 28.1. HRMS Calculated for $\mathrm{C}_{17} \mathrm{H}_{21} \mathrm{~N}_{2} \mathrm{O}_{6}[\mathrm{M}+\mathrm{H}]^{+}:$349.13941, Found: 349.13966 .<smiles>C=C(c1ccc([N+](=O)[O-])cc1)C1(C)CCC(O)C(C(C)=O)C1</smiles>

1-((1S,2R,5R)-2-hydroxy-2-methyl-5-nitro-5-(1-(4-nitrophenyl)vinyl)cyclohexyl)

ethanone (12k-minor) was purified by flash silica gel chromatography $\left(\mathrm{CH}_{2} \mathrm{Cl}_{2} / \mathrm{MeOH}\right.$, 
$\mathrm{v} / \mathrm{v}, 50 / 1)$ as colorless oil, yield: $25 \% .{ }^{1} \mathrm{H}$ NMR $\left(600 \mathrm{MHz}, \mathrm{CDCl}_{3}\right): \delta 8.24(\mathrm{~d}, J=9.0 \mathrm{~Hz}$, $2 \mathrm{H}), 7.40(\mathrm{~d}, J=9.0 \mathrm{~Hz}, 2 \mathrm{H}), 6.03(\mathrm{~s}, 1 \mathrm{H}), 5.79(\mathrm{~s}, 1 \mathrm{H}), 3.79(\mathrm{br}, 1 \mathrm{H}), 2.49-2.56(\mathrm{~m}, 2 \mathrm{H})$, $2.44(\mathrm{dq}, J=13.8 \mathrm{~Hz}, J=3.0 \mathrm{~Hz}, 1 \mathrm{H}), 2.33-2.40(\mathrm{~m}, 2 \mathrm{H}), 1.75(\mathrm{dt}, J=14.4 \mathrm{~Hz}, J=3.6 \mathrm{~Hz}$, $1 \mathrm{H}), 1.34(\mathrm{td}, J=12.6 \mathrm{~Hz}, J=3.6 \mathrm{~Hz}, 1 \mathrm{H}), 1.19(\mathrm{~s}, 3 \mathrm{H}) .{ }^{13} \mathrm{C} \mathrm{NMR}\left(150 \mathrm{MHz}, \mathrm{CDCl}_{3}\right): \delta$ 212.1, 147.7, 145.5, 142.8, 129.3, 125.9, 123.8, 90.5, 68.4, 53.1, 35.8, 31.1, 30.5, 29.1, 28.1. HRMS Calculated for $\mathrm{C}_{17} \mathrm{H}_{21} \mathrm{~N}_{2} \mathrm{O}_{6} \mathrm{Na}[\mathrm{M}+\mathrm{Na}]^{+}: 371.12136$, Found: 371.12125 .

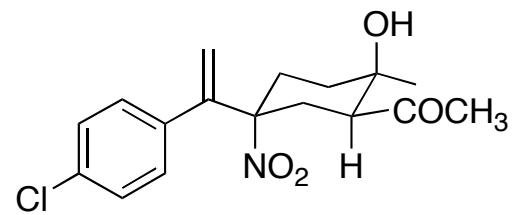

12I-major

1-((1S,2R,5R)-5-(1-(4-chlorophenyl)vinyl)-2-hydroxy-2-methyl-5-nitrocyclohex-

yl)ethanone (121-major) was purified by flash silica gel chromatography (HexaneEtOAc, v/v, 5/1) as white solid, m.p: 78.6-79.9 ${ }^{\circ} \mathrm{C}$, yield: $65 \% .{ }^{1} \mathrm{H}$ NMR $(270 \mathrm{MHz}$, $\left.\mathrm{CDCl}_{3}\right): \delta 7.29(\mathrm{~d}, J=8.4 \mathrm{~Hz}, 2 \mathrm{H}), 7.00(\mathrm{~d}, J=8.4 \mathrm{~Hz}, 2 \mathrm{H}), 5.65(\mathrm{~s}, 1 \mathrm{H}), 5.28(\mathrm{~s}, 1 \mathrm{H})$, $3.85(\mathrm{~d}, J=2.5 \mathrm{~Hz}, 1 \mathrm{H}), 2.48-2.71(\mathrm{~m}, 3 \mathrm{H}), 2.26(\mathrm{~s}, 3 \mathrm{H}), 2.00-2.34(\mathrm{~m}, 2 \mathrm{H}), 1.67-1.78(\mathrm{~m}$, $1 \mathrm{H}), 1.20-1.35(\mathrm{~m}, 1 \mathrm{H}), 1.17(\mathrm{~s}, 3 \mathrm{H}) .{ }^{13} \mathrm{C} \mathrm{NMR}\left(67.5 \mathrm{MHz}, \mathrm{CDCl}_{3}\right): \delta 214.3,147.5,136.6$, $134.1,129.9,128.3,119.4,92.9,68.2,52.6,34.8,32.0,31.6,29.0,28.2$. HRMS Calculated for $\mathrm{C}_{17} \mathrm{H}_{20} \mathrm{ClNO}_{4} \mathrm{Na}[\mathrm{M}+\mathrm{Na}]^{+}: 360.09786$, Found: 360.09710 .

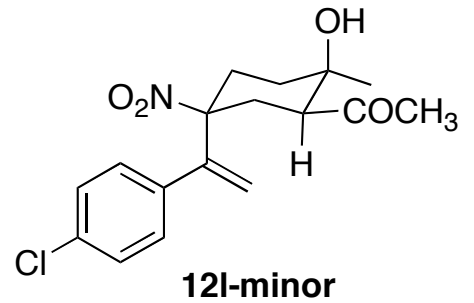

1-((1S,2R,5S)-5-(1-(4-chlorophenyl)vinyl)-2-hydroxy-2-methyl-5-nitrocyclohexyl)

ethanone (12l-minor) was purified by flash silica gel chromatography (Hexane-EtOAc, $\mathrm{v} / \mathrm{v}, 5 / 1)$ as colorless oil, yield: $18 \% .{ }^{1} \mathrm{H}$ NMR $\left(270 \mathrm{MHz}, \mathrm{CDCl}_{3}\right): \delta 7.36(\mathrm{~d}, J=8.4 \mathrm{~Hz}$, $2 \mathrm{H}), 7.14(\mathrm{~d}, J=8.4 \mathrm{~Hz}, 2 \mathrm{H}), 5.93(\mathrm{~s}, 1 \mathrm{H}), 5.72(\mathrm{~s}, 1 \mathrm{H}), 3.86(\mathrm{~d}, J=2.5 \mathrm{~Hz}, 1 \mathrm{H}), 2.30-$ $2.55(\mathrm{~m}, 5 \mathrm{H}), 1.88(\mathrm{~s}, 3 \mathrm{H}), 1.75(\mathrm{dt}, J=14.3 \mathrm{~Hz}, J=3.5 \mathrm{~Hz}, 1 \mathrm{H}), 1.31-46(\mathrm{~m}, 1 \mathrm{H}), 1.18(\mathrm{~s}$, 
$3 \mathrm{H}) .{ }^{13} \mathrm{C} \mathrm{NMR}\left(67.5 \mathrm{MHz}, \mathrm{CDCl}_{3}\right): \delta 212.7,143.1,137.3,134.4,129.5,128.7,124.6$, 90.7, 68.5, 52.8, 35.8, 31.0, 30.3, 29.3, 28.0. HRMS Calculated for $\mathrm{C}_{17} \mathrm{H}_{20} \mathrm{ClNO}_{4} \mathrm{Na}$ $[\mathrm{M}+\mathrm{Na}]^{+}: 360.09786$, Found: 360.09715 .

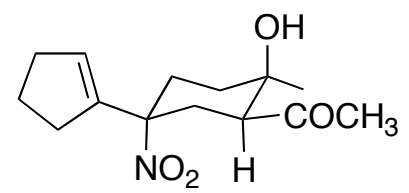

12m-major

\section{1-((1S,2R,5R)-5-cyclopentenyl-2-hydroxy-2-methyl-5-nitrocyclohexyl)ethanone}

(12m-major) was purified by flash silica gel chromatography (Hexane-EtOAc, v/v, 5/1) as colorless oil, yield: $61 \% .{ }^{1} \mathrm{H}$ NMR $\left(270 \mathrm{MHz}, \mathrm{CDCl}_{3}\right): \delta 5.73-5.79(\mathrm{~m}, 1 \mathrm{H}), 3.82(\mathrm{~d}, J=$ $2.7 \mathrm{~Hz}, 1 \mathrm{H}), 2.44-2.72(\mathrm{~m}, 3 \mathrm{H}), 1.95-2.36(\mathrm{~m}, 9 \mathrm{H}), 1.72-1.87(\mathrm{~m}, 2 \mathrm{H}), 1.62(\mathrm{dq}, J=14.6$ $\mathrm{Hz}, J=2.7 \mathrm{~Hz}, 1 \mathrm{H}), 1.09-1.25(\mathrm{~m}, 1 \mathrm{H}), 1.10(\mathrm{~s}, 3 \mathrm{H}) .{ }^{13} \mathrm{C} \mathrm{NMR}\left(67.5 \mathrm{MHz}, \mathrm{CDCl}_{3}\right): \delta$ $214.2,142.1,129.6,91.1,68.4,52.5,34.7,32.3,31.4,31.2,31.0,28.2,28.1,22.7$. HRMS Calculated for $\mathrm{C}_{14} \mathrm{H}_{21} \mathrm{NO}_{4} \mathrm{Na}[\mathrm{M}+\mathrm{Na}]^{+}: 290.13683$, Found: 290.13612 .

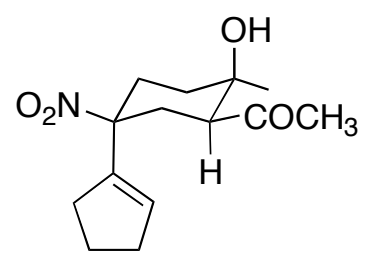

12m-minor

\section{1-((1S,2R,5S)-5-cyclopentenyl-2-hydroxy-2-methyl-5-nitrocyclohexyl)ethanone}

(12m-minor) was purified by flash silica gel chromatography (Hexane-EtOAc, v/v, 5/1) as colorless oil, yield: $15 \% .{ }^{1} \mathrm{H}$ NMR $\left(270 \mathrm{MHz}, \mathrm{CDCl}_{3}\right): \delta 5.77-5.85(\mathrm{~m}, 1 \mathrm{H}), 2.57-$ 2.89(m, 3H), 1.95-2.35(m, H), 1.71-1.89(m, 2H), $1.61(\mathrm{dq}, J=14.4 \mathrm{~Hz}, J=3.0 \mathrm{~Hz}, 1 \mathrm{H})$, 1.01-1.27(m, 1H), 1.12(s, 3H). ${ }^{13} \mathrm{C}$ NMR $\left(67.5 \mathrm{MHz}, \mathrm{CDCl}_{3}\right): \delta 210.7,142.1,130.1$, 90.9, 71.2, 55.3, 38.3, 32.6, 32.5, 32.2, 31.2, 30.7, 22.8, 21.4. HRMS Calculated for $\mathrm{C}_{14} \mathrm{H}_{21} \mathrm{NO}_{4} \mathrm{Na}[\mathrm{M}+\mathrm{Na}]^{+}: 290.13683$, Found: 290.13616 . 


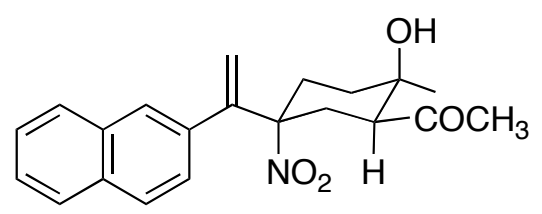

12n-major

1-((1S,2R,5R)-2-hydroxy-2-methyl-5-(1-(naphthalen-2-yl)vinyl)-5-nitrocyclohex-yl)

ethanone (12n-major) was purified by flash silica gel chromatography (Hexane-EtOAc, v/v, 10/1) as white solid, m.p: 133.9-134.8 ${ }^{\circ} \mathrm{C}$, yield: 64\%. ${ }^{1} \mathrm{H}$ NMR (600 MHz, $\left.\mathrm{CDCl}_{3}\right)$ : $\delta 7.77-7.85(\mathrm{~m}, 3 \mathrm{H}), 7.48-7.56(\mathrm{~m}, 3 \mathrm{H}), 7.21(\mathrm{~d}, J=8.4 \mathrm{~Hz}, 1 \mathrm{H}), 5.72(\mathrm{~s}, 1 \mathrm{H}), 5.37(\mathrm{~s}, 1 \mathrm{H})$, 3.81(br, 1H), 2.60-2.71(m, 3H), 2.36(td, $J=13.8 \mathrm{~Hz}, J=4.2 \mathrm{~Hz}, 1 \mathrm{H}), 2.23(\mathrm{~s}, 3 \mathrm{H}), 2.19(\mathrm{t}$, $J=13.8 \mathrm{~Hz}, 1 \mathrm{H}), 1.73(\mathrm{dt}, J=14.4 \mathrm{~Hz}, J=3.6 \mathrm{~Hz}, 1 \mathrm{H}), 1.31(\mathrm{td}, J=13.8 \mathrm{~Hz}, J=3.6 \mathrm{~Hz}$, $1 \mathrm{H}), 1.17(\mathrm{~s}, 3 \mathrm{H}) .{ }^{13} \mathrm{C} \mathrm{NMR}\left(150 \mathrm{MHz}, \mathrm{CDCl}_{3}\right): \delta 214.3,148.7,135.9,132.8,132.7$, $128.0,127.73,127.65,127.5,126.43,126.38,126.3,119.1,93.2,68.3,52.7,34.9,32.3$, 31.5, 29.2, 28.2. HRMS Calculated for $\mathrm{C}_{21} \mathrm{H}_{23} \mathrm{NO}_{4} \mathrm{Na}[\mathrm{M}+\mathrm{Na}]^{+}$: 376.15193, Found: 376.15208 .

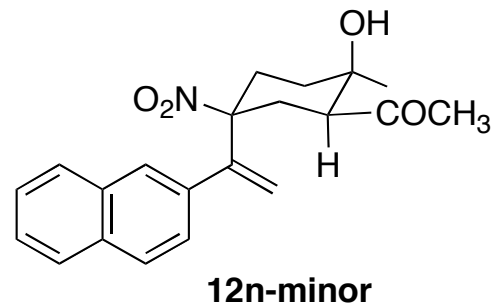

\section{1-((1S,2R,5S)-2-hydroxy-2-methyl-5-(1-(naphthalen-2-yl)vinyl)-5-nitrocyclohex-}

yl)ethanone (12n-minor) was purified by flash silica gel chromatography (HexaneEtOAc, v/v, 10/1) as colorless oil, yield: $18 \%$. ${ }^{1} \mathrm{H}$ NMR (600 $\left.\mathrm{MHz}, \mathrm{CDCl}_{3}\right): \delta 7.81-$ 7.86(m, 3H), 7.67(s, 1H), 7.50-7.54(m, 2H), 7.30(dd, $J=6.4 \mathrm{~Hz}, J=1.8 \mathrm{~Hz}, 1 \mathrm{H}), 6.00(\mathrm{~s}$, $1 \mathrm{H}), 5.82(\mathrm{~s}, 1 \mathrm{H}), 3.82(\mathrm{br}, 1 \mathrm{H}), 2.40-2.61(\mathrm{~m}, 5 \mathrm{H}), 1.76(\mathrm{dt}, J=14.4 \mathrm{~Hz}, J=3.6 \mathrm{~Hz}, 1 \mathrm{H})$, $1.69(\mathrm{~s}, 3 \mathrm{H}), 1.49(\mathrm{td}, J=13.8 \mathrm{~Hz}, J=4.2 \mathrm{~Hz}, 1 \mathrm{H}), 1.19(\mathrm{~s}, 3 \mathrm{H}) .{ }^{13} \mathrm{C} \mathrm{NMR}(150 \mathrm{MHz}$, $\left.\mathrm{CDCl}_{3}\right): \delta 212.9,144.2,136.4,133.0,132.8,128.2,127.9,127.6,127.3,126.8,126.6$, 125.9, 124.4, 91.0, 68.5, 52.9, 35.9, 31.2, 30.1, 29.6, 28.1. HRMS Calculated for $\mathrm{C}_{21} \mathrm{H}_{23} \mathrm{NO}_{4} \mathrm{Na}[\mathrm{M}+\mathrm{Na}]^{+}$: 376.15193, Found: 376.15207 . 


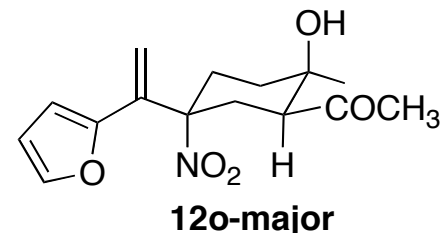

1-((1S,2R,5R)-5-(1-(furan-2-yl)vinyl)-2-hydroxy-2-methyl-5-nitrocyclohexyl)ethanone (120-major) was purified by flash silica gel chromatography (Hexane-EtOAc, v/v, 8/1) as light-yellow solid, m. p: $>70{ }^{\circ} \mathrm{C}$ decomp, yield: $56 \%$. ${ }^{1} \mathrm{H}$ NMR $(270 \mathrm{MHz}$, $\left.\mathrm{CDCl}_{3}\right): \delta 7.29-7.38(\mathrm{~m}, 1 \mathrm{H}), 6.29-6.43(\mathrm{~m}, 2 \mathrm{H}), 5.77(\mathrm{~s}, 1 \mathrm{H}), 5.43(\mathrm{~s}, 1 \mathrm{H}), 3.91(\mathrm{~d}, J=2.5$ $\mathrm{Hz}, 1 \mathrm{H}), 2.81-2.91(\mathrm{~m}, 1 \mathrm{H}), 2.73(\mathrm{td}, J=2.7 \mathrm{~Hz}, J=14.4 \mathrm{~Hz}, 1 \mathrm{H}), 2.41-2.58(\mathrm{~m}, 2 \mathrm{H})$, 2.19-2.31(m 4H), 1.73(td, $J=3.8 \mathrm{~Hz}, J=14.4 \mathrm{~Hz}, 1 \mathrm{H}), 1.34-1.68(\mathrm{~m}, 1 \mathrm{H}), 1.98(\mathrm{~s}, 3 \mathrm{H})$. ${ }^{13} \mathrm{C} \mathrm{NMR}\left(67.5 \mathrm{MHz}, \mathrm{CDCl}_{3}\right): \delta 214.5,150.3,142.65,142.56,138.0,115.6,111.3,108.7$, 92.3, 68.7, 52.7, 34.8, 32.4, 31.7, 29.2, 28.5. HRMS Calculated for $\mathrm{C}_{15} \mathrm{H}_{19} \mathrm{NO}_{5} \mathrm{Na}$ $[\mathrm{M}+\mathrm{Na}]^{+}: 316.11609$, Found: 316.11538.

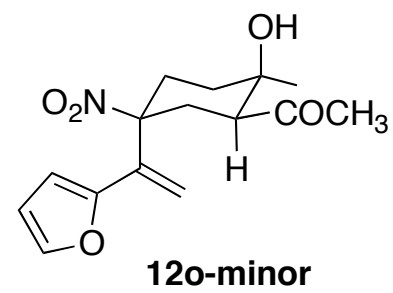

\section{1-((1S,2R,5S)-5-(1-(furan-2-yl)vinyl)-2-hydroxy-2-methyl-5-nitrocyclohexyl)eth-}

anone (120-minor) was purified by flash silica gel chromatography (Hexane-EtOAc, v/v, 8/1) as light-yellow oil, yield: $20 \% .{ }^{1} \mathrm{H}$ NMR $\left(270 \mathrm{MHz}, \mathrm{CDCl}_{3}\right): \delta 7.41(\mathrm{~s}, 1 \mathrm{H}), 6.40(\mathrm{~d}, J$ $=9.7 \mathrm{~Hz}, 2 \mathrm{H}), 6.11(\mathrm{~s}, 1 \mathrm{H}), 5.8(\mathrm{~s}, 1 \mathrm{H}), 3.88(\mathrm{~d}, J=2.2 \mathrm{~Hz}, 1 \mathrm{H}), 2.40-2.76(\mathrm{~m}, 5 \mathrm{H}), 2.03(\mathrm{~s}$, $3 \mathrm{H}), 1.80(\mathrm{td}, J=14.1 \mathrm{~Hz}, J=3.5 \mathrm{~Hz}, 1 \mathrm{H}), 1.41-1.56(\mathrm{~m}, 1 \mathrm{H}), 1.19(\mathrm{~s}, 3 \mathrm{H}) .{ }^{13} \mathrm{C} \mathrm{NMR}$ $\left(67.5 \mathrm{MHz}, \mathrm{CDCl}_{3}\right): \delta 213.0,150.8,142.6,142.5,133.6,121.8,111.1,109.0,90.6,68.7$, 53.2, 36.1, 30.9, 30.5, 29.3, 28.0. HRMS Calculated for $\mathrm{C}_{15} \mathrm{H}_{19} \mathrm{NO}_{5} \mathrm{Na}[\mathrm{M}+\mathrm{Na}]^{+}$: 316.11609, Found: 316.11537. 


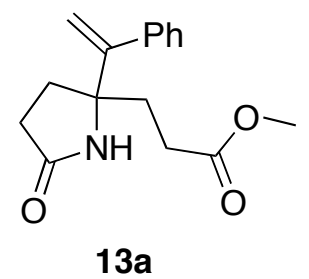

Methyl 3-(5-0xo-2-(1-phenylvinyl)pyrrolidin-2-yl)propanoate (13a) was purified by flash silica gel chromatography (Hexane-EtOAc, v/v, 3/1) as white solid, yield: $91 \% .{ }^{1} \mathrm{H}$ NMR $\left(600 \mathrm{MHz}, \mathrm{CDCl}_{3}\right): \delta$ 7.46(s, $\left.1 \mathrm{H}\right), 7.29-7.34(\mathrm{~m}, 3 \mathrm{H}), 7.21-7.23(\mathrm{~m}, 2 \mathrm{H}), 5.38(\mathrm{~s}$, $1 \mathrm{H}), 5.16(\mathrm{~m}, 1 \mathrm{H}), 3.63(\mathrm{~s}, 3 \mathrm{H}), 2.31-2.44(\mathrm{~m}, 5 \mathrm{H}), 2.07-2.14(\mathrm{~m}, 2 \mathrm{H}), 1.93-1.99(\mathrm{~m}, 1 \mathrm{H})$. ${ }^{13} \mathrm{C}$ NMR $\left(150 \mathrm{MHz}, \mathrm{CDCl}_{3}\right): \delta 177.6,173.5,152.1,140.2,128.2,128.1,127.6,1115.4$, 65.4, 51.7, 34.1, 33.2, 30.0, 29.1. HRMS Calculated for $\mathrm{C}_{16} \mathrm{H}_{20} \mathrm{NO}_{3}[\mathrm{M}+\mathrm{H}]^{+}$: 274.14377, Found: 274.14383 .

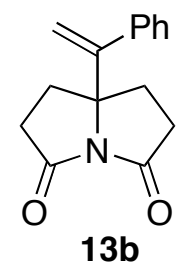

Tetrahydro-7a-(1-phenylvinyl)-6H-pyrrolizine-3,5-dione (13b) was purified by flash silica gel chromatography (Hexane-EtOAc, v/v, 2/1) as white solid, m.p: 141.2-143.0 ${ }^{\circ} \mathrm{C}$, yield: $83 \% .{ }^{1} \mathrm{H}$ NMR $\left(600 \mathrm{MHz}, \mathrm{CDCl}_{3}\right): \delta 7.35-7.39(\mathrm{~m}, 5 \mathrm{H}), 5.52(\mathrm{~s}, 1 \mathrm{H}), 5.40(\mathrm{~s}, 1 \mathrm{H})$, 2.68-2.76(m, 2H), 2.55-2.61(m, 4H), 2.13-2.18(m, 2H). $\left.{ }^{13} \mathrm{C} \mathrm{NMR} \mathrm{(150} \mathrm{MHz,} \mathrm{CDCl}_{3}\right): \delta$ 171.4, 148.0, 138.2, 128.6, 128.2, 127.6, 115.4, 72.0, 34.5, 31.1. HRMS Calculated for $\mathrm{C}_{15} \mathrm{H}_{15} \mathrm{NO}_{2} \mathrm{Na}[\mathrm{M}+\mathrm{Na}]^{+}: 264.09950$, Found: 264.09953.

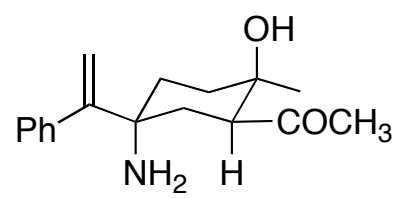

$13 c$ 
1-((1S,2R,5R)-5-amino-2-hydroxy-2-methyl-5-(1-phenylvinyl)cyclohexyl)ethan-one

(13c) was purified by flash silica gel chromatography (Hexane-EtOAc, v/v, 2/1) as colorless oil, yiled: 88\%. ${ }^{1} \mathrm{H}$ NMR (600 MHz, $\left.\mathrm{CDCl}_{3}\right): \delta 7.22-7.29(\mathrm{~m}, 3 \mathrm{H}), 7.11-7.14(\mathrm{~m}$, 2H), 5.28(s, 1H), 4.84(s, 1H), 3.86(br, 1H), 3.09(dd, $J=13.2 \mathrm{~Hz}, J=3.6 \mathrm{~Hz}, 1 \mathrm{H}), 2.15(\mathrm{~s}$, $3 \mathrm{H}), 2.09(\mathrm{td}, J=13.2 \mathrm{~Hz}, J=4.2 \mathrm{~Hz}, 1 \mathrm{H}), 1.98(\mathrm{t}, J=13.2 \mathrm{~Hz}, 1 \mathrm{H}), 1.67(\mathrm{td}, J=10.2 \mathrm{~Hz}$, $J=4.2 \mathrm{~Hz}, 1 \mathrm{H}), 1.44-1.50(\mathrm{~m}, 2 \mathrm{H}), 1.28-1.33(\mathrm{~m}, 1 \mathrm{H}), 1.17(\mathrm{~s}, 3 \mathrm{H}) .{ }^{13} \mathrm{C}$ NMR $(150 \mathrm{MHz}$, $\left.\mathrm{CDCl}_{3}\right): \delta 216.4,159.2,141.4,129.0,127.6,126.7,111.9,69.1,53.7,52.3,35.0,34.0$, 31.4, 31.1, 28.6. HRMS Calculated for $\mathrm{C}_{17} \mathrm{H}_{24} \mathrm{NO}_{2}[\mathrm{M}+\mathrm{H}]^{+}$: 274.18016, Found: 274.18012.

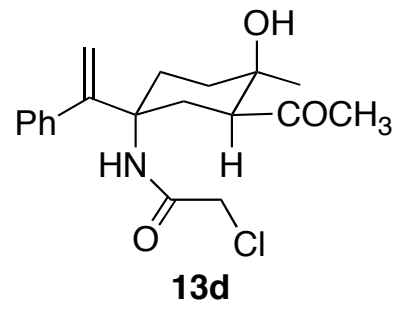

13d was purified by flash silica gel chromatography (Hexane-EtOAc, v/v, 1/1) as white solid, m.p: 154.2-155.9 ${ }^{\circ} \mathrm{C}$, yield: 91\%. ${ }^{1} \mathrm{H}$ NMR (600 MHz, $\left.\mathrm{CDCl}_{3}\right): \delta$ 7.24-7.28(m, 3H), 7.08-7.11(m, 2H), 6.24(s, 1H), 5.36(s, 1H), 5.04(s, 1H), 3.95(d, $J=2.4 \mathrm{~Hz}, 1 \mathrm{H}), 3.94(\mathrm{~d}, J$ $=15.0 \mathrm{~Hz}, 1 \mathrm{H}), 3.87(\mathrm{~d}, J=15.0 \mathrm{~Hz}, 1 \mathrm{H}), 2.90(\mathrm{dt}, J=13.2 \mathrm{~Hz}, J=3.0 \mathrm{~Hz}, 1 \mathrm{H}), 2.56(\mathrm{dd}$, $J=13.2 \mathrm{~Hz}, J=3.0 \mathrm{~Hz}, 1 \mathrm{H}), 2.18(\mathrm{~s}, 3 \mathrm{H}), 2.09(\mathrm{td}, J=13.8 \mathrm{~Hz}, J=4.2 \mathrm{~Hz}, 1 \mathrm{H}), 1.98(\mathrm{t}, J$ $=13.8 \mathrm{~Hz}, 1 \mathrm{H}), 1.71(\mathrm{dq}, J=13.8 \mathrm{~Hz}, J=3.0 \mathrm{~Hz}, 1 \mathrm{H}), 1.60(\mathrm{dq}, J=14.4 \mathrm{~Hz}, J=3.0 \mathrm{~Hz}$, $1 \mathrm{H}), 1.37(\mathrm{tt}, J=13.8 \mathrm{~Hz}, J=3.0 \mathrm{~Hz}, 1 \mathrm{H}), 1.18(\mathrm{~s}, 3 \mathrm{H}) .{ }^{13} \mathrm{C} \mathrm{NMR}\left(150 \mathrm{MHz}, \mathrm{CDCl}_{3}\right): \delta$ $215.2,165.3,152.5,140.6,128.7,127.7,127.1,114.9,68.4,58.9,52.8,42.9,34.4,32.2$, 30.8, 29.8, 28.5. HRMS Calculated for $\mathrm{C}_{19} \mathrm{H}_{25} \mathrm{ClNO}_{3}[\mathrm{M}+\mathrm{H}]^{+}: 350.15175$, Found: 350.15174 . 


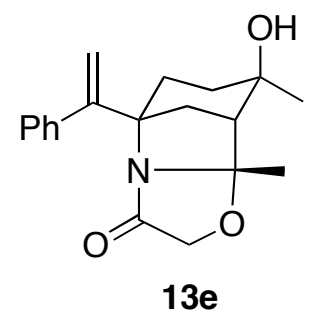

13e was purified by flash silica gel chromatography (Hexane-EtOAc, v/v, 2/1) as colorless oil, yield: $83 \%$. ${ }^{1} \mathrm{H}$ NMR $\left(600 \mathrm{MHz}, \mathrm{CDCl}_{3}\right): \delta 7.27-7.32(\mathrm{~m}, 3 \mathrm{H}), 7.17-7.23(\mathrm{~m}$, $2 \mathrm{H}), 5.44(\mathrm{~s}, 1 \mathrm{H}), 5.10(\mathrm{~s}, 1 \mathrm{H}), 4.65(\mathrm{~d}, J=14.4 \mathrm{~Hz}, 1 \mathrm{H}), 4.98(\mathrm{~d}, J=14.4 \mathrm{~Hz}, 1 \mathrm{H}), 4.04(\mathrm{~s}$, $1 \mathrm{H}), 2.53-2.58(\mathrm{~m}, 1 \mathrm{H}), 2.28(\mathrm{ddd}, J=13.2 \mathrm{~Hz}, J=4.8 \mathrm{~Hz}, J=3.6 \mathrm{~Hz}, 1 \mathrm{H}), 1.98(\mathrm{~d}, J=$ $4.8 \mathrm{~Hz}, 1 \mathrm{H}), 1.86-1.94(\mathrm{~m}, 2 \mathrm{H}), 1.74-1.81(\mathrm{~m}, 1 \mathrm{H}), 1.57-1.64(\mathrm{~m}, 1 \mathrm{H}), 1.28(\mathrm{~s}, 3 \mathrm{H}), 1.25(\mathrm{~s}$, $3 \mathrm{H}) .{ }^{13} \mathrm{C}$ NMR $\left(150 \mathrm{MHz}, \mathrm{CDCl}_{3}\right): \delta 172.2,150.2,140.2,129.1,127.7,127.3,115.1$, 106.7, 71.9, 71.4, 67.2, 51.4, 42.8, 35.8, 29.3, 27.3, 25.5. HRMS Calculated for $\mathrm{C}_{19} \mathrm{H}_{24} \mathrm{NO}_{3} \mathrm{Na}[\mathrm{M}+\mathrm{H}]^{+}: 314.17507$, Found: 314.17518 . 


\section{Part III}

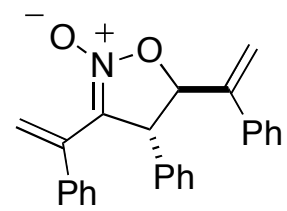

$15 a$

\section{4-Phenyl-3, 5-bis (1-phenylvinyl) isoxazoline- $N$-oxide (15a).}

Purified by flash chromatography (hexane-EtOAc) as colorless oil (yield: 92\%); ${ }^{1} \mathrm{H}$ NMR $\left(600 \mathrm{MHz}, \mathrm{CDCl}_{3}\right) \delta=7.37-7.34(\mathrm{~m}, 5 \mathrm{H}), 7.22-7.17(\mathrm{~m}, 4 \mathrm{H}), 7.15-7.12(\mathrm{~m}, 2 \mathrm{H}), 6.85-$ $6.81(\mathrm{~m}, 4 \mathrm{H}), 6.17(\mathrm{~s}, 1 \mathrm{H}), 5.67(\mathrm{~s}, 1 \mathrm{H}), 5.53(\mathrm{~d}, J=7.8 \mathrm{~Hz}, 2 \mathrm{H}), 5.31(\mathrm{~d}, J=4.2 \mathrm{~Hz}$, $1 \mathrm{H}), 4.15(\mathrm{~d}, J=5.4 \mathrm{~Hz}, 1 \mathrm{H}) ;{ }^{13} \mathrm{C} \mathrm{NMR}\left(150 \mathrm{MHz}, \mathrm{CDCl}_{3}\right): \delta=144.8,138.7,138.0$, $137.1,135.9,128.9,128.7,128.4,128.0,127.9,127.8,127.5,127.1,127.0,121.8,116.2$, 115.0, 82.9, 56.7; HRMS Calculated for $\left[\mathrm{C}_{25} \mathrm{H}_{21} \mathrm{NO}_{2}+\mathrm{H}\right]^{+}: 368.16450$, Found: 368.16474.

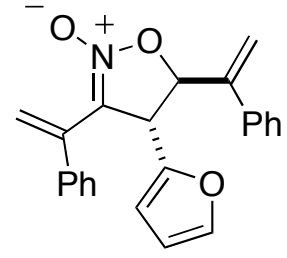

\section{$15 b$}

\section{4-Furyl-3, 5-bis (1-phenylvinyl) isoxazoline- $N$-oxide (15b)}

Purified by flash chromatography (hexane-EtOAc) as colorless oil (yield: 85\%); ${ }^{1} \mathrm{H}$ NMR $\left(600 \mathrm{MHz}, \mathrm{CDCl}_{3}\right) \delta=7.41-7.33(\mathrm{~m}, 5 \mathrm{H}), 7.30-7.29(\mathrm{~s}, 1 \mathrm{H}), 7.23-7.17(\mathrm{~m}, 3 \mathrm{H}), 6.93-$ $6.91(\mathrm{~m}, 2 \mathrm{H}), 6.19(\mathrm{~s}, 1 \mathrm{H}), 6.16(\mathrm{~s}, 1 \mathrm{H}), 5.82(\mathrm{~d}, J=3.0 \mathrm{~Hz}, 1 \mathrm{H}), 5.67(\mathrm{~s}, 1 \mathrm{H}), 5.60(\mathrm{~s}$, $1 \mathrm{H}), 5.57(\mathrm{~s}, 1 \mathrm{H}), 5.57-5.47(\mathrm{~m}, 1 \mathrm{H}), 4.35(\mathrm{~d}, J=4.2 \mathrm{~Hz}, 1 \mathrm{H}) ;{ }^{13} \mathrm{C}$ NMR $(150 \mathrm{MHz}$, $\left.\mathrm{CDCl}_{3}\right): \delta=149.9,144.2,142.7,137.9,136.9,135.8,128.7,128.4,128.1,127.9,127.3$, 127.0, 121.6, 115.7, 113.6, 110.5, 108.0, 80.2, 50.3; HRMS Calculated for $\left[\mathrm{C}_{23} \mathrm{H}_{19} \mathrm{NO}_{3}+\mathrm{H}\right]^{+}:$358.14377, Found: 358.14397. 


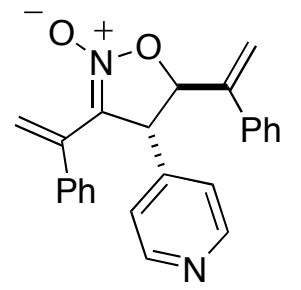

15c

\section{4-Pyridine-3, 5-bis (1-phenylvinyl) isoxazoline- $\mathrm{N}$-oxide (15c)}

Purified by flash chromatography (hexane-EtOAc) as yellowish solid (yield: $91 \%$ ); ${ }^{1} \mathrm{H}$ NMR $\left(600 \mathrm{MHz}, \mathrm{CDCl}_{3}\right) \delta=8.42-8.41(\mathrm{dd}, J=4.8 \mathrm{~Hz}, J=3.6 \mathrm{~Hz}, 2 \mathrm{H}), 7.39-7.36(\mathrm{~m}$, $3 \mathrm{H}), 7.33-7.32(\mathrm{~m}, 2 \mathrm{H}), 7.21-7.18(\mathrm{~m}, 1 \mathrm{H}), 7.15-7.12(\mathrm{~m}, 2 \mathrm{H}), 6.80-6.78(\mathrm{dd}, J=7.2 \mathrm{~Hz}$, $J=1.8 \mathrm{~Hz}, 2 \mathrm{H}), 6.73-6.72(\mathrm{dd}, J=4.2 \mathrm{~Hz}, J=3.0 \mathrm{~Hz}, 2 \mathrm{H}), 6.30(\mathrm{~s}, 1 \mathrm{H}), 5.68(\mathrm{~s}, 1 \mathrm{H})$, $5.58(\mathrm{~s}, 1 \mathrm{H}), 5.56(\mathrm{~s}, 1 \mathrm{H}), 5.27-5.26(\mathrm{~d}, J=4.2 \mathrm{~Hz}, 1 \mathrm{H}), 4.13-4.12(\mathrm{~d}, J=3.6 \mathrm{~Hz}, 1 \mathrm{H}) ;{ }^{13} \mathrm{C}$ NMR (150 MHz, $\left.\mathrm{CDCl}_{3}\right): \delta=150.31,147.42,144.28,137.8,136.8,135.4,128.8,128.6$, 128.1, 128.0, 127.6, 127.0, 122.0, 115.5, 115.0, 82.0, 55.93; HRMS Calculated for $\left[\mathrm{C}_{24} \mathrm{H}_{20} \mathrm{~N}_{2} \mathrm{O}_{2}+\mathrm{Na}\right]^{+}:$391.14170, Found: 391.14190.

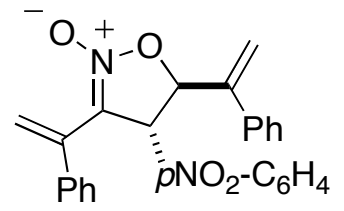

$15 d$

\section{4-p-Nitro-phenyl-3, 5-bis (1-phenylvinyl) isoxazoline- $N$-oxide (15d)}

Purified by flash chromatography (hexane-EtOAc) as colorless oil (yield: 95\%); ${ }^{1} \mathrm{H}$ NMR $\left(600 \mathrm{MHz} \mathrm{CDCl}_{3}\right) \delta=8.03-8.01(\mathrm{~d}, J=9.0 \mathrm{~Hz}, 2 \mathrm{H}), 7.38-7.37(\mathrm{~m}, 3 \mathrm{H}), 7.32-7.31(\mathrm{~m}$, 2H), 7.21-7.18 (t, $J=7.8 \mathrm{~Hz}, 1 \mathrm{H}), 7.14-7.11(\mathrm{t}, J=7.8 \mathrm{~Hz}, 2 \mathrm{H}), 6.93-6.92$ (d, $J=8.4 \mathrm{~Hz}$, 2H), 6.78-6.76 (d, J=7.2 Hz, 2H), $6.34(\mathrm{~s}, 1 \mathrm{H}), 5.69(\mathrm{~s}, 1 \mathrm{H}), 5.59(\mathrm{~s}, 1 \mathrm{H}), 5.56(\mathrm{~s}, 1 \mathrm{H})$, 5.28-5.27 (d, $J=3.6 \mathrm{~Hz}, 1 \mathrm{H}), 4.26-4.25(\mathrm{~d}, J=4.2 \mathrm{~Hz}, 1 \mathrm{H}) ;{ }^{13} \mathrm{C}$ NMR $(150 \mathrm{MHz}$, $\left.\mathrm{CDCl}_{3}\right): \delta=147.5,145.8,144.2,137.9,136.9,135.3,128.9,128.7,128.2,128.1,127.6$, 127.0, 124.1, 122.1, 115.6, 115.3, 82.2, 56.3; HRMS Calculated for $\left[\mathrm{C}_{25} \mathrm{H}_{20} \mathrm{~N}_{2} \mathrm{O}_{4}+\mathrm{H}\right]^{+}$: 413.14958, Found: 413.14967. 


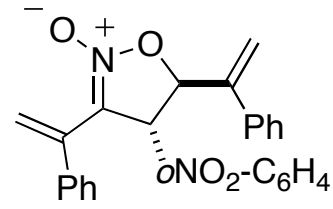

$15 \mathrm{e}$

\section{4-o-Nitro-3, 5-bis (1-phenylvinyl) isoxazoline- $N$-oxide (15e)}

Purified by flash chromatography (hexane-EtOAc) as colorless oil (yield: 86\%); ${ }^{1} \mathrm{H}$ NMR $\left(600 \mathrm{MHz}, \mathrm{CDCl}_{3}\right) \delta=7.68-7.67(\mathrm{~d}, J=8.4 \mathrm{~Hz}, 1 \mathrm{H}), 7.62-7.60$ (t, $\left.J=7.8 \mathrm{~Hz}, 1 \mathrm{H}\right), 7.47-$ $7.36(\mathrm{~m}, 7 \mathrm{H}), 7.16-7.13(\mathrm{t}, J=7.2 \mathrm{~Hz}, 1 \mathrm{H}), 7.05-7.02(\mathrm{t}, J=7.2 \mathrm{~Hz}, 2 \mathrm{H}), 6.47-6.46(\mathrm{~d}, J$ $=7.8 \mathrm{~Hz}, 2 \mathrm{H}), 6.36(\mathrm{~s}, 1 \mathrm{H}), 5.53(\mathrm{~s}, 1 \mathrm{H}), 5.48(\mathrm{~s}, 1 \mathrm{H}), 5.45(\mathrm{~s}, 1 \mathrm{H}), 5.18(\mathrm{~d}, J=3.6 \mathrm{~Hz}$, $1 \mathrm{H}), 5.00(\mathrm{~d}, J=3.6 \mathrm{~Hz}, 1 \mathrm{H}) ;{ }^{13} \mathrm{C}$ NMR $\left(150 \mathrm{MHz}, \mathrm{CDCl}_{3}\right): \delta=147.9,145.2,137.8$, $137.5,135.4,133.9,133.7,129.5,128.8,128.6,128.4,128.1,128.0,127.8,127.3,124.8$, 121.8, 118.5, 115.9, 84.0, 50.6; HRMS Calculated $\left[\mathrm{C}_{25} \mathrm{H}_{20} \mathrm{~N}_{2} \mathrm{O}_{4}+\mathrm{H}\right]^{+}:$413.14958, Found: 413.14967.

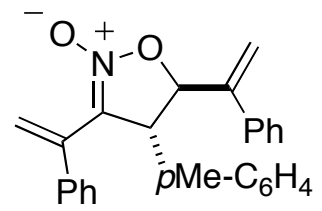

$15 f$

\section{4- p-Toulyl-3, 5-bis (1-phenylvinyl) isoxazoline- $N$-oxide (15f)}

Purified by flash chromatography (hexane-EtOAc) as colorless oil (yield: 93\%); ${ }^{1} \mathrm{H}$ NMR $\left(600 \mathrm{MHz}, \mathrm{CDCl}_{3}\right) \delta=7.36-7.33(\mathrm{~m}, 5 \mathrm{H}), 7.21-7.18(\mathrm{t}, J=7.2 \mathrm{~Hz}, 1 \mathrm{H}), 7.16-7.13(\mathrm{~m}$, 2H), $7.01(\mathrm{~d}, J=7.8 \mathrm{~Hz}, 2 \mathrm{H}), 6.85-6.83(\mathrm{~m}, 2 \mathrm{H}), 6.76-6.75(\mathrm{~d}, J=8.4 \mathrm{~Hz}, 2 \mathrm{H}), 6.11$ (s, $1 \mathrm{H}), 5.66$ (s, 1H), 5.54-5.52 (d, $J=11.8 \mathrm{~Hz}, 2 \mathrm{H}), 5.29-5.28(\mathrm{dd}, J=3.6 \mathrm{~Hz}, J=3.0 \mathrm{~Hz}$, $1 \mathrm{H}), 4.11(\mathrm{~d}, J=3.6 \mathrm{~Hz}, 1 \mathrm{H}), 2.29(\mathrm{~s}, 1 \mathrm{H}) ;{ }^{13} \mathrm{C} \mathrm{NMR}\left(150 \mathrm{MHz}, \mathrm{CDCl}_{3}\right): \delta=144.88$, 138.0, 137.7, 137.1, 135.9, 135.7, 129.6, 128.7, 128.3, 127.9, 127.7, 127.5, 127.0, 121.7, 116.2, 114.8, 83.0, 56.4, 21.0; HRMS Calculated for $\left[\mathrm{C}_{26} \mathrm{H}_{23} \mathrm{NO}_{2}+\mathrm{H}\right]^{+}: 382.18016$, Found: 382.18161. 


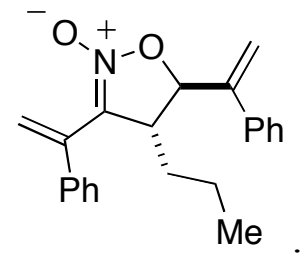

$15 \mathrm{~g}$

\section{4-Propyl-3, 5-bis (1-phenylvinyl) isoxazoline- $\mathrm{N}$-oxide (15g)}

Purified by flash chromatography (hexane-EtOAc) as colorless oil (yield: 61\%); ${ }^{1} \mathrm{H}$ NMR $\left(600 \mathrm{MHz}, \mathrm{CDCl}_{3}\right) \delta=7.38-7.34(\mathrm{~m}, 5 \mathrm{H}), 7.25-7.22(\mathrm{~m}, 3 \mathrm{H}), 6.99-6.89$ (dd, $J=6.0 \mathrm{~Hz}, J$ $=6.0 \mathrm{~Hz}, 2 \mathrm{H}), 6.27(\mathrm{~s}, 1 \mathrm{H}), 5.71(\mathrm{~s}, 1 \mathrm{H}), 5.63(\mathrm{~s}, 1 \mathrm{H}), 5.43(\mathrm{~s}, 1 \mathrm{H}), 5.09(\mathrm{~d}, J=2.4 \mathrm{~Hz}$, $1 \mathrm{H}), 3.06-3.04(\mathrm{~m}, 1 \mathrm{H}), 1.47-1.41(\mathrm{~m}, 1 \mathrm{H}), 1.31-1.25(\mathrm{~m}, 1 \mathrm{H}), 1.22-1.16(\mathrm{~m}, 1 \mathrm{H}), 1.09$ $1.02(\mathrm{~m}, 1 \mathrm{H}), 0.58-0.55(\mathrm{t}, J=7.2 \mathrm{~Hz}, 3 \mathrm{H}) ;{ }^{13} \mathrm{C} \mathrm{NMR}\left(150 \mathrm{MHz}, \mathrm{CDCl}_{3}\right): \delta=145.81$, $138.29,137.88,135.87,128.68,128.28,128.0,127.4,127.2,121.4,116.4,115.1,80.3$, 49.5, 33.85, 18.68, 13.2; HRMS Calculated for $\left[\mathrm{C}_{22} \mathrm{H}_{23} \mathrm{NO}_{2}+\mathrm{H}\right]^{+}$: 334.18016, Found: 334.18031.

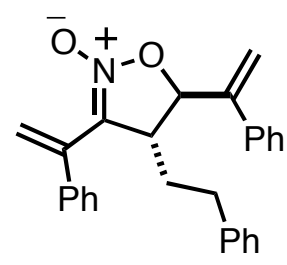

$15 h$

\section{4-Phenyl-ethyl-3, 5-bis (1-phenylvinyl) isoxazoline- $\mathrm{N}$-oxide (15h)}

Purified by flash chromatography (hexane-EtOAc) as colorless oil (yield: 57\%); ${ }^{1} \mathrm{H}$ NMR $\left(600 \mathrm{MHz}, \mathrm{CDCl}_{3}\right) \delta=7.39-7.33(\mathrm{~m}, 5 \mathrm{H}), 7.25-7.22(\mathrm{~m}, 1 \mathrm{H}), 7.20-7.17(\mathrm{~m}, 2 \mathrm{H}), 7.12-$ $7.09(\mathrm{~m}, 3 \mathrm{H}), 6.92-6.90(\mathrm{~m}, 2 \mathrm{H}), 6.73-6.71(\mathrm{dd}, J=8.4 \mathrm{~Hz}, J=6.0 \mathrm{~Hz}, 2 \mathrm{H}), 6.24(\mathrm{~s}, 1 \mathrm{H})$, $5.68(\mathrm{~d}, J=1.2 \mathrm{~Hz}, 1 \mathrm{H}), 5.62(\mathrm{t}, J=1.2 \mathrm{~Hz}, 1 \mathrm{H}), 5.41(\mathrm{~s}, 1 \mathrm{H}), 5.16(\mathrm{~d}, J=2.4 \mathrm{~Hz}, 1 \mathrm{H})$, 3.09-3.07 (m, 1H), 2.51-2.46 (m, 1H), 2.35-2.30 (m, 1H), 1.80-1.74 (m, 1H), 1.69-1.63 $(\mathrm{m}, 1 \mathrm{H}) ;{ }^{13} \mathrm{C} \mathrm{NMR}\left(150 \mathrm{MHz}, \mathrm{CDCl}_{3}\right): \delta=145.7,139.9,138.1,137.8,135.7,128.7$, $128.36,128.34,128.1,127.9,127.4,127.3,125.9,121.6,116.1,115.6,80.4,49.3,33.1$, 31.6; HRMS Calculated for $\left[\mathrm{C}_{27} \mathrm{H}_{25} \mathrm{NO}_{2}+\mathrm{H}\right]^{+}: 396.19581$, Found: 396.19613. 


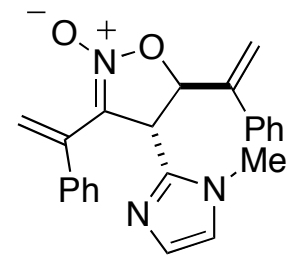

$15 i$

\section{4-Methyl-imidazole-3, 5-bis (1-phenylvinyl) isoxazoline- $N$-oxide (15i)}

Purified by flash chromatography (hexane-EtOAc) as colorless oil (yield: $81 \%$ ); ${ }^{1} \mathrm{H}$ NMR $\left(600 \mathrm{MHz}, \mathrm{CDCl}_{3}\right) \delta=7.28-7.25(\mathrm{~m}, 3 \mathrm{H}), 7.22-7.20(\mathrm{~m}, 2 \mathrm{H}), 7.17-7.12(\mathrm{~m}, 3 \mathrm{H}), 6.96(\mathrm{~d}$, $J=1.2 \mathrm{~Hz}, 1 \mathrm{H}), 6.81-6.79(\mathrm{~m}, 2 \mathrm{H}), 6.47(\mathrm{~s}, 1 \mathrm{H}), 6.36(\mathrm{~d}, J=1.2 \mathrm{~Hz}, 1 \mathrm{H}), 5.67(\mathrm{~s}, 1 \mathrm{H})$, 5.64-5.64 (d, $J=6.6 \mathrm{~Hz}, 1 \mathrm{H}), 5.55(\mathrm{~s}, 1 \mathrm{H}), 5.49(\mathrm{~s}, 1 \mathrm{H}), 4.28-4.26(\mathrm{~d}, J=7.2 \mathrm{~Hz}, 1 \mathrm{H})$, $2.19(\mathrm{~s}, 3 \mathrm{H}) ;{ }^{13} \mathrm{C} \mathrm{NMR}\left(150 \mathrm{MHz}, \mathrm{CDCl}_{3}\right): \delta=144.0,143.5,138.8,137.1,135.2,128.7$, $128.6,128.4,128.1,127.7,127.4,126.9,121.9,120.5,115.4,114.8,81.1,84.2,30.9$; HRMS Calculated for $\left[\mathrm{C}_{23} \mathrm{H}_{21} \mathrm{~N}_{3} \mathrm{O}_{2}+\mathrm{H}\right]^{+}: 372.17065$, Found: 372.17094 .

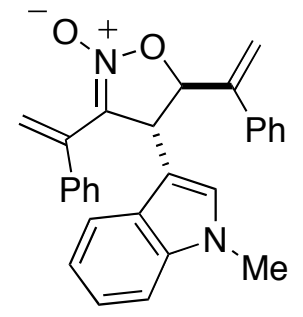

15j

\section{4-N-methyl-indole-3, 5-bis (1-phenylvinyl) isoxazoline- $\mathrm{N}$-oxide (15j)}

Purified by flash chromatography (hexane-EtOAc) as colorless oil (yield: 53\%); ${ }^{1} \mathrm{H}$ NMR $\left(600 \mathrm{MHz}, \mathrm{CDCl}_{3}\right) \delta=7.41-7.39(\mathrm{~m}, 2 \mathrm{H}), 7.38-7.35(\mathrm{~m}, 4 \mathrm{H}), 7.22-7.17(\mathrm{~m}, 3 \mathrm{H}), 7.13-$ $7.10(\mathrm{t}, J=7.8 \mathrm{~Hz}, 2 \mathrm{H}), 7.04-7.02(\mathrm{t}, J=7.8 \mathrm{~Hz}, 1 \mathrm{H}), 6.83-6.81(\mathrm{~m}, 2 \mathrm{H}), 6.49(\mathrm{~s}, 1 \mathrm{H})$, $6.14(\mathrm{~d}, J=1.2 \mathrm{~Hz}, 1 \mathrm{H}), 5.70(\mathrm{~s}, 1 \mathrm{H}), 5.56(\mathrm{~s}, 1 \mathrm{H}), 5.50(\mathrm{~d}, J=1.2 \mathrm{~Hz}, 1 \mathrm{H}), 5.42(\mathrm{~d}, J=$ $3.0 \mathrm{~Hz}, 1 \mathrm{H}), 5.45(\mathrm{~d}, J=3.6 \mathrm{~Hz}, 1 \mathrm{H}), 3.64(\mathrm{~s}, 3 \mathrm{H}) ;{ }^{13} \mathrm{C} \mathrm{NMR}\left(150 \mathrm{MHz}, \mathrm{CDCl}_{3}\right): \delta=$ $145.2,138.2,137.4,137.1,136.0,128.9,128.7,128.3,127.9,127.6,127.5,127.19$, 127.12, 125.7, 122.0, 121.6, 119.6, 118.8, 115.7, 114.6, 111.5, 109.3, 82.29, 48.9, 32.6; HRMS Calculated for $\left[\mathrm{C}_{28} \mathrm{H}_{24} \mathrm{~N}_{2} \mathrm{O}_{2}+\mathrm{H}\right]^{+}: 421.19105$, Found: 421.19143. 


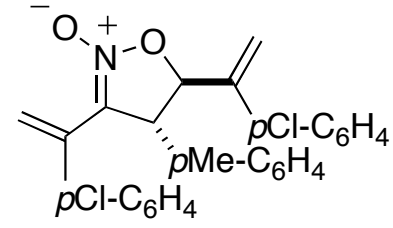

$15 \mathrm{k}$

\section{4-p-Toulyl-3, 5-bis (p-chloribenzylvinyl) isoxazoline- $N$-oxide (15k)}

Purified by flash chromatography (hexane-EtOAc) as colorless oil (yield: 95\%); ${ }^{1} \mathrm{H}$ NMR $\left(600 \mathrm{MHz}, \mathrm{CDCl}_{3}\right) \delta=7.35-7.33(\mathrm{~m}, 2 \mathrm{H}), 7.30-7.28(\mathrm{~m}, 2 \mathrm{H}), 7.15-7.13(\mathrm{dt}, J=8.4 \mathrm{~Hz}, J$ $=4.8 \mathrm{~Hz}, 2 \mathrm{H}), 7.06-7.05(\mathrm{~d}, J=7.8 \mathrm{~Hz}, 2 \mathrm{H}), 6.81-6.79$ (d, $J=7.8 \mathrm{~Hz}, 2 \mathrm{H}), 6.78-6.75$ (dt, $J=8.4 \mathrm{~Hz}, J=4.2 \mathrm{~Hz}, 2 \mathrm{H}), 6.04(\mathrm{~s}, 1 \mathrm{H}), 5.64(\mathrm{~s}, 1 \mathrm{H}), 5,53(\mathrm{~s}, 1 \mathrm{H}), 5.49(\mathrm{~s}, 1 \mathrm{H}), 5.25$ (d, $J=4.2 \mathrm{~Hz}, 1 \mathrm{H}), 4.06(\mathrm{~d}, J=4.2 \mathrm{~Hz}, 1 \mathrm{H}), 2.31(\mathrm{~s}, 3 \mathrm{H}) ;{ }^{13} \mathrm{C} \mathrm{NMR}\left(150 \mathrm{MHz}, \mathrm{CDCl}_{3}\right): \delta=$ $143.6,138.1,136.2,135.48,135.42,134.9,134.5,133.9,129.8,129.0,128.8,128.3$, 128.2, 127.0, 122.3, 115.8, 115.7, 83.0, 56.5, 21.0; HRMS Calculated for $\left[\mathrm{C}_{26} \mathrm{H}_{21} \mathrm{NO}_{2} \mathrm{Cl}_{2}+\mathrm{H}\right]^{+}:$450.10221, Found: 450.10252 .

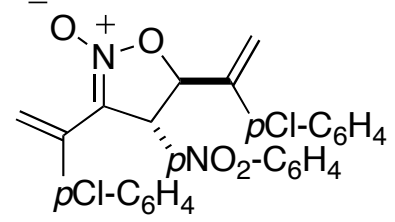

151

\section{4-p-Nitro-phenyl-3, 5-bis (p-chloribenzylvinyl) isoxazoline- $N$-oxide (15l)}

Purified by flash chromatography (hexane-EtOAc) as colorless oil (yield: 95\%); ${ }^{1} \mathrm{H}$ NMR $\left(600 \mathrm{MHz}, \mathrm{CDCl}_{3}\right) \delta=8.10-8.09(\mathrm{~m}, 2 \mathrm{H}), 7.38-7.36(\mathrm{~m}, 2 \mathrm{H}), 7.29-7.26(\mathrm{~m}, 2 \mathrm{H}), 7.15-$ $7.13(\mathrm{~m}, 2 \mathrm{H}), 7.04-7.03(\mathrm{~m}, 2 \mathrm{H}), 6.73-6.71(\mathrm{~m}, 2 \mathrm{H}), 6.21(\mathrm{~s}, 1 \mathrm{H}), 5.68(\mathrm{~s}, 1 \mathrm{H}), 5.56(\mathrm{~s}$, $2 \mathrm{H}), 5.25(\mathrm{~d}, J=4.2 \mathrm{~Hz}, 1 \mathrm{H}), 4.20(\mathrm{~d}, J=4.2 \mathrm{~Hz}, 1 \mathrm{H}) ;{ }^{13} \mathrm{C} \mathrm{NMR}\left(150 \mathrm{MHz}, \mathrm{CDCl}_{3}\right): \delta=$ $147.8,145.4,143.0,136.0,135.1,134.9,134.4,134.3,129.2,128.8,128.5,128.3,128.1$, 124.3, 122.7, 116.7, 114.7,82.3, 56.3; HRMS Calculated for $\left[\mathrm{C}_{25} \mathrm{H}_{18} \mathrm{Cl}_{2} \mathrm{~N}_{2} \mathrm{O}_{4}+\mathrm{H}\right]^{+}$: 481.07164, Found: 481.07172. 


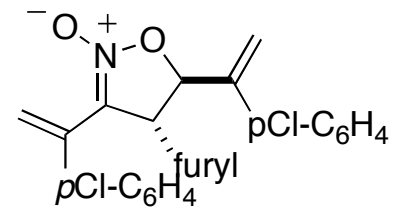

$15 \mathrm{~m}$

\section{4-Furyl-3, 5-bis (p-chloribenzylvinyl) isoxazoline- $N$-oxide (15m)}

Purified by flash chromatography (hexane-EtOAc) as colorless oil (yield: $87 \%$ ); ${ }^{1} \mathrm{H}$ NMR $\left(600 \mathrm{MHz}, \mathrm{CDCl}_{3}\right) \delta=7.36-7.32(\mathrm{~m}, 5 \mathrm{H}), 7.19-7.17(\mathrm{~m}, 2 \mathrm{H}), 6.87-6.85(\mathrm{~m}, 2 \mathrm{H}), 6.23(\mathrm{q}$, $J=3.0 \mathrm{~Hz}, 1 \mathrm{H}), 6.07(\mathrm{~s}, 1 \mathrm{H}), 5.89(\mathrm{~d}, J=3.0 \mathrm{~Hz}, 1 \mathrm{H}), 5.66(\mathrm{~s}, 1 \mathrm{H}), 5.56(\mathrm{~s}, 1 \mathrm{H}), 5.55$ (s, $1 \mathrm{H}), 5.44(\mathrm{~d}, J=4.8 \mathrm{~Hz}, 1 \mathrm{H}), 4.31(\mathrm{~d}, J=4.8 \mathrm{~Hz}, 1 \mathrm{H}) ;{ }^{13} \mathrm{C} \mathrm{NMR}\left(150 \mathrm{MHz}, \mathrm{CDCl}_{3}\right): \delta=$ $149.5,143.0,142.9,136.0,135.2,134.8,134.6,134.0,129.0,128.6,128.4,128.3,122.0$, 116.5, 113.2, 110.7, 108.3, 80.2, 50.3; HRMS Calculated for $\left[\mathrm{C}_{24} \mathrm{H}_{20} \mathrm{NO}_{3} \mathrm{Cl}_{2}+\mathrm{H}\right]^{+}$: 406.12048, Found: 406.12060.

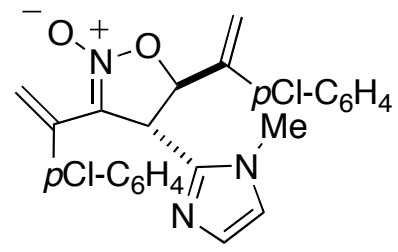

15n

\section{4-2'-methyl-imidazole-3, 5-bis (p-chloribenzylvinyl) isoxazoline- $\mathrm{N}$-oxide (15n)}

Purified by flash chromatography (hexane-EtOAc) as colorless oil (yield: $87 \%$ ); ${ }^{1} \mathrm{H}$ NMR $\left(600 \mathrm{MHz}, \mathrm{CDCl}_{3}\right) \delta=7.27-7.25(\mathrm{~m}, 3 \mathrm{H}), 7.17-7.13(\mathrm{~m}, 4 \mathrm{H}), 6.96(\mathrm{~d}, J=1.2 \mathrm{~Hz}, 1 \mathrm{H})$, 6.77-6.76 (m, 2H), $6.47(\mathrm{~d}, J=1.2 \mathrm{~Hz}, 1 \mathrm{H}), 6.32(\mathrm{~s}, 1 \mathrm{H}), 5.65(\mathrm{~s}, 1 \mathrm{H}), 5.63-5.62(\mathrm{~d}, J=$ $7.8 \mathrm{~Hz}, 1 \mathrm{H}), 5.52(\mathrm{~s}, 1 \mathrm{H}), 4.29(\mathrm{~d}, J=7.2 \mathrm{~Hz}, 1 \mathrm{H}), 2.49(\mathrm{~s}, 3 \mathrm{H}) ;{ }^{13} \mathrm{C}$ NMR $(150 \mathrm{MHz}$, $\left.\mathrm{CDCl}_{3}\right): \delta=143.1,142.7,136.7,135.2,134.6,134.3,133.9,128.9,128.79,128.73$, 128.3, 128.2, 122.2, 120.9, 116.5, 114.4, 81.2, 48.3, 31.3; HRMS Calculated for $\left[\mathrm{C}_{23} \mathrm{H}_{19} \mathrm{~N}_{3} \mathrm{O}_{2} \mathrm{Cl}_{2}+\mathrm{H}\right]^{+}:$440.09271, Found: 440.09301 . 


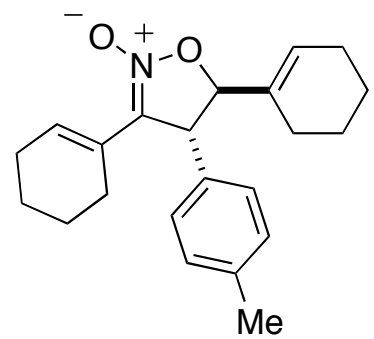

150

\section{4-Toulyl-3, 5-bis (cyclohexenyl) isoxazoline- $N$-oxide (150)}

Purified by flash chromatography (hexane-EtOAc) as white solid (yield: 94\%); ${ }^{1} \mathrm{H}$ NMR $\left(600 \mathrm{MHz}, \mathrm{CDCl}_{3}\right) \delta=7.16-7.15(\mathrm{~d}, J=7.8 \mathrm{~Hz}, 2 \mathrm{H}), 7.10-7.09(\mathrm{~m}, 2 \mathrm{H}), 6.58(\mathrm{t}, J=1.8$ $\mathrm{Hz}, 2 \mathrm{H}), 4.51$ (d, $J=4.2 \mathrm{~Hz}, 1 \mathrm{H}), 4.24$ (d, $J=4.8 \mathrm{~Hz}, 1 \mathrm{H}), 2.34$ (s, 3H), 2.25-1.95 (m, $8 \mathrm{H}), 1.80-1.41(\mathrm{~m}, 8 \mathrm{H}) ;{ }^{13} \mathrm{C} \mathrm{NMR}\left(150 \mathrm{MHz}, \mathrm{CDCl}_{3}\right): \delta=137.5,137.2,134.3,132.4$, $129.8,126.9,126.1,124.8,118.3,86.4,55.0,25.8,24.8,22.8,22.2,22.19,22.15,21.3$, 21.0; HRMS Calculated for $\left[\mathrm{C}_{22} \mathrm{H}_{27} \mathrm{NO}_{2}+\mathrm{H}\right]^{+}: 338.21146$, Found: 338.21159 .

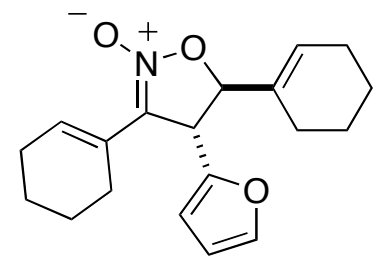

\section{$15 p$}

\section{4-Furyl-3, 5-bis (cyclohexenyl) isoxazoline- $N$-oxide (15p)}

Purified by flash chromatography (hexane-EtOAc) as colorless oil (yield: 89\%); ${ }^{1} \mathrm{H}$ NMR $\left(600 \mathrm{MHz}, \mathrm{CDCl}_{3}\right) \delta=7.38(\mathrm{~s}, 1 \mathrm{H}), 6.60-6.58(\mathrm{~m}, 1 \mathrm{H}), 6.34(\mathrm{~m}, 1 \mathrm{H}), 6.19$ (d, J=3.6 Hz, $1 \mathrm{H}), 5.81(\mathrm{~s}, 1 \mathrm{H}), 4.72(\mathrm{~d}, J=4.2 \mathrm{~Hz}, 1 \mathrm{H}), 4.43(\mathrm{~d}, J=4.2 \mathrm{~Hz}, 1 \mathrm{H}), 2.24-2.23(\mathrm{t}, J=4.2$ $\mathrm{Hz}, 2 \mathrm{H}), 2.17-2.12(\mathrm{~m}, 3 \mathrm{H}), 2.06-2.04(\mathrm{~m}, 2 \mathrm{H}), 1.98-1.92(\mathrm{~m}, 1 \mathrm{H}), 1.76-1.48(\mathrm{~m}, 8 \mathrm{H})$; ${ }^{13} \mathrm{C}$ NMR (150 MHz, $\left.\mathrm{CDCl}_{3}\right): \delta=151.7,142.4,133.8,132.2,126.5,124.8,115.8,110.7$, 107.3, 83.2, 48.8, 25.9, 25.5, 24.8, 22.8, 22.3, 22.1, 22.0, 21.3; HRMS Calculated for $\left[\mathrm{C}_{19} \mathrm{H}_{23} \mathrm{NO}_{3}+\mathrm{H}\right]^{+}:$314.17507, Found: 314.117533 . 


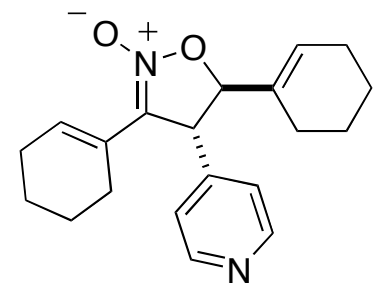

$15 q$

\section{4-Pyridine-3, 5-bis (cyclohexenyl) isoxazoline- $\mathrm{N}$-oxide (15q)}

Purified by flash chromatography (hexane-EtOAc) as orange solid (yield: 92\%); ${ }^{1} \mathrm{H}$ NMR $\left(600 \mathrm{MHz}, \mathrm{CDCl}_{3}\right) \delta=8.61(\mathrm{dd}, J=4.8 \mathrm{~Hz}, J=3.6 \mathrm{~Hz}, 2 \mathrm{H}), 7.17-7.16(\mathrm{dd}, J=4.8 \mathrm{~Hz}, J$ $=3.0 \mathrm{~Hz}, 2 \mathrm{H}), 6.47(\mathrm{~m}, 1 \mathrm{H}), 5.76(\mathrm{~s}, 1 \mathrm{H}), 4.51(\mathrm{~d}, J=3.6 \mathrm{~Hz}, 1 \mathrm{H}), 4.28(\mathrm{~d}, J=4.2 \mathrm{~Hz}$, $1 \mathrm{H}), 2.30-1.92(\mathrm{~m}, 8 \mathrm{H}), 1.78-1.40(\mathrm{~m}, 8 \mathrm{H}) ;{ }^{13} \mathrm{C} \mathrm{NMR}\left(150 \mathrm{MHz}, \mathrm{CDCl}_{3}\right): \delta=150.6$, $148.9,133.7,132.9,127.0,124.5,122.0,117.0,85.4,54.5,25.8,25.7,24.8,22.7,22.1$, 22.05, 22.0, 21.2; HRMS Calculated for $\left[\mathrm{C}_{20} \mathrm{H}_{24} \mathrm{~N}_{2} \mathrm{O}_{2}+\mathrm{H}\right]^{+}$: 325.19105, Found: 325.19129 .

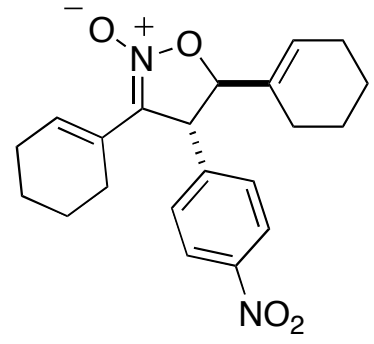

$15 r$

\section{4-p-Nitrophenyl-3, 5-bis (cyclohexenyl) isoxazoline- $\mathrm{N}$-oxide (15r)}

Purified by flash chromatography (hexane-EtOAc) as yellow solid (yield: $92 \%$ ); ${ }^{1} \mathrm{H}$ NMR $\left(600 \mathrm{MHz}, \mathrm{CDCl}_{3}\right) \delta=8.25-8.23(\mathrm{~m}, 2 \mathrm{H}), 7.42-7.40(\mathrm{~m}, 2 \mathrm{H}), 6.47-6.45(\mathrm{p}, J=4.2 \mathrm{~Hz}$, $1 \mathrm{H}), 5.76(\mathrm{~s}, 1 \mathrm{H}), 4.51(\mathrm{~d}, J=4.2 \mathrm{~Hz}, 1 \mathrm{H}), 4.41(\mathrm{~d}, J=4.2 \mathrm{~Hz}, 1 \mathrm{H}), 2.30-1.92(\mathrm{~m}, 8 \mathrm{H})$, 1.78-1.40 (m, 8H); ${ }^{13} \mathrm{C}$ NMR (150 MHz, $\left.\mathrm{CDCl}_{3}\right): \delta=147.6,147.4,133.7,133.1,128.0$, $127.3,124.56,124.53,117.3,85.7,55.0,25.8,24.8,22.7,22.1,22.09,22.04,21.2$; HRMS Calculated for $\left[\mathrm{C}_{21} \mathrm{H}_{24} \mathrm{~N}_{2} \mathrm{O}_{4}+\mathrm{H}\right]^{+}: 369.18088$, Found: 369.18118 . 


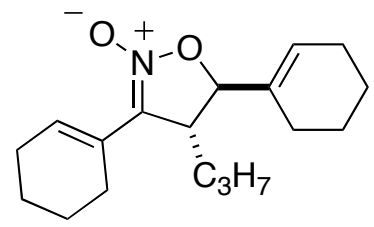

$15 \mathrm{~s}$

\section{4-Propyl-3, 5-bis (cyclohexenyl) isoxazoline- $N$-oxide (15s)}

Purified by flash chromatography (hexane-EtOAc) as white solid (yield: 64\%); ${ }^{1} \mathrm{H}$ NMR $\left(600 \mathrm{MHz} \mathrm{CDCl}_{3}\right) \delta=6.45-6.43(\mathrm{~m}, 1 \mathrm{H}), 4.47(\mathrm{~d}, J=2.4 \mathrm{~Hz}, 1 \mathrm{H}), 3.13-3.11(\mathrm{~m}, 1 \mathrm{H})$, 2.52-2.48 (m, 1H), 2.29-1.88 (m, 6H), 1.89-1.80 (m, 1H), 1.78-1.30 (m, 13H), 0.95 (t, $J=$ $7.2 \mathrm{~Hz}, 3 \mathrm{H}) ;{ }^{13} \mathrm{C} \mathrm{NMR}\left(150 \mathrm{MHz}, \mathrm{CDCl}_{3}\right): \delta=135.3,131.6,125.1,124.9,119.0,82.9$, 48.5, 34.1, 25.9, 25.5, 24.7, 22.8, 22.3, 22.16, 22.13, 21.4, 19.3, 13.9; HRMS Calculated for $\left[\mathrm{C}_{18} \mathrm{H}_{27} \mathrm{NO}_{2}+\mathrm{H}\right]^{+}:$390.21146, Found: 290.21173 .

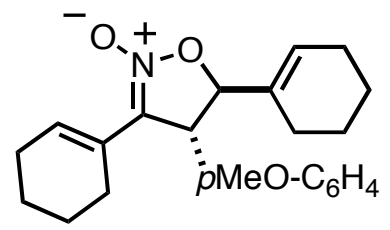

$15 t$

\section{4-p-Anisyl-3, 5-bis (cyclohexenyl) isoxazoline- $N$-oxide (15t)}

Purified by flash chromatography (hexane-EtOAc) as white solid (yield: 90\%); ${ }^{1} \mathrm{H}$ NMR $\left(600 \mathrm{MHz}, \mathrm{CDCl}_{3}\right) \delta=7.26-7.11(\mathrm{~m}, 2 \mathrm{H}), 6.89-6.87(\mathrm{~m}, 2 \mathrm{H}), 6.59-6.58(\mathrm{~m}, 1 \mathrm{H}), 5.73(\mathrm{~s}$, $1 \mathrm{H}), 4.51(\mathrm{~d}, J=4.8 \mathrm{~Hz}, 1 \mathrm{H}), 4.23(\mathrm{~d}, J=4.2 \mathrm{~Hz}, 1 \mathrm{H}), 3.80(\mathrm{~s}, 3 \mathrm{H}), 2.22-1.94(\mathrm{~m}, 8 \mathrm{H})$, 1.73-1.44 (m, 8H); ${ }^{13} \mathrm{C}$ NMR (150 MHz, $\left.\mathrm{CDCl}_{3}\right): \delta=159.1,134.3,132.5,132.2,128.1$, $126.2,124.8,118.4,114.5,86.6,55.2,54.7,25.8,24.8,22.8,22.2,22.1,21.3$; HRMS Calculated for $\left[\mathrm{C}_{22} \mathrm{H}_{27} \mathrm{NO}_{3}+\mathrm{H}\right]^{+}: 354.20637$, Found: 354.20658 .

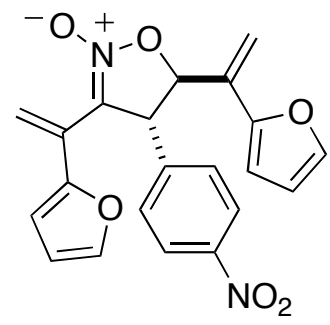

$15 \mathbf{u}$ 


\section{4-p-Nitro-phenyl-3, 5-bis (1-furylvinyl)isoxazoline- $N$-oxide (15u)}

Purified by flash chromatography (hexane-EtOAc) as colorless oil (yield: $46 \%$ ); ${ }^{1} \mathrm{H}$ NMR $\left(600 \mathrm{MHz}, \mathrm{CDCl}_{3}\right) \delta=8.14-8.12(\mathrm{~m}, 2 \mathrm{H}), 7.39(\mathrm{~d}, J=1.8 \mathrm{~Hz}, 1 \mathrm{H}), 7.11-7.09(\mathrm{~m}, 2 \mathrm{H})$, $6.76(\mathrm{~d}, J=1.8 \mathrm{~Hz}, 1 \mathrm{H}), 6.47$ (q, $J=2.4 \mathrm{~Hz}, 1 \mathrm{H}), 6.39$ (q, $J=2.4 \mathrm{~Hz}, 1 \mathrm{H}), 6.17-6.16$ (d, $J=3.0 \mathrm{~Hz}, 1 \mathrm{H}), 5.96-5.65(\mathrm{t}, \mathrm{J}=3.6 \mathrm{~Hz}, 1 \mathrm{H}), 5.77(\mathrm{~s}, 1 \mathrm{H}), 5.64(\mathrm{~d}, J=1.2 \mathrm{~Hz}, 1 \mathrm{H})$, 5.61-5.60 (q, $J=3.6 \mathrm{~Hz}, 1 \mathrm{H}), 5.59(\mathrm{~s}, 1 \mathrm{H}), 5.17(\mathrm{dd}, J=2.4 \mathrm{~Hz}, J=1.2 \mathrm{~Hz}, 1 \mathrm{H}), 4.28(\mathrm{~d}$, $J=3.6 \mathrm{~Hz}, 1 \mathrm{H}) ;{ }^{13} \mathrm{C} \mathrm{NMR}\left(150 \mathrm{MHz}, \mathrm{CDCl}_{3}\right): \delta=150.2,147.7,146.2,142.8,134.5$, $129.3,128.2,126.4,124.7,124.0,120.9,116.0,111.4,110.2,109.7,107.6,107.1,79.6$, 57.6, 46.2, 33.1, 29.6; HRMS Calculated for $\left[\mathrm{C}_{21} \mathrm{H}_{16} \mathrm{~N}_{2} \mathrm{O}_{6}+\mathrm{H}\right]^{+}$: 393.10811, Found: 393.08662 .

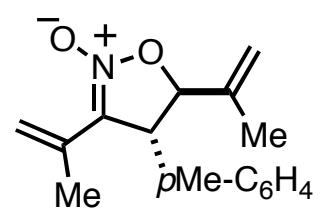

$15 \mathrm{v}$

\section{4-p-Anisyl-3, 5-bis (methylvinyl) isoxazoline- $\mathrm{N}$-oxide (15v)}

Purified by flash chromatography (hexane-EtOAc) as colorless oil (yield: 74\%); ${ }^{1} \mathrm{H}$ NMR $\left(600 \mathrm{MHz}, \mathrm{CDCl}_{3}\right) \delta=7.16-7.13(\mathrm{~m}, 2 \mathrm{H}), 6.90-6.88(\mathrm{~m}, 2 \mathrm{H}), 5.67(\mathrm{~s}, 1 \mathrm{H}), 5.23(\mathrm{t}, J=$ $1.8 \mathrm{~Hz}, 1 \mathrm{H}), 5.07(\mathrm{~s}, 1 \mathrm{H}), 4.97(\mathrm{~s}, 1 \mathrm{H}), 4.64(\mathrm{~d}, J=4.8 \mathrm{~Hz}, 1 \mathrm{H}), 4.26(\mathrm{~d}, J=4.8 \mathrm{~Hz}, 1 \mathrm{H})$, $3.80(\mathrm{~s}, 3 \mathrm{H}), 1.92(\mathrm{~s}, 3 \mathrm{H}), 1.85(\mathrm{~s}, 3 \mathrm{H}) ;{ }^{13} \mathrm{C} \mathrm{NMR}\left(150 \mathrm{MHz}, \mathrm{CDCl}_{3}\right): \delta=159.4,141.0$, 131.6, 130.6, 128.2, 119.7, 117.8, 114.7, 113.8, 85.3, 55.2, 20.8, 16.9; HRMS Calculated for $\left[\mathrm{C}_{16} \mathrm{H}_{19} \mathrm{NO}_{2}+\mathrm{H}\right]^{+}:$258.14886, Found: 258.14896.

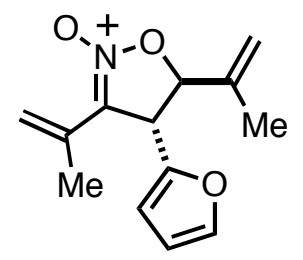

\section{$15 w$}

\section{4-2'-Furyl-3, 5-bis (methylvinyl) isoxazoline- $\mathrm{N}$-oxide (15w)}

Purified by flash chromatography (hexane-EtOAc) as colorless oil (yield: 71\%); ${ }^{1} \mathrm{H}$ NMR $\left(600 \mathrm{MHz}, \mathrm{CDCl}_{3}\right) \delta=7.40-7.39(\mathrm{~m}, 1 \mathrm{H}), 6.36-6.35(\mathrm{dd}, J=3.0 \mathrm{~Hz}, J=1.8 \mathrm{~Hz}, 1 \mathrm{H})$, 
$6.23(\mathrm{~d}, J=3.0 \mathrm{~Hz}, 1 \mathrm{H}), 5.68(\mathrm{~s}, 1 \mathrm{H}), 5.26(\mathrm{~s}, 1 \mathrm{H}), 5.14(\mathrm{~s}, 1 \mathrm{H}), 5.01(\mathrm{~s}, 1 \mathrm{H}), 4.84(\mathrm{~d}, J=$ $4.8 \mathrm{~Hz}, 1 \mathrm{H}), 4.47$ (d, $J=4.8 \mathrm{~Hz}, 1 \mathrm{H}), 1.97$ (s, 3H), 1.85-1.84 (s, 3H); ${ }^{13} \mathrm{C}$ NMR $(150$ $\left.\mathrm{MHz}, \mathrm{CDCl}_{3}\right): \delta=159.4,141.0,131.6,130.6,128.2,119.7,117.8,114.7,113.8,85.3$, 55.2, 20.8, 16.9; HRMS Calculated for $\left[\mathrm{C}_{13} \mathrm{H}_{15} \mathrm{NO}_{3}+\mathrm{H}\right]^{+}: 234.11247$, Found: 234.11261 .

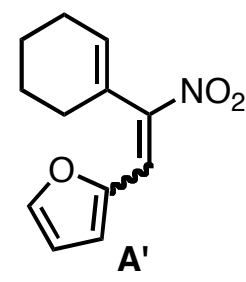

\section{2-(2-cyclohexenyl-2-nitrovinyl) furan ( $\left.\mathrm{A}^{\prime}\right)$}

Purified by flash chromatography (hexane-EtOAc) as clear oil (yield: 55\%); Z/E isomers were not separated. ${ }^{1} \mathrm{H}$ NMR $\left(600 \mathrm{MHz}, \mathrm{CDCl}_{3}\right)$ : set $\mathbf{1}, \delta=7.81(\mathrm{~s}, 1 \mathrm{H}), 7.57(\mathrm{~d}, 1 \mathrm{H})$ 6.85-6.86 (d, 1H), 6.52-6.53 (dd, $J=3.6 \mathrm{~Hz}, 1 \mathrm{H}), 5.95-5.96(\mathrm{~m}, 1 \mathrm{H}), 2.17-2.24(\mathrm{~m}, 4 \mathrm{H})$, 1.72-1.83 (m, 3H), 1.62-1.65 (m, 1H); set 2, $\delta=7.43(\mathrm{~d}, 1 \mathrm{H}), 6.49-6.50(\mathrm{~d}, 1 \mathrm{H}), 6.41$ 6.42 (dd, $J=3.6 \mathrm{~Hz}, J=1.8 \mathrm{~Hz}, 1 \mathrm{H}), 6.15$ (s, 1H), 5.86-5.88 (t, 1H), 2.17-2.24 (m, 4H), 1.72-1.83 (m, 3H), 1.62-1.65 (m, $1 \mathrm{H}) ;{ }^{13} \mathrm{C}$ NMR $\left(150 \mathrm{MHz}, \mathrm{CDCl}_{3}\right)$ : set $\mathbf{1}, \delta=148.0$, 146.1, 144.3, 133.8, 120.8, 117.1, 112.9, 112.0, 26.8, 25.5, 22.5, 21.5; set $2 \delta=149.0$, 148.5, 147.2, 129.1, 128.0, 112.8, 104.9, 29.7, 25.7, 24.5, 22.1; HRMS Calculated for $\left[\mathrm{C}_{12} \mathrm{H}_{13} \mathrm{NO}_{3}+\mathrm{H}\right]^{+}: 220.09682$, Found: 220.09697 .

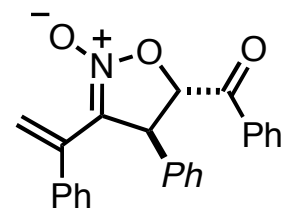

$18 \mathbf{a}$

\section{4-Phenyl-3 (1-phenylvinyl) 5- (Phenylketone) isoxazoline- $N$-oxide (18a)}

Purified by flash chromatography (hexane-EtOAc) as colorless oil (yield: 91\%); ${ }^{1} \mathrm{H}$ NMR $\left(600 \mathrm{MHz}, \mathrm{CDCl}_{3}\right) \delta=7.97-7.95(\mathrm{~m}, 2 \mathrm{H}), 7.62-7.59(\mathrm{tt}, J=7.2 \mathrm{~Hz}, 1 \mathrm{H}), 7.48-7.46$ (t, $J=$ $7.8 \mathrm{~Hz}, 2 \mathrm{H}), 7.26-7.21(\mathrm{~m}, 6 \mathrm{H}), 7.06-7.02(\mathrm{~m}, 4 \mathrm{H}), 6.29(\mathrm{~s}, 1 \mathrm{H}), 5.60(\mathrm{~s}, 1 \mathrm{H}), 5.55(\mathrm{~d}, J=$ $3.6 \mathrm{~Hz}, 1 \mathrm{H}), 5.07(\mathrm{~d}, J=3.6 \mathrm{~Hz}, 1 \mathrm{H}) ;{ }^{13} \mathrm{C} \mathrm{NMR}\left(150 \mathrm{MHz}, \mathrm{CDCl}_{3}\right) \delta=192.4,138.1$, $137.9,135.6,134.3,133.7,129.4,129.1,128.9,128.2,128.1,127.9,127.8,127.5,122.3$, 115.6, 81.0, 52.4; HRMS Calculated for $\left[\mathrm{C}_{22} \mathrm{H}_{27} \mathrm{NO}_{3}+\mathrm{H}\right]^{+}: 370.14377$, Found: 370.14390 . 


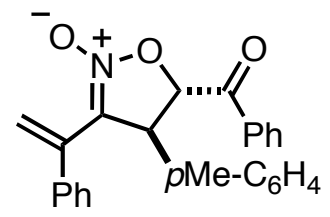

$18 b$

\section{4-p-Toulyl-3 (1-phenylvinyl) 5- (Phenylketone) isoxazoline- $\mathrm{N}$-oxide (18b)}

Purified by flash chromatography (hexane-EtOAc) as colorless oil (yield: 92\%); ${ }^{1} \mathrm{H}$ NMR $\left(600 \mathrm{MHz}, \mathrm{CDCl}_{3}\right) \delta=7.97-7.95(\mathrm{~m}, 2 \mathrm{H}), 7.62-7.60(\mathrm{tt}, J=6.0 \mathrm{~Hz}, 1 \mathrm{H}), 7.49-7.46(\mathrm{t}, J=$ $5.4 \mathrm{~Hz}, 2 \mathrm{H}), 7.26-7.23(\mathrm{~m}, 3 \mathrm{H}), 7.08-7.05$ (m, 4H), 6.93-6.92 (d, J=7.8 Hz, 2H), 6.24 (s, $1 \mathrm{H}), 5.60(\mathrm{~s}, 1 \mathrm{H}), 5.53(\mathrm{~d}, J=3.6 \mathrm{~Hz}, 1 \mathrm{H}), 5.01(\mathrm{~d}, J=3.6 \mathrm{~Hz}, 1 \mathrm{H}), 2.30(\mathrm{~s}, 3 \mathrm{H}) ;{ }^{13} \mathrm{C}$ NMR (150 MHz, $\left.\mathrm{CDCl}_{3}\right): \delta=192.5,138.1,137.9,135.6,135.1,134.3,133.7,129.8$, $129.3,128.9,128.1,127.9,127.7,127.3,122.2,115.6,81.2,52.2,21.0$; HRMS Calculated for $\left[\mathrm{C}_{25} \mathrm{H}_{21} \mathrm{NO}_{3}+\mathrm{H}\right]^{+}: 384.15942$, Found: 384.15955 .<smiles>C=C(C1=[N+]([O-])O[C@H](C(=O)c2ccccc2)[C@@H]1C#N)c1ccccc1</smiles>

$18 \mathrm{c}$

\section{4-Furyl-3 (1-phenylvinyl) 5- (Phenylketone) isoxazoline- $N$-oxide (18c)}

Purified by flash chromatography (hexane-EtOAc) as colorless oil (yield: 73\%); ${ }^{1} \mathrm{H}$ NMR $\left(600 \mathrm{MHz}, \mathrm{CDCl}_{3}\right) \delta=8.03-8.01(\mathrm{~m}, 2 \mathrm{H}), 7.65-7.62(\mathrm{tt}, J=6.0 \mathrm{~Hz}, J=2.4 \mathrm{~Hz}, 1 \mathrm{H})$, 7.52-7.49 (t, $J=7.8 \mathrm{~Hz}, 2 \mathrm{H}), 7.34(\mathrm{~m}, 1 \mathrm{H}), 7.31-7.25(\mathrm{~m}, 3 \mathrm{H}), 7.18-7.16(\mathrm{~m}, 2 \mathrm{H}), 6.28$ (s, 1H), 6.23-6.22 (q, $J=3.6 \mathrm{~Hz}, 1 \mathrm{H}), 5.92(\mathrm{~d}, J=3.0 \mathrm{~Hz}, 1 \mathrm{H}), 5.70(\mathrm{~d}, J=4.2 \mathrm{~Hz}, 1 \mathrm{H})$, $5.67(\mathrm{~s}, 1 \mathrm{H}), 5.35(\mathrm{~d}, J=4.2 \mathrm{~Hz}, 1 \mathrm{H}) ;{ }^{13} \mathrm{C} \mathrm{NMR}\left(150 \mathrm{MHz}, \mathrm{CDCl}_{3}\right): \delta=191.8,186.8$, 149.2 , 143.0, 137.8, 135.5, 134.4, 133.7, 129.5, 128.9, 128.2, 128.1, 127.6, 122.1, 110.7, 108.6, 78.1, 46.0; HRMS Calculated for $\left[\mathrm{C}_{22} \mathrm{H}_{17} \mathrm{NO}_{4}+\mathrm{Na}\right]^{+}:$382.10498, Found: 382.10522 . 
<smiles>C=C(C1=[N+]([O-])O[C@H](C(=O)c2ccccc2)C1CCCC)c1ccccc1</smiles>

\section{8d}

\section{4-Propyl-3 (1-phenylvinyl) 5- (Phenylketone) isoxazoline- $\mathrm{N}$-oxide (18d)}

Purified by flash chromatography (hexane-EtOAc) as colorless oil (yield: 62\%); ${ }^{1} \mathrm{H}$ NMR $\left(600 \mathrm{MHz}, \mathrm{CDCl}_{3}\right) \delta=8.03-8.01(\mathrm{~m}, 2 \mathrm{H}), 7.64-7.61$ (tt, $\left.J=6.0 \mathrm{~Hz}, J=2.4 \mathrm{~Hz}, 1 \mathrm{H}\right)$, 7.52-7.50 (t, $J=7.8 \mathrm{~Hz}, 2 \mathrm{H}), 7.39-7.29(\mathrm{~m}, 5 \mathrm{H}), 6.37(\mathrm{~s}, 1 \mathrm{H}), 5.78(\mathrm{~s}, 1 \mathrm{H}), 5.32(\mathrm{~d}, J=$ $3.0 \mathrm{~Hz}, 1 \mathrm{H}), 4.06-4.04(\mathrm{~m}, 1 \mathrm{H}), 1.56-1.50(\mathrm{~m}, 1 \mathrm{H}), 1.47-1.41(\mathrm{~m}, 1 \mathrm{H}), 1.31-1.30(\mathrm{~m}, 2 \mathrm{H})$, $0.75(\mathrm{t}, J=7.2 \mathrm{~Hz}, 3 \mathrm{H}) ;{ }^{13} \mathrm{C} \mathrm{NMR}\left(150 \mathrm{MHz}, \mathrm{CDCl}_{3}\right): \delta=193.6,138.1,135.6,134.17$, 134.12, 129.3, 128.8, 128.5, 128.2, 127.6, 122.0, 115.8, 78.6, 45.8, 33.3, 18.9, 13.4; HRMS Calculated for $\left[\mathrm{C}_{21} \mathrm{H}_{21} \mathrm{NO}_{3}+\mathrm{H}\right]^{+}: 336.15942$, Found: 336.16080 .<smiles>C=C(C1=[N+]([O-])O[C@H](C(=O)c2ccccc2)C1C(C)C)c1ccccc1</smiles>

$18 \mathrm{e}$

\section{4-Iso-Propyl-3 (1-phenylvinyl) 5- (Phenylketone) isoxazoline- $N$-oxide (18e)}

Purified by flash chromatography (hexane-EtOAc) as colorless oil (yield: 68\%); ${ }^{1} \mathrm{H}$ NMR $\left(600 \mathrm{MHz}, \mathrm{CDCl}_{3}\right) \delta=8.05-8.04(\mathrm{~m}, 2 \mathrm{H}), 7.65-7.62(\mathrm{~m}, 1 \mathrm{H}), 7.53-7.51(\mathrm{~m}, 2 \mathrm{H}), 7.39-$ $7.25(\mathrm{~m}, 5 \mathrm{H}), 7.36(\mathrm{~s}, 1 \mathrm{H}), 5.83(\mathrm{~s}, 1 \mathrm{H}), 5.38(\mathrm{~d}, J=2.4 \mathrm{~Hz}, 1 \mathrm{H}), 4.02-4.01(\mathrm{dd}, J=4.2$ $\mathrm{Hz}, J=1.2 \mathrm{~Hz}, 1 \mathrm{H}), 1.86-1.80(\mathrm{~m}, 1 \mathrm{H}), 0.95-0.89(\mathrm{~m}, 3 \mathrm{H}), 0.82(\mathrm{~d}, J=6.6 \mathrm{~Hz}, 3 \mathrm{H}), 0.74$ $(\mathrm{t}, 3 \mathrm{H}) ;{ }^{13} \mathrm{C} \mathrm{NMR}\left(150 \mathrm{MHz}, \mathrm{CDCl}_{3}\right): \delta=194.0,138.2,135.9,134.2,134.1,129.4$, $128.8,128.5,128.3,127.6,122.2$, 115.0, 75.4, 51.6, 29.3, 19.4, 17.5; HRMS Calculated for $\left[\mathrm{C}_{21} \mathrm{H}_{21} \mathrm{NO}_{3}+\mathrm{H}\right]^{+}: 336.15942$, Found: 358.15967 .

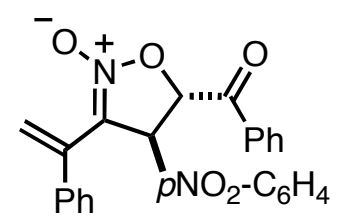

$18 f$ 


\section{4-p-Nitrophenyl-3 (1-phenylvinyl) 5- (Phenylketone) isoxazoline- $N$-oxide (5f)}

Purified by flash chromatography (hexane-EtOAc) as colorless oil (yield: 92\%); ${ }^{1} \mathrm{H}$ NMR $\left(600 \mathrm{MHz}, \mathrm{CDCl}_{3}\right) \delta=8.08-8.06(\mathrm{~d}, J=7.2 \mathrm{~Hz}, 2 \mathrm{H}), 8.00-7.99(\mathrm{~d}, J=8.4 \mathrm{~Hz}, 2 \mathrm{H}), 7.66-$ $7.63(\mathrm{t}, J=8.4 \mathrm{~Hz}, 1 \mathrm{H}), 7.52-7.50(\mathrm{t}, J=7.8 \mathrm{~Hz}, 2 \mathrm{H}), 7.28-7.24(\mathrm{~m}, 3 \mathrm{H}), 7.17-7.15(\mathrm{~m}$, 2H), 7.04-7.03 (d, $J=7.8 \mathrm{~Hz}, 2 \mathrm{H}), 6.46(\mathrm{~s}, 1 \mathrm{H}), 5.67$ (s, 1H), 5.48 (d, J=4.2 Hz, 1H), $5.34(\mathrm{~d}, J=4.2 \mathrm{~Hz}, 1 \mathrm{H}) ;{ }^{13} \mathrm{C} \mathrm{NMR}\left(150 \mathrm{MHz}, \mathrm{CDCl}_{3}\right): \delta=191.7,147.6,145.2,138.0$, $135.0,134.7,133.6,129.5,129.0,128.6,128.4,128.3,127.9,124.2,122.7,114.9,80.4$, 51.5; HRMS Calculated for $\left[\mathrm{C}_{24} \mathrm{H}_{18} \mathrm{~N}_{2} \mathrm{O}_{5}+\mathrm{H}\right]^{+}: 415.12885$, Found: 415.21173.

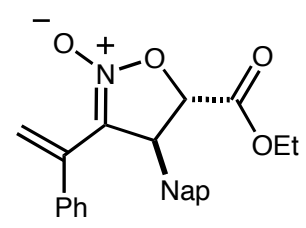

$18 \mathrm{~g}$

\section{4-Napthyl-3 (1-phenylvinyl) 5- (Ethyl ester) isoxazoline- $\mathrm{N}$-oxide (5g)}

Purified by flash chromatography (hexane-EtOAc) as colorless oil (yield: $88 \%$ ); ${ }^{1} \mathrm{H}$ NMR $\left(600 \mathrm{MHz}, \mathrm{CDCl}_{3}\right) \delta=7.85-7.79(\mathrm{~m}, 3 \mathrm{H}), 7.49-7.35(\mathrm{~m}, 4 \mathrm{H}), 7.14-7.12(\mathrm{~m}, 3 \mathrm{H}), 7.03(\mathrm{~d}$, $J=3.0,2 \mathrm{H}), 6.33(\mathrm{~s}, 1 \mathrm{H}), 5.62(\mathrm{~s}, 1 \mathrm{H}), 4.81(\mathrm{~d}, J=3.0 \mathrm{~Hz}, 1 \mathrm{H}), 4.64(\mathrm{~d}, J=3.0 \mathrm{~Hz}, 1 \mathrm{H})$, 4.37-4.33 (m, 2H), 1.37-1.35 (t, $\left.J=7.2,3 \mathrm{H}) ;{ }^{13} \mathrm{C} \mathrm{NMR} \mathrm{(150} \mathrm{MHz,} \mathrm{CDCl}_{3}\right): \delta=169.3$, $137.9,135.5,134.0,133.0,132.8,130.3,129.1$, 129.0, 128.1, 127.9, 127.6, 126.9, 126.0, 125.4, 122.4, 122.1, 78.1, 62.6, 49.1, 14.6; HRMS Calculated for $\left[\mathrm{C}_{20} \mathrm{H}_{19} \mathrm{NO}_{4}+\mathrm{H}\right]^{+}$: 388.15433, Found: 388.15453.

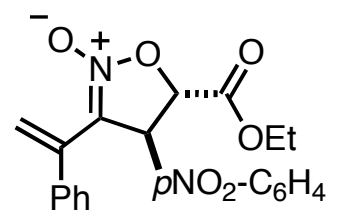

$18 \mathrm{~h}$

\section{4-p-nitrophenyl-3 (1-phenylvinyl) 5- (Ethyl ester) isoxazoline- $N$-oxide (18h)}

Purified by flash chromatography (hexane-EtOAc) as colorless oil (yield: 92\%); ${ }^{1} \mathrm{H}$ NMR $\left(600 \mathrm{MHz}, \mathrm{CDCl}_{3}\right) \delta=8.10-8.07(\mathrm{~m}, 2 \mathrm{H}), 7.30-7.23$ (m, 3H), 7.14-7.12 (m, 2H), 6.99$6.98(\mathrm{~m}, 2 \mathrm{H}), 6.43(\mathrm{~s}, 1 \mathrm{H}), 5.66(\mathrm{~s}, 1 \mathrm{H}), 4.82-4.80(\mathrm{dd}, J=10.2 \mathrm{~Hz}, J=6.6 \mathrm{~Hz}, 2 \mathrm{H})$, 4.39-4.35 (m, 2H), 1.39-1.36 (t, $J=7.2 \mathrm{~Hz}, 3 \mathrm{H}) ;{ }^{13} \mathrm{C} \mathrm{NMR}\left(150 \mathrm{MHz}, \mathrm{CDCl}_{3}\right): \delta=$ 
$168.2,147.7,144.7,137.8,134.8,128.4,128.3,128.1,127.7,124.2,122.5,113.9,62.7$, 54.1, 14.0; HRMS Calculated for $\left[\mathrm{C}_{20} \mathrm{H}_{18} \mathrm{~N}_{2} \mathrm{O}_{6}+\mathrm{H}\right]^{+}: 383.12376$, Found: 383.12396 .

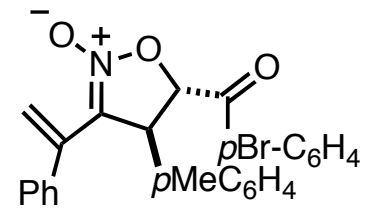

$18 \mathbf{i}$

\section{4-p-Toulyl-3 (1-phenylvinyl) 5- (P-bromophenyl Ketone) isoxazoline- $N$-oxide (18i)}

Purified by flash chromatography (hexane-EtOAc) as colorless oil (yield: 91\%); ${ }^{1} \mathrm{H}$ NMR $\left(600 \mathrm{MHz}, \mathrm{CDCl}_{3}\right) \delta=7.84-7.82(\mathrm{~m}, 2 \mathrm{H}), 7.64-7.62(\mathrm{~m}, 2 \mathrm{H}), 7.27-7.23(\mathrm{~m}, 3 \mathrm{H}), 7.06-$ $7.05(\mathrm{~m}, 4 \mathrm{H}), 6.92-6.90(\mathrm{~d}, J=8.4 \mathrm{~Hz}, 2 \mathrm{H}), 6.25(\mathrm{~s}, 1 \mathrm{H}), 5.60(\mathrm{~s}, 1 \mathrm{H}), 5.45$ (d, $J=4.2$ $\mathrm{Hz}, 1 \mathrm{H}), 5.02(\mathrm{~d}, J=3.6 \mathrm{~Hz}, 1 \mathrm{H}), 2.30(\mathrm{~s}, 3 \mathrm{H}) ;{ }^{13} \mathrm{C} \mathrm{NMR}\left(150 \mathrm{MHz}, \mathrm{CDCl}_{3}\right): \delta=191.7$, $138.2,137.8,135.5,134.9,132.5,132.2,130.8,129.8,128.1,127.9,127.7,127.3,122.4$, 115.5, 81.1, 52.0, 21.0; HRMS Calculated for $\left[\mathrm{C}_{25} \mathrm{H}_{20} \mathrm{NO}_{3} \mathrm{Br}+\mathrm{H}\right]^{+}$: 462.06993, Found: 462.07183 .

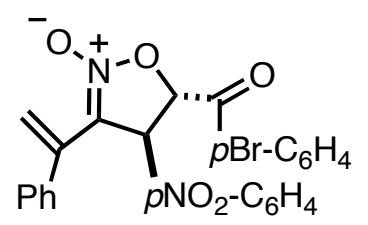

18j

4-p-Nitrophenyl-3 (1-phenylvinyl) 5- (P-bromophenylketone) isoxazoline- $N$-oxide (18j)

Purified by flash chromatography (hexane-EtOAc) as colorless oil (yield: 73\%); ${ }^{1} \mathrm{H}$ NMR $\left(600 \mathrm{MHz}, \mathrm{CDCl}_{3}\right) \delta=8.09-8.06(\mathrm{~m}, 2 \mathrm{H}), 8.00-7.99(\mathrm{~d}, J=7.2 \mathrm{~Hz}, 2 \mathrm{H}), 7.66-7.63$ (tt, $J$ $=6.0 \mathrm{~Hz}, J=2.4 \mathrm{~Hz}, 1 \mathrm{H}), 7.52-7.50(\mathrm{t}, J=6.6 \mathrm{~Hz}, 2 \mathrm{H}), 7.30-7.24(\mathrm{~m}, 2 \mathrm{H}), 7.17-7.15(\mathrm{~m}$, 2H), 7.04-7.03 (d, $J=4.8 \mathrm{~Hz}, 2 \mathrm{H}), 6.46(\mathrm{~s}, 1 \mathrm{H}), 5.67$ (s, 1H), 5.49 (d, J=4.2 Hz, 1H), $5.35(\mathrm{~d}, J=4.2 \mathrm{~Hz}, 1 \mathrm{H}) ;{ }^{13} \mathrm{C} \mathrm{NMR}\left(150 \mathrm{MHz}, \mathrm{CDCl}_{3}\right): \delta=191.7,147.6,145.2,138.0$, $135.0,134.7,133.6,129.5,129.0,128.6,128.4,128.3,127.9,124.2,122.7,114.9,80.5$, 51.5; HRMS Calculated for $\left[\mathrm{C}_{25} \mathrm{H}_{20} \mathrm{BrNO}_{4}+\mathrm{H}\right]^{+}: 478.06485$, Found: 478.06495. 


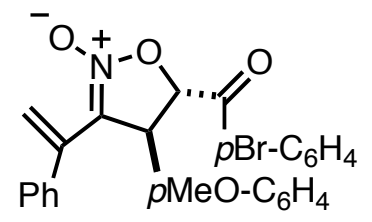

18k

4-p-Anisyl-3 (1-phenylvinyl) 5- (P-bromophenyl Ketone) isoxazoline- $N$-oxide (18k)

Purified by flash chromatography (hexane-EtOAc) as colorless oil (yield: 92\%); ${ }^{1} \mathrm{H}$ NMR $\left(600 \mathrm{MHz}, \mathrm{CDCl}_{3}\right) \delta=7.84-7.82(\mathrm{~d}, J=8.4 \mathrm{~Hz}, 2 \mathrm{H}), 7.64-7.62(\mathrm{~d}, J=9.0 \mathrm{~Hz}, 2 \mathrm{H}), 7.27-$ $7.23(\mathrm{~m}, 3 \mathrm{H}), 7.05-7.04(\mathrm{~m}, 2 \mathrm{H}), 6.93-6.91(\mathrm{~d}, J=8.4 \mathrm{~Hz}, 2 \mathrm{H}), 6.77-6.76(\mathrm{~d}, J=6.6 \mathrm{~Hz}$, 2H), $6.28(\mathrm{~s}, 1 \mathrm{H}), 5.61(\mathrm{~s}, 1 \mathrm{H}), 5.44(\mathrm{~d}, J=3.6 \mathrm{~Hz}, 1 \mathrm{H}), 5.01(\mathrm{~d}, J=3.6 \mathrm{~Hz}, 1 \mathrm{H}), 3.77$ (s, $3 \mathrm{H}) ;{ }^{13} \mathrm{C} \mathrm{NMR}\left(150 \mathrm{MHz}, \mathrm{CDCl}_{3}\right): \delta=191.8,159.5,138.0,135.5,132.5,132.2,130.8$, 129.8, 128.6, 128.1, 128.0, 127.7, 122.4, 115.6, 114.5, 81.2, 55.2, 51.7 HRMS Calculated for $\left[\mathrm{C}_{25} \mathrm{H}_{20} \mathrm{BrNO}_{4}+\mathrm{H}\right]^{+}:$478.06485, Found: 478.06498 .

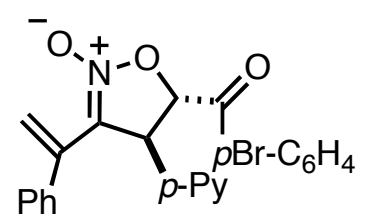

181

\section{4-Pyridine-3 (1-phenylvinyl) 5- (P-bromophenyl Ketone) isoxazoline- $N$-oxide (18I)}

Purified by flash chromatography (hexane-EtOAc) as colorless oil (yield: 92\%); ${ }^{1} \mathrm{H}$ NMR $\left(600 \mathrm{MHz}, \mathrm{CDCl}_{3}\right) \delta=8.41-8.40(\mathrm{dd}, J=4.8 \mathrm{~Hz}, J=3.0 \mathrm{~Hz}, 2 \mathrm{H}), 8.05-8.04(\mathrm{dd}, J=7.2$ $\mathrm{Hz}, J=5.4 \mathrm{~Hz}, 2 \mathrm{H}), 7.66-7.63(\mathrm{t}, J=8.4 \mathrm{~Hz}, 1 \mathrm{H}), 7.52-7.50(\mathrm{t}, J=8.4 \mathrm{~Hz}, 2 \mathrm{H}), 7.26-$ $7.20(\mathrm{~m}, 6 \mathrm{H}), 7.14-7.13(\mathrm{dd}, J=4.2 \mathrm{~Hz}, J=3.0 \mathrm{~Hz}, 2 \mathrm{H}), 5.91(\mathrm{~s}, 1 \mathrm{H}), 5.69(\mathrm{~s}, 1 \mathrm{H}) ;{ }^{13} \mathrm{C}$ NMR $\left(150 \mathrm{MHz}, \mathrm{CDCl}_{3}\right): \delta=182.5,162.8,162.4,149.5,146.9,137.0,136.6,136.3$, $135.7,135.4,134.5,130.2,128.7,128.6,128.4,126.7,124.0,121.8,121.2$; HRMS Calculated for $\left[\mathrm{C}_{23} \mathrm{H}_{17} \mathrm{~N}_{2} \mathrm{O}_{3} \mathrm{Br}+\mathrm{H}\right]^{+}:$449.04953, Found: 449.04984.

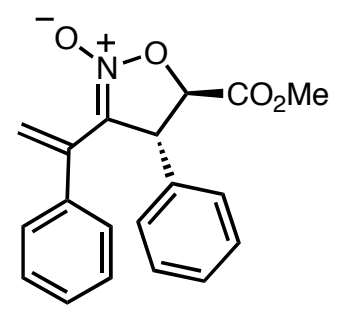

19a 


\section{4-Phenyl-3 (1-phenylvinyl) 5- (methyl ester) isoxazoline- $N$-oxide (18a)}

Purified by flash chromatography (hexane-EtOAc) as colorless oil (yield: 86\%); ${ }^{1} \mathrm{H}$ NMR $\left(600 \mathrm{MHz}, \mathrm{CDCl}_{3}\right) \delta=7.27-7.22(\mathrm{~m}, 6 \mathrm{H}), 7.01-6.98(\mathrm{~m}, 4 \mathrm{H}), 6.29(\mathrm{~s}, 1 \mathrm{H}), 5.61(\mathrm{~s}, 1 \mathrm{H})$, $4.83(\mathrm{~d}, J=3.0 \mathrm{~Hz}, 1 \mathrm{H}), 4.65(\mathrm{~d}, J=3.0 \mathrm{~Hz}, 1 \mathrm{H}), 3.89$ (s, 3H); ${ }^{13} \mathrm{C} \mathrm{NMR}(150 \mathrm{MHz}$, $\left.\mathrm{CDCl}_{3}\right): \delta=169.4,137.9,137.7,135.4,129.1,129.10,128.7,128.3,128.1,128.0,127.7$, 127.0, 122.3, 114.7, 78.2, 54.8, 53.1; HRMS Calculated for $\left[\mathrm{C}_{19} \mathrm{H}_{17} \mathrm{NO}_{4}+\mathrm{H}\right]^{+}:$324.12303, Found: $324.12316 .[\alpha]_{\mathrm{D}}{ }^{26}=104.5^{\circ}\left(\mathrm{c}=1.0 \mathrm{CHCl}_{3}\right)$. Enantiomeric excess was determined by HPLC with a Chiralcel OD-H column; $\lambda=254 \mathrm{~nm}$; eluent: Hexane/Isopropanol = 90/10; Flow rate: $1.0 \mathrm{~mL} / \mathrm{min}$; $\mathrm{t}_{\mathrm{minor}}=10.352 \mathrm{~min}, \mathrm{t}_{\mathrm{major}}=12.964 \mathrm{~min} ; \mathrm{ee} \%=91 \%$.

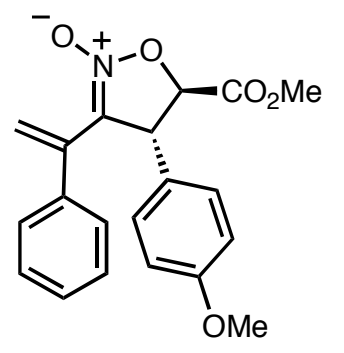

19b

\section{4-p-Methoxyphenyl-3 (1-phenylvinyl) 5- (methyl ester) isoxazoline- $\mathrm{N}$-oxide (19b)}

Purified by flash chromatography (hexane-EtOAc) as colorless oil (yield: 82\%); ${ }^{1} \mathrm{H}$ NMR $\left(600 \mathrm{MHz}, \mathrm{CDCl}_{3}\right) \delta=7.29-7.24(\mathrm{~m}, 3 \mathrm{H}), 7.03-7.01(\mathrm{~m}, 2 \mathrm{H}), 6.91-6.89(\mathrm{~m}, 2 \mathrm{H}), 6.78-$ $6.76(\mathrm{~m}, 2 \mathrm{H}), 6.28(\mathrm{~s}, 1 \mathrm{H}), 5.61(\mathrm{~s}, 1 \mathrm{H}), 4.80(\mathrm{~d}, J=3.0 \mathrm{~Hz}, 1 \mathrm{H}), 4.60(\mathrm{~d}, J=2.4 \mathrm{~Hz}$, $1 \mathrm{H}), 3.89(\mathrm{~s}, 3 \mathrm{H}), 3.77(\mathrm{~s}, 3 \mathrm{H}) ;{ }^{13} \mathrm{C} \mathrm{NMR}\left(150 \mathrm{MHz}, \mathrm{CDCl}_{3}\right): \delta=169.5,159.6,138.0$, $135.4,129.7,128.27,128.21,128.0,127.7,122.3,114.8,114.4,78.4,55.2,54.2,53.1$; HRMS Calculated for $\left[\mathrm{C}_{20} \mathrm{H}_{19} \mathrm{NO}_{5}+\mathrm{H}\right]+:$ 354.13360, Found: 354.13373 . $[\alpha]_{\mathrm{D}}{ }^{26}=105.6^{\circ}$ $\left(\mathrm{c}=1.0 \mathrm{CHCl}_{3}\right)$.

Enantiomeric excess was determined by HPLC with a Chiralcel OD-H column; $\lambda=254$ $\mathrm{nm}$; eluent: Hexane $/ \mathrm{Isopropanol}=90 / 10$; Flow rate: $1.0 \mathrm{~mL} / \mathrm{min} ; \mathrm{t}_{\text {minor }}=15.288 \mathrm{~min}, \mathrm{t}_{\text {major }}$ $=19.468 \mathrm{~min} ; \mathrm{ee} \%=94 \%$. 


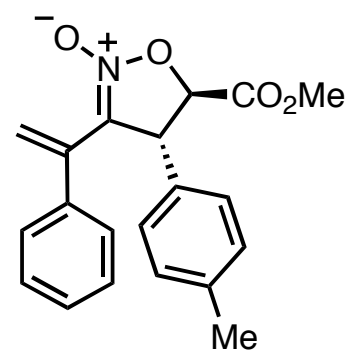

$19 \mathrm{c}$

\section{4-P-Toulyl-3 (1-phenylvinyl) 5- (methyl ester) isoxazoline- $N$-oxide (19c)}

Purified by flash chromatography (hexane-EtOAc) as colorless oil (yield: $89 \%$ ); ${ }^{1} \mathrm{H}$ NMR $\left(600 \mathrm{MHz}, \mathrm{CDCl}_{3}\right) \delta=7.28-7.23(\mathrm{~m}, 3 \mathrm{H}), 7.06-7.02(\mathrm{~m}, 4 \mathrm{H}), 6.89-6.88(\mathrm{~d}, J=8.4$ $\mathrm{Hz}, 2 \mathrm{H}), 6.24(\mathrm{~s}, 1 \mathrm{H}), 5.60(\mathrm{~s}, 1 \mathrm{H}), 4.81(\mathrm{~d}, J=2.4 \mathrm{~Hz}, 1 \mathrm{H}), 4.62(\mathrm{~d}, J=3.0 \mathrm{~Hz}, 1 \mathrm{H})$, $3.89(\mathrm{~s}, 3 \mathrm{H}), 3.30(\mathrm{~s}, 3 \mathrm{H}) ;{ }^{13} \mathrm{C} \mathrm{NMR}\left(150 \mathrm{MHz}, \mathrm{CDCl}_{3}\right): \delta=169.5,138.2,137.9,135.5$, $134.7,129.7,128.1,128.0,127.7,126.9,122.3,114.8,78.4,54.5,53.1,21.0$; HRMS Calculated for $\left[\mathrm{C}_{20} \mathrm{H}_{19} \mathrm{NO}_{4}+\mathrm{H}\right]^{+}: 338.13868$, Found: $338.13879 .[\alpha]_{\mathrm{D}}{ }^{26}=102.5^{\circ}(\mathrm{c}=1.0$ $\left.\mathrm{CHCl}_{3}\right)$.

Enantiomeric excess was determined by HPLC with a Chiralcel OD-H column; $\lambda=254$ $\mathrm{nm}$; eluent: Hexane $/ \mathrm{Isopropanol}=85 / 15$; Flow rate: $0.5 \mathrm{~mL} / \mathrm{min} ; \mathrm{t}_{\text {minor }}=24.012 \mathrm{~min}, \mathrm{t}_{\text {major }}$ $=25.484 \mathrm{~min} ; \mathrm{ee} \%=91 \%$.

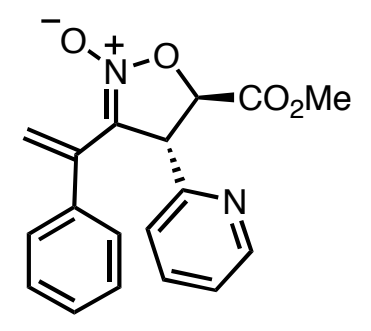

19d

\section{4-2'-pyridine-3 (1-phenylvinyl) 5- (methyl ester) isoxazoline- $N$-oxide (19d)}

Purified by flash chromatography (hexane-EtOAc) as colorless oil (yield: 90\%); ${ }^{1} \mathrm{H}$ NMR $\left(600 \mathrm{MHz}, \mathrm{CDCl}_{3}\right) \delta=8.56-8.55(\mathrm{dt}, J=4.8 \mathrm{~Hz}, 1 \mathrm{H}), 7.50-7.47$ (td, $J=7.8 \mathrm{~Hz}, J=1.8$ $\mathrm{Hz}, 1 \mathrm{H}), 7.26-7.22(\mathrm{~m}, 3 \mathrm{H}), 7.17-7.15(\mathrm{~m}, 1 \mathrm{H}), 7.07-7.05(\mathrm{~m}, 2 \mathrm{H}), 6.76-6.75(\mathrm{~d}, J=7.8$ $\mathrm{Hz}, 1 \mathrm{H}), 6.31(\mathrm{~s}, 1 \mathrm{H}), 5.62(\mathrm{~s}, 1 \mathrm{H}), 5.19(\mathrm{~d}, J=4.2 \mathrm{~Hz}, 1 \mathrm{H}), 4.91(\mathrm{~d}, J=4.2 \mathrm{~Hz}, 1 \mathrm{H})$, $3.88(\mathrm{~s}, 3 \mathrm{H}) ;{ }^{13} \mathrm{C}$ NMR $\left(150 \mathrm{MHz}, \mathrm{CDCl}_{3}\right): \delta=169.3,156.3,150.2,138.0,136.7,135.5$, 
128.21, 128.0, 127.6, 123, 122.4, 122.0, 114.1, 56.3, 53.1; HRMS Calculated for $\left[\mathrm{C}_{18} \mathrm{H}_{16} \mathrm{~N}_{2} \mathrm{O}_{4}+\mathrm{H}\right]^{+}: 325.11828$, Found: $325.11836 .[\alpha]_{\mathrm{D}}{ }^{26}=78.6^{\circ}\left(\mathrm{c}=1.0 \mathrm{CHCl}_{3}\right)$.

Enantiomeric excess was determined by HPLC with a Chiralcel As-H column; $\lambda=254$ $\mathrm{nm}$; eluent: Hexane/Isopropanol $=80 / 20$; Flow rate: $1.0 \mathrm{~mL} / \mathrm{min} ; \mathrm{t}_{\text {minor }}=14.124 \mathrm{~min}, \mathrm{t}_{\text {major }}$ $=11.992 \mathrm{~min} ; \mathrm{ee} \%=80 \%$.

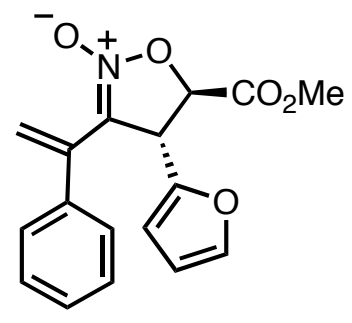

$19 \mathrm{e}$

\section{4-2'-Furyl-3 (1-phenylvinyl) 5- (methyl ester) isoxazoline- $\mathrm{N}$-oxide (19e)}

Purified by flash chromatography (hexane-EtOAc) as colorless oil (yield: $71 \%$ ); ${ }^{1} \mathrm{H}$ NMR $\left(600 \mathrm{MHz}, \mathrm{CDCl}_{3}\right) \delta=7.32-7.25(\mathrm{~m}, 4 \mathrm{H}), 7.12-7.10(\mathrm{~m}, 2 \mathrm{H}), 6.28(\mathrm{~s}, 1 \mathrm{H}), 6.24(\mathrm{dd}, J=$ $3.0 \mathrm{~Hz}, J=1.8 \mathrm{~Hz}, 1 \mathrm{H}), 5.95$ (d, $J=3.0 \mathrm{~Hz}, 1 \mathrm{H}), 5.67(\mathrm{~s}, 1 \mathrm{H}), 4.98(\mathrm{~d}, J=3.0 \mathrm{~Hz}, 1 \mathrm{H})$, 4.88 (d, $J=3.6 \mathrm{~Hz}, 1 \mathrm{H}), 3.89$ (s, $3 \mathrm{H}) ;{ }^{13} \mathrm{C} \mathrm{NMR}\left(150 \mathrm{MHz}, \mathrm{CDCl}_{3}\right): \delta=169.0,148.8$, 143.0, 137.8, 135.3, 128.3, 128.1, 127.5, 122.1, 112.0, 110.7, 108.5, 53.2, 48.4; HRMS Calculated for $\left[\mathrm{C}_{17} \mathrm{H}_{15} \mathrm{NO}_{5}+\mathrm{H}\right]^{+}: 314.10230$, Found: 314.10239. $[\alpha]_{\mathrm{D}}{ }^{26}=94.9^{\circ}(\mathrm{c}=1.0$ $\mathrm{CHCl}_{3}$ ). Enantiomeric excess was determined by HPLC with a Chiralcel OD-H column; $\lambda=254 \mathrm{~nm}$; eluent: Hexane/Isopropanol $=90 / 10$; Flow rate: $1.0 \mathrm{~mL} / \mathrm{min} ; \mathrm{t}_{\mathrm{minor}}=8.208$ $\min , \mathrm{t}_{\text {major }}=7.052 \mathrm{~min} ; \mathrm{ee} \%=90 \%$.

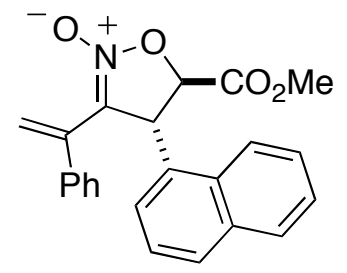

19f

4-2'-Napthyl-3 (1-phenylvinyl) 5- (methyl ester) isoxazoline- $N$-oxide (19f)

Purified by flash chromatography (hexane-EtOAc) as colorless oil (yield: $86 \%$ ); ${ }^{1} \mathrm{H}$ NMR (600 MHz, $\left.\mathrm{CDCl}_{3}\right) \delta=7.85-7.79(\mathrm{~m}, 3 \mathrm{H}), 7.48-7.42(\mathrm{~m}, 4 \mathrm{H})$, 7.14-7.12 (m, 3H), 
$7.02(\mathrm{~d}, J=3.6 \mathrm{~Hz}, 2 \mathrm{H}), 6.34(\mathrm{~s}, 1 \mathrm{H}), 5.62(\mathrm{~s}, 1 \mathrm{H}), 5.51(\mathrm{~s}, 1 \mathrm{H}), 4.83(\mathrm{~s}, 1 \mathrm{H}), 3.96$ (s, $3 \mathrm{H}) ;{ }^{13} \mathrm{C}$ NMR $\left(150 \mathrm{MHz}, \mathrm{CDCl}_{3}\right): \delta=187.3,169.9,137.8,135.8,134.0,132.8,130.2$, 129.1, 129.0, 128.1, 127.9, 127.6, 126.9, 126.0, 125.3, 122.5, 122.0, 114.3, 53.3; HRMS Calculated for $\left[\mathrm{C}_{23} \mathrm{H}_{19} \mathrm{NO}_{4}+\mathrm{H}\right]^{+}: 374.13868$, Found: $374.34876 .[\alpha]_{\mathrm{D}}{ }^{26}=120.1^{\circ}(\mathrm{c}=1.0$ $\left.\mathrm{CHCl}_{3}\right)$.

Enantiomeric excess was determined by HPLC with a Chiralcel As-H column; $\lambda=254$ $\mathrm{nm}$; eluent: Hexane/Isopropanol $=90 / 10$; Flow rate: $1.0 \mathrm{~mL} / \mathrm{min}$; $\mathrm{t}_{\text {minor }}=18.396 \mathrm{~min}, \mathrm{t}_{\text {major }}$ $=23.576 \mathrm{~min} ; \mathrm{ee} \%=96 \%$.<smiles>C=C(C1=C([O-])[N+](=O)OC1C(C)=O)c1ccccc1</smiles>

$19 \mathrm{~g}$

\section{4-Propyl-3-(1-phenylvinyl)-5-(methyl ester) isoxazoline- $N$-oxide (19g)}

Purified by flash chromatography (hexane-EtOAc) as colorless oil (yield: $63 \%$ ); ${ }^{1} \mathrm{H}$ NMR (600 MHz, $\left.\mathrm{CDCl}_{3}\right) \delta=7.37-7.34(\mathrm{~m}, 3 \mathrm{H}), 7.24-7.22(\mathrm{~m}, 1 \mathrm{H}), 6.36(\mathrm{~s}, 1 \mathrm{H}), 6.76(\mathrm{~s}$, $1 \mathrm{H}), 4.66(\mathrm{~d}, J=2.4 \mathrm{~Hz}, 1 \mathrm{H}), 3.87$ (s, 3H), 3.57-3.55 (m, 1H), 1.57-1.19 (m, 4H), 1.010.93 (m, $1 \mathrm{H}), 0.75$ (t, $J=7.2 \mathrm{~Hz}, 3 \mathrm{H}) ;{ }^{13} \mathrm{C}$ NMR $\left(150 \mathrm{MHz}, \mathrm{CDCl}_{3}\right): \delta=170.1,138.1$, 135.4, 128.5, 128.3, 127.6, 122.1, 114.8, 52.9, 48.9, 33.4, 18.8, 13.3; HRMS Calculated for $\left[\mathrm{C}_{16} \mathrm{H}_{19} \mathrm{NO}_{4}+\mathrm{H}\right]^{+}: 290.13868$, Found: $290.13895 .[\alpha]_{\mathrm{D}}{ }^{26}=94.5^{\circ}\left(\mathrm{c}=1.0 \mathrm{CHCl}_{3}\right)$. Enantiomeric excess was determined by HPLC with a Chiralcel As-H column; $\lambda=254$ $\mathrm{nm}$; eluent: Hexane/Isopropanol $=90 / 10$; Flow rate: $1.0 \mathrm{~mL} / \mathrm{min}$; $\mathrm{t}_{\text {minor }}=8.208 \mathrm{~min}, \mathrm{t}_{\text {major }}$ $=7.052 \mathrm{~min} ; \mathrm{ee} \%=88 \%$.

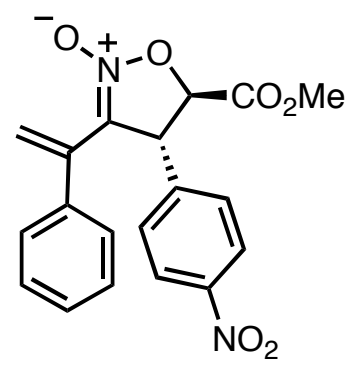

19h 


\section{4-p-Nitrophenyl-3-(1-phenylvinyl)-5-(methyl ester) isoxazoline- $N$-oxide (19h)}

Purified by flash chromatography (hexane-EtOAc) as colorless oil (yield: 92\%); ${ }^{1} \mathrm{H}$ NMR $\left(600 \mathrm{MHz}, \mathrm{CDCl}_{3}\right) \delta=8.12-8.07(\mathrm{~m}, 2 \mathrm{H}), 7.31-7.48(\mathrm{~m}, 3 \mathrm{H}), 7.14-7.11(\mathrm{~m}, 2 \mathrm{H}), 7.0-6.8$ $(\mathrm{m}, 2 \mathrm{H}), 6.45(\mathrm{~s}, 1 \mathrm{H}), 5.65(\mathrm{~s}, 1 \mathrm{H}), 4.82(\mathrm{~m}, 2 \mathrm{H}), 3.91(\mathrm{~m}, 3 \mathrm{H}) ;{ }^{13} \mathrm{C}$ NMR $(150 \mathrm{MHz}$, $\left.\mathrm{CDCl}_{3}\right): \delta=168.8,147.8,144.7,137.8,134.8,128.4,128.3,128.1,127.8,124.2,122.6$, 113.9, 54.2, 53.4; HRMS Calculated for $\left[\mathrm{C}_{19} \mathrm{H}_{16} \mathrm{~N}_{2} \mathrm{O}_{6}+\mathrm{H}\right]^{+}$: 369.10811, Found: 369.10821. $[\alpha]_{\mathrm{D}}{ }^{26}=82.2^{\circ}\left(\mathrm{c}=1.0 \mathrm{CHCl}_{3}\right)$. Enantiomeric excess was determined by HPLC with a Chiralcel OD-H column; $\lambda=254$ nm; eluent: Hexane/Isopropanol = 90/10; Flow rate: $1.0 \mathrm{~mL} / \mathrm{min} ; \mathrm{t}_{\text {minor }}=5.168 \mathrm{~min}, \mathrm{t}_{\text {major }}=5.860 \mathrm{~min} ; \mathrm{ee} \%=81 \%$.

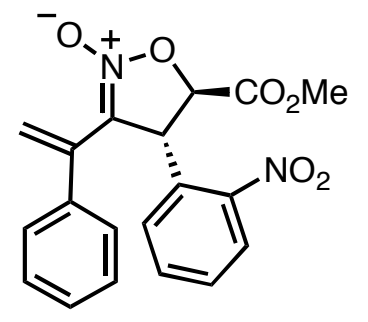

$19 \mathrm{i}$

\section{4-o-Nitrophenyl-3-(1-phenylvinyl) 5- (methyl ester) isoxazoline- $N$-oxide (19i)}

Purified by flash chromatography (hexane-EtOAc) as colorless oil (yield: 82\%); ${ }^{1} \mathrm{H}$ NMR $\left(600 \mathrm{MHz}, \mathrm{CDCl}_{3}\right) \delta=7.81-7.80(\mathrm{dd}, J=8.4 \mathrm{~Hz}, J=7.2 \mathrm{~Hz}, 1 \mathrm{H}), 7.65-7.62(\mathrm{td}, J=7.8$ $\mathrm{Hz}, J=1.2 \mathrm{~Hz}, 1 \mathrm{H}), 7.45-7.41(\mathrm{~m}, 2 \mathrm{H}), 7.26-7.19(\mathrm{~m}, 1 \mathrm{H}), 7.17-7.14(\mathrm{~m}, 2 \mathrm{H}), 6.95-6.93$ $(\mathrm{m}, 2 \mathrm{H}), 6.50(\mathrm{~s}, 1 \mathrm{H}), 5.65(\mathrm{~s}, 1 \mathrm{H}), 5.42(\mathrm{~d}, J=3.0 \mathrm{~Hz}, 1 \mathrm{H}), 4.83-4.82(\mathrm{~d}, J=3.0 \mathrm{~Hz}$, $1 \mathrm{H}), 3.92(\mathrm{~s}, 3 \mathrm{H}) ;{ }^{13} \mathrm{C} \mathrm{NMR}\left(150 \mathrm{MHz}, \mathrm{CDCl}_{3}\right): \delta=168.3,147.8,137.9,135.2,133.9$, 129.4, 129.3, 128.29, 128.26, 127.5, 125.1, 122.4, 113.8, 53.3, 49.7; HRMS Calculated for $\left[\mathrm{C}_{19} \mathrm{H}_{16} \mathrm{~N}_{2} \mathrm{O}_{6}+\mathrm{H}\right]^{+}: 369.10811$, Found: 369.10821. $[\alpha]_{\mathrm{D}}{ }^{26}=72.5^{\circ}\left(\mathrm{c}=1.0 \mathrm{CHCl}_{3}\right)$. Enantiomeric excess was determined by HPLC with a Chiralcel OD-H column; $\lambda=254$ $\mathrm{nm}$; eluent: Hexane/Isopropanol = 90/10; Flow rate: $1.0 \mathrm{~mL} / \mathrm{min} ; \mathrm{t}_{\text {minor }}=29.956 \mathrm{~min}$, $\mathrm{t}_{\text {major }}=24.792 \mathrm{~min} ; \mathrm{ee} \%=70 \%$. 


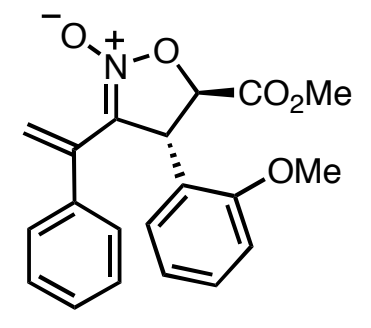

19j

\section{4-o-Anisyl-3-(1-phenylvinyl) 5- (methyl ester) isoxazoline- $N$-oxide (19j)}

Purified by flash chromatography (hexane-EtOAc) as colorless oil (yield: 84\%); ${ }^{1} \mathrm{H}$ NMR $\left(600 \mathrm{MHz}, \mathrm{CDCl}_{3}\right) \delta=7.25-7.20(\mathrm{~m}, 4 \mathrm{H}), 7.06-7.04(\mathrm{~m}, 2 \mathrm{H}), 7.01-6.99$ (dd, $J=7.8 \mathrm{~Hz}, J$ $=6.0 \mathrm{~Hz}, 1 \mathrm{H}), 6.86-6.83(\mathrm{td}, J=7.2 \mathrm{~Hz}, J=1.2 \mathrm{~Hz}, 1 \mathrm{H}), 6.78-6.76(\mathrm{dd}, J=7.8 \mathrm{~Hz}, J=$ $7.2 \mathrm{~Hz}, 1 \mathrm{H}), 6.22$ (s, 1H), 5.59 (s, 1H), 5.08-5.07 (d, $J=3.6 \mathrm{~Hz}, 1 \mathrm{H}), 4.81$ (d, J=4.2 Hz, $1 \mathrm{H}), 3.87(\mathrm{~s}, 3 \mathrm{H}), 3.68(\mathrm{~s}, 3 \mathrm{H}) ;{ }^{13} \mathrm{C} \mathrm{NMR}\left(150 \mathrm{MHz}, \mathrm{CDCl}_{3}\right): \delta=169.5,156.4,137.9$, $135.8,129.6,128.5,128.0,127.7,127.6,125.3,121.9,120.8,114.3,110.7,55.4,52.9$, 49.2; HRMS Calculated for $\left[\mathrm{C}_{20} \mathrm{H}_{19} \mathrm{NO}_{5}+\mathrm{H}\right]^{+}: 354.13360$, Found: 354.13373 . $[\alpha]_{\mathrm{D}}{ }^{26}=$ $99.5^{\circ}\left(\mathrm{c}=1.0 \mathrm{CHCl}_{3}\right)$. Enantiomeric excess was determined by HPLC with a Chiralcel As-H column; $\lambda=254 \mathrm{~nm}$; eluent: Hexane/Isopropanol = 85/15; Flow rate: $1.0 \mathrm{~mL} / \mathrm{min}$; $\mathrm{t}_{\text {minor }}=14.828 \mathrm{~min}, \mathrm{t}_{\text {major }}=10.184 \mathrm{~min} ; \mathrm{ee} \%=88 \%$.

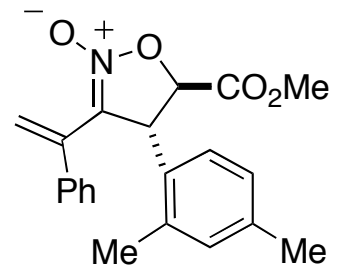

$19 \mathrm{k}$

4-2', 4' dimethylphenyl-3 (1-phenylvinyl) 5- (methyl ester) isoxazoline- $N$-oxide (19k) Purified by flash chromatography (hexane-EtOAc) as colorless oil (yield: 79\%); ${ }^{1} \mathrm{H}$ NMR $\left(600 \mathrm{MHz}, \mathrm{CDCl}_{3}\right) \delta=7.25-7.20(\mathrm{~m}, 3 \mathrm{H}), 6.97-6.95(\mathrm{~m}, 4 \mathrm{H}), 6.90-6.89(\mathrm{~m}, 1 \mathrm{H}), 6.37(\mathrm{~s}$, 1H), 5.60 (d, $J=1.2 \mathrm{~Hz}, 1 \mathrm{H}), 4.83$ (d, $J=3.0 \mathrm{~Hz}, 1 \mathrm{H}), 4.69$ (d, $J=2.4 \mathrm{~Hz}, 1 \mathrm{H}), 3.89$ (s, $3 \mathrm{H}), 2.29(\mathrm{~s}, 3 \mathrm{H}), 1.83(\mathrm{~s}, 3 \mathrm{H}) ;{ }^{13} \mathrm{C} \mathrm{NMR}\left(150 \mathrm{MHz}, \mathrm{CDCl}_{3}\right): \delta=169.7,138.2,136.5$, $135.7,135.4,132.3,130.6,129.0,128.1,127.9,127.7,127.4,122.2,114.9,78.0,53.1$, 50.9, 20.9, 18.2; HRMS Calculated for $\left[\mathrm{C}_{21} \mathrm{H}_{21} \mathrm{NO}_{4}+\mathrm{H}\right]^{+}:$352.15433, Found: 352.15444. $[\alpha]_{\mathrm{D}}^{26}=101.1^{\circ}\left(\mathrm{c}=1.0 \mathrm{CHCl}_{3}\right)$. 
Enantiomeric excess was determined by HPLC with a Chiralcel As-H column; $\lambda=254$ $\mathrm{nm}$; eluent: Hexane/Isopropanol $=90 / 10$; Flow rate: $1.0 \mathrm{~mL} / \mathrm{min}$; $\mathrm{t}_{\text {minor }}=16.352 \mathrm{~min}, \mathrm{t}_{\text {major }}$ $=21.012 \mathrm{~min} ; \mathrm{ee} \%=92 \%$.<smiles>C=C(C1=[N+]([O-])OC(C(C)=O)[C@H]1c1ccco1)c1ccc(Cl)cc1</smiles>

191

\section{4-2' furyl-3 (1-phenylvinyl) 5- (methyl ester) isoxazoline- $N$-oxide (19l)}

Purified by flash chromatography (hexane-EtOAc) as colorless oil (yield: $81 \%$ ); ${ }^{1} \mathrm{H}$ NMR $\left(600 \mathrm{MHz}, \mathrm{CDCl}_{3}\right) \delta=7.35-7.34(\mathrm{~m}, 1 \mathrm{H}), 7.28-7.26(\mathrm{~m}, 2 \mathrm{H}), 7.06-7.05(\mathrm{~m}, 2 \mathrm{H}), 6.27$ (dd, $J=3.6 \mathrm{~Hz}, J=1.8 \mathrm{~Hz}, 1 \mathrm{H}), 6.20(\mathrm{~s}, 1 \mathrm{H}), 6.03(\mathrm{~d}, J=3.6 \mathrm{~Hz}, 1 \mathrm{H}), 5.63(\mathrm{~s}, 1 \mathrm{H}), 4.98$ (d, $J=3.6 \mathrm{~Hz}, 1 \mathrm{H}), 4.86(\mathrm{~d}, J=3.6 \mathrm{~Hz}, 1 \mathrm{H}), 3.89(\mathrm{~s}, 3 \mathrm{H}) ;{ }^{13} \mathrm{C} \mathrm{NMR}\left(150 \mathrm{MHz}, \mathrm{CDCl}_{3}\right)$ : $\delta=168.8,148.7,143.2,136.0,134.4 ; 134.2,128.8,128.5,122.5,111.7,110.8,108.6$, 75.7, 53.3, 48.3; HRMS Calculated for $\left[\mathrm{C}_{17} \mathrm{H}_{14} \mathrm{ClNO}_{5}+\mathrm{H}\right]^{+}:$348.06333, Found: 348.06351. $[\alpha]_{\mathrm{D}}{ }^{26}=69.2^{\circ}\left(\mathrm{c}=1.0 \mathrm{CHCl}_{3}\right)$. Enantiomeric excess was determined by HPLC with a Chiralcel OD-H column; $\lambda=254 \mathrm{~nm}$; eluent: Hexane/Isopropanol $=90 / 10$; Flow rate: $1.0 \mathrm{~mL} / \mathrm{min} ; \mathrm{t}_{\text {minor }}=18.044 \mathrm{~min}, \mathrm{t}_{\text {major }}=23.184 \mathrm{~min} ; \mathrm{ee} \%=76 \%$.<smiles>C=C(C1=C([O-])OC(C(C)=O)C1c1ccccc1OC)c1ccc(Cl)cc1</smiles>

$19 m$

\section{4-o-Anisyl-3 (p-chlorophenylvinyl) 5- (methyl ester) isoxazoline- $N$-oxide (19m)}

Purified by flash chromatography (hexane-EtOAc) as colorless oil (yield: $88 \%$ ); ${ }^{1} \mathrm{H}$ NMR $\left(600 \mathrm{MHz}, \mathrm{CDCl}_{3}\right) \delta=7.26-7.23(\mathrm{~m}, 1 \mathrm{H}), 7.20-7.18(\mathrm{~m}, 2 \mathrm{H}), 7.05-7.03$ (dd, $J=7.8 \mathrm{~Hz}, J$ 
$=5.4 \mathrm{~Hz}, 1 \mathrm{H}), 7.00-6.97(\mathrm{~m}, 2 \mathrm{H}), 6.89-6.86(\mathrm{td}, J=7.8 \mathrm{~Hz}, J=6.6 \mathrm{~Hz}, 1 \mathrm{H}), 6.79(\mathrm{~d}, J=$ $7.2 \mathrm{~Hz}, 1 \mathrm{H}), 6.19$ (s, 1H), 5.55 (s, 1H), 5.07 (d, $J=3.6 \mathrm{~Hz}, 1 \mathrm{H}), 4.81$ (d, $J=4.2 \mathrm{~Hz}, 1 \mathrm{H})$, $3.87(\mathrm{~s}, 3 \mathrm{H}), 3.71(\mathrm{~s}, 3 \mathrm{H}) ;{ }^{13} \mathrm{C} \mathrm{NMR}\left(150 \mathrm{MHz}, \mathrm{CDCl}_{3}\right): \delta=169.4,156.3,136.2,134.9$, 133.8129.8, 129.0, 128.5, 128.2, 125.1, 122.2, 120.9, 114.1, 110.7, 77.7, 55.4, 53.0, 48.9; HRMS Calculated for $\left[\mathrm{C}_{20} \mathrm{H}_{18} \mathrm{NClO}_{5}+\mathrm{H}\right]^{+}: 388.09463$, Found: $388.09473 .[\alpha]_{\mathrm{D}}{ }^{26}=84.5^{\circ}$ $\left(\mathrm{c}=1.0 \mathrm{CHCl}_{3}\right.$ ). Enantiomeric excess was determined by HPLC with a Chiralcel As-H column; $\lambda=254 \mathrm{~nm}$; eluent: Hexane/Isopropanol $=85 / 15$; Flow rate: $1.0 \mathrm{~mL} / \mathrm{min}$; $\mathrm{t}_{\text {minor }}$ $=10.192 \mathrm{~min}, \mathrm{t}_{\text {major }}=8.804 \mathrm{~min} ; \mathrm{ee} \%=81 \%$.

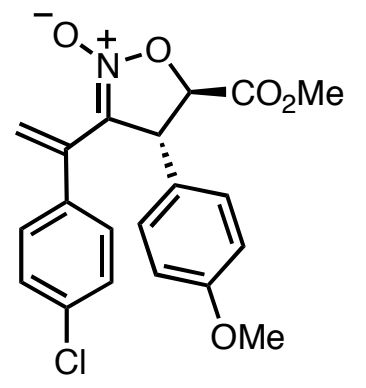

19n

\section{4-p-Anisyl-3 (p-chlorophenylvinyl) 5-(methyl ester) isoxazoline- $N$-oxide (19n)}

Purified by flash chromatography (hexane-EtOAc) as colorless oil (yield: $81 \%$ ); ${ }^{1} \mathrm{H}$ NMR $\left(600 \mathrm{MHz}, \mathrm{CDCl}_{3}\right) \delta=7.24-7.22(\mathrm{~m}, 2 \mathrm{H}), 6.97-6.94(\mathrm{~m}, 4 \mathrm{H}), 6.81-6.79(\mathrm{dt}, J=9.0 \mathrm{~Hz}, J$ $=4.2 \mathrm{~Hz}, 2 \mathrm{H}), 6.21(\mathrm{~s}, 1 \mathrm{H}), 5.57(\mathrm{~s}, 1 \mathrm{H}), 4.80(\mathrm{~d}, J=3.0 \mathrm{~Hz}, 1 \mathrm{H}), 4.58(\mathrm{~d}, J=3.0 \mathrm{~Hz}$, $1 \mathrm{H}), 3.90-3.88$ (s, 3H), 3.78 (s, 3H); ${ }^{13} \mathrm{C}$ NMR (150 MHz, $\left.\mathrm{CDCl}_{3}\right): \delta=169.4,159.7$, 136.2 , 134.5, 134.1, 129.5, 129.0, 128.4, 128.2, 122.6, 114.6, 114.5, 55.3, 54.1, 53.1; HRMS Calculated for $\left[\mathrm{C}_{20} \mathrm{H}_{18} \mathrm{NClO}_{5}+\mathrm{H}\right]^{+}: 388.09463$, Found: $388.09476 .[\alpha]_{\mathrm{D}}{ }^{26}=110.4^{\circ}$ $\left(\mathrm{c}=1.0 \mathrm{CHCl}_{3}\right)$. Enantiomeric excess was determined by HPLC with a Chiralcel OD-H column; $\lambda=254 \mathrm{~nm}$; eluent: Hexane/Isopropanol $=80 / 20$; Flow rate: $1.0 \mathrm{~mL} / \mathrm{min} ; \mathrm{t}_{\mathrm{minor}}$ $=6.920 \mathrm{~min}, \mathrm{t}_{\mathrm{major}}=11.420 \mathrm{~min} ; \mathrm{ee} \%=92 \%$. 


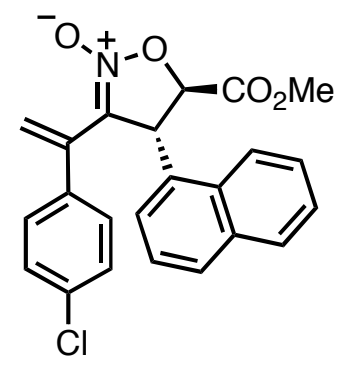

190

\section{4-Napthyl-3 (p-chlorophenylvinyl) 5- (methyl ester) isoxazoline- $\mathrm{N}$-oxide (190)}

Purified by flash chromatography (hexane-EtOAc) as colorless oil (yield: 91\%); ${ }^{1} \mathrm{H}$ NMR $\left(600 \mathrm{MHz}, \mathrm{CDCl}_{3}\right) \delta=7.87-7.81(\mathrm{~m}, 3 \mathrm{H}), 7.52-7.44(\mathrm{~m}, 4 \mathrm{H}), 7.11-7.09(\mathrm{~m}, 2 \mathrm{H}), 6.95(\mathrm{~d}$, $J=7.8 \mathrm{~Hz}, 2 \mathrm{H}), 6.27(\mathrm{~s}, 1 \mathrm{H}), 5.57(\mathrm{~s}, 1 \mathrm{H}), 4.49(\mathrm{~s}, 1 \mathrm{H}), 4.85(\mathrm{~s}, 1 \mathrm{H}), 3.96(\mathrm{~s}, 3 \mathrm{H}) ;{ }^{13} \mathrm{C}$ NMR (150 MHz, $\left.\mathrm{CDCl}_{3}\right): \delta=169.7,136.1,134.8,134.0,132.7,130.2,129.3,129.1$, $128.9,128.3,127.1,126.1,125.3,122.8,121.9,114.1,78.0,53.3,50.1$; HRMS Calculated for $\left[\mathrm{C}_{23} \mathrm{H}_{18} \mathrm{ClNO}_{4}+\mathrm{H}\right]^{+}: 408.09971$, Found: 408.09996. $[\alpha]_{\mathrm{D}}{ }^{26}=120.9^{\circ}(\mathrm{c}=$ $\left.1.0 \mathrm{CHCl}_{3}\right)$.

Enantiomeric excess was determined by HPLC with a Chiralcel As-H column; $\lambda=254$ $\mathrm{nm}$; eluent: Hexane/Isopropanol $=85 / 15$; Flow rate: $1.0 \mathrm{~mL} / \mathrm{min}$; $\mathrm{t}_{\text {minor }}=6.148 \mathrm{~min}, \mathrm{t}_{\text {major }}$ $=9.672 \mathrm{~min} ; \mathrm{ee} \%=93 \%$.

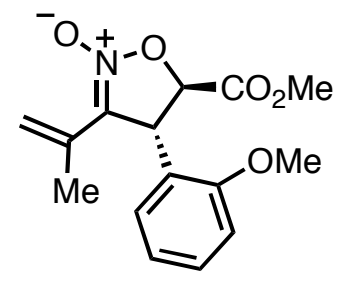

$19 p$

\section{4-o-Anisyl-3 (Methylvinyl) 5- (methyl ester) isoxazoline- $\mathrm{N}$-oxide (19p)}

Purified by flash chromatography (hexane-EtOAc) as colorless oil (yield: 75\%); ${ }^{1} \mathrm{H}$ NMR $\left(600 \mathrm{MHz}, \mathrm{CDCl}_{3}\right) \delta=7.33-7.30(\mathrm{td}, J=7.8 \mathrm{~Hz}, J=6.6 \mathrm{~Hz}, 1 \mathrm{H}), 7.14-7.12(\mathrm{dd}, J=7.8$ $\mathrm{Hz}, J=6.0 \mathrm{~Hz}, 1 \mathrm{H}), 6.96-6.92(\mathrm{~m}, 2 \mathrm{H}), 5.71(\mathrm{~s}, 1 \mathrm{H}), 5.25(\mathrm{~s}, 1 \mathrm{H}), 5.13(\mathrm{~d}, J=3.0 \mathrm{~Hz}$, $1 \mathrm{H}), 4.69(\mathrm{~d}, J=3.0 \mathrm{~Hz}, 1 \mathrm{H}), 3.88(\mathrm{~s}, 3 \mathrm{H}), 3.86(\mathrm{~s}, 3 \mathrm{H}), 1.95(\mathrm{~s}, 3 \mathrm{H}) ;{ }^{13} \mathrm{C} \mathrm{NMR}(150$ $\left.\mathrm{MHz}, \mathrm{CDCl}_{3}\right): \delta=169.6,156.2,130.2,129.7,127.9,126.0,121.1,119.9,115.8,110.9$, 
55.6, 52.9, 48.2, 20.5; HRMS Calculated for $\left[\mathrm{C}_{15} \mathrm{H}_{16} \mathrm{NO}_{5}+\mathrm{H}\right]^{+}: 292.11795$, Found: 292.11810. $[\alpha]_{\mathrm{D}}^{26}=100.1^{\circ}\left(\mathrm{c}=1.0 \mathrm{CHCl}_{3}\right)$.

Enantiomeric excess was determined by HPLC with a Chiralcel As-H column; $\lambda=254$ $\mathrm{nm}$; eluent: Hexane/Isopropanol $=95 / 5$; Flow rate: $1.0 \mathrm{~mL} / \mathrm{min} ; \mathrm{t}_{\text {minor }}=18.072 \mathrm{~min}, \mathrm{t}_{\text {major }}$ $=16.452 \mathrm{~min} ; \mathrm{ee} \%=81 \%$.

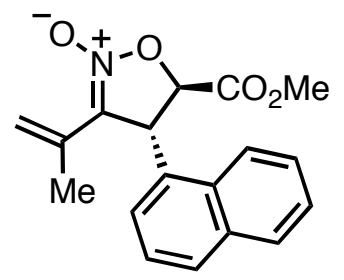

\section{$19 q$}

\section{4-Napthyl-3 (Methylvinyl) 5- (methyl ester) isoxazoline- $N$-oxide (19q)}

Purified by flash chromatography (hexane-EtOAc) as colorless oil (yield: 70\%); ${ }^{1} \mathrm{H}$ NMR $\left(600 \mathrm{MHz}, \mathrm{CDCl}_{3}\right) \delta=8.25(\mathrm{~d}, J=7.2 \mathrm{~Hz}, 1 \mathrm{H}), 7.94-7.93(\mathrm{dd}, J=7.8 \mathrm{~Hz}, J=7.2 \mathrm{~Hz}$, $1 \mathrm{H}), 7.87-7.86(\mathrm{~d}, J=9.6 \mathrm{~Hz}, 1 \mathrm{H}), 7.66-7.63(\mathrm{td}, J=8.4 \mathrm{~Hz}, J=5.4 \mathrm{~Hz}, 1 \mathrm{H}), 7.59-7.56$ (td, $J=8.4 \mathrm{~Hz}, J=6.0 \mathrm{~Hz}, 1 \mathrm{H}), 7.47-7.44$ (t, $J=8.4 \mathrm{~Hz}, 1 \mathrm{H}), 7.31$ (d, $J=6.6 \mathrm{~Hz}, 1 \mathrm{H})$, $5.81(\mathrm{~s}, 1 \mathrm{H}), 5.56(\mathrm{~s}, 1 \mathrm{H}), 5.31(\mathrm{~s}, 1 \mathrm{H}), 4.76(\mathrm{~s}, 1 \mathrm{H}), 3.95(\mathrm{~s}, 3 \mathrm{H}), 1.94(\mathrm{~s}, 3 \mathrm{H}) ;{ }^{13} \mathrm{C} \mathrm{NMR}$ $\left(150 \mathrm{MHz}, \mathrm{CDCl}_{3}\right): \delta=170.1,134.3,132.8,130.3,130.0,129.36,129.33,127.3,126.2$, $125.5,124.6,122.3,120.3,115.6,53.3,50.2,20.7$; HRMS Calculated for $\left[\mathrm{C}_{18} \mathrm{H}_{17} \mathrm{NO}_{4}+\mathrm{H}\right]^{+}:$312.12303, Found: 312.12323. $[\alpha]_{\mathrm{D}}{ }^{26}=114.0^{\circ}\left(\mathrm{c}=1.0 \mathrm{CHCl}_{3}\right)$. Enantiomeric excess was determined by HPLC with a Chiralcel OD-H column; $\lambda=254$ $\mathrm{nm}$; eluent: Hexane/Isopropanol $=90 / 10$; Flow rate: $1.0 \mathrm{~mL} / \mathrm{min}$; $\mathrm{t}_{\text {minor }}=8.148 \mathrm{~min}$, $\mathrm{t}_{\text {major }}$ $=13.664 \mathrm{~min} ; \mathrm{ee} \%=93 \%$.

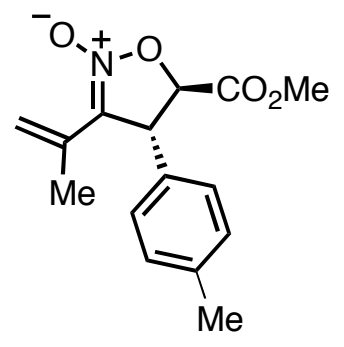

19r

4-Toulyl-3 (Methylvinyl) 5- (methyl ester) isoxazoline- $N$-oxide (19r) 
Purified by flash chromatography (hexane-EtOAc) as colorless oil (yield: 80\%); ${ }^{1} \mathrm{H}$ NMR $\left(600 \mathrm{MHz}, \mathrm{CDCl}_{3}\right) \delta=7.20-7.16(\mathrm{~m}, 4 \mathrm{H}), 5.27(\mathrm{~s}, 1 \mathrm{H}), 4.70(\mathrm{~d}, J=2.4 \mathrm{~Hz}, 1 \mathrm{H}), 4.68(\mathrm{~d}$, $J=2.4 \mathrm{~Hz}, 2 \mathrm{H}), 3.87$ (s, 3H), 2.35 (s, 3H), 1.93 (s, 3H); $\left.{ }^{13} \mathrm{C} \mathrm{NMR} \mathrm{(150} \mathrm{MHz,} \mathrm{CDCl}_{3}\right): \delta$ $=169.6 ; 138.5,135.4,130.1,129.9,126.8,120.2,116.0,78.3,54.2,53.1,21.1,20.7$; HRMS Calculated for $\left[\mathrm{C}_{15} \mathrm{H}_{17} \mathrm{NO}_{4}+\mathrm{H}\right]^{+}: 276.12327$, Found: 276.12357. $[\alpha]_{\mathrm{D}}{ }^{26}=94.5^{\circ}(\mathrm{c}$ $=1.0 \mathrm{CHCl}_{3}$ ). Enantiomeric excess was determined by HPLC with a Chiralcel As- $\mathrm{H}$ column; $\lambda=254 \mathrm{~nm}$; eluent: Hexane/Isopropanol $=85 / 15$; Flow rate: $1.0 \mathrm{~mL} / \mathrm{min}$; $\mathrm{t}_{\text {minor }}$ $=7.640 \mathrm{~min}, \mathrm{t}_{\text {major }}=8.936 \mathrm{~min} ; \mathrm{ee} \%=85 \%$.

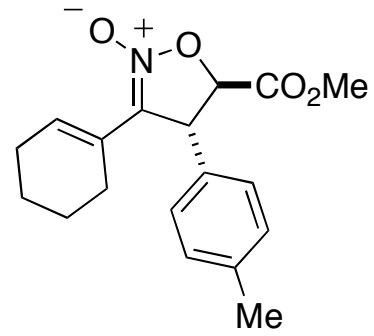

19s

\section{2-p-Toulyl-3 (cyclohexenyl) 5- (methyl ester) isoxazoline- $\mathrm{N}$-oxide (19s)}

Purified by flash chromatography (hexane-EtOAc) as colorless oil (yield: 80\%); ${ }^{1} \mathrm{H}$ NMR $\left(600 \mathrm{MHz}, \mathrm{CDCl}_{3}\right) \delta=7.19-7.15(\mathrm{~m}, 4 \mathrm{H}), 6.64-6.63$ (q, $\left.J=6.0 \mathrm{~Hz}, 1 \mathrm{H}\right), 4.66-4.65$ (q, $J=$ $4.8 \mathrm{~Hz}, 2 \mathrm{H}), 3.86$ (s, 3H), 2.35 (s, 3H), 2.26-2.23 (m, 1H), 2.15-2.12 (m, 3H), 1.60-1.47 $(\mathrm{m}, 4 \mathrm{H}) ;{ }^{13} \mathrm{C}$ NMR $\left(150 \mathrm{MHz}, \mathrm{CDCl}_{3}\right): \delta=169.9,138.2,135.6,133.4,130.0,126.8$, 124.0, 116.2, 54.1, 53.0, 25.9, 25.7, 22.2, 21.2, 21.1; HRMS Calculated for $\left[\mathrm{C}_{18} \mathrm{H}_{21} \mathrm{NO}_{4}+\mathrm{H}\right]^{+}:$316.15433, Found: 316.15441. $[\alpha]_{\mathrm{D}}{ }^{26}=102.8^{\circ}\left(\mathrm{c}=1.0 \mathrm{CHCl}_{3}\right)$. Enantiomeric excess was determined by HPLC with a Chiralcel OD-H column; $\lambda=254$ $\mathrm{nm}$; eluent: Hexane/Isopropanol $=85 / 15$; Flow rate: $1.0 \mathrm{~mL} / \mathrm{min}$; $\mathrm{t}_{\text {minor }}=7.356 \mathrm{~min}, \mathrm{t}_{\text {major }}$ $=12.192 \mathrm{~min} ; \mathrm{ee} \%=90 \%$.<smiles>CC(=O)C1ON=[N+]([O-])C1=C1CCCCC1</smiles>

19t

2-Napthyl-3 (cyclohexenyl) 5- (methyl ester) isoxazoline- $\mathrm{N}$-oxide (19t) 
Purified by flash chromatography (hexane-EtOAc) as colorless oil (yield: $85 \%$ ); ${ }^{1} \mathrm{H}$ NMR $\left(600 \mathrm{MHz}, \mathrm{CDCl}_{3}\right) \delta=8.25(\mathrm{~d}, J=8.4 \mathrm{~Hz}, 1 \mathrm{H}), 7.94(\mathrm{~d}, J=7.8 \mathrm{~Hz}, 1 \mathrm{H}), 7.86(\mathrm{~d}, J=8.4$ $\mathrm{Hz}, 1 \mathrm{H}), 7.66-7.57(\mathrm{td}, J=7.2 \mathrm{~Hz}, J=6.0 \mathrm{~Hz}, 1 \mathrm{H}), 7.58-7.56(\mathrm{td}, J=7.2 \mathrm{~Hz}, J=6.0 \mathrm{~Hz}$, $1 \mathrm{H}), 7.47$ (t, $J=8.4 \mathrm{~Hz}, 1 \mathrm{H}), 7.32$ (d, $J=6.6 \mathrm{~Hz}, 1 \mathrm{H}), 6.72(\mathrm{~s}, 1 \mathrm{H}), 5.53(\mathrm{~s}, 1 \mathrm{H}), 4.71$ (s, 1H), $3.94(\mathrm{~s}, 3 \mathrm{H}), 2.33-2.30(\mathrm{~m}, 1 \mathrm{H}), 2.13-2.04(\mathrm{~m}, 3 \mathrm{H}), 1.59-1.50(\mathrm{~m}, 4 \mathrm{H}) ;{ }^{13} \mathrm{C}$ NMR (150 MHz, $\left.\mathrm{CDCl}_{3}\right): \delta=187.3,173.3,170.4,134.3,133.6,133.0,130.3,129.3,129.1$, 127.2, 126.2, 125.9, 124.6, 124.1, 122.4, 115.8, 53.2, 50.1, 25.9, 25.7, 22.2, 21.2; HRMS Calculated for $\left[\mathrm{C}_{21} \mathrm{H}_{21} \mathrm{NO}_{4}+\mathrm{H}\right]^{+}: 352.15433$, Found: $352.15440 .[\alpha]_{\mathrm{D}}{ }^{26}=101.3^{\circ}(\mathrm{c}=1.0$ $\left.\mathrm{CHCl}_{3}\right)$.

Enantiomeric excess was determined by HPLC with a Chiralcel OD-H column; $\lambda=254$ $\mathrm{nm}$; eluent: Hexane/Isopropanol $=90 / 10$; Flow rate: $1.0 \mathrm{~mL} / \mathrm{min}$; $\mathrm{t}_{\text {minor }}=6.896 \mathrm{~min}, \mathrm{t}_{\text {major }}$ $=11.084 \mathrm{~min} ; \mathrm{ee} \%=91 \%$.

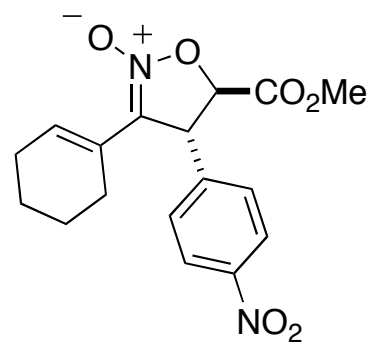

19u

\section{2-p-Nitrophenyl-3 (cyclohexenyl) 5- (methyl ester) isoxazoline- $N$-oxide(19u)}

Purified by flash chromatography (hexane-EtOAc) as colorless oil (yield: 93\%); ${ }^{1} \mathrm{H}$ NMR $\left(600 \mathrm{MHz}, \mathrm{CDCl}_{3}\right) \delta=8.28-8.26(\mathrm{~m}, 2 \mathrm{H}), 7.51-7.48(\mathrm{~m}, 2 \mathrm{H}), 6.54-6.52(\mathrm{p}, J=4.2 \mathrm{~Hz}$, $1 \mathrm{H}), 4.84$ (d, $J=2.4 \mathrm{~Hz}, 1 \mathrm{H}), 4.67$ (d, $J=2.4 \mathrm{~Hz}, 1 \mathrm{H}), 3.89$ (s, 3H), 2.31-2.27 (m, 1H), 2.16-2.04 (m, 3H), 1.67-1.48 (m, 4H); ${ }^{13} \mathrm{C}$ NMR (150 MHz, $\left.\mathrm{CDCl}_{3}\right): \delta=169.3,145.6$, 134.1, 128.1, 124.7, 123.7, 115.2, 53.9, 53.4, 26.0, 25.8, 22.1, 21.1; HRMS Calculated for $\left[\mathrm{C}_{17} \mathrm{H}_{18} \mathrm{~N}_{2} \mathrm{O}_{6}+\mathrm{H}\right]^{+}: 347.12376$, Found: 347.12382. $[\alpha]_{\mathrm{D}}{ }^{26}=62.1^{\circ}\left(\mathrm{c}=1.0 \mathrm{CHCl}_{3}\right)$. Enantiomeric excess was determined by HPLC with a Chiralcel OD-H column; $\lambda=254$ $\mathrm{nm}$; eluent: Hexane/Isopropanol $=80 / 20$; Flow rate: $1.0 \mathrm{~mL} / \mathrm{min}$; $\mathrm{t}_{\text {minor }}=8.376$ min, $\mathrm{t}_{\text {major }}$ $=14.728 \mathrm{~min} ; \mathrm{ee} \%=65 \%$. 
<smiles>CCOC(=O)[C@H]1O[N+]([O-])=C(C(=O)c2ccccc2)C1c1ccccc1</smiles>

22

Purified by flash chromatography (hexane-EtOAc) as white floppy solid (yield: $91 \%$ ); ${ }^{1} \mathrm{H}$ NMR $\left(600 \mathrm{MHz}, \mathrm{CDCl}_{3}\right) \delta=7.74-7.72(\mathrm{~m}, 2 \mathrm{H}), 7.58-7.55(\mathrm{~m}, 1 \mathrm{H}), 7.45-7.43(\mathrm{~m}, 2 \mathrm{H})$, 7.40-7.38 (m, 4H), 7.36-7.33 (m, 1H), 5.10-5.09 (d, $J=3.6 \mathrm{~Hz}, 1 \mathrm{H}), 5.03-5.02(\mathrm{~d}, J=4.2$ $\mathrm{Hz}, 1 \mathrm{H}), 4.39-4.35$ (q, $J=7.2 \mathrm{~Hz}, 2 \mathrm{H}), 1.38-1.36(\mathrm{t}, J=7.2 \mathrm{~Hz}, 3 \mathrm{H}) .{ }^{13} \mathrm{C}$ NMR (150 $\left.\mathrm{MHz}, \mathrm{CDCl}_{3}\right): \delta=184.5,168.1,137.7,135.0,133.6,129.5,128.9,128.8,128.5,127.1$, 115.6, 79.4, 62.7, 53.9, 14.1. HRMS Calculated for $\left[\mathrm{C}_{19} \mathrm{H}_{17} \mathrm{NO}_{5}+\mathrm{H}\right]^{+}: 340.11795$, Found: 340.11786 .<smiles>CC(=O)[C@H]1ON=C(C(=O)c2ccccc2)[C@H]1c1ccccc1</smiles>

26

Purified by flash chromatography (hexane-EtOAc) as colorless oil (yield: 72\%); ${ }^{1} \mathrm{H}$ NMR $\left(600 \mathrm{MHz}, \mathrm{CDCl}_{3}\right) \delta=7.41-7.35(\mathrm{~m}, 3 \mathrm{H}), 7.32-7.30(\mathrm{~m}, 2 \mathrm{H}), 7.28-7.22$ (m, 3H), 7.01$7.00(\mathrm{~m}, 2 \mathrm{H}), 5.19-5.18(\mathrm{~d}, J=4.2 \mathrm{~Hz}, 1 \mathrm{H}), 4.24-4.23$ (d, $J=4.2 \mathrm{~Hz}, 1 \mathrm{H}), 3.65-3.64$ (d, $J$ $=1.8 \mathrm{~Hz}, 3 \mathrm{H}) .{ }^{13} \mathrm{C} \mathrm{NMR}\left(150 \mathrm{MHz}, \mathrm{CDCl}_{3}\right): \delta=207.0,137.6,132.9,129.4,129.1$, 128.9, 128.7, 127.7, 79.2, 44.6. HRMS Calculated for $\left[\mathrm{C}_{18} \mathrm{H}_{15} \mathrm{NO}_{4}+\mathrm{H}\right]^{+}:$314.10738, Found: 314.10728.<smiles>CC(=O)[C@H]1ON=C([C@H](O)c2ccccc2)[C@H]1c1ccccc1</smiles>

27

Purified by flash chromatography (hexane-EtOAc) as colorless oil (yield: $86 \%$ ); ${ }^{1} \mathrm{H}$ NMR $\left(600 \mathrm{MHz}, \mathrm{CDCl}_{3}\right) \delta=7.32-7.27(\mathrm{~m}, 6 \mathrm{H}), 7.20-7.18(\mathrm{~m}, 2 \mathrm{H}), 7.07-7.06(\mathrm{~m}, 2 \mathrm{H})$, 5.26$5.25(\mathrm{~d}, J=4.8 \mathrm{~Hz}, 1 \mathrm{H}) 4.94-4.93(\mathrm{~d}, J=5.4 \mathrm{~Hz}, 1 \mathrm{H}), 4.25-4.24(\mathrm{~d}, J=5.4 \mathrm{~Hz}, 1 \mathrm{H}), 3.74$ (s, 3H), 3.14-3.13 (d, $J=6.8 \mathrm{~Hz}, 1 \mathrm{H}) .{ }^{13} \mathrm{C} \mathrm{NMR}\left(150 \mathrm{MHz}, \mathrm{CDCl}_{3}\right): \delta=169.8,162.4$, 
138.4, 136.3, 129.3, 128.8, 128.7, 128.6, 128.4, 127.6, 126.8, 86.4, 70.2, 58.4, 52.7. HRMS Calculated for $\left[\mathrm{C}_{18} \mathrm{H}_{17} \mathrm{NO}_{4}+\mathrm{H}\right]^{+}: 312.12303$, Found: 312.12296.<smiles>CCOC(=O)C(O)C(C(=O)c1ccccc1)c1ccccc1</smiles>

29

Purified by flash chromatography (hexane-EtOAc) as colorless oil (yield: $>98 \%$ ); ${ }^{1} \mathrm{H}$ NMR (600 MHz, $\left.\mathrm{CDCl}_{3}\right) \delta=9.28(\mathrm{br}, 1 \mathrm{H}), 7.75-7.73(\mathrm{~m}, 2 \mathrm{H}), 7.51-7.48(\mathrm{~m}, 1 \mathrm{H}), 7.37-$ 7.42 (t, $J=7.2 \mathrm{~Hz}, 2 \mathrm{H}), 7.21-7.19$ (m, 2H), 7.03-7.01 (d, $J=7.8 \mathrm{~Hz}, 2 \mathrm{H}), 4.70$ (br, 1H), $4.4(\mathrm{br}, 1 \mathrm{H}), 4.06-4.25(\mathrm{~d}, J=6.6 \mathrm{~Hz}, 1 \mathrm{H}), 4.01-4.05(\mathrm{~m}, 2 \mathrm{H}), 1.09-1.06(\mathrm{~m}, 3 \mathrm{H}) .{ }^{13} \mathrm{C}$ NMR $\left(150 \mathrm{MHz}, \mathrm{CDCl}_{3}\right): \delta=193.6,173.3,173.2,156.3,137.6,134.3,134.2,134.0$, 131.5, 131.4, 129.4, 129.2, 129.1, 128.5, 73.7, 61.71, 51.7, 20.9, 13.7. HRMS Calculated for $\left[\mathrm{C}_{19} \mathrm{H}_{19} \mathrm{NO}_{5}+\mathrm{H}\right]^{+}: 342.13360$, Found: 342.13351 .

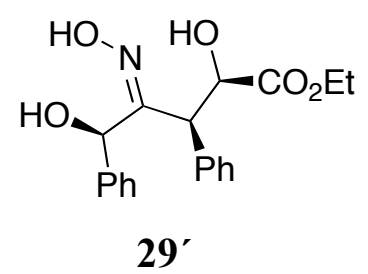

Purified by flash chromatography (hexane-EtOAc) as colorless oil. ${ }^{1} \mathrm{H}$ NMR (600 MHz, DMSO) $\delta=10.9,(\mathrm{~s}, 1 \mathrm{H}), 7.45-7.44(\mathrm{~d}, J=5.6,2 \mathrm{H}), 7.31-7.19(\mathrm{~m}, 8 \mathrm{H}), 5.86-5.85(\mathrm{~d}, J=$ $6.0 \mathrm{~Hz}, 1 \mathrm{H}$ ), $5.39-5.37$ (dd, $J=6.0 \mathrm{~Hz}, J=7.8 \mathrm{~Hz}, 2 \mathrm{H}), 4.46-4.43$ (m, 1H), 4.10-4.09 (d, $J=9.0 \mathrm{~Hz}, 1 \mathrm{H}), 3.69-3.68(\mathrm{~m}, 1 \mathrm{H}), 3.57-3.51(\mathrm{~m}, 1 \mathrm{H}), 0.79-0.77(\mathrm{~m}, 3 \mathrm{H}) .{ }^{13} \mathrm{C}$ NMR $(150$ MHz, DMSO): $\delta=172.9,159.9,142.0,139.4,129.4,128.1,128.0,127.2,127.1,126.8$, 74.5, 67.2, 59.8, 49.3, 13.9. HRMS Calculated for $\left[\mathrm{C}_{19} \mathrm{H}_{21} \mathrm{NO}_{5}+\mathrm{H}\right]^{+}: 344.14925$, Found: 344.14913 .

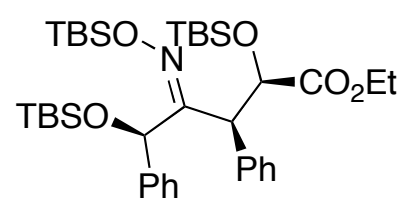

30 
Purified by flash chromatography (hexane-EtOAc) as colorless oil (yield: $>81 \%$ ); ${ }^{1} \mathrm{H}$ NMR (600 MHz, $\left.\mathrm{CDCl}_{3}\right) \delta=7.57-7.56(\mathrm{~d}, J=7.2 \mathrm{~Hz}, 2 \mathrm{H}), 7.43-7.42(\mathrm{~m}, 2 \mathrm{H}), 7.43-7.42$ (t, $J=7.2 \mathrm{~Hz}, 2 \mathrm{H}), 7.26-7.16(\mathrm{~m}, 4 \mathrm{H}), 6.42(\mathrm{~s}, 1 \mathrm{H}), 4.60-4.58(\mathrm{~d}, J=10.2 \mathrm{~Hz}, 1 \mathrm{H}), 4.20-$ $4.18(\mathrm{~d}, J=10.2 \mathrm{~Hz}, 1 \mathrm{H}), 3.76-3.71(\mathrm{~m}, 1 \mathrm{H}), 3.65-3.60(\mathrm{~m}, 1 \mathrm{H}), 1.05(\mathrm{~s}, 9 \mathrm{H}), 0.86-0.85$ (m, 3H), 0.58-0.57 (d, 18H), 0.3 (s, 3H), 0.28 (s, 3H), -0.17 (s, 3H), -0.31 (s, 3H), -0.38 (s, 3H), -0.48 (s, 3H). ${ }^{13} \mathrm{C}$ NMR (150 MHz, $\left.\mathrm{CDCl}_{3}\right): \delta=172.4,163.6,140.5,137.7$, $129.7,127.9,127.8,127.2,126.9,126.8,126.7,68.6,59.9,50.3,26.1,26.0,25.9,25.7$, 25.5, 18.3, 17.9, 17.8, 13.7. HRMS Calculated for $\left[\mathrm{C}_{37} \mathrm{H}_{63} \mathrm{NO}_{5} \mathrm{Si}_{3}+\mathrm{H}\right]^{+}:$686.40868, Found: 686.40860 .

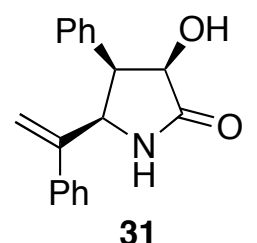

(3R,4R,5R)-3-hydroxy-4-phenyl-5-(1-phenylvinyl) pyrrolidin-2-one (31)

Purified by flash chromatography (Hexane-EtOAc) as clear liquid (yield: 57\%); ${ }^{1} \mathrm{H}$ NMR $\left(600 \mathrm{MHz}, \mathrm{CDCl}_{3}\right) \delta=7.23-7.28(\mathrm{~m}, 3 \mathrm{H}), 7.18-7.20(\mathrm{~m}, 1 \mathrm{H}), 7.13-7.16(\mathrm{~m}, 2 \mathrm{H}), 7.03(\mathrm{~b}$, $1 \mathrm{H}), 6.97-6.99(\mathrm{~m}, 2 \mathrm{H}), 6.82-6.84(\mathrm{~m}, 2 \mathrm{H}), 5.11(\mathrm{~d}, J=0.6 \mathrm{~Hz}, 1 \mathrm{H}), 5.06-5.07$ (d, $J=6.0$ $\mathrm{Hz}, 1 \mathrm{H}), 5.02(\mathrm{~d}, J=0.6 \mathrm{~Hz}, 1 \mathrm{H}), 4.75-4.77$ (dd, $J=7.8 \mathrm{~Hz}, J=6.0 \mathrm{~Hz}, 1 \mathrm{H}), 3.85-3.88$ (dd, $J=7.8 \mathrm{~Hz}, J=6.0 \mathrm{~Hz}, 1 \mathrm{H}), 3.04-3.05(\mathrm{~d}, J=5.4 \mathrm{~Hz}, 1 \mathrm{H}) ;{ }^{13} \mathrm{C}$ NMR $(150 \mathrm{MHz}$, $\left.\mathrm{CDCl}_{3}\right): \delta=177.5,144.0,138.9,133.4,130.0,128.3,128.2,128.1,127.8,127.6,127.5$, 126.6, 113.8, 72.3, 57.2, 50.8; HRMS Calculated for $\left[\mathrm{C}_{18} \mathrm{H}_{17} \mathrm{NO}_{2}+\mathrm{H}\right]^{+}:$: 280.13321, Found: 280.13330 .<smiles>C/C(=C1\NC(=O)C(O)C1c1ccccc1)c1ccccc1</smiles>

(3R, 4R)-3-hydroxy-4-phenyl-5-(1-phenylethylidene) pyrrolidin-2-one (31')

Purified by flash chromatography (Hexane-EtOAc) as white solid (yield: 79\%); ${ }^{1} \mathrm{H}$ NMR $\left(600 \mathrm{MHz}, \mathrm{CDCl}_{3}\right)$ set 1: $\delta=8.69(\mathrm{br}, 1 \mathrm{H}), 7.45(\mathrm{br}, 1 \mathrm{H}), 7.38-7.34(\mathrm{~m}, 4 \mathrm{H}), 7.30-7.21$ (m, 7H), 7.01-7.00 (m, 2H), 6.89-6.87 (m, 2H), 4.79-4.76 (dd, $J=9.0 \mathrm{~Hz}, J=6.0 \mathrm{~Hz}$, 
$1 \mathrm{H}), 4.50-4.48(\mathrm{~d}, J=9.0 \mathrm{~Hz}, 1 \mathrm{H}), 2.54-2.54(\mathrm{~d}, J=5.4 \mathrm{~Hz}, 1 \mathrm{H}), 1.80(\mathrm{~s}, 3 \mathrm{H})$; set $2: \delta=$ 8.63 (br, 1H), 7.45 (br, 1H), 7.38-7.34 (m, 4H), 7.30-7.21 (m, 7H), 7.14-7.12 (m, 4H), $4.67-4.65$ (dd, $J=8.4 \mathrm{~Hz}, J=3.0 \mathrm{~Hz}, 1 \mathrm{H}), 4.17-4.16$ (d, $J=8.4 \mathrm{~Hz}, 1 \mathrm{H}), 2.28-2.27$ (d, $J$ $=6.6 \mathrm{~Hz}, 1 \mathrm{H}), 2.04(\mathrm{~s}, 3 \mathrm{H}) ;{ }^{13} \mathrm{C} \mathrm{NMR}\left(150 \mathrm{MHz}, \mathrm{CDCl}_{3}\right)$ : set $\mathbf{1}: \delta=175.2,140.2,135.3$, $131.6,129.1,128.8,128.5,127.9,127.6,127.3,112.8,71.3,48.1,18.2$; set $2: \delta=176.2$, $140.7,136.9,132.1,128.6,128.5,128.4,128.0,127.7,127.4,126.6,114.3,71.8,49.1$, 18.7; HRMS Calculated for $\left[\mathrm{C}_{18} \mathrm{H}_{17} \mathrm{NO}_{2}+\mathrm{H}\right]^{+}: 280.13321$, Found: 280.13330

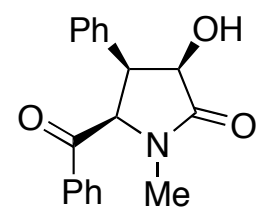

32

\section{(3R, 4R, 5R)-3-hydroxy-4-phenyl-5-(bezoketo) pyrrolidin-2-one (32)}

Purified by flash chromatography (Hexane-EtOAc) as white solid (yield: 79\%); ${ }^{1} \mathrm{H}$ NMR $\left(600 \mathrm{MHz}, \mathrm{CDCl}_{3}\right) \delta=7.64-7.65(\mathrm{~d}, J=7.2 \mathrm{~Hz}, 2 \mathrm{H}), 7.53-7.55(\mathrm{t}, J=7.2 \mathrm{~Hz}, 1 \mathrm{H}), 7.37-$ 7.39 (t, $J=7.8 \mathrm{~Hz}, 2 \mathrm{H}), 7.10-7.12(\mathrm{t}, J=7.8 \mathrm{~Hz}, 1 \mathrm{H}), 7.04-7.06(\mathrm{t}, J=7.2 \mathrm{~Hz}, 2 \mathrm{H}), 6.86-$ $6.87(\mathrm{~d}, J=7.8 \mathrm{~Hz}, 2 \mathrm{H}), 5.43-5.44(\mathrm{~d}, J=7.2 \mathrm{~Hz}, 1 \mathrm{H}), 4.71-4.73(\mathrm{dd}, J=7.2 \mathrm{~Hz}, J=4.2$ $\mathrm{Hz}, 1 \mathrm{H}), 4.11-4.14$ (t, $J=7.2 \mathrm{~Hz}, 1 \mathrm{H}), 3.45-3.46(\mathrm{~d}, J=4.2 \mathrm{~Hz}, 1 \mathrm{H}), 2.99(\mathrm{~s}, 3 \mathrm{H}) ;{ }^{13} \mathrm{C}$ NMR $\left(150 \mathrm{MHz}, \mathrm{CDCl}_{3}\right): \delta=194.5,175.3,135.8,133.7,132.4,129.8,128.7,128.0$, 127.8, 127.8, 71.3, 65.5, 48.0, 30.2; HRMS Calculated for $\left[\mathrm{C}_{18} \mathrm{H}_{17} \mathrm{NO}_{3}+\mathrm{H}\right]^{+}: 296.12812$, Found: 296.12824 .

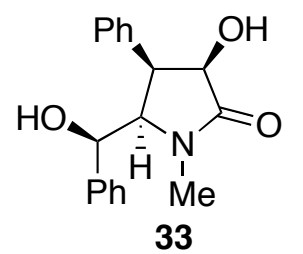

(3R, 4R, 5R)-3-hydroxy-5-((S)-hydroxy(phenyl)methyl)-1-methyl-4-phenylpyrrolidin -2-one (33)

Purified by flash chromatography (EtOAc-MeOH) as white solid (yield: $>98 \%) ;{ }^{1} \mathrm{H}$ NMR $\left(600 \mathrm{MHz}, \mathrm{CDCl}_{3}\right) \delta=7.17-7.28(\mathrm{~m}, 10 \mathrm{H}), 4.81-4.83(\mathrm{t}, J=6.0 \mathrm{~Hz}, 1 \mathrm{H}), 4.51-4.53(\mathrm{t}, J$ $=6.0 \mathrm{~Hz}, 1 \mathrm{H}), 4.14-4.16(\mathrm{dd}, J=6.6 \mathrm{~Hz}, J=0.6 \mathrm{~Hz}, 1 \mathrm{H}), 3.80-3.82(\mathrm{dd}, J=6.6 \mathrm{~Hz}, J=$ 
$0.6 \mathrm{~Hz}, 1 \mathrm{H}), 3.48-3.49(\mathrm{~d}, J=5.4 \mathrm{~Hz}, 1 \mathrm{H}), 2.605(\mathrm{~s}, 3 \mathrm{H}), 2.32-2.33(\mathrm{~d}, J=5.4 \mathrm{~Hz}, 1 \mathrm{H})$; ${ }^{13} \mathrm{C}$ NMR $\left(150 \mathrm{MHz}, \mathrm{CDCl}_{3}\right): \delta=175.8,140.6,134.2,130.0,128.5,128.4,128.1,127.8$, 126.6, 71.8, 71.7, 47.4, 29.5; HRMS Calculated for $\left[\mathrm{C}_{18} \mathrm{H}_{19} \mathrm{NO}_{3}+\mathrm{H}\right]^{+}:$298.14377, Found: 298.14391. Enantiomeric excess was determined by HPLC with a Chiralcel AS-H column; $\lambda=254 \mathrm{~nm}$; eluent: Hexane/Isopropanol $=70 / 30$; Flow rate: $1.5 \mathrm{~mL} / \mathrm{min}$; $\mathrm{t}_{\text {minor }}$ $=17.916 \mathrm{~min}, \mathrm{t}_{\text {major }}=22.848 \mathrm{~min} ; \mathrm{ee} \%=85 \%$. ee $\%>99 \%$, after recrystalization. $[\alpha]_{\mathrm{D}}{ }^{26}=$ $-93.5^{\circ}\left(\mathrm{c}=1.0 \mathrm{CHCl}_{3}\right)$. 


\section{Part IV}

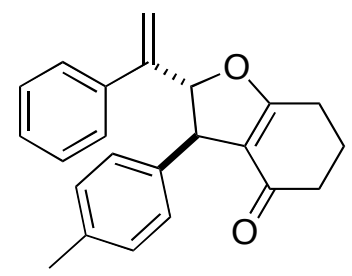

$35 \mathbf{a}$

\section{2,3,6,7-tetrahydro-2-(1-phenylvinyl)-3-p-tolylbenzofuran-4(5H)-one}

Purified by flash chromatography (hexane-EtOAc) as colorless oil (yield: 91\%); ${ }^{1} \mathrm{H}$ NMR $\left(600 \mathrm{MHz}, \mathrm{CDCl}_{3}\right) \delta=7.306-7.295(\mathrm{t}, J=6.6 \mathrm{~Hz}, 3 \mathrm{H}), 7.277-7.253(\mathrm{~m}, 2 \mathrm{H}), 7.052-7.039$ $(\mathrm{d}, J=7.8 \mathrm{~Hz}, 2 \mathrm{H}), 6.902-6.888$ (d, $J=8.4 \mathrm{~Hz}, 2 \mathrm{H}), 5.429-5.420$ (d, $J=5.4 \mathrm{~Hz}, 1 \mathrm{H})$, $5.376(\mathrm{~s}, 1 \mathrm{H}), 5.273(\mathrm{~s}, 1 \mathrm{H}), 4.067-4.060$ (d, $J=4.2 \mathrm{~Hz}, 1 \mathrm{H}), 2.693-2.645$ (dt, $J=18.0$ $\mathrm{Hz}, J=10.8 \mathrm{~Hz}, 1 \mathrm{H}), 2.594-2.538$ (m, 1H), 2.363-2.259 (m, 5H), 2.123-2.075 (m, 2H); ${ }^{13} \mathrm{C} \mathrm{NMR}\left(150 \mathrm{MHz}, \mathrm{CDCl}_{3}\right): \delta=194.550,176.803,146.599,139.236,137.669$, $136.405,129.304,128.415,128.124,127.231,126.918,116.443,113.018,93.774$, 50.611, 36.810, 24.076, 21.752, 21.005; HRMS Calculated for $\left[\mathrm{C}_{23} \mathrm{H}_{22} \mathrm{O}_{2}+\mathrm{H}\right]^{+}$: 331.16924, Found: 331.16946.

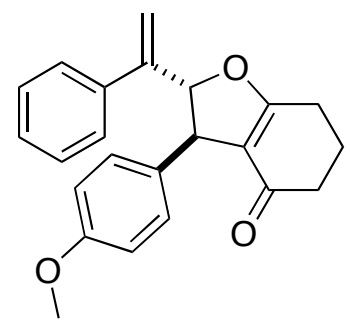

$35 b$

\section{2,3,6,7-tetrahydro-3-(4-methoxyphenyl)-2-(1-phenylvinyl)benzofuran-4(5H)-one}

Purified by flash chromatography (hexane-EtOAc) as colorless oil (yield: 82\%); ${ }^{1} \mathrm{H}$ NMR $\left(600 \mathrm{MHz} \mathrm{CDCl}_{3}\right) \delta=7.308-7.297(\mathrm{t}, J=6.6 \mathrm{~Hz}, 3 \mathrm{H}), 7.268-7.250(\mathrm{~m}, 2 \mathrm{H}), 6.923-6.909$ $(\mathrm{d}, J=8.4 \mathrm{~Hz}, 2 \mathrm{H}), 6.783-6.768(\mathrm{dd}, J=7.2 \mathrm{~Hz}, J=1.8 \mathrm{~Hz}, 2 \mathrm{H}), 5.413-5.405$ (d, $J=4.8$ $\mathrm{Hz}, 1 \mathrm{H}), 5.375$ (s, 1H), $5.271(\mathrm{~s}, 1 \mathrm{H}), 4.056-4.049$ (d, J=4.2 Hz, 1H), 3.753 (s, 3H), 2.694-2.645 (dt, $J=18.0 \mathrm{~Hz}, J=11.4 \mathrm{~Hz}, 1 \mathrm{H}), 2.597-2.541$ (m, 1H), 2.370-2.264 (m, 2H), 2.126-2.082 (m, 2H); ${ }^{13} \mathrm{C}$ NMR (150 MHz, $\left.\mathrm{CDCl}_{3}\right): \delta=194.634,176.810,158.491$, 
$146.596,137.666,134.372,128.426,128.142,128.044,127.224,116.421,114.031$, 112.902, 93.796, 55.173, 50.279, 36.821, 29.658, 24.087, 21.759; HRMS Calculated for $\left[\mathrm{C}_{23} \mathrm{H}_{22} \mathrm{O}_{3}+\mathrm{Na}\right]^{+}: 369.14612$, Found: 369.14627 .

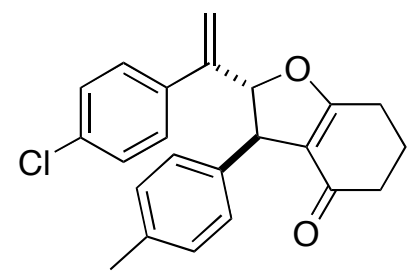

$35 \mathrm{c}$

\section{2-(1-(4-chlorophenyl)vinyl)-2,3,6,7-tetrahydro-3-p-tolylbenzofuran-4(5H)-one}

Purified by flash chromatography (hexane-EtOAc) as colorless oil (yield: 92\%); ${ }^{1} \mathrm{H}$ NMR $\left(600 \mathrm{MHz}, \mathrm{CDCl}_{3}\right) \delta=7.283-7.265(\mathrm{~m}, 2 \mathrm{H}), 7.208-7.190(\mathrm{~m}, 2 \mathrm{H}), 7.071-7.058(\mathrm{~d}, J=$ $7.8 \mathrm{~Hz}, 2 \mathrm{H}), 6.924-6.910$ (d, $J=8.4 \mathrm{~Hz}, 2 \mathrm{H}), 5.389-5.381$ (d, $J=4.8 \mathrm{~Hz}, 1 \mathrm{H}), 5.374$ (s, $1 \mathrm{H}), 5.295(\mathrm{~s}, 1 \mathrm{H}), 4.035-4.028(\mathrm{~d}, J=4.2 \mathrm{~Hz}, 1 \mathrm{H}), 2.693-2.644$ (dt, $J=18.0 \mathrm{~Hz}, J=$ 11.4 Hz, 1H), 2.585-2.529 (m, 1H), 2.366-2.261 (m, 5H), 2.127-2.079 (m, 2H); ${ }^{13} \mathrm{C}$ NMR (150 MHz, $\left.\mathrm{CDCl}_{3}\right): \delta=194.481,176.614,145.485,139.061,136.598,136.114,134.128$, $129.417,128.627,128.499,126.907,116.498,113.703,93.512,50.724,36.817,24.062$, 21.752, 21.027. HRMS Calculated for $\left[\mathrm{C}_{23} \mathrm{H}_{21} \mathrm{ClO}_{2}+\mathrm{H}\right]^{+}: 365.13028$, Found: 365.13042 .

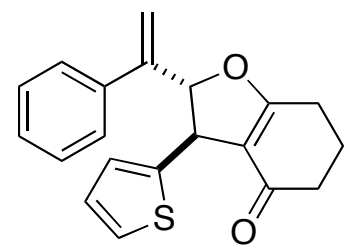

35d

\section{2,3,6,7-tetrahydro-2-(1-phenylvinyl)-3-(thiophen-2-yl)benzofuran-4(5H)-one}

Purified by flash chromatography (hexane-EtOAc) as colorless oil (yield: $81 \%$ ); ${ }^{1} \mathrm{H}$ NMR $\left(600 \mathrm{MHz}, \mathrm{CDCl}_{3}\right) \delta=7.344-7.300(\mathrm{~m}, 5 \mathrm{H}), 7.119-7.108(\mathrm{dd}, J=5.4 \mathrm{~Hz}, J=1.2 \mathrm{~Hz}$, $1 \mathrm{H}), 6.869-6.855(\mathrm{dd}, J=4.8 \mathrm{~Hz}, J=3.6 \mathrm{~Hz}, 1 \mathrm{H}), 6.653-6.646(\mathrm{dd}, J=3.6 \mathrm{~Hz}, J=0.6$ $\mathrm{Hz}, 1 \mathrm{H}), 5.520-5.512$ (d, J=4.8 Hz, 1H), 5.386 (s, 1H), 5.293 (s, 1H), 4.408-4.401 (d, J $=4.2 \mathrm{~Hz}, 1 \mathrm{H}), 2.687-2.638(\mathrm{dt}, J=18.0 \mathrm{~Hz}, J=11.4 \mathrm{~Hz}, 1 \mathrm{H}), 2.584-2.529(\mathrm{~m}, 1 \mathrm{H})$, 2.414-2.367 (dt, $J=16.8 \mathrm{~Hz}, J=11.4 \mathrm{~Hz}, 1 \mathrm{H}), 2.333-2.282(\mathrm{~m}, 1 \mathrm{H}), 2.132-2.065$ (m, 
$2 \mathrm{H}) ;{ }^{13} \mathrm{C}$ NMR $\left(150 \mathrm{MHz}, \mathrm{CDCl}_{3}\right): \delta=194.466,177.156,146.122,146.042,137.498$, $128.525,128.291,127.297,126.991,124.357,123.985,115.973,113.310,93.381$, $46.020,36.828,29.676,24.131,21.643$; HRMS Calculated for $\left[\mathrm{C}_{20} \mathrm{H}_{18} \mathrm{O}_{2} \mathrm{~S}+\mathrm{H}\right]^{+}$: 323.11003, Found: 323.10907.

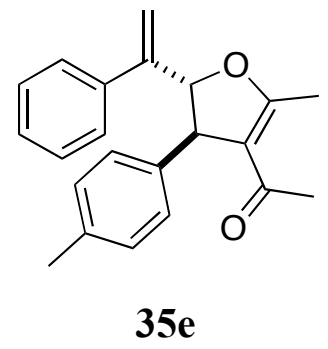

\section{1-(4,5-dihydro-2-methyl-5-(1-phenylvinyl)-4-p-tolylfuran-3-yl)ethanone}

Purified by flash chromatography (hexane-EtOAc) as colorless oil (yield: 86\%); ${ }^{1} \mathrm{H}$ NMR $\left(600 \mathrm{MHz}, \mathrm{CDCl}_{3}\right) \delta=7.334-7.313(\mathrm{~m}, 3 \mathrm{H}), 7.301-7.278(\mathrm{~m}, 2 \mathrm{H}), 7.079-7.066(\mathrm{~d}, J=$ $7.8 \mathrm{~Hz}, 2 \mathrm{H}), 6.897-6.884$ (d, $J=7.8 \mathrm{~Hz}, 2 \mathrm{H}), 5.397$ (s, 1H), 5.284-5.275 (d, $J=5.4 \mathrm{~Hz}$, $1 \mathrm{H}), 5.270(\mathrm{~s}, 1 \mathrm{H}), 4.016-4.006$ (dd, $J=4.8 \mathrm{~Hz}, J=1.2 \mathrm{~Hz}, 1 \mathrm{H}), 2.447-2.444$ (d, $J=1.8$ $\mathrm{Hz}, 3 \mathrm{H}), 2.312(\mathrm{~s}, 3 \mathrm{H}), 1.802(\mathrm{~s}, 3 \mathrm{H}) ;{ }^{13} \mathrm{C} \mathrm{NMR}\left(150 \mathrm{MHz}, \mathrm{CDCl}_{3}\right): \delta=195.285$, $168.390,146.607,140.278,137.706,136.744,129.512,128.485,128.120,127.242$, 127.187, 115.314, 112.698, 91.089, 54.684, 29.556, 21.020, 14.997; HRMS Calculated for $\left[\mathrm{C}_{22} \mathrm{H}_{22} \mathrm{O}_{2}+\mathrm{H}\right]^{+}: 319.16924$, Found: 319.16945 .

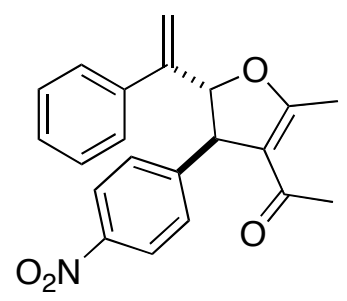

$$
35 f
$$

\section{1-(4,5-dihydro-2-methyl-4-(4-nitrophenyl)-5-(1-phenylvinyl)furan-3-yl)ethanone}

Purified by flash chromatography (hexane-EtOAc) as colorless oil (yield: 94\%); ${ }^{1} \mathrm{H}$ NMR $\left(600 \mathrm{MHz}, \mathrm{CDCl}_{3}\right) \delta=8.124-8.101(\mathrm{~m}, 2 \mathrm{H}), 7.351-7.313(\mathrm{~m}, 3 \mathrm{H}), 7.239-7.223(\mathrm{~m}, 2 \mathrm{H})$, 7.153-7.130 (m, 2H), $5.413(\mathrm{~s}, 1 \mathrm{H}), 5.304(\mathrm{~s}, 1 \mathrm{H}), 5.289-5.280(\mathrm{~d}, J=5.4 \mathrm{~Hz}, 1 \mathrm{H})$, 4.175-4.166 (d, $J=5.4 \mathrm{~Hz}, 1 \mathrm{H}), 2.477$ (s, 3H), 1.949 (s, 3H); ${ }^{13} \mathrm{C}$ NMR $(150 \mathrm{MHz}$, $\left.\mathrm{CDCl}_{3}\right): \delta=193.592,168.762,150.837,147.062,146.071,137.331,128.670,128.466$, 
128.182, 127.155, 124.040, 115.787, 113.007, 90.040, 54.925, 29.425, 15.230; HRMS Calculated for $\left[\mathrm{C}_{21} \mathrm{H}_{19} \mathrm{NO}_{4}+\mathrm{Na}\right]^{+}: 372.12063$, Found: 372.12077 .

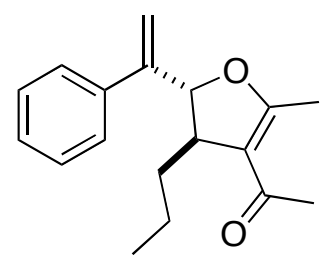

$35 \mathrm{~g}$

1-(4,5-dihydro-2-methyl-5-(1-phenylvinyl)-4-propylfuran-3-yl)ethanone

Purified by flash chromatography (hexane-EtOAc) as colorless oil (yield: $65 \%$ ); ${ }^{1} \mathrm{H}$ NMR $\left(600 \mathrm{MHz}, \mathrm{CDCl}_{3}\right) \delta=7.325-7.285(\mathrm{~m}, 5 \mathrm{H}), 5.235(\mathrm{~s}, 1 \mathrm{H}), 5.188(\mathrm{~s}, 1 \mathrm{H}), 5.105-5.099(\mathrm{~d}$, $J=3.6 \mathrm{~Hz}, 1 \mathrm{H}), 3.000-2.976(\mathrm{~m}, 1 \mathrm{H}), 2.279-2.277$ (d, $J=1.2 \mathrm{~Hz}, 3 \mathrm{H}), 2.159$ (s, 3H), 1.550-1.418 (m, 2H), 1.257-1.197 (m, 1H), 1.119-1.077 (m, 1H), 0.796-0.772 (t, $J=14.4$ $\mathrm{Hz}, 3 \mathrm{H}) ;{ }^{13} \mathrm{C}$ NMR $\left(150 \mathrm{MHz}, \mathrm{CDCl}_{3}\right): \delta=194.328,166.988,147.539,138.602$, $128.390,127.909,127.286,116.505,112.873,87.588,47.846,36.355,29.213,18.983$, 15.289, 13.897. HRMS Calculated for $\left[\mathrm{C}_{18} \mathrm{H}_{22} \mathrm{O}_{2}+\mathrm{H}\right]^{+}: 208.16925$, Found: 208.16949.

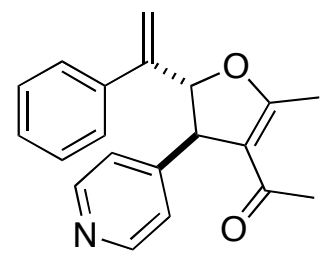

$35 h$

\section{1-(4,5-dihydro-2-methyl-5-(1-phenylvinyl)-4-(pyridin-4-yl)furan-3-yl)ethanone}

Purified by flash chromatography (hexane-EtOAc) as colorless oil (yield: $93 \%$ ); ${ }^{1} \mathrm{H}$ NMR $\left(600 \mathrm{MHz}, \mathrm{CDCl}_{3}\right) \delta=8.496-8.486(\mathrm{dd}, J=4.8 \mathrm{~Hz}, J=1.8 \mathrm{~Hz}, 2 \mathrm{H}), 7.345-7.325$ (m, $3 \mathrm{H}), 7.250-7.234$ (m, 2H), 6.925-6.914 (dd, $J=4.8 \mathrm{~Hz}, J=1.8 \mathrm{~Hz}, 2 \mathrm{H}), 5.409$ (s, 1H), 5.297-5.293 (dd, $J=1.8 \mathrm{~Hz}, J=0.6 \mathrm{~Hz}, 1 \mathrm{H}$ ), 5.272-5.263 (d, $J=5.4 \mathrm{~Hz}, 1 \mathrm{H}$ ), 4.0414.033 (d, $J=4.8 \mathrm{~Hz}, 1 \mathrm{H}), 2.467-2.465$ (d, $J=1.2 \mathrm{~Hz}, 3 \mathrm{H}), 1.918$ (s, 3H); ${ }^{13} \mathrm{C}$ NMR (150 $\left.\mathrm{MHz}, \mathrm{CDCl}_{3}\right): \delta=193.825,168.966,152.166,150.246,146.118,137.363,128.659$, 128.430, 127.173, 122.477, 115.044, 113.033, 89.985, 54.448, 29.443, 15.154; HRMS Calculated for $\left[\mathrm{C}_{20} \mathrm{H}_{19} \mathrm{NO}_{2}+\mathrm{H}\right]^{+}: 306.1488559$, Found: 306.14893 . 


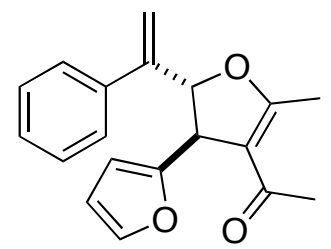

$35 \mathbf{i}$

1-(4-(furan-2-yl)-4,5-dihydro-2-methyl-5-(1-phenylvinyl)furan-3-yl)ethanone

Purified by flash chromatography (hexane-EtOAc) as colorless oil (yield: 89\%); ${ }^{1} \mathrm{H}$ NMR $\left(600 \mathrm{MHz}, \mathrm{CDCl}_{3}\right) \delta=7.341-7.310(\mathrm{~m}, 6 \mathrm{H}), 6.281-6.273(\mathrm{dd}, J=3.0 \mathrm{~Hz}, J=1.8 \mathrm{~Hz}$, $1 \mathrm{H}), 5.979-5.973(\mathrm{~d}, J=3.6 \mathrm{~Hz}, 1 \mathrm{H}), 5.458-5.448$ (dd, $J=5.4 \mathrm{~Hz}, J=0.6 \mathrm{~Hz}, 1 \mathrm{H}), 5.436$ (s, 1H), 5.300-5.297 (dd, $J=1.2 \mathrm{~Hz}, J=0.6 \mathrm{~Hz}, 1 \mathrm{H}), 4.201-4.192(\mathrm{~d}, J=5.4 \mathrm{~Hz}, 1 \mathrm{H})$, 2.395-2.393 (d, $J=1.2 \mathrm{~Hz}, 3 \mathrm{H}), 1.936(\mathrm{~s}, 3 \mathrm{H}) ;{ }^{13} \mathrm{C} \mathrm{NMR}\left(150 \mathrm{MHz}, \mathrm{CDCl}_{3}\right): \delta=$ 194.612 , 168.941, 155.056, 145.951, 141.950, 137.436, 128.492, 128.168, 126.918, 113.164, 112.698, 110.483, 106.540, 87.606, 48.338, 29.122, 15.128; HRMS Calculated for $\left[\mathrm{C}_{19} \mathrm{H}_{18} \mathrm{O}_{3}+\mathrm{Na}\right]^{+}: 317.11428$, Found: 317.11386 .

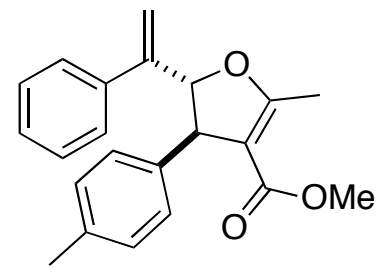

$35 j$

methyl 4,5-dihydro-2-methyl-5-(1-phenylvinyl)-4-p-tolylfuran-3-carboxylate

Purified by flash chromatography (hexane-EtOAc) as colorless oil (yield: 89\%); ${ }^{1} \mathrm{H}$ NMR $\left(600 \mathrm{MHz}, \mathrm{CDCl}_{3}\right) \delta=7.310-7.264(\mathrm{~m}, 5 \mathrm{H}), 7.057-7.044(\mathrm{~d}, J=7.8 \mathrm{~Hz}, 2 \mathrm{H}), 6.922-$ $6.908(\mathrm{dd}, J=6.6 \mathrm{~Hz}, J=1.8 \mathrm{~Hz}, 2 \mathrm{H}), 5.391$ (s, 1H), 5.314-5.305 (dd, $J=4.8 \mathrm{~Hz}, J=0.6$ $\mathrm{Hz}, 1 \mathrm{H}), 5.270-5.268$ (d, $J=1.2 \mathrm{~Hz}, 1 \mathrm{H}), 3.989-3.979$ (dd, $J=4.8 \mathrm{~Hz}, J=1.2 \mathrm{~Hz}, 1 \mathrm{H})$, $3.493(\mathrm{~s}, 3 \mathrm{H}), 2.410-2.408(\mathrm{~d}, J=1.2 \mathrm{~Hz}, 3 \mathrm{H}), 2.300(\mathrm{~s}, 3 \mathrm{H}) ;{ }^{13} \mathrm{C}$ NMR $(150 \mathrm{MHz}$, $\left.\mathrm{CDCl}_{3}\right): \delta=168.212,166.025,146.676,140.664,137.757,136.241,129.169,128.415$, $128.004,127.100,127.013,112.235,106.318,90.068,53.821,50.684,21.030,14.247$; HRMS Calculated for $\left[\mathrm{C}_{22} \mathrm{H}_{22} \mathrm{O}_{3}+\mathrm{H}\right]^{+}: 335.16417$, Found: 335.16429 . 


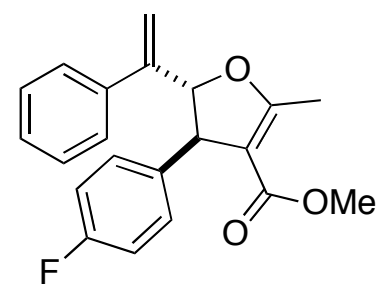

$35 \mathbf{k}$

methyl

4-(4-fluorophenyl)-4,5-dihydro-2-methyl-5-(1-phenylvinyl)furan-3carboxylate

Purified by flash chromatography (hexane-EtOAc) as colorless oil (yield: 90\%); ${ }^{1} \mathrm{H}$ NMR $\left(600 \mathrm{MHz}, \mathrm{CDCl}_{3}\right) \delta=7.313-7.300(\mathrm{~m}, 3 \mathrm{H}), 7.262-7.244(\mathrm{~m}, 2 \mathrm{H}), 6.978-6.907(\mathrm{~m}, 4 \mathrm{H})$, 5.396 (s, 1H), 5.396-5.289 (dd, $J=4.8 \mathrm{~Hz}, J=0.6 \mathrm{~Hz}, 1 \mathrm{H}$ ), 5.279-5.277 (d, $J=1.2 \mathrm{~Hz}$, $1 \mathrm{H}), 4.002-3.994(\mathrm{~d}, J=4.8 \mathrm{~Hz}, 1 \mathrm{H}), 3.502-3.500$ (d, $J=1.2 \mathrm{~Hz}, 3 \mathrm{H}), 2.416-2.412$ (t, $J=$ $2.4 \mathrm{~Hz}, 3 \mathrm{H}) ;{ }^{13} \mathrm{C}$ NMR $\left(150 \mathrm{MHz}, \mathrm{CDCl}_{3}\right): \delta=168.492,165.851,162.557,160.932$, $146.523,139.407,139.389$, 137.659, 128.663, 128.612, 128.488, 128.138, 127.107, 115.350, 115.208, 112.359, 106.169, 90.575, 53.631, 50.735, 14.272. HRMS Calculated for $\left[\mathrm{C}_{21} \mathrm{H}_{19} \mathrm{FO}_{3}+\mathrm{H}\right]^{+}: 339.13909$, Found: 339.13913 .

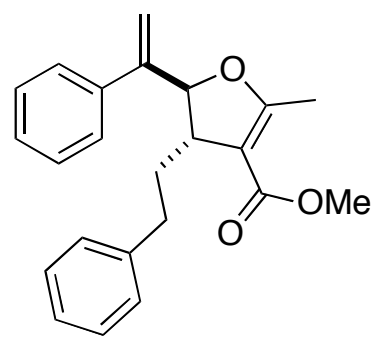

351

methyl 4,5-dihydro-2-methyl-4-phenethyl-5-(1-phenylvinyl)furan-3-carboxylate)

Purified by flash chromatography (hexane-EtOAc) as colorless oil (yield: $71 \%$ ); ${ }^{1} \mathrm{H}$ NMR $\left(600 \mathrm{MHz}, \mathrm{CDCl}_{3}\right) \delta=7.346-7.280(\mathrm{~m}, 5 \mathrm{H}), 7.223-7.198(\mathrm{~m}, 2 \mathrm{H}), 7.142-7.113(\mathrm{~m}, 1 \mathrm{H})$, 7.043-7.028 (dd, $J=8.4 \mathrm{~Hz}, J=1.8 \mathrm{~Hz}, 2 \mathrm{H}), 5.266$ (s, $1 \mathrm{H}), 5.233-5.229$ (d, $J=2.4 \mathrm{~Hz}$, $1 \mathrm{H}), 5.219-5.211$ (d, $J=4.8 \mathrm{~Hz}, 1 \mathrm{H}), 3.641$ (s, 3H), 3.036-3.008 (m, 1H), 2.547-2.497 (m, 1H), 2.393-2.341 (m, 1H), 2.285-2.283 (d, $J=1.2 \mathrm{~Hz}, 3 \mathrm{H}), 1.975-1.918(\mathrm{~m}, 1 \mathrm{H})$, 1.857-1.794 (m, 1H); ${ }^{13} \mathrm{C}$ NMR $\left(150 \mathrm{MHz}, \mathrm{CDCl}_{3}\right): \delta=168.015,166.248,147.426$, $141.786,138.551,128.426,128.219$, 128.182, 127.916, 127.300, 125.665, 113.051, 
105.101, 87.741, 50.607, 47.423, 35.353, 32.004, 14.283; HRMS Calculated for $\left[\mathrm{C}_{23} \mathrm{H}_{24} \mathrm{O}_{3}+\mathrm{H}\right]^{+}:$349.17982, Found: 349.17985 .

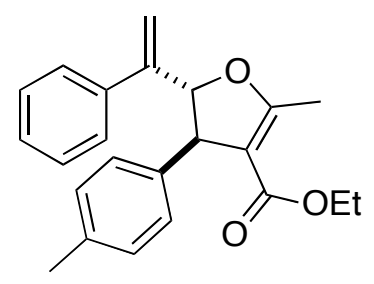

$35 \mathrm{~m}$

ethyl 4,5-dihydro-2-methyl-5-(1-phenylvinyl)-4-p-tolylfuran-3-carboxylate

Purified by flash chromatography (hexane-EtOAc) as colorless oil (yield: 86\%); ${ }^{1} \mathrm{H}$ NMR $\left(600 \mathrm{MHz}, \mathrm{CDCl}_{3}\right) \delta=7.325-7.278(\mathrm{~m}, 5 \mathrm{H}), 7.076-7.062(\mathrm{~d}, J=8.4 \mathrm{~Hz}, 2 \mathrm{H}), 6.884-$ $6.881(\mathrm{~d}, J=1.8 \mathrm{~Hz}, 1 \mathrm{H}), 6.871(\mathrm{~s}, 1 \mathrm{H}), 5.391(\mathrm{~s}, 1 \mathrm{H}), 5.286-5.285$ (d, $J=0.6 \mathrm{~Hz}, 1 \mathrm{H})$, $5.272(\mathrm{~s}, 1 \mathrm{H}), 4.009-4.001(\mathrm{~d}, J=4.8 \mathrm{~Hz}, 1 \mathrm{H}), 2.949-2.852(\mathrm{~m}, 2 \mathrm{H}), 2.308(\mathrm{~s}, 3 \mathrm{H}), 1.788$ (s, 3H), 1.314-1.289 (t, $\left.J=15.0 \mathrm{~Hz}, 3 \mathrm{H}) ;{ }^{13} \mathrm{C} \mathrm{NMR} \mathrm{(150} \mathrm{MHz,} \mathrm{CDCl}_{3}\right): \delta=195.125$, $173.130,146.752,140.413,137.720,136.693,129.497,128.444,128.084,127.195$, $127.180,114.155,112.490,90.797,54.695,29.589,22.025,20.998,11.215$. HRMS Calculated for $\left[\mathrm{C}_{23} \mathrm{H}_{24} \mathrm{O}_{3}+\mathrm{H}\right]^{+}: 349.17982$, Found: 349.17985 .

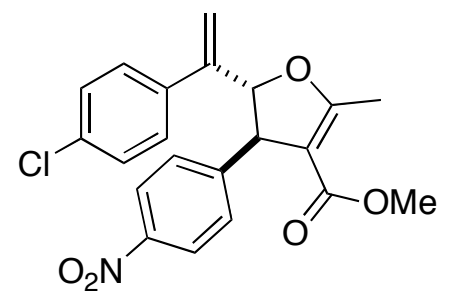

$35 n$

methyl 5-(1-(4-chlorophenyl)vinyl)-4,5-dihydro-2-methyl-4-(4-nitrophenyl)furan-3carboxylate

Purified by flash chromatography (hexane-EtOAc) as colorless oil (yield: 95\%); ${ }^{1} \mathrm{H}$ NMR $\left(600 \mathrm{MHz}, \mathrm{CDCl}_{3}\right) \delta=8.142-8.120(\mathrm{~m}, 2 \mathrm{H}), 7.313-7.290(\mathrm{~m}, 2 \mathrm{H}), 7.201-7.166(\mathrm{~m}, 4 \mathrm{H})$, 5.415 (s, 1H), 5.327-5.325 (d, $J=1.2 \mathrm{~Hz}, 1 \mathrm{H}), 5.278-5.268$ (d, $J=6.0 \mathrm{~Hz}, 1 \mathrm{H}), 4.092-$ 4.080 (dd, $J=5.4 \mathrm{~Hz}, J=1.2 \mathrm{~Hz}, 1 \mathrm{H}), 3.508$ (s, 3H), 2.431-2.429 (d, $J=1.2 \mathrm{~Hz}, 3 \mathrm{H})$; ${ }^{13} \mathrm{C} \mathrm{NMR}\left(150 \mathrm{MHz}, \mathrm{CDCl}_{3}\right): \delta=169.203,165.275,150.851,147.073,144.971$, $135.826,134.394,128.842,128.375,128.084$, 123.949, 113.893, 105.695, 89.293, 
54.265, 50.939, 14.298. HRMS Calculated forC21H18ClNO5 $\left[\mathrm{C}_{21} \mathrm{H}_{18} \mathrm{ClNO}_{5}+\mathrm{H}\right]^{+}$: 400.09463, Found: 400.09476.

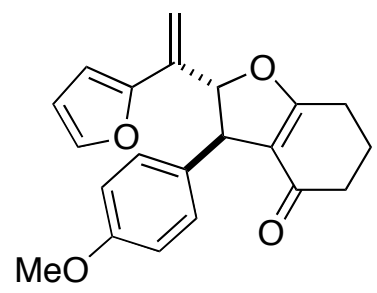

350

2-(1-(furan-2-yl)vinyl)-2,3,6,7-tetrahydro-3-(4-methoxyphenyl)benzofuran-4(5H)one

Purified by flash chromatography (hexane-EtOAc) as colorless oil (yield: 83\%); ${ }^{1} \mathrm{H}$ NMR $\left(600 \mathrm{MHz}, \mathrm{CDCl}_{3}\right) \delta=7.384-7.381(\mathrm{~d}, J=1.8 \mathrm{~Hz}, 1 \mathrm{H}), 7.119-7.104(\mathrm{~d}, J=9.0 \mathrm{~Hz}, 2 \mathrm{H})$, 6.855-6.841 (d, $J=8.4 \mathrm{~Hz}, 2 \mathrm{H}), 6.352-6.343$ (dd, $J=3.6 \mathrm{~Hz}, J=2.4 \mathrm{~Hz}, 1 \mathrm{H}), 6.079-$ $6.073(\mathrm{~d}, J=3.6 \mathrm{~Hz}, 1 \mathrm{H}), 5.656(\mathrm{~s}, 1 \mathrm{H}), 5.286-5.276(\mathrm{~d}, J=6.0 \mathrm{~Hz}, 1 \mathrm{H}), 5.148(\mathrm{~s}, 1 \mathrm{H})$, 4.200-4.191 (d, $J=5.4 \mathrm{~Hz}, 1 \mathrm{H}), 3.785$ (s, 3H), 2.690-2.651 (m, 1H), 2.624-2.580 (m, $1 \mathrm{H}), 2.387-2.335(\mathrm{~m}, 2 \mathrm{H}), 2.151-2.107(\mathrm{~m}, 2 \mathrm{H}) ;{ }^{13} \mathrm{C} \mathrm{NMR}\left(150 \mathrm{MHz}, \mathrm{CDCl}_{3}\right): \delta=$ $194.543,176.559,158.637,150.815,142.504,136.004,134.431,128.273,116.855$, 114.155, 111.204, 109.488, 107.360, 92.178, 55.231, 50.972, 36.908, 24.025, 21.807. HRMS Calculated for $\left[\mathrm{C}_{21} \mathrm{H}_{20} \mathrm{O}_{4}+\mathrm{H}\right]^{+}: 337.14343$, Found: 337.14355 .

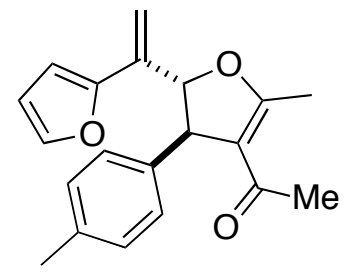

$35 p$

1-(5-(1-(furan-2-yl)vinyl)-4,5-dihydro-2-methyl-4-p-tolylfuran-3-yl)ethanone Purified by flash chromatography (hexane-EtOAc) as colorless oil (yield: 90\%); ${ }^{1} \mathrm{H}$ NMR (600 $\left.\mathrm{MHz}, \mathrm{CDCl}_{3}\right) \delta=7.405-7.403(\mathrm{~d}, J=1.2 \mathrm{~Hz}, 1 \mathrm{H}), 7.154-7.140(\mathrm{~d}, J=8.4 \mathrm{~Hz}, 2 \mathrm{H})$, 7.092-7.079 (d, $J=7.8 \mathrm{~Hz}, 2 \mathrm{H}), 6.377-6.368$ (dd, $J=3.6 \mathrm{~Hz}, J=1.8 \mathrm{~Hz}, 1 \mathrm{H}), 6.115-$ $6.109(\mathrm{~d}, J=3.6 \mathrm{~Hz}, 1 \mathrm{H}), 5.648(\mathrm{~s}, 1 \mathrm{H}), 5.133(\mathrm{~s}, 1 \mathrm{H}), 5.127$ (s, 1H), 4.168-4.161 (d, $J=$ $4.2 \mathrm{~Hz}, 1 \mathrm{H}), 2.451-2.499$ (d, $J=1.2 \mathrm{~Hz}, 3 \mathrm{H}), 2.345$ (s, 3H), 1.862 (s, 3H); ${ }^{13} \mathrm{C}$ NMR (150 
$\left.\mathrm{MHz}, \mathrm{CDCl}_{3}\right): \delta=195.260,168.267,150.910,142.468,140.322,136.955,136.241$, 129.621, 127.457, 115.747, 111.211, 109.222, 107.266, 89.675, 55.453, 29.640, 21.060, 14.877; HRMS Calculated for $\left[\mathrm{C}_{20} \mathrm{H}_{20} \mathrm{O}_{3}+\mathrm{Na}\right]^{+}$: 331.13047, Found: 331.12948 .

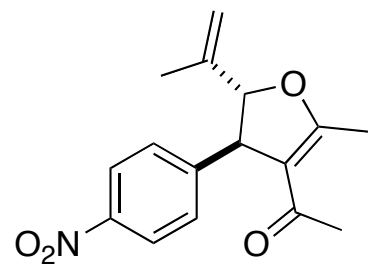

$35 q$

1-(4,5-dihydro-2-methyl-4-(4-nitrophenyl)-5-(prop-1-en-2-yl)furan-3-yl)ethanone

Purified by flash chromatography (hexane-EtOAc) as colorless oil (yield: $85 \%$ ); ${ }^{1} \mathrm{H}$ NMR $\left(600 \mathrm{MHz}, \mathrm{CDCl}_{3}\right) \delta=8.202-8.188(\mathrm{dd}, J=6.6 \mathrm{~Hz}, J=1.8 \mathrm{~Hz}, 2 \mathrm{H}), 7.391-7.376(\mathrm{dd}, J=$ $6.6 \mathrm{~Hz}, J=2.4 \mathrm{~Hz}, 2 \mathrm{H}), 4.940-4.936$ (t, $J=2.4 \mathrm{~Hz}, 1 \mathrm{H}), 4.912-4.911$ (d, $J=0.6 \mathrm{~Hz}, 1 \mathrm{H})$, 4.730-4.720 (d, $J=6.0 \mathrm{~Hz}, 1 \mathrm{H}), 4.274-4.263$ (dd, $J=6.0 \mathrm{~Hz}, J=1.2 \mathrm{~Hz}, 1 \mathrm{H}), 2.423-$ $2.421(\mathrm{~d}, J=1.2 \mathrm{~Hz}, 3 \mathrm{H}), 2.031(\mathrm{~s}, 3 \mathrm{H}), 1.792(\mathrm{~s}, 3 \mathrm{H}) ;{ }^{13} \mathrm{C} \mathrm{NMR}\left(150 \mathrm{MHz}, \mathrm{CDCl}_{3}\right): \delta=$ 193.479, 169.083, 151.157, 147.102, 141.885, 128.171, 124.182, 115.929, 113.157, 92.845, 54.043, 29.447, 16.731, 15.154; HRMS Calculated for $\left[\mathrm{C}_{16} \mathrm{H}_{17} \mathrm{NO}_{4}+\mathrm{H}\right]^{+}$: 288.123035, Found: 288.12317.

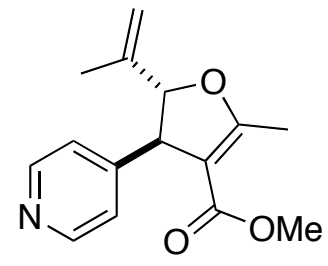

$35 r$

methyl 4,5-dihydro-2-methyl-5-(prop-1-en-2-yl)-4-(pyridin-4-yl)furan-3-carboxylate Purified by flash chromatography (hexane-EtOAc) as colorless oil (yield: $80 \%$ ); ${ }^{1} \mathrm{H}$ NMR $\left(600 \mathrm{MHz}, \mathrm{CDCl}_{3}\right) \delta=8.559$ (b, 2H), 7.143-7.135 (d, $\left.J=4.8 \mathrm{~Hz}, 2 \mathrm{H}\right), 4.914-4.912$ (d, $J$ $=1.2 \mathrm{~Hz}, 2 \mathrm{H}), 4.743-4.733(\mathrm{~d}, J=6.0 \mathrm{~Hz}, 1 \mathrm{H}), 4.069-4.057$ (dd, $J=6.0 \mathrm{~Hz}, J=1.2 \mathrm{~Hz}$, $1 \mathrm{H}), 3.547$ (s, $3 \mathrm{H}), 2.374-2.371$ (d, $J=1.8 \mathrm{~Hz}, 3 \mathrm{H}), 1.779-1.776(\mathrm{t}, J=1.8 \mathrm{~Hz}, 3 \mathrm{H}) ;{ }^{13} \mathrm{C}$ NMR $\left(150 \mathrm{MHz}, \mathrm{CDCl}_{3}\right): \delta=169.746,165.519,152.698,150.057,142.180,122.524$, 
112.869, 104.919, 92.710, 52.928, 50.837, 16.739, 14.159; C15H17NO3 HRMS Calculated for $\left[\mathrm{C}_{15} \mathrm{H}_{17} \mathrm{NO}_{3}+\mathrm{H}\right]^{+}: 260.12812$, Found: 260.12827 .

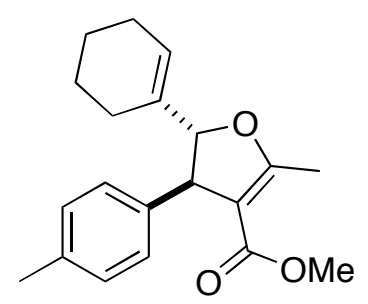

$35 \mathrm{~s}$

methyl 5-cyclohexenyl-4,5-dihydro-2-methyl-4-p-tolylfuran-3-carboxylate

Purified by flash chromatography (hexane-EtOAc) as colorless oil (yield: 85\%); ${ }^{1} \mathrm{H}$ NMR $\left(600 \mathrm{MHz}, \mathrm{CDCl}_{3}\right) \delta=7.106-7.093(\mathrm{~d}, J=7.8 \mathrm{~Hz}, 2 \mathrm{H}), 7.073-7.060(\mathrm{dd}, J=6.0 \mathrm{~Hz}, J=$ $1.8 \mathrm{~Hz}, 2 \mathrm{H}), 5.613$ (s, 1H), 4.681-4.672 (d, $J=5.4 \mathrm{~Hz}, 1 \mathrm{H}), 4.050-4.039$ (dd, $J=5.4 \mathrm{~Hz}$, $J=1.2 \mathrm{~Hz}, 1 \mathrm{H}), 3.540$ (s, 3H), 2.333 (s, 3H), 2.315 (s, 3H), 2.035-1.993 (m, 4H), 1.708$1.550(\mathrm{~m}, 4 \mathrm{H}) ;{ }^{13} \mathrm{C}$ NMR $\left(150 \mathrm{MHz}, \mathrm{CDCl}_{3}\right): \delta=168.700,166.208,141.393,136.114$, 135.964, 129.195, 127.038, 124.550, 106.158, 94.696, 52.633, 50.647, 24.878, 22.765, 22.291, 22.284, 21.030, 14.188; HRMS Calculated for $\left[\mathrm{C}_{20} \mathrm{H}_{24} \mathrm{O}_{3}+\mathrm{H}\right]^{+}: 313.17982$, Found: 313.17986 .

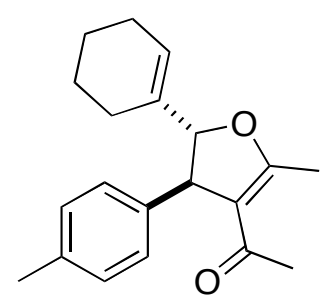

$35 t$

\section{1-(5-cyclohexenyl-4,5-dihydro-2-methyl-4-p-tolylfuran-3-yl)ethanone}

Purified by flash chromatography (hexane-EtOAc) as colorless oil (yield: 83\%); ${ }^{1} \mathrm{H}$ NMR $\left(600 \mathrm{MHz}, \mathrm{CDCl}_{3}\right) \delta=7.129-7.115(\mathrm{~d}, J=8.4 \mathrm{~Hz}, 2 \mathrm{H}), 7.075-7.062(\mathrm{t}, J=7.8 \mathrm{~Hz}, 2 \mathrm{H})$, 5.615 (s, 1H), 4.651-4.641 (d, $J=6.0 \mathrm{~Hz}, 1 \mathrm{H}), 4.098-4.087$ (dd, $J=5.4 \mathrm{~Hz}, J=0.6 \mathrm{~Hz}$, $1 \mathrm{H}), 2.372-2.370$ (d, $J=1.2 \mathrm{~Hz}, 3 \mathrm{H}), 2.320$ (s, 3H), 2.038-1.984 (m, 4H), $1.880(\mathrm{~s}, 3 \mathrm{H})$, 1.714-1.572 (m, 4H); ${ }^{13} \mathrm{C}$ NMR (150 MHz, $\left.\mathrm{CDCl}_{3}\right): \delta=195.166,168.882,140.938$, $136.515,135.633,129.494,127.158,124.834,115.419,94.976,53.424,29.509,24.809$, 
22.754, 22.204, 20.943, 14.895. HRMS Calculated for $\left[\mathrm{C}_{20} \mathrm{H}_{24} \mathrm{O}_{2}+\mathrm{H}\right]^{+}:$297.18490, Found: 297.18503.

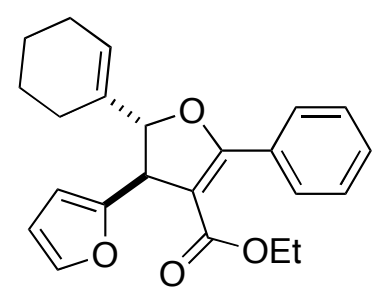

$35 \mathbf{u}$

\section{ethyl 5-cyclohexenyl-4-(furan-2-yl)-4,5-dihydro-2-phenylfuran-3-carboxylate}

Purified by flash chromatography (hexane-EtOAc) as colorless oil (yield: 75\%); ${ }^{1} \mathrm{H}$ NMR $\left(600 \mathrm{MHz}, \mathrm{CDCl}_{3}\right) \delta=7.866-7.846(\mathrm{~m}, 2 \mathrm{H}), 7.445-7.380(\mathrm{~m}, 3 \mathrm{H}), 7.350-7.346(\mathrm{dd}, J=$ $1.8 \mathrm{~Hz}, J=0.6 \mathrm{~Hz}, 1 \mathrm{H}), 6.322-6.314(\mathrm{dd}, J=3.0 \mathrm{~Hz}, J=1.8 \mathrm{~Hz}, 1 \mathrm{H}), 6.150-6.145$ (d, $J=$ $3.0 \mathrm{~Hz}, 1 \mathrm{H}), 5.779-5.778(\mathrm{~d}, J=0.6 \mathrm{~Hz}, 1 \mathrm{H}), 4.991-4.981(\mathrm{~d}, J=6.0 \mathrm{~Hz}, 1 \mathrm{H}), 4.451-$ $4.440(\mathrm{~d}, J=6.6 \mathrm{~Hz}, 1 \mathrm{H}), 4.106-4.052(\mathrm{~m}, 1 \mathrm{H}), 4.022-3.969(\mathrm{~m}, 1 \mathrm{H}), 2.115-2.067$ (m, $4 \mathrm{H}), 1.725-1.573(\mathrm{~m}, 4 \mathrm{H}), 1.082-1.058(\mathrm{t}, J=14.4 \mathrm{~Hz}, 3 \mathrm{H}) ;{ }^{13} \mathrm{C} \mathrm{NMR}(150 \mathrm{MHz}$, $\left.\mathrm{CDCl}_{3}\right): \delta=165.971,164.681,155.963,141.473,135.651,130.521,129.836,129.443$, $127.596,124.910,110.362$, 105.670, 103.717, 90.058, 59.617, 48.159, 24.878, 22.918, 22.262, 13.951; HRMS Calculated for $\left[\mathrm{C}_{23} \mathrm{H}_{24} \mathrm{O}_{4}+\mathrm{Na}\right]^{+}: 387.15668$, Found: 387.15550 .

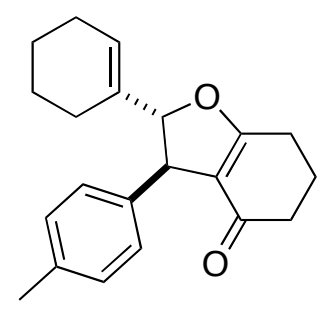

$35 v$

\section{2-cyclohexenyl-2,3,6,7-tetrahydro-3-p-tolylbenzofuran-4(5H)-one}

Purified by flash chromatography (hexane-EtOAc) as colorless oil (yield: 92\%); ${ }^{1} \mathrm{H}$ NMR $\left(600 \mathrm{MHz}, \mathrm{CDCl}_{3}\right) \delta=7.103-7.089(\mathrm{~d}, J=8.4 \mathrm{~Hz}, 2 \mathrm{H}), 7.060-7.046(\mathrm{t}, J=8.4 \mathrm{~Hz}, 2 \mathrm{H})$, 5.659 (s, 1H), 4.834-4.825 (d, $J=5.4 \mathrm{~Hz}, 1 \mathrm{H}), 4.112-4.103(\mathrm{~d}, J=5.4 \mathrm{~Hz}, 1 \mathrm{H}), 2.619$ $2.493(\mathrm{~m}, 2 \mathrm{H}), 2.386-2.281(\mathrm{~m}, 5 \mathrm{H}), 2.112-2.037(\mathrm{~m}, 4 \mathrm{H}), 1.978-1.976(\mathrm{~d}, J=1.2 \mathrm{~Hz}$, 2H), 1.732-1.548 (m, 4H); ${ }^{13} \mathrm{C}$ NMR (150 MHz, $\left.\mathrm{CDCl}_{3}\right): \delta=194.499,177.317,139.743$, 
136.262, 135.363, 129.304, 126.958, 125.461, 116.476, 97.691, 49.197, 36.825, 24.882, 24.022, 22.710, 22.185, 21.766, 20.990; HRMS Calculated for $\left[\mathrm{C}_{21} \mathrm{H}_{24} \mathrm{O}_{2}+\mathrm{H}\right]^{+}$: 309.18491, Found: 309.18498 .

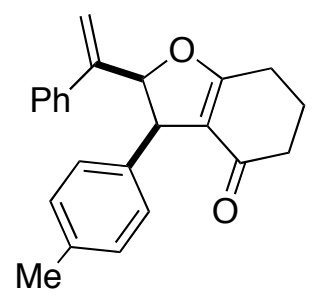

$35 \mathbf{a}^{\prime}$

\section{2,3,6,7-tetrahydro-2-(1-phenylvinyl)-3-p-tolylbenzofuran-4(5H)-one (cis)}

Purified by flash chromatography (hexane-EtOAc) as colorless oil (yield: $12 \%$ ); ${ }^{1} \mathrm{H}$ NMR $\left(600 \mathrm{MHz}, \mathrm{CDCl}_{3}\right) \delta=7.26-7.16(\mathrm{~m} \mathrm{3H}), 6.93-6.90(\mathrm{~m}, 2 \mathrm{H}), 6.82-6.80(\mathrm{~d}, \mathrm{~J}=7.8 \mathrm{~Hz}$, $2 \mathrm{H}), 6.54-6.52(\mathrm{~d}, \mathrm{~J}=7.8 \mathrm{~Hz}, 2 \mathrm{H}), 5.95-5.93(\mathrm{dt}, J=9.6 \mathrm{~Hz}, \mathrm{~J}=1.8 \mathrm{~Hz}, 1 \mathrm{H}), 5.27$ (t, $J=$ $1.8 \mathrm{~Hz}, 1 \mathrm{H}), 5.22(\mathrm{t}, J=1.2 \mathrm{~Hz}, 1 \mathrm{H}), 4.33-4.31(\mathrm{dd}, J=9.6 \mathrm{~Hz}, \mathrm{~J}=1.8 \mathrm{~Hz}, 1 \mathrm{H}), 2.75-$ $2.70(\mathrm{~m}, 1 \mathrm{H}), 2.66-2.60(\mathrm{~m}, 1 \mathrm{H}), 2.40-2.31(\mathrm{~m}, 2 \mathrm{H}), 2.21(\mathrm{~s}, 3 \mathrm{H}), 2.18-2.11(\mathrm{~m}, 2 \mathrm{H}) .{ }^{13} \mathrm{C}$ NMR (150 MHz, $\left.\mathrm{CDCl}_{3}\right): \delta=194.4,176.7,143.3,13836,134.6,128.5,128.1,127.8$, $126.1,118.3,114.1,89.3,48.0,36.9,24.0,21.8,21.03$. HRMS Calculated for $\left[\mathrm{C}_{23} \mathrm{H}_{22} \mathrm{O}_{2}+\mathrm{H}\right]^{+}:$331.16924, Found: 331.16946 .

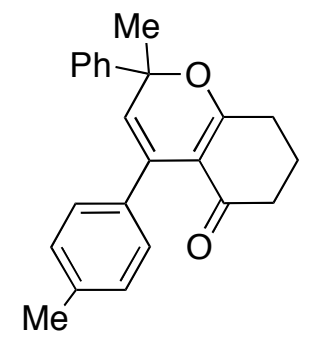

$36 \mathbf{a}$

Purified by flash chromatography (hexane-EtOAc) as colorless oil (yield: $12 \%$ ); ${ }^{1} \mathrm{H}$ NMR $\left(600 \mathrm{MHz}, \mathrm{CDCl}_{3}\right) \delta=7.50-7.48(\mathrm{~m}, 2 \mathrm{H}), 7.36-7.35(\mathrm{~m}, 2 \mathrm{H}), 7.34-7.27(\mathrm{~m}, 1 \mathrm{H}), 7.12$ (m, 4H), $5.55(\mathrm{~s}, 1 \mathrm{H}), 2.63-2.59(\mathrm{~m}, 1 \mathrm{H}), 2.50-2.44(\mathrm{M}, 1 \mathrm{H}), 2.38-2.33(\mathrm{~m}, 4 \mathrm{H}), 2.24-$ $2.19(\mathrm{~m}, 1 \mathrm{H}), 2.05-1.95(\mathrm{~m}, 2 \mathrm{H}), 1.78(\mathrm{~s}, 3 \mathrm{H}) .{ }^{13} \mathrm{C} \mathrm{NMR}\left(150 \mathrm{MHz}, \mathrm{CDCl}_{3}\right): \delta=193.5$, 173.3, 144.6, 136.7, 136.6, 134.2, 128.4, 128.3, 127.8, 126.9, 125.0, 122.2, 113.9, 81.4, 37.5, 29.4, 29.1, 21.2, 20.0. HRMS Calculated for $\left[\mathrm{C}_{23} \mathrm{H}_{22} \mathrm{O}_{2}+\mathrm{H}\right]^{+}: 331.16924$, Found: 331.16946 . 


\section{Part V}

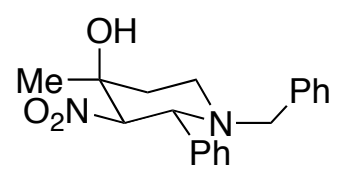

39a-major

(2R，3R，4S)-1-benzyl-4-methyl-3-nitro-2-phenylpiperidin-4-ol (39a-major) was purified by flash silica gel chromatography (Hexane-EtOAc, v/v 10/1) as white solid, yiled: $85 \%$, m.p: $177.7-179.2^{\circ} \mathrm{C} .{ }^{1} \mathrm{H}$ NMR $\left(600 \mathrm{MHz}, \mathrm{CDCl}_{3}\right): \delta 7.45-7.52(\mathrm{~m}, 2 \mathrm{H}), 7.22-$ 7.40(m, 8H), 4.71(d, $J=10.2 \mathrm{~Hz}, 1 \mathrm{H}), 4.10(\mathrm{~d}, J=10.2 \mathrm{~Hz}, 1 \mathrm{H}), 3.73(\mathrm{~d}, J=13.2 \mathrm{~Hz}$, $1 \mathrm{H}), 3.10(\mathrm{~d}, J=2.4 \mathrm{~Hz}, 1 \mathrm{H}), 2.99(\mathrm{~d}, J=13.2 \mathrm{~Hz}, 1 \mathrm{H}), 2.77(\mathrm{dq}, J=12.0 \mathrm{~Hz}, J=2.4 \mathrm{~Hz}$, $1 \mathrm{H}), 2.65(\mathrm{td}, J=12.6 \mathrm{~Hz}, J=3.0 \mathrm{~Hz}, 1 \mathrm{H}), 1.85(\mathrm{dt}, \mathrm{J}=14.4 \mathrm{~Hz}, J=3.0 \mathrm{~Hz}, 1 \mathrm{H}), 1.74-$ $1.78(\mathrm{~m}, 1 \mathrm{H}), 1.32(\mathrm{~s}, 3 \mathrm{H}) .{ }^{13} \mathrm{C} \mathrm{NMR}\left(600 \mathrm{MHz}, \mathrm{CDCl}_{3}\right): \delta 138.7,137.6,128.9,128.8$, 128.5, 128.3, 128.2, 127.0, 97.9, 69.0, 66.2, 58.5, 46.7, 36.7, 27.1. HRMS Calculated for $\mathrm{C}_{19} \mathrm{H}_{23} \mathrm{~N}_{2} \mathrm{O}_{3}[\mathrm{M}+\mathrm{H}]^{+}: 327.17032$, Found: 327.17023 .

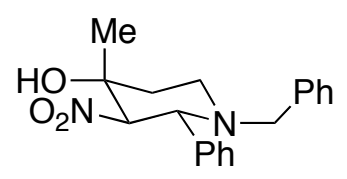

39a-minor

(2R, 3R, 4R)-1-benzyl-4-methyl-3-nitro-2-phenylpiperidin-4-ol (39a-minor) was purified by flash silica gel chromatography (Hexane-EtOAc, v/v 10/1) as white solid, yiled: $12 \%$, m.p: $169.8-171.4^{\circ} \mathrm{C} .{ }^{1} \mathrm{H}$ NMR $\left(600 \mathrm{MHz}, \mathrm{CDCl}_{3}\right): \delta 7.40-7.50(\mathrm{~m}, 2 \mathrm{H}), 7.18-$ 7.37(m, 8H), 4.83(d, $J=10.2 \mathrm{~Hz}, 1 \mathrm{H}), 3.82(\mathrm{~d}, J=10.2 \mathrm{~Hz}, 1 \mathrm{H}), 3.66(\mathrm{~d}, J=13.2 \mathrm{~Hz}, 1 \mathrm{H})$, $2.92(\mathrm{~d}, J=13.2 \mathrm{~Hz}, 1 \mathrm{H}), 2.43(\mathrm{~s}, 1 \mathrm{H}), 2.21(\mathrm{t}, J=12.0 \mathrm{~Hz}, 1 \mathrm{H}), 1.97(\mathrm{t}, J=12.0 \mathrm{~Hz}, 1 \mathrm{H})$, $1.85(\mathrm{dt}, J=13.2 \mathrm{~Hz}, J=3.0 \mathrm{~Hz}, 1 \mathrm{H}), 1.42(\mathrm{~s}, 3 \mathrm{H}) .{ }^{13} \mathrm{C} \mathrm{NMR}\left(600 \mathrm{MHz}, \mathrm{CDCl}_{3}\right): \delta 138.3$, $138.0,128.9,128.7,128.5,128.3,127.1,98.8,71.6,67.4,58.2,48.7,38.9$, 21.4. HRMS Calculated for $\mathrm{C}_{19} \mathrm{H}_{23} \mathrm{~N}_{2} \mathrm{O}_{3}[\mathrm{M}+\mathrm{H}]^{+}: 327.17032$, Found: 327.17031 . 


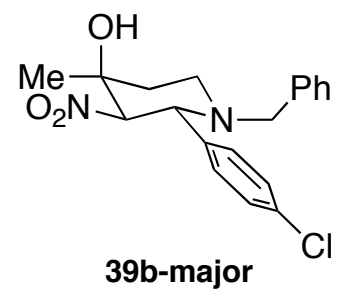

(2R,3R,4S)-1-benzyl-2-(4-chlorophenyl)-4-methyl-3-nitropiperidin-4-ol (4b-major) was purified by flash silica gel chromatography (Hexane-EtOAc, v/v 10/1) as colorless oil, yiled: $86 \%$. ${ }^{1} \mathrm{H}$ NMR (600 MHz, $\left.\mathrm{CDCl}_{3}\right): \delta 7.16-7.42(\mathrm{~m}, 9 \mathrm{H}), 4.61(\mathrm{~d}, J=10.2 \mathrm{~Hz}$, $1 \mathrm{H}), 4.06(\mathrm{~d}, J=10.2 \mathrm{~Hz}, 1 \mathrm{H}), 3.64(\mathrm{~d}, J=13.2 \mathrm{~Hz}, 1 \mathrm{H}), 2.99(\mathrm{~d}, J=2.4 \mathrm{~Hz}, 1 \mathrm{H}), 2.96(\mathrm{~d}, J$ $=13.2 \mathrm{~Hz}, 1 \mathrm{H}), 2.73(\mathrm{dq}, J=12.0 \mathrm{~Hz}, J=2.4 \mathrm{~Hz}, 1 \mathrm{H}), 2.61(\mathrm{td}, J=12.6 \mathrm{~Hz}, J=3.0 \mathrm{~Hz}, 1 \mathrm{H})$, $1.81(\mathrm{dt}, J=13.8 \mathrm{~Hz}, J=3.0 \mathrm{~Hz}, 1 \mathrm{H}), 1.64-1.72(\mathrm{~m}, 1 \mathrm{H}), 1.28(\mathrm{~s}, 3 \mathrm{H}) .{ }^{13} \mathrm{C} \mathrm{NMR}(600 \mathrm{MHz}$, $\left.\mathrm{CDCl}_{3}\right): \delta 138.3,136.2,134.5,129.6,129.2,128.4,128.2,127.1,97.7,69.0,65.5,58.5$, 46.6, 36.6, 27.1. HRMS Calculated for $\mathrm{C}_{19} \mathrm{H}_{22} \mathrm{ClN}_{2} \mathrm{O}_{3}[\mathrm{M}+\mathrm{H}]^{+}$: 361.13135, Found: 361.13148 .

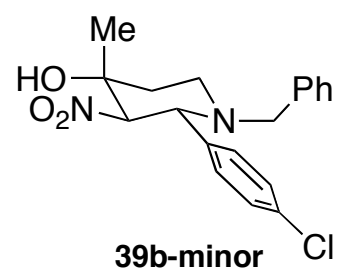

(2R,3R,4R)-1-benzyl-2-(4-chlorophenyl)-4-methyl-3-nitropiperidin-4-ol (39b-minor) was purified by flash silica gel chromatography (Hexane-EtOAc, v/v 10/1) as colorless oil, yiled: $10 \%$. ${ }^{1} \mathrm{H}$ NMR (600 MHz, $\left.\mathrm{CDCl}_{3}\right): \delta 7.14-7.46(\mathrm{~m}, 9 \mathrm{H}), 4.74(\mathrm{~d}, J=10.2 \mathrm{~Hz}$, $1 \mathrm{H}), 3.80(\mathrm{~d}, J=10.2 \mathrm{~Hz}, 1 \mathrm{H}), 3.61(\mathrm{~d}, J=13.2 \mathrm{~Hz}, 1 \mathrm{H}), 2.92(\mathrm{dt}, J=12.6 \mathrm{~Hz}, J=3.0 \mathrm{~Hz}$, $2 \mathrm{H}), 2.40(\mathrm{~s}, 1 \mathrm{H}), 2.20(\mathrm{td}, J=12.6 \mathrm{~Hz}, J=3.0 \mathrm{~Hz}, 1 \mathrm{H}), 1.94(\mathrm{td}, J=13.2 \mathrm{~Hz}, J=4.2 \mathrm{~Hz}$, $1 \mathrm{H}), 1.84(\mathrm{dt}, J=13.2 \mathrm{~Hz}, J=3.0 \mathrm{~Hz}, 1 \mathrm{H}), 1.39(\mathrm{~s}, 3 \mathrm{H}) .{ }^{13} \mathrm{C} \mathrm{NMR}\left(600 \mathrm{MHz}, \mathrm{CDCl}_{3}\right)$ : $\delta .138 .0,136.6,134.5,130.0,129.2,128.4,128.3,127.2,98.7,71.6,66.7,58.2,48.7,38.8$, 21.4. HRMS Calculated for $\mathrm{C}_{19} \mathrm{H}_{22} \mathrm{ClN}_{2} \mathrm{O}_{3}[\mathrm{M}+\mathrm{H}]^{+}:$361.13135, Found: 361.13142 . 


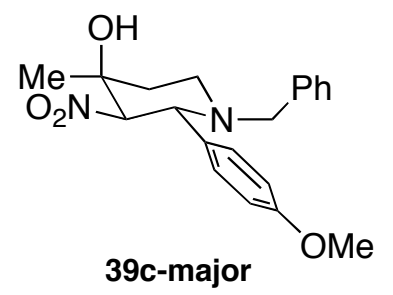

(2R,3R,4S)-1-benzyl-2-(4-methoxyphenyl)-4-methyl-3-nitropiperidin-4-ol

(39c-

major) was purified by flash silica gel chromatography (Hexane-EtOAc, v/v 8/1) as white solid, yiled: 84\%, m.p: $142.4-144.9^{\circ} \mathrm{C} .{ }^{1} \mathrm{H}$ NMR (600 $\left.\mathrm{MHz}, \mathrm{CDCl}_{3}\right)$ : $\delta$ 7.247.41(m, 7H), 6.91(d, $J=8.4 \mathrm{~Hz}, 2 \mathrm{H}), 4.69(\mathrm{~d}, J=10.8 \mathrm{~Hz}, 1 \mathrm{H}), 4.06(\mathrm{~d}, J=10.8 \mathrm{~Hz}, 1 \mathrm{H})$, 3.78(s, 3H), 3.75(d, $J=13.2 \mathrm{~Hz}, 1 \mathrm{H}), 3.13(\mathrm{~s}, 1 \mathrm{H}), 2.98(\mathrm{~d}, J=13.2 \mathrm{~Hz}, 1 \mathrm{H}), 2.75-2.78(\mathrm{~m}$, $1 \mathrm{H}), 2.62-2.67(\mathrm{~m}, 1 \mathrm{H}), 1.81-1.85(\mathrm{~m}, 1 \mathrm{H}), 1.70-1.74(\mathrm{~m}, 1 \mathrm{H}), 1.32(\mathrm{~s}, 3 \mathrm{H}) .{ }^{13} \mathrm{C} \mathrm{NMR}(600$ $\left.\mathrm{MHz}, \mathrm{CDCl}_{3}\right): \delta 159.7,138.7,129.3,128.4,128.1,126.8,114.3,97.9,69.0,65.4,58.2$, 55.0, 46.7, 36.7, 27.1. HRMS Calculated for $\mathrm{C}_{20} \mathrm{H}_{25} \mathrm{~N}_{2} \mathrm{O}_{4}[\mathrm{M}+\mathrm{H}]^{+}$: 357.18088, Found: 357.18083 .

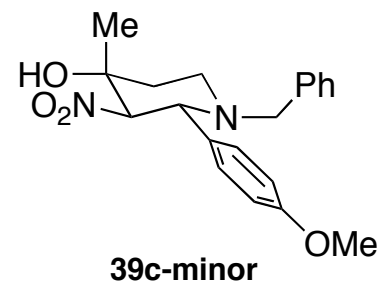

(2R,3R,4R)-1-benzyl-2-(4-methoxyphenyl)-4-methyl-3-nitropiperidin-4-ol(39c-

minor) was purified by flash silica gel chromatography (Hexane-EtOAc, v/v 8/1) as white solid, yiled: 10\%, m.p: $147.3-148.8^{\circ} \mathrm{C} .{ }^{1} \mathrm{H}$ NMR (600 $\left.\mathrm{MHz}, \mathrm{CDCl}_{3}\right)$ : $\delta$ 7.197.41(m, 7H), 6.87(d, $J=8.4 \mathrm{~Hz}, 2 \mathrm{H}), 4.79(\mathrm{~d}, J=10.2 \mathrm{~Hz}, 1 \mathrm{H}), 3.78(\mathrm{~s}, 3 \mathrm{H}), 3.76(\mathrm{~d}, J=$ $10.2 \mathrm{~Hz}, 1 \mathrm{H}), 3.69(\mathrm{~d}, J=13.8 \mathrm{~Hz}, 1 \mathrm{H}), 2.88-2.93(\mathrm{~m}, 2 \mathrm{H}), 2.44(\mathrm{~s}, 1 \mathrm{H}), 2.16-2.24(\mathrm{~m}, 1 \mathrm{H})$, $1.90-1.98(\mathrm{~m}, 1 \mathrm{H}), 1.80-1.1 .87(\mathrm{~m}, 1 \mathrm{H}), 1.41(\mathrm{~s}, 3 \mathrm{H}) .{ }^{13} \mathrm{C} \mathrm{NMR}\left(600 \mathrm{MHz}, \mathrm{CDCl}_{3}\right): \delta$ $159.7,138.4,129.8,128.5,128.2,127.0,114.3,98.9,71.6,66.8,58.0,55.2,48.7,38.9$, 21.4. HRMS Calculated for $\mathrm{C}_{20} \mathrm{H}_{25} \mathrm{~N}_{2} \mathrm{O}_{4}[\mathrm{M}+\mathrm{H}]^{+}: 357.18088$, Found: 357.18081 . 


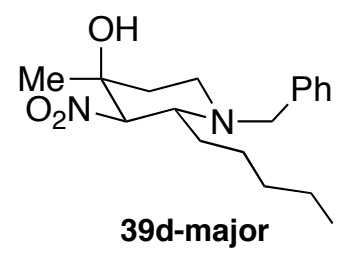

$(2 R, 3 R, 4 S)-1-b e n z y l-4-m e t h y l-3-n i t r o-2-p e n t y l p i p e r i d i n-4-o l \quad$ (39d-major) was purified by flash silica gel chromatography (Hexane-EtOAc, v/v 15/1) as colorless oil, yiled: $70 \%$. ${ }^{1} \mathrm{H}$ NMR (600 MHz, $\left.\mathrm{CDCl}_{3}\right): \delta 7.23-7.34(\mathrm{~m}, 5 \mathrm{H}), 4.59(\mathrm{~d}, J=10.2 \mathrm{~Hz}, 1 \mathrm{H})$, 3.93(d, $J=13.2 \mathrm{~Hz}, 1 \mathrm{H}), 3.30-3.35(\mathrm{~m}, 1 \mathrm{H}), 3.28(\mathrm{~d}, J=13.2 \mathrm{~Hz}, 1 \mathrm{H}), 3.15(\mathrm{~d}, J=1.8 \mathrm{~Hz}$, $1 \mathrm{H}), 2.55-2.65(\mathrm{~m}, 2 \mathrm{H}), 1.20-1.75(\mathrm{~m}, 13 \mathrm{H}), 0.87(\mathrm{t}, J=7.2 \mathrm{~Hz}, 3 \mathrm{H}) .{ }^{13} \mathrm{C}$ NMR $(600 \mathrm{MHz}$, $\left.\mathrm{CDCl}_{3}\right): \delta 138.8,128.5,128.4,127.1,93.4,69.22,58.8,54.2,45.8,35.1,31.9,28.4,27.3$, 22.7, 22.4, 13.9. HRMS Calculated for $\mathrm{C}_{18} \mathrm{H}_{29} \mathrm{~N}_{2} \mathrm{O}_{3}[\mathrm{M}+\mathrm{H}]^{+}$: 321.21727, Found: 321.21740 .

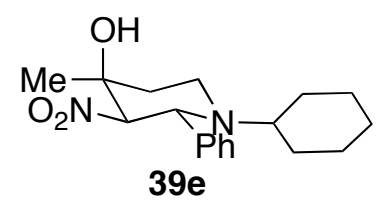

(2R,3R,4S)-1-cyclohexyl-4-methyl-3-nitro-2-phenylpiperidin-4-ol (39e) was purified by flash silica gel chromatography (Hexane-EtOAc, v/v 10/1) as white solid, yiled: 85\%, m.p: $143.3-144.5^{\circ} \mathrm{C} .{ }^{1} \mathrm{H}$ NMR $\left(600 \mathrm{MHz}, \mathrm{CDCl}_{3}\right): \delta$ 7.24-7.35(m, 5H), 4.60(d, $J=$ $10.2 \mathrm{~Hz}, 1 \mathrm{H}), 4.28(\mathrm{~d}, J=10.2 \mathrm{~Hz}, 1 \mathrm{H}), 3.08(\mathrm{~d}, J=2.4 \mathrm{~Hz}, 1 \mathrm{H}), 2.89(\mathrm{td}, J=12.0 \mathrm{~Hz}, J=$ $2.4 \mathrm{~Hz}, 1 \mathrm{H}), 2.77-2.83(\mathrm{~m}, 1 \mathrm{H}), 2.18-2.224(\mathrm{~m}, 1 \mathrm{H}), 1.89(\mathrm{dt}, J=13.8 \mathrm{~Hz}, J=3.0 \mathrm{~Hz}, 1 \mathrm{H})$, $1.60-1.76(\mathrm{~m}, 4 \mathrm{H}), 1.38-1.50(\mathrm{~m}, 3 \mathrm{H}), 1.27(\mathrm{~s}, 3 \mathrm{H}), 1.18(\mathrm{dq}, J=12.0 \mathrm{~Hz}, J=3.6 \mathrm{~Hz}, 1 \mathrm{H})$, 0.90-1.01(m, 2H), 0.70-0.78(m, 1H). ${ }^{13} \mathrm{C} \mathrm{NMR}\left(600 \mathrm{MHz}, \mathrm{CDCl}_{3}\right): \delta 137.2,128.6,128.4$, $128.1,98.3,68.9,63.5,57.7,39.9,37.2,31.6,27.2,26.3,26.1,25.5,24.1$. HRMS Calculated for $\mathrm{C}_{18} \mathrm{H}_{27} \mathrm{~N}_{2} \mathrm{O}_{3}[\mathrm{M}+\mathrm{H}]^{+}:$319.20162, Found: 319.20155 . 


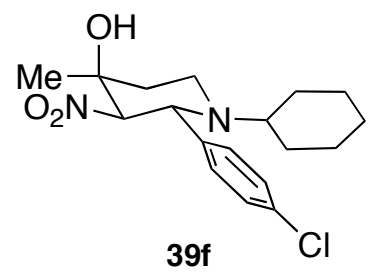

(2R,3R,4S)-2-(4-chlorophenyl)-1-cyclohexyl-4-methyl-3-nitropiperidin-4-ol (39f) was purified by flash silica gel chromatography (Hexane-EtOAc, v/v 10/1) as white solid, yiled: 90\%, m.p: $161.4-163.5^{\circ} \mathrm{C} .{ }^{1} \mathrm{H}$ NMR (600 MHz, $\left.\mathrm{CDCl}_{3}\right): \delta$ 7.22-7.24(m, 4H), $5.43(\mathrm{~d}, J=10.2 \mathrm{~Hz}, 1 \mathrm{H}), 4.27(\mathrm{~d}, J=10.2 \mathrm{~Hz}, 1 \mathrm{H}), 2.98(\mathrm{~d}, J=1.8 \mathrm{~Hz}, 1 \mathrm{H}), 2.88(\mathrm{td}, \mathrm{J}$ $=12.0 \mathrm{~Hz}, J=2.4 \mathrm{~Hz}, 1 \mathrm{H}), 2.79(\mathrm{dq}, J=12.0 \mathrm{~Hz}, J=2.4 \mathrm{~Hz}, 1 \mathrm{H}), 2.17(\mathrm{tt}, J=12.0 \mathrm{~Hz}, J=$ $3.0 \mathrm{~Hz}, 1 \mathrm{H}), 1.89(\mathrm{dt}, J=13.8 \mathrm{~Hz}, J=3.0 \mathrm{~Hz}, 1 \mathrm{H}), 1.62-1.72(\mathrm{~m}, 4 \mathrm{H}), 1.38-1.50(\mathrm{~m}, 3 \mathrm{H})$, 1.14-1.23(m 1H), 0.91-1.04(m, 2H), 0.73-0.82(m, 1H). $\left.{ }^{13} \mathrm{C} \mathrm{NMR} \mathrm{(600} \mathrm{MHz,} \mathrm{CDCl}_{3}\right): \delta$ 136.0, 134.1, 129.5, 129.0, 98.3, 69.0, 62.9, 58.0, 39.8, 37.2, 31.7, 27.2, 26.4, 26.1, 25.5, 24.1. HRMS Calculated for $\mathrm{C}_{18} \mathrm{H}_{26} \mathrm{ClN}_{2} \mathrm{O}_{3}[\mathrm{M}+\mathrm{H}]^{+}:$353.16265, Found: 353.16285.

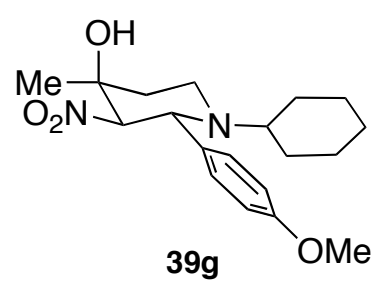

(2R,3R,4S)-1-cyclohexyl-2-(4-methoxyphenyl)-4-methyl-3-nitropiperidin-4-ol

(39g) was purified by flash silica gel chromatography (Hexane-EtOAc, v/v 8/1) as white solid, yiled: $93 \%$, m.p:152.3-154.1 ${ }^{\circ} \mathrm{C} .{ }^{1} \mathrm{H}$ NMR (600 MHz, $\left.\mathrm{CDCl}_{3}\right): \delta 7.20(\mathrm{~d}, J=8.4 \mathrm{~Hz}, 1 \mathrm{H})$, $6.82(\mathrm{~d}, J=8.4 \mathrm{~Hz}, 1 \mathrm{H}), 4.57(\mathrm{~d}, J=10.2 \mathrm{~Hz}, 1 \mathrm{H}), 4.21(\mathrm{~d}, J=10.2 \mathrm{~Hz}, 1 \mathrm{H}), 3.77(\mathrm{~s}, 3 \mathrm{H})$, $3.05(\mathrm{~d}, J=1.8 \mathrm{~Hz}, 1 \mathrm{H}), 2.86(\mathrm{t}, J=12.0 \mathrm{~Hz}, 1 \mathrm{H}), 2.78(\mathrm{t}, J=12.0 \mathrm{~Hz}, 1 \mathrm{H}), 2.22(\mathrm{t}, J=$ $10.2 \mathrm{~Hz}, 1 \mathrm{H}), 1.87(\mathrm{dt}, J=13.8 \mathrm{~Hz}, J=2.4 \mathrm{~Hz}, 1 \mathrm{H}), 1.58-1.72(\mathrm{~m}, 4 \mathrm{H}), 1.35-1.48(\mathrm{~m}, 3 \mathrm{H})$, $1.26(\mathrm{~s}, 3 \mathrm{H}), 1.14(\mathrm{qd}, J=12.0 \mathrm{~Hz}, J=3.0 \mathrm{~Hz}, 1 \mathrm{H}), 0.90-1.02(\mathrm{~m}, 2 \mathrm{H}), 0.72-0.80(\mathrm{~m}, 1 \mathrm{H})$. ${ }^{13} \mathrm{C}$ NMR $\left(600 \mathrm{MHz}, \mathrm{CDCl}_{3}\right): \delta 159.4,129.3,129.0,114.1,98.4,69.0,62.9,57.6,55.0$, 40.0, 37.2, 31.7, 27.3, 26.4, 26.1, 25.6, 24.1. HRMS Calculated for $\mathrm{C}_{19} \mathrm{H}_{29} \mathrm{~N}_{2} \mathrm{O}_{4}[\mathrm{M}+\mathrm{H}]^{+}$: 349.21219, Found: 349.21207. 


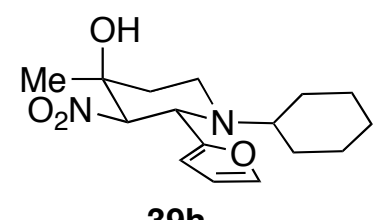

$39 \mathrm{~h}$

(2S,3R,4S)-1-cyclohexyl-2-(furan-2-yl)-4-methyl-3-nitropiperidin-4-ol (39h) was purified by flash silica gel chromatography (Hexane-EtOAc, v/v 8/1) as white solid, yiled: $82 \%$, m.p $>130^{\circ} \mathrm{C}\left(\right.$ decomposed). ${ }^{1} \mathrm{H}$ NMR $\left(600 \mathrm{MHz}, \mathrm{CDCl}_{3}\right): \delta 7.40(\mathrm{~s}, 1 \mathrm{H})$, $6.27(\mathrm{~d}, J=1.2 \mathrm{~Hz}, 2 \mathrm{H}), 4.89(\mathrm{~d}, J=10.2 \mathrm{~Hz}, 1 \mathrm{H}), 4.43(\mathrm{~d}, J=10.2 \mathrm{~Hz}, 1 \mathrm{H}), 2.99(\mathrm{br}, 1 \mathrm{H})$, $2.74-2.85(\mathrm{~m}, 2 \mathrm{H}), 2.03(\mathrm{tt}, J=12.0 \mathrm{~Hz}, J=3.0 \mathrm{~Hz}, 1 \mathrm{H}), 1.86(\mathrm{dt}, J=13.8 \mathrm{~Hz}, J=3.0 \mathrm{~Hz}$, $1 \mathrm{H}), 1.75-1.80(\mathrm{~m}, 1 \mathrm{H}), 1.65-1.73(\mathrm{~m}, 3 \mathrm{H}), 1.49-1.56(\mathrm{~m}, 2 \mathrm{H}), 1.40(\mathrm{qd}, J=12.0 \mathrm{~Hz}, J=$ $33.6 \mathrm{~Hz}, 1 \mathrm{H}) .1 .03-1.18(\mathrm{~m}, 1 \mathrm{H}), 0.98(\mathrm{qt}, J=13.2 \mathrm{~Hz}, J=3.6 \mathrm{~Hz}, 1 \mathrm{H}), 0.89(\mathrm{qt}, J=13.2 \mathrm{~Hz}$, $J=3.6 \mathrm{~Hz}, 1 \mathrm{H}) .{ }^{13} \mathrm{C} \mathrm{NMR}\left(600 \mathrm{MHz}, \mathrm{CDCl}_{3}\right): \delta 149.6,143.1,110.7,110.1,95.1,69.1$, 59.1, 56.9, 40.0, 37.1, 31.6, 27.2, 26.5, 26.1, 25.7, 24.5. HRMS Calculated for $\mathrm{C}_{16} \mathrm{H}_{25} \mathrm{~N}_{2} \mathrm{O}_{4}[\mathrm{M}+\mathrm{H}]^{+}: 309.18088$, Found: 309.18092.

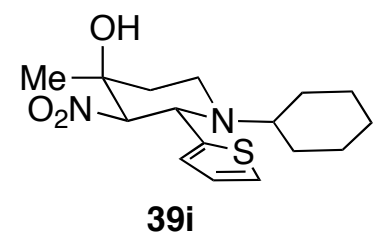

(2S,3R,4S)-1-cyclohexyl-4-methyl-3-nitro-2-(thiophen-2-yl)piperidin-4-ol (39i) was purified by flash silica gel chromatography (Hexane-EtOAc, v/v 6/1) as white solid, yiled: 84\%, m.p: $139.5-141.7^{\circ} \mathrm{C} .{ }^{1} \mathrm{H}$ NMR (600 MHz, $\left.\mathrm{CDCl}_{3}\right): \delta$ 7.27-7.29(m, $\left.1 \mathrm{H}\right)$, $6.91(\mathrm{dd}, J=3.6 \mathrm{~Hz}, J=1.2 \mathrm{~Hz}, 1 \mathrm{H}), 6.86(\mathrm{dd}, J=5.4 \mathrm{~Hz}, J=3.6 \mathrm{~Hz}, 1 \mathrm{H}), 4.62(\mathrm{~d}, J=$ $10.2 \mathrm{~Hz}, 1 \mathrm{H}), 4.59(\mathrm{~d}, J=10.2 \mathrm{~Hz}, 1 \mathrm{H}), 2.99(\mathrm{~d}, J=1.8 \mathrm{~Hz}, 1 \mathrm{H}), 2.88(\mathrm{td}, J=12.0 \mathrm{~Hz}, \mathrm{~J}=$ $3.0 \mathrm{~Hz}, 1 \mathrm{H}), 2.76(\mathrm{dq}, J=12.0 \mathrm{~Hz}, J=3.0 \mathrm{~Hz}, 1 \mathrm{H}), 2.41(\mathrm{tt}, J=12.0 \mathrm{~Hz}, J=3.0 \mathrm{~Hz}, 1 \mathrm{H})$, $1.87(\mathrm{dt}, J=13.8 \mathrm{~Hz}, J=2.4 \mathrm{~Hz}, 1 \mathrm{H}), 1.64-1.78(\mathrm{~m}, 4 \mathrm{H}), 1.47-1.53(\mathrm{~m}, 2 \mathrm{H}), 1.40(\mathrm{qd}, \mathrm{J}=$ $12.0 \mathrm{~Hz}, J=3.6 \mathrm{~Hz}, 1 \mathrm{H}), 1.26(\mathrm{~s}, 3 \mathrm{H}), 1.19(\mathrm{qd}, J=12.0 \mathrm{~Hz}, J=3.6 \mathrm{~Hz}, 1 \mathrm{H}), 0.93-1.08(\mathrm{~m}$, $2 \mathrm{H}), 0.86(\mathrm{qt}, J=12.6 \mathrm{~Hz}, J=3.6 \mathrm{~Hz}, 1 \mathrm{H}) .{ }^{13} \mathrm{C} \mathrm{NMR}\left(600 \mathrm{MHz}, \mathrm{CDCl}_{3}\right): \delta 141.4,127.4$, 
126.24, 126.16, 99.2, 69.0, 58.9, 58.0, 39.9, 36.9, 31.6, 27.11, 26.4, 26.1, 25.6, 24.1. HRMS Calculated for $\mathrm{C}_{16} \mathrm{H}_{25} \mathrm{~N}_{2} \mathrm{O}_{3} \mathrm{~S}[\mathrm{M}+\mathrm{H}]^{+}: 325.15804$, Found: 325.15803 .

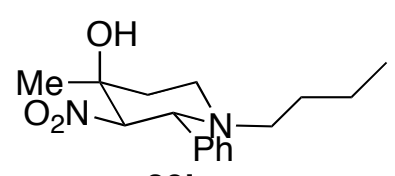

39j

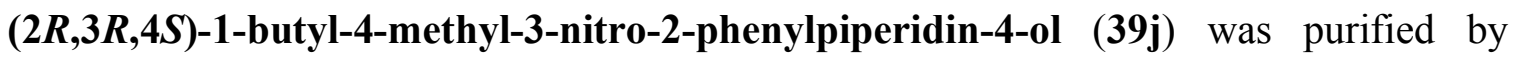
flash silica gel chromatography (Hexane-EtOAc, v/v 8/1) as white solid, yiled: 93\%, m.p: 76.1-78.8 ${ }^{\circ} \mathrm{C} .{ }^{1} \mathrm{H}$ NMR (600 MHz, $\left.\mathrm{CDCl}_{3}\right): \delta 7.26-7.32(\mathrm{~m}, 5 \mathrm{H}), 4.58(\mathrm{~d}, J=10.2 \mathrm{~Hz}, 1 \mathrm{H})$, $3.95(\mathrm{~d}, J=10.2 \mathrm{H}, 1 \mathrm{H}), 3.09(\mathrm{br}, 1 \mathrm{H}), 2.91(\mathrm{dq}, J=12.0 \mathrm{~Hz}, J=2.4 \mathrm{~Hz}, 1 \mathrm{H}), 2.72(\mathrm{td}, J=$ $12.6 \mathrm{~Hz}, J=3.0 \mathrm{~Hz}, 1 \mathrm{H}), 2.32-2.38(\mathrm{~m}, 1 \mathrm{H}), 2.01-2.07(\mathrm{~m}, 1 \mathrm{H}), 1.91(\mathrm{dt}, J=14.4 \mathrm{~Hz}, J=$ $3.0 \mathrm{~Hz}, 1 \mathrm{H}), 1.77(\mathrm{td}, J=12.6 \mathrm{~Hz}, J=3.0 \mathrm{~Hz}, 1 \mathrm{H}), 1.26-1.40(\mathrm{~m}, 2 \mathrm{H}), 1.29(\mathrm{~s}, 3 \mathrm{H}), 1.15-$ $1.19(\mathrm{~m}, 1 \mathrm{H}), 0.99-1.08(\mathrm{~m}, 1 \mathrm{H}), 0.75(\mathrm{t}, J=7.2 \mathrm{~Hz}, 3 \mathrm{H}) .{ }^{13} \mathrm{C} \mathrm{NMR}\left(600 \mathrm{MHz}, \mathrm{CDCl}_{3}\right): \delta$ $137.5,128.7,128.5,128.4,97.9,68.9,65.9,53.7,46.5,36.9,28.1,27.2,20.2,13.8$. HRMS Calculated for $\mathrm{C}_{16} \mathrm{H}_{25} \mathrm{~N}_{2} \mathrm{O}_{3}[\mathrm{M}+\mathrm{H}]^{+}:$293.18597, Found: 297.18593.

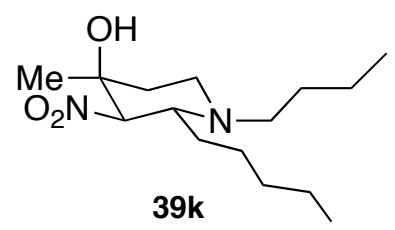

$(2 R, 3 R, 4 S)-1-b u t y l-4-m e t h y l-3-n i t r o-2-p e n t y l p i p e r i d i n-4-o l ~(39 k)$ was purified by flash silica gel chromatography (Hexane-EtOAc, v/v 10/1) as light-yellow oil, yiled: $68 \%$. ${ }^{1} \mathrm{H}$ NMR $\left(600 \mathrm{MHz}, \mathrm{CDCl}_{3}\right): \delta 4.47(\mathrm{~d}, J=10.2 \mathrm{~Hz}, 1 \mathrm{H}), 3.21(\mathrm{dt}, J=10.2 \mathrm{~Hz}, J=$ $4.2 \mathrm{~Hz}, 1 \mathrm{H}), 3.16(\mathrm{br}, 1 \mathrm{H}), 2.81(\mathrm{td}, J=12.0 \mathrm{~Hz}, J=3.0 \mathrm{~Hz}, 1 \mathrm{H}), 2.73(\mathrm{dq}, J=12.6 \mathrm{~Hz}, J=$ $2.4 \mathrm{~Hz}, 1 \mathrm{H}), 2.55-2.61(\mathrm{~m}, 1 \mathrm{H}), 2.36-2.42(\mathrm{~m}, 1 \mathrm{H}), 1.71(\mathrm{dt}, J=13.8 \mathrm{~Hz}, J=3.0 \mathrm{~Hz}, 1 \mathrm{H})$, $1.52-1.64(\mathrm{~m}, 3 \mathrm{H}), 1.19-1.50(\mathrm{~m}, 19 \mathrm{H}), 0.92(\mathrm{t}, J=7.2 \mathrm{~Hz}, 3 \mathrm{H}), 0.88(\mathrm{t}, J=7.2 \mathrm{~Hz}, 3 \mathrm{H}) .{ }^{13} \mathrm{C}$ NMR (600 MHz, $\left.\mathrm{CDCl}_{3}\right): \delta$ 93.5, 69.1, 58.0, 49.9, 45.9, 35.4, 32.0, 28.2, 27.9, 27.4, 22.8, 22.4, 20.6, 14.02, 13.96. HRMS Calculated for $\mathrm{C}_{15} \mathrm{H}_{31} \mathrm{~N}_{2} \mathrm{O}_{3}[\mathrm{M}+\mathrm{H}]^{+}:$287.23460, Found: 287.23303. 


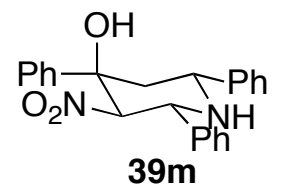

$(2 R, 3 R, 4 R, 6 R)-3-n i t r o-2,4,6-t r i p h e n y l p i p e r i d i n-4-o l ~(39 m)$ was purified by flash silica gel chromatography (Hexane-EtOAc, v/v 10/1) as white solid, yiled: 83\%, m.p: 153.5$155.2^{\circ} \mathrm{C} .{ }^{1} \mathrm{H}$ NMR $\left(600 \mathrm{MHz}, \mathrm{CDCl}_{3}\right): \delta 7.41-7.57(\mathrm{~m}, 6 \mathrm{H}), 7.22-7.40(\mathrm{~m}, 9 \mathrm{H}), 5.22(\mathrm{~d}, J=$ $10.2 \mathrm{~Hz}, 1 \mathrm{H}), 4.90(\mathrm{~d}, J=10.2 \mathrm{~Hz}, 1 \mathrm{H}), 4.64(\mathrm{dd}, J=10.2 \mathrm{~Hz}, J=3.0 \mathrm{~Hz}, 1 \mathrm{H}), 4.31(\mathrm{~d}, J$ $=3.0 \mathrm{~Hz}, 1 \mathrm{H}), 2.17(\mathrm{dd}, J=13.8 \mathrm{~Hz}, J=2.4 \mathrm{~Hz}, 1 \mathrm{H}), 2.01-2.07(\mathrm{~m}, 2 \mathrm{H}) .{ }^{13} \mathrm{C}$ NMR $(600$ $\left.\mathrm{MHz}, \mathrm{CDCl}_{3}\right): \delta 142.8,142.3,138.00,1129.1,128.9,128.7,128.6,128.1,127.7,127.6$, 126.8, 124.5, 95.6, 74.9, 61.2, 56.4, 48.2. HRMS Calculated for $\mathrm{C}_{23} \mathrm{H}_{23} \mathrm{~N}_{2} \mathrm{O}_{3}[\mathrm{M}+\mathrm{H}]^{+}$: 375.17032, Found: 375.17041.

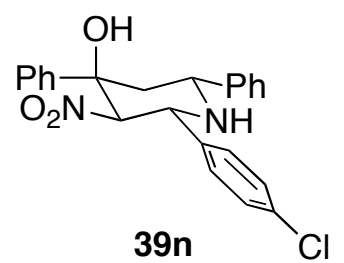

$(2 R, 3 R, 4 R, 6 R)-2-(4-c h l o r o p h e n y l)-3-n i t r o-4,6-d i p h e n y l p i p e r i d i n-4-o l \quad(39 n) \quad$ was purified by flash silica gel chromatography (Hexane-EtOAc, v/v 10/1) as colorless oil, yiled: $81 \%$. ${ }^{1} \mathrm{H}$ NMR $\left(600 \mathrm{MHz}, \mathrm{CDCl}_{3}\right): \delta$ 7.46-7.52(m, 6H), 7.32-7.38(m, 6H), 7.24$7.30(\mathrm{~m}, 2 \mathrm{H}), 5.16(\mathrm{~d}, J=10.2 \mathrm{~Hz}, 1 \mathrm{H}), 4.89(\mathrm{~d}, J=10.2 \mathrm{~Hz}, 1 \mathrm{H}), 4.62(\mathrm{dd}, J=10.2 \mathrm{~Hz}, J=$ $3.0 \mathrm{~Hz}, 1 \mathrm{H}), 2.16(\mathrm{dd}, J=14.4 \mathrm{~Hz}, J=3.0 \mathrm{~Hz}, 1 \mathrm{H}), 2.00-2.07(\mathrm{~m}, 2 \mathrm{H}) .{ }^{13} \mathrm{C} \mathrm{NMR}(600 \mathrm{MHz}$, $\left.\mathrm{CDCl}_{3}\right): \delta 142.6,142.1,136.6,134.9,129.1,128.9,128.7,128.6,128.2,127.77,126.66$, 124.4, 95.5, 74.9, 60.5, 56.3, 48.1. HRMS Calculated for $\mathrm{C}_{23} \mathrm{H}_{22} \mathrm{ClN}_{2} \mathrm{O}_{3}[\mathrm{M}+\mathrm{H}]^{+}$: 409.13135, Found: 409.13141. 


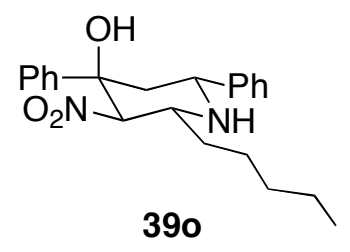

$(2 R, 3 R, 4 R, 6 R)-3-n i t r o-2-p e n t y l-4,6-d i p h e n y l p i p e r i d i n-4-o l ~(390)$ was purified by flash silica gel chromatography (Hexane-EtOAc, v/v 10/1) as colorless oil, yiled: $65 \% .{ }^{1} \mathrm{H}$ NMR $\left(600 \mathrm{MHz}, \mathrm{CDCl}_{3}\right): \delta 7.27-7.51(\mathrm{~m}, 10 \mathrm{H}), 4.76(\mathrm{~d}, J=3.0 \mathrm{~Hz}, 1 \mathrm{H}), 4.27(\mathrm{dd}, J=$ $12.0 \mathrm{~Hz}, J=3.0 \mathrm{~Hz}, 1 \mathrm{H}), 3.64(\mathrm{td}, J=7.2 \mathrm{~Hz}, \mathrm{~J}=3.0 \mathrm{~Hz}, 1 \mathrm{H}), 3.02(\mathrm{dd}, J=13.8 \mathrm{~Hz}, J=$ $12.0 \mathrm{~Hz}, 1 \mathrm{H}), 1.96(\mathrm{dd}, J=13.8 \mathrm{~Hz}, J=3.0 \mathrm{~Hz}, 1 \mathrm{H}), 1.25-1.56(\mathrm{~m}, 9 \mathrm{H}), 0.90(\mathrm{t}, J=7.2 \mathrm{~Hz}$, $3 \mathrm{H}) .{ }^{13} \mathrm{C} \mathrm{NMR}\left(600 \mathrm{MHz}, \mathrm{CDCl}_{3}\right): \delta 143.2,142.4,128.9,128.8,128.7,127.5,126.6$, 125.4, 91.0, 73.1, 55.13, 55.12, 38.6, 32.5, 31.6, 25.9, 22.4, 14.0. HRMS Calculated for $\mathrm{C}_{22} \mathrm{H}_{29} \mathrm{~N}_{2} \mathrm{O}_{3}[\mathrm{M}+\mathrm{H}]^{+}: 349.21727$, Found: 349.21722 .

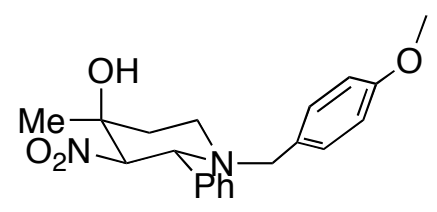

39r-major

(2R,3R,4S)-1-(4-methoxybenzyl)-4-methyl-3-nitro-2-phenylpiperidin-4-ol(39r-

major) was purified by flash silica gel chromatography (Hexane-EtOAc, v/v 10/1) as white solid, yiled: 79\%, m.p: $130.1-131.9^{\circ} \mathrm{C} .{ }^{1} \mathrm{H}$ NMR (600 MHz, $\left.\mathrm{CDCl}_{3}\right)$ : $\delta$ 7.297.46(m, 5H), 7.12(d, $J=8.4 \mathrm{~Hz}, 2 \mathrm{H}), 6.83(\mathrm{~d}, J=8.4 \mathrm{~Hz}, 2 \mathrm{H}), 4.67(\mathrm{~d}, J=10.2 \mathrm{~Hz}, 1 \mathrm{H})$, $4.05(\mathrm{~d}, J=10.2 \mathrm{~Hz}, 1 \mathrm{H}), 3.78(\mathrm{~s}, 3 \mathrm{H}), 3.64(\mathrm{~d}, J=13.2 \mathrm{~Hz}, 1 \mathrm{H}), 3.08(\mathrm{~d}, J=2.4 \mathrm{~Hz}, 1 \mathrm{H})$, $2.92(\mathrm{~d}, J=13.2 \mathrm{~Hz}, 2 \mathrm{H}), 2.73-2.78(\mathrm{~m}, 1 \mathrm{H}), 2.61(\mathrm{td}, J=12.0 \mathrm{~Hz}, J=2.4 \mathrm{~Hz}, 1 \mathrm{H}), 1.84(\mathrm{dt}$, $J=13.8 \mathrm{~Hz}, J=2.4 \mathrm{~Hz}, 1 \mathrm{H}), 1.68-1.74(\mathrm{~m}, 1 \mathrm{H}), 1.30(\mathrm{~s}, 3 \mathrm{H}) .{ }^{13} \mathrm{C} \mathrm{NMR}\left(600 \mathrm{MHz}, \mathrm{CDCl}_{3}\right)$ : $\delta 158.7,137.7,130.5,129.7,128.9,128.7,128.3,113.6,97.9,69.0,66.1,57.8,55.2,46.5$, 36.7, 27.1. HRMS Calculated for $\mathrm{C}_{20} \mathrm{H}_{25} \mathrm{~N}_{2} \mathrm{O}_{4}[\mathrm{M}+\mathrm{H}]^{+}: 357.18088$, Found: 357.18078 .

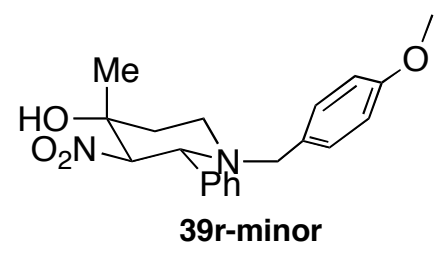


minor) was purified by flash silica gel chromatography (Hexane-EtOAc, v/v 10/1) as white solid, yiled: $11 \%$, m.p: $152.1-153.8^{\circ} \mathrm{C} .{ }^{1} \mathrm{H}$ NMR $\left(600 \mathrm{MHz}, \mathrm{CDCl}_{3}\right)$ : $\delta$ 7.267.49(m, 5H), 7.09(d, $J=8.4 \mathrm{~Hz}, 2 \mathrm{H}), 6.81(\mathrm{~d}, J=8.4 \mathrm{~Hz}, 2 \mathrm{H}), 4.81(\mathrm{~d}, J=5.4 \mathrm{~Hz}, 1 \mathrm{H})$, 3.77-3.82(m, 1H), 3.78(s, 1H), 3.59(d, $J=13.2 \mathrm{~Hz}, 1 \mathrm{H}), 2.93(\mathrm{~d}, J=12.6 \mathrm{~Hz}, 1 \mathrm{H}), 2.88(\mathrm{~d}$, $J=12.6 \mathrm{~Hz}, 1 \mathrm{H}), 2.50(\mathrm{br}, 1 \mathrm{H}), 2.20(\mathrm{t}, J=12.0 \mathrm{~Hz}, 1 \mathrm{H}), 1.90-2.02(\mathrm{~m}, 1 \mathrm{H}), 1.85(\mathrm{dt}, J=$ $13.2 \mathrm{~Hz}, J=2.4 \mathrm{~Hz}, 1 \mathrm{H}), 1.40(\mathrm{~s}, 3 \mathrm{H}) .{ }^{13} \mathrm{C} \mathrm{NMR}\left(600 \mathrm{MHz}, \mathrm{CDCl}_{3}\right): \delta 158.8,129.7$, $129.2,128.8,128.7,127.6,113.7,98.8,71.6,67.3,57.5,55.2,48.5,38.8,21.4$. HRMS Calculated for $\mathrm{C}_{20} \mathrm{H}_{25} \mathrm{~N}_{2} \mathrm{O}_{4}[\mathrm{M}+\mathrm{H}]^{+}: 357.18088$, Found: 357.18090 .

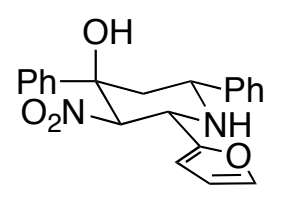

$39 q$

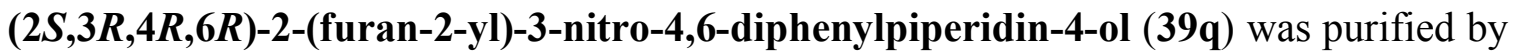
flash silica gel chromatography (Hexane-EtOAc, v/v 10/1) as colorless oil, yield: 75\%. ${ }^{1} \mathrm{H}$ NMR (600 MHz, $\left.\mathrm{CDCl}_{3}\right): \delta$ 7.51-7.54(m, 2H), 7.41-7.46(m, 3H), 7.24-7.39(m, 6H), $6.40(\mathrm{~d}, J=3.00 \mathrm{~Hz}, 1 \mathrm{H}), 6.33(\mathrm{dd}, J=3.0 \mathrm{~Hz}, J=1.8 \mathrm{~Hz}, 1 \mathrm{H}), 5.43(\mathrm{~d}, J=10.2 \mathrm{~Hz}, 1 \mathrm{H})$, $5.07(\mathrm{~d}, J=10.2 \mathrm{~Hz}, 1 \mathrm{H}), 4.61(\mathrm{dd}, J=11.4 \mathrm{~Hz}, J=3.0 \mathrm{~Hz}, 1 \mathrm{H}), 4.24(\mathrm{~d}, J=3.0 \mathrm{~Hz}, 1 \mathrm{H})$, $2.17(\mathrm{dd}, J=13.8 \mathrm{~Hz}, J=3.0 \mathrm{~Hz}, 1 \mathrm{H}), 2.00-2.09(\mathrm{~m}, 2 \mathrm{H}) .{ }^{13} \mathrm{C} \mathrm{NMR}\left(600 \mathrm{MHz}, \mathrm{CDCl}_{3}\right): \delta$ $150.7,143.2$, 142.12, 142.06, 128.8, 128.6, 128.2, 127.7, 126.7, 124.4, 110.3, 108.7, 92.8, 74.9, 56.0, 54.8, 47.7. HRMS Calculated for $\mathrm{C}_{21} \mathrm{H}_{21} \mathrm{~N}_{2} \mathrm{O}_{4}[\mathrm{M}+\mathrm{H}]^{+}:$365.14958, Found: 365.14957.

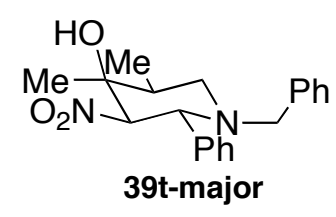


$(2 R, 3 R, 4 S, 5 S)-1-b e n z y l-4,5-d i m e t h y l-3-n i t r o-2-p h e n y l p i p e r i d i n-4-o l ~(39 t-m a j o r)$ was purified by flash silica gel chromatography (Hexane-EtOAc, v/v 8/1) as colorless oil, yield: $53 \%$. ${ }^{1} \mathrm{H}$ NMR $\left(600 \mathrm{MHz}, \mathrm{CDCl}_{3}\right): \delta 7.25-7.62(\mathrm{~m}, 10 \mathrm{H}), 5.21(\mathrm{~d}, J=10.2 \mathrm{~Hz}, 1 \mathrm{H})$, $4.09(\mathrm{~d}, J=10.2 \mathrm{~Hz}, 1 \mathrm{H}), 4.04(\mathrm{~d}, J=11.4 \mathrm{~Hz}, 1 \mathrm{H}), 3.73(\mathrm{dd}, J=13.8 \mathrm{~Hz}, J=10.2 \mathrm{~Hz}, 1 \mathrm{H})$, $2.99(\mathrm{~d}, J=13.8 \mathrm{~Hz}, 1 \mathrm{H}), 2.88(\mathrm{dd}, J=12.0 \mathrm{~Hz}, J=3.6 \mathrm{~Hz}, 1 \mathrm{H}), 2.31-2.40(\mathrm{~m}, 1 \mathrm{H}), 2.22(\mathrm{t}, J$ $=12.0 \mathrm{~Hz}, 1 \mathrm{H}), 1.21(\mathrm{~s}, 3 \mathrm{H}), 1.00(\mathrm{~d}, J=6.6 \mathrm{~Hz}, 3 \mathrm{H}) .{ }^{13} \mathrm{C} \mathrm{NMR}\left(600 \mathrm{MHz}, \mathrm{CDCl}_{3}\right): \delta$ $139.8,138.6,138.5,128.8,128.6,128.54,128.48,128.36,128.2,127.1,127.0,94.2$, 67.2, 59.0, 58.3, 55.0, 44.6, 34.4, 15.9, 11.6. HRMS Calculated for $\mathrm{C}_{20} \mathrm{H}_{25} \mathrm{~N}_{2} \mathrm{O}_{3}[\mathrm{M}+\mathrm{H}]^{+}$: 341.18597, Found: 341.18594.

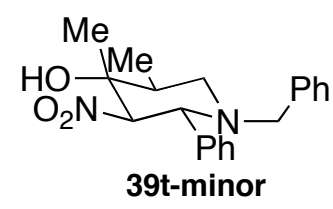

$(2 R, 3 R, 4 R, 5 S)-1-b e n z y l-4,5-d i m e t h y l-3-n i t r o-2-p h e n y l p i p e r i d i n-4-o l ~(4 q-m i n o r)$ was purified by flash silica gel chromatography (Hexane-EtOAc, v/v 8/1) as white solid, yield: 22\%. m.p: $195.3-197.4{ }^{\circ} \mathrm{C} .{ }^{1} \mathrm{H}$ NMR (600 $\left.\mathrm{MHz}, \mathrm{CDCl}_{3}\right): \delta$ 7.20-7.50(m, $\left.10 \mathrm{H}\right)$, $4.70(\mathrm{~d}, J=10.2 \mathrm{~Hz}, 1 \mathrm{H}), 4.11(\mathrm{~d}, J=10.2 \mathrm{~Hz}, 1 \mathrm{H}), 3.70(\mathrm{~d}, J=13.8 \mathrm{~Hz}, 1 \mathrm{H}), 2.96(\mathrm{~d}, J=$ $13.8 \mathrm{~Hz}, 1 \mathrm{H}), 2.91(\mathrm{~d}, J=1.8 \mathrm{~Hz}, 1 \mathrm{H}), 2.63(\mathrm{dd}, J=12.0 \mathrm{~Hz}, J=4.2 \mathrm{~Hz}, 1 \mathrm{H}), 2.41(\mathrm{t}, J=$ $12.0 \mathrm{~Hz}, 1 \mathrm{H}), 1.75-1.781(\mathrm{~m}, 1 \mathrm{H}), 1.27(\mathrm{~s}, 3 \mathrm{H}), 0.96(\mathrm{~d}, J=6.6 \mathrm{~Hz}, 3 \mathrm{H}) .{ }^{13} \mathrm{C} \mathrm{NMR}(600$ $\mathrm{MHz}_{\mathrm{CDCl}}$ ): $\delta 138.7,137.7,129.0,128.8,128.5,128.2,127.0,98.9,70.7,66.2,58.4$, 54.1, 38.6, 23.8, 11.5. HRMS Calculated for $\mathrm{C}_{20} \mathrm{H}_{25} \mathrm{~N}_{2} \mathrm{O}_{3}[\mathrm{M}+\mathrm{H}]^{+}$: 341.18597, Found: 341.18589 .

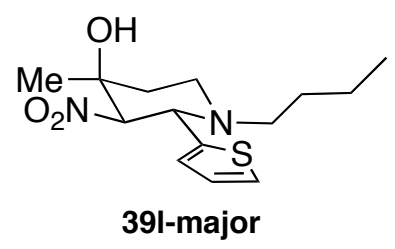

(2S,3R,4S)-1-butyl-4-methyl-3-nitro-2-(thiophen-2-yl)piperidin-4-ol (391-major) was purified by flash silica gel chromatography (Hexane-EtOAc, v/v 10/1) as colorless oil, 
yield: $85 \% .{ }^{1} \mathrm{H}$ NMR $\left(600 \mathrm{MHz}, \mathrm{CDCl}_{3}\right): \delta 7.27(\mathrm{~d}, J=4.8 \mathrm{~Hz}, 1 \mathrm{H}), 6.91(\mathrm{~d}, J=4.8 \mathrm{~Hz}$, $1 \mathrm{H}), 6.85-6.88(\mathrm{~m}, 1 \mathrm{H}), 4.57(\mathrm{~d}, J=10.2 \mathrm{~Hz}, 1 \mathrm{H}), 4.31(\mathrm{~d}, J=10.2 \mathrm{~Hz}, 1 \mathrm{H}), 3.04(\mathrm{br}, 1 \mathrm{H})$, $2.90(\mathrm{dq}, J=12.0 \mathrm{~Hz}, J=2.4 \mathrm{~Hz}, 1 \mathrm{H}), 2.70(\mathrm{td}, J=15.6 \mathrm{~Hz}, J=3.0 \mathrm{~Hz}, 1 \mathrm{H}), 2.54($, dt, $J=$ $13.2 \mathrm{~Hz}, J=8.4 \mathrm{~Hz}, 1 \mathrm{H}), 2.08-2.14(\mathrm{~m}, 1 \mathrm{H}), 1.88(\mathrm{dt}, J=13.8 \mathrm{~Hz}, J=2.4 \mathrm{~Hz}, 1 \mathrm{H}), 1.75(\mathrm{td}, J$ $=13.2 \mathrm{~Hz}, J=4.8 \mathrm{~Hz}, 1 \mathrm{H}), 1.36-1.42(\mathrm{~m}, 1 \mathrm{H}), 1.28(\mathrm{~s}, 3 \mathrm{H}), 1.06-1.22(\mathrm{~m} 2 \mathrm{H}), 0.79(\mathrm{t}, J=$ 7.2Hz, 3H). ${ }^{13} \mathrm{C}$ NMR (600 MHz, $\left.\mathrm{CDCl}_{3}\right): \delta 141.6,127.5,126.2,98.7,68.9,61.4,53.9$, 46.6, 36.6, 28.2, 27.1, 20.2, 13.9. HRMS Calculated for $\mathrm{C}_{14} \mathrm{H}_{23} \mathrm{~N}_{2} \mathrm{O}_{3}[\mathrm{M}+\mathrm{H}]^{+}: 299.14239$, Found: 299.14237.

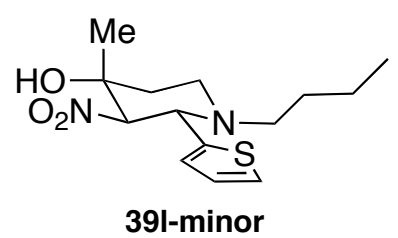

(2S,3R,4R)-1-butyl-4-methyl-3-nitro-2-(thiophen-2-yl)piperidin-4-ol (39l-minor) was purified by flash silica gel chromatography (Hexane-EtOAc, v/v 10/1) as colorless oil, yield: $5 \% .{ }^{1} \mathrm{H}$ NMR $\left(600 \mathrm{MHz}, \mathrm{CDCl}_{3}\right): \delta 7.26(\mathrm{~d}, J=5.4 \mathrm{~Hz}, 1 \mathrm{H}), 6.97(\mathrm{~d}, J=3.0 \mathrm{~Hz}, 1 \mathrm{H})$, $6.87-6.89(\mathrm{~m}, 1 \mathrm{H}), 4.70(\mathrm{~d}, J=10.2 \mathrm{~Hz}, 1 \mathrm{H}), 4.06(\mathrm{~d}, J=10.2 \mathrm{~Hz}, 1 \mathrm{H}), 3.08(\mathrm{dt}, J=12.6 \mathrm{~Hz}$, $J=3.6 \mathrm{~Hz}, 1 \mathrm{H}), 2.52(\mathrm{dt}, J=13.2 \mathrm{~Hz}, J=6.4 \mathrm{~Hz}, 1 \mathrm{H}), 2.37(\mathrm{br}, 1 \mathrm{H}), 2.30(\mathrm{td}, J=12.6 \mathrm{~Hz}, J$ $=3.0 \mathrm{~Hz}, 1 \mathrm{H}), 2.04-2.09(\mathrm{~m}, 1 \mathrm{H}), 2.00(\mathrm{td}, J=12.6 \mathrm{~Hz}, J=3.0 \mathrm{~Hz}, 1 \mathrm{H}), 1.91(\mathrm{dt}, J=$ $13.2 \mathrm{~Hz}, J=3.0 \mathrm{~Hz}, 1 \mathrm{H}), 1.34-1.40(\mathrm{~m}, 4 \mathrm{H}), 1.07-1.23(\mathrm{~m}, 2 \mathrm{H}), 0.79(\mathrm{t}, J=7.8 \mathrm{~Hz}, 3 \mathrm{H}) .{ }^{13} \mathrm{C}$ NMR (600 MHz, $\left.\mathrm{CDCl}_{3}\right): \delta 141.9,127.9,126.3,126.1,99.7,71.4,62.6,53.6,48.5,38.8$, 28.1, 21.4, 20.2, 13.9. HRMS Calculated for $\mathrm{C}_{14} \mathrm{H}_{23} \mathrm{~N}_{2} \mathrm{O}_{3}[\mathrm{M}+\mathrm{H}]^{+}:$299.14239, Found: 299.14234 .

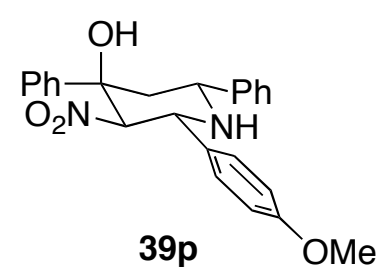

$(2 R, 3 R, 4 R, 6 R)-2-(4-m e t h o x y p h e n y l)-3-n i t r o-4,6-d i p h e n y l p i p e r i d i n-4-o l \quad(39 p)$ was purified by flash silica gel chromatography (Hexane-EtOAc, v/v 8/1) as colorless oil, 
yield: $80 \% .{ }^{1} \mathrm{H}$ NMR $\left(600 \mathrm{MHz}, \mathrm{CDCl}_{3}\right): \delta 7.52(\mathrm{~d}, J=8.4 \mathrm{~Hz}, 2 \mathrm{H}), 7.44-7.49(\mathrm{~m}, 4 \mathrm{H})$, 7.24-7.37(m, 6H), 6.89(d, $J=8.4 \mathrm{~Hz}, 1 \mathrm{H}), 5.18(\mathrm{~d}, J=10.2 \mathrm{~Hz}, 1 \mathrm{H}), 4.85(\mathrm{~d}, J=10.2 \mathrm{~Hz}$, $1 \mathrm{H}), 4.62(\mathrm{dd}, J=11.4 \mathrm{~Hz}, J=3.0 \mathrm{~Hz}, 1 \mathrm{H}), 4.30(\mathrm{~d}, J=3.0 \mathrm{~Hz}, 1 \mathrm{H}), 3.79(\mathrm{~s}, 3 \mathrm{H}), 2.15(\mathrm{dd}, J$ $=13.8 \mathrm{~Hz}, J=3.0 \mathrm{~Hz}, 1 \mathrm{H}), 2.00-2.06(\mathrm{~m}, 2 \mathrm{H}) .{ }^{13} \mathrm{C}$ NMR $\left(600 \mathrm{MHz}, \mathrm{CDCl}_{3}\right): \delta 160.1$, $1142.9,142.4,130.0,128.7,128.5,128.1,127.6,126.7,124.4,114.2,95.7,74.9,60.6$, 56.4, 55.2, 48.2. HRMS Calculated for $\mathrm{C}_{24} \mathrm{H}_{25} \mathrm{~N}_{2} \mathrm{O}_{4}[\mathrm{M}+\mathrm{H}]^{+}$: 405.18088, Found: 405.18082 .

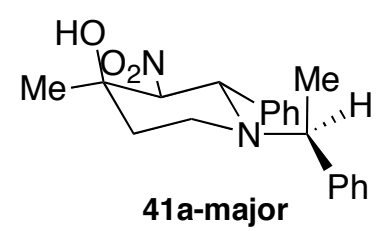

(2S,3S,4R)-4-methyl-3-nitro-2-phenyl-1-((S)-1-phenylethyl)piperidin-4-ol

(41a-

major) was purified by flash silica gel chromatography (Hexane-EtOAc, v/v 10/1) as colorless oil, yield: 55\%. ${ }^{1} \mathrm{H}$ NMR (600 MHz, $\left.\mathrm{CDCl}_{3}\right): \delta 7.20-7.55(\mathrm{~m}, 10 \mathrm{H}), 4.75(\mathrm{~d}, J=$ $10.2 \mathrm{~Hz}, 1 \mathrm{H}), 4.39(\mathrm{~d}, J=10.2 \mathrm{~Hz}, 1 \mathrm{H}), 3.81(\mathrm{q}, J=7.2 \mathrm{~Hz}, 1 \mathrm{H}), 3.06(\mathrm{~d}, J=3.0 \mathrm{~Hz}, 1 \mathrm{H})$, $2.89(\mathrm{td}, J=12.0 \mathrm{~Hz}, J=2.4 \mathrm{~Hz}, 1 \mathrm{H}), 2.38(\mathrm{dq}, J=12.0 \mathrm{~Hz}, J=2.4 \mathrm{~Hz}, 1 \mathrm{H}), 1.79(\mathrm{dt}, J=$ $13.8 \mathrm{~Hz}, J=2.4 \mathrm{~Hz}, 1 \mathrm{H}), 1.57-1.65(\mathrm{~m}, 1 \mathrm{H}), 1.31(\mathrm{~s}, 3 \mathrm{H}), 1.28(\mathrm{~d}, J=7.2 \mathrm{~Hz}, 3 \mathrm{H}) .{ }^{13} \mathrm{C} \mathrm{NMR}$ $\left(600 \mathrm{MHz}, \mathrm{CDCl}_{3}\right): \delta 143.3,136.9,129.0,128.8,128.0,127.4,126.5,98.2,69.3,63.8$, 54.9, 38.7, 36.9, 27.3, 8.6. HRMS Calculated for $\mathrm{C}_{20} \mathrm{H}_{25} \mathrm{~N}_{2} \mathrm{O}_{3}[\mathrm{M}+\mathrm{H}]^{+}$: 341.18597, Found: $341.18592 .[\alpha]_{\mathrm{D}}{ }^{26}=-95.4^{\circ}(\mathrm{c}=1.0 \mathrm{in} \mathrm{MeOH})$.

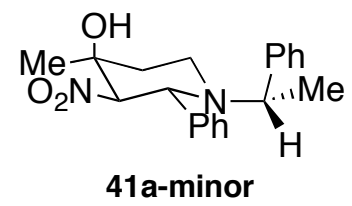

(2R,3R,4S)-4-methyl-3-nitro-2-phenyl-1-((S)-1-phenylethyl)piperidin-4-ol

(41aminor) was purified by flash silica gel chromatography (Hexane-EtOAc, v/v 10/1) as white solid, yield: $10 \%$. m.p $=135.5-137.8^{\circ} \mathrm{C} .{ }^{1} \mathrm{H}$ NMR $\left(600 \mathrm{MHz}, \mathrm{CDCl}_{3}\right): \delta 7.22-$ $7.45(\mathrm{~m}, 8 \mathrm{H}), 7.01(\mathrm{~d}, J=7.2 \mathrm{~Hz}, 2 \mathrm{H}), 4.53(\mathrm{~d}, J=10.2 \mathrm{~Hz}, 1 \mathrm{H}), 3.97(\mathrm{~d}, J=10.2 \mathrm{~Hz}, 1 \mathrm{H})$, 
$3.90(\mathrm{q}, J=7.2 \mathrm{~Hz}, 1 \mathrm{H}), 2.93(\mathrm{dq}, \mathrm{J}=12.0 \mathrm{~Hz}, J=3.0 \mathrm{~Hz}, 1 \mathrm{H}), 2.85(\mathrm{~d}, J=3.0 \mathrm{~Hz}, 1 \mathrm{H})$, $2.45(\mathrm{td}, J=12.0 \mathrm{~Hz}, J=3.0 \mathrm{~Hz}, 1 \mathrm{H}), 1.88(\mathrm{dt}, J=13.8 \mathrm{~Hz}, J=3.0 \mathrm{~Hz}, 1 \mathrm{H}), 1.72(\mathrm{tq}, J=$ $12.0 \mathrm{~Hz}, J=2.4 \mathrm{~Hz}, 1 \mathrm{H}), 1.37(\mathrm{~d}, J=7.2 \mathrm{~Hz}, 3 \mathrm{H}), 1.23(\mathrm{~s}, 3 \mathrm{H}) .{ }^{13} \mathrm{C} \mathrm{NMR}(600 \mathrm{MHz}$, $\left.\mathrm{CDCl}_{3}\right): \delta 137.9,137.8,129.0,128.7,128.6,127.9,127.2,99.2,68.8,64.2,56.4,39.7$, 37.0, 27.3, 18.8. HRMS Calculated for $\mathrm{C}_{20} \mathrm{H}_{25} \mathrm{~N}_{2} \mathrm{O}_{3}[\mathrm{M}+\mathrm{H}]^{+}:$341.18597, Found: 341.18606. $[\alpha]_{\mathrm{D}}{ }^{26}=-33.7^{\circ}(\mathrm{c}=1.0 \mathrm{in} \mathrm{MeOH})$.

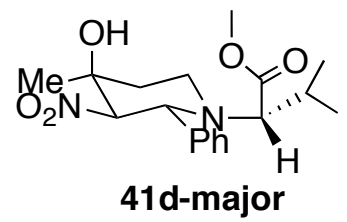

(S)-methyl-2-((2R,3R,4S)-4-hydroxy-4-methyl-3-nitro-2-phenylpiperidin-1-yl)-3methylbutanoate (41d-major) was purified by flash silica gel chromatography (HexaneEtOAc, v/v 5/1) as white solid, yield: 48\%. m.p $=295.4-297.9{ }^{\circ} \mathrm{C} .{ }^{1} \mathrm{H}$ NMR $(600 \mathrm{MHz}$, $\left.\mathrm{CDCl}_{3}\right): \delta 7.26-7.35(\mathrm{~m}, 5 \mathrm{H}), 4.63(\mathrm{~d}, J=10.2 \mathrm{~Hz}, 1 \mathrm{H}), 4.24(\mathrm{~d}, J=10.2 \mathrm{~Hz}, 1 \mathrm{H}), 3.72(\mathrm{~s}$, $1 \mathrm{H}), 2.98(\mathrm{br}, 1 \mathrm{H}), 2.75-2.82(\mathrm{~m}, 2 \mathrm{H}), 2.63(\mathrm{~d}, J=11.4 \mathrm{~Hz}, 1 \mathrm{H}), 2.05-2.13(\mathrm{~m}, 1 \mathrm{H}), 1.91(\mathrm{dt}$, $J=13.8 \mathrm{~Hz}, J=3.0 \mathrm{~Hz}, 1 \mathrm{H}), 1.66-1.72(\mathrm{~m}, 1 \mathrm{H}), 1.29(\mathrm{~s}, 3 \mathrm{H}), 0.90(\mathrm{~d}, J=6.6 \mathrm{~Hz}, 3 \mathrm{H})$, $0.67(\mathrm{~d}, J=6.6 \mathrm{~Hz}, 1 \mathrm{H}) .{ }^{13} \mathrm{C} \mathrm{NMR}\left(600 \mathrm{MHz}, \mathrm{CDCl}_{3}\right): \delta 171.1,136.2,128.9,128.7,98.1$, $68.8,67.3,64.5,50.8,41.0,37.0,27.3,26.5,20.1,19.1$. HRMS Calculated for $\mathrm{C}_{18} \mathrm{H}_{27} \mathrm{~N}_{2} \mathrm{O}_{5}[\mathrm{M}+\mathrm{H}]^{+}: 351.19234$, Found: $351.19133 .[\alpha]_{\mathrm{D}}{ }^{26}=-43.2^{\circ}(\mathrm{c}=1.0$ in $\mathrm{MeOH})$.

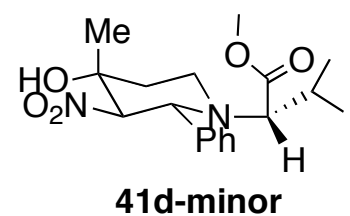

(S)-methyl-2-((2R,3R,4R)-4-hydroxy-4-methyl-3-nitro-2-phenylpiperidin-1-yl)-3-

methylbutanoate (41d-minor) was purified by flash silica gel chromatography (HexaneEtOAc, v/v 5/1) as white solid, yield: $14 \%$. m.p $=131.1-132.9{ }^{\circ} \mathrm{C} .{ }^{1} \mathrm{H}$ NMR $(600 \mathrm{MHz}$, $\left.\mathrm{CDCl}_{3}\right): \delta 7.26-7.34(\mathrm{~m}, 5 \mathrm{H}), 4.76(\mathrm{~d}, J=10.2 \mathrm{~Hz}, 1 \mathrm{H}), 3.99(\mathrm{~d}, J=10.2 \mathrm{~Hz}, 1 \mathrm{H}), 3.72(\mathrm{~s}$, $3 \mathrm{H}), 3.00(\mathrm{dt}, J=12.6 \mathrm{~Hz}, J=3.6 \mathrm{~Hz}, 1 \mathrm{H}), 2.56(\mathrm{~d}, J=11.4 \mathrm{~Hz}, 1 \mathrm{H}), 2.42-2.48(\mathrm{~m}, 1 \mathrm{H})$, 
2.35(s, 1H), 2.02-2.19(m,1H), 1.92-1.96(m, $1 \mathrm{H}), 1.36(\mathrm{~s}, 3 \mathrm{H}), 0.86(\mathrm{~d}, J=6.6 \mathrm{~Hz}, 3 \mathrm{H})$, $0.66(\mathrm{~d}, J=6.6 \mathrm{~Hz}, 3 \mathrm{H}) .{ }^{13} \mathrm{C} \mathrm{NMR}\left(600 \mathrm{MHz}, \mathrm{CDCl}_{3}\right): \delta 171.3,136.6,128.8,128.6,98.9$, 71.4, 67.1, 65.3, 50.7, 42.9, 39.0, 26.7, 21.3, 20.0, 19.1. HRMS Calculated for $\mathrm{C}_{18} \mathrm{H}_{27} \mathrm{~N}_{2} \mathrm{O}_{5}[\mathrm{M}+\mathrm{H}]^{+}: 351.19234$, Found: 351.19141. $[\alpha]_{\mathrm{D}}{ }^{26}=-80.8^{\circ}(\mathrm{c}=1.0$ in $\mathrm{MeOH})$.

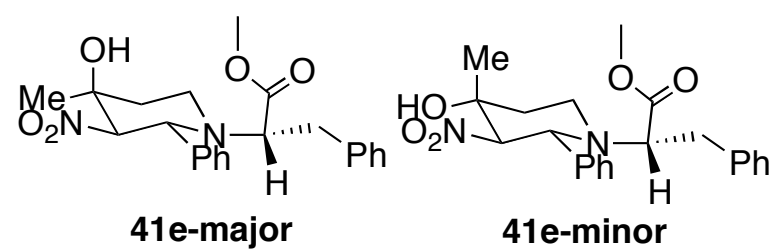

(S)-methyl-2-((2R,3R,4S(R))-4-hydroxy-4-methyl-3-nitro-2-phenylpiperidin-1-yl)-3-

phenylpropanoate (41e) was purified by flash silica gel chromatography (HexaneEtOAc, v/v 5/1) as colorless oil with overall isolated yield 65\% (two diasterisomers cannot be separated). ${ }^{1} \mathrm{H}$ NMR $\left(600 \mathrm{MHz}, \mathrm{CDCl}_{3}\right): \delta 6.80-37(\mathrm{~m}, 10 \mathrm{H}+1.7 \mathrm{H}), 4.63(\mathrm{~d}, J=$ $10.2 \mathrm{~Hz}, 0.17 \mathrm{H}), 4.98(\mathrm{~d}, J=10.2 \mathrm{H}, 1 \mathrm{H}), 4.39(\mathrm{~d}, J=10.2 \mathrm{~Hz}, 1 \mathrm{H}), 4.31(\mathrm{~d}, J=10.2 \mathrm{~Hz}$, $0.17 \mathrm{H}), 3.72(\mathrm{~s}, 3 \mathrm{H}), 3.55(\mathrm{~s}, 0.42 \mathrm{H}), 3.50(\mathrm{dd}, J=9.6 \mathrm{~Hz}, J=3.0 \mathrm{~Hz}, 0.17 \mathrm{H}), 3.28(\mathrm{dd}, J=$ $9.6 \mathrm{~Hz}, J=6.0 \mathrm{~Hz}, 1 \mathrm{H}), 3.23(\mathrm{td}, J=12.0 \mathrm{~Hz}, J=3.0 \mathrm{~Hz}, 0.17 \mathrm{H}), 2.78-3.06(\mathrm{~m}, 5 \mathrm{H}+0.85 \mathrm{H})$, 2.01-2.004(m, 0.17H), 1.90-1.96(m, 1H), 1.73-1.80(m, 0.17H), 1.65-1.72(m, $1 \mathrm{H}), 1.29(\mathrm{~s}$, $0.42 \mathrm{H}), 1.26(\mathrm{~s}, 3 \mathrm{H}) .{ }^{13} \mathrm{C} \mathrm{NMR}\left(600 \mathrm{MHz}, \mathrm{CDCl}_{3}\right): \delta 171.8(172.2), 138.3(138.2)$, 135.6(135.9), 129.7, 129.2, 129.1, 129.0, 128.7, 128.6, 128.2, 127.9, 126.3, 126.2, 97.5(97.9), 68.9(69.0), 64.6(63.8, 63.7), 62.1, 51.3(51.8), 40.7(41.7), 36.9(36.8), 35.3, (30.1), 27.15(27.12). HRMS Calculated for $\mathrm{C}_{22} \mathrm{H}_{27} \mathrm{~N}_{2} \mathrm{O}_{5}[\mathrm{M}+\mathrm{H}]^{+}$: 399.19145, Found: 399.19194.<smiles></smiles> 


\section{(S)-methyl-2-((5S,6R)-5-acetamido-5,6-dihydro-4-methyl-6-phenylpyridin-1(2H)-yl)-}

3-phenylpropanoate (42a) was purified by flash silica gel chromatography (HexaneEtOAc, v/v 3/1) as white solid with overall isolated yield $80 \%$. m.p $>160{ }^{\circ} \mathrm{C}$ (decomposed). ${ }^{1} \mathrm{H}$ NMR (600 MHz, $\left.\mathrm{CDCl}_{3}\right): \delta 7.17-7.27(\mathrm{~m}, 6 \mathrm{H}), 7.04-7.10(\mathrm{~m}, 4 \mathrm{H})$, $5.65(\mathrm{br}, 1 \mathrm{H}), 5.98(\mathrm{~d}, J=9.6 \mathrm{~Hz}, 1 \mathrm{H}), 5.44(\mathrm{dd}, J=9.0 \mathrm{~Hz}, \mathrm{~J}=4.8 \mathrm{~Hz}, 1 \mathrm{H}), 3.76(\mathrm{~d}, J=$ $4.8 \mathrm{~Hz}, 1 \mathrm{H}), 3.58(\mathrm{t}, J=7.2 \mathrm{~Hz}, 1 \mathrm{H}), 3.42-3.52(\mathrm{~m}, 1 \mathrm{H}), 3.47(\mathrm{~s}, 1 \mathrm{H}), 3.30-3.35(\mathrm{~m}, 1 \mathrm{H})$, $2.98(\mathrm{dd}, J=13.8 \mathrm{~Hz}, J=7.2 \mathrm{~Hz}, 1 \mathrm{H}), 2.88(\mathrm{dd}, J=13.8 \mathrm{~Hz}, J=7.2 \mathrm{~Hz}, 1 \mathrm{H}), 1.82(\mathrm{~s}, 3 \mathrm{H})$, 1.73(s, 3H). ${ }^{13} \mathrm{C} \mathrm{NMR}\left(600 \mathrm{MHz}, \mathrm{CDCl}_{3}\right): \delta 173.0,169.2,138.2,138.0,133.0,129.2$, $128.9,128.25,128.21,127.7,126.4,122.6,66.8,64.7,53.5,51.11,44.9,35.4,23.2,20.2$. HRMS Calculated for $[\mathrm{M}+\mathrm{H}]^{+}$:, Found:. $[\alpha]_{\mathrm{D}}{ }^{26}=-18.4^{\circ}(\mathrm{c}=1.0$ in $\mathrm{MeOH})$.

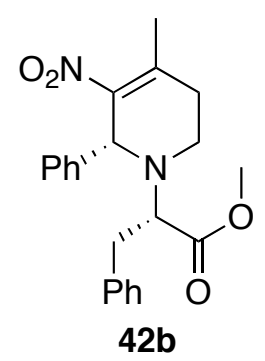

\section{(S)-methyl-2-((R)-5,6-dihydro-4-methyl-3-nitro-2-phenylpyridin-1(2H)-yl)-3-phenyl}

propanoate (7b). $7 \mathbf{b}$ was purified by flash silica gel chromatography (Hexane-EtOAc, v/v 10/1) as colorless oil, yield: 65\%. ${ }^{1} \mathrm{H}$ NMR (600 MHz, $\left.\mathrm{CDCl}_{3}\right): \delta 7.10-7.22(\mathrm{~m}, 6 \mathrm{H})$, $6.98-7.01(\mathrm{~m}, 2 \mathrm{H}), 6.81-6.86(\mathrm{~m}, 2 \mathrm{H}), 4.97(\mathrm{~s}, 1 \mathrm{H}), 3.74(\mathrm{~s}, 3 \mathrm{H}), 3.42(\mathrm{dd}, J=9.0 \mathrm{~Hz}, J=$ $6.6 \mathrm{~Hz}, 1 \mathrm{H}), 3.22(\mathrm{qd}, J=5.4 \mathrm{~Hz}, J=3.0 \mathrm{~Hz}, 1 \mathrm{H}), 2.93(\mathrm{dd}, J=13.8 \mathrm{~Hz}, J=6.0 \mathrm{~Hz}, 1 \mathrm{H})$, $2.78(\mathrm{dd}, J=13.8 \mathrm{~Hz}, J=6.0 \mathrm{~Hz}, 1 \mathrm{H}), 2.68(\mathrm{td}, J=12.0 \mathrm{~Hz}, J=3.0 \mathrm{~Hz}, 1 \mathrm{H}), 2.52-2.60(\mathrm{~m}$, 1H), 2.14-2.19(m, 1H), 1.97(s, 3H). $\left.{ }^{13} \mathrm{C} \mathrm{NMR} \mathrm{(600} \mathrm{MHz,} \mathrm{CDCl}_{3}\right): \delta 172.00,147.7$, $137.7,136.9,135.1,129.4,129.2$, 128.3, 128.2, 128.1, 126.2, 63.8, 62.2, 51.3, 41.2, 35.6, 32.2, 19.8. HRMS Calculated for $\mathrm{C}_{22} \mathrm{H}_{25} \mathrm{~N}_{2} \mathrm{O}_{4}[\mathrm{M}+\mathrm{H}]^{+}$: 381.18088, Found: 381.18092 . $[\alpha]_{\mathrm{D}}^{26}=-21.3^{\circ}(\mathrm{c}=1.0$ in $\mathrm{MeOH})$. 


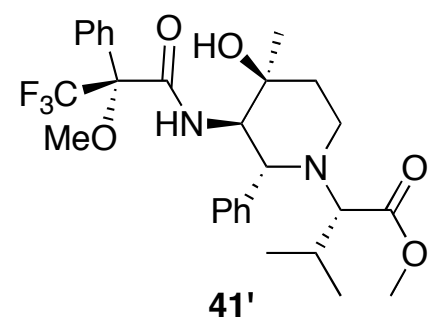

41' was purified by flash silica gel chromatography (Hexane-EtOAc, v/v 2/1) as colorless oil with overall isolated yield $81 \% .{ }^{1} \mathrm{H}$ NMR $\left(600 \mathrm{MHz}, \mathrm{CDCl}_{3}\right): \delta 7.10-7.41(\mathrm{~m}, 11 \mathrm{H})$, $6.56(\mathrm{~d}, J=7.8 \mathrm{~Hz}, 2 \mathrm{H}), 4.20(\mathrm{t}, J=10.2 \mathrm{~Hz}, 1 \mathrm{H}), 3.70(\mathrm{~s}, 3 \mathrm{H}), 3.64(\mathrm{~d}, J=10.2 \mathrm{~Hz}, 1 \mathrm{H})$, 3.05 (s, $3 \mathrm{H}), 2.82(\mathrm{dt}, J=12.6 \mathrm{~Hz}, J=3.0 \mathrm{~Hz}, 1 \mathrm{H}), 2.58(\mathrm{td}, J=12.6 \mathrm{~Hz}, J=3.0 \mathrm{~Hz}, 1 \mathrm{H})$, $2.49(\mathrm{~d}, J=12.6 \mathrm{~Hz}, 1 \mathrm{H}), 2.00-2.08(\mathrm{~m}, 1 \mathrm{H}), 1.91(\mathrm{td}, J=13.2 \mathrm{~Hz}, J=4.8 \mathrm{~Hz}, 1 \mathrm{H}), 1.75(\mathrm{dt}$, $J=13.8 \mathrm{~Hz}, J=1.2 \mathrm{~Hz}, 1 \mathrm{H}), 1.26(\mathrm{~s}, 3 \mathrm{H}), 0.86(\mathrm{~d}, J=6.6 \mathrm{~Hz}, 1 \mathrm{H}), 0.63(\mathrm{~d}, J=6.6 \mathrm{~Hz}, 1 \mathrm{H})$. ${ }^{13} \mathrm{C}$ NMR $\left(600 \mathrm{MHz}, \mathrm{CDCl}_{3}\right): \delta 171.7,165.5,139.3,131.2,129.0,128.4,128.3,127.9$, $125.1,123.2,84.2(\mathrm{q}, J=103.2 \mathrm{~Hz}, 1 \mathrm{C}), 71.0,70.9,67.8,66.4,58.8,54.5,50.5,41.5$, 38.5, 27.7, 26.7, 20.1, 19.2. HRMS Calculated for $\mathrm{C}_{28} \mathrm{H}_{35} \mathrm{~F}_{3} \mathrm{~N}_{2} \mathrm{O}_{5}[\mathrm{M}+\mathrm{H}]^{+}$: 537.25708, Found: 537.25337. $[\alpha]_{\mathrm{D}}{ }^{26}=-112.6^{\circ}(\mathrm{c}=1.0$ in $\mathrm{MeOH})$. 


\section{Part VI}

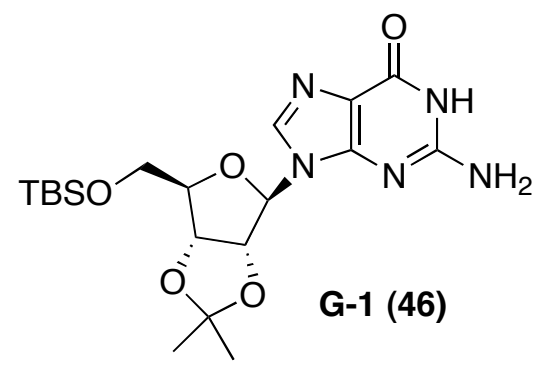

G-1 (46). ${ }^{1} \mathrm{H}$ NMR (600 MHz, d 6 -DMSO): $\delta 10.45$ (br, s, 1 H, NH1), 7.89 (s, 1 H, H8), $\left.6.51 \mathrm{~s}, 2 \mathrm{H}, \mathrm{NH}_{2}\right), 5.91$ (d, 1H, H1', $\left.J=2.4 \mathrm{~Hz}\right), 5.24$ (dd, 1H, H2', $J=6.0,2.4 \mathrm{~Hz}$ ), 4.88 (dd, 1 H, H3', $J=6.0,2.8 \mathrm{~Hz}), 4.13$ (m, 1 H, H4', $J=10.0,2.4 \mathrm{~Hz}), 3.69$ (m, 2 H, H5', $J=$ 11.2, $6.0 \mathrm{~Hz}), 1.50\left(\mathrm{~s}, 3 \mathrm{H}, \mathrm{CH}_{3}\right), 1.31\left(\mathrm{~s}, 3 \mathrm{H}, \mathrm{CH}_{3}\right), 0.81(\mathrm{~s}, 9 \mathrm{H}, \mathrm{t}-\mathrm{Bu}),-0.03(\mathrm{~s}, 6 \mathrm{H}$, $\left.\mathrm{Si}\left(\mathrm{CH}_{3}\right)_{2}\right) .{ }^{13} \mathrm{C}$ NMR (600 MHz d $\mathrm{d}_{6}$-DMSO) $\delta-5.63,17.85,25.18,25.63,26.88,63.39$, $80.89,83.62,86.84,88.36,112.90,116.75,135.68,150.49,153.68,156.71$

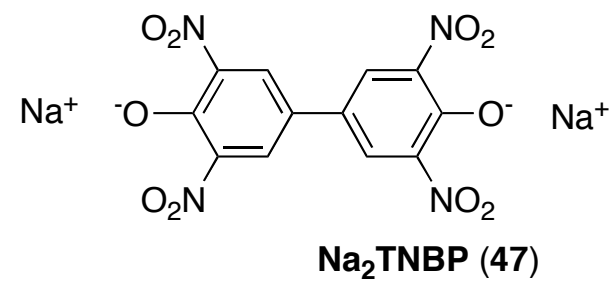

Na 2 TNBP (47) ${ }^{1} \mathrm{H}$ NMR (600 MHz, d 6 -DMSO): $\delta 8.00$ (s, $4 \mathrm{H}, 3,5,3$, , 5' H). ${ }^{13} \mathrm{C}$ NMR (600 MHz d 6 -DMSO) $\delta 115.19,127.54,143.92,158.96$.<smiles>CCCC[Nb+2]</smiles>

$\left(\mathbf{B u}_{4} \mathbf{N}\right)_{2} \cdot$ TNBP (48) Dissolve $\mathrm{Bu}_{4} \mathrm{NF}(250 \mathrm{mg}, 0.958 \mathrm{mmol})$ in $20 \mathrm{ml}$ distilled water followed by adding $\mathrm{Na}_{2}$ TNBP (500mg, $1.222 \mathrm{mmol}$ ), stir to dark red clear solution. $\mathrm{CH}_{2} \mathrm{Cl}_{2}(20 \mathrm{ml})$ was applied to extract the $\left(\mathrm{Bu}_{4} \mathrm{~N}\right)_{2} \bullet \mathrm{TNBP}$. Then the dark red organic solution was washed by distilled water $(20 \mathrm{ml} \times 3)$ to remove $\mathrm{NaF}$ and dried under vacuum to black solid (763mg, 90\%). ${ }^{1} \mathrm{H}$ NMR (600 MHz, d6-DMSO): $\delta 7.93$ (s, $4 \mathrm{H}$, 
TNBP), 3.18-3.12 (m, $\left.16 \mathrm{H}, \mathrm{CH}_{2}-\alpha\right), 1.61-1.49\left(\mathrm{~m}, 16 \mathrm{H}, \mathrm{CH}_{2}-\beta\right), 1.33-1.25(\mathrm{~m}, 16 \mathrm{H}$, $\left.\mathrm{CH}_{2}-\gamma\right), 0.92$ (t, $\left.24 \mathrm{H}, \mathrm{CH}_{3}, J=7.5\right) .{ }^{13} \mathrm{C}$ NMR (600 MHz d $\mathrm{d}_{6}$-DMSO) $\delta$ 13.42, 19.14, $22.99,57.43,113.95,126.62,143.26,157.87$. 


\section{ORTEP Drawings of the X-ray Crystal Structures}

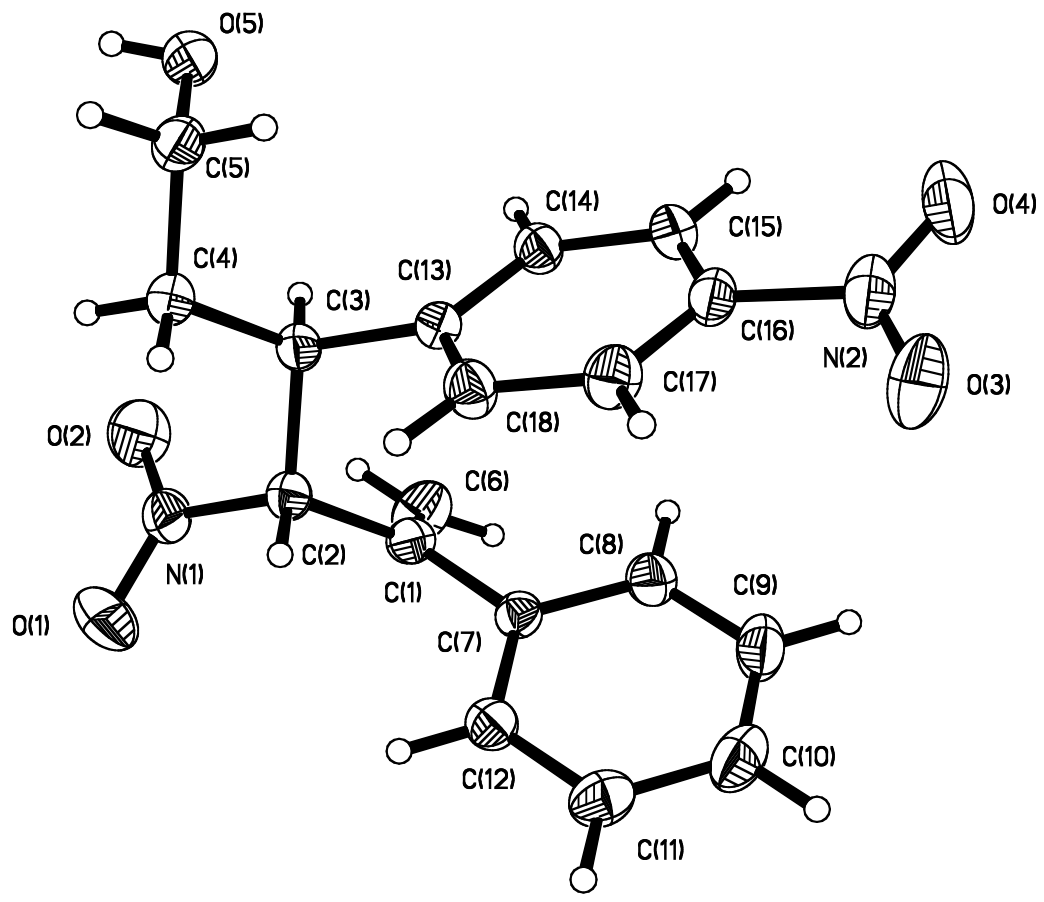

Figure 1. Perspective view of the molecular structure of $4 \mathrm{e}-$ cis with the atom labeling scheme. The thermal ellipsoids are scaled to enclose $30 \%$ probability.

CCDC :704977

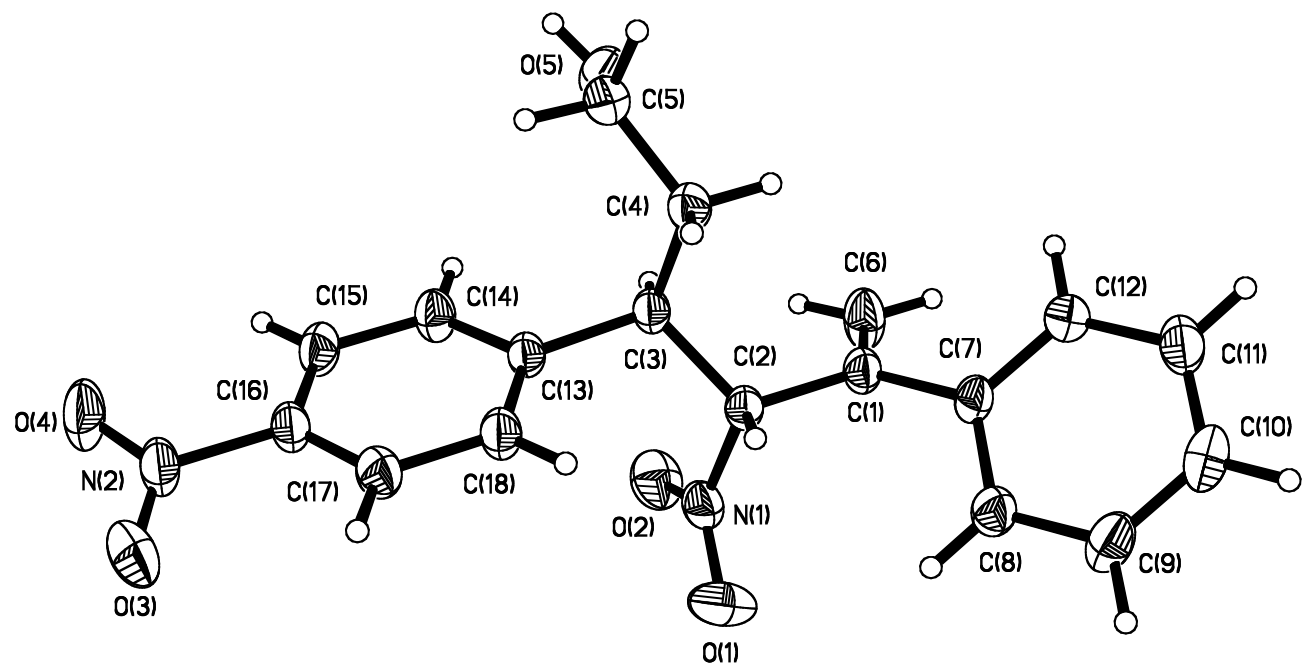

Figure 2. Perspective view of the molecular structure of 4e-trans with the atom labeling scheme. The thermal ellipsoids are scaled to enclose $30 \%$ probability. CCDC: 704978 


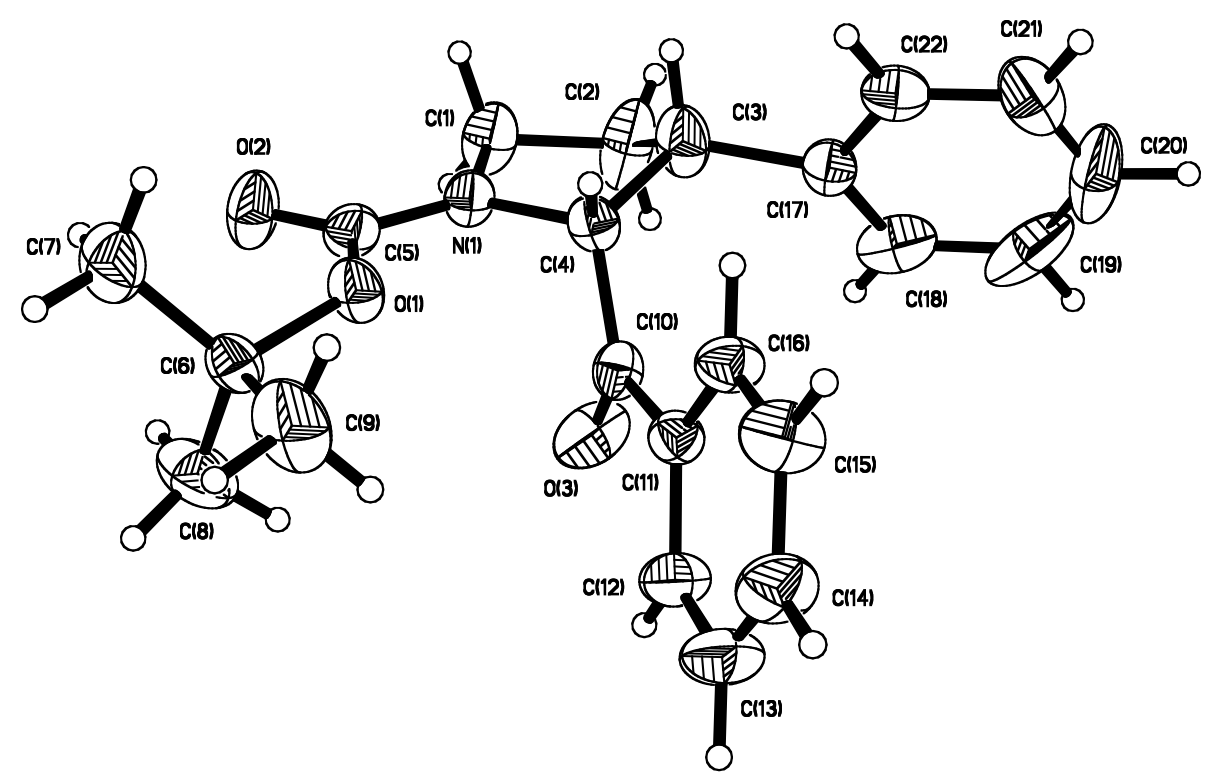

Figure 3. Perspective view of the molecular structure 6a-cis showing the syn relationship of the hydrogen atoms bound to $\mathrm{C}(3)$ and $\mathrm{C}(4)$ within molecule 6a of $\mathrm{C}_{22} \mathrm{H}_{25} \mathrm{NO}_{3}$ with the atom labeling scheme. The thermal ellipsoids are scaled to enclose $30 \%$ probability.

CCDC: 704979

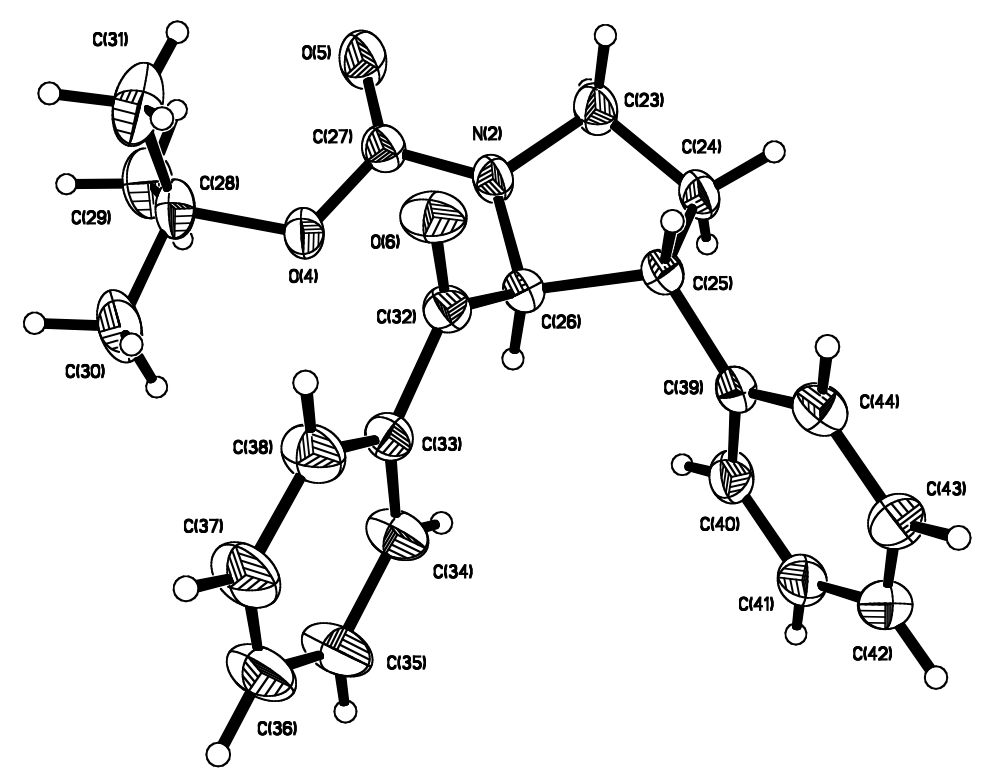

Figure 4. Perspective view of the molecular structure 6a-trans showing the anti relationship of the hydrogen atoms bound to $\mathrm{C}(25)$ and $\mathrm{C}(26)$ within molecule 6a of $\mathrm{C}_{22} \mathrm{H}_{25} \mathrm{NO}_{3}$ with the atom labeling scheme. The thermal ellipsoids are scaled to enclose $30 \%$ probability.

CCDC 704979 


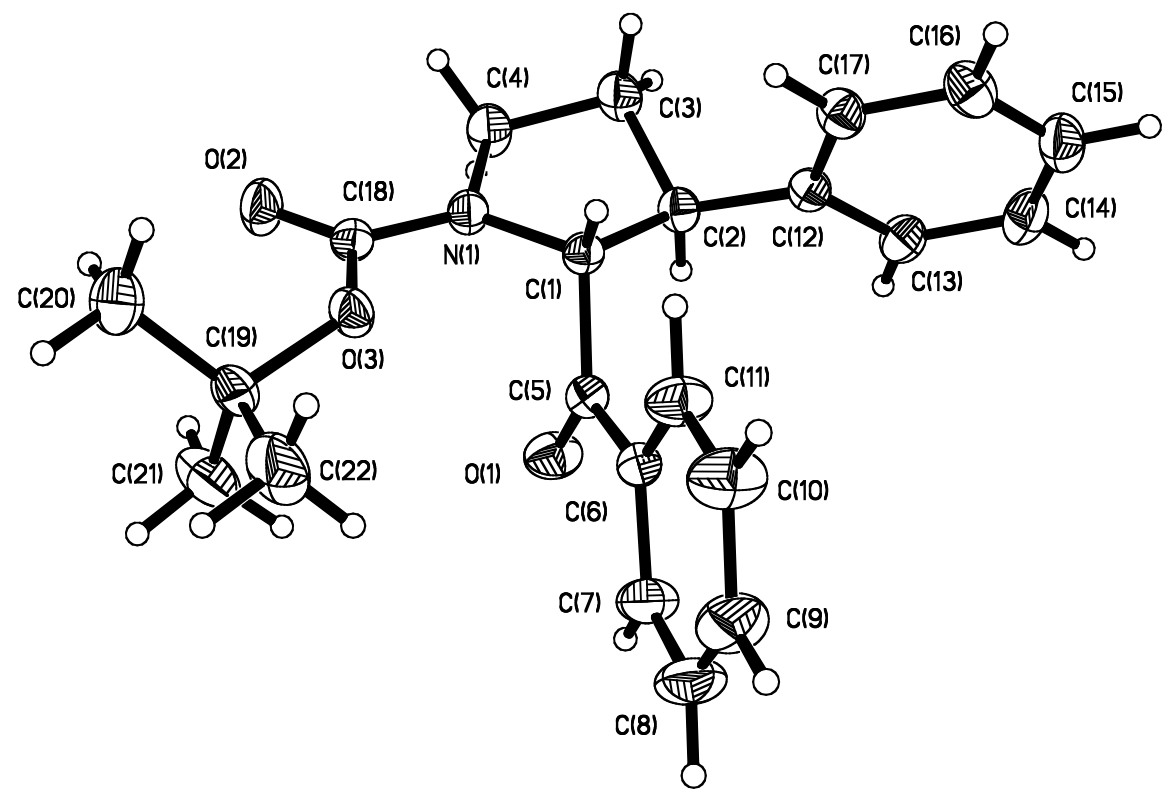

Figure 5. Perspective view of the molecular structure of 6a-trans with the atom labeling scheme. The thermal ellipsoids are scaled to enclose $30 \%$ probability. CCDC 704980

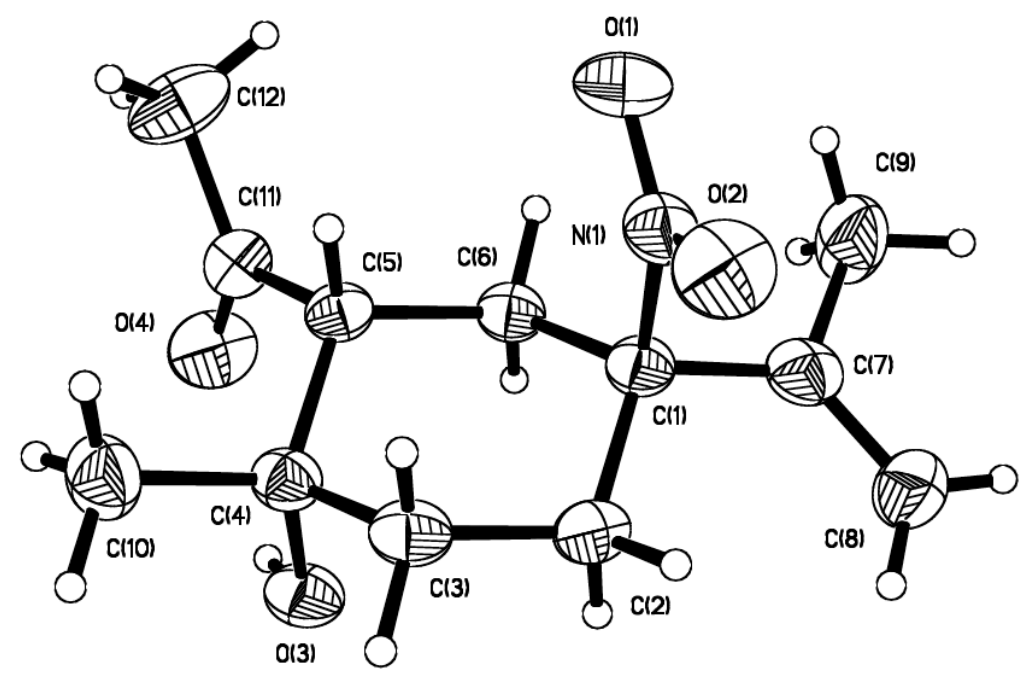

Figure 6. Perspective view of the molecular structure of 12g-major with the atom labeling scheme. The $\mathrm{CMe}=\mathrm{CH}_{2}$ substituent containing carbons $\mathrm{C}(7)-\mathrm{C}(9)$ exhibits a two-site rotational disorder. The thermal ellipsoids are scaled to enclose $30 \%$ probability. 


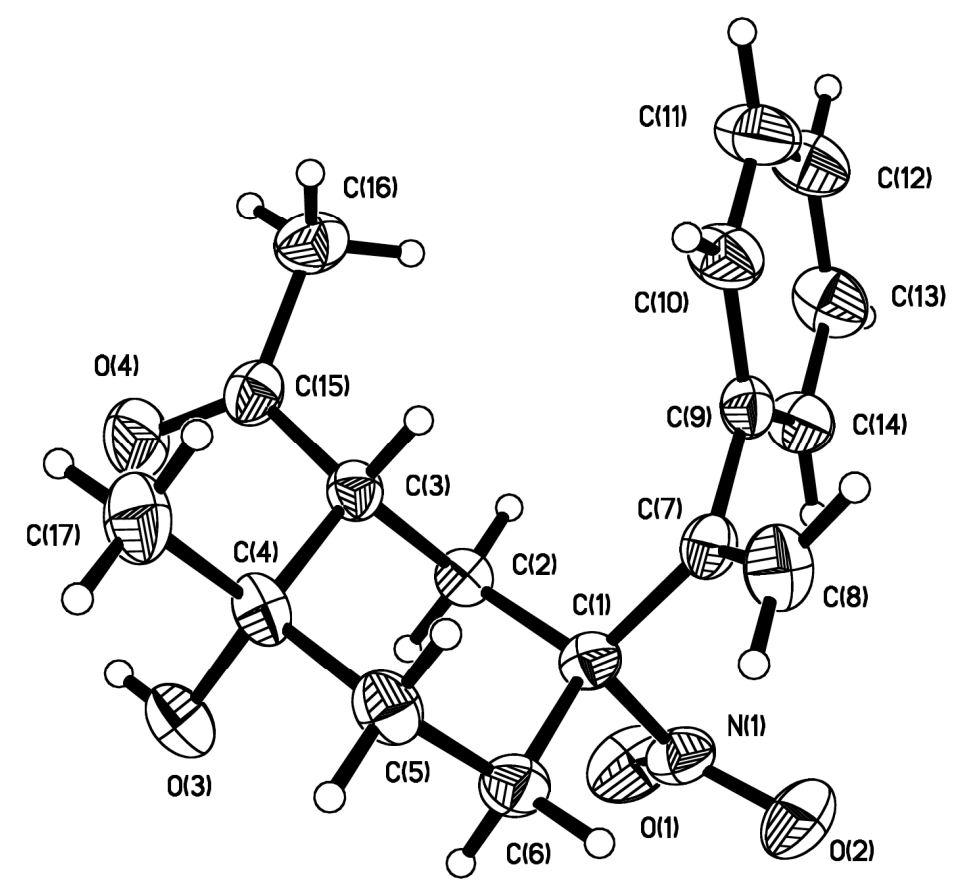

Figure 7. Perspective view of the molecular structure of 12a-minor with the atom labeling scheme. The thermal ellipsoids are scaled to enclose $30 \%$ probability.

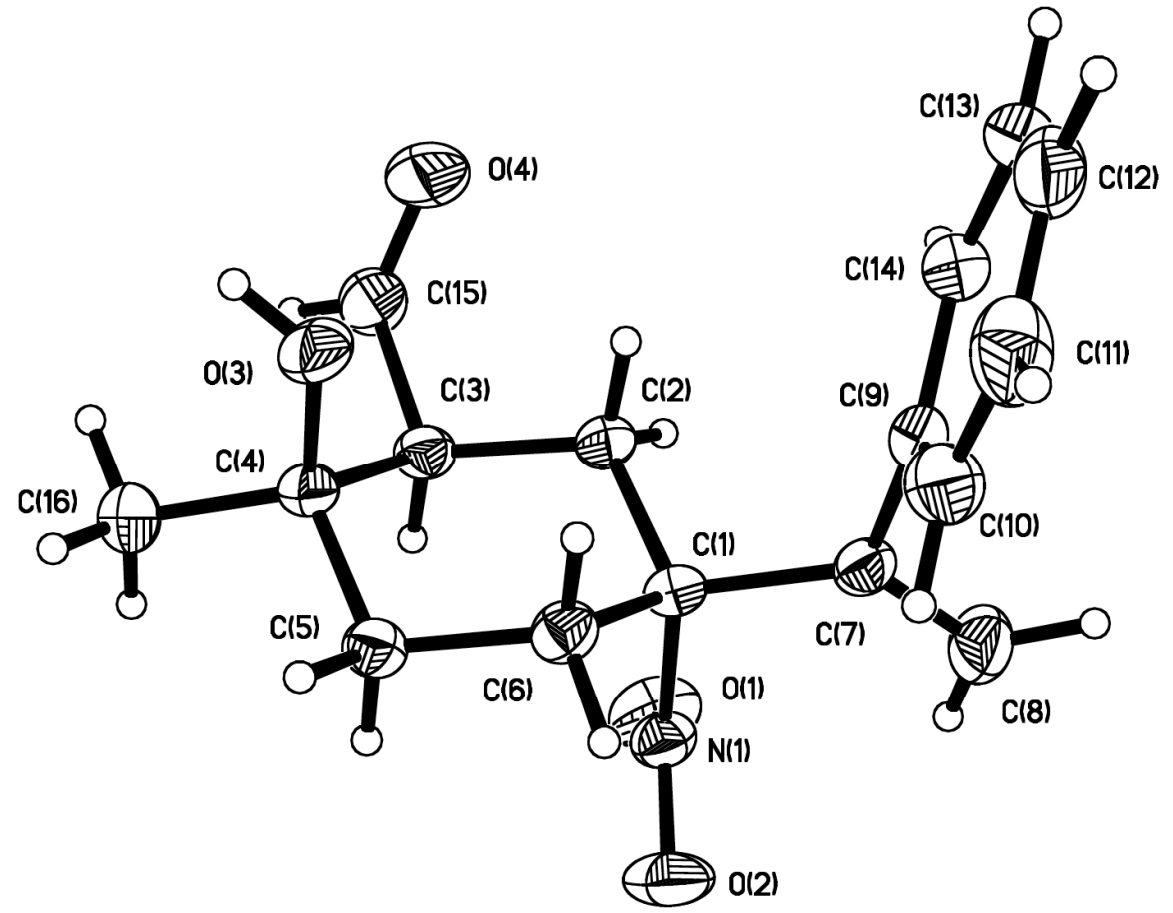

Figure 8. Perspective view of the molecular structure of 11a-major with the atom labeling scheme. The thermal ellipsoids are scaled to enclose $30 \%$ probability. 


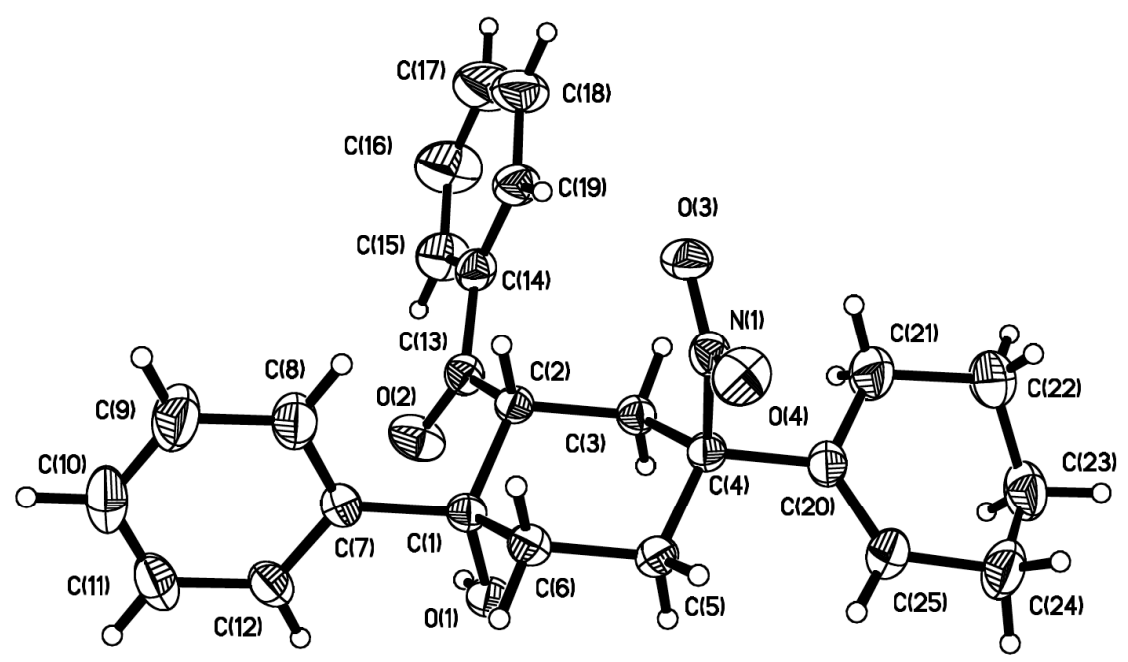

Figure 9. Perspective view of the molecular structure of 12f-major with the atom labeling scheme. The thermal ellipsoids are scaled to enclose $30 \%$ probability.

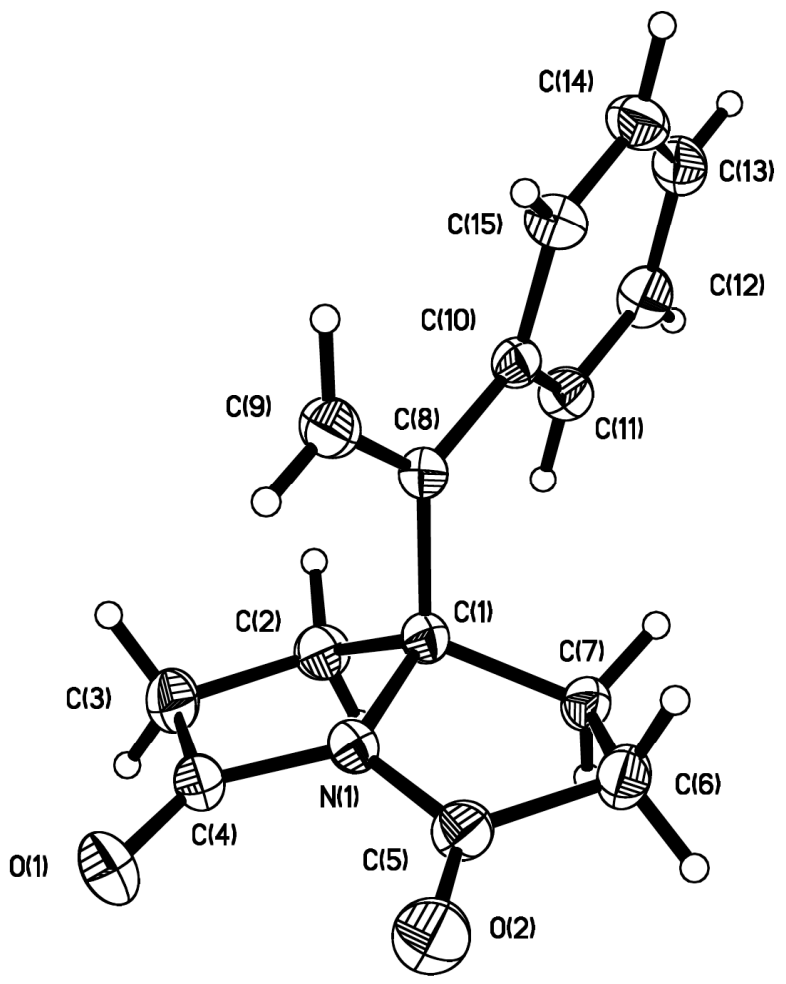

Figure 10. Perspective view of the molecular structure of $\mathbf{1 3 b}$ with the atom labeling scheme. The thermal ellipsoids are scaled to enclose $30 \%$ probability. 


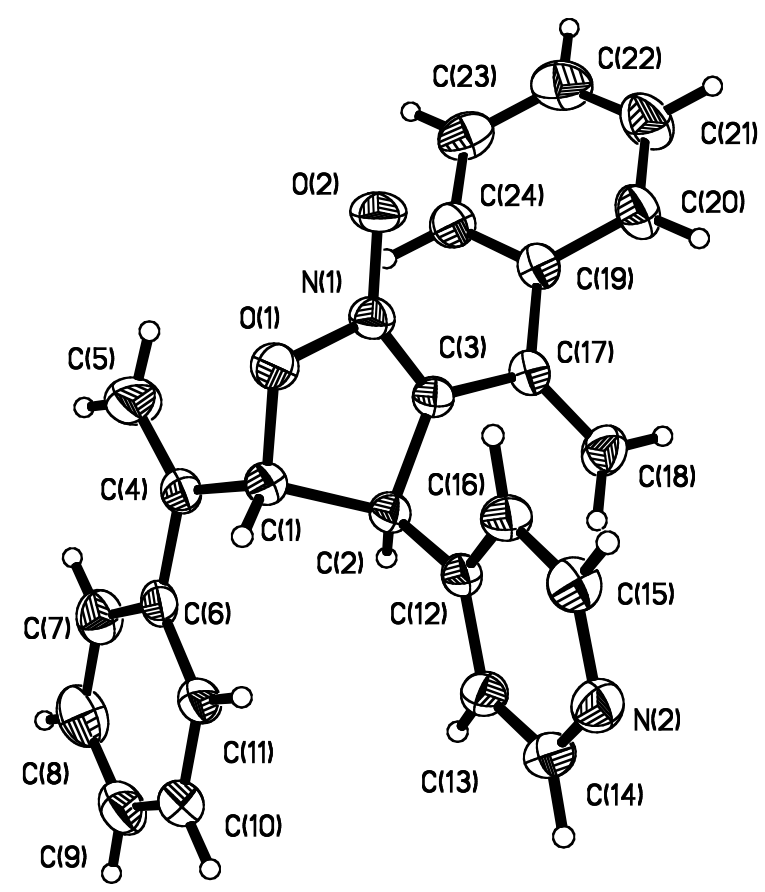

Figure 11. Perspective view of the molecular structure of $\mathbf{1 5 c}$ with the atom labeling scheme. The thermal ellipsoids are scaled to enclose $30 \%$ probability. CCDC: 755528 .

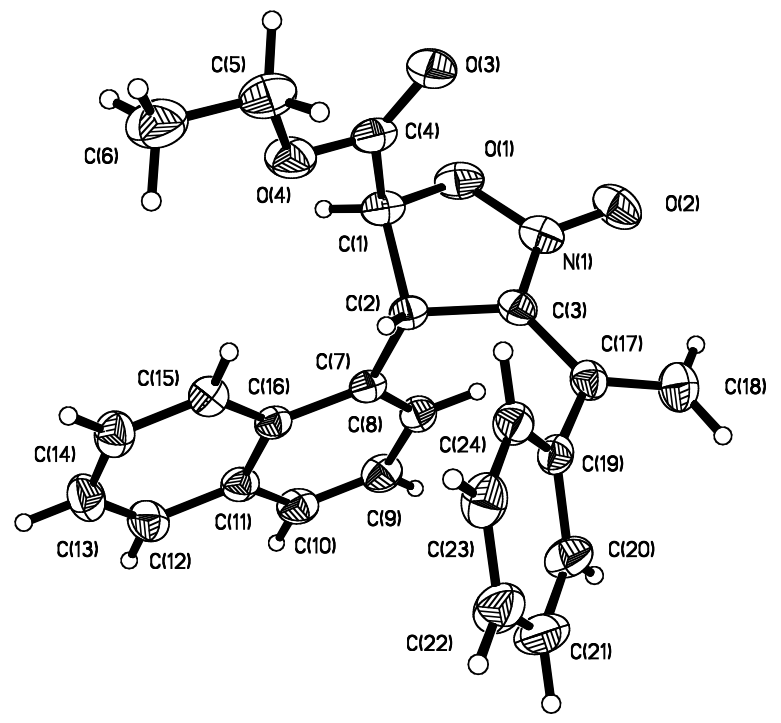

Figure 12. Perspective view of the molecular structure of $\mathbf{1 8 g}$ with the atom labeling scheme. The thermal ellipsoids are scaled to enclose $30 \%$ probability. CCDC: 755527. 

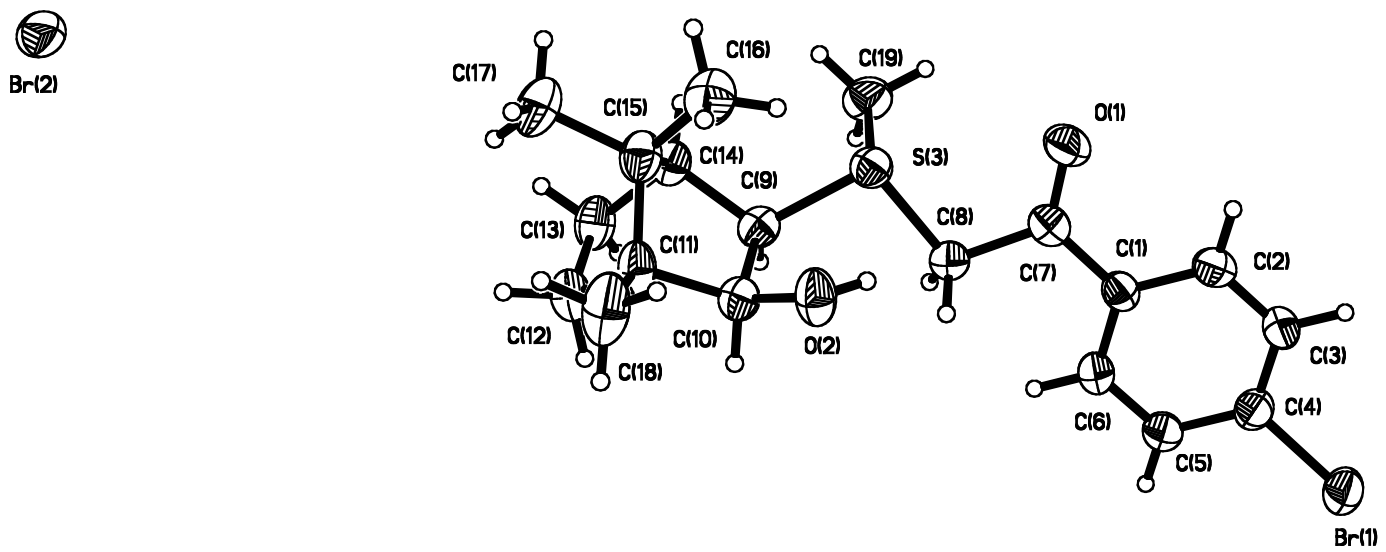

Figure 13. Perspective view of the molecular structure of aux-g with the atom labeling scheme. The thermal ellipsoids are scaled to enclose 30\% probability. CCDC: 755526 .

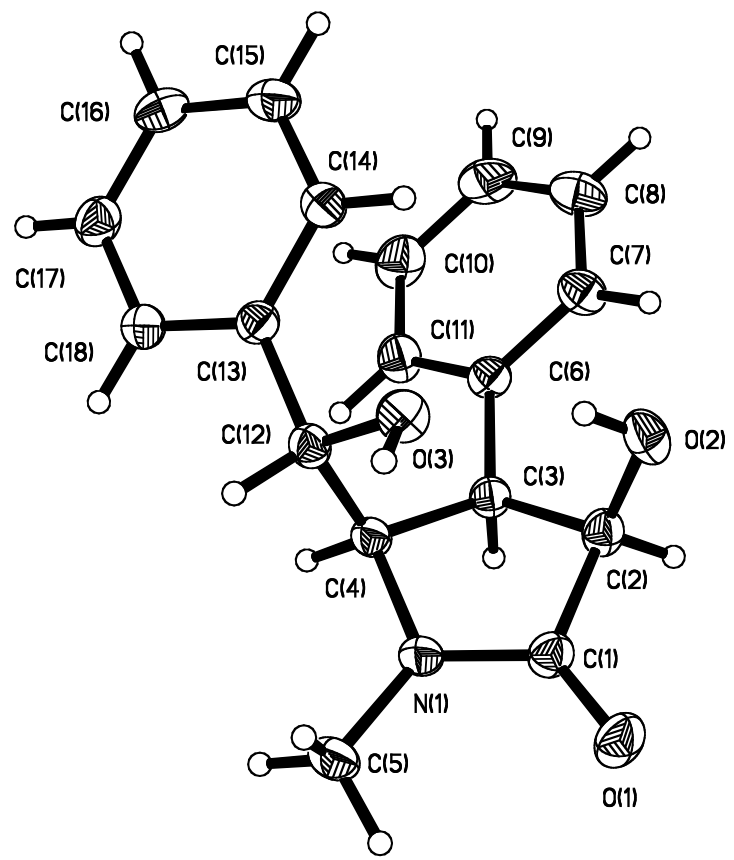

Figure 14. Perspective view of the molecular structure of $\mathbf{3 3}$ with the atom labeling scheme. The thermal ellipsoids are scaled to enclose $30 \%$ probability. CCDC: 755529 . 


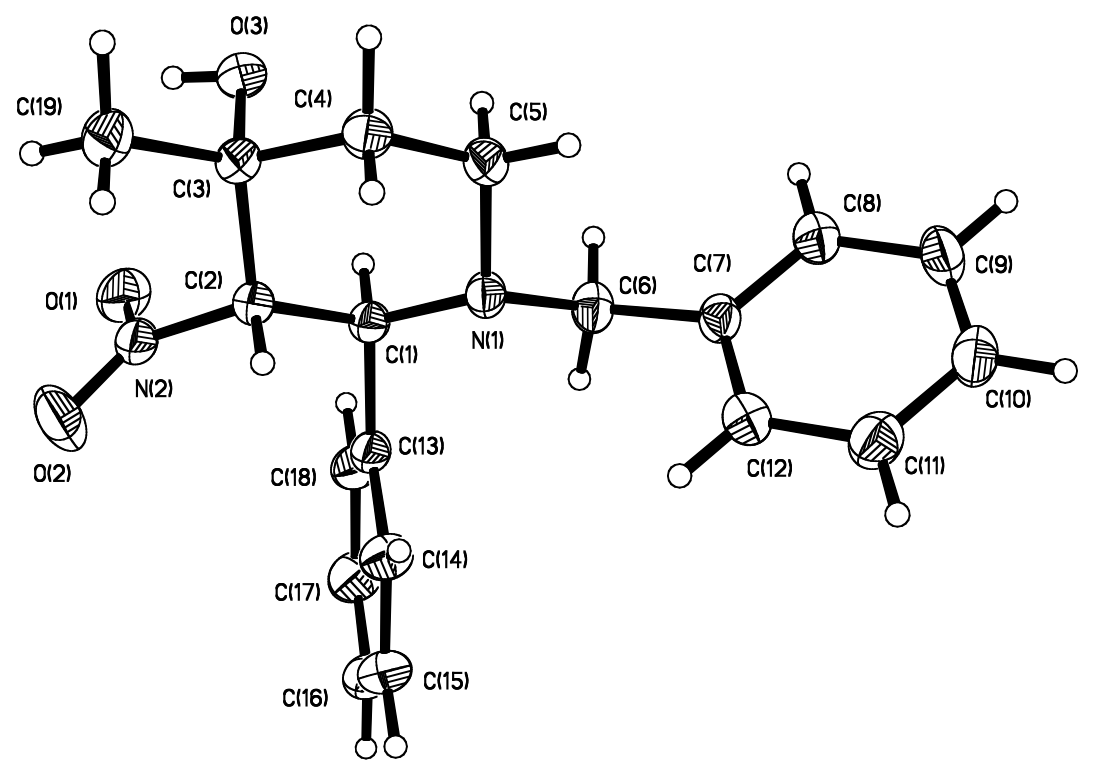

Figure 15. Perspective view of the molecular structure of 39a-major with the atom labeling scheme. The thermal ellipsoids are scaled to enclose $30 \%$ probability. CCDC Number: 712066

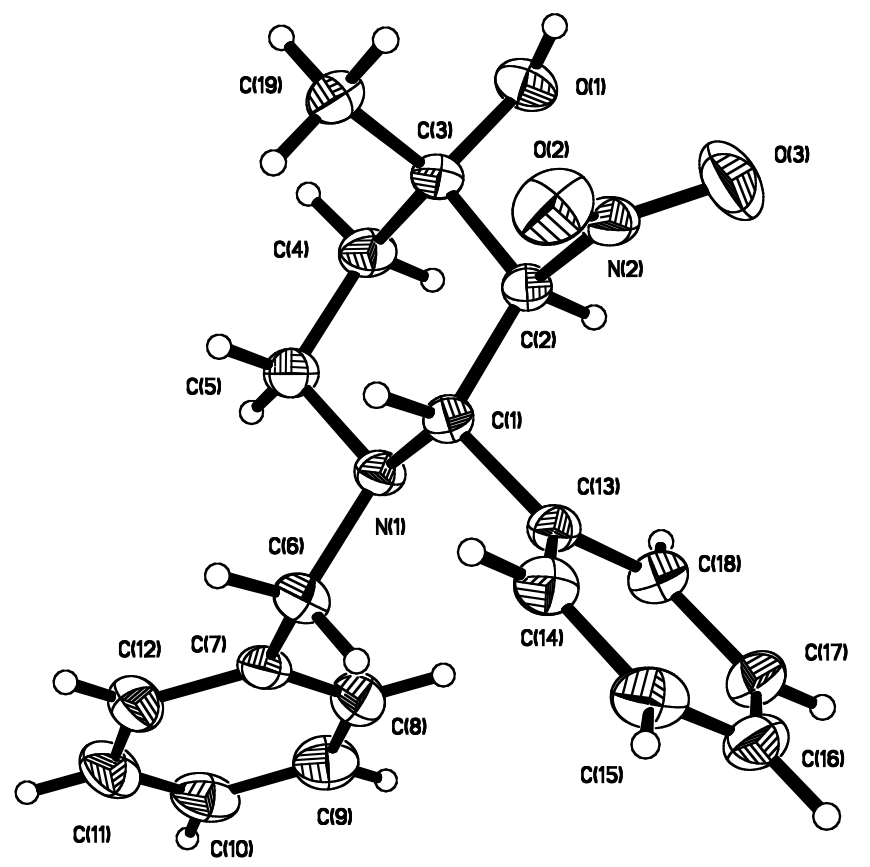

Figure 16. Perspective view of the molecular structure of 39a-minor with the atom labeling scheme. The thermal ellipsoids are scaled to enclose $30 \%$ probability. CCDC Number: 712068 


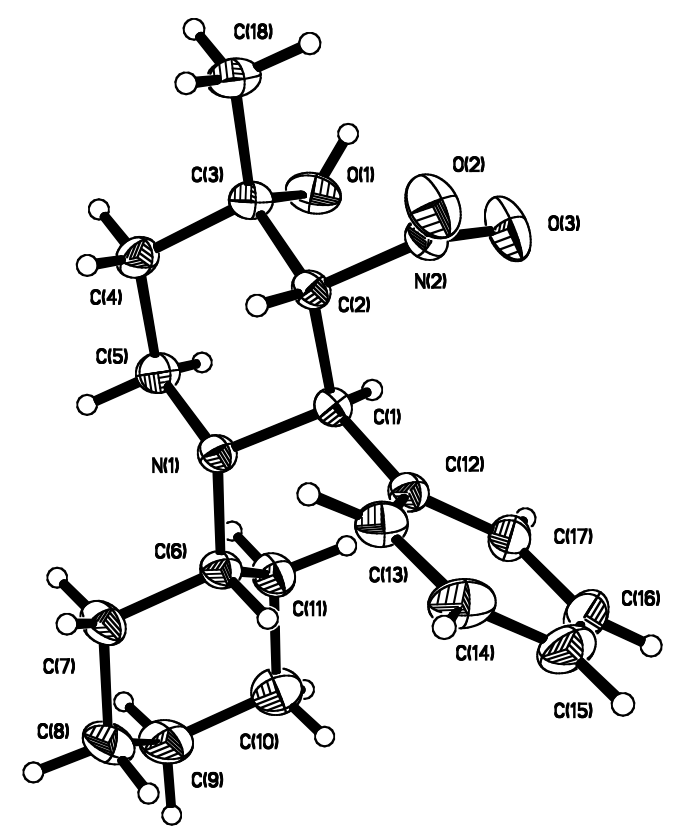

Figure 17. Perspective view of the molecular structure of 39e with the atom labeling scheme. The thermal ellipsoids are scaled to enclose $30 \%$ probability CCDC Number: 712067

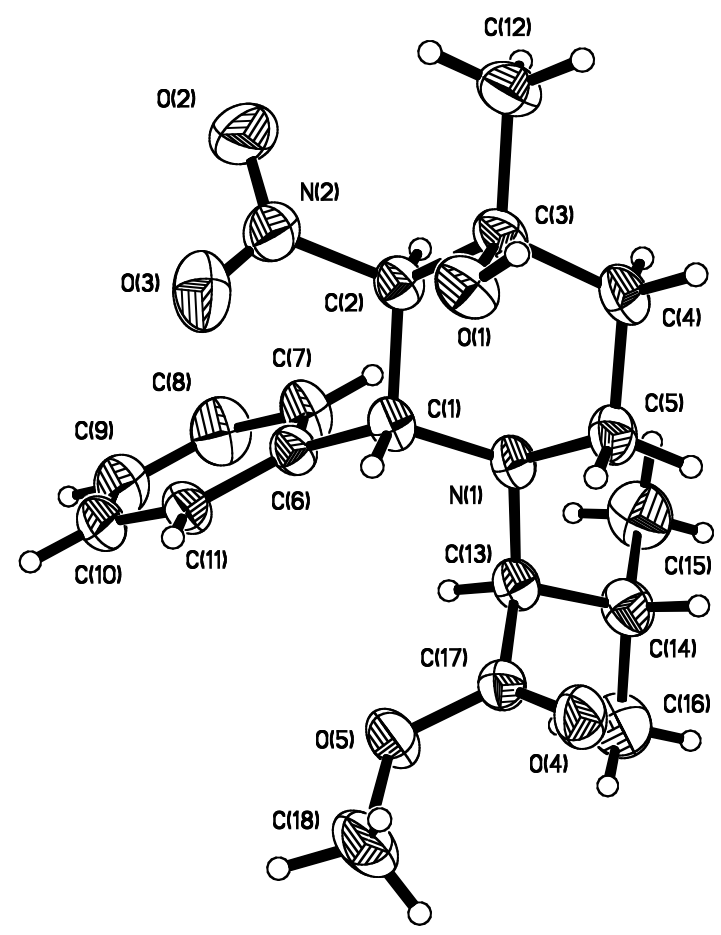

Figure 18. Perspective view of the molecular structure of 41d-major with the atom labeling scheme. The thermal ellipsoids are scaled to enclose $30 \%$ probability. CCDC Number: 712069 


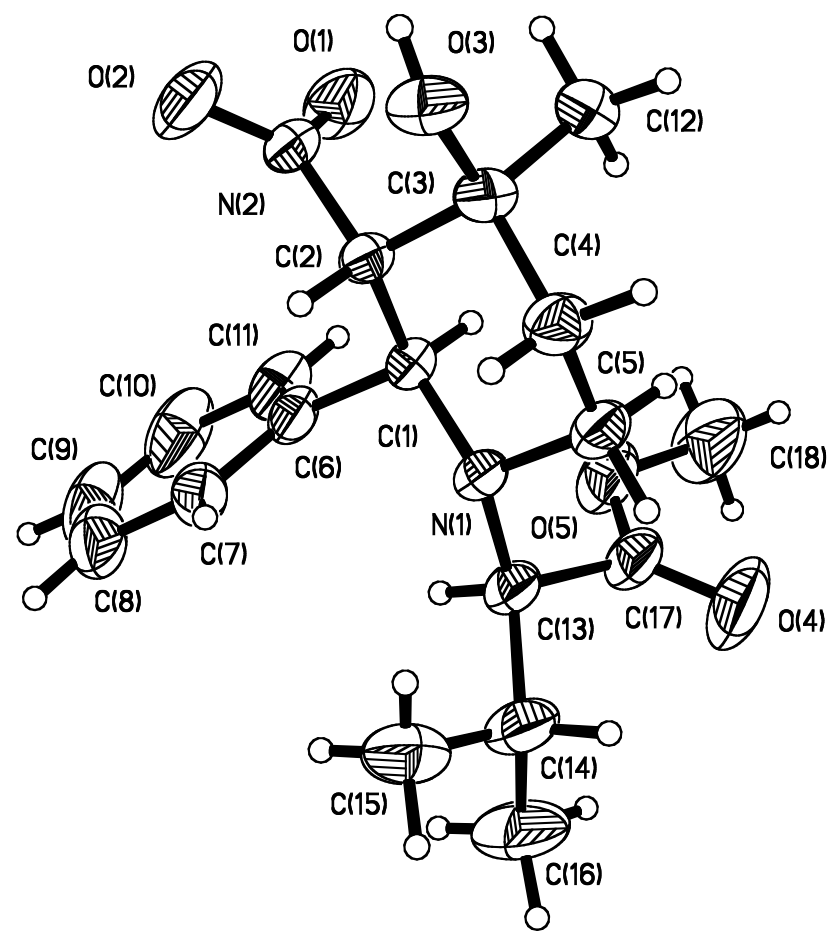

Figure 19. Perspective view of the molecular structure of 41d-minor with the atom labeling scheme. The thermal ellipsoids are scaled to enclose $30 \%$ probability. CCDC Number: 712070

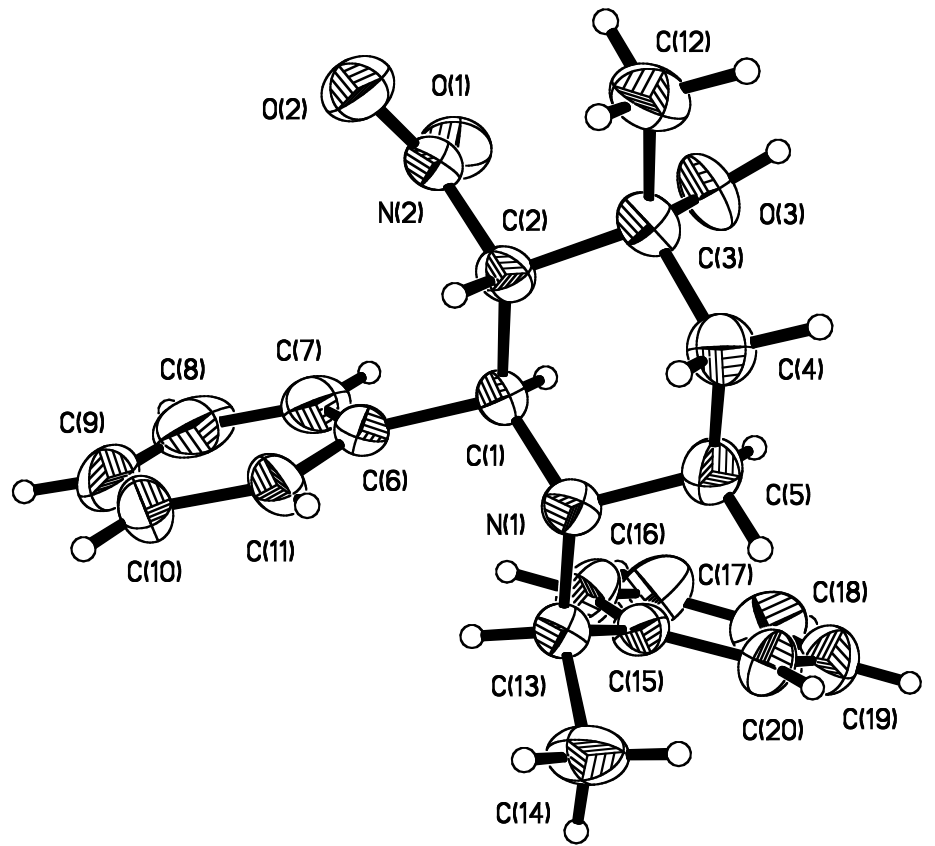

Figure 20. Perspective view of the molecular structure of 41a-minor with the atom labeling scheme. The thermal ellipsoids are scaled to enclose $30 \%$ probability. CCDC Number: 712071 